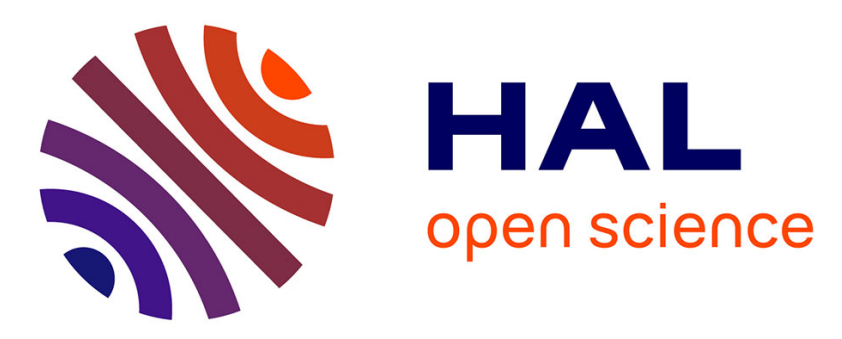

\title{
Antiviral effects of HSV1-specific meganucleases in a mouse model of relapsing herpes keratitis
}

M. Labetoulle, E. Gabison, A. Rousseau, S. Arnould, R. Galetto, D. Pasdeloup

\section{To cite this version:}

M. Labetoulle, E. Gabison, A. Rousseau, S. Arnould, R. Galetto, et al.. Antiviral effects of HSV1specific meganucleases in a mouse model of relapsing herpes keratitis. EVER 2014 - European Association for Vision and Eye Research Conference 2014, Oct 2014, Nice, France. WILEY-BLACKWELL, Acta Ophthalmologica, 92, pp.3, 2014, 10.1111/j.1755-3768.2014.T064.x . hal-02742511

\section{HAL Id: hal-02742511 https://hal.inrae.fr/hal-02742511}

Submitted on 3 Jun 2020

HAL is a multi-disciplinary open access archive for the deposit and dissemination of scientific research documents, whether they are published or not. The documents may come from teaching and research institutions in France or abroad, or from public or private research centers.
L'archive ouverte pluridisciplinaire HAL, est destinée au dépôt et à la diffusion de documents scientifiques de niveau recherche, publiés ou non, émanant des établissements d'enseignement et de recherche français ou étrangers, des laboratoires publics ou privés. 


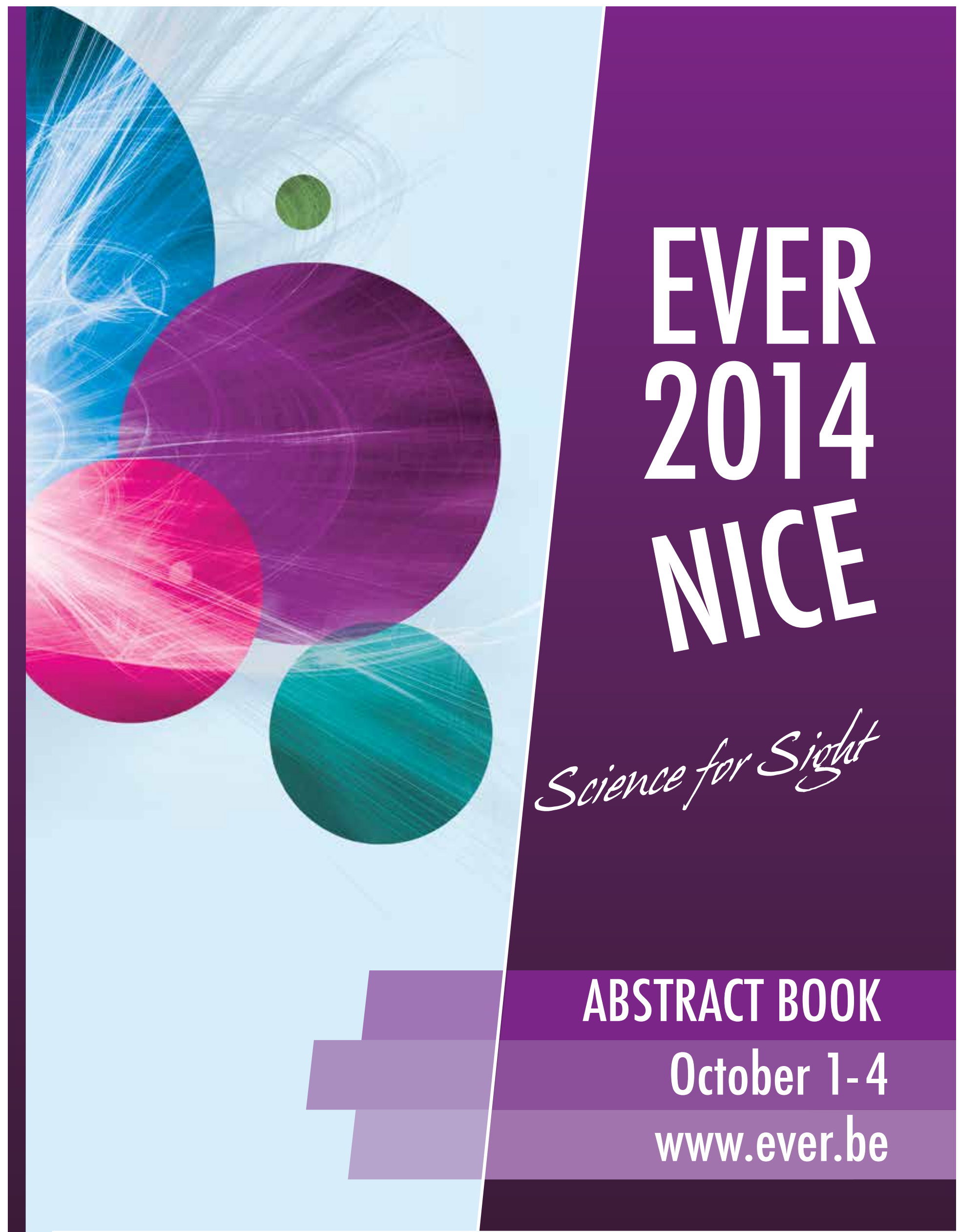




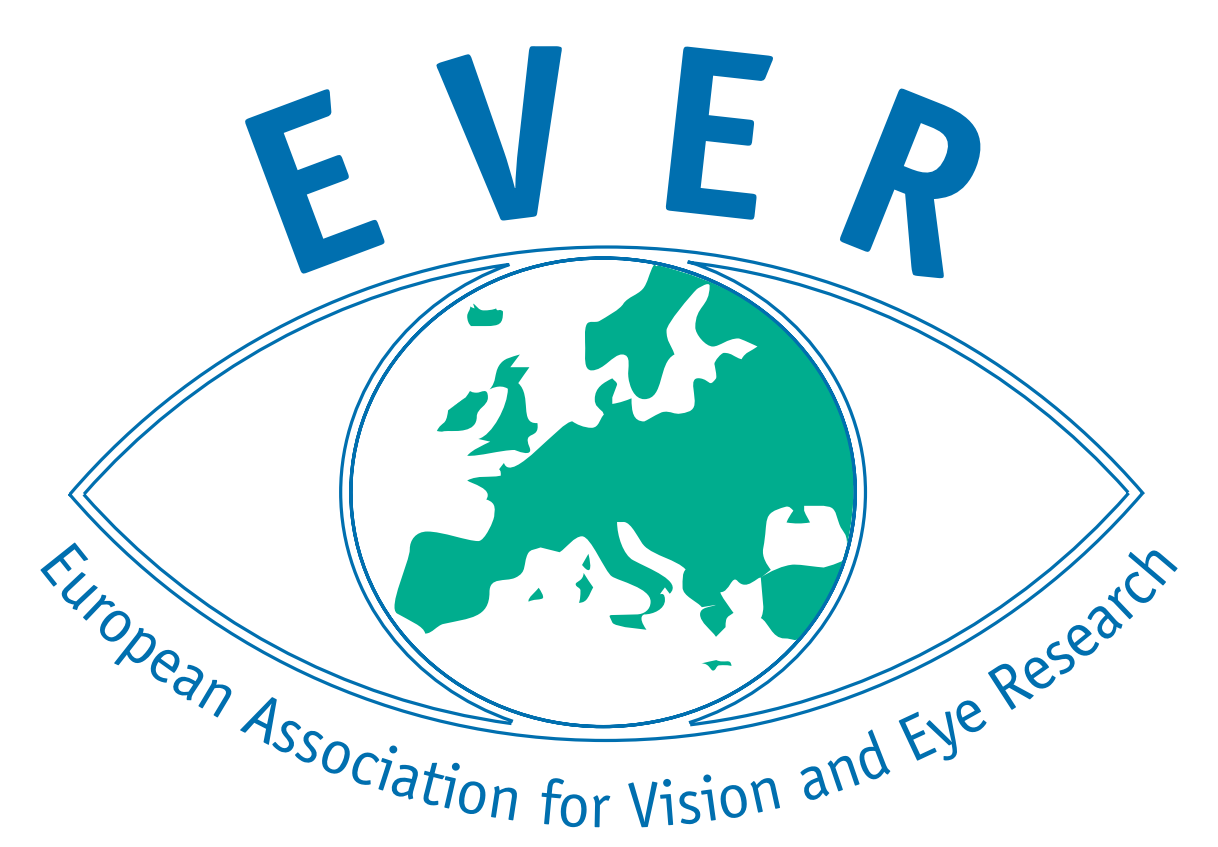


EVER PAST PRESIDENT LECTURE

- Philippe KESTELYN

\section{EUROPEAN OPHTHALMOLOGY HERITAGE LECTURE}

- Daniel SCHORDERET

EVER-ACTA LECTURE

- Russell G FOSTER

\section{OPHTHALMIC RESEARCH LECTURE}

- Bahram BODAGHI

\section{KEYNOTE LECTURES}

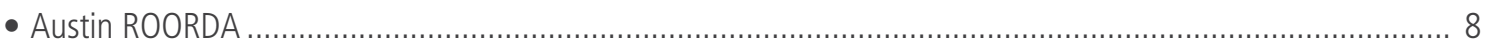

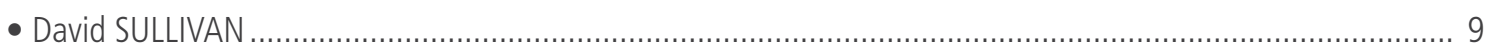

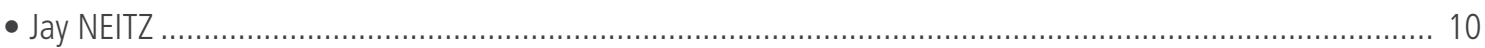

ABSTRACTS

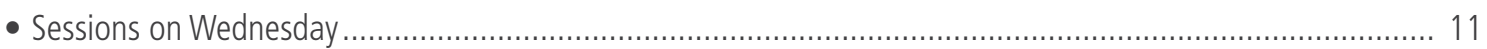

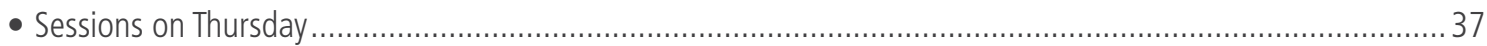

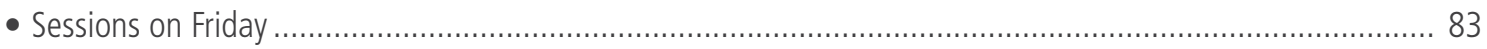

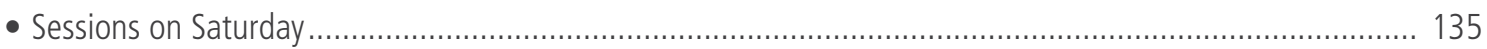

POSTERS

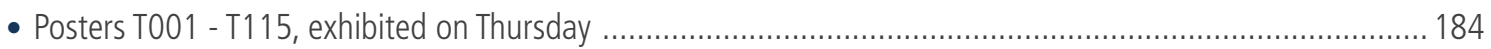

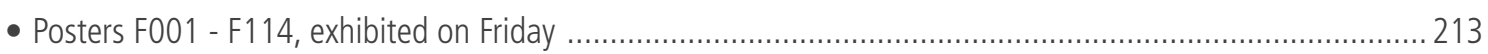

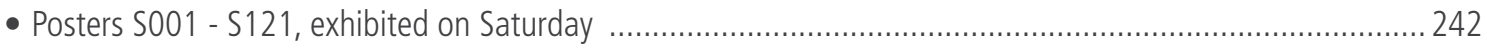

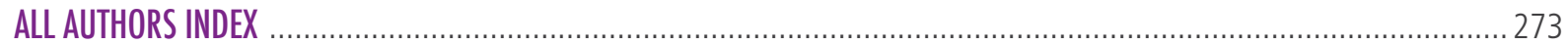




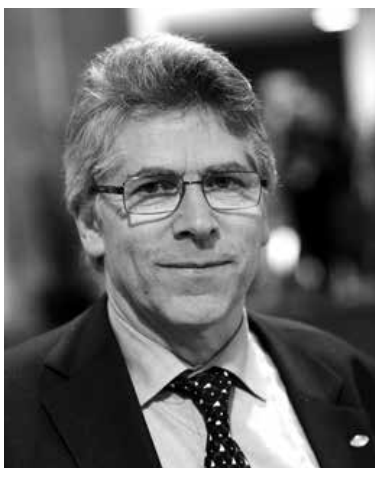

EVER Past-President Lecture - Philippe KESTELYN

AIDS thirty years later, lessons from a pandemic

\section{Philippe KESTELYN}

UZ Gent, Ophthalmology, Gent, Belgium

\section{Summary}

At the start of the AIDS epidemic in the early eighties panic, fear and disbelief prevailed. A new "Great Plague" had arrived! Now, thirty years later, we know the enemy, we have devised effective weapons that transform a deadly disease into a chronic condition, and very recently we made our first attempts at engineering cellular resistance to HIV. This lecture will explore the many new insights we gained during our fight against AIDS, with some emphasis on the ophthalmic aspects of the disease. 


\section{European Ophthalmology Heritage Lecture - Daniel SCHORDERET}

Eye and genes: the new ophthalmology

\section{Institute for Research in Ophthalmology (Sion)}

Faculty of Life Sciences, Ecole polytechnique fédérale de Lausanne (Lausanne)

Faculty of Biology and Medicine (Lausanne)

\section{Summary}

For many years, ophthalmology has based its practice on observing, trying, deducing and remembering. For many pioneers, this approach was not sufficient and tools were designed to improve the diagnostic and intervention capacities for these ocular diseases. Although inheritable eye disorders have been identified many years ago-Dalton described color blindness in 1798 - it is only in the second part of the 20th century that genetics flourished. Franceschetti and Klein from Geneva published numerous reports on dysmorphology, and were part of a great effort to categorize all syndromes so that nature's experiments were not lost. Although both were ophthalmologists, they founded the first Institute of Medical Genetics in Switzerland and made significant contributions to the field. I was blessed to discover genetics through David Klein and to further do my doctoral thesis with Eric Engel, his successor, in Geneva. Fifteen years later, the identification of the genes responsible for inherited diseases was done either by karyotyping or by linkage analysis followed by physical mapping. The former was based on luck, and often, we didn't have any. For the latter, we needed large families and new technologies, like autoradiograms or automated

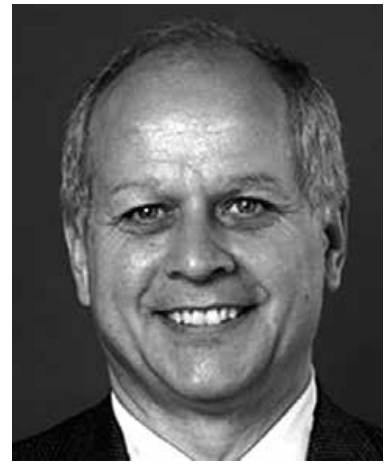




\section{EVER/ACTA LECTURE}

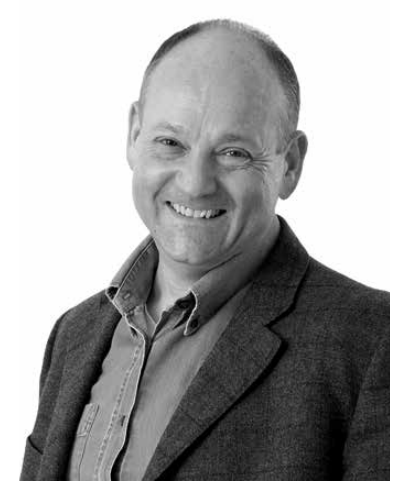

\section{Acta Ophthalmologica}

\section{EVER-Acta Lecture - Russell G FOSTER}

Regulating the body clock - The unrecognised role of the eye

\section{FOSTER $R$}

University of Oxford (Oxford)

\section{Summary}

Little more than a decade ago discussion that the eye might contain another photoreceptor, different from the rods and cones, generated either polite amusement or a hostile rebuttal. The dogma was that all light detection took place by these photoreceptors whilst the other cells of the retina act only to process visual signals. However, several lines of research led to the discovery that the vertebrate eye, including humans, contains another class of photoreceptor based upon a small number of photosensitive retinal ganglion cells (pRGCs). These specialised neurons detect environmental irradiance and regulate a wide range of physiology and behaviour including the regulation of $24 \mathrm{~h}$ body clocks, sleep, alertness, mood and even pupil size. Furthermore, the pRGCs have been shown to utilize a novel light signalling pathway based upon the photopigment melanopsin. Collectively these findings have transformed our understanding of how the eye detects light and are redefining our assessment, treatment and care of individuals with eye disease. The discovery and current understanding of this "third" class of ocular photoreceptor will be reviewed in this presentation. 


\section{OPHTHALMIC RESEARCH LECTURE}

\section{Ophthalmic Research Lecture - Bahram BODAGHI}

\section{Revisiting infectious triggers of chronic intraocular immunity}

\section{BODAGHI B}

University of Pierre and Marie Curie (Paris)

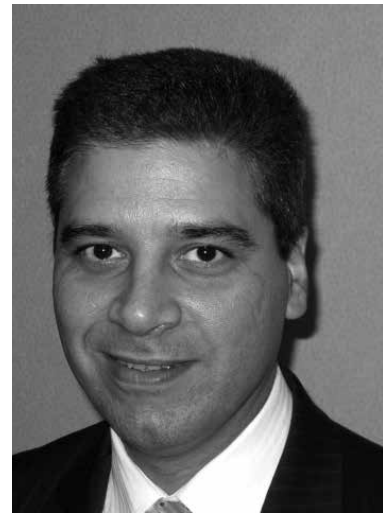

\section{Summary}

The pathogenesis of intraocular inflammation has been extensively revisited on the basis of different sophisticated experimental models but also new molecular tools applied to ocular fluids or tissues. Experimental ocular inflammation may not be induced in germ-free animal facilities. Moreover, an infectious agent has been identified in patients presenting with different uveitis entities, previously considered as autoimmune and therefore treated with corticosteroids and aggressive immunosuppressive agents. Molecular mimicry or expression of foreign antigens may trigger an inflammatory reaction, masquerading as an autoimmune uveitis. Fuchs cyclitis, Posner-Schlossman syndrome, serpiginous choroiditis, nonnecrotizing herpetic retinopathies have been recently characterized, expanding the spectrum of infectious uveitis. Microbiological identification remains the first step prior to initiation of a specific therapy, reducing the risk of relapse and achieving a better visual outcome in challenging situations. 


\section{KEYNOTE LECTURE}

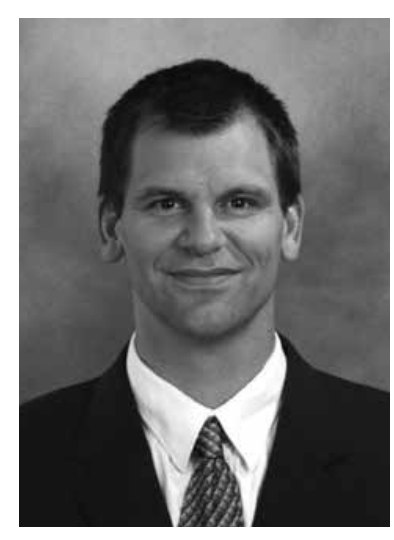

\section{Austin ROORDA}

Testing vision: one cone at a time

\section{ROORDA A}

UC Berkeley School of Optometry (Berkeley)

\section{Summary}

In humans, gaining experimental access to single sensory receptors - photoreceptors specifically - is a challenge, yet it is crucial for learning how the signals arising from each receptor are transformed into perception. By combining adaptive-optics microstimulation with high-speed eye tracking, we show that retinal function can be probed at the level of the individual cone photoreceptor in living eyes. This talk will describe the development and applications of our adaptive optics confocal imaging system which we use to track, target and deliver visible light stimuli to single cones or selections of cones. Highlighted applications will include our ongoing efforts to learn about the neural circuits that underlie human spatial and color vision. 


\section{David SULLIVAN}

New therapeutic approaches and challenges for the treatment of dry eye disease

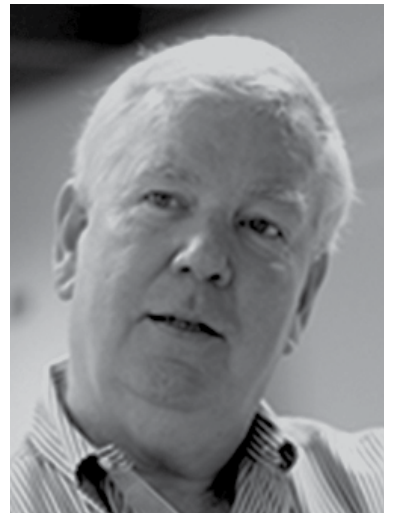

\section{SULLIVAN DA}

Schepens Eye Research Institute and Harvard Medical School (Boston, MA)

\section{Summary}

Innumerable individuals suffer from tear film dysfunctions, which are collectively diagnosed as dry eye disease (DED). In the United States alone DED afflicts tens of millions of people, especially women, and is one of the most frequent causes of patient visits to eye care practitioners. DED is characterized by a vicious cycle of tear film hyperosmolarity and instability and ocular surface stress, leading to increased friction, inflammation and damage to the eye. DED is caused primarily by meibomian gland dysfunction, and is associated with significant pain, decreased vitality and poorer general health. Because of diminished worker productivity, the burden of DED for the United States is estimated to be over $\$ 55.4$ billion. There is no safe and effective global treatment for DED, and its therapy remains a profound unmet need throughout the world. A virtual kaleidoscope of new therapeutic approaches have been proposed to treat the mucin, aqueous and/or lipid tear film deficiencies, as well as the ocular surface damage, associated with DED. My presentation will highlight these approaches, and also address the significant clinical endpoint challenges that serve as barriers to the successful development of potential treatments for DED. 


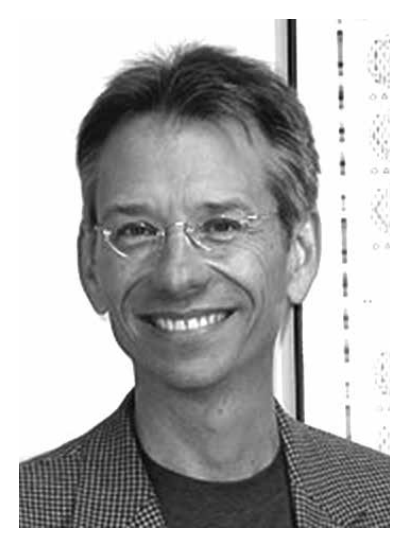

Jay NEITZ

Lessons learned from gene therapy for color blindness in primates

NEITZ J

Department of Ophthalmology, University of Washington Medical School (Seattle)

\section{Summary}

Color blindness is the most common genetic disorder. The possibility of curing color blindness using gene therapy was explored by adding a third type of cone pigment to dichromatic retinas of squirrel monkeys. This opened a new avenue to explore the requirements for establishing the neural circuits for a new dimension of color sensation. The addition of a third opsin in adults was sufficient to produce trichromatic color vision. Thus, trichromacy can arise from a single addition of a third cone class in a primate and it does not require an early developmental process. This has implications for understanding how our brain processes conscious visual information and it illuminates the opportunities and limits of gene therapy for treating human vision disorders. 
WEDNESDAY OCTOBER 1
2014 
- 1611

Challenges for interpreting retinal morphology

BEK T

Aarhus $C$

Diseases in the retina are the most frequent causes of visual impairment and blindness in the Western World. However it is a paradox that these diseases that are debilitating because of the effects on retinal function, are mainly diagnosed and monitored on the basis of their morphological appeareance in the ocular fundus.The most widespread methods for evaluating retinal morphology are based on the study of light reflected from the fundus background and imaged through the optics of the eye. This imaging principle depends on image contrast, on spatial resolution of the optics of the eye, and on the temporal resolution of the recording system used to image the fundus The morphological lesions observed in the ocular fundus are often heterogenous with varying number, size, shape, density, location and dynamics. In most cases information about these features are aggregated into more simple qualitative or semi-quantitative grading systems that can be handled in simple rule based clinical decision models. However, the advent of computerized image processing has allowed a more detailed quantitative evaluation of retinal morphology. Examples will be given of how this may help improving the diagnosis and management of retinal disease
$-1613$

\section{OCT elastography}

LEITGEBR

Center for Medical Physics and Biomedical Engineering, Vienne

Functional extensions of optical coherence tomography (OCT) are today extensively studied as they have the potential to further enhance the diagnostic capabilities of OCT. Modern Doppler OCT, as the most prominent functional extension, yields outstanding contrast for the vascular plexus down to even smallest capillaries. Optical or OCT elastography is a very young functional OCT approach that gained from the developments of high speed OCT technology. It is capable to sense smallest structural tissue deformation during mechanical stress. The achieved deformation sensitivity is on the nanometer scale even in living retinal tissue. Due to the high sensitivity, OCT elastography is capable to observe tissue derformation caused by the natural pulse of the perfusion bed. This avoids external mechanical stress applied to the eye bulb and allows studying the biomechanical properties of tissue in its natural environment. Of particular interest is the motion of the fundus and of the lamina cribrosa (LC). First results indicate differential motion within the retina and LC of a few micrometer/second. This might be the basis for using the biomechanical status of the LC as new diagnostic biomarker of disease for example for glaucoma.

\section{- 1612}

Doppler OCT

WERKMEISTER R

Center for Medical Physics and Biomedical Engineering, Vienna

OCT is a non-invasive imaging modality capable of providing information on sample structure with micrometer scale resolution. Doppler OCT (DOCT), a functional extension of OCT, provides knowledge about the motion of the sample and its substructures. It found its main application in biomedical imaging in the assessment of ocular blood flow. Doppler OCT allows for both measurement of blood flow velocities in larger retinal vessels and visualization of the vascular network.Recent developments such as dual beam DOCT make it possible to measure absolute blood flow velocity. Measurements are independent of the a priori unknown Doppler angle and further enable calculation of total blood flow when taking vessel diameter estimation via OCT or fundus camera based approaches into account. In addition, Doppler OCT allows for visualization of blood flow profiles and their alterations in retinal vessels and venous junctions and to further gain insight into hemodynamics as well as to study flow characteristics of the non-Newtonian fluid blood.Results will be presented that show the potential of DOCT to provide crucial information for diagnosis, monitoring and treatment planning of ocular diseases related to alterations in ocular perfusion.
- 1614

\section{Choroidal thickness}

SOUBRANEG

Paris

The role of the choroid has been established in a number of chorioretinal diseases. The choroid is qualitatively assessed with Fluorescein Angiography and even better with ICG angiography that explores the choroidal vasculature and the different originating pathologies. The quantitative assessment is yet clinically performed with OCT.The normal choroidal thickness ranges from $287 \pm 74.2$ to $345 \pm 111 \mu$ depending on the instrument, the OCT technic, of the delineation of the choroidal limit. Factors affect choroidal thickness:age (and weight), axial length, possibly gender. Diurnal variations have been noted as well as a relation with water drinking. A number of chorioretinal diseases are associated with changes in the choroidal thickness. Central Serous Chorioretinopathy (CSC) exhibiting a choroidal hyperpermeabilityon ICG is associated with an increased choroidal thickness that elevates globally the retina from the RPEchoriocapillaris band. The choroid may be so thickened as to lose signal penetration and intensity at increasing depth. The implication in CSC of the choroid is further supported by the aspect of the fellow eye. Polypoidal Choroidal Vasculopathy (PCV) a vascular choroidal abnormality has a thick subfoveal choroid.Decreased choroidal thickness is found in Age related Macular Degeneration. In dry AMD, it is possible to visualize the hyperreflective choroid in the area of RPE atrophy.Similarly in exudative AMD, the choroid can be visualized beneath choroidal neovascularization. The choroid is very thin in degenerative myopiaand related to the axial lenght. The comparison of the choroidal thickness among eyes with neovascular AMD, PCV, CSC and normal eyes demonstrated that the choroid was thicker in PCV and CSC. The thicker choroid could be partially attributed to the dilation of middle and large choroidal vessels or an increase in the choroidal vascular permeability. Choroidal thickness may thus be very helpfull to differentiate border line cases with different treatment approaches. Post treatment analysis demonstates choroidal thinning, following PDT for CSC, anti-VEGF therapy for wet/exudative AMD, and PDT + antiVEGF for PCV.Choroidal thickness can be an aadditional parameter to assist diagnosis and may indicate the activity of the disease and the response to treatment. 
- 1621

Kaplan-Meier analysis and Cox regression

KIVELÄ T

Department of Ophthalmology, Helsinki Iniversity Central Hospital, Helsinki

Kaplan-Meier analysis and Cox regression are nonparametric techniques with wide applicability. They are needed when time-to-event data are analysed (e.g. time to loss of vision, filtration bleb failure, corneal graft rejection). They are especially necessary when follow-up times vary, which is common in clinical research. The entry data are time to event or last follow-up, last status (experienced the event, under follow-up, lost to follow-up, or died) and any explanatory or confounding variables (e.g. sex, age). Subjects who did not experience the event are "censored" at last follow-up. Censoring must be independent of the probability of experiencing the event, and the subject must remain at risk of the event after censoring. Kaplan-Meier analysis produces stepped curves which show the cumulative probability of experiencing the event over time. Cox regression is a related multivariate model that provides a numerical hazard ratio (e.g. increased or decreased risk of the study group to experience the event relative to the control group) adjusted for the effect of other variables included in the regression model. After this talk, participants should be able to recognize time-to-event data and interpret studies using these methods.

- 1623

Cumulative incidence and interval censoring

KIVELÄT

Department of Ophthalmology, Helsinki Iniversity Central Hospital, Helsinki

Kaplan-Meier and Cox regression return biased estimates in the presence of competing events that render subjects immune to the event of interest (e.g. a filtration bleb can not fail after dying). Kaplan-Meier can then be supplemented with cumulative incidence analysis and Cox regression with competing risks regression. The data needed are timeto-event or last follow-up, last status, and explanatory or confounding variables. Subjects who did not experience any event are "censored" at last follow-up. A set of stepped curves is produced which show the cumulative incidence of each event analysed as a function of time; groups can be compared using dedicated tests. Competing risks regression provides a hazard ratio, adjusted for the effect of other variables in the model. In addition to competing risks, another consideration is the follow-up interval. Events are usually recorded at planned review visits but actually have taken place earlier, which causes bias. Interval censoring is a method that accounts for this fact. After this talk, participants should be able to recognise competing events and interval censoring assess whether Kaplan-Meier and Cox regression were appropriate methods and know alternatives to them.
- 1622

Relative survival

DAMATO B

San Francisco, $C A$

ABSTRACT NOT PROVIDED 
- 1631

\section{Anterior corneal dystrophies}

JANISZEWSKA D $(1,2)$

(1) Ophth. Dep St.Barbara Hospital, Trauma Centre, Sosnowiec

(2) Ophth. Dep. Distric Railway Hospital, Katowice

In pursuance of the IC3D classification the group of the anterior corneal dystrophies include: Epithelial and Subepithelial Dystrophies: (( Epithelial basement membrane dystrophy (EBMD)-majority degenerative, some C1; Epithelial recurrent erosion dystrophy (ERED) C4, (Smolandiensis variant) C3;Subepithelial mucinous corneal dystrophy (SMCD) C4; Mutation in keratin genes: Meesmann corneal dystrophy (MECD) C1; Lisch epithelial corneal dystrophy (LECD) C2; Gelatinous drop-like corneal dystrophy (GDLD) C10 and Bowman Layer Dystrophies: (( Reis-Bücklers corneal dystrophy (RBCD) - Granular corneal dystrophy type 3 C1;Thiel-Behnke corneal dystrophy (TBCD) $\mathrm{C} 1$, potential variant $\mathrm{C} 2$; Grayson -Wilbrandt corneal dystrophy (GWCD) C4). Foregoing course summarizes genetic background, invasive examination (histological, confocal) and non invasive modern visualization techniques such as AS SOCT. Above lecture will also assume clinical approach.

- 1633

Posterior corneal dystrophies

LISKOVA P

Prague

Posterior corneal dystrophies represent a clinically and genetically heterogeneous group of disorders. The International Committee for Classification of Corneal Dystrophies (C3D) lists autosomal dominant congenital hereditary endothelial dystrophy (CHED1), autosomal recessive congenital hereditary endothelial dystrophy (CHED2), posterior polymorphous corneal dystrophy (PPCD) and Fuchs endothelial corneal dystrophy (FECD) as separate clinical entities. The course will provide an overview of posterior corneal dystrophies with a focus on recent clinical and genetic findings and their possible implications for future classification.

\section{- 1632}

Stromal corneal dystrophies

NOWINSKA A

Bytom

According to the IC3D classification the group of the stromal corneal dystrophies include: TGFBI corneal dystrophies, Macular corneal dystrophy (MCD), Schnyder corneal dystrophy (SCD), Congenital stromal corneal dystrophy (CSCD), Fleck corneal dystrophy (FCD), Posterior amorphous corneal dystrophy (PACD), Central cloudy dystrophy of Francois (CCDF) and Pre-Descemet corneal dystrophy (PDCD).The lecture summarizes the clinical data of each corneal dystrophy with the referral to the genetic analysis, histological exam and modern non invasive optical imaging techniques as long as presents current treatment options.

1634

Advantages and disadvantages of PTK

DOBROWOLSKID $(1,2)$

(1) II Ophthalmology Clinic, Medical University of Silesia, District Railway Hospital, Katowice

(2) Ophthalmology with Pediatric Unit, St. Barbara Hospital, Trauma Center, Sosnowiec

Phototherapeutic keratectomy (PTK) became more popular laser assisted procedure in cornel surgery in recent years. Superficial degenerations, corneal dystrofies, stromal opacities can be easily removed increasing visual comfort for the patients. Procedure however, should be carefully planed considering not only depth of stromal involvement as well as the condition of the ocular surface. Presentation includes actual guidelines for PTK and overview of potential complications of the surgery. 
- 1635

Surgical treatment options for corneal dystrophies

WYLEGALA E $(1,2)$

(1) Ophthalmology Clinic Medical University of Silesia, Katowice

(2) Railway Hospital Ophthalmology Clinic, Katowice

Corneal dystrophies are the group of hereditary disorders affecting all corneal layers The choice of the surgical treatment option depends on the corneal dystrophy type and includes: anterior lamellar keratoplasty for dystrophies without Descemet membrane involvement, descemet stripping endothelial keratoplasty for Fuchs corneal dystrophy and penetrating keratoplasty for dystrophies characterized by full corneal thickness involvement. All types of keratoplasty could be performed traditionally or with the assistance of the femtosecond laser. The corneal graft survival depends on the corneal dystrophy type. The recurrence of the disease is a possible complication of the surgery related to the diagnosis of the corneal dystrophy. 
- 1641

Pathophysiology of uveitis

DICK A

Bristol

This talk will overview the pathophysiology of non-infectious uveitis in relation to recent SUN (standardised uveitis nomenclature) disease classification. The experimental and translational human evidence of autoimmunity and activation of immunity will be discussed. In addition the talk will highlight the pathways and mechanisms of tissue damage that results in sight-threatening disease.Traditionally, despite active immune regulatory mechanisms operative within the ocular environment, inflammation still occurs. Activated antigen and non-antigen specific $\mathrm{T}$ cells are generated in uveitis The interplay with innate immunity and in particular cells of myeloid lineage both systemically and within the local environment dictate the severity and extent of pathology we observe. The understanding of immune responses during the uveitis open many avenues to potential novel immunotherapies that not only suppress inflammation but attempt to redress immune balance, tolerance and local homeostasis within ocular tissues.

\section{- 1642}

\section{Classification of uveitis}

ANDROUDIS

Thessaloniki

Classification and standardization of uveitis is important, as it enhances the precision and comparability of clinical research from different centers and assists in the development of a complete picture of the course of the disorders and their response to treatment.Attempts have been made to standardize some aspects of uveitis, and various classification criteria, inflammation grading schema, and outcomes criteria have been described.The most widely used classification of uveitis is the one devised by the International Uveitis Study Group (IUSG) in 1987, based on the anatomical location of the inflammation. This classification includes anterior uveitis (iritis, iridocyclitis, and anterior cyclitis), intermediate uveitis (pars planitis, posterior cyclitis, and hyalitis), posterior uveitis (focal, multifocal, or diffuse choroiditis, chorioretinitis, retinitis, and neuroretinitis) or panuveitis (anterior chamber, vitreous, retina, and choroid).In 2005, the Standardization of Uveitis Nomenclature (SUN) Working Group standardized a grading schema for aspects of intraocular intraocular inflammation, that is, anterior chamber cells, anterior chamber flare, and vitreous haze, was developed. Standardized definitions of outcomes, including reporting visual acuity outcomes, were approved. Today's uveitis nomenclature has been revised regarding the anatomical location and the grade of inflammation, and supplemented by the inclusion of definitions for onset, duration and course.
- 1643

Signs and symptoms of uveitis

NERI P, ARAPI I, PIRANI V, ZUCCHI M, PRIMAVERA L

The Eye Clinic, Polytechnic University of Marche, Ancona

Inflammation of uveal tract can be divided into: anterior, intermediate, posterior, and panuveitis. Uveitis can be a sight-threatening disease. The commonest ocular symptoms are: blurred vision, ocular pain, photophobia and floaters, depending on the type of uveitis. The onset of uveitis can be either acute or insidious, involving one or both eyes. Posterior uveitis is usually associated with vitritis. Anterior chamber cells and flare should be graded according to standardized uveitis nomenclature (SUN) working group. Binocular indirect ophthalmoscopy (BIO) score is used to grade vitreous involvement. Vitreous changes may comprehend: vitreous hemorrhage, vitreous strands, and vitreous traction. A further classification of posterior uveitis depends on the primary site of inflammation, which can identify: retinitis, choroiditis, retinochoroiditis, and chorioretinitis. Posterior pole uveal involvement can be: focal, multifocal, and placoid. Retinal vasculitis can be associated with several sub-types of posterior uveitis. Uveitis can present several complications such as, anterior and posterior synechiae, which can lead to uveitic glaucoma, cystoid macular oedema, retinal and choroidal neovascularizations, and retinal ischemia.
- 1644

\section{Laboratory work-up and specialized investigations}

PLEYERU

Berlin

Based on the anatomical involvement of the eye intraocular inflammation is classified into anterior, intermediate, posterior and panuveitis. All subtypes of uveitis are potentially related to infectious and noninfectious etiologies.This presentation will assist the participants in accurately diagnosing uveitis in a step latter approach including physical and laboratory investigations.In addition, a tailored approach based on confounding clinical observations with specialized investigations will help to further differentiate clinical entities. In cases of suspected intraocular infections the option of intraocular fluid evaluation for antibody testing and polymerase chain testing against the causative agent will be presented. Taken together, this part of the course will provide a rational decision-making strategy for diagnosis of patients with uveitis. 
- 1645

Imaging in uveitis: techniques and indications

HERBORT C

Lausanne

This tutorial will address the main complementary imaging techniques used in the field of (posterior) uveitis. In case imaging work-up is decided, fluorescein angiography (FA) is performed routinely since a few decades. FA gives information on the superficial structures and lesions of the fundus including pathology of the retina, retinal vessels, optic disc, and subretinal fluid collection, as well as the RPE for which it is the examination method of choice, and the choriocapillaris in the first seconds of angiography. Most of the time it only confirms and gives the precise extension of lesions already identified by the clinical examination. The choroid is however involved at least as often as the retina and often all or part of choroidal lesions are occult and not detected by the clinical exam or FA. Only indocyanine green angiography (ICGA) gives visual access to choroidal inflammatory pathology where it can distinguish stromal choroidits (birdshot, VKH) from choriocapillaritis (MEWDS, APMPPE, etc). So if angiography is deemed necessary during initial appraisal of a case dual FA/ICGA should be performed as choroiditis can not be excluded "à priori." Other methods addressed will be OCT, UBM, FAF as well as anterior segment OCT.
- 1646

Therapeutic management of uveitis

DICK A

Bristol

This talk will overview the contemporary therapeutic approaches to treatment of noninfectious non-infective ocular inflammatory disease. Treatment of non-infectious uveitis has over past 15 years expanded from the use of traditional therapies including corticosteroids and immunosuppressants to the deployment of targetting the immune response with biologic therapies with monoclonal antibodies and immunoadhesins. Such use will be exemplified with case reports during the talk. Evidence of efficacy of immunosuppressants in the treatment of uveitis, the role of predicting steroid responsiveness, the use of monotherapy with immunosuppression and finally the pathways and evidence of success of biologic therapy will be provided. 
- 1651

Genetic causes of acute visual loss

YU-WAI-MANP $(1,2)$

(1) Institute of Genetic Medicine, Newcastle University, Newcastle upon Tyne

(2) Newcastle Eye Centre, Royal Victoria Infirmary, Newcastle upon Tyne

Genetic causes of acute visual loss are relatively rare, but these still need to be considered in the differential diagnosis, especially in the relevant clinical context. In this presentation, classical genetic disorders affecting the optic nerve will be discussed with a particular focus on Leber hereditary optic neuropathy (LHON). LHON is a primary mitochondrial DNA (mtDNA) disorder and three point mutations within the mitochondrial genome account for the vast majority of cases, namely: m.3460G>A, m.11778G $>\mathrm{A}$ and $\mathrm{m} .14484 \mathrm{~T}>\mathrm{C}$. It typically affects young adult males who present with bilateral severe visual loss in the second or third decade of life In addition to the canonical LHON mutations, other pathogenic mtDNA mutations have been reported that can result in both syndromic and non-syndromic optic neuropathies. Acute visual loss in genetic disorders can also be secondary to pathology involving the retrochiasma visual pathways, for example, in MELAS (mitochondrial encephalomyopathy, lactic acidosis, and stroke-like episodes). The cardinal clinical manifestations in this group of patients are homonymous hemianopic visual field defects or cortical blindness.

- 1653

Neoplastic causes of acute visual loss

BOSCHI A

Brussels

Visual loss in relation with cancer has greatly increased in adult as in child. Not only because improvement of diagnostic techniques, but because survival rate has dramatically improved.Acute visual loss may be associated to direct or metastatic involvement of the eye or may be a consequence of cancer therapy including chemotherapy, radiation as newer modality like intra-arterial injections.Considering the possible immunodysfunction in these patients infection as autoimmune disorders should be considered in the differential diagnosis of acute uni or bilateral visual loss. Clinical features and therapeutic options will be discussed through clinical cases.

\section{2}

Inflammatory and infectious causes of acute visual loss

SZATMARY G (1), POLGAR T (2)

(1) Hattiesburg

(2) Neuro_ophthalmology, Hattiesburg

In this course comprehensive review of inflammatory and infectious causes of acute visual loss will be presented. This will include pre-and retrochiasmal-chiasmal visual pathway lesions. The lecture will focus on clinical presentation with localizing symptomatology and signs, recommended diagnostic tests, differential diagnosis and treatment possibilities. Also, known pathophysiology of various disorders will be discussed. At the conclusion of presentation the audience will be familiar with the wide range of neuro-ophthalmological disease states that can result in acute visual loss.

1654

Vascular causes of acute visual loss

KAWASAKIA

Lausanne

Acute optic neuropathy due to a vascular cause is essentially a syndrome of ischemic optic neuropathy. The mechanism of ischemic optic neuropathy involves a fall in perfusion pressure to the optic nerve which, in susceptible individuals, leads to infarction of the prelaminar or retrolaminar portion of the optic nerve. Ischemic optic neuropathy can be subdivided into anterior ischemic optic neuropathy and posterior ischemic optic neuropathy . A special category is post-operative ischemic optic neuropathy. This lecture will discuss the clinical features that distinguish these forms of ischemic optic neuropathy, the risk factors associated with them and the approach to their management 


\section{- 1661}

The effect of a rock inhibitor ama0428 in a model of wet agerelated macular degeneration

HOLLANDERS K (1), VAN BERGEN T (1), VANDEWALLE E (2), STALMANS I (1, 2) (1) KULeuven Ophthalmology, Leuven

(2) UZLeuven Ophthalmology, Leuven

Purpose Rho kinase (ROCK) is associated with VEGF-driven angiogenesis and is involved in inflammation and fibrosis. Therefore, the effect of a locally acting ROCK inhibitor, AMA0428, was studied in wet age-related macular degeneration (AMD).

Methods The effect of AMA0428 on human brain microvascular endothelial cells (HBMEC), human brain vascular pericytes (HBVP) and human tenon fibroblasts (HTF) was determined by measuring cell viability (WST-1), apoptosis (caspase 3/7) and 2 migration assays (scratch and under-agarose) The in vivo response was investigated using a laser-induced choroidal neovascularization (CNV) mouse model. Intravitreal injections were given on day 0, 4, 10 and 20 with AMA0428, murine anti-VEGFR $\mathrm{Ab}$ (DC101) or placebo. Outcome was assessed by analysis of inflammation (CD45), angiogenesis (FITC-dextran), vessel leakage (Texas Red-conjugated Dextran and FITClabeled lectin) and fibrosis (Collagen I).

Results AMA0428 dose-dependently reduced proliferation and VEGF-induced migration of HBMEC and HTF. No significant effect was seen on HBVP proliferation; however, migration and pericyte recruitment were increased. There was no apoptosis induction. AMA0428 significantly reduced CNV and vessel leakage 2 weeks after lasering, comparable to DC101. In addition, AMA0428 inhibited inflammation on day 5 by $20 \%$ and collagen deposition on day 30 by $39 \%$ while DC101 had no effect on inflammation nor fibrosis.

Conclusion Our data suggest that targeting ROCK with AMA0428 not only reduces neoangiogenesis, but also blocks inflammation and fibrosis (contrary to anti-VEGF). These results point to a potential therapeutic benefit of ROCK inhibition in wet AMD.

\section{- 1663 / F109 \\ Variations and repeatability of macular pigment and its spatial profiles in south Asian and white subjects}

HUNTJENS B, CTORII

Applied Vision Research Centre, The Henry Wellcome Laboratories for Vision Sciences, City University London, London

Purpose To investigate variations in macular pigment optical density (MPOD) between south Asian and white subjects and explore the repeatability of the MPOD spatial profile.

Methods MPOD was measured by heterochromatic flicker photometry in 77 south Asian and 60 white healthy volunteers aged 18 to 39 years, and repeated at a second visit in 24 subjects. The MPOD spatial profile was classified as typical exponential, or atypical ring-like or central dip (IOVS 2014;55:1440-1446).

Results We report excellent MPOD spatial profile repeatability (96\%) between visits Our findings showed $67 \%$ of all subjects with atypical ring-like or central dip profiles were south Asian. Average integrated MPOD up to $1.8^{\circ}$ (MPODav0-1.8) was increased in subjects with atypical profiles $(\mathrm{P}<0.0005)$. Half peak MPOD value occurred at an average $1.8+0.7^{\circ}$ for the typical profile group, which was significantly broader than that for the atypical ring-like $\left(1.5 \pm 0.3^{\circ}\right)$ or central dip profiles $\left(1.4 \pm 0.3^{\circ}, \mathrm{P}=0.01\right)$. South Asian subjects had greater MPODav0-1.8 $(0.39 \pm 0.13)$ compared to whites $(0.32 \pm 0.13$ $\log$ units, $\mathrm{P}=0.01$ ) after controlling for age. White subjects with dark eyes had higher MPODav0-1.8 than those with light eyes $(\mathrm{P}=0.012)$.

Conclusion Classification of the MPOD spatial profile was highly repeatable. Since half peak MPOD value occurred within $1.8^{\circ}$ eccentricity, the spatial profile and MPODav0-1.8 together best describe the distribution of MPOD over the central area where macular pigment has its maximum effect. Further, atypical MPOD profiles offer increased MPOD over a more concentrated area centred on the fovea. Both parameters showed significant variations between the two ethnic groups.
- 1662

Evaluation of potential retinal toxicity after intravitreal administration of a novel neuroprotecive agent, synthetic analog of dehydroepinadrosterone (DHEA)

TSIKA C (1), NIAVIS (1), GRAVANIS A (2), TSILIMBARIS M (1)

(1) Department of Ophthalmology, Medical School of the University of Crete, Heraklion (2) Department of Pharmacology, Medical School of the University of Crete, Heraklion

Purpose To investigate the effect of the intravitreal administration of a synthetic analog of DHEA on retinal anatomy and function of the rabbit eye

Methods All experiments complied with the ARVO Statement for the Use of Animals in Ophthalmic and Vision Research. We injected $0.6 \mathrm{mg} / 0.1 \mathrm{ml}$ of a synthetic DHEA analog in the right eye of 9 pigmented rabbits. The left eye received $0.1 \mathrm{ml}$ of the solvent

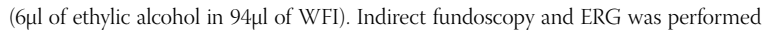
before injection (baseline) and one month post injection. This was the end time point of the study. Histological samples were prepared for photonic microscopy after euthanasia and enucleation. Additionally, fundoscopy was performed weekly to follow up any macroscopic changes.

Results At one month, no significant macroscopic retinal changes were observed. No significant difference in scotopic b-wave amplitude of the ERG was recorded one month after injection of the neuroprotective solution $(\mathrm{p}=0.441)$ or the solvent alone $(\mathrm{p}=0.767)$. Histology revealed minor changes in retinal anatomy that were not attributed to any substance effect. Control eyes showed similar findings. Interocular differences in ERG were not significant at baseline $(\mathrm{p}=0.136)$ or at one month $(\mathrm{p}=0.666)$.

Conclusion BNN 93 exhibited no toxicity on retinal anatomy or function after intravitreal administration of a high concentration in the rabbit eye. This is a promising preliminary finding for potential use in ocular neurodegenerative disease.

\section{- 1664}

Macular pigment optical density in a French population over 75 years old (MONTRACHET's study: Maculopathy, Optic Nerve, nuTRition, neurovAsCular and HEarT diseases)

FLECK O (1), KOEHRER P (1), DANIEL S (2), BINQUET C (2), BRETILLON L (3), ACARN (3), TZOURIO C (4), BRON AM (1), CREUZOT-GARCHERC (1)

(1) Department of Ophthalmology, CHU, Dijon

(2) Department of Epidemiology, Inserm, Dijon

(3) UMR 1324, CSGA, Dijon

(4) Department of Epidemiology, Inserm unité 708, Bordeaux

Purpose The aim of our study was to describe the frequency of macular pigment distribution patterns in a french population-based study over 75 years old.

Methods Three city study (3C) included 9294 subjects aged 65 years or more from three cities (Bordeaux, Dijon and Montpellier) in 1999. After a 10 year follow-up, Dijon's cohort had a complete ophthalmic examination in the MONTRACHET study 1153 patients over 75 years were included. All patients had fundus photographs and macular pigment optical density measured by two-wavelength autofluorescence. Pseudophakic patients without any retinal pathological conditions were included. In each patient, the eye with the best autofluorescence image quality was included.

Results One hundred fourty three eyes were selected (81 right eyes and 62 left eyes). Mean age was $82.0 \pm 3.9$ years, $71 \%$ (102) were women. Macular pigment optical density was $0.93 \pm 0.22$ density unit at the foveal center, $0.71 \pm 0.20$ density unit at $0.5^{\circ}$ eccentricity $0.59 \pm 0.17$ at $1^{\circ}, 0.36 \pm 0.12$ at $2^{\circ}$ and $0.09 \pm 0.03$ at $6^{\circ}$. Nineteen patients $(13.3 \%)$ had ringlike structure at mean $0.87 \pm 0.19^{\circ}$ eccentricity, 21 patients had intermediate distribution in density profile, 103 patients had strictly monotonic decline of macular pigment from the center to the periphery. There was no significant correlation between gender and macular pigment pattern $(\mathrm{p}=0.36)$

Conclusion This is the first study describing the frequency of the patterns of macular pigment distribution in an elderly pseudo-phakic population. It would be useful to establish normative values in this age subgroup. 
- 1665

Does foveal anatomy influence macular pigment and its spatial profile? A bi-racial study

CTORI I, HUNTJENS B

Applied Vision Research Centre, The Henry Wellcome Laboratories for Vision Sciences, City University London, London

Purpose To investigate the relationship of foveal architecture with macular pigment optical density (MPOD) and its spatial profile in south Asian and white females.

Methods Foveal anatomy measurements, obtained using optical coherence tomography (Spectralis OCT; Heidelberg), were collected from 46 south Asian (age 21 \pm 3 years) and 38 white healthy females (age $26 \pm 5$ years). Spatial distribution of MPOD, measured by heterochromatic flicker photometry, was classified as a typical exponential or an atypical profile.

Results South Asian compared to white females had thinner central retinas (220 \pm $14 \mu \mathrm{m}$ vs. $228 \pm 19 \mu \mathrm{m} ; \mathrm{P}=0.02)$, wider foveas $(2526 \pm 247 \mu \mathrm{m}$ vs. $2296 \pm 228 \mu \mathrm{m} ; \mathrm{P}<$ $0.0005)$ and higher central MPOD $(0.56 \pm 0.18$ vs. $0.44 \pm 0.23 ; \mathrm{P}=0.001)$, after controlling for age. Central MPOD was greater in those with an atypical $(0.61 \pm 0.21)$ compared to a typical MPOD profile $(0.47 \pm 0.17, \mathrm{P}<0.005)$. Only white females presenting a typical MPOD profile showed a significant correlation between central MPOD and central retinal thickness $(\rho=0.403, P=0.03)$ and between the foveal pit and MPOD profile slope gradients from 0 to $0.8^{\circ}(\mathrm{r}=-0.522, \mathrm{P}=0.004)$. We found no association between foveal width and central MPOD in either ethnicity regardless of MPOD profile type $(\mathrm{P}=0.90)$.

Conclusion Our findings show that there are significant differences in retinal morphology and central MPOD between south Asian and white females. However, foveal anatomy seems to correlate with central MPOD only in white females with typical MPOD profiles. We hypothesize that higher central MPOD associated with atypical MPOD profiles and south Asian ethnicity is not related to central foveal anatomy, but a feature of an individual's congenital constitution

- 1667

Relation between the retinal reflectivity on SD-OCT and the cone density measured using adaptive optics

FLORES M, DEBELLEMANIERE G, TUMAHAI P, KOEHL A, MEILLAT M, SCHWARTZ C, DELBOSC B, SALEHM

Ophthalmology Department, Besançon

Purpose To assess the relation between the relative reflectivity of the inner segment ellipsoid (ISe) band on Spectral domain (SD)-OCT and the cone density measured using adaptive optics.

Methods Twenty eyes of 10 subjects were studied. Two subjects were healthy and the others were randomly chosen from a pool of patients presenting retinal diseases impairing bilaterally the ISe layer. Horizontal B-scans were acquired using SD-OCT (Spectralis, Heidelberg, Germany). The relative reflectivity, defined as the ratio between the reflectivity of the ISe layer and the reflectivity of the full retinal thickness was measured, together with ISe thickness, using Image). Cone counts were performed at $2^{\circ}$ eccentricity from the fovea (nasal, temporal, superior and inferior areas) using an Adaptive Optics retinal camera (RTX1, Imagine Eyes, Orsay, France) and its dedicated software (AOdetect v0.1" ,Imagine Eyes, Orsay, France). Correlation coefficients (Pearson $\mathrm{r}$ ) between the cone density and the others parameters were calculated for each area.

Results Cone densities were significantly correlated with the ISe relative reflectivity (r $=0,70[0,59 ; 0,79] \mathrm{p}<0,01)$, and to a lesser extent with the ISe thickness $(r=0,32[0,04$ $0,56] \mathrm{p}<0,01)$ and with BCVA $(\log M A R)(r=-0,38[-0,55 ;-0,21] p<0,01)$.

Conclusion The reflectivity on SD-OCT of the outer retina brings valuable information on photoreceptor density in absence of adaptive optics camera. Further developments in OCT software enabling it uses in a clinical setting would be a useful advance.
- 1666 / F030

Macular response to a carotenoid supplement in healthy eyes

MARQUINA I, BRAUTASET R, NILSSONM

Karolinska Institutet, Unit of Optometry, Stockholm

Purpose Reduced macular pigment (MP) density is related to an increased risk of developing age-related macular degeneration (AMD). The aim of this study was to measure the macular response to a MP supplement by measuring macular pigment optical density (MPOD) before and after consumption.

Methods Fifty-one healthy subjects (age 18-62 years) participated in the study and were divided in two groups, one control group and one supplementary group. MPOD values were measured using heterochromatic flicker photometry (HFP) at baseline and after 90 days of supplementation with the dietary supplement MacuShield ${ }^{\circ}(10 \mathrm{mg}$ lutein, $10 \mathrm{mg}$ meso-zeaxanthin and $2 \mathrm{mg}$ zeaxanthin).

Results Mean MPOD values did not change significantly in any of the groups. A correlation between low MPOD values at baseline and a larger increase of MPOD values after supplementation was found in the supplementary group $(r=-0.52, p=$ $0.008)$. Increased MPOD values also correlated with increasing age $(r=0.40, p=0.0454)$.

Conclusion The results of the present study indicate that older subjects with low MPOD values increase their MPOD values after supplementation. As increased age and low MPOD values increase the risk of developing AMD, it might be beneficial for these individuals to consume macular pigments to reduce the risk of developing AMD. 
- 1671

Human retinal pigment epithelial cells dying through autophagy are engulfed by professional and non-professional phagocytes

TOTHM (1), KRISTOFE (1), DORO Z (1), VERÉB Z (2), ALBERT R (2), FÉSÜS L (1), PETROVSKIG $(2,1)$

(1) Stem Cells and Eye Research Laboratory, Department of Biochemistry and Molecular Biology, University of Debrecen, Debrecen

(2) Department of Ophthalmology, University of Szeged, Szeged

Purpose Inefficient removal of dying retinal pigment epithelial (RPE) cells by professional and non-professional phagocytes can result in debris formation and development of age-related macular degeneration (AMD). We aimed to study the clearance of autophagic dying RPE cells serving as a model for dry and wet type of AMD, respectively.

Methods Autophagic cell death was induced by serum deprivation and $\mathrm{H} 2 \mathrm{O} 2 \mathrm{CO}^{-}$ treatment in ARPE-19 and primary human RPE (hRPE) cells. Annexin-V FITC PI labelling was used to determine the cell death rate, while autophagy detection was achieved by Western blot quantification of LC3 II/LC3 I ratio. The clearance of autophagic dying cells by non-professional and professional phagocytes was quantified using flow cytometry.

Results An increasing percentage of dying RPE cells was observed in a time- and concentration dependent manner upon $\mathrm{H} 2 \mathrm{O} 2$ treatment. Paralelly, an induction of autophagy could be detected within 2 hrs of $\mathrm{H} 2 \mathrm{O} 2$ treatment. Phagocytosis studies found autophagic dying cells to be efficiently and increasingly engulfed by both professional and non-professional phagocytes over time.

Conclusion The clearance of autophagic dying RPE cells can be used as a model for studying both dry and wet type of AMD in vitro, as well as for testing future pharmacological agents for treating this disease.

- 1673

Microglia in mice retina contralateral to experimental glaucoma exhibit qualitative signs of activation in all retinal layers

$\operatorname{GALLEGOB}(1,2), \operatorname{SALAZARJJ}(1,2), \operatorname{DEHOZ} R(1,2), \operatorname{ROJAS} B(1,3)$, RAMIREZ AI (1, 2), VALIENTE-SORIANO FJ (4), AVILÉS-TRIGUEROS M (4), VILLEGAS PEREZMP (4), VIDAL-SANZM (4), TRIVINO A (1,3), RAMIREZ IM (1,3)

(1) Instituto de Investigaciones Oftalmológicas Ramón Castroviejo. UCM, Madrid

(2) Facultad de Óptica y Optometría. UCM, Madrid

(3) Facultad de Medicina. UCM, Madrid

(4) Exp. Ophthalmol. Lab;, Dept. Ophthalmology, College of Medicine, University of Murcia, Murcia

Purpose To analyse qualitative effects of two weeks of laser-induced ocular hypertension $(\mathrm{OHT})$ in the retinal microglial of OHT-eyes and their contralateral eyes

Methods Adult albino Swiss mice were divided into two groups: naive $(\mathrm{n}=6)$ and lasered $(n=9)$. Retinal whole-mounts immunolabelled with anti-Ibal were used to analyse the distribution and the morphology of Iba 1+ microglial cells

Results In naïve, contralateral and OHT-eyes, Iba 1+ microglia was evenly distributed throughout the retina in the photoreceptor laver (PRL), outer plexiform laver (OPL) inner plexiform layer (IPL), nerve-fibre layer (NFL) and ganglion-cell layer (GCL). In comparison with naive, the main differences in the microglia of contralateral eyes and OHT-eyes were: i) the presence of ameboid microglia; ii) the retraction of cell processes; iii) a higher level of branching; iv) soma displacement; v) reorientation of processes; vi) MHC-II up-regulation; vii) increased CD68 immunostaining and; viii) CD86 immunolabelling in ameboid cells. In addition, rounded Iba-1+ CD86+ cells in the NFL-RGC laver and PRL and Ym1+ cells and rod-like microglia in the NFL-RGC were restricted to OHT-eyes

Conclusion Two weeks of laser induced-OHT triggered several signs of microglia activation that extended beyond GCL. The same features were also observed in contralateral normotensive untreated eyes. Contralateral eyes appear to be a potential of discovering points of intervention to which future therapeutics can be directed (Support: OFTARED (Grant RD12/0034/0002 and RD12/0034/0014, ISCIII, Spanish Ministry of Science and Innovation)
- 1672

Study of anti-proliferative activity of ranibizumab, bevacizumab, pegaptanib and aflibercept on fibroblast-like cell strain in vitro

LYTVYNCHUK L (1), SA (2)

(1) Kyiv Eye Microsurgery Center, Kyiv

(2) National medical academy of postgraduating education, Kyiv

Purpose To evaluate the anti-proliferative action of drugs that block vascular endothelial growth factor (anti-VEGF: ranibizumab, bevacizumab, pegaptanib and aflibercept) upon fibroblast-like cells, as in vitro model for studying their implication on fibroblast-containing choroidal neovascular membranes (CNV).

Methods The cellular anti-proliferative effects of the four anti-VEGFs (cellular survival, mitotic- and polykaryocyte index, level of apoptosis) were evaluated over 5 days on the fibroblast-like cell strain L929. Cellular growth-kinetics indices (specific growth velocity $(\mu)$, population number doubling time (td) and reproduction velocity $(\mathrm{n}))$ were calculated and used to reveal dose-dependence of their anti-proliferative activity.

Results The anti-VEGFs could inhibit cellular survival, mitotic activity, and increase cellular heterogeneity of L929 cells in a dose-dependent manner. The antiproliferative activity of the anti-VEGFs was dose-dependent, while the apoptosis level was proportional to the dose increase in all four cases. The cellular growth-kinetics distinguished ranibizumab for having less aggressive anti-proliferative action with increasing doses, which was due to compensation of cell death by proliferation; the rest of the anti-VEGFs had anti-proliferative and apoptotic activity prevail the cellular survival. Different concentrations of aflibercept caused insignificant effect upon the cellular mitotic index compared to controls.

Conclusion All four anti-VEGF drugs exhibited marked anti-proliferative and apoptotic activity upon fibroblast-like cells in vitro. Overall, the explored effects might explain the fibroblast-like response to anti-VEGF drugs in vivo and be the cause for involution of CNVs.

- 1674

Energy supply is critical for Müller cells ability to transport glutamate

$\operatorname{KOLKOM}(1,2)$

(1) Roskilde University Hospital, Hellerup

(2) Neuroscience and Pharmacology, Copenhagen

Purpose To study the effect of energy deprivation in Müller cellsability to maintain their function as well as their ability to protectretinal ganglion cells (RGC).

Methods The human Müller cell line, MIO-M1 and primarymouse Müller cells were used to study changes in glutamate uptake,glutamate release, excitatory amino acid transporter (EAAT) proteinexpression, ATP levels and glycogen content, when cells werecompromised from energy. Moreover, a co-culture system of primaryRGC cultures and primary Müller cells, separated by an insert, wasused to evaluate the role of Müller cells in RGC survival.

Results EAAT1 and EAAT2 proteins were up-regulated in energydeprivedMüller cells and glutamate uptake was significantlyincreased in the absence of glucose, as opposed to conditions withsodium depletion or in the presence of the EAAT-inhibitor tfb-TBOA,in which glutamate uptake was decreased. The intracellular glycogencontent decreased in a time-dependent manner, whereas the ATPlevels were sustained following energy deprivation. Co-cultures ofRGC and Müller cells revealed better survival of glutamate treatedRGC in the presence of Müller cells compared to controls. Energydeprivation of Müller cells enhanced their ability to protect RGC.

Conclusion The present findings revealed an up-regulation ofEAAT1 and EAAT2 in energy compromised Müller cells as wellas an increased ability to remove glutamate from the extracellularspace. Co-cultures of RGC and Müller cells revealed a protectiverole of Müller cells when RGC were exposed to glutamate. Hence,energy failure may result in an increased ability to protect RGC fromglutamate-induced excitotoxicity, whereas malfunction of glutamateuptake in Müller cells may contribute to RGC death. 


\section{- 1675}

Activation of cannabinoid receptor CB2 in ARPE-19 cells stimulates the release of inflammatory cytokines via activation of the ERK pathway

HYTTIM (1), PIIPPO N (1), KORHONENE (1), SALMINENA (2),

KAARNIRANTA K $(1,3)$, KAUPPINENA $(1,3)$

(1) Department of Ophthalmology, Kuopio

(2) Department of Neurology, Kuopio

(3) University Hospital, Kuopio

Purpose Endocannabinoid receptors have been suggested to be a possible therapeutical target in age-related diseases. Particularly CB2, the receptor primarily responsible for the immune modulatory effects of cannabinoids, might be a valuable target in diseases, such as age-related macular degeneration, where inflammation is known to be a key player.In this study, we have evaluated the effects of CB2 activation on the viability and inflammatory response of retinal pigment epithelial cells.

Methods We cultured ARPE-19 cells until confluent and treated the cells with the selective CB2 agonist JWH-133. To assess the effect of CB2 activation on oxidatively stressed cells we treated some cells with the lipid peroxidation end product 4-Hydroxynonenal (HNE) after the JWH-133 stimulation. The effects of the treatments on cell viability were assessed and inflammatory cytokine expression and signaling protein activation were measured by ELISA.

Results Our results show that $10 \mu \mathrm{M} J \mathrm{WH}-133$ robustly increased the production of interleukin (IL) 6 and IL-8 in both HNE-treated and untreated cells. Investigation of the MAPK signaling pathway showed that JWH-133 at this concentration increased the phosphorylation of ERK1/2.

Conclusion Our results show that JWH-133, a selective agonist of the cannabinoid receptor CB2, stimulates an inflammatory response in ARPE-19 cells. The release of inflammatory cytokines seems to be mediated by the increased activity of the MAPKinase ERK1/2. Taken together, our results suggest that CB2's role as a potentia therapeutical target in retinal pigment epithelium should be more carefully analyzed in future studies.
- 1676

The best corrected visual acuity and retinal thickness are associated with improved cortical visual processing in treated wet-AMD patients

VOTTONEN P (1), KAARNIRANTA K (1), TARKKA I (2), PÄÄKKÖNENA (3)

(1) Department of Ophthalmology, Kuopio

(2) Department of Health Sciences, Jyväskyla

(3) Department of Clinical Neurophysiology, Kuopio

Purpose Age-related macular degeneration is the most common cause of blindness in the western world among elderly people. In response to anti-VEGF treatment for wet-AMD retinal anatomy and visual acuity is often remedied. In our previous study, we showed that also visual evoked potentials (VEP) improve following successful antiVEGF treatment. The aim of the present study was to investigate, if visual acuity and retinal thickness changes are associated with VEP parameters.

Methods Fifteen patients enrolled this study. Patients received three bevacizumab intravitreal injections. At the beginning of the study and 4-6 weeks after the last injection, the best corrected visual acuity (BCVA) test, full biomicroscope examination, OCT analysis and VEP were performed.

Results In treated eyes, $\log$ MAR visual acuity decreased on average $0.2 \pm 0.3$ units, OCT retinal thickness decreased $170 \pm 200$ micrometers and VEP amplitude increased $1.0 \pm 1.4$ microvolts. All changes were significant at $\mathrm{p}<0.05$. The relative changes of VEP amplitude and retinal thickness correlated significantly $\mathrm{r}=-0.630(\mathrm{p}<0.05)$, as well visual acuity (logMAR) and retinal thickness $\mathrm{r}=0.576(\mathrm{p}<0.05)$. VEP latency did not correlate with retinal thickness or visual acuity

Conclusion We showed that both the increase in VEP amplitude and the improvement in visual acuity are correlated with the decrease in retinal thickness in treated wet-AMD patients indicating that successful intravitreal anti-VEGF treatment initiates significant changes in upstream visual processing in the brain. 


\section{- 1711}

Pathophysiologic mechanisms based treatment for RVO related macular edema

POURNARASC

Memorial Rothschild Clinical Rechearch Group, Colline Ophthalmology Center, Geneva

The retinal vein occlusion (RVO) related to visual loss, results from changes in the blood-retina barrier leading to the formation of an extracellular retinal edema; and conconmmitant arteriolar vasoconstruction, leading to tissue hypoxia, intracellular retinal edema and neuronal cell death.Patient's evaluation requires the definition of the pathogenic role of systemic diseases affecting the retinal vessels wall. In addition, hemodynamic modifications of the retinal blood flow and primary and secondary thrombophilias, may trigger the manifestation of the RVO occlusive event.Current treatment of acute RVO aims to restore venous circulation. In that sense, isovolemic hemodilution leads to an increase of ocular blood flow and regression of tissue hypoxia. Grid macular photocoagulation, reversing the inner retinal tissue hypoxia, improves visual prognosis in eves with macular edema following branch RVO. The intra-vitreal treatments using anti-VEGF and anti-inflammatory drugs, reverse the veins abnormal permeability, relieve the macular edema, either in branch or central RVO, offering new insights for the management of those pathologies.

- 1713

BRVO treatments in 2014

PETROPOULOSIK

Ophthalmological Center of Rive, Geneva

Until recently, laser photocoagulation was the standard of care for branch retinal vein occlusion (BRVO). The recent development of intravitreal pharmacotherapy has revolutionized the management of BRVO and has expanded our treatment options. Intravitreal anti-VEGF agents (ranibizumab, bevacizumab, aflibercept) and a sustainedrelease dexamethasone implant have been shown effective to treat BRVO-related macular edema by randomized studies and/or case series, offering favorable anatomical and visual outcomes. Still, long-term repeated injections may be needed and headto-head comparisons of these treatment modalities have not been performed. As for treatment duration, new data suggest that half of the patients may be cured after 2 years from the onset of the disease, whereas the other half will probably need repeated injections for at least 4 years. A better control of the underlying systemic disease (mostly arterial hypertension) and the identification of the proper time to perform laser photocoagulation could further improve prognosis. Safety and efficacy profiles and updated indications of each treatment modality with representative examples will be discussed.
- 1712

\section{CRVO treatments in 2014}

\section{GLACET-BERNARD A}

Creteil

Although central retinal vein occlusion (CRVO) accounts for a minority of retinal vein occlusions, it is associated with more severe vision loss and with the vision-threatening risk of neovascular glaucoma. The newly available treatments for macular edema have considerably improved visual outcome of many CRVO patients. Nevertheless, the evaluation of the ischemic component remains essential. The presentation will include when to perform laboratory workup for systemic disease and how macular edema treatment could be combined with classical treatment (etiologic treatment such as hemodilution, treatment of non-perfusion area with laser photocoagulation).

Commercial interest

1714

The (new) place of laser treatment in RVO

PUCHEN

Montbonnot/Grenoble

The appearance of injections in the treatment of macular edema has upsets the management of RVO. Now, What is the place of laser ?We will present the place of focal laser treatment of macular edema and remember the importance of panretinalphotocoagulation in the treatment of ischemic forms. The injection delay anti VEGF ischemic CRVO conversion. 
- 1715

RVO related macular edema: Rules for the patients follow up; how to choose the applied treatment?

LOEWENSTEINA

Department of Ophthalmology, Tel Aviv Medical Centeo, Tel Aviv

The treatment of RVO has dramatically evolved during the last few years. The main evidence base therapies are antiVEGF agents (Ranibizumab, Aflibercept, and level 2 evidence for bevacizumab) and steroids (Ozurdex). Each approach has advantages and limitations, while both have good efficacy results. In the decision making of the needed treatment inflammatory as well as VEGF pathways need to be taken into consideration. The treatment is to be tailored to the individual patient taking into consideration both ocular (existence of glaucoma, if it is controlled, existence of cataract, pseudophakia, ischemia), systemic (existence of cardiovascular risk factors) and social (ability to come monthly and availability of the health care system). Results of head to head trials are awaited. 
- 1721

\section{A standardised 'off the shelf' substrate for enhanced tissue} engineering

HOPKINSON A, BRANCH MJ, DUA A

Academic Ophthalmology, University of Nottingham, Nottingham

Purpose Amniotic membrane (AM) is a popular ophthalmic tissue engineering adjunct. We previously developed a highly effective thermolysin-based denuding technique that preserves basement membrane integrity This technique has been validated clinically using fresh frozen AM (FAM). We now propose a standardised dryprepared 'off the shelf' AM tissue engineering substrate.

Methods Dry preserved thermolysin denuded AM (DAM), and FAM denuded with ethylenediaminetetraacetic acid (EDTA), and dispase -based methodologies were prepared. Denuding efficiencies were compared using electron microscopy. The effect of denuding on AM molecular composition was investigated and characterised using proteomics. The propensity of DAM to support stem cells was explored using fluorescent immunohistochemistry for defined markers

Results Electron microscopy demonstrated thermolysin denuding efficiency was comparable in FAM and DAM. Proteomic analyses showed effective removal of epithelial cell proteins in DAM, but not EDTA-based denuding techniques. Whilst similar enzymatic activity to thermolysin, mechanical scraping reduces the efficacy of dispase denuding. Collagens IV, VI, periostin, Big-h3 and VLA-6 are targets of thermolysin activity. DAM maintains stem cell characteristics and is most effective in preventing differentiation.

Conclusion Conventional EDTA and dispase procedures for preparing AM for tissue engineering are ineffective at removing cells whilst preserving the basement membrane Combining our novel thermolvsin denuding and dry preservation techniques improves the overall quality of AM for tissue-engineered constructs. Therefore, we propose a fist of its kind, stable and dry-stored 'off the shelf' construct for enhanced ocular surface tissue engineering.

\section{- 1723}

\section{Corneal decellularisation: recycling tissue for transplantation}

WILSONSL

Academic Ophthalmology, Univeristy of Nottingham, Queen's Medical Centre,

Nottingham

Purpose There is a clinical need for reliable, reproducible biomimetic corneas Decellularised tissues are advantageous compared to synthetic/semi-synthetic tissues in that the native matrix ultrastructure and intrinsic cues including growth factors cytokines and glycosaminoglycans (GAGs) may be retained. However, there is currently no reliable, standardised human corneal decellularisation method. Here, we provide a systematic study of commonly used decellularisation methods and assess their appropriateness for corneal applications.

Methods Eye-bank tissue unsuitable for transplantation was used to test decellularisation methods: Dispase removal of the epi- and endothelium; Mechanical agitation; Hypertonic $\mathrm{NaCl}$; Ionic detergent (SDS); Non-ionic detergent (Triton-X100); all followed by nuclease treatment. Removal of cellular material, preservation of transparency, retention of corneal architecture and GAGs was assessed via histological, immunofluorescence and quantitative analysis. Potential cytotoxicity in vitro of the treated tissue was also assessed.

Results No decellularisation technique investigated successfully removed $100 \%$ of cellular components. The techniques which had the least residual DNA, SDS and Triton-X100, were most structurally compromised with reduced GAG content. Dispase treated, $\mathrm{NaCl}$ and mechanically agitated corneas had better preservation of structure transparency and GAGs, but had higher residual DNA.

Conclusion The ability to reprocess and regenerate tissues deemed "unsuitable" for transplantation allows us to salvage valuable tissue. However, in order to progress, we may need to take a step back to establish a "decellularisation" criterion; which should balance effective removal of immune reactive material with maintenance of tissue functionality.
- 1722

Human induced pluripotent stem cells (hiPSC)-derived endothelial cells: new opportunity for corneal bioengineering

THURET G (1, 2), HEZ (1), SUFFEE N (1), FOREST F (1,3), BERNARD A (1), PISELLIS (1), PERRACHE C (1), DUMOLLARD IM (1,3), PEOC'H M (1,3), GAIN P (1)

(1) Corneal Graft Biology, Engineering and Imaging Laboratory, EA2521, Federative Institute of Research in Sciences and Health Engineering, Faculty of Medicine, Jean Monnet University, Saint-Etienne

(2) Institut Universitaire de France, Bd St Michel, Paris

(3) Department of Pathology, University Hospital, Saint-Etienne

Purpose hiPSCs can self-renew indefinitely, while maintaining the capacity to differentiate into somatic cells. As such, they represent an essentially inexhaustible source of committed corneal endothelial cells (CECs) of potential use in cell-based corneal therapies. We present a method of differentiation of hiPSCs into CE-like cells.

Methods hiPSCs reprogrammed from adipocytes by retrovirus (SBI) were expanded in mTSR1 medium. Differentiation was induced by sequential treatment mimicking the normal embryonic endothelial development, using co-cultures during 3 weeks (not detailed because of ongoing patent application). Cells were sorted using magnetic beads with negative selection with anti-K3/K12. Sorted cells were subsequently cultivated in maintenance medium for 3-4 weeks. Differentiation was assessed by immunolabeling of ZO-1, Glypican-4, CD200R, Na/K/ATPase, CLCN3, MiTF, K3/K12. Stemcellness was assessed using anti-OCT-4.

Results hiPSCs were successfully and stably differentiated into a monolayer of tightly packed cells expression typical ECs makers and neither keratocytes nor epithelial markers. We observed the consecutive formation of embryoïd bodies after 4 days (D), neural crest cells after 7D, mesenchymal cells after 10D and cells with CEC phenotype after 15D. They lost expression of OCT-4.

Conclusion hiPSCs are a promising source of CECs. In addition to the short-circuit current measurement using a Ussing chamber, their ability to deswell corneas is being studied using our innovative bioreactor. Safe sources of cornea-derived hiPSCs are also under study to facilitate clinical translation.Grants: ABM2013, IUF2012-17, UJM2013

\section{- 1724}

Corneal endothelial cells from old donors: differentiation, senescence, proliferative capacities and optimized culture conditions

HA THI B (1), HEZ (1), FOREST F (1, 2), THURET JY (3), PISELLIS (1), PERRACHEC (1), PEOC'HM (1, 2), DUMOLLARDIM (1,2), ACQUART S (4), GAIN P (1, 5),

THURET G $(1,5,6)$

(1) Corneal Graft Biology, Engineering and Imaging Laboratory, EA2521, Federative Institute of Research in Sciences and Health Engineering, Faculty of Medicine, Jean Monnet University, Saint-Etienne

(2) Department of Pathology, University Hospital, Saint-Etienne

(3) Institute of Biology and Technology Saclay (iBiTec-S)/ Units/Integrative Biology and Molecular Genetics (SBiGeM), Gif sur Yvette

(4) Eye Bank, French Blood Centre, Saint-Etienne

(5) Department of Ophthalmology, University Hospital, Saint-Etienne

(6) Institut Universitaire de France, Bd St Michel, Paris

Purpose The intrinsically low proliferative capacity of human corneal endothelial cells (CECs) and their rapid senescence in in vitro culture constitute major limitations for cell bioengineering destined to treat endothelial dysfunctions. In Europe, the mean donor age of 70 adds a supplementary difficulty to the complex equation. Aim: to clarify whether old donor corneas could be however used for cell expansion

Methods Morphology and differentiation of central and peripheral CECs were analyzed by immunostaining of ion transport-related proteins, progenitor and neuronal markers in corneas retrieved in donor $>50$ years. The proliferative capacity of CECs from organ-cultured corneas was quantified by EdU proliferation assay. The differences of central vs peripheral CECs were further characterized in vitro by studying their migration, proliferation and differentiation in serum-containing medium.

Results Central CECs highly expressed specific ion transport-related proteins, and had a high senescence level. On the contrary, CECs located in the periphery or extreme periphery were less specialized, expressed progenitors and neuronal markers and were more prone to endothelial-mesenchymal transformation in serum-containing medium. With a new culture sequence that limited proliferation and maintained differentiation, corneas from donors $>70$ yo, could successfully be used to reconstitute a monolayer of stable endothelial phenotype with ECD of 2000 cells $/ \mathrm{mm} 2$ but require pooling of corneas.

Conclusion This work could allow to "recycle" the $20 \%$ of corneas usually discarded by eye banks because of age-related ECD decline 


\section{- 1725 / S013 \\ Influence of pressure on in vitro human corneal endothelial cells derived from human induced pluripotent stem cell (hIPSC)}

IUMELLEC (1), SUFFEEN (1), FOREST F (1, 2), HE Z (1), BERNARD A (1), NANGOUM-FOSSO T (1), NAIGEONN (1), PERRACHEC (1), PEOC'H M (1, 2) $\operatorname{GAINP}(1,3)$, THURET G $(1,3,4)$

(1) Corneal Graft Biology, Engineering and Imaging Laboratory, EA2521, Federative Institute of Research in Sciences and Health Engineering, Faculty of Medicine, Jean Monnet University, Saint-Etienne

(2) Department of Pathology, University Hospital, Saint-Etienne

(3) Department of Ophthalmology, University Hospital, Saint-Etienne

(4) Institut Universitaire de France, Bd St Michel, Paris

Purpose Human induced pluripotent stem cells (hIPSC) have infinite self-renewal capacity and can differentiate into somatic cells. We obtained hIPSC-derived corneal endothelial-like cells (CE-likeC) using a sequential supply of growth factors. As, in vivo, the functionality of CEC may depend on various environmental factors, including intraocular pressure, we investigated whether pressure could influence the hIPSC differentiation into EC

Methods We developed a specific device for pressurizing cell cultures placed in a $\mathrm{CO} 2$ incubator. The device consists of a pump injecting the gas mixture from the incubator into a sealed container equipped with a pressure sensor and an electronic control system. hIPSC-derived CEC were cultured in the differentiation medium for 1 week either in a standard incubator at atmospheric pressure or under a pressure of 20 $\mathrm{mmHg}$. The medium was changed every 2 days. Differentiation was determined by cell morphology analysis and ionic pumps immunostaining (CLCN3, VDAC3, SLC4A and $\mathrm{Na}+/ \mathrm{K}+$ /ATPase) and tight junctions (ZO-1).

Results At D8, the 2 cultures showed similar endothelial morphology, ZO-1 and SLC4A, but expression of ionic pumps CLCN3, VDAC3, and Na+/K+/ATPase were increased under pressure.

Conclusion The device developed is fully functional. Our results show that, in vivo, a pressure of $20 \mathrm{mmHg}$ did not modify the cell morphology. However, it tends to modulate the expression of some ionic pumps. Pressure level seems to be an important paramete in the differentiation into $\mathrm{EC}$ Considering this, it could improve the $\mathrm{EC}$ functionality, i.e. deswelling capacityGrants: ABM2013, IUF2012-2017 and postdoc UJM 2013

\section{- 1727 / S015}

Ocular chronic graft versus host disease after allogeneic hematopoietic stem cell transplantation - preliminary data

JEPPESEN H (1, 2), LINDEGAARD J (2), SENGELOEV H (3), JULIAN HO (2), PRAUSE J (4), HEEGAARD S (2, 4)

(1) Dept of ophthalmology, Copenhagen

(2) Dept of ophthalmology, Glostrup

(3) Dept of hematology, Copenhagen

(4) Dept of neuroscience and pharmacology, Eye pathology section, University of Copenhagen, Copenhagen

Purpose Allogeneic hematopoietic stem cell transplantation(HSCT) is a curative therapy for a number of malignant and non-malignant hematological diseases. Allogeneic HSCT can be performed in several ways; donor cells can be obtained from bone marrow(BMT), peripheral blood(PBSCT) or cord blood(CBT). Ocular chronic graft versus host disease(cGVHD) is a major contributor to long-term morbidity after HSCT. The purpose of this study was to report the frequency of ocular cGVHD after allogeneic HSCT.

Methods Retrospective examination of 233 charts of patients (adults $>15 y e a r s)$ who underwent consecutive allogeneic HSCT from January 2000-june 2011 at Copenhagen University Hospital (Rigshospitalet). All allogeneic HSCT in Denmark are performed at this hospital. All patients were examined ophthalmologically before the transplant, yearly after the transplant and ad hoc if any ophthalmic problems occurred. The diagnosis of ocular cGVHD was made according to the NIH consensus criteria.

Results Fifty-eight(25.3\%) fulfilled the diagnostic criteria of ocular cGVHD, 16 out of $78(20.5 \%)$ in the BMT group, 41 out of $152(27.0 \%)$ in the PBSCT group and 1 out of 3(33.3\%) in the CBT group. Male:female ratio was 40:18(2.22) (in the cohort 146:87(1.68)). Median time of onset was 24.3 months after HSCT(range 4.9-146.1). In the cohort median age was 45.2 years(rage 15.2-71) at transplantation time and 55.5 years(18.43-69.0) when ocular cGVHD was diagnosed. Median time of follow-up was 24.8 months(range 0.4-147.6)

Conclusion Out of 233 patients who received allogeneic HSCT, 59(25.3\%) developed ocular CGVHD. The median time of onset was 24.3 months after transplantation and median age at onset was 55.5 years.
- 1726 / S014

Development of an ECM hydrogel for corneal tissue engineering

AHEARNEM $(1,2)$

(1) Trinity Centre for Bioengineering, Trinty College Dublin, Dublin

(2) Department of Mechanical and Manufacturing Engineering, Trinity College Dublin Dublin

Purpose Tissue engineering has been proposed as a method of dealing with the shortage of suitable tissue available for transplants. Many biomaterials under investigation for engineering corneal tissue lack the native corneas biochemical composition. The aim of this study was to fabricate and test a biomimetic hydrogel derived from corneal extracellular matrix (ECM) to be used for corneal tissue engineering.

Methods Porcine corneas were decellularized using several different techniques. The corneas were then freeze dried and milled into a fine powder. This ECM powder was dissolved using an acidic pepsin digest solution for 72 hours. A crosslinked hydrogel was formed after neutralizing the $\mathrm{pH}$ of the solution. Human corneal stromal cells were mixed into the hydrogels prior to gelation and cultured over 14 days. Biochemical assays, mechanical and optical testing, RT-PCR and immunohistochemical staining were performed on the cell seeded hydrogels. Rat-tail collagen hydrogels were used as controls.

Results The hydrogels were highly transparent and able to maintain stromal cell viability. When compared to standard collagen hydrogels, the ECM hydrogels retained corneal GAGs and appeared to enhance the cells native keratocyte phenotype. The amount of GAG present was dependent on the decellularization technique used. The ECM hydrogels exhibited similar viscoelastic characteristics to collagen hydrogels.

Conclusion A new type of hydrogel has been demonstrated that could be used for engineering corneal tissue. We plan further develop these hydrogels by optimizing the stromal decellularization process and examining different crosslinking techniques to improve the hydrogels mechanical strength and stiffness.

- 1728 / S016

Positive cultures in corneas stored with cold storage technique to be used for corneal grafting

JULIAN HO (1), LINDEGAARD J (1), HØJGAARD-OLSEN K (1), HEEGAARD S (2) (1) Eye Department, Glostrup Hospital, Copenhagen

(2) Eye Pathology Institute, Department of Neuroscience and Pharmacology, University of Copenhagen, Copenhagen

Purpose Tissue for cornea grafting processed in the US is stored using cold storage method whereas corneas processed in the EU is stored in organ culture.The two preservation techniques differ in technical aspects, evaluation techniques, storage time and microbiological safety.Both techniques are considered to result in similar graft survival.Due to low availability of Danish donor tissue, we have used US donor tissue for more than seven years in Denmark.

Methods Routine microbiological culturing of the cornea-scleral ring was performed at the time of DSAEK surgery.

Results From April 2013 to March 2014 a total of 11 donor rims tested after grafting were positive for bacteria or fungi with the cold storage technique. Microbiological testing of donor rims stored with organ culture were all negative. Total number of grafts, $\mathrm{n}=210$. Six candida, one MRSA, two E. Faecium and two E.coli were cultured. Two eyes had severe and vision threatening infection (fungi). Four eyes showed primary graft failure. Five eyes showed no signs of infection.

Conclusion Corneas prepared with the cold storage technique were more prone to microbiological contamination, some with vision-threatning fungal infection. Antimycotics are now added to the current hypothermic storage solution. 


\section{- 1731}

What is driving the decision to perform a filtering procedure

BRONAM (1), DE LAZZER A (1), KOEHRER P (1), BONNABEL A (1), AHOS (2), CREUZOT-GARCHER C(1)

(1) Ophthalmology, University Hospital, Dijon

(2) Epidemiology, University Hospital, Dijon

Purpose To assess what is driving the decision to perform a filtering procedure in a patient suffering from glaucoma.

Methods Retrospective study in one single University centre with one glaucoma specialist. All the indications for filtering glaucoma surgeries (alone or combined with cataract extraction) undertaken during 2013 with the same surgeon were reviewed. The indications were as follows: clinical evaluation of the optic disc, visual field progression, progression on imaging (OCT and HRT), poor tolerance to medical treatment, non controlled intraocular pressure (IOP) and cataract.

Results Retrospective study in one single University centre with one glaucoma specialist. All the indications for filtering glaucoma surgeries (alone or combined with cataract extraction) undertaken during 2013 with the same surgeon were reviewed. The indications were as follows: clinical evaluation of the optic disc, visual field progression, progression on imaging (OCT and HRT), poor tolerance to medical treatment, non controlled intraocular pressure (IOP) and cataract.

Conclusion The indications for glaucoma surgery may be highly variable according to the country, the practice and the surgeon. A multicentre study should be useful to better define what is driving the indications for glaucoma surgery
- 1733

Inhibition of $\alpha 5 \beta 1$-integrin significantly improves the surgical outcome of glaucoma surgery in mice compared to MMC

VANBERGEN T (1), ZAHNG (2), CALDIROLA P (2), FSADNIM (2, 3),

CARAM-LELHAM N (2), VANDEWALLE E (1,4), STALMANS I $(1,4)$

(1) Lab of Ophthalmology, KU Leuven, Leuven

(2) Clanotech, Stockholm

(3) International Pharm-Med Ltd, Bramhall

(4) Ophthalmology, UZ Leuven, Leuven

Purpose The aim of this study was to evaluate the therapeutic potential of $\alpha 5 \beta 1$ integrin inhibitor (CLT-28643) to improve the outcome of filtering surgery in a mouse model. Different dose regimen and administration routes of the inhibitor were compared to mitomycin $\mathrm{C}(\mathrm{MMC})$, the gold standard in clinical practice

Methods The efficacy of CLT-28643 on surgical outcome was studied in a mouse model for filtering surgery ( $\mathrm{n}=40$ eyes from 20 mice per group). Group 1 received a single subconjunctival (SC) injection of $2 \mu \mathrm{g}$ of the integrin inhibitor immediately after surgery, whereas repeated SC injections were administered on day $0,3,7,14$ and 21 in groups 2 and 3 ( 2 and $1 \mu \mathrm{g}$, respectively). Group 4 received topical eye drops containing $10 \mu \mathrm{g}$ of the compound 3 times daily. MMC $0.02 \%$ was applied for 2 minutes in group 5 and repeated SC injections of $\mathrm{NaCl}$ were given in the last group. Treatment outcome was studied by a masked observer every other day by clinical investigation of the bleb until postoperative day 28.

Results Surgical outcome was improved in all treatment groups compared to $\mathrm{NaCl}$ treated eyes $(\mathrm{P}<0.001)$. A dose-response curve was observed in the efficacy of the subconjunctival injections, with the repetitive administration of $1 \mu \mathrm{g}$ being significantly inferior to, the single injection of $2 \mu \mathrm{g}$ or topical administration $(10 \mu \mathrm{g})$ comparable to, and the repetitive injection of $2 \mu \mathrm{g}$ significantly superior to MMC.

Conclusion These data suggest that administration of the $\alpha 5 \beta 1$-integrin inhibitor CLT-28643 has therapeutic potential as an adjunct to glaucoma surgery, possibly with a superior efficacy to MMC when used at the optimal dose.

- 1732

Rho Kinase Inhibitor AMA0526 improves surgical outcome in a rabbit model of glaucoma filtration surgery

VANDE VELDE S (1), VAN BERGEN T (1), VANDEWALLE E (2), STALMANS I (2, 1) (1) Lab of Ophthalmology, Leuven

(2) UZ Leuven, Leuven

Purpose To elucidate the effect of the ROCK inhibitor AMA0526 on the wound healing process and surgical outcome of glaucoma filtration surgery.

Methods The in vitro effect of ROCK inhibitor AMA0526 on human brain microvascular endothelial cells (HBMEC) and human Tenon fibroblasts (HTF) was determined using a proliferation assay. Secondly the in vivo effect of topical AMA0526 $0.3 \%$ TID was investigated in a rabbit model of glaucoma filtration surgery $(\mathrm{n}=5 /$ time point). Treatment outcome was studied by clinical investigation of the bleb area as well as immunohistological analyses for inflammation (CD45), angiogenesis (CD31) and collagen deposition at day 8,14 and 30 after surgery. Contralateral eyes were used as control and were treated with vehicle.

Results A dose-dependent reduction of HBMEC and HTF proliferation was measured after incubation with AMA0526. Incubation with the highest concentration of AMA0526 reduced proliferation of HBMEC and HTF to 22 and $35 \%$. In the surgery model, AMA0526 significantly improved bleb area and survival compared to vehicle treated eyes. Immunohistological analyses showed significant reduction of inflammation, angiogenesis and collagen deposition after treatment with the ROCK inhibitor. Compared to vehicle, inflammation was decreased by $33 \%$ at 8 days and angiogenesis by $52 \%$ on day 8 and by $29 \%$ at 14 days. Collagen deposition was significantly reduced by 11 and $42 \%$ on day 14 and 30

Conclusion This study shows that AMA0526 is able to inhibit proliferation of microvascular endothelial cells and Tenon fibroblasts in vitro, and to improve glaucoma surgery outcome in rabbits. In addition to improved bleb area, AMA0526 led to decreased inflammation, angiogenesis and fibrosis.

\section{- 1734}

Selective laser trabeculoplasty in pseudophakic eyes with glaucoma

MAGOURITSAS G, PORTALIOU DM, MOSCHONAS K, GEORGOPOULOS V Ophthalmology Department, Red Cross Hospital, Athens

Purpose To evaluate the efficacy and safety of selective laser trabeculoplasty(SLT) in patients with open angle glaucoma, which had previously undergone uneventful phacoemulsification with intraocular lens implantation. We also studied the intraocular pressure(IOP) lowering effect in association with the stage of glaucoma and the customized for each patient target-IOP.

Methods In this prospective study we enrolled 30 consecutive pseudophakic patients 30 eves) with uncontrolled glaucoma under maximum tolerated medical treatment and without any previous filtering surgery. We performed a $360^{\circ}$ treatment using the Ellex Solo laser system. Mean IOP measurements were carried out month 1, month 6 and month 12 post-laser treatment. Glaucoma stage was classified according to visual field loss as early, moderate or advanced. A range of target IOP was determined for each glaucomatous eye and used as an absolute success criterion. An IOP $<21 \mathrm{mmHg}$ was defined as a relative success

Results During the mean follow up of $15 \pm 2$ months $12(40 \%)$ eyes required either acetazolamide per os or surgical intervention. Clinically significant complications did not occur. At the last visit, the mean post-laser IOP reduction of the remaining 18 eyes was $4.4 \pm 0,7(20 \%) \mathrm{mmHg}$. An IOP- reduction of $<21 \mathrm{mmHg}$ was found in $73 \%$ (month6) and in $57 \%$ (month 12) of the eyes, whereas the overall absolute success was $50 \%$ and $37 \%$ respectively. SLT was significantly successful in eyes with early glaucoma.

Conclusion SLT is useful and safe for pseudophakic eyes with early to moderate glaucoma, which cannot be controlled under maximum tolerated medical treatment 
- 1735 / T051

Filtering blebs functionality after trabeculectomy: a clinical and in vivo confocal microscopy study

VIEIRA L, SANTOS A, LISBOA M, AMARAL A, CUNHA JP, MADURO V, REINA M Centro Hospitalar Lisboa Central, Lisbon

Purpose To evaluate macroscopic and microscopic morphological features of filtering blebs after trabeculectomy with Mitomycin C (MMC) or 5-Fluorouracil (5-FU) and correlate them with function.

Methods Retrospective case control study of 28 eyes (21 patients) submitted to trabeculectomy (15 with MMC and 13 with 5-FU) and 11 eyes (10 patients) treated medically. Ophthalmologic examinations included Goldmann applanation tonometry, slit-lamp examination and photography (Moorfields bleb grading system classification) and in vivo confocal microscopy (Heidelberg Retina Tomograph II, Rostock Cornea Module). Eves were classified into 3 groups: control, functioning blebs (Intraocular pressure $(\mathrm{PIO})<21 \mathrm{mmHg}$ without therapy) and nonfunctioning blebs (PIO $<21 \mathrm{mmHg}$ with therapy or $\geq 21 \mathrm{mmHg}$.

Results Biomicroscopically, functioning blebs, in comparison to nonfunctioning blebs showed a significant increase in the central $(\mathrm{p}=0,009)$ and maximal $(\mathrm{p}=0,017)$ bleb area and a decrease in central $(\mathrm{p}=0,021)$ and maximal $(\mathrm{p}=0,028)$ bleb vascularization When comparing successful with failed blebs, in vivo confocal microscopic showed a significant increased number of epithelial microcysts $(\mathrm{p}=0,014)$, a liquid content of epithelial microcysts $(\mathrm{p}=0,008)$, a lower density of stromal connective tissue $(\mathrm{p}=0,039)$ and a decreased number $(\mathrm{p}=0,041)$ and diameter $(\mathrm{p}=0,045)$ of the vessels. Al morphological findings were similar when comparing the usage of $\mathrm{MMC}$ with the usage of 5 -FU $(\mathrm{p}>0,05)$

Conclusion This study suggests that both biomicroscopy and in vivo confocal microscopy findings can lead to a better understanding and management of filtering blebs.

- 1737 / T052

Different aspects of IOP measurements with GAT versus DCT in glaucoma patients

MEIER-GIBBONS F

Private office, Rapperswil

Purpose 1. To compare the intraocular pressure (IOP) measurements with Goldmann Applanation Tonometry (GAT) versus Dynamic Contour Tonometry (DCT) in patients with glaucoma or ocular hypertension $(\mathrm{OHT})$.2. To evaluate whether the measurements of GAT versus DCT are influenced firstly by the type of glaucoma (Primary Open Angle Glaucoma, Pseudoexfoliative Glaucoma, Angle Closure Glaucoma or OHT), secondly by the type of glaucoma therapy and thirdly by the Central Corneal Thickness (CCT).

Methods Office based clinical study of 282 consecutive patients with glaucoma or OHT. Consecutive IOP measurements with GAT and DCT and CCT measurements (with ultrasound pachymetry) by one examiner.A statistical analysis evaluates whether the type of glaucoma, the type of glaucoma therapy or the CCT influence the difference between the IOP measurements with GAT and DCT.

Results Ongoing study, preliminary results show a difference between GAT and DCT in the range of other studies (DCT slightly higher in most of the patients). The type of glaucoma therapy does not seem to influence the difference between GAT and DCT, nor does the CCT or the type of glaucoma.

Conclusion The GAT is still the gold standard, but the IOP measurements with the CCT independent DCT become more and more important. Preliminary results show that in this study, unlike in another study with much less patients, the glaucoma therapy did not influence the difference between GAT and DCT.

Commercial interest
- 1736 / T050

Self-tonometry is useful to detect IOP elevations in DALK patients with apparently normal intraocular pressures

SMEDOWSKIA (1), TARNAWSKA D (1,2), WYLEGALA E (1)

(1) Clinical Department of Ophthalmology, Faculty of Medicine and Department of Dentistry in Zabrze, Medical University of Silesia, Katowice

(2) Department of Biophysics and Molecular Physics, Institute of Physics, University of Silesia, Katowice

Purpose To perform increased intraocular pressure (IOP) screening in nonglaucomatous patients after deep anterior lamellar keratoplasty (DALK) due to keratoconus with normal IOP measured during control visits.

Methods Ten non-glaucomatous patients, who underwent DALK procedure due to keratoconus were included into study. Patients were measuring IOP using self-tonometer care One (Icare, Finland) continuously for 30 days, 3 times per each day. Additionally patients were checked by ophthalmologist 3-times during follow-up time in Outpatient Clinic, where applanation tonometry and central corneal thickness measurements were performed. After 30-days, self-measured IOP was evaluated for rises above $21 \mathrm{mmHg}$ as well as rises above mean GAT values measured during control visits.

Results Mean IOP measured with GAT and adjusted according to CCT was $15 \mathrm{mmHg}$ and ranged between 11 and $19 \mathrm{mmHg}$. There were no values above $21 \mathrm{mmHg}$ reported during follow-up time. In Icare One measurements, all patients showed incidents of IOP values higher than measured with GAT, however mean values ranged between 10$18 \mathrm{mmHg}$. Six out of 10 patients revealed to have IOP elevations higher than $21 \mathrm{mmHg}$ between control visits (with maximum values up to $46 \mathrm{mmHg}$ ), and this elevated values constituted from 3.3 up to $20.0 \%$ of all self-measurements.

Conclusion Self-tonometry with Icare One might be useful tool to identify patients who develop undetectable IOP increases between control visits. This can help in prevention of vision loss and transplant procedure failure in DALK patients. 


\section{- 1741}

B27-associated uveitis, Fuchs uveitis

\section{WILLERMAINF}

Bruxelles

B27-associated uveitis is a very frequent form of non infectious intraocular inflammation which account for approximately $50 \%$ of acute anterior uveitis. Its main clinical features, natural history and association with seronegative arthritis are well known. Fuchs uveitis is another frequent cause of anterior and intermediate uveitis. Its natural history is well characterised as well as its association with intraocular production of anti-rubella antibodies. Both diseases are thus often considered as easy diagnosis. However, several aspects of those diseases remain challenging and debated. In this interactive course, based on clinical cases, we will insist on those difficult aspects as well as on the more recent issues discussed in the literature.

\section{- 1743}

\section{Behçet's disease, VKH, sarcoidosis}

KHAIRALLAHM (1), KAHLOUNR (2), BEN YAHIA S (2)

(1) Monastir

(2) Ophthalmology, Monastir

Ocular involvement associated with Behçet disease is characterized by a relapsing remitting panuveitis with diffuse vitritis, retinal infiltrates, and occlusive vasculitis. Proper management relies on the early use of immunosuppressive drugs in combination with corticosteroids and administration of biologic agent in resistant and severe posterior segment involvement. VKH disease is a bilateral panuveitis that may be associated with extraocular manifestations. Exudative retinal detachment, associated with typical imaging findings, is the most specific feature to acute VKH disease. Sunset glow fundus is typical to chronic VKH disease. Complications are more likely to occur in the chronic recurrent phase. The mainstay of treatment for acute VKH disease relies on systemic corticosteroid therapy for at least 6 months. Immunosuppressive therapy is mainly used in chronic recurrent disease. Main ocular features of sarcoidosis include bilateral granulomatous anterior uveitis, vitritis with snowballs, multifocal chorioretinitis, and segmental periphlebitis. Diagnosis may be challenging in the absence of apparent systemic involvement. Treatment of sarcoidosis is based on corticosteroids and immunosuppressive agents, in severe cases
- 1742

\section{Infectious uveitis}

PLEYERU

Berlin

The differential diagnosis of infectious uveitis is broad and an essential step in any initial work-up. Underlying organisms include all types of infectious agents. The more common infectious causes of uveitis include viruses, T. gondii, T. pallidum, Mycobacterium tuberculosis that will be covered in this course. Based on clinical features further diagnostic tools will be discussed and critically reviewed. In particular newer evolving techniques in the investigations will be included, e.g. intraocular fluid evaluation for polymerase chain testing for the genome and antibody synthesis against the causative organisms.
- 1744

White dot syndromes

HERBORTC

Lausanne

"White dot syndromes" (WDS)is a term introduced around 1995 to describe posterior uveitis syndromes that were poorly understood such as MEWDS, APMPPE, multifocal choroiditis (MFC), serpiginous choroiditis (SC), birdshot retinochoroiditis (BRC) and many others depending on the extension with which the term is used. Unfortunatelly the term is of no utility as it is purely based on the the white dots most posterior uveitis exhibit and as it emcompasses entities that look alike but have nothing in common as far as mechanism is concerned. Thanks to indocyanine green angiography (ICGA) it became possible to get away from this pot-pourri terminology and allowed to sort out choroiditis entities according to the pathophysiological mechanism subdividing choroiditis into diseases of the choriocapillaris (primary choriocapillaritis) including MEWDS, APMPPE, MFC, SC and atypical and overlapping entities on one side and stromal choroiditis on the other side including Vogt-Koxanangi-Harada disease (VKH), $\mathrm{BRC}$, sarcoid and tubercular choroiditis. The appraisal of these diseases and the rationale of their new classification will be explained and examples will be given to illustrate this new comprehensive approach that should make WDS obsolete. 
- 1745

Pediatric uveitis

BODAGHIB

Paris

The etiology and treatment of uveitis in children remains different from adults. Infectious and auto-immune conditions must be identified.Juvenile idiopathic arthritisassociated uveitis is the main etiology of chronic anterior uveitis. Pars planitis is another frequent etiology of bilateral auto-immune uveitis. On the other hand, toxoplasmic retinochoroiditis, ocular toxocariasis and cat scratch disease should be excluded in children with unilateral posterior uveitis. Case reports will be presented in order to illustrate the management of different pediatric uveitis entities. 
- 1751

A transcriptional network underlies the identity and diversity of tissue macrophages

GAUTIERE

INSERM U1166, University of Pierre and Marie Curie, Hôpital de La Pitié, Paris

We recently assessed gene expression in tissue macrophages, which do not derive from monocytes, extracted from various mouse organs and found that the diversity in gene expression among different populations of macrophages was considerable. Only a few hundred mRNA transcripts were selectively expressed by macrophages rather than dendritic cells, and many of these were not present in all macrophages. Nonetheless, well-characterized surface markers, including MerTK and F $c \gamma$ R1 (CD64), along with a cluster of previously unidentified transcripts, were distinctly and universally associated with mature tissue macrophages. We further demonstrated how these transcripts and the proteins they encode facilitated distinguishing macrophages from dendritic cells, and showed that they were turned on during monocyte to inflammatory macrophage differentiation. Furthermore, in support of the high diversity observed among tissue resident macrophages, the mRNAs encoding several transcription factors were associated with single macrophage populations and we provided evidence that Pparg and Gata6 specifically controls the homeostasis of specific tissue resident macrophage populations.

\section{- 1752}

The aging phenotype of microglia in the retina and its relationship to AMD

WONG W

Bethesda

The association between age-related retinal diseases and chronic inflammatory change in the retina raises the hypothesis that microglia, the resident immune cell in the retina, may undergo aging related changes that drive pathological change. In live imaging experiments, we discovered that "resting" microglia in the inner retina undergo aging changes in the forms of decreased ramification and slowed dynamic behavior. Also, dynamic responses to injury in aged microglia were slowed in the acute phase, but more prolonged in the chronic phase. These changes indicated that aged microglia may be less able to carry constitutive functions and have abnormal and more chronic responses to injury. Also, the age-associated translocation of microglia into the subretinal space has been associated with intracellular lipofuscin accumulation. We found that microglial uptake of A2E, a key bisretinoid component of lipofuscin, increases activation, decreases chemotaxis, and dysregulates complement activation. Taken together, aging-related changes in retinal microglia may result in increased neuronal vulnerability, dysregulated injury responses, and altered complement regulation in the outer retina that together contribute to AMD pathogenesis.
- 1753

Damaged photoreceptor cells initiate microglial activation in a mouse model of retinal degeneration

MAEDA A $(1,2)$

(1) Ophthalmology \& Visual Sciences, Cleveland

(2) Pharmacology, Cleveland

Many degenerative retinal diseases, including AMD, illustrate retinal inflammatory changes that include infiltration of microglia/macrophages into the subretinal space. We reported microglial/macrophage activation in a Stargardt disease and AMD (SGD/AMD) mouse model. Notably, when microglia/macrophages and RPE cells phagocytosed isolated photoreceptor outer segments, these cells produced cytokines and chemokines via TLR4. Inactivation of these cells by pharmacological approaches attenuated mouse retinal degeneration. PCR array analysis of 84 chemokines and related molecules revealed 84.6-fold elevated expression of Ccl3/MIP-1a after light injury in SGD/AMD mice. MIP-1 chemokines displayed a differential temporal profile compared to the other chemokines, and deficiency of $\mathrm{Ccl} 3$ uniquely modulated severity of diseases. Our study demonstrates an important contribution of TLR4-mediated monocyte activation by endogenous photoreceptor proteins in retinal inflammation that aggravates retinal cell death, and that CCL3 has an essential role in regulating the severity of retinal inflammation and degeneration in these mouse models.
- 1754

Photoreceptor toxicity of subretinal Mononuclear Phagocytes

GUILLONNEAU X, HU S, CALIPPE B, LAVALETTE S, SENNLAUB F Institut de la Vision, Paris

Growing evidences indicates that inflammation play an important role in AMD. In particular, subretinal mononuclear phagocytes (MPs) accumulate in the vicinity of the atrophic lesion of GA patients and are thought to contribute to photoreceptor degeneration. The mechanism by which MP participates to neuronal cell loss is currently unknown and no treatment is available to date to delay degeneration. We have recently shown that subretinal MPs that originate from the blood circulation and accumulate in the subretinal space are particularly detrimental in animal models of subretinal inflammation. Defects in the CX3CR1/CX3CL1 axis have been associated in animals with cardinal features of AMD including photoreceptor loss. We here show that CX3CR1 deficiency resulted in an exacerbated neurotoxicity of subretinal MPs. we present evidences that CX3CR1 /- deficiency lead to the differentiation of subretinal MPs into an exacerbated pro-inflammatory profile. Inhibiting neurotoxic mediators produced by subretinaly differentiated monocytes efficiently reduces photoreceptor loss in vitro and in animal models where MP accumulates. Our result provides new rationales to protect retina from damaging age-dependent subretinal inflammation. 
- 1755

Subretinal Mononuclear Phagocyte survival and accumulation in AMD

SENNLAUB F

Paris

The subretinal space, located between the RPE and the photoreceptor outer segments, is a zone of immune privilege as a consequence of immunosuppressive RPE signals, possibly to limit or subvert inflammatory damage. Age related macular degeneration (AMD) is associated with a breakdown of the subretinal immunosuppressive environment and infiltration and activation of mononuclear phagocytes (MPs, a family of cells that include microglial cells, monocytes, and macrophages) in both late forms of AMD. The molecular mechanisms that provoke the breakdown of local immunosuppression in AMD are unknown. Our recent data indicates that Apolipoprotein E (APOE), a lipoprotein that exists in three isoforms in human, participates in this process We demonstrate that increased APOE secretion from MPs, observed in MPs carrying the AMD-associated APOe2 allele, leads to prolonged subretinal MP survival and accumulation. We show that subretinal MP accumulation in APOe2 mice is associated with photoreceptor degeneration and excessive experimentally induced choroidal neovascularization, major hallmarks of late AMD. Our results provide a molecular mechanism of local breakdown of immuno-suppression rationale for the increased AMD-risk of APOe2 carriers 
- 1761

\section{The KORA-AGE Eye Study: Genetic Susceptibility}

GRAWJ (1), MOLNOS S (2, 3), HEIERM (2), LINKOHR B (4), BREIER M (4, 3), HOLLER (5), GRILL E (6, 7), GRALLERT H (3, 2), PETERS A (2, 3)

(1) Helmholtz Center Munich, Institute of Developmental Genetics, Neuherberg

(2) Helmholtz Center Munich, Institute of Epidemiology-II, Neuherberg

(3) Helmholtz Center Munich, Research Unit Molecular Epidemiology, Neuherberg

(4) Helmholtz Center Munich, Institute of Epidemiology II, Neuherberg

(5) Helmholtz Center Munich, Institute of Health Economics and Health Care Management, Neuherberg

(6) LMU Munich, Institute for Medical Information Processing, Biometry and Epidemiology, Munich

(7) LMU Munich, German Center for Vertigo and Balance Disorders, Munich

Purpose To estimate the genetic susceptibility of major age-related eye diseases in a population-based study in the region of Augsburg, Southern Germany (KORA).

Methods 822 persons aged 68-96 years from the KORA-AGE study were asked in a follow up 2012 in a standardized interview for the presence of major eye disorders like cataracts, glaucoma and age-related macula degeneration (AMD). In validated cases we investigated genetic susceptibility for major eye diseases by association with 31 functional candidate genes; association was calculated using logistic regression adjusted for age and gender.

Results 465 persons reported any eye disorder (57\%); $71 \%$ of them could be validated and specified. There were 68 cases of AMD and 72 cases of glaucoma; $90 \%$ of glaucoma and $93 \%$ of AMD are overlapping with cataracts resulting in 182 pure cataracts and 117 cataracts with glaucoma and/or AMD. In a recessive model, only ARMS2 (age-related maculopathy susceptibility gene 2 ) showed significant $(\mathrm{p}=0.0000175)$ association with $\mathrm{AMD}$ (OR 9.0; 95\%-CI 3.8 -21.4). This gene is present only in humans and chimpanzees, but not in rodents. Additionally, CRYBA1 (encoding $\beta A 1$-crystallin) showed an increased risk for glaucoma (OR 5.8; 95\%-CI $1.7-20.5)$, however it is statistically not significant ( $\mathrm{p}=0.144$ ). These effects might be due to a relatively small sample size.

Conclusion Age-related eye diseases frequently do not occur in their "pure" form; cataracts overlap frequently with glaucoma and/or AMD. The association of AMD with ARMS2 strongly supports previous findings of ARMS2 as a major risk gene for AMD. This study was supported by the BMBF (FKZ 01ET1003A)

\section{- 1763}

Ocular biometry and its relationship to incident myopia and hyperopia in children

ROSE K

University of Sydney, Sydney

Purpose Establish relationship of changes in ocular biometry in children with the development of refraction

Methods Sydney Myopia Study participants aged 6 and 12 years were re-examined 5-6 years later. Axial length (AL), anterior chamber depth (ACD) and corneal radius (CR) were measured using the IOLMaster (Carl Zeiss, Germany). Spherical equivalent refraction (SER) in dioptres (D) was calculated from cycloplegic autorefraction (cyclopentolate 1\%; Canon RK-F1) and myopia was defined SER $\leq-0.50 \mathrm{D}$. All measures were made at baseline and follow-up using the same methodology

Results Longitudinal data was available 860 children at follow-up, mean age 12.8 years and 1150 aged 17.2 years. There was significant change $(\mathrm{p}<0.0001)$ in SER from baseline to follow-up for both cohorts and for all biometric measures, other than ACD in the older cohort ( $\mathrm{p}=0.854)$. Marginal flattening of $\mathrm{CR}(0.05,0.02 \mathrm{~mm}$, in younger and older cohorts respectively) was not clinically significant. Change in SER was significantly correlated in both the younger and older cohorts with $\mathrm{AL}$ and $\mathrm{AL} / \mathrm{CR}(\mathrm{p}<0.0001)$ with $\mathrm{AL} / \mathrm{CR}$ the biometric parameter most strongly correlated with SER at both baseline and follow-up in both cohorts (younger $r=-0.63,0.77$, older $r=-0.81,-0.86$ respectively). In the younger cohort an $\mathrm{AL} / \mathrm{CR}$ ratio $\leq 2.75$ was associated with a baseline refraction of $\geq+2 \mathrm{D}$ in all cases and $87.5 \%$ of these remained significantly hyperopic at age 12 .

Conclusion Baseline AL/CR was the best biometric measure for predicting 5 year incidence myopia in both cohorts. In principle $\mathrm{AL} / \mathrm{CR}$ also has the potential to resolve the ambiguity of visual acuity measures for the detection of hyperopia in younger children using a non-invasive technique.

\section{- 1762}

Assessment of ocular surface microbiota in keratoconus patients - a pilot study

GAJECKA M (1, 2), MATYSIAKA (1), KUBICKA M (1), SZAFLIKJP (3)

(1) Department of Genetics and Pharmaceutical Microbiology, Poznan University of Medical Sciences, Poznan

(2) Institute of Human Genetics, Polish Academy of Sciences, Poznan

(3) Department of Ophthalmology II, Medical Faculty, Medical University of Warsaw, Warsaw

Purpose Both genetic and environmental factors are associated with keratoconus (KTCN). The hypothesis of this project is that genetic factors together with environmental aspects, including the presence of specific ocular surface (OS) microbiota, influence the etiology of KTCN. Currently numerous studies focus on describing the variant microbe populations that occur in specific disease states, or the temporal microbial changes that are observed over the course of a disease. Microbiota at KTCN eve has not been evaluated so far. Therefore, the study question is whether there is a causal link between eye microbiome variation and KTCN.

Methods Because of inter-individual microbiota variation revealed at the same anatomical sites, only male individuals in similar age (30-40 yrs old) are being ascertained. Individuals are divided into four study subgroups ( $\mathrm{n}=30$ each): 1) patients with bilateral KTCN, 2) patients with KTCN and atopy, 3) patients with atopy, and 4) individuals without both KTCN and atopy. Culture-based microbiology methods are used in the investigation to preliminary examine the OS microbiota. Simultaneously, microbial genetic material is extracted from the eye swabs for further qualitative and quantitative analyzes using molecular biology methods (real-time PCR, NGS).

Results Preliminary results indicate a diversity of OS microbiota in both healthy and affected individuals. The most common isolated bacteria were Staphylococcus epidermidis, Corynebacterium spp., and Propionibacterium spp. Detailed profiles of OS microbiota in the studied groups are under investigation.

Conclusion The results of this project will widen current knowledge about environmental aspects of KTCN etiology.Support: National Science Centre in Poland, Grant no. 2012/05/E/NZ5/02127.

\section{- 1764}

Use of antibiotic-resistance-free plasmids for non-viral transfection of pigment epithelium-derived factor in rat primary cells

GARCIA GARCIA L (1), RECALDES (1), FERNÁNDEZ-ROBREDO P (1), HERNANDEZ M (1) RODRIGUEZ IR (2), BEZUINARTEA J (1), MARIE C (3) SCHERMAND (3), IZSVAKZ (4), JOHNEN S (5), THUMANNG (6), GARCIA-LAYANA A (1)

(1) Experimental Ophthalmology Laboratory, School of Medicine, Clinica Universidad de Navarra, Pamplona

(2) Cell Therapy Area.Division of Cancer. Center for Applied Medical Research (CIMA), Pamplona

(3) Unité de Technologies Chimiques et Biologiques pour la Santé, INSERM U1022 CNRS UMR8258, Paris

(4) Max Delbrück Center for Molecular Medicine, Berlin

(5) Department of Ophthalmology, University Hospital RWTH Aachen, Aachen

(6) Département des neurosciences cliniques, Service d'ophtalmologie, Hôpitaux universitaires de Genève, Genève

Purpose We aim to assess the pigment epithelium-derived factor (PEDF) production in cells after transfection with plasmids free of antibiotic resistance markers (pFAR4) in a non-viral Sleeping Beauty transposon system (SB100X).

Methods ARPE-19 cell line and primary rat iris and retinal pigment epithelial (IPE and RPE) cells were transfected with SB100X transposase and PEDF transposon encoding pFAR4 plasmids (1:16 ratio). Control cells were transfected with SB100X and pFAR4Venus plasmids. Supernatants were taken every 24 hours for kinetics. The effect of transfecting different amount of cells was also analyzed. PEDF production was tested by Western blot and ELISA

Results A cumulative PEDF secretion over time in ARPE-19 and primary cells was shown, reaching almost 3-fold higher PEDF production in ARPE-19 cells. Control cells did not express recombinant PEDF, but showed fluorescence. The transfection of 10,000 cells clearly showed higher PEDF expression than cells that were transfected as a group of 70,000 and subsequently divided.

Conclusion Rat primary cells are efficiently transfected and produced PEDF as it happens in ARPE-19 cell line. Merging of the SB100X and pFAR4 technologies led to increased PEDF expression and improved safety by avoiding the transfer and potential integration of antibiotic resistance genes. This study demonstrates the feasibility of our approach based on ex vivo transfection of a low number of primary autologous pigment epithelial cells. 
- 1765 / T072 new obesity-related genetic loci are associated with advanced AMD

PATERNO JJ (1), HELISALMIS (2), TOKARZ P (3), BLASIAKJ (3), HILTUNENM (2) UUSITUPA M (4), KAARNIRANTA K (1)

(1) Department of Ophthalmology, University of Eastern Finland (UEF), Kuopio

(2) Department of Neurology, University of Eastern Finland (UEF), Kuopio

(3) Department of Molecular Genetics, University of Lodz, Lodz

(4) Department of Clinical Nutrition, University of Eastern Finland (UEF), Kuopio

Purpose AMD is the leading cause of central blindness in the elderly in Western countries. It has a complex multifactorial etiology, including aging, genetic factors, smoking, hypertension and atherosclerosis. Moreover, obesity has been considered to increase the risk for AMD. We hypothesize that the genetic variations associated with obesity might also be involved in the pathogenesis of AMD.

Methods Cross-sectional clinical data from 348 advanced AMD cases and 722 controls genotyped 40 newly associated obesity related loci using the Sequenom iPlex platform. Results Of the 40 SNPs analysed, PTBP2 (rs1555543), GNPDA2 (rs10938397), HOXC13 (rs1443512) and MAP2K5 (rs2241423) showed a nominally ( $<<0.05)$ significant association with advanced AMD in this local Finnish population.

Conclusion These new findings suggest that the link between AMD and obesity could be partly explained by genetic factors. Besides providing new insights into pathogenic mechanisms of AMD, these findings may also help to reveal possible targets for therapeutic intervention and useful diagnostic biomarkers in this disease.
Biomarkers of Age-Related Macular Degeneration (AMD): Four were collected. All subjects were over 65 years old, and without diabetes mellitus. WWe

- 1766 / T078

Analysis of Keratoconus genetic factors within Keratoconus Loci and mtDNA

NOWAKDM (1, 2), KAROLAKJA (2, 1), KUBICKA M (2), KULINSKA K (2, 3 , POLAKOWSKIP (4) SZAFLIKJP (4) GAJECKA M (2 1)

(1) Institute of Human Genetics, Polish Academy of Sciences, Poznan

(2) Department of Genetics and Pharmaceutical Microbiology, Faculty of Pharmacy, Poznan University of Medical Sciences, Poznan

(3) Department of Experimental Anaesthesiology, Poznan University of Medical Sciences, Poznan

(4) Department of Ophthalmology II, Medical Faculty, Medical University of Warsaw, Warsaw

Purpose Keratoconus (KTCN) is a multifactorial disorder in which both environmental and genetic factors are involved. Genetic studies have led to the identification of several loci on different chromosomes, linked to KTCN. However, only few reports indicated causative genes in these loci. The aim of this project was to analyze the DNA sequence information available in the databases of SNVs located within known KTCN loci. We extended the analyzes to include miRNA genes located. Additionally, sequencing of mitochondrial genome in $\mathrm{KTCN}$ patients from Polish population was performed.

Methods The list of SNVs, which are located in KTCN loci were obtained on the basis of Ensemble. For analyzed SNVs allele frequencies were obtained from 1,000 Genomes Project. The list of miRNA genes was obtained from the miRBase. The potential impact of nonsynonymous amino acid substitutions on protein structure and function was assessed with PolyPhen and SIFT. Additionally, mtDNA was analyzed in samples from 93 people with the Polish population (42 - KTCN, 51 - controls)

Results In this study 94 miRNA-encoding genes and over 2 million SNVs were identified within known KTCN loci. From these SNVs, over 4,500 were located within exons and almost $600 \mathrm{SNVs}$ were indicated as deleterious. Sequencing analysis of fragments of the mitochondrial genome revealed a number of changes, including several new polymorphisms.

Conclusion The KTCN development does not depend on a single change in the gene, but on the accumulation of numerous sequence variants. The complexity of KTCN etiology causes the need to find appropriate approach to investigate this disease. Support: National Science Centre, Poland, grant no. 2011/03/N/NZ5/01470

- 1768 / T074

- 1767 / T069

Visual dysfunction and pupillary responses are dissociated in the Opa3 mutant mouse with retinal degeneration

VOTRUBA M (1, 2), DAVIES JR (1), DOUGLAS RH (3), DAVIES VJ (1)

(1) Cardiff Centre for Vision Sciences, Cardiff University, Cardiff

(2) University Hospital of Wales Eye Department, Cardiff

(3) Department of Optometry \& Visual Sciences, City University, London

Purpose To assess the affect of the missense mutation p.L122P in a mouse model of 3-methylglutaconic aciduria (MGA-III), a neuro-metabolic syndrome which presents with retinal and optic atrophy and neurological impairment.

Methods Visual acuity was quantified in an optokinetic nystagmus drum by increasing the spatial frequency of the grating until an optomotor response could not be elicited. Pupillary light responsiveness was assessed by video pupillometry. Time course and phenotype of retinal degeneration was examined using haematoxylin and eosin and terminal dUTP transferase nick end labelling.

Results Opa3+/+ and Opa3+/- mice showed normal visual acuity by tracking a $2^{\circ}$ grating. However, Opa3-/- mice displayed no optomotor response at any grating frequency. Despite this, their pupil response was little affected. Pupil response/intensity curves of Opa3-/- and Opa3+/+ mice diverged somewhat at lower intensities although they only differed significantly at two irradiance levels. At 50\% constriction Opa3-/mice were only $0.61 \log$ units less sensitive than wildtypes. Histology showed panretinal degeneration in adult Opa3-/- mice and TUNEL revealed increased cell death in postnatal and adult Opa3-/-retinae

Conclusion The Opa3-/- mouse is a useful model of the human disease. Retina atrophy in Opa3-/- animals is associated with widespread retinal thinning. However, dissociation between visual perception and pupillary function implies that the intrinsically photosensitive retinal ganglion cells (ipRGCs) subserving pupillary function may be less susceptible to damage by mutations of Opa 3 than the retinogeniculate fibres underlying visual perception. and $B C L-2$ genes in patients with Retinal Detachment with and without Proliferative Vitreoretinopathy. The Retina 4 project

GONZALEZ-BUENDIA L (1), PASTOR-IDOATES $(1,2)$

RODRIGUEZ-HERNANDEZI (2, 3), ROJASJ (1),

GONZALEZ-SARMIENTOR (2,3), PASTORJC (1)

(1) Instituto de Oftalmobiologia Aplicada (IOBA), University of Valladolid, Valladolid

(2) Unidad de Medicina Molecular, University of Salamanca, Salamanca

(3) Instituto de Biologia Molecular y Celular del Cancer (IBMCC). Consejo Superior

de Investigaciones Cientificas (CSIC). Instituto de Investigacion Biomedica de Salamanca (IBSAL), University of Salamanca, Salamanca

Purpose To compare the distribution of BAX G(-248)A and BCL-2 C(-938)A genotypes among European subjects undergoing rhegmatogenous retinal detachment surgery in relation to further development of proliferative vitreoretinopathy (PVR).

Methods A case-control gene association study as part of the Retina 4 Project was designed. Two promoter single nucleotide polvmorphisms (rs2279115 and rs4645878) were analysed by TaqMan 5'exonuclease allelic discrimination assay, using a StepOne system in 134 samples from patients with PVR and 421 without PVR. Proportions of genotypes and AA homozygote groups of these polymorphisms were analysed. Genotypic and allelic frequencies were compared in global sample and in sub-samples. Results BAX gene: In the comparison of proportions of genotypes in Spain, Spain+Portugal and in the global sample, significant differences were found. The odds ratio (OR) for A carriers in Spain and Spain+Portugal was 1.8, and 1.7 in the global sample. BCL-2 gene: Significant differences were observed regarding proportions of genotypes in Spain+Portugal. Furthermore, a protective effect was found in the analysis of A carriers from Spain+Portugal with an OR of 0.6 .

Conclusion The A allele of BAX is associated with a higher risk of developing PVR, pathway) is proposed as a possible new target in PVR prophylaxis. suggesting that a down-regulation in the apoptosis pathway could be an important key in PVR pathogenesis. Additionally, the role of BCL-2 gene (inhibitor of necroptosis 


\section{- 1771}

Optical and morphological characterization of a 3D printed intraocular lens

DEBELLEMANIERE G, FLORES M, MONTARD M, DELBOSC B, SALEH M Service d'Ophtalmologie, CHU Jean Minjoz, Besançon

Purpose Three-dimensional printing (or "additive manufacturing") is a technology which allows to create a three dimensional object by building it layer by successive layer It is especially interesting for objects that are produced in little series and that require a high degree of customization, as it allows economies of scale and potentially unlimited design possibilities. Those characteristics are particularly relevant to intraocular lenses used in cataract surgery, in a personalized medicine approach. Despite technical barriers, additive manufacturing of good quality lenses without post-processing is now achievable. We aimed to determine if the historical Ridley lens was able to be reproduced with current $3 \mathrm{D}$ lens printing technology

Methods We realized a digital reproduction of the Ridley's IOL using a 3D modeling program. Lenses were then printed. No post-processing was used. Photographs and electron microscopy images of the printed lens were obtained. Dimensional measurements, weight and dioptric power were determined.The data was compared with the data available in the literature concerning the original Ridley's intraocular lens.

Results The morphological study showed a shape similar to the original lens Transparency was satisfying. A good optical surface regularity was obtained. The lens power was 1.31 diopters different than expected, due to inaccuracies in curved surfaces printing, however general properties of a lens were achevied.

Conclusion This is the first attempt to produce an intraocular lens using a 3D printer Despite major technological barriers, 3D printing of optical lenses could have important implications in tomorrow's ophthalmic surgery by allowing potentially unlimited optical and morphological lens designs.
- 1773

\section{Strategies to reduce cytokine levels could suppress PCO progression}

ELDRED JA (1), SPALTON DJ (2), WORMSTONE IM (1)

(1) UEA, Norwich

(2) King Edward VII Hospital, London

Purpose Posterior capsule opacification (PCO) develops after cataract surgery. Open bag IOLs, in contrast to current IOL devices, separate anterior and posterior capsules (PC) and are proposed to aid wash out of cytokines in the capsular bag that may promote PCO. The current study assessed the correlation between cytokine availability and cell growth on the human capsular bag.

Methods To assess cell growth in different volumes (1.5 Vs 6ml) of Serum-Free (SF) EMEM we employed a human capsular bag model. We further adapted the capsular bag system, such that radial incisions were made in the anterior capsule, which was then folded back and secured to a dish (a fully open bag model). Cell growth was observed by phase-contrast microscopy and quantified by Imagel software. Cytokines were detected by Bioplex suspended bead array at culture day 2. Immunocytochemistry was used to detect myofibroblasts (using the marker alpha smooth muscle actin; $\alpha \mathrm{SMA}$ ) and cell density.

Results Increasing media volume significantly reduced the cell coverage rate on the central PC. A significant cytokine reduction was observed $(9$ of the 27 assessed) in $6 \mathrm{~m}$ cultures compared to $1.5 \mathrm{ml}$ counterparts; no cytokines were significantly elevated. At end-point (28 days) capsular bags maintained in $6 \mathrm{ml}$ of media exhibited decreased cell density in the peripheral PC and reduced $\alpha$ SMA expression. Fully open capsular bags cultured in 1.5 or $6 \mathrm{ml}$ SF EMEM had limited cell progression onto the PC in both volumes. Fully open capsular bags exposed to $5 \%$ human serum demonstrated rapid colonisation of the PC

Conclusion Reduced cytokine availability is associated with impaired cell growth on the capsular bag. It is likely that open bag devices will restrict cytokine levels and improve the management of $\mathrm{PCO}$
- 1772

Radiation effects on light scattering and cataract formation in the lenses of interventional radiologist and cardiologists

WEGENERA (1), HÖCKA (1), SCHEIDEMANN-WESP U (2), CÁMARA R (2), HAMMER GP (3), VOSSMERBÄUMER U (4), LASER-IUNGA H (1), SINGER S (2)

(1) Universitätsklinikum Bonn, Augenklinik, Bonn

(2) Medizinische Biometrie, Epidemiologie und Informatik, Mainz

(3) Laboratoire National de Santé, Luxembourg

(4) Johannes Gutenberg Universität, Augenklinik, Mainz

Purpose There is little information about exposure to dosages below $500 \mathrm{mSv}$. This pilot study aimed at investigating the effects of low dose radiation on lens transparency in occupationally exposed interventional cardiologists and radiologists.

Methods Physicians who have performed fluoroscopically guided procedures for at least 5 years were recruited in the areas of Cologne-Bonn and Rhine-Main together with their non-exposed colleagues. A questionnaire has been developed to estimate occupational exposure. Exclusion criteria comprised corticosteroid treatment, radiotherapy, chemotherapy and glaucoma. Changes in lens transparency and cataract formation were determined by slit lamp microscopy including LOCS III grading, slit lamp photography and Scheimpflug/Pentacam" imaging.

Results The prevalence of lens opacification mainly found in the nuclear region was $52 \%$ (95\% CI: 30-74\%) among interventional physicians and 31\% (95\% CI: 9-61\%) among their non-exposed colleagues. Densitometric analysis of the cornea demonstrated a mean value $52,8 \%$ (SD: 7,4) in exposed participants compared to $45,9 \%$ (SD: 7,2) in non-exposed colleagues. In the lenses layers 4 (deep cortex) and 5 (supra-nuclear layer) evidenced higher mean densities (40,5\% (SD: 3,9\%) vs. 31,6\% (SD: 6,9\%) in layer 4 and $27,5 \%$ (SD: $1,9 \%$ ) vs. $23,7 \%$ (SD: 4,3\%) in layer 5 ).

Conclusion The data from the ophthalmological examinations demonstrated generally normal findings. However, classification of the mostly small cataracts and densitometric analysis of cornea and lens evidenced that low dosage exposure to ionizing radiation has an effect on corneal and lenticular transparency, albeit on a subclinical level.

- 1774

Safety and efficacy of intra-operative sub-Tenon injection of $2 \%$ lidocaine in cases of unexpected intra-operative floppy iris syndrome (IFIS)

KLYSIK A (1), KORZYCKA D (2)

(1) Department of Ophthalmology. Medical University of Lodz., Lodz

(2) Department of Ophthalmology. Hospital of the Ministry of Internal Affairs and Administration, Lodz

Purpose To report safety and efficacy of intra-operative injection of $2 \%$ lidocaine during small incision cataract surgery in cases of unexpected intra-operative floppy iris syndrome.

Methods 4 patients, undergoing routine cataract surgery, who were exposed to alfa-adrenergic inhibitors, and it was not known pre-operatively, and in whom intraoperative floppy iris syndrome occurred unexpectedly. $2.5 \mathrm{ml}$ of $2 \%$ lidocaine was injected into the sub-Tenon space in response to intra-operative iris prolapse and pupillary constriction. Injection was performed following hydrodissection in 3 of 4 cases and hollowing capsulorrhexis in 1 of 4 cases. The surgery was video-taped from the beginning of the injection.

Results No further iris prolapse was noted in any case, nor further pupillary constriction. Iris plane was stabilized. There were no further complications of cataract surgery. Patients were comfortable and pain free for the rest of the procedure.

Conclusion Intra-operative injection of $2 \%$ lidocaine into the sub-Tenon space is a safe way of reversing iris floppiness and the tendency to iris prolapsed in cases of unexpected intra-operative floppy iris syndrome. It is an alternative to other intra-operative techniques to manage IFIS, which provides pain relief as well as iris stabilization. 
- 1775

Topical caffeine induces mydriasis in animals under ketaminexylaxine anesthesia

SÖDERBERG P, YUZ, TALEBIZADEH N, KRONSCHLÄGER M Gullstrand lab, Ophthalmology, Dept. of Neuroscience, Uppsala university, Uppsala

Purpose To determine the effect of topically applied caffeine on the pupil diameter during ketamine-xylazine anesthesia

Methods Six-week-old Sprague-Dawley rats were anesthetized with ketamine xylazine intraperitoneally. Caffeine, $72 \mathrm{mM}$, dissolved in vehicle, $9 \mathrm{mg} / \mathrm{ml}$, hydroxypropylmethyl cellulose dissolved in water, or the vehicle only, was topically applied. Animals were kept under room light conditions. In experiment I, caffeine in vehicle was topically applied to one eye and vehicle only to the other eye in 10 animals. In experiment II, caffeine in vehicle was applied to both eyes in one group of 10 animals and vehicle only to another group of 10 animals. In both experiments, pupil diameter was measured on both eyes. Measurements were taken immediately after initiation of the anesthesia, and 10, 20, 40 and $60 \mathrm{~min}$. later

Results In experiment I, vehicle only caused a slight transient increase of pupil diameter, peaking around $20 \mathrm{~min}$. after initiation of anesthesia and then decreased below baseline. Caffeine in vehicle caused an initial fast decrease in pupil diameter and the decrease rate leveled out towards $60 \mathrm{~min}$. after initiation of anesthesia. In experiment II, vehicle to both eyes induced a transient increase in pupil diameter that returned to baseline. Caffeine and vehicle on both eyes induced an exponentially declining decrease towards asymptote miosis. The miosis reversed to mydriasis after topical application of tropicamide $5 \mathrm{mg} / \mathrm{ml}$

Conclusion Topically applied caffeine induces miosis in a ketamine-xylazine anesthetized rat. There is some systemic effect on pupil diameter of the currently used topical application of caffeine. The miotic effect of topically applied caffeine is reversed by tropicamide.

- 1777 / S097

Comparison of optical and psychophysical methods to measure intraocular straylight

PENNOS A, GINIS HS, ARTALP

Laboratorio De Optica, University of Murcia, Murcia

Purpose To quantify intraocular forward scattering (straylight) by using three different methods in a group of normal subjects.

Methods Intraocular straylight was measured in the dominant eye of 10 emmetropic subjects without any known ocular pathology ( $30 \pm 5$ years).Two different, commercially available, scatter filters (Black Pro-Mist 1 $1 / 4$ and Black Pro-Mist $1 / 2$ from Tiffen) where used in order to recreate the effects of higher scattering. Three measurement techniques were used:a commercially available straylight meter (C-quant, Oculus),a prototype of an optical double-pass based method (Sigma) and a psychophysical method that we developed.We used a Bayesian method (quick-CSF) in a range of spatial frequencies (2 to 20 cycles/degree) with 50 2-alternative forced choice trials.Each set of trials was repeated 2 times for each condition (with and without glare).As a glare source, a fluorescent lamp was positioned concentrically to the test area where the sinusoida gratings were projected.All tests were presented on a screen placed at 1 meter distance from the subject and the test area was covering a total of 6.4 degrees of visual field.For Sigma and C-Quant tests,the logarithm of Scatter coefficient was determined, whereas for the q-CSF measurements the difference between the areas below the log contrast sensitivity curves was used as scatter metrics.

Results In the three methods, the effects of straylight were accordingly increasing with the addition of the scatter filters. A correlation between all three methods was verified. Conclusion These results suggest the potential use of an even simplified method based on the q-CSF to evaluate straylight complementing other existing methods
- 1776 / S091

Change in intraocular pressure in the early period after cataract phacoemulsification

YUGAY MARIA, RYABTSEVA ALLA

Moscow Regional Research Clinical Institute, Moscow

Purpose Described decrease of IOP after phaco is $2-5 \mathrm{~mm} \mathrm{Hg}$. At the same time, in the early period after cataract extraction frequent increase in IOP is described. The purpose of our study was to analyse the dynamics of IOP after uncomplicated phacoemulcification

Methods The study included 67 eyes of 66 patients who underwent uncomplicated phaco with foldable IOL implantation . All patients underwent tonography resulting tonographic IOP (Po) and ease outflow coeffitient $(\mathrm{C})$, a study of corneal - compensated IOP (IOPcc) and Goldmann IOP (IOPg) with Ocular Response Analyzer. Which were repeated before surgery, the first two days , 2 weeks , 1 and 3 months after operation.

Results Average Po before surgery was 15,67 $\pm 0,85 \mathrm{~mm} \mathrm{Hg}$, IOPcc 15,91 $\pm 0,69 \mathrm{~mm}$ $\mathrm{Hg}$ and IOPg $14,51 \pm 0,78 \mathrm{~mm} \mathrm{Hg}$. On the first day after surgery IOPcc increased by 2,84 $\mathrm{mm} \mathrm{Hg}$, and IOPg by $1.53 \mathrm{~mm} \mathrm{Hg}$. Two weeks after surgery Po decreased from baseline by $3.45 \mathrm{~mm} \mathrm{Hg}$, IOPcc still increasing by $0.62 \mathrm{~mm} \mathrm{Hg}$, IOPg also further increased by $1.44 \mathrm{~mm} \mathrm{Hg} .1$ month after surgery Po virtually unchanged compared with data from 2 weeks, IOPcc returned to preoperative values, IOPg reduced compared with the preoperative figures by $1.0 \mathrm{~mm} \mathrm{Hg}$. Finally, after 3 months Po became lower then preoperative by $3.23 \mathrm{~mm} \mathrm{Hg}$, IOPcc reduced compared with preoperative by $1.51 \mathrm{~mm}$ $\mathrm{Hg}$, and IOPg by $1.92 \mathrm{~mm} \mathrm{Hg}$

Conclusion IOP rise after phaco begins on the first postoperative day, reaching maximum in 2 weeks. Then towards the end of the first month after surgery IOP returns to preoperative values. IOP reduction 1,5-2 $\mathrm{mm} \mathrm{Hg}$ starts after decrease of postoperative reactive syndrome 1 month after surgery 


\section{THURSDAY \\ OCTOBER 2 \\ 2014}


- 2211

Contribution of "en face" SD-OCT in inflammatory diseases

WOLFF B (1), VASSEUR V (1), BASDEKIDOU C (1), DE BATS F (2), SAHEL J (1), MAUGET-FAYSSEM (1)

(1) Paris

(2) Lyon

To evaluate the retinal and choroidal changes in White Dot syndromes (WDS) using "en face" and B-scan OCT images. All the study patients underwent a comprehensive ophthalmologic examination including biomicroscopic fundus examination, color photography, fundus autofluorescence, fluorescein angiography, indocyanin green angiography and B scan and "en face" SD-OCT.A total of 10 patients with Multiple evanescent white dot syndrome (MEWDS), 4 cases diagnosed with acute posterior multifocal placoid pigmentepitheliopathy (AMPPE), 1 case of serpiginous choroidopathy, 4 cases of multifocal choroiditis, 3 cases of punctuate inner choroidopathy (PIC) and 3 cases diagnosed with Birdshot disease were analysed and included in the study. The B-scan findings were correlated with the angiography findings as well as with the "en face" OCT scans. In all the cases the "en face" OCT, providing a laver-by-layer view of the involved retina, enabled depicting in detail the IS/OS envolvement and the extent of the outer retinal damage. The patho-physiologic mechanism of the WDS remains poorly understood. All these entities probably share a common pathway leading to external retinal involvem

- 2213

\section{Contribution of "en face" SD-OCT in retinal and choroidal} vascular abnormalities

SCEMAMA TIMSIT C, MANASSEHN, MAUGET-FAYSSEM, VASSEUR V, WOLFF B, SAHEL J

Paris

PURPOSE : To evaluate the retinal and choroidal aspect in retinal or choroidal vascular abnormalities using "en face " OCT images.METHODS : All the study patients underwent a complete ophthalmologic examination including biomicroscopic fundus examination, color photography, fundus autofluorescence, fluorescein angiography, indocyanin green angiography, B-scan and « en face " SD-OCT.RESULTS : A total of 11 patients with choroidal or retinal vascular abnormalities, 4 cases diagnosed with submacular choroidal varix, 2 cases of central serous chorioretinopathy with choroida cavitations, 2 cases of retinal vein occlusion (RVO) with perivenular whitening and retinal ischemia, 3 cases of retinal arterial macroaneurysm were analysed. The $\mathrm{B}$ scan findings were correlated with the angiography findings as well as with the " en face " OCT scans. In all the cases the " en face » OCT providing a layer-by laver view of the involved retina and choroid (with EDI mode: enhanced-depth imaging) CONCLUSIONS : " En face " OCT imaging enables an easy analyse of the structural retinal and choroidal vascular abnormalities which are correlated with B scan OCT and angiography findings.

\section{- 2212}

Contribution of "en face" SD-OCT in MacTel type 2

\section{BASDEKIDOUC}

Paris

Purpose:To comprehensively evaluate the retinal and choroidal changes in eyes with type 2 idiopathic macular telangiectasia (MacTel) using "en face" and B-scan SDOCT.Methods:Eyes with a diagnosis of proliferative or nonproliferative type $2 \mathrm{MacTel}$ were prospectively studied. All the study patients underwent a comprehensive ophthalmologic examination including biomicroscopic fundus examination, color photography, fundus autofluorescence, fluorescein angiography, and B scan and "en face" SD-OCT.Results: 20 eyes of 10 patients were included in this study. "En face" OCT C-scans and conventional B-scans were both able to show inner crystalline deposits $(15 \%)$, retinal capillary anomalies $(100 \%)$, intraretinal cysts $(80 \%)$, hyperreflective spots in the outer nuclear layer $(100 \%)$ and external limiting membrane (80\%), hyperplastic pigment plaques (30\%), intraretinal neovascularization (20\%), photoreceptor loss (100\%) and choroidal cavitations (30\%). "En face" OCT C-scans provided more information than B-scans on intraretinal neovascularization, photoreceptor loss and Choroidal cavitations. "En face" OCT is a non-invasive, and reproducible technique that helps to better assess and follow up retinal and choroidal processes in type $2 \mathrm{MacTel}$

- 2214

Contribution of "en face" SD-OCT in retinal surgical diseases

CHIOUET C (1, 2), GALLICE M (1)

(1) Department of Ophthalmology, University Hospital of Grenoble, Grenoble (2) UJF-Grenoble 1, Grenoble

En face OCT is complementary Imaging procedure useful for the analysis of vitreoretinal disorders, including macular hole, epiretinal membrane, or retinal detachment. This procedure is informative before and after vitreoretinal surgery. Before surgery, characteristics of the retinal surface may be defined. After vitrectomy, en face OCT reveals changes in the retinal nerve fibers layer (dimples), residual epiretinal tissue, cystic changes, disruption of external limiting membrane and/or outer segment laver, or persistent subretinal fluid. This procedure helps us to refine high resolution imaging of each layer of the retina during the healing process of macular hole or retinal detachment. 
- 2215

Contribution of "en face" SD-OCT in retinal toxicity

CAHUZACA, VASSEUR V, PAQUES M, WOLFF B, MAUGET-FAYSSE M

Paris

PURPOSE: To assess retinal and choroidal changes in drug-induced retinal toxicity using "en-face" and BScan OCT images.METHODS: All patients underwent complete ophthalmologic examination, including fundus examination and autofluorescence, color photography, fluorescein and indocyanin green angiography. Type, amount and time of drug intake was precisely assessed.RESULTS: 8 patients were analysed and included retrospectively. Retinal toxicity was induced by tamoxifen in 2 cases, by synthetic antimalarial (SAM) drug in 3 cases and by inhaled amyl nitrite ("poppers") in 3 cases. The angiographic features, B-Scan and "en-face" OCT scans findings were correlated. In all cases, "en-face" OCT provided precise anatomic location of the retinal layers involved, the extend of retinal damage, as well as follow-up of these changes. CONCLUSION: "En-face" OCT allows full imaging and better understanding of retinal toxicity induced by tamoxifen, SAM drugs and amyl nitrite. 
- 2221

The role of ocular perfusion pressure in glaucoma

TOPOUZISF

Thessaloniki

In hemodynamics, perfusion pressure (PP) is the driving force for blood flow determining the nutritive delivery of arterial blood to capillary beds. When describing blood flow for an organ, PP is generally expressed as the difference between the arterial and the venous pressure. In the eye, OPP is calculated as systemic blood pressure, which is a surrogate for ocular arterial pressure, minus IOP, which conventionally equals ocular venous pressure.There is increasing evidence on an association between low Ocular Perfusion Pressure (OPP) and glaucoma. Population-based studies conducted in different geographical locations and in populations of different ethnic and socioeconomic backgrounds have consistently reported that low diastolic OPP is associated with the prevalence of OAG. In addition, low OPP at baseline presented with increased risk of developing glaucoma in incidence studies. The presentation will discuss whether the existing evidence could be translated in clinical practice and how further research could better define the role of OPP in glaucoma pathogenesis, risk factors assessment and management.

- 2223

Plasmalogens, astrocytes, and retinal vascular development

ACARN (1), SAAB S (1), CREUZOT-GARCHER C (2, 1), BRONAM (2, 1), BRETILLONL (1)

(1) Eye and Nutrition Research Group, INRA, UMR CSGA, Dijon

(2) Department of Ophthalmology, University Hospital, Dijon

Sufficient oxygen and nutrient supplies to the retina are dependent on a correc development of blood vessels. Polyunsaturated fatty acids (PUFAs) were shown to modulate factors involved in tissue vascularization. A congenital deficiency in etherlinked phospholipids also termed as "plasmalogens" was shown to lead to abnormal ocular vascularization. Because plasmalogens are considered as reservoirs of PUFAs, we investigated whether they may influence retinal vascular development through the release of fatty acids by a specific phospholipase A2 (PLA2). Studies on mouse models of plasmalogen deficiency and of retinal PLA2 inhibition have shown that plasmalogens regulate retinal angiogenesis during early phases of retinal vascular development by influencing glial activity and primary astrocyte template formation. Subsequent modifications in the expression of genes involved in retinal angiogenesis impacted endothelial cell proliferation and retinal vessel outgrowth. These early defects in retinal vascular development led to a disorganized and dysfunctional retinal vascular network at adult age. These data suggest a crucial role of plasmalogens in retinal vascularization processes through a signalization involving PUFAs and PLA2.
- 2222

The coupling between neuronal activity and blood flow What can we learn about glaucoma?

GARHOFER G

Department of Clinical Pharmacology, Vienna

The coupling between neural activity and blood flow is an essential physiological mechanism to assure constant supply of the tissue with oxygen and nutrients. Although the exact molecular mechanisms are currently still focus of research, it has been shown that several ocular diseases are accompanied by a breakdown of neuro-vascular coupling. As such, it has been shown that in patients with early stage glaucoma, the hyperemic response to visual stimulation is reduced. The talk aims to summarize our current knowledge on the physiological importance of neuro-vascular coupling. In addition, the potential underlying physiological mechanism will be covered. Finally the role of neuro-vascular coupling and its potential application in the diagnosis and follow up in neuro-degenerative diseases such as glaucoma will be discussed.

Commercial interes

2224

Ocular rigidity: Biological importance and clinical implications

SCHMETTERER L $(1,2)$

(1) Medical Physics and Biomedical Engineering, Vienna

(2) Clinical Pharmacology, Vienna

Ocular rigidity is a biomechanical property of the eye coats that depends on the relationship between pressure and volume changes in the eyeball. Generally, the relation between volume and pressure changes in the eye is characterized by the Friedenwald equation and its modifications. In glaucoma, alterations in the biomechanical properties of the eye coats have been hypothesized to play a critical role. The basic concept is hat dependent on the stiffness of the eyeball the individual eve can tolerate higher or lower intraocular pressures. This concept has received support from model calculations as well as from animal studies. In humans, however, assessment of ocular rigidity is difficult. The present talk will summarize techniques that have been proposed for the measurement of the ocular volume and pressure pulse and discuss clinical applicability. 
- 2231

An update on corneal bacterial infections

GICQUELJ

Poitiers

Because of their potential to permanently impair vision or perforate the eye, bacterial corneal ulcers are an ophthalmologic emergency. They usually follow an insult (sometimes minor) in the corneal epithelium that provides an entry for bacteria. The increased use of soft contact lenses in recent years has led to an important rise in the occurrence of bacterial ulcers (especially aggressive Gram- bacteria). The goa of this presentation is to propose a practical, systematic approach of the management of bacterial corneal ulcers, as well as an update on new concepts in the diagnosis and treatment of those conditions.
- 2232

Corneal infectious diseases in children

BREMOND-GIGNACD $(1,2)$

(1) Ophthalmology, University Hospital, Amiens

(2) CNRS, FR3636, Paris

Corneal infections in children can affect subjects of all ages with a high frequency in newborns and infants. Severe visual impairment can result from corneal infectious disease especially if late diagnosed with delayed treatment. In infant, children and teenagers the most common ocular pathogens, that differ from the adult, are Haemophilus Influenzae, Staphylococcus aureus, Streptococcus pneumonia and also Moraxella species. These infections infections could lead to ulcers and sight-threatening complications. In children corneal infections can be difficult to diagnose because the pathology can develop without pain. In addition the examination may be difficult to perform. The treatment should be provided earlier and aims to eliminate the bacteria, virus or fungus pathogens. Specific epidemiology of pathogens will be detailed according to age. Risks of visual impairment and amblyopia must be integrated. An update on topical antibiotics and current options will be reviewed with practical aspects, diverse clinical cases and considering quality of life of children and parents.

Commercial interest

- 2234

Acanthamoeba keratitis: a modern approach

YEUNG A

Nottingham

ABSTRACT NOT PROVIDED

ABSTRACT NOT PROVIDED 


\section{- 2241}

Types and functions of retinal glial

OSBORNENN $(1,2)$

(1) Nuffield Dept.Clinical Neurosciences, Oxford University, Oxford

(2) Fundación de Investigación Oftalmológica, Oviedo

This symposium will highlight some the functions associated with the three glial celltypes of the retina. Glial cells actively interact with each other as well as with neurons and the vasculature and participate in the generation of the electroretinogram and spreading depression. Particularly prominent are the specialised Müller cells that span nearly the entire depth of the retina. Müller and astrocytes are resident immune cells constituting the "macroglial" and both express a wide variety of ion channels, receptors, and transporters regulating the microenvironment by controlling extracellular glutamate and potassium levels and modulate neuronal activity by release of gliotransmitters. Astrocytes also play a crucial part in retinal vascularisation, in the mature retina and are prominent in the nerve fibre and ganglion cell layers. Microglia, are resident macrophages and thus act as the first and main form of active immune defence in the retina. They are particularly associated with nerve processes occurring in the nerve fibre and plexiform layers.
- 2243

Microglial cell reaction after optic nerve lesions

VILLEGAS PEREZMP $(1,2)$, GALINDO-ROMERO C $(1,2)$

VALIENTE-SORIANO FI $(1,2)$, IIMENEZ-LOPEZM $(1,2)$

SOBRADO-CALVOP (1, 2), AGUDO-BARRIUSO M $(1,2), \operatorname{VIDAL-SANZM}(1,2)$

(1) Oftalmología, Optometría, Otorrinolaringología y Anatomía Patológica, Murcia

(2) Instituto Murciano de Investigación Biosanitaria-Virgen de la Arrixaca, El Palmar Murcia

Microglial cells may be involved in the degeneration and regeneration processes of Retinal Ganglion Cells (RGCs). Optic nerve (ON) lesions induce microglial cell activation both in goldfish (an animal with important regeneration capabilities; Salvador-Silva et al., 2000) and rodents (animals with limited regenerating capabilities; Sobrado-Calvo et al., 2007; Galindo-Romero et al., 2013). The microglial cell reaction after an ON lesion is common to all the animal models studied and typically includes: increased enzyme activities, morphologic changes and cell hyperplasia. Because these reactions are found in regenerating and non-regenerating animal models, they may represent a general response of the microglia that is directed towards helping the regeneration process However, the location, severity and timing of the reaction varies depending on the animal model, amount of RGC death and type of lesion used and on other factors such as whether other eye lesions, trophic or other factors are administered concomitantly Therefore the microglial cell reaction after an $\mathrm{ON}$ lesion can be modified externally and may subsequently influence the degeneration and regeneration processes.

\section{- 2242}

Müller cells - structural and physiological functions

REICHENBACH A

Leipzig

In 1851, Heinrich Müller discovered what he called 'radial fibers' and what we now call Müller cells, as the principal glial cells of the vertebrate retina. During the past decades it became evident that they are essential constituents of the retinal tissue. Müller cells appear to constitute the 'core' of columnar units of clonally and functionally related groups of neurons. Their primary function is to support neuronal functioning (by increasing the signal-to-noise ratio of information processing) and survival (by maintaining a metabolic 'symbiosis' with the neurons). Much of this dual role of glial cells involves specific homeostatic mechanisms. Current progress in the field is achieved by the application of various novel experimental methods (cellular electrophysiology, imaging methods, optical and biomechanical measurements, and others). Herewith it was shown that Müller cells increase the signal-to-noise ratio of retinal information processing by, for instance, (i) guiding the light towards the photoreceptor cells, (ii) removing excess neurotransmitter molecules from extracellular space, and (iii) performing an efficient clearance of excess extracellular potassium ions after neuronal excitation.
- 2244

Retinal astrocytes -characteristics and role in retinal blood vessel development

FRUTTIGERM

UCL Institute of Ophthalmology, London

During development retinal astrocytes emerge from the optic nerve as a proliferating and migrating cell population that spreads across the inner surface of the retina. This process is driven by retinal ganglion cells secreting platelet derived growth factor (PDGFA). The resulting retinal astrocyte network is essential for the developing retinal vasculature. The retinal astrocytes provide a critical substrate and trophic support via the secretion of vascular endothelial growth factor (VEGF) for the growing blood vessels

Commercial interest 
- 2245

The influence of ocular hypertension on retinal glial cells

ROJAS B (1), GALLEGO B (1), DE HOZ R (2), RAMIREZ AI (2), SALAZARJJ (2), TRIVINO A (1), RAMIREZ IM (1)

(1) Inst Inves Oftalmol Ramon Castroviejo. Facultad Medicina. UCM, Madrid

(2) Inst Inves Oftalmol Ramon Castroviejo. Facultad Optica Optometria. UCM, Madrid

We describe the effects of unilateral laser-induced ocular hypertension (OHT) in the macroglia and microglia of eyes with OHT and their contralateral normotensive untreated eyes. In both eyes, the glia was reactive even though, contrary to OHT-eyes, no retinal ganglion cell loss or abnormalities in the electroretinogram were found in contralateral eyes. With respect GFAP immunostaining there were differences between contralateral and OHT-eye. Signs of microglial activation in contralateral and in OHTeyes affected all retinal layers, including: morphological changes, migration, increased cell number, upregulation of activation markers and quantification of the area occupied by Iba- $1+$ cells and of the arbor area of Iba- $1+$ cells. In microglia, MHC-II upregulation in contralateral eves was similar to that in OHT-eyes. By contrast, MHC-II upregulation in macroglia was observed mainly in astrocytes in contralateral eyes and in Müller cells in OHT-eyes. Only eyes with OHT had rod-like microglia and rounded Iba- $1+$ CD68+ CD86+ cells. Glial differences between contralateral and OHT-eyes could help us to understand glaucoma pathophysiology and develop new strategies for treatment. 
- 2251

Ocular immunology and vitreoretinal surgery "face to face"

NERI P (1), ARAPI I (2), PIRANI V (2), CARBONARI G (2)

(1) Agugliano

(2) The Eye Clinic, Polytechnic University of Marche, Ancona

The clinical assessment of most of vitreo-retinal (VR) inflammations is often challenging the clinical picture of such diseases can be overlapping and, despite the advances in medical technologies, the diagnostic tools might not provide data which can be conclusive. At this point, the possibility to take a vitreous tap via pars plana can help the ocular immunologist to differentiate between different sub-sets of infectious diseases and masquerade syndrome. The term "Masquerade Syndrome" was first used in 1967 to describe a case of conjunctival carcinoma that manifested as chronic conjunctivitis Masquerade syndromes are disorders that occur with intraocular inflammation and are often misdiagnosed as a chronic idiopathic uveitis. Very often mesquerade syndrome is a synonymous of intraocular lymphoma which can present insidious clinical pictures, which are very often confused. The possibility to test the interleukines' ratio and to analyze the cytology represents a great scientific advance. A clearer role of VR surgery is played for all the complications of uveitis, such as uveitic retinal detachment, inflammatory macular pucker and dense persistent vitritis. VR surgery can warrant an important contribution to the clinical outcome.

- 2253

The state of the art of diagnostic vitrectomy

DICK A

Bristol

The emergence of increasingly sensitive molecular and biological assays has facilitated our diagnostic ability in uveitis. Together with the advances in surgical vitrectomy procedures, the risk to biopsy is much reduced. Combined therefore we are currently at an unprecedented state of ability to rapidly intervene and increase our diagnostic yield. The talk will highlight indications, simple techniques and evidence of how improved diagnostics have altered outcomes and discuss how diagnostic sampling should be employed in management of uveitis.

\section{- 2252}

Vitrectomy in uveitis

MARIOTTI C, SAITTA A, NICOLAIM

Eye Clinic - Università Politecnica delle Marche, Ancona

Today, pars plana vitrectomy (PPV) for ocular inflammatory diseases represents a powerful tool for those cases non responding to medical treatment. PPV has been increasingly performed for both diagnostic and therapeutic purposes in the management of patients with uveitis. PPV offers a surgical option in clearing vitreous opacities and repairing inflammatory related complications, such as epiretinal membrane, retinal detachment, vitreous haemorrhage, cyclitic membrane removal for non-responsive hypotony. The evolution of both technology and surgical techniques for PPV has been very important in order to develop a step forward in approaching and treating ocular inflammatory diseases.

2254

The role of retinal and choroidal biopsy

DA CRUZL

Vitreo-retinal, London

Chorio-retinal biopsy is relatively infrequently used for the diagnosis of infiltrative processes of the posterior segment. It is used in cases where local and systemic clinical features are insufficient to determine whether the infiltration is inflammatory, infective or neoplastic. In most of the few cases described in the literature the aim is to exclude or diagnose lymphoma. The cases published to date suggest the diagnostic pick-up rate is good relative to vitreous biopsy and it is possible that the diagnosis is confirmed from Chorioretinal tissue biopsy when the vitreous biopsy from the same operation is negative. Diagnoses of many types of pathology including viral and non-viral retinitis, lymphoma, tuberculosis and non malignant inflammatory infiltration have all been described in the literature. In the majority of cases reported in the literature the diagnosis of lymphoma is clearly made or excluded definitively. The technique of carrying out the surgery has been well described and the reported morbidity of the procedure is minimal. In this presentation The technique, surgical issues and complications will be discussed as well as the outcomes from the published literature concerning these cases. 
- 2261

\section{Clinical manifestations of NMO}

YU-WAI-MANP $(1,2)$

(1) Institute of Genetic Medicine, Newcastle University, Newcastle upon Tyne

(2) Newcastle Eye Centre, Royal Victoria Infirmary, Newcastle upon Tyne

Neuromyelitis optica (NMO), also known as Devic's disease, is an idiopathic inflammatory demyelinating disease of the central nervous system (CNS) that preferentially targets the optic nerve and the spinal cord. Due to the significant risk of permanent visual and neurological impairment in $\mathrm{NMO}$, rapid diagnosis and the prompt initiation of the most appropriate treatment are essential The current diagnostic criteria require a clinical history of optic neuritis and transverse myelitis accompanied by at least two of the following three supportive criteria: (i) brain MRI not diagnostic of multiple sclerosis (MS); (ii) spinal cord MRI with contiguous lesion over three or more vertebral segments; and (iii) IgG seropositivity against the aquaporin-4 (AQP4) water channel. The development of optic neuritis and transverse myelitis can be simultaneous or sequential. In this presentation, the classical clinical features of NMO will be reviewed and put in the context of other demyelinating CNS disorders. Recent clinicopathological studies have revealed striking differences between NMO and prototypic MS, establishing them as distinct entities, and with important practical implications for the management of these two disorders.

\section{- 2263}

\section{Using OCT to distinguish NMO and other types of optic} neuropathy

$\operatorname{KARDONR}(1,2)$

(1) Ophthalmology and Visual Sciences, University of Iowa, Iowa City

(2) Veterans Administration Center of Excellence for the Prevention and Treatment of Vision Loss, Iowa City

Acute and chronic vision loss due to neuromyelitis optica (NMO) can be difficult to distinguish from optic neuritis associated with multiple sclerosis (MS) and other causes of visual loss. This is especially true in cases where classic findings of multiple spinal cord level involvement with transverse myelitis is not present and in cases where an IgG1 autoantibody (NMO-IgG) that binds aquaporin 4 (AQP4) is not present at the time of visual loss. A number of studies have compared features of the inner retina defined by optical coherence tomography (OCT) in $\mathrm{NMO}$ and in MS and have found that patients with NMO have much greater axonal loss when matched for final visual acuity at the chronic phase. During acute visual loss, scanning laser polarimetry (SLP$\mathrm{GDx}$ ) is abnormal before changes in OCT features are apparent. This is due to an acute disorganization of axon microfilaments and microtubules that appears to predict final outcome. At the sub-acute phase (2-4 weeks), the retinal ganglion cell layer thickness shows thinning before the retinal nerve fiber layer becomes thin. The literature will be reviewed on structural features in the acute, sub-acute and chronic phases of vision loss in optic neuropathy.

Commercial interest

\section{- 2262}

Antibodies, MRI and unusual clinical features of NMO

DESEZEJ

Strasbourg

Neuromyelitis optica (NMO) is a rare disease including both optic neuritis and myelitis together or successively. Recently, a specific antibody named anti-NMO has been discovered allowing a more precise diagnosis. This antibody is directed againbst abtiAQP4 protein, a watter chanel protein. This discovery enlarged the spectrum of NMO to patients with recurrent optic neuritis and positive antibodies, now include in the NMO spectrum disorder (NMOSD). MRI frequently shows intense hypersignal of optic nerve and spinal cord with normal or atypical brain MRI for MS.NMO is frequently associated with other auto-immune diseases such as Sjogren syndrome ou lupus. The outcome of the disease may be severe with blindness and para/tetraplegia implying that an intensive treatment should be propose as soon as the diagnosis has been made.

\section{- 2264}

\section{Overview of treatment of NMO}

DESEZEJ

Strasbourg

Recent studies has shown that neuromyelitis optica (NMO) is clearly a different disease compared with multiple sclerosis (MS). Several MS drugs may increase inflammation process in NMO, especially interferon beta, natalizumab and fingolimod. This fact has 2 important consequences : First the diagnosis of NMO has to been done as soon as possible in order to differentiate NMO and MS patients. Second, as NMO is an antibody mediated disease immunosuppressive grugs should be proposed if the diagnosis is done. First step therapy is based on azathioprine, mycophenolate mofetil and methotrexate. Second step therapy are Rituximab, cyclophosphamide and mitoxantrone. Several drugs (with atarget on complement subunites or several cytokine, especially IL6 or IL17) are currently tested in phase II or III trials.Symptomatic treatments directed against fatigue, urinary or bowel dysfunctions, pain remains also of importance.

Commercial interest 
- 2271

From protein aggregation to inflammasome activation in RPE cells

\section{KAUPPINENA}

Kuopio

Retinal pigment epithelium (RPE) cells play the principal role in maintaining the functionality of overlying photoreceptors, and their degeneration is the primary event resulting in the deterioration of central vision in age-related macular degeneration (AMD). Multifunctional RPE cells also take care of immune defence in macula. Due to high metabolic rate, lipid peroxidation products accumulating from ingested photoreceptor outer segments (POS), and almost continuous exposure to light, RPE cells are exposed to constant oxidative stress. Intralysosomal lipofuscin further promotes the production of reactive oxygen species (ROS), and chronic oxidative stress contributes to the age-related decline of intracellular degradation systems, such as proteosomal clearance and autophagy. Dysfunctional degradation results in the intracellular accumulation of protein aggregates and ROS-producing mitochondria, which can serve as danger signals and result in the inflammasome activation.
- 2273

Tale of lysosome in RPE cells and in the pathogenesis of AMD

SINHA D

Ophthalmology, The Johns Hopkins University School of Medicine, Baltimore

In RPE cells, the lysosomal system is a crucial regulator of both phagocytosis and autophagy. We have recently shown in the Nucl rat (a spontaneous mutation in the Crybal gene encoding for betaA3/A1-crystallin) and in mice lacking betaA3/A1crystallin specifically in the RPE, that impaired lysosomal clearance decreases both autophagy and phagocytosis. This leads to the accumulation of lipofuscin within RPE cells, subretinal lesions in the posterior pole, and deposits between the RPE and Bruch's membrane. It is likely that disturbances in the homeostasis of RPE cells, due to accumulation of undegraded intracellular material in the mice lacking betaA3/A1crystallin, trigger a para-inflammatory response in an attempt to restore normal RPE function. Severe or prolonged impairment of lysosomal-mediated clearance may also convert para-inflammation to chronic inflammation, leading to retinal degeneration. Several studies support the hypothesis that the immune system is involved in the pathogenesis of $\mathrm{AMD}$, in concert with, or in addition to, other factors. We have generated a genetically engineered mouse model that exhibits a slowly progressing form of AMD-like pathology associated with inefficient lysosomal clearance.

\section{- 2272}

Investigating AMD mechanisms using primary RPE cultures and donor tissue

FERRINGTOND (1), TERLUKM (1), EBELING M (1), ROEHRICH H (2) MONTEZUMA S (1)

(1) Ophthalmology and Visual Neurosciences, Minneapolis

(2) Histology Core for Vision Research, Minneapolis

Stimuli that activate inflammasomes include mitochondrial $(\mathrm{mt})$ reactive oxygen species, release of damaged mtDNA into the cytosol, or disruption of cellular ionic balance. These stimuli can arise from mt dysfunction, which may be a key pathogenic event in age-related macular degeneration (AMD). This hypothesis is consistent with increased mtDNA damage reported in macular retinal pigment epithelium (RPE) of human donors with AMD. To investigate inflammasome activation, we developed primary cultures of RPE from donors with or without AMD. Cells express the RPEspecific proteins bestrophin, CRALBP, and RPE65, are pigmented, express ZO-1 at cell margins and Na-K ATPase apically. These results show our primary cultures phenocopy the RPE in vivo. To examine inflammasome activation, cells were stimulated with LPS as a priming event, then treated with either the proteasome inhibitor MG132 to initiate formation of protein aggregates or rotenone to generate reactive oxygen species. We observed differences between cells from control and AMD donors in IL-18 and IL1beta production and also caspase-1 cleavage, the prototypic marker of inflammasome activation. These results suggest AMD affects inflammasome activation in the RPE.

Commercial interest

\section{- 2274}

Dysregulated para-inflammation in the pathogenesis of AMD

XUH

Centre for Experimental Medicine, Belfast

Para-inflammation is an adaptive response of the immune system to low-degree noxious tissue insults. The physiological purpose is to maintain homeostasis and restore functionality. During ageing, retina suffers from low levels of sustained oxidative insults. As a result, a para-inflammatory response characterised by microglial activation, subretinal migration and complement activation, is initiated. We propose that a dysregulated para-inflammatory response may turn into a detrimental chronic inflammation, which may contribute to tissue damage and disease progression. The role of dysregulated para-inflammation in the pathogenesis of age-related macular degeneration will be discussed in this presentation. 
EVER Keynote Lecture 1 - Austin ROORDA

- 2311

Testing vision: one cone at a time

ROORDA A

UC Berkeley School of Optometry, Berkeley

In humans, gaining experimental access to single sensory receptors - photoreceptors specifically - is a challenge, yet it is crucial for learning how the signals arising from each receptor are transformed into perception. By combining adaptive-optics microstimulation with high-speed eye tracking, we show that retinal function can be probed at the level of the individual cone photoreceptor in living eyes. This talk will describe the development and applications of our adaptive optics confocal imaging system which we use to track, target and deliver visible light stimuli to single cones or selections of cones. Highlighted applications will include our ongoing efforts to learn about the neural circuits that underlie human spatial and color vision. 
- 2411

Classification, clinical pattern, multimodal imaging

COSCAS G

Creteil

To suggest a clinical distinction between idiopathic typical polypoidal choroidal vasculopathy $(\mathrm{PCV})$ and secondary polyps associated with late $\mathrm{AMD}$ and its therapeutic application.Retrospective case series of 30 eyes of 30 consecutive patients ( $x x$ females and $\mathrm{xx}$ males) with PCV. Initial diagnosis was based on SLO-ICGA, associated with FA and EDI SD-OCT, which allowed a clinical distinction between two subtypes of polyps. This multimodal imaging and visual acuity testing were repeated then at regular follow-up visits. The central macular and choroidal thickness, presence and number of hyper-reflective dots in retina and in choroid were also evaluated.The two groups were clinically different, based on demographic and ethnical characteristics, imaging findings and topography of lesions, presence of drusen, of true CNV, and changes in choroidal thickness. Therapeutic response was different in the two groups. After treatment, hyper-reflective dots decreased significantly but choroidal morphology did not change significantly. Functional and anatomical changes after treatment are analyzed and discussed.The clinical distinction between idiopathic typical PCV and secondary polyps associated with late AMD and results of treatment, sugges
- 2413

\section{Genetics in polypoidal choroidal vasculopathy}

ZERBIB J (1, 2), SEMOUNO (1), LALLOUM F (1), SIKORAV A (1), TILLEUL J (1),

COSCAS G (1), SOUIEDE (1)

(1) Department of Ophthalmology, Hôpital Intercommunal de Créteil, Créteil

(2) Nice Retina, Nice

Genetic factors have been identified these last years in polypoidal choroidal vasculopathy Because genetic factors play an important role in the pathogenesis of AMD and because AMD and PCV share similarities, the genes involved in AMD have been investigated in PCV. To date, a lot of studies and even reviews have been published on the genetic of PCV, trying to answer if the genetic background between PCV and AMD could help classifying PCV as a subtype of AMD or a distinct disease. Several genes associated to AMD have been analyzed in PCV showing significantly association such as rs10490924 of ARMS2, Y402H of CFH, C2 and C3 genes... But some differences, in particulary, in the rs10490924 of the ARMS2 gene in the allele frequency and the oddsratio between $\mathrm{PCV}$ and $\mathrm{AMD}$ are observed. The genes associated with PCV are the same genes than those associated with AMD, underligning that $\mathrm{PCV}$ and $\mathrm{AMD}$ share common pathway of development. Genetic results to date do not help clearly to answer the controversy whether PCV is a subtype of AMD or a specific entity but show interestingly that AMD and PCV, share, at least partially similar molecular mechanisms. Additional genetic and environmental factors might be implicated in the pathophysiology of PCV
- 2412

Secondary polypoidal choroidal vasculopathy (PCV)

DONATI G (1), POURNARAS C (2)

(1) Geneva

(2) Centre Ophtalmologique de la Colline, Clinique La Colline, Geneva

Polypoidal choroidal vasculopathy (PCV) was first described as polypoidal, subretinal, vascular lesions associated with serous and hemorrhagic detachments of the retinal pigment epithelium (RPE) in a series of patients (10/11 were women) by Yannuzzi. PCV is most commonly diagnosed in patients between the ages of 50 and 65 years. Although more frequently being an primary disease associated to high blood pressure, it may also be secondary to others diseases such as AMD, Central Serous Chorioretinopathy (CSC), ocular radiotherapy, Pseudoxanthoma Elasticum not related to systemic hypertension. Secondary PCV was first reported to occur in AMD patients. Prevalence rates of $4 \%$, $7.8 \%, 8.2 \%$, and $9.8 \%$ have been reported in Caucasian patients with presumed age-related macular degeneration (AMD). In Asian patients with diagnosed AMD the prevalence rates are even higher, between $23.9 \%$ and $54.7 \%$. PCV was categorized as a subtype of neovascular AMD. Vascular proliferative changes associated with PCV in AMD form a network of vessels ending with saccular polypoidal lesions. ICG angiography can be used to distinguish it from classic or occult CNV as usually seen in AMD. PCV is demonstrated by ICG angiography as a prominent vascular network in the early stages of the study and an area of clearing or so called "washout" of the dye in the late stage. On OCT examination the polypoidal lesions are seen as more steep domelike elevations of the highly reflective RPE layers with underlying moderate reflectivity then seen in PEDs. The regions of the branching choroidal vascular networks are visualized as double reflective layers comprising nodular RPE and another highly reflective layer beneath the RPE.According to the EVERST and LAPTOP studies either Anti-VEGF monotherapy alone or associated to Photodynamic therapy is the best therapeutic option actually available to treat PCV in AMD patients and should be considered as first line therapy in all secondary cases

- 2414

Therapeutic approaches- aflibercept first french results of intravitreous aflibercept for active naïve polypoidal choroidal vasculopathy- 6 months follow up

DIRANI A (1), COSCAS F (2,3), COSCAS G (2), SEMOUNO (2), SOUIED E (2)

(1) Saint-Joseph University, Faculty of Medecin, Beirut

(2) Service hospitalo universitaire CHI Creteil, Creteil

(3) Centre Ophtalmologique de l'Odeon, Paris

To evaluate the anatomical and functional effect of intravitreous aflibercept (IAI) for the treatment of polypoidal choroidal vasculopathy (PCV) associated with exudative AMD, with a follow up of 3 months. This is a case series study that included 22 eyes with active PCV. All eyes were naive to treatment before inclusion in the study. The treatment protocol consisted of an induction phase of $3 \mathrm{IAI}$, then the injections were done every 2 months. A complete ophthalmologic workup was done for every patient and included: best corrected visual acuity (ETDRS scale), Fluorescein and Indocyanine Green Angiography, EDI-OCT (Spectralis HRA+OCT", Heidelberg engineering). All results were analyzed at inclusion and at 3 months. The mean age was $76.3+/-11.3$. Male to female ratio was 1 . The BCVA improved at 3 months $(0.58+/-0.39 \log M A R$ vs $0.20 \pm 0.27 \log$ MAR; $p=0.01$ ). The patients gained a mean of 15 letters in the BCVA. No loss of BCVA was noted in any patient. The central macular thickness improved significantly from $376.77+/-99.54$ to $279.80 \pm 82.53$ at 3 months $(\mathrm{p}=0.043)$.The polyp's regression started at one month after the first injection and was almost complete at 3 months. A significant improvement of retinal pigment epithelium detachment 
- 2415

Therapeutic approaches 2: Ranibizumab

SEMOUN O, ZERBIB J, LALLOUM F, SIKORAV A, COSCAS F, COSCAS G, SOUIED E Department of Ophthalmology, Hôpital Intercommunal de Créteil, Creteil

Polypoidal choroidal vasculopathy (PCV) is an exudative maculopathy, with clinical features distinct from neovascular age-related macular degeneration. The EVEREST trial is the only published randomized controlled clinical trial in PCV.Ranibizumab has been proposed for the treatment of PCV and has become one of the standard of care in this particular pathology.To date, a lot of studies have been published, trying to analyze the early and late morphological and functional response to intravitreal ranibizumab, in different ethnic populations, as well as predictive factors of outcome.Imaging characteristics and their changing after treatment, including choroidal modifications have been also investigated.These literature data may help to answer the controversy whether PCV is a subtype of AMD or a specific entity.
- 2416

Therapeutic approaches 3: PDT , Laser and combined treatments UZZANJ (1), COSCAS G (2)

(1) Rouen

(2) Paris

Laser photocoagulation was initially, the first and unique modality of treatment available for PCV, with useful results but risk of hemorrhage and recurrences. Verteporphin-PDT rapidly became the first line of the treatment, mainly for sub foveal lesions with some good results.Intravitreous injections of antiVEGF (ranibizumab and bevacizumab) agents showed immediate good results and achieved regression of fluid accumulation and improvement in vision and became the only treatment of neovascular AMD. But, it has shown less efficiency on polypoidal lesions themselves.Combination therapy with PDT and anti-VEGF became the first line treatment of many authors, beside the lack of comparative randomized study. Laser photocoagulation remains a second line option after that combination of PDT and anti-VEGF, to lower the number of PDT in case of extra-foveal recurrent lesions.The recent disponibility of Aflibercept with probably, a better efficiency on polypoidal lesions, may become the first line therapeutic options where Laser and PDT combinations will need to be specially evaluated.

Commercial interest 
- 2421

Modulators for autophagy: implications for ageing and neurodegeneration

BEHL C

Mainz

Autophagy is a key player in the cellular proteostasis and a degradative pathway that sequesters and transports cytosolic cargo in autophagosomes to lysosomes. This process is dependent on complex membrane dynamics and its deregulation leads to a disturbance in proteostasis contributing to several diseases, including neurodegeneration. In addition to selective macroautophagy pathways such as the "Bag3-mediated selective macroautophagy" different key regulator proteins control the canonical autophagy process. In an RNAi screen in C. elegans designed to identify novel modifiers of proteostasis, we isolated RBG1, the homolog of mammalian RAB3GAP1 and found that the RAB3GAP complex previously known to be involved in cellular endocytosis is required for basal and rapamycin-induced autophagy. The analysis of such distinct key modulators of macroautophagy will help to understand the involvement of the degradation pathways in ageing and disease.
- 2423

Autoimmunity and neuroprotection in glaucoma

GRUSF

Mainz

Immunoproteomics could play a significant role in glaucoma. There are many hints for an involvement of autoantibodies in the pathogenesis of the disease. This paper will address the changes in immunoreactivities in glaucoma pateints. If will further demonstrate the findings about immunological changes in human glaucomatous retinae and the possible involvement of cellular components. Furthermore, the paper will address the clinical use for diagnosis and new approaches to neuroprotection in future.

\section{- 2422}

Autophagy in retina and axonal degeneration

BOYA P

CIB CSIC, Madrid

Autophagy is a catabolic pathway that degrades and recycles intracellular components inside lysosomes to maintain cell homeostasis. This pathway is essential to keep postmitic cells such are neurons free of garbaje and damaged cell components. There are several types of autophagy. During macroautophagy, simply called autophagy, parts of the cytosol and even entire organelles are engulfed in an autophagosome that will finally fuse with a lysosome where degradation will take place. In chaperone mediated autophagy (CMA) proteins harbouring a specific aminoacid sequence will be recognized by a chaperone $\mathrm{Hsc70}$ and delivered inside lysosomes thanks to the lysosomal receptor LAMP-2A.The role of autophagy in the physiology and pathology of the retina is just starting to be investigated. We have demonstrated the cytoprotective role of autophagy in a model of optic nerve axotomy in mice. We show that autophagydeficient animals have reduced number of surviving RGCs after ONT and that pharmacological induction of autophagy delays neuronal cell death. We have also observed a reduction in the levels of autophagy during retinal aging and Atg 5 deficient animals in the retina show increased photoreceptor death and reduced scotopic vision. Unexpectedly CMA seems to increase in these conditions and we postulate that this is a compensatory response to sustain cell survival. Thus balance in autophagic pathways plays important roles to maintain retinal homestasis.

- 2424

Reactive gliosis along the visual system in rodent models of ocular hypertension

VIDAL-SANZM (1), GALINDO-ROMEROC (1), SALINAS-NAVARROM VALIENTE-SORIANO FJ, GALLEGO B (2), ROJAS B (2), DEHOZR (2), RAMIREZ AI (2), SALAZAR IJ (2), AVILES M (1), TRIVINO A (2), RAMIREZ IM (2), AGUDOM (1), VILLEGAS PEREZMP(1)

(1) Dpto. Oftalmologia, Universidad de Murcia, Murcia

(2) Instituto Investigaciones Oftalmológicas Ramón Castroviejo, Universidad Complutense de Madrid, Madrid

Glaucoma is a complex disease that causes the loss of retinal ganglion cells (RGCs) and eventually blindness. The mechanisms leading to the progressive RGC death in glaucoma are still a matter of debate. It is known that the main risk factor is an elevated intraocular pressure (IOP) that triggers the initial insult: presumably a crush-like injury to the RGC axons at the level of the optic nerve head. Current research indicates that neuroinflammation, mediated by the activation of glial cells and the release of cytokines, pro-inflammatory and neurotoxic molecules, plays an important role in the pathogenesis of glaucoma, aggravating and spreading the initial insult. Ocular hypertension in rats and mice causes permanent damage to the outer and inner retinal layers that cannot be the result of RGC axonal damage and may thus be secondary to an ischemic or neurotoxic insult. Ocular hypertension also induces a glial response that may affect the whole visual system. Activated microglial and macroglial cells are found not only in the ocular hypertensive but also in the contralateral retina and these cells may be the mediators of neuroprotective or neurotoxic reactions to injury. 


\section{- 2431}

Electron microscopic evaluation of the surfaces of variable depth lenticules in SMILE cases

OSMANI

Ophthalmology, Alexandria

Purpose Evaluating lenticule surfaces using electron microscopy in deeper corneal layers and comparing them to the superficial ones. This aims at determining the possibility of preserving the stronger superficial layers in the cornea by removing deeper lenticules in SMILE cases while attaining the same visual results and with the same ease.

Methods 40 eyes underwent SMILE refractive procedure using femtosecond laser by VisuMax (Zeiss Meditech). 2 groups were used, one with lenticules at $100 \mu \mathrm{m}$ and the other with lenticules at $160 \mu \mathrm{m}$. Lenticules were immediately processed (fixed, dehydrated, dried and mounted with gold coating). JSM-5300 scanning electron microscope was used for observations.

Results Deeper lenticules had more irregular surfaces and were more difficult to dissect. Comparing the central and peripheral thickness of lenticules to the intended thickness was significantly more accurate in the superficial group. The visual results of the two groups were comparable.Surface irregularities were resulting from the break of bridges between the femtosecond cavities.

Conclusion Energy settings still needs further adjustment to be able to create deeper predictable lenticules in SMILE cases

\section{- 2433}

Peripheral neuropathy in Parkinson disease: An in vivo confocal microscopy study

ANJOS R, VIEIRA L, SOUSA A, MADURO V, ALVES N, CANDELARIA P Centro Hospitalar Lisboa Central, Lisboa

Purpose To assess whether corneal confocal microscopy (CCM) can detect alterations in corneal nerve morphology in patients with Parkinson disease.

Methods Twenty-five patients with Parkinson disease and 25 control subjects were examined. Patients underwent neurological and complete ophthalmological evaluation. Corneal sensitivity testing was performed with a Cochet-Bonnet esthesiometer and corneal sub-basal nerve morphology assessment with confocal microscopy (Heidelberg Retina Tomograph II/Rostock Cornea Module). CCM was used to quantify corneal nerve fibre density, tortuosity, length and branch density.

Results Patients with Parkinson disease had decreased corneal sensation in comparison with controls. Corneal nerve fiber morphology differed in both groups, with a lower global fiber density, branch density and higher tortuosity in Parksinson patients $(\mathrm{p}<0,05)$. These parameters were found to be related to dopaminergic medication exposure.

Conclusion We found a decreased corneal sensation and corneal nerve fiber changes in patients with Parkinson disease. CCM may be used to detect signs of peripheral neuropathy and follow patients in dopaminergic therapy.
- 2432

Handheld reflectance in vivo confocal microscopy for the diagnosis of conjunctival tumors

GRIVET D (1), HAOUAS M (1), JULIENNE R (1, 2), PERROT JL (3), LABEILLE B (3) CAMBAZARD F (3) FOREST F (2 4) DUMOLLARDIM (2 4) PEOC'HM(2 4 ) GAINP $(2,1)$, THURET G $(2,1,5)$, CINOTTIE (3)

(1) Department of Ophthalmology, University Hospital, Saint-Etienne

(2) Corneal Graft Biology, Engineering and Imaging Laboratory, EA2521, Federative Institute of Research in Sciences and Health Engineering, Faculty of Medicine, Jean Monnet University, Saint-Etienne

(3) Department of Dermatology, University Hospital, Saint-Etienne

(4) Department of Pathology, University Hospital, Saint-Etienne

(5) Institut Universitaire de France, Bd St Michel, Paris

Purpose We evaluated whether the handheld in vivo confocal microscope (IVCM) that has been recently developed for the study of skin tumors is suitable for the diagnosis of conjunctival tumors.

Methods Prospective study, observational case series. We prospectively evaluated the IVCM features of 53 conjunctival lesions clinically suspicious of tumors of 46 patients referred to our University Hospital, by using the handheld device (vivascope 3000, Caliber/Mavig). Twenty-three lesions were excised (3 nevi, 10 melanomas, 5 squamous cell carcinoma, 2 lymphomas and 3 pinguecula/pterygium) while the other 30, presenting no IVCM malignant features, were under follow-up for at least 1 year Clinical, IVCM and histological diagnosis were compared. Sensitivity and specificity of the IVCM for the diagnosis of conjunctival malignant tumors was calculated using as reference 1) the histolological diagnosis and 2) the histological diagnosis plus the clinical diagnosis at follow-up greater than one year

Results IVCM showed sensitivity and specificity for malignant tumors of $100 \%$ using both methods

Conclusion IVCM with a handheld dermatology-dedicated microscope can play a rôle in the non-invasive diagnosis of conjunctival lesions. Further studies should be performed to better define the diagnostic ability of this technique.Grants: GIRCI2012

- 2434 / S029

Reproducibility of keratometric measurements obtained with OCULUS $^{\oplus}$ Pentacam ${ }^{\circ}$ HR in the evaluation of keratoconus progression

SILVA N (1, 2), VIEIRA L (1, 3), ANJOS R (1), ALVESN (1), MADURO V (1), FEIJAOI (1) CANDELARIA P(1)

(1) Centro Hospitalar Lisboa Central, Lisboa

(2) Centro Hospitalar do Funchal, Funchal

(3) Hospital Divino Espirito Santo, Ponta Delgada

Purpose To determine the reproducibility of keratometric parameters obtained by OCULUS" Pentacam "HR in the evaluation of keratoconus progression.

Methods Thirty nine eyes of 25 patients from the Cornea Department of the Centro Hospitalar de Lisboa Central were included in the study. Fourteen eyes presented with clinical and topographic diagnosis of keratoconus (stage 1-3) and were defined as the "keratoconus without ring" group; 9 eyes had already an intrastromal ring implanted and were defined as the "keratoconus with ring"; the remaining 16 eyes, without clinical or topographic evidence of disease, were used as controls. Every eye was submitted to identical methodology: 5 consecutive keratometric measurements obtained with OCULUS Pentacam HR; central keratometry, SKmax, minimal pachymetry and anterior chamber depth were used to determine the existence of statistically significant reproducibility.

Results Overall significant measurement variability was obtained in the "keratoconus without ring" group (Km 0.129 D vs 0.0375 D, SKmax 0.507 D vs 0.0625 D, Minpach $5.429 \mu \mathrm{m}$ vs $2.5625 \mu \mathrm{m}$. ACD $0.024 \mathrm{~mm}$ vs $0.0194 \mathrm{~mm}$ ), with highest variability in the subgroup of patients with more advanced disease. In the "keratoconus with ring" group statistically significant variability was found for all parameters except for anterior chamber depth (Km 0.289 D vs 0.0375 D, SKmax 0.444 D vs 0.0625 D, Minpach 4.333 m vs $2.5625 \mu \mathrm{m}$, ACD $0.024 \mathrm{~mm}$ vs $0.0194 \mathrm{~mm}$ )

Conclusion Keratometric measurements obtained with OCULUS" Pentacam ${ }^{\circ}$ HR should be assessed cautiously in the evaluation of keratoconus progression, with or without intrastromal ring; the use of single measurements is unadvised. 
- 2435 / S030

Peripheral neuropathy in chronic renal failure: an in vivo confocal microscopy study

VIEIRA L (1), ANJOSR (1), SOUSA H (2), SOUSA A (3), BORGES A (2), BRANCO P (2), ALVES N (1), MADURO V (1). CANDELARIA P (1)

(1) Centro Hospitalar Lisboa Central - Ophthalmology, Lisboa

(2) Centro Hospitalar Lisboa Ocidental - Nephrology, Lisboa

(3) Centro Hospitalar Lisboa Central - Neurology, Lisboa

Purpose To analyse corneal nerve morphology alterations in patients with chronic renal failure by in vivo corneal confocal microscopy (CCM).

Methods A prospective study was conducted in 30 patients (30 eyes) with pre-dialysis chronic renal failure (CRF) and 30 aged-matched healthy volunteers (30 eyes). A complete ophthalmologic examination, esthesiometry (Cochet-Bonnet esthesiometer) in vivo CCM (Heidelberg Retina Tomograph II/Rostock Cornea Module) and neurological evaluation were performed

Results Corneal sensation was reduced in pre-dialysis CRF patients compared with control subjects. Nerve fiber density, length and branch density were reduced and tortuosity was increased in the CRF group $(\mathrm{p}<0,05)$. These parameters were related with severity of neuropathy.

Conclusion In vivo CCM may be a valuable method for diagnosis of uremic neuropathy and may be used to grade its severity.

\section{- 2437 / S032}

Investigation of anterior chamber flare and corneal Langerhans cells in rheumatoid arthritis and in dry eye

MARSOVSZKY L, BAUSZ M, NEMETH J, RESCH M

Department of Ophthalmology, Semmelweis University, Budapest

Purpose To examine the anterior chamber flare and corneal Langerhans cells (LC)in rheumatoid arthritis (RA) and compare the results to those found in patients with dry eye disease (DED) and in age matched control (C).

Methods 15 patients with RA (11 women, és 4 men, mean age: $67.3 \pm 8.6$ years), 20 DED patients (11 women, 9 men, mean age: $57.2 \pm 14.4$ years) and 15 control subjects ( 8 women, 7 men, mean age: $49.6 \pm 19.1$ years) were enrolled in this study. Anterior chamber flare was evaluated using Kowa FC-600 laser flare meter and corneal LC densities were investigated at the corneal centre with confocal microscopy (Heidelberg Retina Tomograph with Rostock Cornea Modul (HRT II RCM)) in all groups

Results There was a significant difference in the flare values between RA and DED patients $(6,65 \mathrm{f} / \mathrm{ms}$ in RA and 4,75 f/ms in DED ( $<<0.01)$. Central LC density was: $57.6 \pm 34.4 / \mathrm{mm} 2$ in RA and $52.3 \pm 31.3 / \mathrm{mm} 2$ in DED respectively. Both values were significantly lower than that of in control $(24.6 \pm 18.6 / \mathrm{mm} 2, \mathrm{p}<0.05)$

Conclusion The blood aqueous barrier might be altered in RA even without signs of clinically significant ocular inflammation. This alteration in the blood aqueous barrier might contribute to the accumulation of LCs at the corneal centre. The greater LC density and flare values found in RA might represent novel players in the pathogenesis of dry eve.
- 2436 / S031

Mathematical model of corneal reendothelialization after inadvertent descemetorhexis

TRONEM (1, 2), JULIENNER (1, 2), RANNOU K (3), BERNARD A (1), HEZ (1), GAVET Y (3), THURET G (1, 4) GAINP $(1,2)$

(1) Corneal Graft Biology, Engineering and Imaging Laboratory, EA2521,

Federative Institute of Research in Sciences and Health Engineering

Faculty of Medicine, Jean Monnet University, Saint-Etienne

(2) Ophthalmology dpt, University Hospital, Saint-Etienne

(3) Ecole Nationale Supérieure des Mines, Saint-Etienne

(4) Institut Universitaire de France, Bd St Michel, Paris

Purpose To report a case of inadvertent descemetorhexis during phacoemulsification and our conservative approach based on the endothelial cells (EC) self-healing capacity Methods A 85-year-old man presented with an unusual complication of cataract surgery. During visco injection, the Descemet was accidentally dissected and removed during the capsulorhexis. On the next day, a massive corneal edema was observed. Based on the self-healing capacity of the residual peripheral ECs, we proposed to observe the natural course. The posterior corneal surface geometry was measured in OCT (CASIASS-1000) and the residual endothelial area was calculated from a mathematical hemiellipsoid model. Considering the EC density (ECD) from the fellow eye, we estimated the theoretical residual EC pool and thus the final post redistribution ECD in the patient.

Results The theoretical ECD after endothelial cell redistribution was $1680 \mathrm{cells} / \mathrm{mm} 2$, reinforcing the wait and see strategy. Visual acuity improved from counting fingers

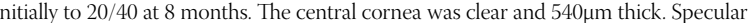
microscopy and confocal microscopy confirmed the repopulation of the central defect by the ECs over the denuded posterior stroma. The central ECD measured at 5 months and 8 months were 550 and 516 cells $/ \mathrm{mm} 2$ respectively.

Conclusion The ECD was less than expected suggesting a higher traumatic loss and a arger defect. The originality lies in the mathematical modeling of the spontaneous selfhealing capacity of the endothelium, deferring penetrating or endothelial keratoplasty. Interestingly, despite the 85 years old, ECs keep important migration and enlargement capacities. Grant: ANR/CORRIMO3D 


\section{- 2441}

Developing a clinically viable protocol for measuring and analysing the human contrast sensitivity function on a standard computerised chart

DONALD J (1), LIJ (1), SAROSSYM (1, 2), WEYMOUTHA (1)

(1) The University of Melbourne, Parkville

(2) RMIT University, Melbourne

Purpose Contrast sensitivity testing is an important part of visual assessment and can identify visual deficits that go undetected by measuring visual acuity (VA) alone. Despite this, contrast sensitivity testing is rarely performed clinically due to the expensive processes required and limited test availability, with few spatial frequencies used. Further, gratings can be difficult for subjects to learn and results are often not standardized. We developed and tested a convenient, inexpensive protocol using Snellen optotypes to measure the CSF using a widely available computerised VA chart. Effects of blur and neutral density (ND) light attenuation were analysed to evaluate the technique under different conditions.

Methods 34 eyes of 34 subjects were tested monocularly under photopic conditions. Subjects had corrected VA of 6/4.8 (or better) and normal ocular health. A computerised chart (Luneau) was used to measure contrast sensitivity thresholds across 19 spatial frequencies between 0.9 and $60.0 \mathrm{cpd}$ using an ascending Method of Limits. This was repeated with +1.50DS blur and a 2.9 ND filter. CSFs were analysed and modeled using $R$ and Prism to extract useful parameters: the area under the log 10 CSF (AULCSF), CSF peak, high- and low-spatial frequency limits.

Results A quadratic function was found to best fit the pooled log CSF data, minimising cross-validation error. The AULCSF was calculated between log spatial frequencies of 0.6 and 1.4 using integration of the fitted quadratic function. Mean AULCSF was $1.82 \pm 0.133$.

Conclusion Our method can measure, display and calculate the normal CSF. This can be used to compare and assess the CSFs of patients with ocular diseases.

- 2443

\section{Simulation of DME changes on OCT}

CORREIA A (1), ARAÚJO A (2), BARBEIROS (2), CARAMELOF (3),

SERRANHO P (4), MORGADOM (3), BERNARDES R (3)

(1) AIBILI - Association for Innovation and Biomedical Research on Light and Image, Coimbra

(2) CMUC, Department of Mathematics, Faculty of Science and Technology, University of Coimbra, Coimbra

(3) IBILI - Institute for Biomedical Imaging and Life Sciences, Coimbra

(4) Mathematics Section, Department of Science and Technology, Universidade Aberta, Lisboa

Purpose To identify the changes at the cellular level responsible for the differences seen in OCT scans of DME patients.

Methods Cirrus HD-OCT (Carl Zeiss Meditec, Dublin, CA, USA) scans of DME

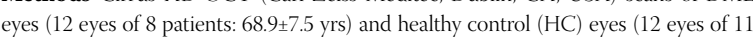
controls: $63.0 \pm 3.6$ yrs) were acquired from our database.The ONL was segmented by a human grader $(\mathrm{AC})$ and each segmentation was aligned to its upper boundary, averaged into an A-scan and then normalized by the intensity at the RPE to discard the effect of media opacities.To understand the significant differences found between the profiles of DME and $\mathrm{HC}$ a Monte Carlo method for simulating light scattering with a model of the ONL was implemented.The inputs for the method are calculated using Mie theory by assuming that the ONL may be modelled as a homogeneous medium filled with nuclei.Changes in each model parameter (eg nucleus' size) were tested and compared with the measured profile to identify which cellular change could best reproduce the OCT differences found between HC and DME patients.Moreover, the DME group was divided into those without significant visible changes and those with increased ONL volume.

Results The simulation method successfully reproduced the differences observed between the HC and DME groups.For the DME cases with increased volume, it was possible to match the simulated and acquired profiles by using the measured increase in ONL thickness. In the DME case with no significant changes, it was necessary to increase only the nucleus size to be able to reproduce the measured profiles.

Conclusion The method proposed is capable of reproducing the reflectivity changes measured on OCT scans of the ONL of DME eyes and to justify these at the subcellular level.

\section{- 2442}

Flash visual evoked potentials (FVEP) in various stimulation conditions

POJDA-WILCZEKD

Ophthalmology Clinic and Department of Ophthalmology, School of Medicine in Katowice, Medical University of Silesia in Katowice, Katowice

Purpose Comparison between the results of FVEP examinations conducted with various stimulators (Ganzfeld, Mini Ganzfeld i Flash Goggles) as well as for opened and closed eyes.

Methods 17 volunteers (34 eyes), including 11 females, aged 23-56 (average age: 30 years) have been examined with Roland Consult electrophysiological equipment. Basing on Wilcoxon signed-rank test the amplitude and the latency of P2 wave have been compared: 1 . of results acquired with each of three stimulators; 2 . of results acquired with eyes opened and eyes closed

Results Mean P2 amplitude (latency) acquired with Ganzfeld stimulation amounted to $12 \mu \mathrm{V}$ (130 ms), acquired with Mini Ganzfeld stimulation amounted to $7 \mu \mathrm{V}$ (124 ms) and acquired with Flash Goggles amounted to $9 \mu \mathrm{V}(113 \mathrm{~ms})$. The results statistically significantly differed $(\mathrm{p}<0,0001)$ between all compared stimulators. Mean P2 amplitude (latency) acquired with Ganzfeld stimulation amounted to $12 \mu \mathrm{V}(130 \mathrm{~ms})$, acquired with Mini Ganzfeld stimulation amounted to $7 \mu \mathrm{V}(124 \mathrm{~ms})$ and acquired with Flash Goggles amounted to $9 \mu \mathrm{V}(113 \mathrm{~ms})$ with eyes opened and accordingly $11 \mu \mathrm{V}(131 \mathrm{~ms})$, $9 \mu \mathrm{V}(126 \mathrm{~ms})$ and $6 \mu \mathrm{V}(120 \mathrm{~ms})$ with eyes closed. The results for eyes opened and eyes closed statistically significantly differed $(\mathrm{p}<0,001)$ for Mini Ganzfeld as well as for Flash Goggles stimulators.

Conclusion The amplitude and latency of P2 FVEP acquired with different stimulators cannot be compared. Keeping eyes closed does not significantly alter the amplitude and latency of P2 FVEP only in case of Ganzfeld stimulation. The results acquired with other stimulators can be faulty if the patient closed eyes during examination.

\section{- 2444}

Visual Search Test (VST) - reaction time perimetry with fixation objects detectable only by foveal vision

LEINONENM (1), KNAAPI L (2)

(1) Ophthalmology, Turku

(2) Ophthalmology, Pori

Purpose To validate a novel perimetry method (Visual Search Test, VST) which is based on reaction times in visual decision-making task, where the subject is allowed to move eyes and where the fixation is verified by the subject himself in contrast to standard automatic perimetry (SAP) where prolonged stationary fixation and external fixation monitoring apparatus is required.

Methods Device (Ocusweep prototype, Ocuspecto Ltd, Turku, Finland) consisting of $35^{\circ}$ arc (radius $54 \mathrm{~cm}$ ) was constructed with six $3 \times 5$ LED arrays displaying $0.4^{\circ}, 100 \mathrm{~ms}$ suprathreshold stimulus (Saccade Triggering Stimulus, STS) followed by an arrow ( $<$ or $>$ ) as a fixation object (FO) the recognition of which was reported by pressing a correct button. The intensity of FO was 3-5 dB above the foveal threshold. With a correct button press a new peripheral STS \& FO stimulus sequence was displayed in order to initiate a reflex saccade towards the STS. The time periods between button presses were recorded. 18 locations (up to $28^{\circ}$ temporal, $20^{\circ}$ nasal) along the meridians $0^{\circ}$ and $8^{\circ}$ of the visual field of left eye were tested. The center of the physiological blind spot of 11 healthy volunteers was measured with Octopus perimeter by custom program $\left(1^{\circ}\right.$ oval grid of 139 test points). Reaction times within the blind spot area were compared to other locations.

Results Reaction times within the blind spot area were slower compared to other locations in 10 of 11 subjects (AOV, $\mathrm{p}<0.001$ in 9 subjects, $\mathrm{p}<0.006$ in one subject). Conclusion Visual Search Test (VST) can be used to detect visual field defects. Commercial interest 


\section{- 2445}

Visual impairment in diabetic patients with and without established blood-retinal barrier leakage and relation with the status of the blood-brain barrier

QUENDERA B (1), AMORIM A (1), CORDEIROM (2), FERREIRA C (1)

MARQUES J (1), BERNARDES R (1), REIS A (1), RIBEIRO L (3)

CASTELO-BRANCOM (1)

(1) Visual Neuroscience Laboratory, IBILI - Institute of Biomedical Imaging and Life Sciences, Coimbra

(2) Coimbra University Hospital, Coimbra

(3) Association for Innovation and Biomedical Research on Light, Coimbra

Purpose To assess visual impairment in diabetic patients with and without established blood-retinal barrier leakage and relation with the status of the blood-brain barrier

Methods We assessed 37 diabetic patients (29 type2; mean Hb1 Ac 7.93[1.44]) using four different approaches: a two-alternative forced-choice multi-psychophysical channel test for speed discrimination and achromatic contrast as well as chromatic L, M and S cone contrast discrimination; multifocal electrorretinography(mfERG) and brain barrier integrity assessment using a permeability measure Ktrans for dynamic contrast enhanced MRI (Cramer et. al, 2013) in 4 regions of interest(ROIs). We selected two diabetic groups with and without measured retinal barrier leakage.Spearman's rank were used for correlations and Mann-Whitney $\mathrm{U}$ test for comparison between groups for a set $\mathrm{p}$ value threshold of 0.05

Results We found that presence of retinal leakage was not associated with changes in blood brain barrier permeability at any of the studied ROIs. Interestingly we found in particular in diabetic type 2 patients, significant negative correlations between the amplitude and/or implicit time of N1 and P1 components of the multifocal electrorretinogram and brain leakage, for the most external stimulation rings Psychophysical performance was impaired in particular for $\mathrm{S}$ but was not correlated with barrier status.

Conclusion Our findings suggest that retina and brain blood barriers are patho physiologically unrelated in diabetes. We found a direct correlation between neurophysiological measures and brain barrier damage, suggesting that both may have a direct common cause.
- 2446

Chromatic pupillometry for assessment functional rod and cone patterns in Usher syndrome patients

SLIESORAITYTE I, MACHIN C, MAKOWIECKA K, MOHAND-SAID S, SAHEL INSERM, CIC 1243, Institute de la Vision, Paris

Purpose The aim of the study was to characterize the functional rod and cone patterns using chromatic pupillometry in Usher syndrome patients.

Methods Prospective case series study enrolled 45 Usher syndrome patients. All patients underwent in-depth phenotypic examination. Functional rod and cone patterns were obtained using custom-made chromatic pupillometer(AMTech, Dosenheim, Germany). Pupil diameter was recorded monocular in response to red (640 $\mathrm{nm})$ and blue $(462 \mathrm{~nm})$ light stimuli for varied intensity levels (ranged from $1000 \mathrm{~cd} /$ $\mathrm{m} 2$ to $0.001 \mathrm{~cd} / \mathrm{m} 2$ ). Response amplitudes were analyzed and compared to the clinical phenotype.

Results Pupil responses to rod- and cone-weighted stimulation were significantly diminished in all Usher syndrome patients for all intensity levels (mean decrease 60\% and $30 \%$, respectively $\mathrm{p}<0.001$ ). Lower rod-weighted responses were related with decreased $b$-wave amplitude in full field ERG $(r=0.37, p<0.001)$. Decreased coneweighted responses were correlated with diminished photopic ERG b-wave amplitude $(\mathrm{r}=0.43, \mathrm{p}<0.001)$ and mfERG amplitudes $(\mathrm{r}=0.65, \mathrm{p}<0.001)$. Higher cones-weighted responses were associated with preserved cone mosaic in adaptive optics and presence of inner/outer segment in optical coherence tomography, although not always related with preserved visual field and visual acuity. Rod- and cone-weighted responses were recordable even in a very advanced Usher syndrome cases associated with abolished ERG.

Conclusion Chromatic pupillography is an objective tool to assess functional rod and cone patterns in Usher syndrome, which potentially could be used as an outcomes measure to assess safety and efficacy in therapy trials, in particularly in advanced cases. 


\section{- 2451}

New concepts concerning the neural mechanisms of infantile strabismus and amblyopia

TEN TUSSCHER M

Ophthalmology, Brussels

Infantile strabismus results in a loss of binocularity in the primary visual cortex. Beyond the primary visual cortex, the contralateral eye dominates while the temporal retinal signal appears to lose influence. This may either be caused by inadequate retinotopic matching between the nasal and temporal retinal signals (e.g. albinism or agenesis of the corpus callosum), or the result of a lack of normal sensory input. Dominance of the crossing retinal signal might also explain the motor characteristics of infantile esotropia (asymmetric OKN, latent nystagmus, DVD). Only a normal binocular cortical signal will predominate over the evolutionary older, originally non-binocular, retinal projections to the superior colliculi (CS) and the accessory optic system (AOS). Amblyopia seems to be the result of active suppression by the crossing ganglion cells of the non-amblyopic eve. Early-onset monocular deprivation is thought to produce competitive imbalance for synaptic sites, which in turn results in a reduced proportion of functionally binocular neurons.Amblyopia treatment may aim at an optimal binocular balance and pharmacological treatment after the critical period.

\section{- 2452}

Curable forms of childhood strabismus

MOHNEYB

Department of Ophthalmology, Rochester

Background: The literature on the management of childhood strabismus emphasizes some subtypes while ignoring others, based upon the experience of the author or the popularity of the topic, and are often from single-institution, tertiary-care referral centers. The purpose of this study is to review which forms of childhood strabismus are potentially curable.Methods: This overview identified surgically curable forms of strabismus, defined as a life-long result of orthotropia to small angle strabismus with > 50 seconds or better of stereoacuity and normal ocular motility.Results: Children with a developmental disorder, sensory strabismus, convergence insufficiency, dissociated strabismus and Browns syndrome were excluded. Curable forms of childhood strabismus included some children with partial sixth nerve palsy, accommodative and acquired nonaccommodative estropia and, in rare instances, a small percentage of children with intermittent exotropia. Conclusions: In declining order, some children with partial sixth nerve palsy, accommodative esotropia, acquired nonaccommodative esotropia, and only a handful with intermittent exotropia, can be said to be curable forms of childhood strabismus.
- 2453

\section{Assessing stereopsis in preverbal children}

HOUTMANAC

ophthalmology, Brussels

Stereovision became a hype when Wheatley presented his theory in 1838. Stereoscopes became popular. Stereoperception was recognized as the ultimate stage of binocular vision in strabismus theories of the 19th century.Stereopsis is assessed as part of the normal strabismologic examination. Most tests depend on the subject's active response. In babies the assessment of stereopsis is more cumbersome and rarely done outside a research setting.Stereopsis can be demonstrated from the age of around 3 months by FCPL techniques. Interestingly, this can also be demonstrated to a similar degree in babies with infantile esotropia but the number in which this can be done declines rapidly over months. In the age-old controversy regarding the timing of surgery for this condition the potential for stereovision is rarely taken into account. Yet this may be an important prognostic factor; it would seem that in the presence of demonstrable stereopsis offering very early surgery makes sense. However, a quick and reliable test for stereopsis in babies is hard to find.This part of the work-shop will look into the history and background of stereotesting, available tests, recent advances and our own research into developing such a test. $\cdot 2454$

\section{Amblyopia treatment trials}

HOLMES JM

Ophthalmology, Mayo Clinic, Rochester MN

Recent randomized clinical trials and observational studies conducted by the Pediatric Eye Disease Investigator Group, and other research teams, have led to an evidencebased approach for treating anisometropic and strabismic amblyopia. Starting spectacle correction first, as optical treatment alone, results in resolution of amblyopia in at least a quarter of children. Starting low intensity treatment is the next reasonable step, offering parents a choice between 2 hours a day of patching the fellow eye or atropine drops to the fellow eye at the weekend. If amblyopia does not completely respond to these lowintensity approaches, then more intense patching is warranted, such as patching 6 to 8 hours a day or changing the spectacle lens to plano while using atropine to increase penalization. We also have evidence that treating older children (13-17 years old) has merit, particularly if not previously offered treatment. But despite these advances in treatment of amblyopia, one of our ongoing challenges is that a proportion of children are still left with residual amblyopia, so we continue to investigate alternative approaches such as oral levodopa as an adjunct to patching and a new treatment approach based on binocularity. 
- 2461

What genetics has taught us about keratoconus

VINCENT AL $(1,2)$

(1) Ophthalmology, FMHS New Zealand National Eye Centre, University of Auckland, Auckland

(2) Eye Department, Auckland District Health Board, Auckland

Keratoconus most likely represents complex disease, with different modes of inheritance, genetic heterogeneity, and environmental factors contributing to the disease manifestation. The relative contribution of each is subject to debate and likely varies between individuals. At one end of the spectrum, disease in some individuals is probably due entirely to environmental influences, yet at the other end is solely under the control of genetic mechanisms. However the majority of cases will most likely be the result of a genetic predisposition with precipitating environmental stressors or modifiersOver the last decade significant progress has been made in identifying genetic risk factors for keratoconus. These genetic risk factors have been identified using multiple approaches including candidate gene studies, linkage analvsis, genome-wide studies and new sequencing technologies. This presentation will summarize how the genes implicated provide clues to the molecular etiology and pathways involved in the pathogenesis of keratoconus.

- 2463

Twin studies in keratoconus

LISKOVA P

Prague

Genetic determinants of keratoconus are still largely unknown. Over the last two decades there has been a shift from regarding keratoconus as a monogenic disease with variable expression towards a general consensus that it is most likely to be a multifactorial disorder with multiple genetic and environmental components contributing to its development. Twins are useful in assessing the heritability of traits. Discordant monozygotic twin pairs can be used to look for environmental and epigenetic factors The contribution of twin studies to the understanding of keratoconus genetics will be discussed.
- 2462

Genome-wide association studies for the identification of keratoconus susceptibility regions

HYSIP

London

Consensus seems to be building that most diseases are of complex nature and the result of interaction of a polygenic architecture with the environment. A great deal on the genetic architecture of the disease can be learned from population-based and amily studies. This presentation will explore the influence of heritable factors over keratoconus as well as shared genetic elements that influence both abnormal cornea and other phenotypic anomalies of the eye. 
SIS: You don`t always need a knife ... - scleral lenses in ocular surface disease

- 2471

Ocular surface anatomy and physiology underlying scleral lens application

KNOPE (1), KNOPN (2)

(1) Ocular Surface Center Berlin (OSCB), Dept. for Cell and Neurobiology, Charité University School of Medicine, Berlin, Berlin

(2) Hannover

Contact lenses $(\mathrm{CL})$ are in the public interest mainly for cosmetic reasons. Because of supposed simplicity of fitting, adaption, care and safety, soft CL are the predominant type world-wide. Only few rigid gas permeable (RGB) CL are applied even though it may appear that they have less side effects. Another type of CL rests on the sclera and is hence termed scleral lenses. In contrast to the aforementioned CL, that cover only the cornea (RGB) or extend just beyond, scleral lenses have a wide diameter and cover also a large part of the bulbar conjunctiva. This leads to different impact on the anatomy, physiology and pathology of the ocular surface. Below their apex is a deep tear pond that allows to cover even major deformations of the cornea as occur in keratokonus, recurrent surface defects or scars without touching them. New sophisticated diagnostic techniques e.g. for measuring ocular surface topography as well as advanced designs and materials of standard or custom made lenses have significantly improved the fitting and the wearing comfort of scleral lenses. Therefore, scleral lenses have emerged in recent years as a new and EVER more exciting medical tool for the dedicated ocular surface specialist.

- 2473

\section{Scleral Lenses in the United States}

$\operatorname{IACOBSD}(1,2,3)$

(1) Boston Foundation for Sight, Needham

(2) Harvard Medical School, Boston

(3) Mass Eye \& Ear Cornea Service, Boston

There is growing acceptance of scleral lenses by ophthalmologists, optometrists, and patients in the United States. Options among mini-scleral and scleral lenses will be reviewed. PROSE treatment (prosthetic replacement of the ocular surface ecosystem) as a therapeutic approach will be introduced. Success rate for PROSE treatment among patients with ectasia/astigmatism and in patients with ocular surface disease will be presented, as will experience with PROSE treatment in pediatric patients. Evidence for reversal of corneal scarring with PROSE treatment of ocular surface disease will be presented. Scleral lens and PROSE treatment are important alternatives to keratoplasty for corneal scarring and to tarsorrhaphy for exposure. Clinicians and researchers should be aware of scleral lens and PROSE treatment as important options for patients with ocular surface disease or contact lens intolerance, who may be at at higher risk of complications, contact lens dependence, or graft failure with penetrating keratoplasty.
- 2472

Coming back to the basics - overview on therapeutic contact lenses and scleral lenses

MELYR

Valmont

Scleral lenses were introduced at the end of the 19th century but these first glass blown shells had a very limited success related to the corneal hypoxia they induced after a short wearing time.Therapeutic contact lenses (TCL) did not become widespread until the introduction of hydrogel lenses in the 1960s. The availability of high gas permeable silicone hydrogel materials (1999) was a major breakthrough and these lenses are now the most popular TCL used in everyday practice.Thanks to a better understanding of the scleral shape and the availability of high gas permeable materials, scleral lenses have made a significant comeback in recent years. These lenses rest on the sclera and are designed not to touch the cornea, creating a reservoir filled with fluid that prevents any desiccation and mechanical irritation of the cornea. Sclerals are often the only effective non-surgical treatment option in a wide range of severe ocular conditions, including graft-versus-host disease, pellucid marginal degeneration, Stevens-Johnson syndrome, ocular cicatricial pemphigoïd, severe dry eye, non-healing epithelial defects.Scleral lenses dramatically improve the visual acuity and the quality of life of these patients.

- 2474

The rebirth of sclearl lenses in Europe - 20 years of experience in treating ocular surface disease with scleral lenses

MANCZAKH

Specialty Medical Contact Lens Practice, Poznan

Concept of scleral lens implementation in diseased corneas can be probably dated at 1859 when William White Cooper used "glass mask" in patient with chemical burn. Scleral lens implementation was greatly limited due to oxygen deprivation under glass lenses then PMMA lenses causing significant adverse reactions. First article on implementation of GP scleral lens was published in 1983. Since then many designs of scleral lenses where developed. Besides neutralizing irregular astigmatism while fitting cases of KC, PMD, post trauma post PK etc. scleral lenses are used to heal and protect corneal epithelium and at the same time to correct refractive error. Scleral lenses have wide medical indications among them: Lagophthalmos, Ocular Cicatricial Pemphigoid, TEN Syndrome, Neurotrophic Ulcer, Chronic GVHD, Bullous Keratopathy. In the presentation: visual, healing and comfort outcomes of scleral lenses implementation in above diseases will be described and illustrated with pictures. In addition rotationally asymmetrical changes enabling achievement of designs which provide a maximally customized fit for each cornea will be discussed. Such modifications are the solution to overcoming the need for fitting compromises in distorted corneas. 
- 2475

Scleral lenses as a high tech, versatile and cost efficient tool for the treatment of ocular surface disease in less developed countries

MEKKIMB (1), YAHIAOUIS (1), BELAOUDMOUR R (2), MOKHTARIMA (3)

(1) Ibn Al Haythem Center, Algiers

(2) Epidemiology unit, CHU Lamine Debaghine, Algiers

(3) Ophthalmology unit, CHU Mustapha Bacha, Algier

Scleral lens has a double interest: Thanks to its anterior optically perfect surface without any contact with the cornea nor the limbus, it erases the irregularities of cornea whatever the degree of ectasia. On the other hand, it provides a favorable microenvironment for regeneration of diseased corneal epithelium. We present our short Algerian experience in cases of challenging cornea, typically requiring keratoplasty (expensive, often unavailable and with unpredictable results), fitted with scleral lens: Primary corneal ectasia (stage IV keratoconus, pellucid marginal degeneration, globus), secondary post Lasik ectasia, failure of corneal reshaping surgery (Lasik, PKR, radial keratotomy and intracorneal ring segment implantation), irregular corneal graft and traumatic or post infectious corneal scar. We present the functional outcome and the quality of life of these patients. Therapeutic scleral lenses have a magical result, we illustrate it with trophic ulcer cases of various etiologies, resistant to conventional treatment and who healed very quickly under scleral lens wear alone. Finally, we present our pilot study on total UV filter scleral lens to protect ocular surface of children with Xeroderma Pigmentosum.

Commercial interest 
Industry-sponsored symposium 2: What's new in micronutrition for AMD patients?

- 2531

Effect of resveratrol on RPE cells and its potential role in AMD prevention

KAARNIRANTA K

Kuopio

ABSTRACT NOT PROVIDED
- 2532

New evidence from French investigation

KOROBELNIKJ

Bordeaux

ABSTRACT NOT PROVIDED

- 2533

How to identify patients with nutritional risk factors for AMD?

ASLAM T

Manchester

ABSTRACT NOT PROVIDED 


\section{Revisiting infectious triggers of chronic intraocular immunity}

BODAGHI B

University of Pierre and Marie Curie, Paris

The pathogenesis of intraocular inflammation has been extensively revisited on the basis of different sophisticated experimental models but also new molecular tools applied to ocular fluids or tissues. Experimental ocular inflammation may not be induced in germ-free animal facilities. Moreover, an infectious agent has been identified in patients presenting with different uveitis entities, previously considered as autoimmune and therefore treated with corticosteroids and aggressive immunosuppressive agents. Molecular mimicry or expression of foreign antigens may trigger an inflammatory reaction, masquerading as an autoimmune uveitis. Fuchs cyclitis, Posner-Schlossman syndrome, serpiginous choroiditis, nonnecrotizing herpetic retinopathies have been recently characterized, expanding the spectrum of infectious uveitis. Microbiological identification remains the first step prior to initiation of a specific therapy, reducing the risk of relapse and achieving a better visual outcome in challenging situations. 
- 2611

Classification of DR

MASSINP

Paris

\section{ABSTRACT NOT PROVIDED}

- 2613

\section{Anti VEGF}

POURNARASC

Memorial Rothschild Clinical Rechearch Group, Colline Ophthalmology Center. Genève

The diabetic macular edema (DME) related to visual loss, results mainly from changes in the affected blood-retina barrierVEGF, originally described as vascular permeability factor, is considered not only as a critical stimulus of retinal neovascularization but also as the primary inducer of increased vascular permeability involved in the formation of diabetic macular edema. Increased levels of VEGF in retina, vitreous and aqueous humor were found in diabetic patients with macular edema (DME). There is increasing evidence that favorable outcomes can be achieved by the use of antivascular endothelial growth factor therapy. Anti-VEGF therapy combined with laser photocoagulation, is superior to laser photocoagulation for the treatment of moderateto-severe visual impairment caused by DME. Data provided from large phase III clinical trials for ranibizumab showed evidence of visual improvement and favorable safety profile. Further studies for bevacizumab, aflibercept and pegaptanib in DME, although limited, also proved the benefits of using anti-VEGF therapy instead of laser. As a result, the use of anti-VEGF therapy as the first line of treatment,is recommended instead of laser photocoagulation in the management of DME.
- 2612

\section{Macular edema}

LOEWENSTEIN A

Tel Aviv

Diabetic macular edema (ME) is a severe complication of diabetic retinopathy (DR). The increasing worldwide prevalence of DR is fueling sustained efforts in devising new ways to prevent as well as to diagnose diabetic ME at an early stage in order to allow effective management to preserve or restore patient's visual acuity. The extant knowledge of the pathophysiology underlying ME points to an interplay among systemic factors, retinal vascular dysfunction, chronic inflammation, angiogenic hyperactivity that promotes permeability and other pathological changes at cellular level, all of which may contribute to the breakdown of the blood-retina barrier and accumulation of extracellular fluid, with subsequent leakage and ME formation. We overview the signs and symptoms which may help diagnose the pathology at different stages (reduced visual acuity, leakage from microaneurysms and nonperfusion) by assessing the role of imaging modalities such as FA and OCT in detecting these pathological changes.

2614

\section{Steroids}

KODIKIANL

Lyon

Diabetic macular edema (DME) is the most common cause of moderate vision loss in working-age individuals in developed countries. Corticosteroids reduce expression of VEGF and other permeability, providing good rationale for their use in DME.The first steroid used was the triamcinolone by intravitreal injection. Efficacy was good but the adverse events were rather important (elevated intraocular pressure being the most common).Different steroids implant with sustained ocular delivery are available.The Fluocinolone Acetonide for Macular Edema (FAME) studies permitted to the ILUVIEN" implant $(190 \mu \mathrm{g})$ has received its marketing authorization approval in different European countries. It is indicated for the treatment of vision impairment associated with chronic DME, considered insufficiently responsive to available therapies.Dexamethasone Intravitreal Implant (OZURDEX $)$ provided long-term vision improvement, with a mean of 4.1 injections over 3 years (phase III MEAD study). Its label is not yet obtained (March 2014). It safety profile was better or equivalent than that of steroids currently used in clinical practice.In summary, two platforms for sustained steroids drug delivery are available in DME. 
- 2615

Clinical cases

CREUZOT-GARCHER C

Dijon

Clinical cases of diabetic retinopathy with or without diabetic macular edema will be

discussed with the audience. These cases will define the role and the pros and cons of different therapeutic options. Cases with macula edema with proliferative diabetic retinopathy, without diabetic retinopathy in phakic or pseudophakic patients will be presented. 


\section{- 2621}

Neuroprotection in the real world

KURYSHEVA N

Ophthalmological department of Medical and Biological Agency of Russian Federation, Moscow

Glaucoma causes vision loss through the degeneration of retinal ganglion cells and their axons. Lowering IOP does not necessarily stop degeneration. Therefore neuroprotection is directed at neurons independently of its effects on IOP [1].As the topical ocular hypotensive treatment is the main option in glaucoma nowadays, the direct neuroprotective effect of hypotensive eye drops is important. It has been postulated that PG analogues may have an IOP independent neuroprotective effect at clinically available intracameral concentration [2]. In vivo experiments demonstrated the neuroprotective properties of betaxolol [3]. The recent study of LoGTS demonstrates that the $\alpha 2$ agonist brimonidine may have a beneficial effect on visual function independent of IOP lowering [4]. Our recent research revealed the high antioxidant activity of fixed combinations of carboanhydrase inhibitors with timolol [5].References 1. Cheung W, Guo L, Cordeiro MF. Optom Vis Sci. 2008;85:406-416.2. Yamagishi R., et al. Exp. Eye Res. 2011;93:265-270.3.Osborne NN, et al. Brain Res. 1997;751:113-123. 4.Krupin T, et al. 2011;151(4):671- 681. 5.Kurysheva N.I., et al. Rus. Ophthalm. J. 2013;6(2):41 - 45.

- 2623

\section{Possible future strategies in Neuroprotection}

PICAUD S (1), FROGER N (1), MATONTI F (1), FORSTER V (1), IVKOVICI (1) BRUNEL N (2), SAHEL J (1)

(1) Institut de la vision, INSERM, UPMC UMR_S 968, CNRS UM7210, Paris (2) INSERM IFR65, Hôpital St Antoine, Paris

Retinal ganglion cell (RGC) degeneration is causing blindness in retinal diseases like glaucoma. However, all therapeutic strategies in glaucoma are targeting the IOP increase but they do not aim at retinal ganglion cell neuroprotection. Using purified retinal ganglion cells, we are screening for neuroprotective molecules. Two natural protective molecules were thus identified.This investigation started when finding retinal ganglion cell degeneration in taurine-deprived animals, we wanted to define if this taurine was acting directly on retinal ganglion cells. Taurine not only increased RGC survival in a pure culture but also in retinal explants exposed to NMDA. Taurine was also found to increase RGC survival in animal models of glaucoma and retinitis pigmentosa. In addition, we had observed that media used to culture mixed retinal cells can promote RGC survival. Using the luminex technology, we have found the presence of VEGF in such media. In fact, recombinant VEGF was able to stimulate RGC survival. RGCs were found to express VEGF receptors in vivo suggesting that this mechanism could have a role in control and pathological conditions. We therefore demonstrated that both taurine and VEGF can provide direct neuroprotective effects on RGCs. If the administration of VEGF is unlikely to become a treatment for glaucoma, these results raise questions on the generalized use of anti-VEGF strategies for retinal diseases such as age-related macular degeneration.

\section{- 2622}

Current and emerging concepts in neuroprotection

CORDEIRO $M$

London

The realisation that lowering IOP is unable to prevent progressive vision loss in all glaucoma patients, has led to investigation of neuroprotection as an alternative or additional treatment option. Neuroprotection has gained renewed interest recently as a therapeutic approach to prevent neuronal degeneration and loss of function in glaucoma, since the publication of the LoGTS study. Although confirmation of neuroprotective effects by other randomized clinical trials is needed, there is now a demonstration of positive non-IOP dependent effects. Furthermore, it has been proposed as a treatment strategy in other diseases, such as AMD, Alzheimer's and Parkinson's Disease. Imaging biomarkers are used in medicine to provide measures that correlate with a clinically significant outcome in disease. By definition they should identify disease early, be easy to assess, have the ability to help predict disease severity and have high enough sensitivity and specificity to be useful. Retinal ganglion cell imaging in glaucoma should fulfil all these criteria. Ideally, a good end-point in glaucoma would be one that could directly measure RGC dysfunction and disease. Continuing developments in imaging technologies offer great potential in glaucoma. More recently, advances in this field have allowed unprecedented in vivo access to the retinal lavers, using many different properties of light to differentiate cellular structures. Over the next few years, advances in therapy \& diagnostic methodologies, such as imaging, offer great potential in future for glaucoma.
- 2624

\section{NP in glaucoma - pitfalls and promises?}

SCHMETTERER L $(1,2)$

(1) Clinical Pharmacology, Vienna

(2) Center of Medical Physics and Biomedical Engineering, Vienna

In the last 25 years the pharmacological spectrum for reducing intraocular pressure (IOP) has increased drastically. As such many patients have their IOP lowered using topical medications. Evidence has, however, accumulated that some patients show visual field deterioration despite low IOP values. As such there is a clinical need for neuroprotective strategies that rescue retinal ganglion cells. Implementation of this concept is, however, not easy. A variety of mechanisms that may contribute to ganglion cell death have been identified, but the interplay of these pathways is largely unknown. As such doubts have been raised whether targeting a single pathway can be successful, which is a major problem in terms of drug approval. In addition, it is not entirely clear which end points should be used in clinical trials focusing on neuroprotection. Nevertheless, the hope to treat progressive glaucoma at low IOPs comes for neuroprotective strategies. 
- 2631

\section{Wavefront Aberrometry: from basics to daily practice}

GICQUELJ

Poitiers

Quality of vision is a new comprehensive set of measures that determines visual performance at near, far and in variable light conditions. Good vision is no longer defined by $20 / 20$ vision. The necessity of evaluating vision beyond Snellen acuity has led clinicians and vision scientists to utilize contrast sensitivity, wavefront aberrometry and straylight measurement in most clinical trials related to vision. Recently,the advent of 3D movies and 3D TVs brought us new challenges regarding 33 Dimensional Quality of Vision.In this course, experts will share with you their experience on this new frontier of modern ophthalmology.

\section{- 2633}

Straylight for dummies: applications in your daily practice

\section{VANDENBERG T}

Amsterdam

If we consider limitations and defects in the optical apparatus of the eye, two distinct types of defect must be distinguished: (1) errors and irregularities in refraction, and (2) light scattering. Those two effects together determine retinal image quality. Light scattering is recognized from the radiation of light around bright light sources against a dark background, leading to glare. The intensity of this "false" light can be assessed and is called "straylight". It disturbs not only night driving, but also day time situations, and can be voiced by patients as "hazy vision" face recognition problems, spatial orientation problems, etc. It is of great importance to realize that these problems often do not coincide with visual acuity loss. So, if visual acuity is good, you may need to assess straylight in order to understand your patient's complaint. This occurs often in case of early cataract. Studies have shown the existence of a large proportion of non-acuity cataract that needs treatment all the same. But also in other conditions straylight can be the more important problem. Practical examples and population data will be discussed, including Fuch's dystrophy, pigmentation defects, IOL deposits and inclusions, etc.

Commercial interest
- 2632

Customization of Intraocular Lenses: a practical approach

PISELLAP

Tours

ABSTRACT NOT PROVIDED 
- 2641

Cytokine gene expression and protein production in West Nile virus-infected retinal pigment epithelium

MUNOZ-ERAZOL (1, 2), MADIGANM (3), KINGNJC (1)

(1) Department of Pathology, Charles Perkins Centre, University of Sydney., Sydney

(2) Save Sight Institute, University of Sydney

(3) Optometry \& Vision Science, UNSW, Sydney

Purpose To investigate if West Nile virus (WNV) infection of human retinal pigment epithelium (RPE) cells induces differential expression of mRNA and proteins for cytokines important for leucocyte recruitment (e.g. CCL5) and integrity of the outer blood retinal barrier (BRB) (e.g. TNF)

Methods Primary human RPE cells (P3-5) were cultured until a complete monolayer was observed, and then infected with 1,5 or 10 plaque-forming units (p.f.u.) of WNV (Sarafend) for 24, 48 and 72hrs. Supernatants were collected for Luminex-based cytokine assay. RNA was prepared from cells and processed for real-time (RT)-PCR. Pro-inflammatory, leucocyte recruitment and viral defense cytokines and chemokines were studied.

Results CCL5, IL8, IL6 (pro-inflammatory) and IFNB1 (anti-viral) showed increases in mRNA expression from $24 \mathrm{~h}$ post-infection (p.i.) and protein levels from $48 \mathrm{~h}$ p.i However in WNV-infected RPE, protein levels of VEGF and TNF were similar to mockinfected cells, despite significant increases in mRNA (TNF 24 h p.i., p $<0.05$; VEGF $48 \mathrm{~h}$, $\mathrm{p}<0.01)$. Interestingly, CCL2 mRNA and protein showed no change in expression postWNV infection at 24,48 and $72 \mathrm{~h}$

Conclusion We previously observed that WNV induced transepithelial electrical resistance (TEER) changes in human RPE (outer BRB) after 48 hours, although the mechanisms(s) remain unclear Studies in mice show that both TNF and CCL2 are important for blood-brain barrier integrity and leucocyte recruitment following WNV infection. Surprisingly we find little or no effect on TNF or CCL2 expression in WNVinfected RPE, suggesting alternate mechanisms operate in RPE post-viral infection. Funding- NFMRI and NHMRC

- 2643

A new and standardized method to sample and analyse vitreous biopsies in unsolved uveitis by the Cellient ${ }^{\circ}$ automated cell block system

VAN CALSTER J, VAN GINDERDEURENR

Dept. of Ophthalmology, University Hospitals Leuven, Leuven

Purpose In this prospective study a universal protocol for sampling and analyzing vitreous material was investigated. Vitreous biopsies are difficult to handle because of the paucity of cells and the gelatinous structure of the vitreous. Histopathological analysis of the vitreous is useful in difficult uveitis cases to differentiate uveitis from lymphoma or infection and to define the type of cellular reaction.

Methods After isolation of vitreous content by a single port biopsy or by a standardized $23 \mathrm{G}$ vitrectomy, 170 consecutive vitreous samples were analyzed with the Cellient tissue processor (Hologic). This machine is a fully automated processor starting from a specified container with PreservCyt' (fixative fluid) with cells to paraffin. Cytology was compared with fixatives Cytolyt" (contains a mucolytic agent) and Preservcyt'. Routine histochemical and immunostainings were evaluated.

Results In 79 cases (46\%) a single port biopsy and in 91 cases (54\%) a tree port pars plana vitrectomy was performed. In $94 \%$ of the cases, sufficient material was found for establishing the diagnosis. In 18\%, a Cytolyt" wash was necessary to prevent clotting of the tubes in the Cellient due to the viscosity of the sample. In $21 \%$ the diagnosis was an acute inflammation (presence of granulocytes), in $32 \%$ chronic active inflammation (presence of T-lymphocytes), in 35\% low-grade inflammation (presence of CD68 cells, without T-lymphocytes); and in 5\% a malignant process. In 9\% a granulomatous inflammation was detected, in $5 \%$ glial tissue (floaters). In $6 \%$ no diagnosis was found.

Conclusion A standardized protocol for sampling and handling vitreous biopsies, fixing in PreservCyt" and processing by the Cellient" system gives a superior result in morphology, number of cells, and possibility of immuno-histochemical stainings. The diagnosis can be established or confirmed in more than $90 \%$ of cases.

\section{- 2642}

Aqueous humor cytokine profile of patients with primary or recurrent ocular toxoplasmosis

THIEME C (1), PLEYER U (2), METZNER S (2), DAMES C (3), SCHLICKEISER S (3) DOBNER B (1), VOLKHD (3)

(1) University Eye Hospital Charité, Berlin

(2) University Eye Hospital Berlin, Berlin

(3) Immunology Institute University Hospital Charité, Berlin

Purpose Toxoplasma gondii infection remains the most frequent cause of infectious posterior uveitis and a significant cause of visual impairment worldwide. Still, the immune mechanisms are largely unexplored. The aim of this study is to investigate the cytokine profiles of aqueous humor samples in patients with first or recurrent manifestation of ocular toxoplasmosis and to correlate findings with clinical characteristics

Methods Using the BioRad Multiplex immunoassay, we determined the concentrations of 27 cytokines/chemokines in aqueous humor specimens obtained from 16 patients with active $(n=7)$ or recurrent $(n=9)$ ocular toxoplasmosis. Results were compared to 5 matched control patients without intraocular infection

Results Using the Wilcoxon-Mann-Whitney-Test significant changes could be identified in both groups affected by either initial or recurrent ocular toxoplasmosis compared to controls. Patients with initial manifestation revealed a significant increase $(\mathrm{p}<0.05)$ of IL-1RA, IL-7, MIP1a and IP-10. In addition significant changes in individuals affected by recurrent disease also differed from controls and became significant $(\mathrm{p}<$ $0.05)$ for TNF-alpha, MIP-1b and IP-10. The cytokine/chemokine profiles however did not differ significantly between initial or recurrent ocular toxoplasmosis subgroups.

Conclusion Our data suggests that MIP1 and IP-10 are associated with both primary and recurrent ocular toxoplasmosis. These findings may support the important role of IP-10 and MIP, as specific chemoattractants for activated T cells in parasitic disorders. In contrast to a recent report we were not able to correlate IL-5 and VEGF with recurrences of the disease. A larger cohort is recruited to underline the results.

\section{- 2644}

Specifics of viral entry in epidemic keratoconjunctivitis

RAIAIYA J, YOUSUF MA, MUKHERJEE S, LEE IS, ZHOU X, RAMKE M, LEE IY, CHODOSHJ

Department of Ophthalmology, Massachusetts Eye and Ear Infirmary, Harvard Medical School, Boston

Cellular entry pathways for viruses dictate the types of immune responses elicited upon infection. Human adenovirus species D type 37 (HAdV-D37) causes epidemic keratoconjunctivitis (EKC), associated with severe ocular surface inflammation. However, viral entry is known to be cell type and virus specific. To examine basic mechanisms of HAdV-D37 pathogenesis in EKC, we studied entry of HAdV-D37 in vivo in our mouse corneal adenovirus keratitis model, and in vitro in cultured human corneal cells. In the mouse model at 1 hour post infection, viruses appeared to be entering cells by macropinocytosis. By 8 hours post infection, viruses formed regular packed intracytoplasmic arrays adjacent to cell nuclei, with minimal capsid uncoating. Therefore, viral entry occurred, but transport of virus to the cell nucleus appeared abortive, possibly explaining the failure of HAdV-D37 replication in the mouse cornea. In vitro, inhibition of PKCo reduced viral entry and phosphorylation of both Src and caveolin-1 in lipid raft fractions, suggesting that PKC $\alpha$ activity occurs upstream of both molecules. Phosphorylated PKC $\alpha$ and Src were found in same endosomal fractions and may be physically associated. These results suggest a central role for PKC in HAdV-D37 entry and regulation of downstream molecules in corneal cells, including caveolin-1. Interestingly, the small GTPase dynamin II, did not appear necessary for HAdV-D37 entry into corneal epithelial cells, but was critical for viral entry in corneal fibroblasts. 
- 2645 / T055

FGF $\beta$ and TGF $\beta$ contribute to tissue remodeling, fibrosis and inflammation in the orbital tissue of severe Grav

PAWLOWSKI P (1, 2), RESZECJ (1), ECKSTEINA (3), JOHNSON K (3), GRZYBOWSKI A (4, 5), CHYCZEWSKIL (1), MYSLIWIEC I (6)

(1) Department of Medical Pathomorphology, Cathedral of Biostructure, Medical University of Bialystok, Bialystok

(2) Department of Paediatric Ophthalmology with Strabismus Treatment Unit, Medical University of Bialystok, Bialystok

(3) Department of Ophthalmology, University Hospital Essen, University of DuisburgEssen, Essen

(4) Department of Ophthalmology, Poznań City Hospital, Poznan

(5) Department of Ophthalmology, University of Warmia and Mazury, Olsztyn

(6) Department of Nuclear Medicine, Medical University of Bialystok, Bialystok

Purpose To assess FGF- $\beta$, TGF- $\beta$, COX2 expression and immunocompetent cells in the orbital fat/connective tissue of patients with severe and mild Graves' orbitopathy as possible prognosting factors of the course of disease.

Methods Orbital tissue from 27 patients with GO undergoing orbital decompression (26 females and 1 male): 1) patients with severe GO $(n=18)$, the mean clinical activity score (CAS) was 8,5 (SD 2.5); 2) Patients with mild GO (n=9), CAS was 2.2 (SD 0.8). 10 individuals undergoing blepharoplasty with no history of orbital inflammation served as controls. We evaluated the expression of CD4+, CD8+. CD20+, CD68 and FGF- $\beta$, TGF- $\beta$, COX 2 in the orbital tissue by immunohistochemical staining

Results The robust intraorbital CD4 + T cells infiltration in severe GO more than with mild GO with absence of CD20+ B lymphocytes and less CD8 infiltration were seen. CD68 expression (fibroblasts and macrophages staining) observed in the fibrous connective area of the adipose tissue of the mild GO and robust in severe GO. Increased FGF $\beta$ expression was observed in the fibroblasts and adipocytes in the connective tissue of the severe GO. The prominent TGF $\beta$ expression was observed in the fibrous connective tissue of the severe and mild GO. No expression of COX2 in the orbital tissue specimen was found in patients with $\mathrm{GO}$

Conclusion Macrophages and CD4 T lymphocytes are both engaged in the active/ severe and long stage of inflammation in the orbital tissue. FGF $\beta$ and TGF $\beta$ expression may contribute to tissue remodeling, fibrosis and perpetuation of inflammation in the orbital tissue of GO especially in severe GO. Their high expression may suggest lack of effect of anti-inflammatory treatment.

\section{- 2647 / T062}

Eswab ${ }^{\circ}$ transport media and 16S RNA PCR in the microbiological diagnosis of infectious keratitis

BAUDONNET R (1, 2, 3), GARNIER F (4, 2, 3), ROBERT PY (1, 2, 3)

(1) Limoges University Hospital, Departement of Ophtalmology, Limoges

(2) Inserm, UMR 1092, Limoges

(3) Limoges University, UMR-S1092, Limoges

(4) Limoges University Hospital, Departement of Bacteriology and Virology, Limoges

Purpose The microbiological diagnosis of infectious keratitis based on a corneal scrapping is necessary in order to adapt the treatment. Our study aimed to determinate if the use of Eswab transport media and 16S RNA PCR increase the sensitivity of this microbiological diagnosis

Methods Retrospective cohort study including all patients who benefited from a corneal scrapping for an infectious keratitis suspicion from June 1, 2007 to June 1, 2011. They were divided into two groups according to the methods of microbiological analysis. In non-Eswab" (NES) group, four samples per patient were collected, directed to laboratories: bacteriology (direct exam and culture); virology (PCR and culture); mycology and parasitology (direct exam and Acanthamoeba PCR). In the Eswab (ES) group, three samples per patient were collected. The first one, on Eswab" transport media, was directed to PCR analysis, including 16S RNA, viral and Acanthamoeba research and bacteriological culture. The two others samples were analysed in mycology and parasitology. Infectious gravity factors and the use of antibiotics before corneal scraping, were reported. The main criteria of judgement was the number of patients with a microbiological diagnosis

Results 190 patients were included, 84 in the NES group and 106 in the ES group. Infectious keratitis gravity factors ( $47,6 \%$ vs $56,6 \% \mathrm{p}=0,356)$ and use of antibiotics before the corneal scrapings ( $23,8 \%$ vs $29,2 \% \mathrm{p}=0,5)$ was not significantly different between two groups. The diagnosis sensibility in the NES group was 36,9\% versus $60,4 \%$ in the ES group ( $\mathrm{p}=0,002)$

Conclusion The usage of a transport media such as Eswab" and 16S RNA PCR research on corneal scrapping significantly improves the sensibility of microbiological diagnosis
- 2646 / T059

Study of TOXO KO vaccine efficiency on the ophtalmological damage bound to Toxoplasmaa gondii, on a mouse model of ocular toxoplasmosis

LEMEE G(1), MORISSE S (2), TARFAOUI N (1), DIMIER-POISSON I (2), SECHEE (2), PISELLA P (1)

(1) ophtalmology, Tours

(2) immunology, Tours

Purpose The toxoplasmosis is one of the most wild-spread parasitoses, affecting almost a third of the world population, and as the main symptomatic demonstration : eye infringement.The aim of this study is to test, on a murin model, the efficiency of a vaccine candidate named Toxo $\mathrm{KO}$, developped by genetic engineering against this pathology.

Methods Ninety Swiss Webster mice (OF1), were filled up with fifty cysts of the 76K strain of Toxoplasmaa gondii (type II). A part of these mice received the TOXO KO vaccine intraperitoneally before. Ophtalmological examination and immunological investigation were performed on aqueous humor samples. The intra retinal cysts were also counted after dissection of the entire retinal tissue

Results After vaccination, we observed a decrease of $69 \%$ of ophtalmological damage and $90 \%$ of intra-retinal cysts. Besides, this group presented no secretion of IFN- $\gamma$ in aqueous humor, testifying of an absence of deleterious inflammation in these eyes unlike those of the control group.

Conclusion Our results are highly significant. This proof of concept lets think that the forward use of such a tool of prevention is possible, to fight more effectively and prematurely against ocular toxoplasmosis.

\section{- 2648 / T058 \\ Infectious keratitis: 4 years in a tertiary hospital}

ANIOS R, VICENTE A, BORGES B, VIEIRA L, PINTO M, MADURO V, ALVES N, CANDELARIA P

Centro Hospitalar Lisboa Central, Lisboa

Purpose To evaluate the clinical presentation, microbiological results and treatment used in cases of infectious keratitis diagnosed in Centro Hospitalar Lisboa Central (CHLC) between July 2009 and July 2013

Methods Retrospective analysis of data from patients who underwent microbiological examination for suspected infectious keratitis between July 2009 and July 2013 in the CHLC ( $\mathrm{n}=86$ patients)

Results The most prevalent risk factor was the use of contact lenses. Of the 93 samples taken, $59(63.4 \%)$ were positive for at least one pathogen. A total of 64 microorganisms were isolated with 35 (54.8\%) being Gram-negative, 25 (41.9\%) Gram-positive and 2 (3.2\%) fungi. The most prevalent pathogen was Pseudomonas aeruginosa. In two patients, Acanthamoeba cysts were isolated by confocal microscopy. Hospitalization was needed in $64.0 \%$ of cases. The treatment of choice was the use of fortified eyedrops of vancomycin and ceftazidime. In seven patients surgical therapy was needed for infection control

Conclusion Our work draws attention to the morbidity associated with this disease and reinforces the importance of conducting microbiological studies in these patients. The main predisposing factor in our population was the use of contact lens, with Gramnegative microorganisms being isolated in the majority of cases. 
- 2651

Advanced glycation end-products induced activation of NFkB and its suppression by different neurotrophic factors

BIKBOVA G, OSHITARI T, YAMAMOTOS

Ophthalmology and Visual Science, Chiba

Purpose To determine the effect of high doses of AGEs on neurite regeneration in isolated rat retinas, and the regenerative effects of different neurotrophic factors.

Methods Retinal explants of 4 adult SD rats were three-dimensionally cultured in collagen gel, and incubated in: 1) serum free control culture media; 2) $100 \mu \mathrm{g} / \mathrm{ml}$ glucose-AGE-BSA, glycolaldehyde-AGE-BSA, glyceraldehyde-AGE-BSA media; 3) glucose, glycol, glycer+100 ng/ml neurotrophin 4 (NT-4)media; 4) glucose, glycol, glycer+100 ng/ml hepatocyte growth factor (HGF) media; 5)glucose, glycol, glycer+100 $\mathrm{ng} / \mathrm{ml}$ glial cell line derived neurotrophic factor (GDNF) media; or 6) glucose, glycol, glycer+100 ng/ml tauroursodeoxycholic acid (TUDCA) media. After 7 days, the number of regenerating neurites were counted under a phase-contrast microscope. The explants were immunostained for NFkB transcription factor. Statistical analyses were performed by one-way ANOVA.

Results In retinas incubated with AGEs, the number of regenerating neurites were fewer than in control. Neurotrophic factors increased the number of neurites, but more significantly in the NT4 group. The number of NFkB immunopositive cells was higher in retinas exposed to AGEs than in control $(53,2 \pm 7,2 \%$ vs.31, $6 \pm 16,08 \%, \mathrm{P}=$ $0,0146)$. Neurotrophic factors decreased the number of immunopositives cell, but more significantly in the NT4 group.

Conclusion High dose AGEs induce activation of NFkB and NT4 significantly suppresses this activation and enhances neurite regeneration in isolated retinas compared with other neurotrophic factors such as HGF,GDNF,and TUDCA

\section{- 2653}

Focal venous hypertension as a pathophysiologic mechanism for port-wine stains and the Sturge-Weber syndrome

PARSA C $(1,2)$

(1) Quinze-Vingts National Ophthalmology Hospital, Paris

(2) UPMC-Sorbonne Universities

Purpose To provide an in-depth re-examination of assumed causes of tissue hypertrophy, port-wine stains and the Sturge-Weber and related syndromes to support an alternative unifying pathophysiologic mechanism of venous dysplasia producing focal venous hypertension with attendant tissue responses and to propose a novel etiological hypothesis for the venous dysplasia in these syndromes with supportive evidence.

Methods Data from twenty patients with port-wine stains was collected prospectively by the author in an institutional referral-based practice. The literature was searched using Medline.

Results Newly obtained dermatologic, corneal pachymetry, fundus ophthalmoscopic, ocular and orbital venous Doppler ultrasonography, and magnetic resonance imaging findings in patients with the Sturge-Weber syndrome or isolated port-wine stains, along with published data, reveal diffusely thickened tissues and neural atrophy in all areas associated with venous congestion.

Conclusion Contrary to traditional understanding, signs and symptoms in the SturgeWeber and related syndromes, including both congenital and acquired port-wine stains are shown to arise from effects of localized primary venous dysplasia or acquired venous obstruction rather than neural dysfunction, differentiating these syndromes from actual phacomatoses. Effects of focal venous hypertension are transmitted to nearby areas via compensatory collateral venous channels in the above conditions.Conclusion: A novel underlving etiology - prenatal venous thrombo-occlusion-may be responsible for the absence of veins with persistence and enlargement of collateral circulatory pathways with data in the literature backing this offshoot hypothesis. The mechanism for isolated pathologic tissue

\section{- 2652}

Retinal vessel pathologies in a rat model of periventricular leucomalazia

LORENZ B (1), STECKJ (1), BLUEML C (2), KAMPMANN S (2), GERSTNER B (3), MAIER RF (2), STIEGER K (1)

(1) Department of Ophthalmology, Justus-Liebig-University Giessen, Giessen

(2) Department of Pediatrics, Phillips-University, Marburg

(3) Department of Pediatric Cardiology, Pediatric Heart Center, Justus-Liebig-University, Giessen

Purpose Alternating oxygen levels during delivery and supplemental oxygen therapies can cause pathological vascularization of the retina (retinopathy of prematurity, ROP) and/or loss of oligodendrocytes in the periventricular area of the brain with subsequent white matter damage (periventricular leukomalacia (PVL). The aim of this study was to examine retinal vessel pathologies in the standard PVL rat model in order to identify uniform damage pathways.

Methods Ischemia was induced in post natal day (P) 6 Long Evans rats with unilateral (left side) carotid ligation (UCL) and exposure to different oxygen concentrations. In total, four different groups were examined: (A) hypoxia/ischemia $(\mathrm{UCL}+6 \% \mathrm{O} 2$ $1 \mathrm{~h})$, (B) hyperoxia $(80 \% \mathrm{O} 2,24 \mathrm{~h})$, (C) hypoxia/ischemia + hyperoxia ( $\mathrm{UCL}+6 \% \mathrm{O} 2$, $1 \mathrm{~h}+80 \% \mathrm{O} 2,24 \mathrm{~h}$ ), (D) normoxia. Morphological analysis of vessel development was performed at P11 and P21. Quantitative PCR was performed at P7, P11, and P21 (VEGF, HIF1a, EPOR, TNFa, iNOS and BMP-9).

Results Distinct retardation in deeper vascular plexus development was observed most prominent in left eyes of groups $\mathrm{A}$ and $\mathrm{C}$. Diameters of big retinal arteries were significantly increased in central retinae at P21 and showed retardation in vascular remodeling. Left retinae of groups A and C at P11 displayed reduced capillary free zones (CFZ) and an increase in the number of branching points (BP). Quantitative gene expression analysis showed down regulation of angiogenic factors at P7.

Conclusion This is the first report on concurring damage to the retina that was evaluated in a model of white matter injury in the neonatal brain. We observed mild damage to the vessel development and maturation, which is the basis for a model for moderate forms of ROP.

\section{- 2654}

Incidence and characteristics of congenital cataract surgery in France from 2010 to 2012

DAIEN V (1), ELIAOU-MALRIEU C (1), LEPAPE A (2), HEVE D (2), VILLAINM (1) (1) Universitary Hospital, Ophthalmology, Montpellier

(2) Regional agency of health, Languedoc Roussillon, Montpellier

Purpose Prevention of visual impairment due to congenital cataract is an international priority. Estimates of incidence are required for implementation and assessment of preventive strategies, but are lacking, despite routine monitoring of birth defects at a national level in many industrialized countries. The purpose of this study was to determine the incidence of congenital cataract in France between 2010 and 2012. Secondary objective was to assess the proportion of unilateral and bilateral cataract surgeries, age of surgery and implantation of intraocular lens or not.

Methods All children who undergo cataract surgery before 1 year of age in France between January 2010 and December 2012 were identified using the french national database of information systems. Incidence rate per year was estimate using the French database from the national institut of statistics.

Results In 3 years, 590 eves in 464 children with $46.6 \%$ of girls had a cataract surgery with $27.0 \%$ of them having a bilateral surgery. In the first year of age, cataract surgery incidence was $1.87 / 10000$ birth in this period. Age (median [interquartile]) of surgery was 3.5 (2.2-4.8) months. Among the 590 operated eyes, 28\% (168 eyes) were not implanted with intraoculair lens.

Conclusion The incidence of congenital cataract in France is close to what was previously assessed in literature in industrialized world. Median age of surgery corresponds to recommendations. An important proportion of children was not primary implanted with an intraoculair lens. 


\section{- 2655 / T092}

A new pediatric vision screener employing polarizationmodulated, retinal-birefringence-scanning-based strabismus detection and bull's eye focus detection

IRSCH K (1, 2), GAMATIKOV B (1), WU YK (1), GUYTOND (1)

(1) The Wilmer Eye Institute, The Johns Hopkins University School of Medicine, Baltimore

(2) Centre d'Investigation Clinique, CHNO des Quinze-Vingts, Paris

Purpose Commercially available pediatric vision screening devices that test for amblyopiás primary causes (strabismus and defocus) can detect strabismus only indirectly and inaccurately via assessment of the positions of external light reflections from the cornea, but they cannot detect the anatomical feature of the eyes where fixation actually occurs (the fovea).

Methods Our laboratory has been developing technology to detect true foveal fixation, by exploiting the birefringence of the uniquely arranged Henle fibers delineating the fovea, using retinal birefringence scanning (RBS), and we recently described a new, polarization-modulated approach to RBS that enables entirely direct and reliable detection of true foveal fixation, with greatly enhanced signal-to-noise ratio and essentially independent of corneal birefringence. Here we present a new pediatric vision screener (PVS) that employs polarization-modulated RBS for strabismus detection, and double-pass focus detection using a bull's eye photodetector and improved target system with accommodative control.

Results Performance tests in adult volunteers and pediatric patients demonstrate that our new PVS 1) is capable of detecting even small angles of eye misalignment (at least $0.75^{\circ}$ or $1.3 \mathrm{PD}$ ), 2) has the potential to detect spherical focus within $\pm 1.00 \mathrm{D}$, and 3 ) has the potential to detect the misalignment in patients with accommodative esotropia who only develop a crossed eye when focusing intently on a near target.

Conclusion In conclusion, the new PVS shows real promise in supplying the demand for a reliable, automated strabismus/defocus screening tool for children at risk for amblyopia.
- 2656 / T093

Neosynephrine and tropicamide eyedrops for pupil dilation in premature infants is almost twice as efficient as tropicamide eyedrops alone

LUX AL (1), GUILLOIS B (2), DEGOUMOIS A (1), MOURIAUX F (1), DENIONE (1) (1) Ophthalmology, Caen

(2) Neonatology, Caen

Purpose Retinopathy of prematurity screening requires pupil dilation of premature infants. While essential for reliable fundus examination, proper dilatation may sometimes be difficult to achieve in this population. The aim of our study was to compare the efficacy of two mydriatic regimens: one consisting in three drops of tropicamide $0.5 \%$ and the other one consisting in two drops of tropicamide $0.5 \%$ with one drop of phenylephrine $5 \%$

Methods Thirty premature infants were enrolled and each infant was dilated with one regimen in one eye and the other regimen in the other eye. The side of each regimen was determined by randomization. A photograph of each eye was taken. The photographs were anonymised and randomly presented to two independant readers. The effect of the two regimens was determined by calculating the ratio of pupil diameter divided by cornea diameter. Assuming that pupil and cornea are circle-shaped, we calculated the ratio of pupil surface area relative to cornea surface area for both regimens.

Results The pupil to cornea diameters ratio was 46.7\% (+/-8.8) with tropicamide alone and $65.2 \%(+/-6.0)$ with tropicamide-phenylephrine combination. The ratio of pupil to cornea surface areas was $21.8 \%$ with tropicamide alone and $42.6 \%$ with tropicamidephenylephrine combination. In other words, the pupil surface area was 1.95 fold greater with tropicamide-phenylephrine combination than with tropicamide alone

Conclusion The use of a combination of two drops of tropicamide $0.5 \%$ with one drop of phenylephrine $5 \%$ is far more efficient than tropicamide alone to achieve proper pupil in premature infants.

\section{- 2657 / T094}

3D shape perception in strabismus subjects

SAWAMURA H (1), GILLEBERT C (2), TODD IT (3), VANDENBERGHER (4) ORBANGA $(5)$

(1) Department of Ophthalmology, Tokyo University, Tokyo

(2) Department of Experimental Psychology, Oxford University, Oxford

(3) Department of Psychology. The Ohio State University, Columbus

(4) Laboratory for cognitive neurology, Department of. Neuroscience, KU Leuven, Leuven (5) Department of Neruoscience, Parma University, Parma

Purpose To evaluate 3D shape perception in strabismus subjects, psychophysical experiment was performed using 3D shapes extracted from four different visual cues (shading, texture, motion and disparity), comparing normal and strabismus subjects.

Methods The 11 randomly generated complex 3D shape visual stimuli were created and rendered in 4 modalities (3D shape from shading, texture, motion and disparity) and displayed on a PC monitor. The subjects were required to indicate the global maximum of the 3D shape as accurately as possible by displacing a red cross using the computer mouse. The distance in depth $(\mathrm{cm})$ between the global maximum indicated by the subject and the true global maximum was calculated. Strabismus subjects $(\mathrm{n}=43$; 6-65 years of age) and normal subjects ( $\mathrm{n}=18: 6-57$ years of age) participated in the task All the subjects have normal or corrected normal visual acuity. Stereo acuity was also evaluated by titmus stereo test.

Results 3D shapes extracted from disparity were more difficult to perceive than those extracted from texture (ANOVA, $\mathrm{p}<0.03)$, motion $(\mathrm{p}<0.01)$ and shading $(\mathrm{p}<0.01)$ in both normal and strabismus subjects. The 3D shape perception in normal and strabismus subjects differed only for the binocular stereopsis cue, not the other visual cues of motion, shading and texture. In addition, 3D shape perception of motion, shading and texture was not affected by the stereo acuity in either normal or strabismus subjects.
- 2658 / T111

Clinical experience with Raxone ${ }^{\circ}$ (Idebenone) in the treatment of patients with Leber's hereditary optic neuropathy (LHON)

METZ G(1), GALLENMÜLLER C (2), VONLIVONIUS B (2), LOB F (2), COPPARD N (1), MEIER T (1), KLOPSTOCK T (2)

(1) Santhera Pharmaceuticals, Liestal

(2) Ludwig-Maximilians-University, Munich

Purpose $\mathrm{LHON}$ is an orphan mitochondrial disorder affecting the retinal ganglion cells leading to permanent blindness from which recovery is rare. Here we report visual acuity outcomes for patients with recent onset who received Raxone' (idebenone) under an ongoing global Expanded Access Program (EAP).

Methods Visual acuity was measured in 3-monthly intervals. Clinically relevant recovery was defined as (i) improvement from nadir by at least 10 letters on the ETDRS chart or (ii) improvement from "off-chart" at nadir to being able to read at least 5 letters on-chart.

Results Currently there are $61 \mathrm{LHON}$ patients enrolled, of which 48 patients have been treated for an average of 11 months. So far, 24 of 48 patients (50\%) have experienced a clinically relevant and stable recovery in VA (89\% for T14484C, 70\% for G3460A and $31 \%$ for G11778A). The average treatment effect size in patients with recovery was 29 letters and $84 \%$ recovered within 12 months of the initiation of the EAP

Conclusion A high proportion of LHON patients treated with idebenone under a global EAP experienced a clinically meaningful recovery of vision, further demonstrating the therapeutic potential of idebenone in the treatment of LHON.

Commercial interes 
- 2661

Normal pigment production

ARVEILER B $(1,2)$

(1) Rare Diseases - Genetic and Metabolism, University of Bordeaux, Bordeaux

(2) Medical Genetics, Universitary Hospital, Bordeaux

Melanin is synthesized in melanocytes and retinal pigment epithelial cells. Two main types of melanin are produced: eumelanin (brown, black) and phaeomelanin (yellow, orange). Pigmentation is a complex process involving various steps from normal development of melanocytes to the synthesis of melanin itself. In the skin and choroid, precursors of melanocytes are melanoblasts which are derived from the neural crest. Retinal pigment epithelium cells are generated directly from the optic neuroepithelium. Melanoblasts migrate and colonize the epidermis, hair follicles, and ocular, ear and nervous structures. The differentiation of melanoblasts into melanocytes is controlled by numerous transcription factors and signalling pathways, and is characterized by the acquisition of Tyrosinase activity, the presence of mature melanosomes, and the formation of dendrites. Melanin production results from catalytic activities by Tyrosinase, TRP-1 and TRP-2 and occurs in melanosomes which follow a maturation process involving four stages (I - IV). Melanosomes are transported from the perinuclear region to the end of melanocytic dentrites, where they are eventually transferred to keratinocytes.

- 2663

\section{Genotypes \& molecular strategies in albinism}

ARVEILER B (1, 2), LASSEAUX E (2), MORICE-PICARD F $(1,2$

(1) Rare Diseases - Genetic and Metabolism, University of Bordeaux, Bordeaux

(2) Medical Genetics, Universitary Hospital, Bordeaux

Oculocutaneous albinism (OCA) affects $1 / 17000$ person. It is characterized by hypopigmentation of the skin, hair and eyes. The main handicap in patients is at the ophthalmologic level. There are 6 known OCA genes (TYR, OCA2, TYRP1, SLC45A2, SLC24A5, C10ORF11) (OCA1,2,3,4,6,7 respectively). An OCA5 locus has been localized, but the gene is not known yet. Apart from the OCA types, an X-linked ocular albinism gene (OA1, GPR143) as well as 17 genes involved in syndromic forms (HPS CHS, WS, GS) of albinism have been identified. We routinely search for mutations (point mutations, deletions) in the OCA1, 2, 3, 4, 6,7, OA1 and HPS1 genes. Our data in more than 400 patients show $36 \%$ OCA $1,25 \%$ OCA2, 2\% OCA3, 11\% OCA $4,1.25 \%$ OCA6, 0\% OCA7, 6\% OA1, 1\% HPS1. 17.75\% of patients remain without a molecular diagnostic either because only one mutation (6\%)or no mutation at all (11.75\%) was found.This suggests two main alternative hypotheses. First of all the patient may have a hitherto clinically undiagnosed form of syndromic albinism. For this reason, we are now heading towards analyzing all 24 known albinism genes by next generation sequencing. Secondly, it is likely that additional OCA genes remain to be identified.

\section{- 2662}

The array of clinical albinism subtypes

LEROY BP

Ghent

Purpose:To illustrate the different types of clinical subtypes of albinism. Methods:A systematic overview of the different clinical subtypes of albinism will be given. The relationship between the different phenotypes and their respective genotypes will be explained.Results:In general, a distinction is made between ocular albinism and oculocutaneous albinism. The latter can be isolated or syndromic. Examples of syndromic albinism include the Hermansky-Pudlak and Chediak-Higashi syndromes. Conclusions:Ocular and oculocutaneous types of albinism exist. The latter can isolated or can be part of syndromic oculocutaneous albinism.

2664

Electrophysiology in the diagnosis of albinism

$\operatorname{HOLDER~GE~}(1,2), \operatorname{NEVEUMM}(1,2)$

(1) Moorfields Eye Hospital, London

(2) UCL Institute of Ophthalmology, London

Albinism is a genetically determined disorder of melanin synthesis associated with intracranial visual pathway misrouting, with the majority of fibres from each eye usually decussating to the contralateral cerebral hemisphere. The presentation will detail the use of flash and pattern appearance VEPs in the demonstration of this albino misrouting. It will be demonstrated that the misrouting has a highly variable expression, including uniocular misrouting, and is a spectrum of disease rather than an all-or-none phenomenon. 


\section{- 2671}

Innovative treatments for MGD - EVER more important for ocular surface disease

KNOPN (1), KNOPE (2)

(1) Ocular Surface Center Berlin (OSCB), Dept. for Cell- and Neurobiology, Charité Universitätsmedizin Berlin, Hannover

(2) Ocular Surface Center Berlin (OSCB), Dept. for Cell- and Neurobiology, Charité Universitätsmedizin Berlin, Berlin

The 2011 TFOS MGD Report has translated into several new developments in the therapy of Dry Eye Disease with a focus on MGD. Lipid supplementation is recognized as crucial for dry eye therapy either as liposomal spray or in many new tear substitutes. Physical therapy is recognized as a causative treatment in order to re-liquify inspissated secretum before the application of mechanical pressure in order to eventually release obstructed glands. Since the application of adequate heat is time consuming and tricky, pre-heated gel applicators or controlled heating googles can be used but must still be repeated every day. Even then manual expression of the multitude of meibomian glands can be painful or ineffective. A new automated device that allows the computer controlled application of heat and pressure under the supervision of the ophthalmologist in his practice presently occurs as the most advance and effective therapy option that can lead to longterm restoration of meibomian gland function over several months. Azithromycin, a new topical drug has, besides a long-term tissue uptake, not only an antibiotic but also an anti-inflammatory and a newly observed promotive effect on gland function and is widely used for MGD.

- 2673

Automatic application of warmth and expression - a breakthrough in physical therapy of dry eye

GREINER

Schepens Eye Research Institute, Massachusetts Eye and Ear Infirmary, 20 Staniford Street, Boston, MA, Boston

The most common form of dry eve disease is evaporative dry eye resulting from meibomian gland dysfunction (MGD) based on obstructed MG.A prospective, randomized, crossover, multicenter clinical study employed a single treatment with the automated computer controlled LipiFlow thermal pulsation system (LTPS). Improvement in signs \& symptoms and its duration in patients with evaporative dry eye secondary to MGD was investigated over several time points. Initial results verified significant improvement of MG secretion at 2 \& 4 weeks compared to conventional warm compress method. This was based on evaluation of tear film break up time (TFBUT), ocular surface staining, patient symptoms (OSDI \& SPEED test). The secretion score improved significantly from baseline $(4.4 \pm 4.0)$ to 1 - month post-treatment $(11.3 \pm$ 6.2; $\mathrm{p}<0.0001)$ and this improvement was maintained with no significant regression at 9 months $(11.7 \pm 5.9)$ and even at 3 -years $(18.4 \pm 6.2)$ after therapy.Treatment with the Lipiflow device is a technological advance that exceeds any known form of dry eye therapy in duration and efficacy, since it is efficacious even for extended durations (9mos, 1-, 2- and 3-years) following a single 12-minute treatment.
- 2672

Individual physical therapy of MGD in private setting by a moist heat device

PURSLOWC

Monmouth

There is a frequent observation from patients that dry eye symptoms improve after time spent in a steam room or sauna. This presentation will outline the challenges in delivering heat to the meibomian glands and why moist heat is the gold standard in heat therapy in meibomian gland dysfunction (MGD). This understanding led to the invention of a medical device by a British ophthalmologist that was commercially developed to deliver a controlled amount of latent, moist heat (Blephasteam, Laboratoires Thea, France). Its use in patients will be presented: patient satisfaction and compliance are increased compared to conventional methods, whilst clinical signs are also improved.

- 2674

Restoration of meibomian gland function in severe dry eye disease due to obstructive MGD with an automated physical therapy device

FINIS D, HAYAINEH J, KÖNIG C, BORRELLI M, SCHRADER S, GEERLINGG Dusseldorf

We compared the effectiveness of a single Lipiflow" treatment with combined lid warming and massage in patients with Meibomian gland dysfunction (MGD) in a prospective, randomized, crossover, observer-masked trial. Subjects were randomized to receive either a single 12-min Lipiflow" treatment or to perform combined twice-daily lid warming and massage for 3 months. All subjects received an examination pattern of subjective symptoms, lipid layer thickness, Meibomian gland assessment, tear break-up time, tear osmolarity, corneal and conjunctival staining, Schirmer test values, and tear meniscus height before, and 1 and 3 months after initiation of treatments.A total of 31 subjects completed the 3-month follow up. At 1 and 3 months, patients in the Lipiflow treatment group had a significant reduction in subjective symptomes compared with those in the lid-margin hygiene group. With regard to objective parameters, both treatments produced a significant improvement in expressible Meibomian glands; no other significant differences were noted between the two groups. Results of our study show that both a single Lipiflow treatment and a 3-month, twice-daily lid margin hygiene regimen are effective therapies for MGD.

Commercial interest 
- 2675

Azithromycin and MGD: Is it possible to turn an off-label treatment into an on-label therapy?

SULLIVAN DA, KAM WR, DING J, LIU Y

Schepens Eye Research Institute and Harvard Medical School, Boston

Meibomian gland dysfunction (MGD) is the primary cause of dry eye disease (DED), which afflicts hundreds of millions of people, predominantly women, and is a leading cause of patient visits to eye care practitioners. The impact of moderate to severe DED is comparable to conditions such as dialysis and severe angina, and is associated with significant pain, role limitations, low vitality and poor general health. There is no cure for MGD. The most frequent pharmaceutical treatment in the United States for the management of MGD is the off-label use of topical azithromycin. This antibiotic is presumed to be effective because of its anti-inflammatory and anti-bacterial actions, which may suppress the MGD-associated posterior blepharitis and growth of lid bacteria. We hypothesize that azithromycin can act directly on human meibomian gland epithelial cells to stimulate their differentiation, enhance the quality and quantity of their lipid production, promote their holocrine secretion and ameliorate MGD pathophysiology. Our results support our hypotheses and may help lead to the approval of topical azithromycin as a safe and effective therapy for human MGD and thereby improve the quality of life of countless people throughout the world.

Commercial interest
- 2676

Clinical experiences with short-term delivery of azithromycin in conjunctival inflammation and MGD

DOANS

Hopital Bichat and Fondation A de Rothschild, Paris

Azithromycin is an azalide antibiotic, derived from the macrolides family. This is a good candidate for treating meibomian gland dysfunction and MGD-related ocular surface inflammation for several reasons : (1) its bacterial spectrum is mainly oriented against gram positive cocci ; (2)it has a long tissular and lachrymal half life, because it accumulates in the intracellular compartment ; (3) it has anti-inflammatory, anti-MMP and lipase inhibiting properties.We tested azithromycin $1.5 \%$ eyedrops in blepharitis patients in two clinical studies. - The first study was a multicenter randomized placebo controlled study, involving chronic moderate-to-severe blepharitis patients. -The second study was a single center non controlled prospective study in children suffering from ocular rosacea with chronic phlyctenular keratoconjunctivitis. In both studies, topical azithromycin was effective in reducing signs and symptoms. We will discuss the results of these studies in detail, and provide practical clinical schemes for the use of this innovative treatment.

Commercial interest 
- 2711

Biological mechanism of myopia development

SCHAEFFEL F

Tübingen

Purpose. Describing biological mechanisms of visual control of eye growth, based on experiments in animal models.

Methods. To induce refractive errors, emmetropic eyes are treated with positive lenses (causing development of hyperopia), negative lenses or diffusers (causing myopia) Ocular biometry by ultrasonography or low coherence interferometry, refractions measured by infrared photoretinoscopy, transcription of candidate genes in fundus by realtime PCR, dopamine and metabolites by HPLC, proteins by immunohistochemistry. Receptor agonists or antagonists are intravitreally injected.

Results. Eye growth is locally controlled by the retina which detects the sign of defocus to accelerate or reduce axial eye growth but underlying image processing is not understood. Major retinal signals are dopamine, glucagon (in chick), acetylcholine, $\mathrm{NO}$ and retinoic acid. Signals can be divided in image blur, light and diurnally controlled (dopamine) or sign of defocus controlled (glucagon, ZENK/Egr1, retinoic acid).

Conclusions. The amount of knowledge accumulated on visual control of eye growth over the past 25 years is abundant but cannot yet prevent myopia that is induced by changes in visual experience in our industrialized world.

- 2713

Interventions to slow the progression of myopia

OHLENDORFA

ZEISS Vision Science Lab, Tuebingen

Purpose The purpose of the talk is to assess the effectiveness of different strategies including optical refractive corrections with either spectacles or contact lenses as well as pharmaceutical approaches, to control the progression of myopia.

Methods Interventions that have been explored to slow the progression of myopia include monofocal, bifocal and multifocal spectacles, cycloplegic drops, drugs to lower the intraocular pressure, muscarinic receptor antagonists and contact lenses. The scientific background for the different interventions will be presented and results of clinical trials will be reviewed.

Results Clinical trials have shown negligible positive effects of progressive additiona and bifocal lenses on myopia progression compared to single vision lenses. Observed beneficial effects of rigid gas permeable contact lenses could be related to the ethnicity of participants. The largest positive effects for slowing myopia progression were exhibited with anti-muscarinic medications.

Conclusion Further investigations of myopia control must be conducted in order to find treatments which are clinically meaningful and beneficial. The leading potentia candidates at this time are anti-muscarinic topical medications.

Commercial interest

\section{- 2712}

Myopia and high myopia - genetics and environmental factors

MORGANI $(1,2)$

(1) Research School of Biology, Australian National University, Canberra

(2) Division of Preventive Ophthalmology, Sun Yat-sen University, Guangzhou

Prevention of myopia is now urgent, since there is an epidemic of high myopia, as well as ordinary myopia, in East Asia. The prevalence of high myopia is now around 20\%, compared to about $1 \%$ in older generations. This new, acquired, high myopia has an unusual developmental pattern, sitting at around 1\% up to the age of 11-13, and then increasing over the next 10 years, presumably as a result of early onset environmental myopia progressing to high myopia. A critical unanswered question is whether this acquired high myopia carries the same pathological burden as the older genetic form. This question is generally important, because the prevalence of myopia appears to be rising in Europe and North America, and the prevalence of high myopia is probably increasing as well. Fortunately, it is now generally accepted that environmental change in educational pressures and the time that children spend outdoors must be responsible for the 3-4-fold increase in the prevalence of myopia in East Asia. In contrast, identified SNPs currently account for less than $5 \%$ of phenotypic variation. Recent trials based on increasing the amount of time that children spend outdoors suggest that incident myopia, and hence high myopia, can be prevented.

- 2714

Progressive myopia and their associated pathologies (definitions and therapies)

MAIERM

Munich

Pathological myopia (PM) is a usually progressive eye disease causing significant visual loss. PM predominantly affects younger active emploved patients therefore its complications have major individual and sozioeconomic consequences.Common and special myopia associated pathologies will be reviewed and their treatments will be discussed.Progressive chorioretinal distension results in pathognonomic changes at the fundus called "myopic retinopathy". Myopic conus, posterior staphyloma, rupture of Bruch `s membrane (lacquer cracks), myopic choroidal neovascularization (mCNV), chorioret-inal atrophy, myopic vitreopathy, myopic traction maculopathy and Fuchs spot repre-senting the scar stage of myopic CNV can be found. Myopic choroidal neovasculari-zation (mCNV) is the major cause of visual impairment in myopic patients. In the past, photodynamic therapy (PDT) as treatment of mCNV was only able to stabilize visual acuity. A randomiced, multicenter prospective Phase 3 study (RADIANCE) compared photodynamic therapy (PDT) and ranibizumab treatment and showed that immediate treatment of mCNV with a VEGF-inhibitor (ranibizumab) significantly im-prove visual acuity.There are suitable treatments of myopia associated pathologies and further studies using other VEGF-inhibitor agents (Bevacizumab, Aflibercept) are underway. 
SIS: Myopia and pathological myopia - etiology and therapeutic approaches

- 2715

Scleral crosslinking as a therapeutic approach to treat progressive myopia

ISELI HP

Ophathalmology, Zurich

IntroductionElongation of the bulbus is the main cause of high myopia. Light induced scleral crosslinking is suggested as a therapeutic approach to prevent extensive eye elongation . Data about efficient light intensities and light-induced damage threshold of retinal tissue are not conclusive. There are no data available about the effect on eye growth of light induced crosslinking in an animal model.Material an methodsin vitro and in vivo experiments in rabbits were performed with riboflavin/blue light application for scleral crosslinking. Mechanical stiffening effects were studied by means of rheology and tissue damage threshold were characterized by histology and electron microscopy.ResultsCollagen Crosslinking by riboflavin/blue light stiffens the scleral tissue and induced a significant reduction of eye growth in young rabbits without damaging effects on retinal tissue. ConclusionRiboflavin/Blue light treatment might be a promissing treatment to control eye growth and therefore, to prevent high myopia. More investigations are needed to establish optimal treatment parameters.

Commercial interest 


\section{- 2721}

Experiences with developing virtual clinics in a specialist centre

KOTECHA A

Moorfields Eye Hospital and UCL Institute of Opthalmology, London

A challenge facing many ophthalmology departments is how to manage the increasing numbers of glaucoma patients efficiently without compromising patient care. There are a number of approaches to meeting the increasing demand for services. One is to increase clinic capacity, which is not a viable long term solution. Another is to implement community eye care schemes, whereby 'stable' patients may be discharged from secondary care to be followed up within the community. However, there will always be a number of patients who are not suitable for, or who do not want, community monitoring. These patients need to be managed efficiently within the secondary-care hospital setting. The 'virtual clinic consultation' has traditionally thought of as a model to manage patients in remote areas, who have limited access to specialist care. However, as information technologies improve, there is scope for this style of 'consultation' to be used within the hospital setting. This talk will discuss the practical aspects of developing a 'virtual' service within a UK National Health Service hospital, the challenges faced and overcome, when developing the service, and the overall impact the service has had within the Glaucoma Service itself.
- 2723

\section{Virtual glaucoma services in Finland}

TUULONENA

Tampere

The number of patients using reimbursed glaucoma medications in Finland has increased 2.4-fold since 1986 and increases by $2.5 \%$ every year. In 2020, 100000 patients are expected to be using glaucoma drops in a population of 5.4 million. Simultaneously the gap between diagnostic and therapeutic possibilities and available resources is broadening more rapidly. It is obvious that radical changes and new insights in attitudes and policies are mandatory to make the glaucoma processes more cost-effective and to be able to guarantee access to high quality, high volume and low cost care. There is a continuous trade-off 'right' level or services. Paradoxically, just adding more care may not always be better. What is too early vs. too late diagnosis? What represents under- vs. over treatment in glaucoma care? Is 'good enough' enough?With limited resources more resources are allocated for patients with greatest risk of visual disability. Physicians' time is spent in developing the health care system to find and segment the aggressive and rapidly progressive glaucoma patients from 'usual' stable patients - as well as organizing and supervising the work of other professionals and care processes As it is very expensive - time and money wise - to receive simple care from highly trained professionals (e.g. tonometry-based follow-up of glaucoma), shared care is of utmost importance. Whatever we do, we start by asking whether the intervention (test, treatment, care protocol etc) is necessary, i.e. beneficial to the patient's well being. If the answer is 'yes' - and the society affords the intervention - the next question asks who should do it and could technology substitute professionals (e.g. imaging, hometonometer etc). As it is especially the cumulative effect of small changes in clinical practices (e.g. adding new diagnostic tests or therapy) that has a massive impact on the healthcare budgets, clinician weighs not only their benefits and risks but should also consider the costs. Whenever we wish to add more care, we simultaneously consider removing unnecessary old interventions and preventing the adoption of useless new interventions. The principles of virtual glaucoma virtual glaucoma services in Finland can be summarized as the priorization and segmentation (most resources allocated to prevent permanent visual disability), standardization and efficient ways to produce services as well as shared care among different professionals and patients.

\section{- 2722}

Virtual glaucoma clinic in Wales

MORGANJE

Ophthalmology, Cardiff

The effective clinical review of increasing numbers of glaucoma patients remains a major challenge. To meet this, we have developed a virtual clinic review module using the OpenEyes electronic medical record for Ophthalmology. OpenEyes is Open Source and browser-based, allowing considerable flexibility in where patients are seen. The virtual clinic module allows the review of condensed data for any given patient and acts as a link to a summary containing patient visual fields, clinical diagnosis and IOP data. Data are collected by technicians appointed at a level appropriate to the task. Patient data are then reviewed by the supervising clinician. To date the system has been used to managed over 100,000 visual field examinations and 11,000 patient events on over 3,500 patients. The data handling requirements for modern browser based applications (LINUX, Apache, MySQL, PHP) are low, ensuring a rapid data refresh and robust data curation.OpenEyes is highly scaleable and can be run over fixed or wireless networks to provide clinical data provision at the point of care regardless of geographic location. It is available royalty free and provides a firm foundation for collaborative software development.
- 2724

Enhanced access to glaucoma diagnosis and management via patient centered collaborative teleglaucoma (TG) approaches in Northern Alberta and in Ethiopia

$D A M I I K F$

Department of Ophthalmology and Visual Sciences, Alberta

We describe patient centered collaborative TG care models in Northern Alberta \& Ethiopia (1-3). Optometrists or technicians select high risk patients \& those with possible early glaucoma, \& upload structured history, exam, \& diagnostic tests. Data is stored on a secure web platform (teleophthalmology.com) \& graded by glaucoma specialists with recommendations for care. Validation included comparison of optic nerve features with digital 3D \& 2D vs 3D slide film, and in person vs virtual exam. Stakeholder feedback improved front line \& grading protocols. Some TG consults are ineffective due to patient cooperation, media opacities, \& technology challenges (4). Opportunities exist for mobile phone photography of the anterior \& posterior segment \& leveraging ' $m$ health' for e-learning, research \& patient education. Future research could focus on alidation of TG models \& optimal use of finite resources, including cost \& comparative effectiveness. 1. J Telemed Telecare 2012;18(7):367-732. MEAJO;2013:20(2):142-9 3. Clin Exp Optom. 2013 Jun 134. MEAJO 2013;20(2):150-7Acknowledgements: Drs A Giorgis, A Mulugeta, F Kassam, S Arora, A Kurji, M Edwards, A Moalin \& Secure Diagnostic Imaging 
- 2731

An update on ocular surface reconctruction with the amniotic membrane

GICQUEL J

Poitiers

Cryopreserved and Dried amniotic membrane (AM) can be a useful therapeutic adjunct in ophthalmic surgery. Differences in preservation techniques can significantly improve biochemical composition and physical properties of AM, potentially affecting clinical efficacy. There is a considerable variation in growth factors content of the Amniotic Membrane, between and within donors. This is further affected by handling of the AM. Such variations could affect the clinical efficacy of tissue constructs. Current use of AM for ex vivo expansion or surgery is not standardized and remains an area of concern. In this session, through practical clinical cases, we will apprehend the various modern surgical techniques, involving the use of amniotic membrane in ophthalmology.

- 2733

Customised amniotic membrane: a surgeon's dream come true

HOPKINSON A

Nottingham

Amniotic membrane (AM) is a proven ophthalmic clinical adjunct, but crude manufacturing and cold chain storage, damage its functional properties and greatly restricts clinical access. Overcoming these problems, Nottingham have developed an innovative, effective and accessible ophthalmic bandage with enhanced wound healing properties. Their AM is produced in a dry, stable and sterilised format, which allows long-term room temperature storage and distribution, creating an 'off the shelf' product. Their AM has improved function over conventional frozen AM, and has the potential to become the first effective, scientifically and clinically validated, low cost treatment for ocular injury. As well as a conventional surgical adjunct for chronic indications, their pre-clinical data suggests a 'first of its kind' acute and emergency application to treat superficial ocular surface injuries. It can potentially be opened and applied directly to the surface of the injury without the need for expensive surgery. As well as pain relief and protecting the wound from infection, the greatly improved profile of trophic factors of their AM facilitates wound healing to limit visual loss and reduce the burden of longterm clinical treatment.
- 2732

Variation and limitations of the amniotic membrane

DUA H

Nottingham

ABSTRACT NOT PROVIDED

- 2734

Amniotic membrane applications in children

BREMOND-GIGNACD $(1,2)$

(1) Ophthalmology, University Hospital, Amiens

(2) CNRS, FR3636, Paris V

Amniotic membrane is the innermost, multilayered part of the placenta. Its properties, stemming from its biological composition, make it a useful tissue for ophthalmic surgery. It promotes epithelization, has antimicrobial effects, decreases inflammation, fibrosis and neovascularization. Reconstruction of conjunctival and corneal defects, treatment of corneal ulcers demonstrated the beneficial effect of amniotic membrane or different ophthalmological indications. The various properties and its current clinical use in ophthalmology are studied in children. Stem cell characteristics have a potential contribution to cell-based treatment of ocular surface disease such as ocular burns and limbal deficiency. Some specific clinical cases are exposed and show the interest of the application of amniotic membranes in children. 
- 2741

Introduction - Descriptions of the techniques

ZOGRAFOS L

Lausanne

\section{ABSTRACT NOT PROVIDED}

- 2743

Proton beam radiotherapy of uveal melanomas

DESIARDINS L

Paris

Abstract: Proton beam therapy is a conservative treatment of choroidal melanoma This study reports our results in terms of overall survival, metastasis-free survival and secondary enucleation in 3404 patientsWe present a retrospective, single-center study of patients treated for choroidal melanoma with proton beam therapy between January 1992 and December 2010 (total dose of 60 cobalt Grays equivalent delivered in 4 fractions over 4 days). The initial clinical tumor characteristics, treatment and follow-up were recorded prospectively. Overall survival, metastasis-free survival and enucleation free survival and their prognostic factors were statistically analyzed.3404 patients were included during this period. The median follow-up was 98 months. The overall survival rate, metastasis-free survival rate, local recurrence and secondary enucleation at 5 years were respectively $64 \%, 74 \%, 3.82 \%, 6.79 \%$. The prognostic factors for metastasis-free survival: the location, the diameter and tumor thickness, and cytogenetic factors body Risk factors of secondary enucleation are essentially tumor size (diameter, thickness and TNM).Treatment with proton therapy provides excellent local control of choroidal melanoma
- 2742

Brachytherapy of uveal melanomas

KIVELÄ T

Department of Ophthalmology, Helsinki Iniversity Central Hospital, Helsinki

Radioactive plaque brachytherapy is currently the most common treatment modality for primary uveal melanoma. The American Brachytherapy Society consensus guidelines for brachytherapy of uveal melanoma, revised in 2014, are summarised. Several isotopes are used for brachytherapy of the eye, such as strontium-90 and ruthenium-106 (beta ays, embedded within the plaque) and palladium-103 and iodine-125 (gamma rays, seeds attached to the plaque). The isotope determines how thick tumours can be irradiated (strontium up to $3 \mathrm{~mm}$, ruthenium up to $6 \mathrm{~mm}$, iodine and palladium over $6 \mathrm{~mm}$ ) and to which extent scatter radiation will affect neighbouring tissues. The plaque is implanted under local or general anaesthesia, localising the tumor by indirect ophthalmoscopy and scleral depression or transillumination. The size and shape of the plaque are chosen according to the size and shape of the melanoma. Most common complications are radiation cataract, retinopathy and optic neuropathy, depending on the location of the tumour, and iris rubeosis and neovascular glaucoma when managing larger tumours. Brachytherapy results for small melanomas irradiated with ruthenium and large melanomas irradiated with iodine are presented as examples.

2744

Irradiation treatment of choroidal hemangiomas

ZOGRAFOS L

Lausanne

The advanced course of radiotherapy in ophtalmology covers all the technical and medical details of irradiation treatment in occular oncology. Following an historical overview of the evolution of occular radiotherapy all the modern technics of occular radiotherapy are described.The Brachytherapy and Teletherapy with Proton beam of uvual melanomas the irradiation treatment of choroidal hemangiomas, the irradiation treatment of metastatic tumors and lymphomas as well as radiotherapy of pediatric tumors are clearly illustrated. 
- 2745

Irradiation treatment of metastatic tumors and lymphomas

SCHALENBOURGA

Lausanne

Intraocular metastases are usually uveal, secondary to breast or lung carcinoma. Lymphoma is most frequently vitreoretinal, in a context of primary central nervous system lymphoma, while a uveal location points towards systemic lymphoma. Management is influenced by a palliative context and bilateral ocular involvement.Close collaboration with the oncologist is required as to the histopathology of the primary tumor, its global extent, oncologic treatment strategy and life expectancy. Stereotactic irradiation is often the principal ocular treatment. A circumscribed treatment of individual lesions carries the risk of local recurrences. The hemato-ocular barrier often prevents chemotherapy from being effective within the uvea. Radiotherapy usually allows a quick reattachment of the retina and preservation of vision. Evolution towards a painful globe and enucleation should be avoided. With the increasing life expectancy of oncologic patients, treatment of ischemic retina with anti-VEGFs and PRP becomes more indicated.Conclusion: Irradiation of intraocular metastases and lymphomas is the most important tool in preserving vision and the eye(s). Its indication should be made within a global oncologic treatment strategy.
- 2746

Radiotherapy in pediatric oncology

MUNIER F

Lausanne

ABSTRACT NOT PROVIDED 
- 2751

Innate immunity and retinal angiogenesis

XUH

Centre for Experimental Medicine, Belfast

The innate immune system plays an important role in tissue homeostasis under chronic insult conditions such as ageing and diabetes. Uncontrolled or dysregulated innate immune response to retinal insults in the elderly or diabetes may result in retinal pathologies such as age-related macular degeneration (AMD) or diabetic retinopathy (DR). Patients suffering from AMD or DR may lose visual function due to the abnorma growth of new blood vessels. Growing evidence suggests that innate immune cells are critically involved in retinal angiogenesis. The role of monocytes/macrophages and neutrophils in retinal angiogenesis will be discussed.

- 2753

Inflammation and angiogenesis in age-related macular degeneration

SENNLAUB F

Paris

It is becoming increasingly clear that an altered immune response, which leads to lowgrade persistent inflammation, plays an important role in AMD pathogenesis in general and in choroidal neovascularization (CNV) in particular. Markers of inflammation, such as plasma levels of complement components and activation-fragments, inflammatory cytokines and inflammatory monocytes are increased in AMD patients. Locally, mononuclear phagocytes (MPs, a family of cells that include microglial cells, monocytes, and macrophages) accumulate subretinally and possibly promote CNV. Data from different laboratories including our own indicate that the recruitment of inflammatory MPs, factors that promote their subretinal survival and the lack of tonic inhibitory signalling on MPs promotes CNV development. We will here present a summary of the current state of knowledge and its impact on the development of future therapies.
- 2752

Oxidative stress and retinal vascular damage in diabetic retinopathy

LYONS T

Queen's University, Belfast

\section{ABSTRACT NOT PROVIDED}

- 2754

Regenerating the retinal vasculature using endothelial progenitor cells

$\operatorname{STITT} A(1,2)$

(1) Centre for Experimental Medicine, Belfast

(2) Queen's University Belfast

Hypoxia-related stimuli from oxygen-deprived retinal neurons and glia networks can drive expression of growth factors and cytokines which induce leakage from the surviving vasculature and/or pre-retinal and papillary neovascularisation. Current therapies for ischemic retinopathies include laser photocoagulation, injection of corticosteroids or VEGF-antibodies and vitreoretinal surgery. Unfortunately these treatments fail to address the underlying retinal vasodegeneration and they also involve significant side effects. An alternative approach is to regenerate the retinal vasculature using endothelial progenitor cells (EPCs) to promote vascular repair and reversal of ischemia. This presentation emphasises the molecular and phenotypic nature of EPCs and how they become altered in disease. There will also be discussion about the potential for some EPC sub-types to be harnessed for cell therapy and the building evidence for how these cells could eventually lead to exciting new therapeutic options for retinal ischemia 
- 2755

What do retinal telangiectasia tell us about the control of vasculature in the retina?

FRUTTIGERM

UCL Institute of Ophthalmology, London

Neurodegenerative changes in the retina and retinal vasculature abnormalities are two closely linked processes. In addition, both of these processes have been linked to inflammation numerous times in humans and in model systems. We have studied Macular Telangiectasia type 2 (MacTel), which is an uncommon eye disease that is characterised by both neurodegenerative and vascular changes. However, histological analysis on post-mortem tissue provided no evidence for inflammation in the retina of MacTel patients. This demonstrates that inflammation is not necessarily linked to neurodegeneration and vascular changes.

Commercial interest 
SIS: Grand rounds in ophthalmic genetics

- 2761

Cases

HAMEL C

Montpellier

There will be presentation of clinical cases in the field of retinal genetics

- 2763

Cases

LISKOVA P

Prague

Interesting Czech cases with monogenic ocular disorders will be presented.
- 2762

Cases

LEROY BP

Ghent

Clinical cases will be shown. Everyone is encouraged to present unknown of interesting diagnostic cases in inherited eye disease. Submission of cases can be done in the room where the Grand Rounds in Ophthalmic Genetics are being held.
- 2764

Cases

AUDOI

Paris

Presentation of case report 
- 2771

The corneal epithelial basement membrane: Master regulator of the stromal myofibroblast response to injury

WILSON S, TORRICELLIA

Cole Eye Institute, Cleveland

The corneal epithelial basement membrane (EBM) is positioned between basal epithelial cellsand the stroma. This highly specialized extracellular matrix functions not only to anchorepithelial cells to the stroma but also during migration, differentiation and maintenance of the differentiated epithelial phenotype. Basement membranes are composed of a diverse assemblage of extracellular molecules, some of which are likely specific to the cornea. In general, they are composed of four primary componentscollagens (types IV and VII), laminins, heparan sulfate proteoglycans, and nidogens. Our recent studies have demonstrated defective regeneration of the EBM in corneas that develop haze after high correction photorefractive keratectomy (PRK) in rabbits or irregular PTK in mice. Likely this is attributable to myofibroblast generation from bone marrow-derived and keratocyte-derived precursor cells that persist in the subepithelial stroma and block keratocyte/corneal fibroblast contribution of EBM components. In turn, persistent EBM defects facilitate the development and persistence of myofibroblasts via augmented TGF beta and PDGF penetration into the anterior stroma. Similar mechanisms underlie corneal scaring after most injuries.

- 2773

The role of inflammation and extracellular matrix in the wound healing after glaucoma surgery

UUSITALO H $(1,2)$

(1) SILK, Department of Ophthalmology, University of Tampere, Tampere

(2) Tauh Eye Center, Tampere

Wound healing is a complex process involved in ocular surgery, trauma and pathogenesis of several eye diseases. Due to the delicate and sensitive structures of the eye wound healing is playing a essential role in ophthalmology. In glaucoma surgery well controlled wound healing process is as important for the creation of a functioning passage to aqueous humor out of the eye. There are growing body of evidence that the preoperative state of the conjunctiva is of great importance for the success of glaucoma surgery. This has been demonstrated in trabeculectomy and in non-penetrating glaucoma surgery like deep sclerectomy. The presence of inflammatory cells in the conjunctival specimens taken during the surgery and the success of the operation points out the role of inflammation in wound healing processes. The data obtained from the specimens taken from the eyes with good and poor ontrol of IOP after glaucoma implants demonstrating the accumulation of extracellular components and activated myofibroblasts further indicates the activation of the whole wound healing cascade. The role of matrix metalloproteinases and their endogenous inhibotors (TIMP's) is evident but seem to be rather specifically involving certain MMP's.

Commercial interest
- 2772

Cytoskeletal regulators are implicated in fibrosis in mechanical injury and infection in the mouse cornea

BEUERMANR

Singapore

Purpose: Mechanical injury as well as infection can result in corneal fibrosis and loss of transparency. We have used a mouse model of cornea insult using sterile mechanical injury and infection.

Methods: Corneas of 8 week old C57BL6 mice underwent either an anterior keratectomy (AK) wound or infection with Pseudomonas aeruginosa (PA), and animals were sacrificed at 2 and 7 days, as well as 2 and 4 weeks after the procedures. The isolated stroma was used to monitor the expression and location of moesin, phospho-moesin, TGF- $\beta 1$ and $\alpha$-SMA.

Results: TGF- $\beta 1$ and phospho-moesin were not detected in normal corneal stromas. However, after either treatment, TGF- $\beta 1$ expression increased, along with phosphomoesin and moesin in the wounded corneal stroma from day 2 to 7 , and decreased after 2 weeks. No expression of TGF- $\beta 1$ and phospho-moesin was found at PO week 4. Myofibroblasts positive for $\alpha$-SMA associated with either treatment were detected from day 2 to week 4 and peaked at week 2 .

Conclusion: The results suggest a role for moesin signaling in corneal fibrosis. Interfering with the action of moesin is a potential target for interventive strategies to avert corneal fibrosis.

- 2774

Matrix wound healing in the optic nerve

O'BRIENC

Dublin

The risk factors associated with optic disc cupping in glaucoma include intraocular pressure and ocular perfusion pressure. The mechanisms of optic disc cupping result in significant extra-cellular matrix alterations for the lamina cribrosa. A number of cell types play a key role in this ECM re-modelling including lamina cribrosa cells and astrocytes. A dysfunction in these cell types include high intra-cellular calcium, mitochondrial dysfunction, and oxidative stress. A greater understanding of the mechanisms involved lead to new therapeutic approaches. 
82

Industry-sponsored symposium 3: What's new in ocular surface disorders ?

- 2831

The burden of dry eye

PURSLOWC

Monmouth

ABSTRACT NOT PROVIDED
- 2832

Trehalose and hyaluronic acid association; added benefits

PINTO-BONILLA I

Castellon

ABSTRACT NOT PROVIDED

- 2833

Visualization of tear film thickness after eyedrop instillation

SCHMETTERER L

Vienna

ABSTRACT NOT PROVIDED 


\section{FRIDAY \\ OCTOBER 3 \\ 2014}


- 3211

VEGF family members (alternatively, molecular identities of antiVEGF agents)

\section{SENNLAUB F}

Paris

The vascular endothelial growth factors (VEGFs), comprise a family of growth factors that include VEGF-A, VEGF-B and Placental growth factor (PIGF) and promote angiogenesis and neovascularization. The important role of VEGF-A in the development of choroidal neovascularization (CNV) and retinal edema has been demonstrated by the clinical success of VEGF-inhibiting agents in wet AMD. An aptamer, an antibody, an antibody-fragment and a soluble receptor have been developed for ophthalmological use to inhibit CNV in AMD. These therapeutic molecules differ in their spectrum of VEGF family members they inhibit, and in the structure of the molecules. We will here provide an overview of VEGF family members and their involvement in CNV and discuss the differences in the therapeutic molecules to bind VEGFs, inhibit CNV and to produce off target effects.

\section{- 3212}

The role of ER stress in regulation of retinal angiogenesis and VEGF signaling

ZHANGS

Buffalo

The endoplasmic reticulum (ER) is the central hub for lipid and protein biosynthesis, protein folding and intracellular calcium storage in eukaryotic cells. Apart from these important and traditional roles, emerging evidence suggests that the ER functions as a principal stress sensor and signal transducer in the cell and this novel function is fulfilled primarily by the unfolded protein response (UPR). The UPR is an intricate set of adaptive signaling pathways activated upon aberrant accumulation of unfolded and/ or misfolded proteins in the ER, or ER stress. Increased ER stress has been implicated in the pathogenesis of many human diseases such as neurodegenerative diseases, cancer and diabetes. The dynamic activation and differential regulation of the UPR signaling pathways plays a central role in coordinating the cellular responses to various metabolic changes and regulates a broad range of physiological and pathophysiological processes such as cell differentiation, proliferation, migration, apoptosis, inflammation, and angiogenesis. This presentation will summarize the role of ER stress in retinal angiogenesis and discuss the novel mechanisms of regulation of VEGF signaling by UPR pathways in relation to diabetic retinopathy
- 3213

Systemic safety of VEGF antagonists and their influence on vascular homeostasis

MACHALINSKA A

Pomeranian Medical University, Szczecin

Intravitreal vascular endothelial growth factor (VEGF) inhibitors constitutes currently the first-line treatment in neovascular age-related macular degeneration (AMD) and diabetic oedema. By intravitreal application of the drug, the dosage can be kept low while maximizing its effect on retina and minimizing potential adverse systemic effects. However, the eye-blood barrier is often disrupted in ophthalmic neovascular disorders and the drugs can be detected in circulating blood despite being administered as an intraocular injection. As patients with AMD or diabetes mellitus constitute a highrisk population for cardiovascular events, the safety of anti-VEGF therapies must be precisely and thoroughly assessed. Here, the recent reports documenting systemic safety of intravitreal VEGF inhibitors will be reviewed. Moreover, the novel methods to assess the potential systemic effect on vascular homeostasis as the consequence of such therapy will be also discussed.
- 3214

\section{TGF-beta in ocular angiogenesis}

WANG X (1, 2), ABRAHAM S (3), JEFFSN (3), SWIREM (3), LUHMANN U (4), LANGE C (4, 5, 6), BAINBRIDGEJ (4, 5), MOSS S (3), GREENWOODJ (3)

(1) Metabolic Medicine, Lee Kong Chian School of Medicine, Nanyang Technological University, Singapore

(2) Institute of Molecular and Cell Biology, Agency for Science, Technology and Research ( $\left.A^{*} S T A R\right)$, Singapore

(3) Cell Biology, UCL Institute of Ophthalmology, London

(4) Department of Genetics, UCL Institute of Ophthalmology, London

(5) NIHR Biomedical Research Centre for Ophthalmology, Moorfields Eye Hospital,

London

(6) University Eye Hospital Freiburg, Freiburg

Abnormal angiogenesis is associated with many blinding eye diseases. Targeting VEGF, a master regulator of blood vessel formation, has shown improved outcomes in ocular vascular disorders. However, many patients do not respond or respond poorly to anti-VEGF therapy, which is not surprising as angiogenesis is regulated by a delicate balance of diverse molecular signals. Combination therapeutics targeting alternative or complementary pathways may circumvent resistance and enhance efficacy of anti-VEGF treatment.TGF $\beta 1$, a multifunctional cytokine, plays an important but contradictory role during angiogenesis. We recently identified a novel regulator of TGF $\beta$ signaling pathway, namely leucine rich $\alpha$-2-glycoprotein-1 (LRG1). LRG1 defines the contextdependent effect of TGF $\beta 1$ and promotes blood vessel formation by driving the proangiogenic TGF $\beta 1 / \mathrm{Smad} 1 / 5 / 8$ pathway. Unlike VEGF, LRG1 is specifically required for abnormal blood vessel formation in the eye. LRG1 antibody blockade attenuates the ocular angiogenesis either on its own or in combination with an anti-VEGF neutralizing antibody. Together, our study provided compelling evidence that LRG1 is an attractive target for therapeutic intervention in ocular vascular complications. 
- 3215

Glial cells as producers of VEGF and opponents in the retina

EICHLER WOLFR

Leipzig

Vascular endothelial growth factor (VEGF) is a key cytokine for the development of pathologic neovascularization in the retina. While Müller glial cells upregulate VEGF in the ischemic retina they constitutively secrete anti-angiogenic factors such as pigment epithelium-derived factor (PEDF) and transforming growth factor (TGF)- $\beta 2$, thereby determining an anti-proliferative milieu for microvascular endothelial cells. Neutralization of PEDF or TGF- $\beta$ partially abrogates the angiostatic action of Müller cells. PEDF and TGF- $\beta 2$ are able to control retinal endothelial cell proliferation via suppressing the activity of ERK-1/-2 MAP kinases, even in VEGF-stimulated cells.

TGF- $\beta 2$ exerts its anti-proliferative effect through increased Smad phosphorylation. Exposure of retinal endothelial cells to TGF- $\beta 2$ or Müller-cell derived secreted factors causes an increased activation of $\operatorname{Smad} 2 / 3$, which leads to a decreased ERK-1/-2 phosphorylation. Given their impact on endothelial signaling and cell growth. Mülle cells have emerged as an important cellular element for the control of angiogenesis and retinal neovascularization
- 3216

Anti-VEGF in the retina

STAHL A

Freiburg

Vascular endothelial growth factor A (VEGF-A) was initially discovered as an endothelial cell-specific growth factor, and current evidence suggests that it also plays numerous roles outside the vasculature, perhaps most significantly in the nervous system. We previously determined a neuroprotective role for both exogenous and endogenous VEGF-A in the adult rodent retina during acute ischemia-reperfusion injury. Furthermore, we demonstrated that endogenous VEGF-A is critical for maintaining neuron survival in normal adult retina, that long-term systemic and local VEGF antagonism resulted in significant loss of retinal ganglion cells. More recently, a primary retinal ganglion cell culture model was used to investigate the molecular basis of VEGF-A's neuroprotective function, and the importance of VEGF-A to neuron survival was evaluated in models of glaucoma. Our findings reinforce the essential role for VEGF-A in retinal neuron survival and have potential implications for therapeutic anti-VEGF-A in conditions associated with retinal ganglion cell distress. 
- 3221

Wet lab training of trabeculectomy and tube surgery

$\operatorname{SHAHP}(1,2)$

(1) University Hospitals Birmingham NHS Foundation Trust, Birmingham

(2) NIHR Biomedical Research Centre (Moorfields Eye Hospital / UCL Institute of Ophthalmology), London

This lecture covers Professor Shah's experience of developing a glaucoma surgery wetlab training facility in Birmingham, UK. Topics covered include: (a) Facility set-up, (b) Safety issues, (c) Preparation of surgical material, (d) Instrumentation, (e) Mentormentee ratios, (f) Faculty briefing, (g) Logistical difficulties and (h) Future developments. Preliminary data on the impact of trabeculectomy wet-lab training will be presented.

- 3223

Simulated ocular surgery: cataract, glaucoma, strabismus and VR surgery

FERRIS J

Gloucestershire

The emergence of high fidelity virtual reality simulators has helped to raise the profile of simulation for cataract and vitreoretinal surgery. However with the advent of the EYESi there is a misconception that all simulation needs to be high tech and expensive and is out of the reach of many training units across the globe. We will describe how innovative model eves, can be used to teach the same skills and in many instances offer a more realistic and versatile simulation experience than virtual reality simulators and how to incorporate this form of simulation into your training programmes. With reference to cataract surgery we will demonstrate how the building blocks of phacoemulsification, such as wound construction, continuous curvilinear capsulorhexis, nucleus sculpting and IOL implantation can be practice on the model eyes. We will also demonstrate how one can simulate intraoperative complications of cataract surgery, such as posterior capsule rupture with vitreous loss, zonular dialysis and suprachoroidal haemorrhage We will also show how these eyes can be used to simulate rectus muscle and oblique muscle surgery in a highly life-like fashion.

Commercial interest

\section{- 3222}

Simulated Ocular Surgery (VR)

HAYNESR

Bristol Eye Hospital, Bristol

Simulation of ocular surgery has advanced in recent years with the advent of high quality virtual reality platforms such as the Eyesi vitreoretinal module, which simulate the look and feel of ocular tissues \& various pathological conditions encountered during pars plana vitrectomy surgery. However, computer simulation has not yet extended to the simulation of Cryobuckling surgery that is used to treat retinal detachment \& involves techniques \& manoeuvres that are often outside the comfort zone of even quite senior ophthalmic trainees. This session will demonstrate how model eyes can be used to train surgeons in all of the steps involved in cryobuckle surgery, including the location \& treatment of retinal breaks with the indirect ophthalmoscope, peritomy incisions, slinging of rectus muscles, marking the external location of breaks, measuring the break position \& choosing an appropriately sized buckle, placing scleral buckle sutures of the correct length \& depth, \& tightening them with the appropriate tension to create an adequate indent effect. Transcleral drainage of subretinal fluid \& injection of intraocular gas can also be practiced, reducing the stress of performing these crucial steps when first performed for real.

3224

Plastic eye surgery simulator for trabeculectomy

MCNAUGHT A

Gloucestershire Eye Unit, Cheltenham

Glaucoma surgical training is becoming difficult for surgical trainees to access, because of increasing subspecialisation, reduced practical experience in suturing using 100 nylon, and a proliferation of new glaucoma surgical procedures. Traditional early practical experience eg surgical wet-labs, using perhaps porcine eyes, are not always easily accessible. The presentation outlines the continued importance of training in trabeculectomy surgery, and describes recent innovations in plastic artificial eye technology. Parallels between actual trabeculectomy surgery in patients, versus modern plastic eye simulated trabeculectomy surgery are described. The potential value, and easy availability, of this new simulated eye surgery training is highlighted 
SIS: Surgical training in glaucoma, and other sub-specialities: wet-lab, virtual reality, and novel technology

- 3225

European Board of Ophthalmology new subspecialty diploma; example: glaucoma surgery

SUNARIC MEGEVAND G $(1,2)$

(1) Mémorial A de Rothschild, Geneva

(2) Société Médicale de Beaulieu, Geneva

The main goal of the European Board of Ophthalmology is promoting education in Ophthalmology and harmonizing medical care in the field of Ophthalmology in European countries. The current EBO diploma ensures a minimum standard of knowledge in general Ophthalmology and is actually attended by a growing number of candidates, reaching in 2014 more than 500 from 28 countries. As there is a growing interest and real need for subspecialty training in different fields, the EBO is currently creating, with the help of European specialist societies , a sub-specialty exam in order to award Ophthalmologists who complete sub-specialty training and pass the exam with a formal recognition of their expertise by a Diploma. The aim is also to protect SubSpecialists and for the benefit of our patients in general, by inviting interested colleagues to obtain a distinction as a proof of excellence. During this course the example of the EBO-Glaucoma Subspecialty exam with particular emphasis on surgical training, will be discussed. 
- 3231

The science from ocular war injuries

SCOTT ROBER

Royal Centre for Defence Medicine, Birmingham

Approximately $10 \%$ of war injuries with major trauma have eye injuries. Primary ocular blast injuries are from the transfer of kinetic energy from the explosive blast wave alone. Secondary ocular blast injuries are the most common type of blast injury (42\%) from the impact of shrapnel from the explosive device itself or from exogenous debris propelled by the explosion. Tertiary ocular blast injuries (1\%) are caused by indirect damage to the eye when an individual is thrown by the blast wind. Quaternary blast injures (3\%) are from any other mechanism related to the blast, usually thermal burns. Quinary blast injuries are a new entity that describes a hyperinflammatory state occurring after an explosion that is thought to cause retinal vascular occlusion. IOFB injuries are given prophylactic systemic antibiotics to protect against endophthalmitis, before surgical removal. Combat eye protection (CEP) significantly reduces secondary and tertiary blast injuries, but increased the proportion with commotio retinae. We rehabilitate blind patients with the BrainPort (Wicab, USA) vision device that substitutes the sense of sight with electrotactile stimulation of the tongue and improves navigation and object recognition
- 3232

French military ophthalmologists experience in Afghanistan War $-2006-2013$

GIRAUD IM (1), EL CHEHAB H (2), VALERO B (1),VIGNAL R (1), RENARD JP (2) (1) Ophthalmology, Hôpital d'Instruction des Armées Sainte Anne, Toulon (2) Ophthalmology, Hôpital d'Instruction des Armées du Val de Grâce, Paris

French military ophthalmologists were deployed in Kabul, Afghanistan, since 2006 till december 2013. Their activity first focus was traumatic ocular injuries, concerning NATO forces and Afghan National Army soldiers. They also provided humanitary support to Afghan civilians, offering medical and surgical care. Kabul's NATO French leaded hospital was equipped with high level examination, investigation and surgical treatment facilities, giving possibility to care all type of ocular emergencies.During 7 years, ophthalmologists realised 1000 surgical interventions. One third were done for traumatic injuries, the two other thirds were mainly cataract surgeries for Afghan population. 95\% of all surgeries were for Afghan peoples and 5\% interested NATO soldiers and contractors Consultations were 25\% for NATO forces, foreign civilians from embassies, international organisations and contractors and 75\% for Afghan population. $10 \%$ of patients presented post traumatic ocular pathologies. The high evel of equipement in the new NATO Hospital offered possibility to recieve Afghan ophthalmologists for mentoring and practical training.

- 3234

War ocular trauma and burns in Kaboul NATO Hospital

Ophthalmic combat injuries in the German Sector of Northern Afghanistan 2003 - 2013

GÜMBEL H

Ulm

During the last decade the German Armed Forces (Bundeswehr) were needed in various missions. Especially in Afghanistan the German Army found their greatest challenge on military medicine. The medical treatment of injured soldiers of all nations, who were on missions, with the highest possible quality, relies to a great extent on the unbroken chain at emergency and trauma therapy from the theater of deployment up to the following medical care and rehabilitation in the homeland.In this talk we will tell the story how to manage the mass of casualities in ophthalmology or how to deal with difficult cases we have seen during our mission. So mostly we have to deal with simple irritations of the cornea by constructions in the camp, but on the other hand blunt trauma by IED (improvised explosive devices) play a key role in case of emergency We report about our experience in Field Hospitals (Role 3) which provide in-patient and out-patient specialist medical care. In severe ophtalmic casualities patients were stabilised for direct evacuation back to Germany by StratAIRMedEvac. So we could give you a follow up of the further treatment of patients and casualities who have been evacuated from the area of deployment.
RIGAL SASTOURNE JC

Paris

\section{ABSTRACT NOT PROVIDED}


- 3241

Experimental retinal vein occlusion, a model of non-neovascular microvascular remodeling

PAQUESM

Paris

The main cause of visual loss after retinal vein occlusion and diabetes is microvascular remodelling, a spectrum of functional and morphological changes affecting vessels Some are beneficial, such as collateral vessel development. The other manifestations of microvascular remodelling are usually detrimental such as chronic rupture of the blood-retinal barrier, capillary closure or macroaneurysms. The processes underlying microvascular remodelling are poorly understood, in part because of the absence of a clinically meaningful animal model. We have been investigating the effet of retinal vein occlusion in rodents using laser photocoagulation, which eanbled us to reproduce many of the features of human microvascular remodelling. Based on our experience, we will discuss issues related to interspecies variability and clinical relevance.

- 3243

Learning about herpes infections by using animal models

ATHERTONS

Augusta, Georgic

Ocular infections caused by members of the herpesvirus family may have serious and sometimes, sight-threatening, consequences. As has been for so many disease processes, animal models have been used to better understand the pathogenesis of herpetic keratitis and retinitis in human patients. While no animal model has absolute fidelity to its human counterpart, careful interpretation and extrapolation of results from animal studies may provide valuable information/insight about the pathogenesis of human disease. This presentation will provide an overview of animal models of keratitis and retinitis, summarize the strengths and weaknesses of the models, and discuss how what has been learned from these models has advanced our knowledge of the mechanisms of human ocular disease and/or has led to new therapeutic approaches to these diseases.
- 3242

Animal models of glaucoma

BRONAM

Dijon

Animal models of glaucoma are still useful to better understand the pathogenesis of glaucoma, to decipher biochemical pathwavs and to evaluate drugs at a preclinical development. Many species have been used but due to practical and economical reasons, rodents have been the most studied.We will detail the methods used to mimic glaucoma with the most common techniques with an elevation of IOP or not. Then we will review the in vivo endpoints mostly used to assess IOP elevation and glaucoma damage. Rebound tonometry has been a great improvement in the IOP recording accuracy in rodents. Electrophysiology is a surrogate of retina and optic nerve function in these nimals. The structure is mainly evaluated with optic disc photography, fluorescein angiography SLO and more recently with OCT.We will end this presentation with the pros and cones of different in vitro techniques mainly used to characterise glaucoma damage at the level of the retina and the optic nerve head.

3244

Using animal models for the study of uveitis

DICK A

Bristol

Our understanding of inflammatory responses and mechanisms of immune-mediated tissue damage in man has been greatly facilitated by our animal models systems. The talk will discuss the utility of animal model in uveitis from two aspects. Firstly, permitting fundamental elucidation of immune pathogenic mechanisms that are at play in uveitis and secondly, delivering a platform for preclinical assessment of drug or biologic intervention. We have been fortunate to be able to further manipulate animal models to create spontaneous models of uveitis and also humanised models of uveitis to assess antigen repertoires and responsiveness. Further we now have persistent models of disease activity, which have illuminated mechanisms of tissue repair and also mechanisms of immune mediated angiogenesis. To this end. the models permit us to look at and translate through to man interventions that have benefitted uveitic patients, and such successes will be highlighted throughout the talk 
- 3245

Animal models in ocular oncology

CASSOUXN (1), JAGER M (2)

(1) Paris

(2) Leiden

Retinoblastoma is a rare cancer in childhood. The goal of current and future therapeutic strategies is to conserve the eye and visual function without using external beam radiotherapy that increases the risk of second cancer in genetically predisposed patients. Multimodality therapy (usual intravenous but also, intra-arterial, intra-vitreal chemotherapy, transpupillary thermotherapy, cryotherapy, brachytherapy) has recently improved eye salvage rate and allowed decreased need for external beam radiotherapy. However treatment of advanced intraocular retinoblastoma remains a real challenge, especially in case of vitreous and sub-retinal seeding. There is a need of alternative and less toxic therapy and a need of new ways for administration of the drugs. Animal models are an integral part of preclinical research in the field of oncology. This paper described the different xenograft rodent models published in the literature. We have also developed a new orthotopic xenografted retinoblastoma model in immunodeficient mice, suitable for pre- clinical assays. 


\section{- 3251}

Corneal stromal cells: A potential cell source for ocular surface regeneration

SIDNEY LE, BRANCH MJ, MCINTOSH OD, DUA H, HOPKINSON A

Academic Ophthalmology, Nottingham

Purpose Keratocytes of the corneal stroma are quiescent, dendritic cells, which maintain the extracellular matrix. It is believed that there is a subpopulation of keratocytes that act as multipotent progenitor cells. These cells repopulate the stroma after damage and may play a role in the regeneration of other lavers of the cornea such as the epithelium. Optimisation of culture of these cells is integral for developing potential cell therapies.

Methods Primary human corneal stromal stem cells (hCSC) were extracted from the limbal region of the cornea. A range of culture media were tested for the effect on stem cell phenotype and differentiation potential. The effect of extended passaging and three-dimensional culture of the cells as spheroids was assessed by RT-qPCR and immunocytochemistry.

Results The phenotype of hCSC is strongly affected by extraction, passage and the culture medium. The cells form spheroids and upregulate markers, such as CD34. Under the correct conditions, hCSC show potential as a multipotent stem cell, expressing the indicative markers and differentiating in vitro down osteogenic, chondrogenic and adipogenic lineages. The CD34+ population of hCSC demonstrate a higher stem cell potential and differentiate to corneal epithelial cells, with a rounded morphology and high expression of key markers such as cytokeratin 3, HES1 and desmoglein 3.

Conclusion Corneal stromal stem cells demonstrate a multipotent stem cell potential, independent of the role they are traditionally associated with in the cornea In vitro, this potential depends on the culture environment and on isolation of certain subpopulations. In future, these cells show potential in the regeneration of the ocular surface in cases of disease or trauma.

\section{- 3253}

Functional and molecular characterization of ex vivo cultured neuronal- and glial- like cells from idiopathic epiretinal membranes

PETROVSKI G (1, 2), LUMIX (3), YAN X (4), GRAWJ (4), FACSKO A (1) HAWLINA M (3) ANDIELIC S(3)

(1) Department of Ophthalmology, University of Szeged, Szeged

(2) Stem Cells and Eye Research Laboratory, Department of Biochemistry and Molecular Biology, University of Debrecen, Debrecen

(3) Eye Hospital, University Medical Centre, Ljubljana

(4) Research Eye Diseases Group, Institute of Development Genetics, Helmholtz Center, Münich

Purpose To test the proliferating potential and characterize the functional and molecular profile of cells growing out of human idopathic epiretinal membranes (iERMs) ex vivo.

Methods iERMs were obtained from uneventful vitrectomies from cases with iERM. Preoperative and postoperative BCVA, CFT and TMV were compared. Ex vivo cultivation under adherent conditions was carried out and followed up to 6 days. Morphological and immunocytochemical characterization of the cells was carried out. The dynamics of the intracellular calcium was measured using fluorescent dye Fura-2 and imaged in response to pharmacological stimulation by acetylcholine (ACh) or mechanostimulation.

Results Visual acuity improved in 9/10 patients. CFT and TMV dereased significantly 3 months after surgery. The cells from the iERMs formed sphere-like structures when cultured ex vivo. The diameter of the spheres increased by 5\% at day 6 and kept an increasing tendency over a month. The cells growing out of the iERM spheres had mainly a glial- and some neuronal- like morphology. Stimulation of these cells with ACh or mechanostimulation induced intracellular calcium propagation in both cell phenotypes - in the neuronal-like cells resembling action potential from the soma to the dendrites. Immunocytochemistry confirmed presence of glial- and neuronal cell phenotype in the iERMs, as well as presence of pluripotency marker.

Conclusion iERMs contain cells of neuronal- and glial- like origin which have proliferative and pluripotent potential, show functionality reflected through calcium dynamics upon ACh and mechano- stimulation, and a corresponding molecular phenotype
- 3252

Effect of hydration on the ultra-structural distribution of collagen fibrils and proteoglycans in human corneal stroma

AKHTARS (1), KHANA (1), PETROVSKI G (2, 3), ALBERT R (3), KIRAT O (4) ALMUBRAD T(1)

(1) Cornea Research Chair, Optometry, College of Applied Medical Sciences, Riyadh

(2) Ophthalmology, University of Szeged, Szeged

(3) Stem Cells and Eye Research Laboratory, Debrecen

(4) King Khalid Specialist Eye Hospital, Riyadh

Purpose We investigated the ultra-structural changes in the distribution of collagen fibrils (CF) and proteoglycans (PGs) due to hydration in the human corneal stroma.

Methods Six fresh human scleral corneal rings were hydrated separately in deionized water for $2 \mathrm{hrs}$ and $48 \mathrm{hrs}$. Corneas were divided into two parts. The first part of each cornea was fixed in $2.5 \%$ glutaraldehyde containing cuprolinic blue in sodium acetate buffer and processed for electron microscopy to assess proteoglycans. A second part of each cornea was fixed in $2.5 \%$ glutaraldehyde in $0.1 \mathrm{M}$ phosphate buffer and post fixed in $1 \%$ osmium tetroxide to assess collagen fibril diameter

Results In the hydrated corneas the mean diameter of CF in the anterior stroma $(23.40 \pm 2.61 \mathrm{~nm})$, middle stroma $(22.48 \pm 3.28 \mathrm{~nm})$ and posterior stroma $(23.58 \pm 2.46 \mathrm{~nm})$ was less than the CF diameter in the anterior $(28.27 \pm 4.05 \mathrm{~nm})$, middle $(28.22 \pm 3.09 \mathrm{~nm})$ and posterior stroma $(29.73 \pm 3.72 \mathrm{~nm})$ of the normal cornea. Micro-fibrils within the $\mathrm{CF}$ in the hydrated cornea were degenerated. In the hydrated cornea, the mean area of the PGs in the anterior stroma was reduced whereas it increased in the middle stroma compared to those in the normal cornea. The density of the PGs in the hydrated cornea was reduced in the anterior, middle and posterior stroma compared to those in the normal cornea.

Conclusion The effect of hydration on the uniform organisation of CF and PGs was higher in the middle and posterior stroma than in the anterior stroma. This could be due to less penetration of water in the interlacing lamellae of the anterior stroma. The reduction in the collagen fibrils diameter could be due to degeneration of microfibrils within the collagen fibrils.

- 3254 / S011

Predegenerated Schwann cells promote neuroprotection and regeneration of retinal ganglion cells in ex vivo rat retinal explants

SMEDOWSKIA (1, 2, 3), PIETRUCHA-DUTCZAKM (1), KAARNIRANTA K $(3,4)$, LEWIN-KOWALIKJ 1

(1) Department of Physiology, Medical University of Silesia, Katowice

(2) Clinical Department of Ophthalmology, Medical University of Silesia, Katowice

(3) Department of Ophthalmology, University of Eastern Finland, Kuopio

(4) Department of Ophthalmology, Kuopio University Hospital, Kuopio

Purpose To investigate neuroprotective and pro-regenerative potential of predegenerated Schwann cells towards RGCs in ex vivo retinal explants.

Methods In this study we used two models of rat retinal explants culture - reversed explants from P2 Wistar rats pups and insert explants from 4 weeks old Wistar rats. Both types of explants were cultured in Neurobasal A medium with supplements (B-27. N2 and GlutaMax). In this way we prepared 32 insert explants (16 were cultured with 2 $\mu \mathrm{l}$ of Schwann cells suspension (104 cells) dropped on the each explants surface, another 16 served as control treated with $2 \mu \mathrm{l}$ of DPBS) and 30 reversed explants (10 of them were cultured in standard medium, 10 explants were with addition of $5 \mathrm{ng} / \mathrm{ml}$ BDNF into medium and 10 were co-cultured with 104 of Schwann cells). After proper culture time, explants were fixed and processed for immunostainings and stereological analyses. Results In retinal explants cultured in inserts system, there was significantly higher density of RGCs in group treated with Schwann cells in comparison to PBS treated (independent samples t-test, $\mathrm{p}<0.05$ ). In reversed retinal explants, there were significant differences between groups expressed in mean and total length and surface of neurites, as well as in number and arboration rate of neurites (U-Mann Whitney test, $\mathrm{p}<0.05$ ). Immunofluorescent staining using GAP43 additionally revealed intense neurites outgrowth (also in explants from adult animals).

Conclusion Predegenerated Schwann cells exert neuroprotective action and support regeneration of RGCs in neonatal and adult retina in ex vivo conditions. 
- 3255 / S012

Pinosylvin protects retinal pigment epithelial cells from oxidative stress by activating Nrf2-mediated antioxidant defence system

KOSKELA A (1,2), REINISALO M (1), HYTTINEN I (1), KAARNIRANTA K (1), KARJALAINENR (3)

(1) Department of Ophthalmology, University of Eastern Finland, Kuopio

(2) Department of Biology, Kuopio

(3) Department of Biology, University of Eastern Finland, Kuopio

Purpose The constant light exposure, high metabolic rate and high lipid concentration expose retinal pigment epithelial (RPE) cells to chronic oxidative stress, which may lead to RPE cell degeneration and evokes secondarily photoreceptor damage and visual loss. In this work, we assessed the protective role of wood derived polyphenol, pinosylvin (PS), in the prevention of oxidative stress and the molecular mechanisms behind these effects

Methods ARPE-19 cells were exposed either to PS or hydroquinone (HQ) or both simultaneoulsy. Toxicity and protective effects of PS against HQ-induced oxidative stres were determined by colorimetric cell viability test (MTT-test). Signalling mechanism of PS was studied by analyzing mRNA levels of Nrf2 (nuclear factor-erythroid 2-related factor-2) and its target genes heme oxygenase-1 (HO-1), glutathione S-transferase pi 1 (GSTP1) and sequestosome 1 (p62) by PCR. Moreover, Nrf2 and p62 were silenced by using siRNA technology.

Results PS treatment protected ARPE-19 cells from HQ when analyzed by MTT assay. PS treatment significantly increased the expression of HO-1 that was abolished by Nrf2 siRNA . PS did not protect p62 siRNA-treated cells from HQ induced oxidative stress.

Conclusion Our results suggest that PS treatment conferred protection against HQinduced oxidative stress through the induction of HO-1 in ARPE-19 cells. Consequently, PS-stilbene compounds may possess health-promoting properties against oxidative stress-related diseases such as AMD.

- 3257 / S009

Cytotoxicity of mesoporous silicon microparticles with different surface modifications on ARPE-19 cells

KORHONENE (1), RIIKONENJ (2), XU W (2), LEHTO VP (2), KAUPPINENA (1, 3) (1) University of Eastern Finland, Department of Ophthalmology, Kuopio

(2) University of Eastern Finland, Department of Applied Physics, Kuopio

(3) Kuopio University Hospital, Kuopio

Purpose Retinal pigment epithelial (RPE) cells play a crucial role in the pathogenesis of age-related macular degeneration (AMD). However, RPE is a challenging target in a therapeutical sense. In addition to its location, the possible hydrophobicity of drug molecules makes its medication even more challenging. Biodegradable porous silicon (PSi) microparticles could be a promising possibility for delivering hydrophobic drugs to RPE cells. In the present study, we aimed at testing the cytotoxicity of thermally oxidized (ToPSi) and thermally carbonized porous silicon (TCPSi) particles with and without additional surface modification with amino groups on human ARPE-19 cells. Since the cytotoxicity of PSi particles cannot be tested by the commonly used MTT (3-[4,5-dimethylthiazol-2-yl]-2,5-diphenyltetrazolium bromide) assay due to nonspecific redox reactions, our aim was also to search for alternative assays for working with mesoporous silicon materials.

Methods ARPE-19 cells were treated with microparticles at several concentrations for $24 \mathrm{~h}$. Thereafter, cell viability was measured using a protease viability marker assay (CellTiter-FluorTM) and a lactate dehydrogenase (LDH) release assay. Cells were also evaluated ocularly under an inverted microscope.

Results All tested particles were well tolerated by ARPE-19 cells. CellTiter-Fluor assay seemed slightly more reliable than the LDH test.

Conclusion Our results show that the tested porous silicon particles did not cause major cytotoxicity on ARPE-19 cells, and CellTiter-Fluor assay, especially together with ocular examination, could be suitable for testing cytotoxicity in association with mesoporous silicon particles.
- 3256 / S008

Autophagy stimulus promotes HuR protein phosphorylation and SQSTM1/p62 protein up-regulation in ARPE-19 cells

AMADIO M (1), MARCHESIN (1), GOVONI S (1), PASCALE A (1)

KAARNIRANTA K (2)

(1) Dept of Drug Sciences, Pavia University, Pavia

(2) Dept. of Ophthalmology, School of Medicine, Eastern Finland University, Kuopio

Purpose Age-related macular degeneration (AMD) pathogenesis involves impaired protein degradation in retinal pigment epithelial (RPE) cells. Our recent observation reveals that the expression of autophagy receptor SQSTM1/p62 is positively regulated by HuR protein (mRNA-stabilizing human Embryonic Lethal Abnormal Vision protein) (Viiri et al. 8(7):e69563, 2013, PLoS One). In this study, we investigated the effects of AICAR (autophagy inducer, 5-aminoimidazole-4-carboxamide-1- $\beta$-D-ribofuranoside) with/without MG-132 (proteasome inhibitor) on HuR post-translational activation and p62 protein levels in ARPE-19 cells.

Methods ARPE-19 cells were treated with MG-132 $(1 \mu \mathrm{M})$ and/or AICAR $(2 \mathrm{mM})$ for increasing times (from $15 \mathrm{~min}$ to $2 \mathrm{~h}$ ) and subjected to cell fractionation. HuR and SQSTM1/p62 protein levels were measured by Western blotting. HuR phosphorylation in threonine residues was evaluated by Western blotting following immunoprecipitation.

Results AICAR+MG-132 co-treatment induces early translocation of HuR protein from the nucleus to the cytoplasm (already after $15 \mathrm{~min}$ ). In this latter cellular fraction, after $2 \mathrm{~h}$ an increase of HuR protein phosphorylation ( $+94 \%$ vs control) and an upregulation of p 62 protein levels are observed (+85\% vs control)

Conclusion In the cytoplasm of ARPE-19 cells AICAR+MG-132 co-treatment leads to HuR protein activation, which in turn may determine the increased p62 protein expression. 


\section{- 3261}

Cellular \& molecular origins of the electro-oculogram

CONSTABLE P

City University London, London

The clinical electro-oculogram has been used to assess retinal pigment epithelium (RPE) function using the changes in the standing potential of the eye in response to light. However, only a part of this mechanism is fully understood. One important clinical finding is that individuals with Best's macular dystrophy display a reduced light-rise and therefore a role for bestrophin in the generation of the light-rise is necessary. It is now evident that bestrophin acts a regulator of intracellular calcium stores interacting with the L-type-Ca2+ channel in the basolateral membrane of the RPE to drive the changes in basolateral chloride conductance. This new role helps to explain the cases of Best's macular dystrophy in which the light-rise is normal. However, the initiating events surrounding the rise in intracellular calcium are unknown. One possibility is that light itself is able to elevate intracellular Inositol tri-phosphate that releases calcium from intracellular stores and initiate the light-rise. The talk will elaborate on the role of bestrophin in the generation of the light-rise, and examine light and the onset of phagocytosis as possible triggers for the light-rise.

- 3263

\section{RPE Disease beyond BEST1}

MEUNIER I, HAMEL C

Centre National de Référence Maladies rares, Affections sensorielles génétiques, CHU Gui de Chauliac., Montpellier

This presentation will focus on one RPE disease, the vitelliform dystrophies. Vitelliform macular dystrophies include a juvenile form i.e., Best vitelliform macular dystrophy and, an adult form apart from the previous one by Gass. These dystrophies represent the second most common cause of inherited maculopathy, after Stargardt disease. Based on recent human iPS cell studies, impairment in phagocytosis was confirmed, as RPE from BEST1 mutant human iPS-RPE have disrupted fluid flux and increased accumulation of autofluorescent material after long-term outer segment feeding when compared with human iPS-RPE from unaffected siblings. Secondly, the role of the protein in chloride currents activated by intracellular calcium was linked to the regulation and release of endoplasmic reticulum calcium stores. Beyond the major gene BEST1 and a minor gene PRPH2, we will point out the frequencies, the clinical spectrum and mode of inheritance linked to two new causal genes: IMPG1, IMPG2. These new genes introduce a third actor in the causes of vitelliform macular dystrophies; namely, this actor is the interphotoreceptor matrix that joins together the photoreceptors and the RPE.

\section{- 3262}

Phenotypes of bestrophinopathies

LEROY BP

Ghent

Purpose:To describe the phenotypes of conditions due to mutations in BEST1, the gene encoding bestrophin-1.Methods:A case presentation format will be used to illustrate the phenotypes and genotypes of the different bestrophinopathies, with special attention to both the clinical and electrophysiological features that distinguish one phenotype from the other, and those they have in common. In addition, the different BEST1 genotypes will be discussed.Results:The phenotypes of Best vitelliform macular dystrophy (BVMD), autosomal dominant vitreoretinochoroidopathy (ADVIRC), and autosomal recessive bestrophinopathy (ARB) are very different. Nevertheless, they share an abnormal electro-oculography (EOG) as a common feature. Electroretinography is normal in BVMD, whereas a rod-cone dystrophy is evident in the later stages of ADVIRC and ARB. BVMD is due to a heterozygous missense mutation in BEST1, ADVIRC is due to interaction of several bestrophin protein isoforms, and ARB is probably the null phenotype.Conclusions:The phenotypes of the bestrophinopathies are diverse, although they share an abnormal EOG as the common feature. The specific genotypes are also different, leading to different molecular pathogenetic mechanisms.

- 3264

\section{Gene therapy for bestrophinopathies}

GUZIEWICZ K (1), BELTRAN W (1), CIDECIYANA (2), KOMÁROMY A (3),

IWABES (1), DUTROWE (1), ZANGERL B (4), HAUSWIRTH W (5),

JACOBSON S (2), AGUIRRE G (1)

(1) University of Pennsylvania, Philadelphia

(2) Scheie Eye Institute, Philadelphid

(3) Michigan State University, East Lansing

(4) University of New South Wales, Kensington

(5) University of Florida, Gainesville

Human bestrophinopathies, a group of inherited retinal disorders caused by mutations in BEST1, are one of the most common early-onset macular dystrophies still considered incurable. The disease is usually diagnosed in early childhood or adolescence, and primarily affects macular region leading to major declines in central vision later in life. Canine multifocal retinopathy, a spontaneous animal model of BEST1-associated retinopathies in man, captures the full spectrum of clinical and molecular features observed in human disease, including the salient predilection of lesions to the canine fovea-like region, and constitutes an important translational model for development and testing of therapeutic strategies. We have previously shown that rAAV2-mediated BEST1 gene delivery controlled by human VMD2 promoter specifically targets RPE cells, and is well tolerated in the wt canine retina. Here, we demonstrate that rAAV2mediated BEST1 gene augmentation reverses the characteristic BEST1 lesions and pathology in cmr models up to 23 months post-injection. The talk will highlight the natural history of the disease and elaborate on the potential of rAAV2-mediated BEST1 gene replacement therapy for prevention as well as reversal of disease. 
- 3271

IgE, anti-IgE, and allergic keratoconjunctivitis - part 1

DOANS

Paris

Vernal and atopic keratoconjunctivis $(\mathrm{VKC}, \mathrm{AKC})$ are severe types of ocular allergy characterized by acute and chronic corneoconjunctival inflammation that may lead to visual sequelae. Although topical immunosuppressive drugs such as cyclosporine and tacrolimus are usually effective, some severe may be refractory. Omalizumab is a monoclonal anti IgE antibody, administered systematically and authorized for severe asthma. We will report our clinical experience of omalizumab in AKC and VKC patients, and will also detail a review of the literature.

- 3273

\section{Food allergy and ocular surface}

FAUQUERT IL $(1,2)$

(1) Pediatric Allergy Unit, Clermont-Ferrand

(2) Centre d'Investigation Clinique, Clermont-Ferrand

Allergic diseases of the ocular surface are in most cases associated with allergic triggers. Food allergens are more and more frequently involved in children. We analyzed the incidence of ocular symptoms associated with food allergy. The airway contact with some particular food is known to result in ocular symptoms (fish, peeling of various fruits). Ocular symptoms are frequently associated with food oral syndrome. Moreover according to allergic patients' questioning, the intake of food results in ocular symptoms in an important amount of cases, reaching almost $20 \%$ of the cases of food allergy (rhino conjunctivitis or conjunctivitis). In patients suspected for food allergy, Oral Food Challenge (OFC) to various allergens revealed ocular involvement in $14 \%$ of the patients. Furthermore some authors compared the safety and efficiency of the OFC and the Conjunctival Provocation Test to assess food allergy in children. They justify this practice on the frequency of instillation of cow's milk on the ocular surface and its absence of side effect on one hand and on the difficulties to practice oral food challenges in young children on the other hand. Thus the eye should be considered as an interesting target for food allergy investigation
- 3272

IgE, anti-IgE, and allergic keratoconjunctivitis - part 2

CHIAMBARETTA F

Clermont Ferrand

Vernal and atopic keratoconjunctivis (VKC, AKC) are severe types of ocular allergy, characterized by acute and chronic corneoconjunctival inflammation that may lead to visual sequelae. Although topical immunosuppressive drugs such as cyclosporine and tacrolimus are usually effective, some severe may be refractory. Omalizumab is a monoclonal anti IgE antibody, administered systematically and authorized for severe asthma. We will report our clinical experience of omalizumab in AKC and VKC patients, and will also detail a review of the literature.

- 3274

Molecular allergens and tear investigation

CHABANEH

Paris

Tears immunological investigations are limited in routine practice because of the small volume of fluid collected. Only few tests can be performed in tears: Total IgE, screening test or specific IgE to aeroallergen to confirm allergic sensitization. Inflammatory mediators in lachrymal fluid are only measured for a research purpose.During the last decade, an increasing number of specific IgE to molecular allergens became available for routine use. Molecular allergens largely contributed to an improvement of allergy diagnosis by distinguishing between true sensitization and cross-reactivity and a better indication of allergen immunotherapy in polysensitized patients. However, each of these single tests needs 50-100 $\mu$ l of serum. More recently, allergen arrayed biochip technology became routinely available (ImmunoCAP ISAC) enabling the quantification of specific IgE against 112 allergens, using only $30 \mu \mathrm{l}$ of serum. The allergen biochip technology seems to be a promising tool to assess local synthesis of IgE in tears in case of suspicion of allergy and the negativity of both skin prick tests and serum specific IgE. Large-scale studies are needed to confirm the usefulness of this tool for tears testing in allergic conjunctivitis. 
- 3311

New therapeutic approaches and challenges for the treatment of dry eye disease

SULLIVANDA

Schepens Eye Research Institute and Harvard Medical School, Boston, MA

Innumerable individuals suffer from tear film dysfunctions, which are collectively diagnosed as dry eye disease (DED). In the United States alone DED afflicts tens of millions of people, especially women, and is one of the most frequent causes of patient visits to eve care practitioners. DED is characterized by a vicious cycle of tear film hyperosmolarity and instability and ocular surface stress, leading to increased friction, inflammation and damage to the eye. DED is caused primarily by meibomian gland dysfunction, and is associated with significant pain, decreased vitality and poore general health. Because of diminished worker productivity, the burden of DED for the United States is estimated to be over $\$ 55.4$ billion. There is no safe and effective global treatment for DED, and its therapy remains a profound unmet need throughout the world. A virtual kaleidoscope of new therapeutic approaches have been proposed to treat the mucin, aqueous and/or lipid tear film deficiencies, as well as the ocular surface damage, associated with DED. My presentation will highlight these approaches, and also address the significant clinical endpoint challenges that serve as barriers to the successful development of potential treatments for DED.

Commercial interest 
- 3411

Pigmentary retinopathy

$\operatorname{ASCASOFJ}(1,2)$

(1) Department of Ophthalmology, University Clinic Hospital., Zaragoza

(2) Aragon Health Sciences Institute, Zaragoza

Numerous medications can cause pigmentary retinopathies (PR). Firstly, we discuss the retinal toxicity in patients taking quinolones (chloroquine and hydroxychloroquine), which are traditional antimalarial agents now used to treat autoimmune diseases such as rheumatoid arthritis and systemic lupus erythematosus. Both medications bind to melanin in the retinal pigment epithelium (RPE), altering normal physiologic function and developing a bilateral bull's-eye maculopathy. PR has also been shown to be related to high dosages of typical antipsychotics, including chlorpromazine and specially thioridazine. The frequency of occurrence of retinal effects seems to be proportional to the total amount of drug used over a long period of time. Furthermore deferoxamine, an iron-chelating agent used to treat conditions with excessive serum iron levels, produces a toxicity which is reversible with drug cessation. Other drugs such as etetrinate, quinine, methoxyflurane, didanosine, and clofazimine can be implied in PR. Psychiatrists, ophthalmologists, rheumatologists and patients need to be aware of any medication-induced PR. Early prevention and intervention can avoid most of the serious and potentially irreversible ocular toxicities.

- 3413

\section{Retinal vasculopathies}

GRZYBOWSKIA $(1,2)$

(1) Department of Ophthalmology. Poznan City Hospital, Poznan

(2) Chair of Ophthalmology, University of Warmia and Mazury, Olsztyn

Drug-induced retinal vasculopathies constitute a group of rare disorders, most often produced by quinine, aminoglycosides, hormone preparations, and interferon. IFNassociated retinopathy includes cotton wool spots, retinal hemorrhages and retinal micro aneurysms. Aminoglycosides were often used subconjunctivally after different ocular surgeries as a postoperative prevention of endophthalmitis and intravitreally as a method of endophthalmitis treatment. Both clinical and animal studies have shown vascular changes, including whitening of the retina, retinal hemorrhages, multiple areas of vascular leakage and macular infarction. Although the true pathogenesis remains unknown, the clinical presentation of capillary nonperfusion has suggested a primarily vascular mechanism. The mechanism of quinine-induced retinal toxicity, although still not well understood, includes probably ischemic/occlusive type of events occurring in the posterior retinal circulation with relative sparing of choroidal circulation. Hormonerelated retinopathy, mainly due to oral contraceptives, include among others retinal hemorrhage or emboli, macular edema, and papillary edema.

\section{- 3412}

\section{Crystalline retinopathy}

NADIMN

Beirut

Refractile crystalline deposits in the retina may be a manifestation of drug toxicity.The five drugs known to produce this side effect are:(1)Tamoxifen-non steroidal estrogen antagonist used in the treatment of breast cancer.(2)Canthaxanthine-tanning agent for skin pigmentation in vitiligo and for the treatment of photosensitivity in psoriasis and erythrotic porphyrias. (3)Crashed tablets of Methadone, Mepiridine hydrochloride and Methylphenylate that are injectedintravenously in drug abusers-Talc Retinopathy. (4)Methoxyflurane-an inhalational anesthetic agent .(5)Nitrofurantoin-an antibiotic used in the treatment of urinary tract infections.Daily doses and cumulative doses that give rise to these deposits will be presented and the fundus/clinical features of each drug as well.Since primary ocular disorders (Bietti's crystalline retinopathy, idiopathic parafoveal telengiectasis, calcified macular drusen) and systemic disorders (oxalosis, cystinosis,Sjogren Larsson syndrome) are known to be associated with crystals in the retina,the differential diagnosis of this clinical entity will be discussed.
- 3414

Fingolimod-associated cystoid macular oedema

LIU L (1), CUTHBERTSON F (2)

(1) Academic Unit of Ophthalmology, Bristol

(2) Department of Ophthalmology, Royal United Hospital Bath, Bath

Fingolimod-associated cystoid macular oedema has been reported in treatment of MS and following renal transplantation. This seems to be dose-dependent with an incidence of approximately $0.5 \%$ with $0.5 \mathrm{mg}$ oral dose. Although most patients develop macular oedema between 3 to 4 months after initiation, we reported an incidence as early as 5 days. The mechanism underlying fingolimod-associated macular oedema is not entirely clear. It has been shown that fingolimod, the S1P receptor, not only plays a role in recruiting $\mathrm{T}$ lymphocytes, but also in regulating vascular permeability. Patients on fingolimod should be advised of the possible adverse visual symptoms. It may be helpful for patient to self-monitor with an Amsler chart, and urgent ophthalmology review is suggested for patients with painless reduced vision. 
SIS: Drug retinal toxicity

- 3415

Retinal drug toxicity in the absence of visible fundus changes

LAWRENSONJ

Division of Optometry and Visual Science, City University London, London

A variety of systemic medications cause retinal drug toxicity associated with characteristic ophthalmoscopic changes e.g. pigmentary retinopathies, crystalline retinopathies, retinal vasculopathies, or retinopathies associated with macula edema. However, altered retinal function can occur in the absence of visible fundus changes. For example, the cardiac glycosides, digoxin and digitoxin, are frequently associated with disturbances of colour vision mediated through the inhibition of sodium-potassium ATPase. Similarly, phosphodiesterase inhibitors such as sildenafil can also act on the photoreceptor transduction cascade to cause reversible changes in colour perception blurred vision and increased glare sensitivity. Exposure to antiepileptic drugs that modulate GABA, can also lead to altered visual function. The most significant adverse reaction occurs with vigabatrin which can cause irreversible concentric visual field loss in susceptible individuals. It is important for eye care professionals to be aware of the potential for adverse reactions associated with systemic drugs and consider the use of functional vision tests for their detection and monitoring. 


\section{- 3421}

Glaucoma pathology: an eye on the brain

DEKEYSTER E (1), AERTS J (2), VALIENTE-SORIANO FJ (3), DE GROEF L (1), SALINAS-NAVARROM (1), VIDAL-SANZM (3), ARCKENS L (2), MOONS L (1)

(1) Neural Circuit Development and Regeneration Research Group, Leuven

(2) Laboratory of Neuroplasticity and Neuroproteomics, Leuven

(3) Department of Ophthalmology, Murcia

Purpose Glaucoma is a group of optic neuropathies characterized by loss of retinal ganglion cells (RGC). Since ocular hypertension (OHT) is one of the main risk factors, current therapies are merely based on lowering eye pressure. As a subset of treated patients continue losing vision, more research into the pathological mechanisms underlying glaucoma is needed in order to develop novel therapeutic strategies. Here, we studied the impact of $\mathrm{OHT}$ on the main visual areas in the mouse brain: superior colliculus (SC) and primary visual cortex (V1)

Methods Temporary monocular hypertension ( 5 davs) was induced in mice by laserin the episcleral and perilimbal vessels. The impact on the retina and its target areas was examined via immunohistochemistry (Brn3a, VGluT2 and GFAP). Alterations in neuronal activity in $\mathrm{V} 1$ were assessed via in situ hybridization for zif268.

Results Transient OHT resulted in diffuse and sectorial RGC death. In the SC contralateral to the $\mathrm{OHT}$ eye, we detected a strong decrease in synaptic connections at 1 week after lasering, which was retinotopically linked to RGC degeneration patterns in the retina. Similar retinotopic dropouts were discerned in activity patterns of V1 at 1 week post lasering, while $\mathrm{V} 1$ activity resumed 3 weeks later. In the SC, however no recovery of synapse density was observed and inflammation was not resolved at 4 weeks post lasering. Nevertheless, SC neurons appeared healthy and cell density and cell morphology looked normal.

Conclusion Besides RGC death, OHT also induces loss of synaptic connections and neuronal activity in visual brain centers, accompanied by extensive immune responses. This stresses the importance of including the whole visual system in glaucoma research

\section{- 3423}

Visual acuity loss in open-angle glaucoma patients: causes and risk factor

AYCHOUA N, JANSONIUS NM

Ophthalmology, Groningen

Purpose To identify causes of visual acuity loss in open angle-glaucoma (OAG) patients and to determine associated risk factors.

Methods From the visual field database of the University Medical Center Groningen 400 patients with at least 3 reliable visual fields were randomly selected. Of these, 233 had OAG. OAG patients with a visual acuity of 0.5 or less in at least one eye were considered as cases; controls had to have a visual acuity above 0.5 in both eyes. Causes of visual acuity loss were determined from the medical records. Potential risk factors for visual acuity loss in OAG (age, gender, myopia, baseline IOP, corneal thickness, and visual field mean deviation $[\mathrm{MD}]$ ) were assessed by comparing cases with acuity loss due to OAG with the controls, using logistic regression.

Results Of the 233 patients with OAG, 134 (57\%) were cases and 99 (43\%) controls Concerning the cases, visual acuity loss was caused by OAG in $40 \%$, cataract 17\%, AMD $14 \%$, vitreo-retinal surgery $10 \%$, corneal pathology $4.5 \%$, acquired retinal disorders $10 \%$ and neuro-ophthalmological disorders $4.5 \%$. Among the assessed risk factors, cases had a lower $\mathrm{MD}(\mathrm{OR}=0.90 ; \mathrm{P}<0.001)$ and a higher age $(\mathrm{OR}=1.05 ; \mathrm{P}=0.015)$.

Conclusion Visual acuity loss in OAG is common but mostly due to other eye comorbidities. A lower MD and an older age were highly significant risk factors.
- 3422

Performance of advanced technologies for community-based glaucoma case-finding

LAWRENSONJ (1), DABASIA P (1), EDGAR D (1), GARWAY-HEATHD (2)

(1) City University London, London

(2) National Institute for Health Research (NIHR) Biomedical Research Centre at Moorfields Eye Hospital NHS Foundation Trust and UCL Institute of Ophthalmology, London

Purpose To identify if the Frequency Doubling Technology perimeter (FDT), Moorfields Motion Displacement Test (MDT), iVue Optical Coherence Tomographer (OCT) and Ocular Response Analyser (ORA), alone or in combination, improve case detection of primary open angle glaucoma (POAG) in a population aged over 59 years.

Methods 505 volunteers aged 60 to 92 years (median age 68) completed all 4 screening tests conducted without knowledge of subjects' ocular status. FDT and MDT were used in suprathreshold mode. iVue OCT measured ganglion cell complex and retinal nerve fibre layer thickness. Each subject then received a full ophthalmic examination by an experienced clinician, masked to preceding test results. Based on this evaluation, subjects were classified as normal, glaucoma suspects or POAG (defined as an open drainage angle, glaucomatous optic neuropathy, and glaucomatous field defects).

Results Based on the reference standard examination, 26 (5.1\%) subjects were classified as POAG, and $32(6.4 \%)$ suspect glaucoma. Sensitivity at $90 \%$ specificity for detection of POAG was 63\% (95\% CI: 39 to 82 ) for FDT, 54\% (33 to 74) for MDT, and 82\% (64 to 95) for best performing OCT parameter (inferior RNFL thickness). Using these criteria, partial AUROC was significantly greater for inferior RNFL thickness than other tests $(\mathrm{p}=<0.01)$.The probability of having POAG increased substantially when bestperforming criteria were combined for perimetry, iVue OCT and ORA.

Conclusion Diagnostic performance of individual tests gave acceptable accuracy for POAG detection in a representative sample of elderly subjects. For case-finding, our study supports combining retinal nerve fibre layer thickness analysis with performance on vision-function tests.
- 3424

Lateral inhibition in the human visual system in healthy subjects and in patients with glaucoma

JUNOY MONTOLIO FG (1), MEENS W (1), JANSSENS MSA (1), STAM L (1), JANSONIUS NM $(1,2)$

(1) Ophthalmology, University of Groningen, University Medical Center Groningen, Groningen

(2) Epidemiology, Erasmus Medical Center, Rotterdam

Purpose In glaucoma, the density of retinal ganglion cells (RGCs) is reduced. It is largely unknown whether and how this influences the spatial interactions between the remaining RGCs. A decrease in lateral inhibition might arise and could result in a larger than expected response to some stimuli, which could mask RGC loss on functional glaucoma testing. The aim of this study was to compare lateral inhibition in the visual system between glaucoma patients and healthy subjects.

Methods A case-control study was performed with 14 glaucoma patients and 50 healthy subjects. Cases were selected to have advanced visual field loss in combination with a normal visual acuity. Controls had to have a negative ophthalmic and family history, normal eye pressure, and a normal acuity. Experiments were performed monocularly. Lateral inhibition was measured psychophysically on a computer screen, with (1) a modified illusory movement experiment and (2) a contrast sensitivity (CS) test. The illusory movement was quantified by nulling it with a real movement; measure of lateral inhibition was the amount of illusory movement. Log CS was measured at 1 and 4 cpd; measure of lateral inhibition was the difference between $\log$ CS at 1 and 4 cpd. Both measures of lateral inhibition were compared between cases and controls; analyses were adjusted for age with multiple linear regression

Results There was no difference in lateral inhibition between cases and controls $(\mathrm{P}=0.76$ for illusory movement; $\mathrm{P}=0.84$ for $\mathrm{CS}$ testing). Log CS was lower in the cases than in the controls at both $1(-0.21 ; \mathrm{P}=0.006)$ and $4(-0.22 ; \mathrm{P}=0.016) \mathrm{cpd}$.

Conclusion Lateral inhibition is not decreased in glaucoma patients, at least not in patients with a normal visual acuity. 


\section{- 3425}

Angle closure glaucoma in Asians: comparison of biometric and anterior segment parameters between Japanese and Chinese subjects

HOH (1), OZAKIM (2), MIZOGUCHI T (3), NONGPIUR M (1), HOW A (1), HE M (4), WONG T (1), PERERA S (1), AUNG T (1)

(1) Ophthalmology, Singapore

(2) Ophthalmology, Hyuga

(3) Ophthalmology, Sasebo

(4) Ophthalmology, Guangzhou

Purpose To compare anterior segment parameters in Chinese and Japanese subjects using anterior segment optical coherence tomography (ASOCT) and to determine if the associations between these parameters and angle closure vary between the two ethnic groups.

Methods We conducted a case-control study among 117 Japanese and 102 Chinese patients with primary angle closure (PAC) or primary angle closure glaucoma (PACG) and 117 Japanese and 176 Chinese controls. All participants underwent A-scan biometry and ASOCT imaging (Visante, Carl Zeiss Meditec, Dublin, Ca). ASOCTderived biometric parameters were compared between groups. Multivariate analysis was performed to determine predictors of angle opening distance at $750 \mu \mathrm{m}$ from the scleral spur (AOD750).

Results Japanese were older $(\mathrm{p}<0.001)$ and consisted of more females $(\mathrm{p}<0.001)$. Age and gender adjusted analysis of covariance showed that Japanese eyes with angle closure had a significantly shallower anterior chamber depth $(\mathrm{p}=0.015)$, shorter axial length $(\mathrm{p}<0.001)$, greater lens thickness $(\mathrm{p}<0.001)$, lens vault $(\mathrm{LV})(\mathrm{p}=0.001)$ and iris curvature (I-Curv) $(\mathrm{p}<0.001)$, and a smaller anterior chamber area ( $\mathrm{p}=0.044)$ and volume $(\mathrm{p}=0.028)$; but no significant difference was noted for anterior chamber width ( $\mathrm{ACW}$ ). In Japanese, the predictors of AOD750 were LV $(\mathrm{p}=0.004), \mathrm{ACW}(\mathrm{p}=0.049)$ and ICurv $(\mathrm{p}=0.017)$; in Chinese, only the LV $(\mathrm{p}<0.001)$ was a determinant factor

Conclusion Japanese eyes with angle closure were smaller, with thicker lenses. LV is an important factor for angle closure in both races. These results suggest differing mechanisms of angle closure pathogenesis between the two ethnic groups.
- 3426

IOP measurement from corneal vibration parameters analysis: first clinical trial partial results

BITOUN P (1), BENZACKEN L (2), BOUAFIA K (2), HAOUCHINE B (2), CHAPELLE P (3), CHAMBARD IP (4), JOUROT P (4). PEAN V (5)

(1) Ophthalmic Genetics Hopital Jean Verdier, Bondy

(2) Ophthalmology, Aulnay

(3) Engineering, Gifs/y

(4) Engineering, Saint Loui

(5) Biostatistics, Etavigny

Purpose A novel non-contact, non-invasive Vibration Tonometry technology with excitation of corneal vibration and their correlation to GAT IOP was tested in a large clinical trial reported

Methods 413 eyes from 207 volunteers from various origins at a suburban Paris hospital eye clinic were enrolled. History, vibration tonometry measurements in triplicate GAT, NIDEK air puff IOP, refractometry, keratometry and Tomey pachymetry. Algorithm discovery was performed by random draw splitting runs into a discovery sample and a separate validation sample performed 10 times separately on males and females.

Results 40/207 19.3\% patients had glaucoma,50\% were females.993 measurement runs were performed of which $83.5 \%$ were in correct position. Population aged 23-92 yo with $42 \%$ females with mean $59.7(+/-15.7)$ in females and $60.4(+/-13.8)$ in males. GAT IOP mean was $16.0 \mathrm{~mm} \mathrm{Hg}(+/-3.46)$ in females and $15.8(+/-3.95)$ in males. CCT mean was $530.2 \mu(+/-35.1)$ in females and $526.3 \mu(+/-42.4)$ in males, AL mean was $23.29 \mathrm{~mm}$ $(+/-1.68)$ in females and $23.73 \mathrm{~mm}(+/-1.68)$ in males. None of these differences were significant. 46 eyes had GAT IOP greater $>/=20 \mathrm{~mm} \mathrm{Hg}$. No adverse events were reported during or post trial. Algorithm for women yielded $95,4 \%$ of IOP mean within $5 \mathrm{~mm} \mathrm{Hg}$ of mean GAT IOP. For men 91\% of IOP mean was within $5 \mathrm{~mm} \mathrm{Hg}$ of mean GAT IOP

Conclusion Current algorithm seems to show no evidence of effect of CCT on measurement precision. Vibration tonometry is a valuable method for noninvasive measurement of IOP without contact with reasonable precision and good reproducibility and independently of CCT. It may thus prove to be a valuable tool for IOP measurement in thin and surgical corneas

Commercial interest 
- 3431

Intraocular pressure modulation by neuropeptides - the effect of vasopressin

BOGNER B (1), RUNGE C (1), STROHMAIER C (1), TROST A (1), KASER-EICHBERGER A (1), KREFFT K (1), GRABNER G (1), KIEL JW (2), SCHROEDL F (1,3), REITSAMER HA (1)

(1) Ophthalmology and Optometry and Research Program for Exp Ophthalmology, SALKIParacelsus Medical University, Salzburg

(2) Ophthalmology, UTHSCSA, San Antonio, TX

(3) Anatomy, PMU, Salzburg

Elevated intraocular pressure (IOP) is a major risk factor for glaucoma. Currently, the primary strategy to slow down glaucoma progression is IOP reduction. Numerous studies investigated the IOP lowering effects of neuropeptides, but the complex physiological mechanisms involved are difficult to interpret. Therefore, we investigated the effect of arginine-vasopressin (AVP) on IOP in a multiparameter approach.In a rabbit model the effect of i.v.-applied AVP on IOP, ciliary blood flow (CilBF) and aqueous flow $(\mathrm{AqF})$ was measured simultaneously by manometry, laser-Doppler flowmetry and fluorophotometry.AVP caused a dose-dependent decrease of IOP, CilBF and AqF. Based on the Goldmann equation the decrease of AqF at $0.08 \mathrm{ng} / \mathrm{kg} / \mathrm{min}$ AVP (low dose) accounted for $77.1 \%$ of the IOP reduction $(9.56 \pm 2.35 \%)$, while $\mathrm{AqF}$ at 1.33 $\mathrm{ng} / \mathrm{kg} / \mathrm{min}$ AVP (high dose) accounted for $28.4 \%$ of the IOP decline (41.02 $\pm 3.19 \%$ ). The results indicate that AqF reduction by low dose AVP is mainly caused by inhibition of ciliary epithelium secretion. At high dose AVP AqF reduction is caused by decreased CilBF or more likely by a combination of CilBF decline and secretory inhibition. This conclusion is only possible with the multiparameter measurement approach presented.

- 3433

Reconstitution of a corneal endothelium by intracameral injection of cultured endothelial cells

BRLNETTE I, BOSTAN C, THERIAULT M, FORGET K, PROULX S Montréal

The only currently available treatment for irreversible corneal endothelial failure is allotransplantation, which is overshadowed by optical distortion of the posterior surface, postoperative donor corneal endothelial cells attrition and risk of rejection. Assessment of the in vivo functionality of a corneal endothelium reconstituted by the injection of corneal endothelial cells in the anterior chamber of an endothelial deficiency model allows to consider cell-injection as a possible therapy for corneal endotheliopathies.
- 3432

Novel cell therapeutical approaches to treat the corneal endothelium

FUCHSLUGERT

Düsseldorf University Hospital, Heinrich-Heine-University, Düsseldorf

The talk will give an overview of cell therapeutical treatments of corneal endothelial cells. The use of different vectors in nano-scale will be presented. Functional data on transfering nucleic acids to the corneal endothelium will be discussed.

- 3434

Function follows morphology? New aspects of macula OCT analysis

GUTHOFF R

Dusseldorf

Factors that are predictive for the outcome of medical or surgical therapy to anatomic success are desirable. Clinical tools such as opticalcoherence tomography and autofluorescence provide to demonstrate early morphological alterations. The value in identifying subclinical pathologies that affects the macula may help to understand better the natural course. The examination results of these tools may be useful for the correlation with post-treatment visual acuity to better predict the eventual visual function 
- 3435

Diabetic macular edema - back to laser therapy?

JOUSSENA

Berlin

Treatment of diabetic macular edema has changed considerably since approval of several intravitreal drugs. Several studies demonstrated superiority of intravitreal injections over laser therapy. Nevertheless laser approaches are currently reconsidered with adapted protocols. This overview critically discusses the effectiveness of the treatment approaches on the background of the pathophysiology of diabetic macular edema.
- 3436

Noninvasive drug delivery to the retina

STEFANSSONE

Reykjavik

In general, only a small fraction of drug administered as eye drops enters the eye. This is due to permeability barriers of the eve as well as effective rinsing of the ocular surface. Additionally, lipophilic drug molecules are difficult to dissolve in adequate concentrations in eye drops.We have developed a drug delivery platform, based on cyclodextrins, which can be used to increase drug delivery with eye drops. The cyclodextrins are lipophilic on the inside and hydrophilic on the outside. This allows formation of water-soluble complexes of cyclodextrins and lipophilic drug molecules, thereby increasing the possible concentration of drug in the eye drop. Cyclodextrins may also increase drug delivery by enhancing mucoadhesion and by facilitating transport of drug molecules through the unstirred water layer at the surface of the eye. We have formulated and tested cyclodextrin-dexamethasone eye drops. The eye drops have been shown to decrease diabetic macular edema. A recent study also shows higher and extended concentration of dissolved dexamethasone after using cyclodextrindexamethasone, compared to a regular formulation of dexamethasone.

Commercial interest 


\section{- 3441}

Healthcare resource use and costs in persistent non-anterior non-infectious uveitis

THORNEJ (1, 2), TUNDIA N (3), SKUPM (3), MACAULAY D (4), REVOL C (4), CHAO I (3) BAO Y (3), DICKA (5)

(1) Ophthalmology, Wilmer Eye Institute, Baltimore

(2) Epidemiology, Baltimore

(3) AbbVie Inc., North Chicago

(4) Analysis Group, Inc., New York

(5) Ophthalmology, Bristol Eye Hospital, Bristol

Purpose To assess direct and indirect economic burden for privately insured US employees with persistent non-anterior non-infectious uveitis (NIU) compared with matched controls without uveitis.

Methods Persistent cases were 18-64 years old with $\geq 2$ ICD-9 diagnoses for intermediate-, posterior- or pan-NIU (OptumHealth, 01/1998-03/2012) with $\geq 90$ days of corticosteroid, immunosuppressant, and/or biologic use. Cases were matched 1:1 by sex, age, region and index date to controls. Direct (medical services and prescription drugs) and indirect (disability and medically-related absenteeism) healthcare resource use (HRU) and costs (2012 USD) were compared using Wilcoxon/McNemar tests and multivariate regression (adjusting for baseline characteristics)

Results Annual unadjusted mean direct HRU and costs, including inpatient ( 0.3 vs. 0.1 stays; $\$ 3,628$ vs $\$ 586$ ), outpatient ( 26.3 vs 9.6 visits; $\$ 12,038$ vs $\$ 3,065)$, ophthalmology/ optometry (6.6 vs 0.5 visits; $\$ 3,486$ vs $\$ 83$ ), emergency (0.6 vs 0.1 visits; $\$ 267$ vs $\$ 31$ ), and prescription drugs (13.3 vs $4.5 ; \$ 10,345$ vs $\$ 1,499$ ) were significantly higher for cases vs controls (all $\mathrm{P}<.05)$. Indirect HRU and costs ( 35.5 vs 11.5 days; $\$ 6,624$ vs $\$ 1,816$ ), specifically absenteeism (14.3 vs 4.0 days; $\$ 3,818$ vs $\$ 862$ ), also were higher for cases vs controls (all $\mathrm{P}<.05)$. Adjusted total direct costs ( $\$ 35,739$ vs $\$ 7,670)$ and indirect costs ( $\$ 6,902$ vs $\$ 1,612$ ) were significantly higher for cases vs controls (all $\mathrm{P}<.05)$.

Conclusion Persistent non-anterior NIU is associated with substantial direct and indirect costs.

Cотпегcial interest
- 3442

The effect of intravitreal adalimumab on the treatment of active noninfectious ocular inflammation: A pilot study

HAMAM R, MANSOURA

American University of beirut, Beirut

Purpose To evaluate the short term efficacy of intravitreal adalimumab (IVA) for the treatment of eyes with active noninfectious uveitis

Methods In a prospective noncomparative interventional case series, consecutive eyes with active noninfectious uveitis were injected with IVA at 0,2 then every 4 weeks for total of 6 months. Change in VA, grade of inflammation (cells, haze, leakage on FA adopted from the angiography scoring for uveitis working group), and central retinal thickness (CRT) were recorded. No systemic or ocular therapies were added to the IVA treatment during the study period.

Results Six out of seven patients ( 12 of 13 eyes) completed 26 weeks of treatment. One patient (1 eye) failed treatment. Median logMar VA at baseline was 0.243 and improved to 0.049 at 26 weeks. Seven out of twelve eves had improvement of $\geq 2$ ETDRS lines. All hree eyes with anterior chamber cells grade $\geq 1$ at baseline had zero cells at 26 weeks. Also, all nine eyes with vitreous haze grade $\geq 1$ at baseline had zero haze on last follow up. Median CRT at baseline was 317 microns and decreased to 277 microns. Five out of eight eyes with macular edema had complete resolution. Median FA score at baseline was 14 and improved to 4. Using Wilcoxon Signed Rank test, the decrease in logMar, CRT and FA score at 26 weeks compared to baseline was significant $(n=12$ eyes; $p=0.003$, $0.021,0.002$ respectively). No local or systemic side effects were detected. Stratifying the data to include one eye per patient revealed similar results.

Conclusion IVA was safe in this pilot study; was effective in improving the VA in 7/12 eyes, resolving macular edema in $5 / 8$ eyes \& decreasing AC cells, vit haze and FA score in 12 eyes while 1 patient ( 1 eye) failed treatment.
- 3443

Diagnostic and prognostic value of cerebrospinal fluid analysis in Vogt-Koyanagi-Harada syndrome

MOUALLEM A (1), TOUITOU V (1), BODAGHI B (1), CHAMPIONE (1), DARUGAR A (1), FARDEAU C (1), CASSOUXN (2), LE HOANG P (1)

(1) Ophtalmology, Hôpital Pitié Salpétrière, Paris

(2) Ophtalmology, Institut Curie, Paris

Purpose To evaluate the diagnostic and prognostic values of lumbar puncture in VogtKoyanagi-Harada(VKH) syndrome

Methods Medical records of patients seen in a single tertiary center between 1995 and 2013, with confirmedVKH syndrome according to the Revised Diagnostic Criterias, were retrospectively reviewed. Clinical stage of the disease, cerebrospinal fluid (CSF) analysis, therapeutic strategy, and prognosis were compared.

Results 68 patients with a confirmed diagnosis of $\mathrm{VKH}$ disease were initially reviewed. Among them, 29patients (42\%) had undergone a lumbar puncture (LP). 21 LP (72.4\%) were performed at the early stage of the disease, and 11 were performed at the chronic stage of the disease (37.6\%). 16 patients (55\%) had a pleiocytosis. LP was abnormal in $66,7 \%$ at the acute phase and $27 \%$ at the chronic phase. An average of 62.3 lymphocytes $/ \mathrm{mm} 3$ was observed when LP was performed during the acute phase and an average of 9.5 lymphocytes $/ \mathrm{mm} 3$ was observed during the chronic phase $(\mathrm{p}=0,03)$. For two cases, the patient met the diagnostic criteria because of the LP findings. Patients dependant on low doses of steroids ( $<5 \mathrm{mg} / \mathrm{d}$ ) had more lymphocytes (93,8 lymphocytesmm3) into the initial CSF analysis compared to the patients dependant on high doses of steroids $(>5 \mathrm{mg} / \mathrm{d})(29,3$ lymphocytesmm 3$)(\mathrm{p}=0.03)$ when LP was performed at the acute phase of the disease.

Conclusion The number of lymphocytes in the CSF at the diagnostic phase seems to be one of theprognostic factors for the outcome of $\mathrm{VKH}$. Patients with a low pleiocytosis in the initial lumbar puncture should be closely monitored and may require a more

\section{- 3444}

Characterization of uveitis in association with multiple sclerosis

MACKENSEN F (1), MESSENGER W (2), HILDEBRANDT L (1), SUHLER E (2), ROSENBAUM JT $(2,3)$

(1) Interdisciplinary Uveitis Center, Heidelberg

(2) Ophthalmology, Casey Eye Institute, OHSU, Portland

(3) Legacy Devers Eye Institute, Portland

Purpose Characterize uveitis in association withmultiple sclerosis (MS) including anatomic presentations, age, gender, and outcome.

Methods Retrospective chart review of patients with uveitis and MS at two uveitis centers (Portland, Oregon, USA and Heidelberg, Germany). Baseline characteristics and ophthalmic data were collected at the patient's first and last visits. Additionally, neurologic records were obtained when possible. In a sub-analysis, controls with idiopathic intermediate uveitis were compared to cases with intermediate uveitis and MS

Results We identified 113 patients (196 eyes), 83 (73\%) female and a mean age of presentation of 41 years (range 13-64). Vision was fair with mean visual acuity of 20/39. After classifying anatomically, intermediate, anterior, posterior and pan-uveitis comprised $80 \%, 15 \%, 3 \%$, and $2 \%$, respectively. Fifty-four patients (56\%) were diagnosed with uveitis before MS, 15 patients (15\%) simultaneously, and 28 patients (29\%)after having MS. Optic neuritis was present in $33 \%$ of our patients. During an average followup of 4.2 years (range $0.04-21$ ), visual acuity improved -0.09 $\log M a r / y e a r$. Compared to our location-matched controls with idiopathic intermediate uveitis $(\mathrm{n}=16)$, patients with MS and intermediate uveitis were significantly older when diagnosed with uveitis $(\mathrm{p}=0.027)$ and more likely to be female $(\mathrm{p}=0.01)$. There was no statistical difference in visual acuity or rate of vision change between our cases and controls.

Conclusion Uveitis with MS generally presents as intermediate uveitis with a minority presenting with anterior uveitis. Patients are significantly older and more likely to be female than patients with idiopathic intermediate uveitis. The visual prognosis is generally favorable.

Commercial interest 
- 3445

The Dublin Uveitis Evaluation Tool (DUET) - an algorithm for earlier diagnosis of spondyloarthropathies by ophthalmologists in acute anterior uveitis.

O'ROURKEM (1, 2), HAROONM (3), RAMASAMY P (1), FITZGERALDO (3) MURPHY C (1)

(1) RCSI Dept of Ophthalmology, Royal Victoria Eye and Ear Hospital, Dublin

(2) Education and Research Centre, St Vincent's University Hospital, Dublin

(3) Department of Rheumatology, St Vincent's University Hospital, Dublin

Purpose The association between anterior uveitis (AU) and spondyloarthritis (SpA) is well established. However, no algorithm exists to advise the ophthalmologist of which patients with AU should be referred to the rheumatolgist as a suspect SpA. Early diagnosis in $\mathrm{SpA}$ is key as disease severity and morbidity is related to disease duration. This study firstly establishes the incidence of SpA in an Irish cohort of patients attending eye casualty with previously unknown SpA. It also establishes a highly effective predictive algorithm to advise which patients should be referred to a rheumatologist as potential $\mathrm{SpA}$

Methods 104 consecutive patients with non-infectious $\mathrm{AU}$ were recruited prospectively and subsequently screened by a rheumatologist for SpA. The most statically significant features were used to generate a predictive algorithm which was subsequently validated in a further cohort of 80 patients.

Results The incidence of SpA is 41.5\% with average duration of backache 9.36 years Multiple regression analysis with detailed step wise post-hoc analysis identified that patients $<45$ years, with backache $>3$ months should have HLA-B27 checked. If positive, a referral is appropriate. If negative, a history of psoriasis should be ascertained and if present, the patient should be referred. This algorithm has sensitivity of $95 \%$ and specificity of $98 \%$. Validation of this algorithm in a second cohort had comparable sensitivity and specificity.

Conclusion The ophthalmologist has a necessary role in identifying SpA. Close collaboration between ophthalmologists and rheumatologists utilizing our algorithm will result in earlier treatment intervention to improve disease outcome.
- 3446

Changes in vitreous haze over time with intravitreal sirolimus in noninfectious posterior-segment uveitis (NI-PSU): results of SAKURA Study 1

BODAGHI B (1), ALI Y (2), YANG Y (2), CHERNOCKM (2), THURAUS (3), PAVESIOC (4)

(1) Ophthalmology, Paris

(2) Santen, Emeryville

(3) Ophthalmology, Munich

(4) Ophthalmology, London

Purpose To evaluate changes in vitreous haze $(\mathrm{VH})$ scores over time in in SAKURA Study 1, a randomized, double-masked, multicenter, international phase III study of subjects with active NI-PSU.

Methods 347 subjects with active NI-PSU (VH score $\geq 1.5+$ ) were randomized to intravitreal sirolimus $(44,440$, or $880 \mu \mathrm{g})$, given every 2 months. VH scores were assessed at Week 2 and Months 1-5.

Results At baseline, the mean (SD) VH scores were 1.9 (0.44) for the $440 \mu \mathrm{g}$ group and $1.9(0.50)$ for the $44 \mu \mathrm{g}$ group. The risk:benefit profile was most favorable with $440 \mu \mathrm{g}$ : Significantly more patients achieved a VH score of 0 at Month 5 (the primary endpoint) with $440 \mu \mathrm{g}$ vs $44 \mu \mathrm{g}(22.8 \%$ vs $10.3 \%$; $\mathrm{p}=025) .440 \mu \mathrm{g}$ was also significantly more effective than $44 \mu \mathrm{g}$ in achieving a VH score of $0 / 0.5+(51.8 \% \mathrm{vs} 35 \% ; \mathrm{p}=.012)$. Improvement was seen as early as Week 2 with $440 \mu \mathrm{g}(22.8 \%$ with $\mathrm{VH}=0 / 0.5+)$ and continued improvement with this dose was seen at every analysis visit $(36.0 \%, 40.4 \%$, $49.1 \%, 42.1 \%$, and $51.8 \%$ with $\mathrm{VH}=0 / 0.5+$ at Months $1-5$, respectively).

Conclusion Intravitreal sirolimus $440 \mu \mathrm{g}$ significantly improved inflammation in this diverse NI-PSU population. VH improvement was seen as early as 2 weeks after the first injection and continued through Month 5. Treatment with intravitreal sirolimus $440 \mu \mathrm{g}$ appears to be effective in treating NI-PSU, with a balanced safety profile.

Commercial interes 
- 3451

Long-term follow-up of the bag-in-the-lens in children

TASSIGNONMJ

Department of Ophthalmology, Edegem

Over the years the visual prognosis of pediatric cataract has significantly improved Final visual outcome depends upon many factors: age of onset, uni- or bilaterality, pre- and coexisting ocular abnormalities, timing of surgery, postoperative course and complications, and adherence to amblyopia treatment.We present a series of 46 eyes implanted with the bag-in-the-lens intraocular lens (BIL IOL), of which 30 bilateral and 16 unilateral eyes. A significant improvement in best-corrected visual acuity (BCVA) was obtained in all but one case (PFV case with glaucoma) though with a significant difference between uni- and bilateral cases. BCVA above 0.5 was achieved in 26, and of 10 in 17 of the bilateral cases. Of the unilateral cases only 5 achieved a BCVA above 0.5 while none achieved 1.0.The likely reasons for the poorer outcome in unilateral cases include a higher rate of ocular comorbidity (4 cases of PFV in this series) and the increased tendency towards amblyopia in unilateral cases despite early and intensive visual rehabilitation treatment.In conclusion, the BIL IOL can be considered a safe and well-tolerated approach for pediatric cataract with favorable visual outcomes and a low rate of VAR and other complications.

Commercial interest
- 3452

Cataract surgery in infants: should I implant an IOL?

VANDERVEEND

Boston

Implantation of an intraocular lens (IOL) in infancy remains controversial, and the decision about implantation may be driven by many factors. Some of these factors include age of the infant, unilateral vs bilateral status, presence of other ocular abnormalities, age at surgery, socioeconomic status of the family, environmental conditions, and surgeon preference and expertise. The risks and benefits of alternatives for correction of aphakia in infants will be reviewed, and current practice strategies in the United States discussed. The results of the Infant Aphakia Treatment Study, a randomized prospective multicenter study, can also help decision making in this context This study showed to age 5 years, similar visual outcomes for infants who had IOL vs. contact lens correction of unilateral aphakia. However, there was a higher rate of adverse events ( $81 \%$ vs $56 \%$ eyes) and additional intraocular surgeries (72\% vs $21 \%$ ) in the group receiving primary IOL implantation compared to the group treated with an aphakic contact lens. These risks should be weighed with regard to the other factors at play in each case of infantile cataract.
- 3453

Cataract surgery in children with syndromes or mental retardation

BREMOND-GIGNACD $(1,2)$

(1) Ophthalmology, University Hospital, Amiens

(2) CNRS, FR3636, Paris $V$

Specific complications of cataract surgery in children are well known. Refractive errors strabismus, amblyopia, glaucoma, posterior capsule opacification, inflammation, retinal complication. Young patients with syndromes or mental retardation can present some particular features that need to be evaluated and anticipated. Essentially they come from two directions. One is linked to ocular conditions that can be associated with the syndrome, such as microphthalmia, nanophthalmos, anterior segment dysgenesia, aniridia, frequency of capsular opacification, IO L calculation and complications, persistent fetal vasculature needs complex ultrasound examination to evaluate the surgical options, ocular misalignement, glaucoma, coloboma, retinal anomalies. The other condition is linked to mental retardation and the post-operative capacity of the child. The different surgical procedures with each step and aphakia correction are discussed following specific ocular conditions and should be adjusted with the level of mental retardation.
- 3454

Pars plana vitrectomy and IOL implantation in uveitic children with cataract

NYSTRÖM A, PÅLSSON S, SJÖDELL L, JAKOBSSON G, BYHR E, ANDERSSON GRÖNLUND M, ZETTERBERGM

Department of clinical neuroscience and rehabilitation, Mölndal

We have performed a study with the purpose to evaluate the outcome of combined cataract surgery with primary intraocular lens (IOL) implantation and pars plana vitrectomy (PPV) in children with uveitis. This paper is accepted for publication in Journal of ocular immunology and inflammation. 
- 3455

Update from the Swedish national pediatric cataract quality registry

TORNQVIST K

Dept of Ophthalmology, Lund University, Lund

Purpose: To present recent data from the Swedish/Danish pediatric cataract register (PECARE), a register with the aim of improving pediatric cataract care. Methods: A web-based register, the Pediatric cataract register, PECARE, with data on cataract operations in children $<8$ years old in Sweden and Denmark is used. The participating clinics have access to their own results together with the results of the total database online at any time.Results: On Dec 312013 the register contained data on 562 registered operations in 403 children, 209 (52\%) boys and 194 (48\%) girls.In 199 cases (49\%) the cataract was bilateral and in 204 (51\%) unilateral. Altogether 159 individuals (39\%) had undergone bilateral operation and 244 (61\%) unilateral operation. In 67/159 cases (42\%) operation had been performed in both eyes the same day. Totally 209 patients (300 eves) have at least one registered follow-up at 1,2,5 or 10 years of age. Data from these follow up registrations will be presented. Conclusion: The web based register "Pediatric cataract register, PECARE" gives the participating clinics up dated results on line. Aggregated data provide valuable information which can be used to improve the care of children with cataract. 
- 3461

The initial consultation

SPILEERS W

Leuven

A patient reporting flashing lights, shimmering, positive scotoma, hallucinations, needs a full (neuro-) ophthalmological work-up.The ophthalmological work-up includes visual acuity testing, biomicroscopy of the anterior segment, examination of the fundus. In selected cases, more specific ophthalmic testing such as visual electrophysiology is needed. Sometimes a general work up is indicated.The different neuro-ophthalmological causes of reporting positive phenomena will be introduced.

- 3463

Neurological considerations

KAWASAKIA

Lausanne

Seeing light flashes, scintillations, movement, simple or complex forms which are not based on actual sensory visual input from the external world represents a group of positive visual phenomena , often referred to as "hallucinations ». Such visual experiences arise from abnormal endogenously- activated neural discharges and can originate from any part of the visual system. In certain cases of disordered spatial perception, the lesion may even arise from the brainstem vestibular pathway. This lecture will examine the possible central mechanisms by which hallucinations are generated and give clinical examples of different types of positive visual phenomena.
- 3462

\section{Genetically determined disorders}

LEROY B

Ghent

Purpose:To describe genetically determined conditions which affect retinal and optic nerve function and can lead to seeing things which aren't there.Methods:A case presentation format will be used to illustrate different genetically determined conditions leading to visual hallucinations. Both clinical and electrophysiological phenotypes as well as genotypes will be discussed. Results:Phenotypes of genetically determined retinal diseases leading to visual hallucinations are very different. Often, the ophthalmologist can make a real difference by explaining the cause of the complaints.Conclusions:Very diverse conditions may give rise to seeing things which aren't there. Clinical examination and electrophysiology allow making a correct diagnosis.

- 3464

The role of electrophysiological investigation

HOLDER GE $(1,2)$

(1) Moorfields Eye Hospital, London

(2) UCL Institute of Ophthalmology, London

Purpose: To describe the diagnostic electrophysiological features associated with disorders that may present with positive phenomena.

Methods: Standardised electrophysiological testing, performed to incorporate and exceed the Standards of the International Society for Clinical Electrophysiology of Vision.

Results: Illustrative cases will be used to demonstrate diagnostic electrophysiological features in a variety of disorders.

Conclusions: The objective functional data provided by electrophysiological testing are of considerable importance to the diagnosis and management of conditions associated with positive phenomena. 
- 3471

Prognostic factors in myopic choroidal neovascularisation

SILVA R (1), WONG TY (2), ASMUS F (3)

(1) Faculty of Medicine, University of Coimbra (FMUC) and Ophthalmology Department, Coimbra

(2) Singapore Eye Research Institute, Singapore National Eye Center, Duke-NUS Graduate Medical School, National University of Singapore, Singapore

(3) Bayer HealthCare Pharmaceuticals, Berlin

Purpose To assess effects of age and baseline axial length on best-corrected visual acuity (BCVA) in patients (pts) with myopic CNV receiving intravitreal aflibercept (IVT-AFL)

Methods MYRROR was a 48 week, double-masked, sham-controlled, Phase 3 multicentre study (NCT01249664). Pts were randomised 3:1 to IVT AFL or sham/IVTAFL (sham to Week 20 [W20]; IVT-AFL from W24 to W48). Post hoc analyses assessed effects of baseline pt age and axial length on mean BCVA change at W24 and W48. Subgroups were based on median cut-offs (age: $\leq 62$ y vs $>62$ y; axial length: $\leq 28.6 \mathrm{~mm}$ vs $>28.6 \mathrm{~mm}$ ).

Results 121 pts were analysed (IVT-AFL, $n=90$ / sham/IVT-AFL, n=31). With age $\leq 62 \mathrm{y}$, mean BCVA changes were 13.0/14.7 letters (W24/W48) for IVT-AFL vs -0.8/ 8.1 for sham/IVT-AFL. With age $>62 \mathrm{y}$, changes were 11.1/12.2 letters for IVT-AFL vs $-3.6 /-1.2$ for sham/IVT-AFL. With axial length $\leq 28.6 \mathrm{~mm}$, mean BCVA changes were 12.6/13.8 letters (W24/W48) for IVT-AFL vs $-2.1 / 1.7$ for sham/IVT-AFL. With axial length $>28.6 \mathrm{~mm}$, changes were 11.6/13.2 letters for IVT-AFL vs -2.0/7.0 for sham/IVTAFL Correlation coefficients based on age were -0.234 and -0.215 (W24/W48) (both $\mathrm{P}<0.05)$; coefficients based on axial length were non significant. Ocular AEs were mainly mild and occurred at a similar rate with IVT-AFL and sham/IVT-AFL (37.4\% vs 38.7\%; most common, respectively: conjunctival haemorrhage $[11.0 \%]$ and punctate keratitis $[12.9 \%]) .7(5.7 \%)$ pts had a serious AE (all IVT-AFL; only one SAE in study eye [macular hole]). No deaths were reported.

Conclusion IVT-AFL was well tolerated and associated with clinically meaningful BCVA improvements in pts stratified by age or baseline axial length. Treatment benefits may be greater among younger myopic CNV pts. Baseline axial length did not appear to affect outcomes.

Commercial interest

- 3473

Correlation between peripapillary choroidal thickness and retinal vessel oxygen saturation in young healthy individuals

VAN KEER K (1), ABEGÃO PINTO L (2), WILLEKENS K (1), STALMANS I (1, 3), VANDEWALLEE (1)

(1) Department of Ophthalmology, University Hospitals Leuven, Leuven

(2) Department of Pharmacology and Neurosciences, Faculty of Medicine of Lisbon, Lisbon

(3) Department of Ophthalmology Neurosciences, Laboratory of Ophthalmology KULeuven, Leuven

Purpose To investigate the correlation between the peripapillary choroidal thickness (CT) and the retinal vessel oxygen saturation (SO2) in young healthy individuals

Methods Young healthy volunteers were recruited in this observational, cross-sectional study. Peripapillary CT was obtained using enhanced depth imaging optical coherence tomography (EDI-OCT). Arterial $(\mathrm{SaO} 2)$ and venous retinal oxygen saturation $(\mathrm{SvO} 2)$ were measured by a noninvasive spectrophotometric retinal oximeter in all four quadrants. Spearman's rank correlation and multiple regression analysis were used to determine relationships between choroidal thickness and oxymetric parameters.

Results 54 eyes of 54 individuals aged 21.6 \pm 1.1 years were analyzed. Average $\mathrm{SaO} 2$ $(92.3 \pm 3.0 \%)$ and $\mathrm{SvO} 2(55.4 \pm 4.6 \%)$ were positively correlated with the average peripapillary CT at 500 $\mu \mathrm{m}$ from the optic disc margin (Spearmans's R $=0.477, \mathrm{p}<0.001$ and $0.414, \mathrm{p}<0.002$ respectively). These results were confirmed to be independent of intraocular pressure and hemodynamic parameters on multivariate linear regression analysis $(\mathrm{p}<0.01)$.

Conclusion In young healthy individuals, retinal vessel oxygen saturation appears to be positively correlated with the peripapillary choroidal thickness.
- 3472

Visual outcomes following intravitreal aflibercept in patients with myopic choroidal neovascularisation

KOROBELNIKJ (1, 2, 3), IKUNO Y (4), ASMUS F (5)

(1) Service d'ophtalmologie, Hôpital Pellegrin, Bordeaux

(2) Université Bordeaux Segalen, Bordeaux

(3) Centre INSERM U897-Epidemiologie-Biostatistique, Bordeaux

(4) Department of Ophthalmology, Osaka University School of Medicine, Osaka

(5) Bayer HealthCare Pharmaceuticals, Berlin

Purpose To evaluate visual acuity outcomes in patients (pts) with myopic CNV receiving intravitreal aflibercept (IVT-AFL) or sham injection in the international, 48 week, Phase 3 MYRROR trial (NCT01249664).

Methods Pts were randomised 3:1 to IVT AFL or sham/IVT-AFL (sham to Week 20; IVT-AFL from Week 24 to Week 48). We report on exploratory post hoc analyses evaluating proportions of pts achieving visual gains (ETDRS letters) and the associated time course

Results 121 pts were analysed (IVT-AFL, n=90/ sham/IVT-AFL, n=31). At Week 24, $63.3 \%$ and $38.9 \%$ of IVT-AFL pts had gained $\geq 10$ or $\geq 15$ letters from baseline. In the sham/IVT-AFL group, $12.9 \%$ and $9.7 \%$ had gained $\geq 10$ or $\geq 15$ letters from baseline. By Week 48 , the proportion of IVT-AFL pts gaining $\geq 10$ or $\geq 15$ letters was $68.9 \%$ and $50.0 \%$, respectively. At Week 48 in the sham/IVT-AFL group (i.e., 24 weeks after IVTAFL initiation), $41.9 \%$ and $29.0 \%$ of pts had gained $\geq 10$ or $\geq 15$ letters from baseline, respectively. Ocular AEs were mainly mild and occurred at a similar rate with IVTAFL and sham/IVT-AFL (37.4\% vs 38.7\%; most common, respectively: conjunctival haemorrhage [11.0\%] and punctate keratitis [12.9\%]). 7 (5.7\%) pts had a serious $\mathrm{AE}$ (all IVT-AFL; only one SAE in study eye [macular hole]). No deaths were reported.

Conclusion The proportion of IVT-AFL pts who gained $\geq 10$ or $\geq 15$ letters by Week 24 was well maintained and even slightly increased at Week 48. Clinically meaningful benefits from IVT-AFL were also observed at Week 48 in the sham/IVT-AFL group. However, the proportion of gainers did not reach the extent seen in the initial IVT-AFL group, suggesting that earlier IVT-AFL treatment should be recommended.

Commercial interest

\section{- 3474}

Findings in low luminance kinetic and static perimetry

KRASTEL H (1), GAGYI-PALFFYZ (2), BEUTELSPACHERS (3),

SCHLICHTENBREDE F (1), JONAS J (3)

(1) Dept. of Ophthalmology, University Medical Center, Mannheim

(2) Oculus Optik Geräte GmbH, Wetzlar

(3) Mannheim Medical Faculty, University of Heidelberg, Mannhein

Purpose To transfer the neutral density filter test to perimetry; i.e. to show that the diagnostic battery can be supplemented by low luminance visual fields, if the standard procedures yield unsatisfactory information.

Methods The Goldmann perimeter uses one single incandescent light source for both stimulus and background illumination (BI). By dimming the luminance was reduced by $0.6 \log$ units. The accompanying change in colour temperature was ignored. The i) Twinfield and ii) Tübingen automatic perimeters were modified to low luminance conditions using neutral grey filters i) in front of the eye, or ii) in the stimulus and the BI light paths. Special attention was paid to perform glaucoma fields at normal IOP level as proven by GAT and/or by Corvis tonometry (the latter to avoid affection of the tear film by anesthetics)

Results Low luminance perimetry produces more pronounced scotomas in kinetic, and more distinct loss of sensitivity in static perimetry than otherwise identical perimetric procedures in standard luminance. Cases concern inflammatory, ischemic and compressive optic nerve diseases, glaucoma, and ischemic and diabetic retinopathies. Results in hereditary, dystrophic and toxic retinal conditions vary depending on whether the rods and the scotopic system are mainly affected, or the cones and the photopic system. Cone diseases lack visual field deterioration in low luminance and nstead may show improvement.

Conclusion Low luminance perimetry is a supplementary diagnostic tool which may add helpful diagnostic information, particularly if standard results are insignificant.

Commercial interest 
- 3475

Silencing HuR: a new potential pharmacological strategy to target VEGF

AMADIOM (1), PASCALEA (1), GOVONIS (1), DRAGO F (2), BUCOLOC (2) (1) Dept of Drug Sciences, Pavia University, Pavia

(2) Dept of Clinical and Molecular Biomedicine, Catania University, Catania

Purpose VEGF, a key element in the pathogenesis of proliferative diabetic retinopathy (DR), is regulated at post-transcriptional level by HuR protein (mRNA-stabilizing human Embryonic Lethal Abnormal Vision protein), which binds to VEGF mRNA and, following diabetes-induction, positively affects its expression. We investigated the effect of the in vivo administration of a silencing RNA (siRNA) which down-regulates HuR expression on the diabetes-induced VEGF increase.

Methods Diabetes was induced in rats by streptozotocin (STZ) injection (Amadio et al., Biochem. Pharmacol. 80: 1230-1237, 2010). After 24 h from STZ injection, 2.5uM siRNA (commercially available) against HuR was intravitreally administered. Animals were sacrificed $48 \mathrm{~h}$ after siRNA injection and retinal tissues collected. HuR and VEGF retinal protein levels were analyzed by Western blotting and ELISA, respectively.

Results siRNA treatment blunts HuR protein increase in STZ rat retina (mean gray level ratio of HuR $/ \alpha$-tubulin \pm S.E.M.: CTR 100 $\% \pm 4.8$; CTR + siRNA 99\% \pm 9.3; STZ $147 \%$ $\pm 7.5 ; \mathrm{STZ}+$ siRNA $109 \% \pm 7.3 ; \mathrm{p}<0.0001 \mathrm{STZ}+$ siRNA vs. STZ) and, as a consequence, dampened VEGF production (STZ+siRNA $9.5 \mathrm{pg} / \mathrm{mg} \pm 3.5 \mathrm{vs}$. STZ $18.2 \mathrm{pg} / \mathrm{mg} \pm 4.2$; $\mathrm{p}<0.05)$.

Conclusion HuR protein represents a novel pharmacological target useful to counteract pathologies implicating VEGF deregulation, such as DR
- 3476

Ghrelin's effects in diabetic retinopathy: Inhibition of choroid retinal cells migration cultured under a hyperglycemic environment.

ROCHA DE SOUSA A (1), SILVA-GOMES R (2), PEREIRA-SILVA P (2) AZEVEDO-PINTOS (2), MOLEIROA (2), CONCEIÇÃOG (2) LEITE-MOREIRA AF (2)

(1) Department of Senses Organs; Faculty of Medicine; University of Porto, Porto

(2) Department of Physiology and Thoracic Surgery; Faculty of Medicine; University of Porto, Porto

Purpose The purpose of this work is to investigate the effect of ghrelin in a primate choroid retinal cell line cultured under hyperglycemic conditions and its effect on the early changes of diabetic retinopathy in an animal model of type1 diabetes mellitus (DM1).

Methods A RF/6a cell line was used in the in vitro assay. Cell migration was assessed using the wound healing assay under increasing $(0-300 \mathrm{mM})$ glucose concentrations. To test its effect, ghrelin was added (10-5-10-10nM) to the cell cultures for 24h. Positive controls had VEGF added to the medium. For the in vivo studies, diabetic Wistar rats received intravitreal injections of either ghrelin $(81 \mathrm{nM})$ or saline every 4 weeks for 3 months. Vascular permeability was assessed using the Evans blue assay.

Results Increasing concentrations of glucose show a reduction in cell migration distance. We defined $10 \mathrm{mM}$ of glucose as the basal and $250 \mathrm{mM}$ as the hyperglycemic condition. At a concentration of 10-8 $\mathrm{nM}$ ghrelin potentiates the reduction of migration induced by the hyperglycemic medium, and reduces the migration induced by VEGF. Regarding the in vivo model, diabetic animals treated with intravitreal ghrelin injections showed no alteration in vascular permeability, when compared with diabetic controls.

Conclusion Ghrelin reduces cell migration in choroid-retinal cells under hyperglycemic media, but appears to have no effect on the vascular permeability in a DM1 animal model. 
Industry-sponsored symposium 4: Aflibercept: Setting its sight on DME

- 3531

Science behind the disease

SCHLINGEMANNR

Amsterdam

ABSTRACT NOT PROVIDED
- 3532

Managing the DME patient

PEARCE I

Liverpool

ABSTRACT NOT PROVIDED

- 3533

VIVID and VISTA: Clinical trial results and implications

KOROBELNIKJ

Bordeaux

ABSTRACT NOT PROVIDED 
- 3611

Pulsatile versus non-pulsatile choroidal blood flow in Primary Open-Angle Glaucoma (POAG)

RIVA C (1), ABOU SAMRA W (2), POURNARAS C (3)

(1) Medicine of University of Lausanne, Lausanne

(2) Ophthalmic Center, Mansoura University, Mansoura

(3) Colline Ophthalmology Center, Memorial Rothschild Clinical Research Group, Geneva

Purpose After demonstrating altered subfoveal choroidal blood flow(F)in POAG (AbouSamra et al,Acta Ophthal 2012,we now studied the waveform of the pulsatile component of $\mathrm{F}(\mathrm{FP})$ in normal subjects and POAG patients.

Methods Using laser Doppler Flowmetry (LDF,F and FP were measured in 17 healthy controls and 19 age- and sex-matched POAG patients. We determined the waveform amplitude $\mathrm{A}(=1$-FPdiast/FPsyst) during the heart beat and the times $\mathrm{T}(\mathrm{d}-\mathrm{s})$ and $\mathrm{T}(\mathrm{s}-\mathrm{d})$ to increase from the diastolic to the systolic and to decrease from the systolic to the diastolic values of FP, respectively.

Results Both mean F and mean FP were significantly $(\mathrm{p}<0.001$ and $\mathrm{p}=0.02$ respectively) reduced in the POAG compared to the control group. However mean $\mathrm{FP} /$ mean $\mathrm{F}$ was not significantly different between groups. Relative to the brachial artery value, the $\mathrm{A}$-value at the subfovea was significantly increased in POAG compared to controls. The mean vascular resistance $(\mathrm{R}=$ mean perfusion pressure $/$ mean $\mathrm{F})$ was markedly increased $(\mathrm{p}<0.0001$ in the POAG group. There was no significant differences in $\mathrm{T}(\mathrm{d}-\mathrm{s})$ and $\mathrm{T}(\mathrm{s}-\mathrm{d})$ between the groups

Conclusion These LDF-based findings further support the presence of hemodynamic alterations in the subfoveal choroid in POAG.

- 3613

Effect of acute hypoxia or hyperoxia on the optic nerve head and choroidal blood flow in healthy humans

CHIQUeTC C (1, 2, 3), ZHOU T (1, 3), GALLICEM $(1,3), \operatorname{APTEL} F(1,3)$, TRUFFER F (4), GEISER M (4)

(1) Department of Ophthalmology, University Hospital of Grenoble, Grenoble

(2) UJF-Grenoble 1, Grenoble

(3) INSERM 1042, Grenoble

(4) HES-SO//Valais, Engineering system, Sion

This presentation deals with the ocular vasoreactivity to acute hypoxia and hyperoxia in young healthy subjects $(\mathrm{n}=16)$. The protocol consisted in a 15-min inhalation of $100 \%$ $\mathrm{O} 2$ (hyperoxia) or $10.5 \% \mathrm{O} 2+\mathrm{N} 2$ (for a stable $\mathrm{SpO} 2$ of $85 \%$, hypoxia) in normocapnic conditions. Blood flow was measured using laser Doppler flowmetry in the subfoveal choroid or at the temporal site of the optic nerve head $(\mathrm{ONH})$. Hypoxia induced a significant $30 \pm 10 \%$ increase in ONH blood flow (due to simultaneous VEL and VOL rise). Hyperoxia significantly decreased ONH blood flow by $12 \pm 7 \%$ (due to both VEL and VOL decrease). Choroidal blood flow was not affected by hypoxia or hyperoxia. We will discuss the mechanisms associated with the effect of gas and the difference of response between choroid and $\mathrm{ONH}$
- 3612

LDF and assessment of autoregulation

SCHMETTERER L $(1,2)$

(1) Medical Physics and Biomedical Engineering, Vienna

(2) Clinical Pharmacology, Vienna

The main advantage of laser Doppler flowmetry is its excellent time resolution. This allows for continuous measurement of tissue blood flow during changes in perfusion pressure. As such the technique is optimally suitable to assess autoregulation, referring to the ability of a vascular bed to maintain its blood flow in response to changes in perfusion pressure. In the eye the technique has been applied for both measuring optic nerve head blood flow and subfoveal choroidal blood flow. For both vascular beds data have been obtained using either an experimental increase or decrease in ocular perfusion pressure. In addition, data are available showing the complex regulation of ocular blood flow when both mean arterial pressure and intraocular pressure are modified.

3614

Choroidal blood flow and choroidal thickness

POURNARAS C(1), DONATI G (2), FROUNTZOUE (2), GEISER M (3),

RIVA CE (4)

(1) Memorial Rothschild Clinical Rechearch Group, Colline Ophthalmology Center. Geneva

(2) Colline Ophthalmology Center, Geneva

(3) University of Applied Sciences of Western Switzerland, Sion

(4) University of Lausanne, Lausanne

A progressive decrease of the choroidal blood flow related to macular degeneration was observed with glaucoma and aging. Abnormal regulation of the choroidal blood flow in patients suffering from neovascular AMD or Diabétic microangiopathy was also reported. Recent clinical studies haveshown a decrease of the choroidal thicknessof in high myopia, AMD or in retinal dystrophies and increased choroidal thickness in central serous chorioretinopathy.Continuous real time Laser Doppler Flowmetry (LDF) has been used in humans for the evaluation of the subfoveal choroidal circulation and the investigation of the regulatory processes of the choroidal blood flow in response to various physiological stimuli, such as increase and decrease of the ocular perfusion pressure.The correlation of the subfoveal choroidal blood flow with the anatomical changes of the choroidal circulation, as observed by enhanced depth imaging optical coherencetomography(EDI-OCT), was investigated in healthy volonteeres.No significant correlation was found between the subfoveal choroidal thickness and the choroidal blood flow. However, decreased subfoveal choroidal thickness was associated with decreased refractive error and axial length. 
SIS: Recent developments in laser Doppler flowmetry

- 3615

Laser Doppler flowmetrie on small animal

GEISER M (1), TRUFFER F (1), MENTEKM (2), BERNABEIM (3), APTEL F (2, 4)

CHIQUET C $(2,4)$

(1) HES-SO//Valais, Engineering system, Sion

(2) INSERM 1042, Grenoble

(3) University of Modena, Modena

(4) Department of Ophthalmology, University Hospital of Grenoble, Grenoble

We present a compact fundus camera designed to measure the blood flow in smal animals based on laser Doppler flowmetry (LDF). The small animal is immobilized on a modified stereotaxic table and the pupil of the LDF device is superposed by translation to the pupil's eye of the animal. Then rotation centered at the pupil is used to point the probing beam to the optic nerve head $(\mathrm{ONH})$ or on retinal arteries (RA). LDF parameters Vel, Vol and F were assessed with a rotating teflon wheel simulating Doppler signal. Experiments were conducted on anesthetized adult male Wistar and Brown Normay rats. We demonstrated stable LDF parameters over 1 hours on RA and optic ONH for 6 and 10 rats respectively. Reproducibility is good for Vel on RA and ONH and moderate for Vol and F ONH. Transient ocular blood flow was blocked by increasing intra-ocular pressure (IOP). Vel decreases significantly by $70 \%$ of baseline. Vel on RA was recorded during rat euthanasia. Vel decreases to the same level as for increased IOP. Vel measurement on RA are feasible, however measurement on ONH are more difficult probably due to a scare capillary network. 
- 3621

How to get your research published?

PLEYER U (1), STEFANSSONE (2), KIVELÄ T (3), DUA H (4)

(1) Berlin

(2) Reykjavik

(3) Helsinki

(4) Nottingham

Researchers often face the challenge of getting their work published. The goal of this session will be to provide some keys to write a high quality paper that will help to transform your ideas and findings into a research article. The panelists will discuss major aspects of the editorial process including basic decisions,e.g. where to submit your work how to organize and prepare a manuscript and how to deal with the review process.We will raise the discussion on issues like:- How to choose a journal?- How to organize your paper?- What are the characteristics of a good manuscript?- How to interpret the letter from the editor?- How to write a good reply to the reviewers?- What to do when your paper is rejected?In addition, the personal view from an authors and editors perspective will be given in a vivid discussion with the participants.

- 3623

How to get your research published?

KIVELÄ T

Department of Ophthalmology, Helsinki Iniversity Central Hospital, Helsinki

A primary criterion for publishing a paper is novelty of its data. This extends to the introduction and discussion of the paper. These can not consequently be cut and pasted from published literature, not even from the author's previously published papers. The journals typically require the authors to confirm that their submission is unique and that the data are new. Violations of these rules may today result in retraction of the paper after publishing, even years or decades after its publication, if plagiarism or self plagiarism is later detected. One must also be certain to submit only unaltered images and data. Many universities and some journals provide today on-line tools that can be used to check whether the manuscript has borrowed verbatim from the literature. The advent of on-line manuscript review and publishing mav tempt to copv from previous papers without rephrasing or attribution, habits which should carefully be avoided. On the other hand, electronic publishing is low cost and easy to start, which recently has resulted in proliferation of so-called "predatory" gold open access publishers. Guidelines are discussed which may help authors to identify such publishing forums and to avoid plagiarism
- 3622

How to get your research published?

STEFANSSONE

Reykjavik

The quality of an article depends first and foremost on its scientific content. However, the way in which the scientific material is presented may determine whether it will receive proper attention. The aim of this presentation is to point out general characteristics of good papers.The title can decide whether a potential reader will give your article a proper look. A good title is descriptive and may also give a statement if the ournal style allows.The abstract is the most important part of your article. People will proceed to read the article only if they find the abstract interesting.Describe the material or patients and the methods in brief. It is advisable to specify the study design.Present the most important results in a brief manner. Give quantitative data with confidence limits whenever applicable. The reader wants to know what your results were, not just whether they were "statistically significant".In the discussion, put your data into context with the clinical practice, literature or both. What have we learned? A common mistake is to include general conclusions which are not specifically supported by the data presented. Please check that the conclusions are in line with the stated aim of the study.

- 3624

How to get your research published?

DUA H

Nottingham

ABSTRACT NOT PROVIDED 
- 3631

Excimer vs Femtosecond laser for nonmechanical keratoplasty Where are the true benefits?

SEITZB (1), EL-HUSSEINY M (1), JANUNTS E (2), LANGENBUCHER A (2) SZENTMARYN (1)

(1) Department of Ophthalmology, Saarland University Medical Center, Homburg

(2) Experimental Ophthalmology, Saarland University, Homburg

Purpose: To compare the intraoperative feasibility and postoperative outcome of the excimer (EXL) and femtosecond laser (FSL) for penetrating keratoplasty (PKP).

Methods: Since 1989 more than 3500 non-contact nonmechanical PKPs using the Zeiss-Meditec MEL70 $193 \mathrm{~nm}$ EXL along metal masks with eight "orientation teeth/ notches" (PKP) were performed. Since 2006 various groups applied the FSL for PKP with various 3D-configurations.

Results: The technique of EXL PKP may improve donor and recipient centration, reduce "vertical tilt" and "horizontal torsion" of the graft in the recipient bed, thus resulting in significantly less "all-sutures-out" astigmatism, higher regularity of the topography and better visual acuity. Clinical results of FSL PKP - requiring variable amounts of applanation thus causing distortion of corneal tissue - are still very limited and not yet finally convincing.

Conclusions: Because of undisputed clinical advantages, especially in eyes with keratoconus, EXL trephination is still favoured in Homburg/Saar in daily practise. To determine potential benefits of FSL over EXL PKP, a prospective randomized study for keratoconus and Fuchs dystrophy is ongoing in Homburg/Saar.

- 3633

Corneal dystrophies related to cholesterol metabolism (fish eye and Schnyder dystrophy)

KESTELYNP

Gent

Schnyder corneal dystrophy (SCD) is a rare corneal dystrophy characterized by abnormally increaseddeposition of cholesterol and phospholipids in the cornea. SCD is inherited as an autosomal dominant trait with high penetrance and has been mapped to the UBIAD1 gene on chromosome 1p36.3.Diseases of systemic lipid metabolism that cause corneal opacification, such as lecithin- cholesterolacyltransferase (LCAT) deficiency and fish eye disease (FED), should be considered in the differential diagnosis LCAT deficiency and FED are both entities that result from deficiency of the LCAT enzyme. LCAT deficiency is defined by deficient LCAT activity towards HDL and LDL whereas FED is defined by decreased LCAT activity against HDL only. When comparing LCAT deficiency and FED to SCD, there are numerous differences to help distinguish all 3 entities. Both LCAT deficiency and FED are inherited in an autosomal recessive manner while SCD has autosomal dominant inheritance. Systemically, SCD has a normal level of LCAT enzyme activity and normal HDL levels. Both LCAT deficiency and FED show abnormally low levels of HDL. SCD is often associated with a true arcus lipoides, unlike FED and LCAT deficiency.

\section{- 3632}

New insights into genetics of Fuchs dystrophy

BARATZK

Rochester

Fuchs endothelial corneal dystrophy (FECD) is a common, familial trait. Several genes have been associated with the disease including LOXHD1, SLC4A11, TCF4, ZEB-1 and COL8A2 which has been identified as a cause of an early-onset Fuchs-like disease. The evidence for these genetic associations will be reviewed and new data will be shared. To date, the most sensitive biomarker for FECD is an intronic TGC trinucleotide expansion in the TCF4 gene which is present in the majority of FECD cases and in a small proportion of normal control subjects. Within families, this tricnucleotide expansion tends to segregate with the disease phenotype. The biology of trinucleotide expansion diseases and possible mechanisms by which TGC expansion may conribute to FECD will be presented. A brief discussion of recent advances in our understanding of the pathophysiology of FECD, such as the unfolded protein response and oxidative stress, will also be presented in order to highlight the challenges in linking the implicated causative genes with the biochemical pathways involved in the disease.

3634

Current revision of the international classification of corneal dystrophies (IC3D)

LISCH W

Hanau, Augenklinik

The International classification of corneal dystrophies (CD) was published by the International committee for classification of corneal dystrophies (IC3D) in the Journal CORNEA in 2008.In the meantime, we propose some revisions. I' $d$ like to present the main points:1. New general classification of CD2. Spliting of ERED (epithelial recurrent erosion dystrophy) into distinct entities3. The so-called epithelial-stromal TGFBI dystrophies4. Revision of Thiel-Behnke CD5. Lattice lines in granular CD, type 2 ?6. Revision of congenital hereditary endothelial dystrophy (CHED): the so-called CHED 1 = eponym of posterior polymorphous corneal dystrophy (PPCD). 
114

SIS: Corneal dystrophy \& laser keratoplasty: updates

- 3635

Confocal microscopy and optical coherence tomography imaging of corneal dystrophies

WYLEGALA E $(1,2)$

(1) Ophthalmology Clinic Medical University of Silesia, Katowice

(2) Railway Hospital Ophthalmology Clinic, Katowice

Corneal dystrophies are the group of hereditary, slowly progressive disorders affecting all corneal layers. In the past, histological examination was crucial in proper diagnosis of corneal dystrophies. In recent years, non invasive optical imaging systems such as optical coherence tomography (OCT) and confocal microscopy (CM) along with genetic testing have become a new standard procedures in the diagnostic process.

Advances in diagnosis and treatment of corneal dystrophies including genetic analysis and confocal microscopy findings were included in the IC3D classification in 2008 . The lecture summarizes the basic principles of the confocal microscopy and optical coherence tomography. It describes the pathological corneal changes revealed by slit amp and histological examination in relation to non contact imaging techniques. The structural changes observed with the histological methods give an account and provide an explanation for pathological changes demonstrated by the OCT and CM. This may allow for a better understanding of the pathological process involved in the corneal dystrophies progression. 
- 3641

Characteristic features of choroidal tumors in Deep Range

ROMANOWSKA DIXON B, JAKUBOWSKA B, MARKIEWICZ A

Ophthalmological Department of Jagiellonian University, Krakow

Purpose To find and analyze characteristic features of choroidal tumors and

Methods Patients with choroidal tumors (choroidal nevus, choroidal melanoma choroidal metastses, choroidal hemangioma, choroidal osteoma and others) diagnosed and treated in Department of Ophthalmology and Ocular Oncology of University Hospital were included. Swept Source OCT (DRI OCT-1) developed by Topcon was used for imaging.

Results Deep range imaging DRI-OCT-1 displavs detail structures of not only retina but choroid and even sclera. The characteristic features, of intraocular tumors can improve the differential diagnosis and estimation of progression or treatment effects.

Conclusion The new diagnostic modality may be useful for the evaluation of abnormalities observed intratumoral and in a border area and can improve diagnostic accuracy. Imaging OCT (DRI-OCT) surrounding tissues, retina, choroid and vessels.

- 3642

Diagnostic contribution of ocular imaging techniques in a multimodal diagnostic approach of small pigmented choroidal tumors

ZOGRAFOS L, PETROVICA, SCHALENBOURG A

Lausanne

Purpose The differential diagnosis of small pigmented choroidal tumors is based on the evaluation of the tumor diameter, thickness, retinal detachment, pigment epithelium changes, and possible associated symptoms.

Methods The diagnostic contribution of color fundus photography, fluorescein angiography (FA), indocyanine green angiography (ICGA), fundus autofluorescence (FAF) and optical coherence tomography (OCT), was analyzed in 85 eyes with a small pigmented choroidal tumors, measuring $2-10 \mathrm{~mm}$ in diameter and $0.8-2.5 \mathrm{~mm}$ in thickness. The presumed diagnosis was a large choroidal nevus in 60 cases $(71 \%)$, and a small choroidal melanoma in 25 cases (29\%). The identification of the tumor border, the presence of any retinal detachment on the tumor surface or outside the tumor borders, the presence of orange pigment, drusen, pigment epithelium changes and/or pin points were analyzed on each imaging technique.

Results The highest identification score for the tumor margins was reached by color fundus photography. An associated retinal detachment was best identified on OCT Orange pigment was best detected on fundus color photography and then by FAF. Drusen identification reached the same score on fundus color photography and FA. The appearance of pin points was exclusively seen on FA. Pigment epithelium changes showed the best score on FAF

Conclusion The majority of the investigated ocular imaging techniques played an important role in the identification of diagnostic elements that contribute to the distinction between a small choroidal melanoma and a large nevus. Multimodal imaging techniques have to be used in order to obtain a higher efficacy in differentiating small pigmented choroidal tumors.

- 3644

Aquaporin-4 and potassium channel kir4.1 in amd and serous retinal detachment secondary to choroidal melanoma

MADIGANM (1, 2), DIEPM (1), JUNGHANS B (1), VALTER K (3)

(1) Optometry \& Vision Science, UNSW, Sydney

(2) Save Sight Institute Uni Sydney, Sydney

(3) ANU Medical School, ARC Centre of Excellence in Vision Science, John Curtin School of Medical Research, Canberra

Methods All the evisceration specimens from a 15 year period were analyzed fo malignancies. A literature search on the accidental finding of intraocular malignancy after evisceration was performed

Results We found an incidence of intraocular malignant melanoma of $0.7 \%(2 / 315)$. In these 2 cases, a standard evisceration procedure with implantation of an acrylic ball had been performed for pain in a longstanding blind eye with opaque media. Both patients had a history of glaucoma and massive intraocular hemorrhage, confirmed by repetitive ocular ultrasound (US) and orbital CT. Histopathology showed a large intraocular uveal melanoma with substantial necrosis and haemorrhageAfter negative screening for metastases, one week following the evisceration an enucleation of the scleral shell and implant was performed, with implantation of a new acrylic ball with donor sclera. External beam radiation of 5,000 cGy was given to the orbital socket. The firs patient died from liver metastases 3 years after the diagnosis, without evidence of local recurrence. The second patient has a too short follow-upThe literature is scarce on this subject, with only 9 published cases. US is the mainstay in the screening for intraocular tumors in opaque eyes. There are no guidelines on the management after accidental evisceration of uveal melanoma

Conclusion Ultrasound can not always distinguish primary choroidal hemorrhage from secondary hemorrhage due to uveal melanoma with necrosis. An atypical clinical history of a blind painful opaque eye with prolonged massive intraocular hemorrhage and ocular hypertension may be suspect for malignant melanoma, in which case evisceration should be avoided

Purpose Aquaporin-4 (AQP4) and potassium channel Kir4.1 are normally expressed in retinal Müller glia, and play a major role in controlling retinal fluid and maintaining retinal integrity and function. We examined AQP4 and Kir4.1 distribution and expression in human eyes with late age-related macular degeneration (AMD) or longterm serous retinal detachment secondary to choroidal melanoma.

Methods Paraffin sections of central and peripheral choroid/retina from young $(<40$ years, $\mathrm{n}=5)$, aged ( $>70$ years, $\mathrm{n}=4$ ) and AMD $(>70$ years, $\mathrm{n}=5$ ) human post-mortem eyes, and whole human eyes with choroidal melanoma $(\mathrm{n}=5)$, were co-immunolabelled with antibodies to AQP4, Kir4.1 and GFAP, and visualised using immunofluoresence and confocal microscopy.

Results We found differential expression of AQP4 and Kir4.1 in detached retina of melanoma eyes, with upregulation of AQP4 and downregulation of Kir4.1 compared to unaffected eyes. This was most obvious in the retina overlying melanomas and in detached retina separated from underlying choroid. GFAP immunoreactitvity was upregulated in areas of serous retinal detachment, and retinal thinning; Müller cell processes extended along the anterior surface of melanomas. No apparent difference in distribution or expression of AQP4 or Kir4.1 was observed for retinas with late stage AMD compared to controls.

Conclusion Decreased Kir4.1 expression in long term serous retinal detachment may be associated with Müller cell proliferative gliosis, as suggested in earlier animal studies. Increased AQP4 expression suggests efforts to restore retinal fluid balance in areas with photoreceptor degeneration and compromised outer blood-retinal barrier:Support: NFMRI (MCM), Lions NSW Eye Eyebank 
- 3645

Preclinical scheduling for metastatic ocular melanoma treatment by using a xenograft model approach

LEMAITRE S, NEMATI F, THULEAU A, LABIOD D, RODRIGUES M, DE PLATER L PIPERNOS, SASTRE X MARIANI P ROMANROMANS GENTIEND, DECAUTIND, CASSOUXN

Institut Curie, Paris

Purpose The prognosis for patients with disseminated ocular melanoma is poor Responses to standard chemotherapy are low and serious toxicities are common. Here, we determine the feasibility of treating melanoma xenograft models in mice in order to guide the selection of treatments in metastatic patients.

Methods A patient-derived xenograft model was established in SCID mice for metastatic choroidal and conjunctival melanoma. Mice bearing growing tumors were randomly assigned to the control or treatment groups. Mice were treated with dacarbazine and everolimus in the choroidal melanoma model. Dacarbazine and dabrafenib were used in the conjunctival melanoma model.

Results Everolimus resulted in tumor stasis in the choroidal melanoma model while dacarbazine had no antitumor effect. In the conjunctival melanoma model the relative tumor volumes in mice treated with dacarbazine and dabrafenib were similar to the control group. Since this is a feasibility study we included only a limited number of cancer drugs. More drugs will have to be screened in future studies. The oncologists in our institution were aware of the results but it had no influence on the choice of treatments for the patients. To our knowledge this is the first report of a conjunctival melanoma xenograft model in SCID mice.

Conclusion Our results suggest that ocular melanoma xenograft models can be used to determine which drugs achieve antitumor activity. These data could help oncologists in the choice of treatments for metastatic patients.

\section{- 3647}

Orbital solitary fibrous tumor - Case series and new perspectives

IRION L, BONSHEK R, KUMAR A, QURESHI F, COOK A, ATAULLAHS, BARBIER J, LEATHERBARROW B Central Manchester University Hospitals, Manchester

Purpose To present a case series of orbital solitary fibrous tumour (OSFT) from a ertiary referral centre focussing on diagnostic features including assessment of STAT6, a recently described antibody for immunohistochemistry (IHC) in which positivity is said to indicate NAB2-STAT6 gene fusion.

Methods This is a retrospective case series. Among all orbital tumours resected between 2001-2014, 12 OSFT cases were identified. Data collected included patient demographics, clinical presentation, morphological and IHC features, management and clinical outcome. IHC for STAT6 was also performed in other soft tissue tumours to assess its specificity.

Results There were 3 males and 9 females. The average age was 46 years $(18-81)$. The mean follow-up was 39.3 months (3-156 months). Presenting symptoms included $\operatorname{proptosis}(\mathrm{n}=6)$, periocular mass $(\mathrm{n}=4)$, foreign body sensation $(\mathrm{n}=1)$, and epiphora $(\mathrm{n}=3)$. Cellularity hyalinisation and myxoid areas were variable. Necrosis was seen in one recurrent case. Giant cells were present in 3 cases. $<4 / 10 \mathrm{HPF}$ mitoses were seen in 6 cases. All cases showed diffuse strong nuclear positivity with STAT6. In contrast. STAT6 expression was not demonstrated in other pathologies (e.g. neurofibroma schwannoma, haemangioma, nodular fasciitis). CD34, CD99 and BCL2 ware positive in most cases but with varied staining pattern. One case was CD31 positive and 2 cases expressed SMA focally. All cases underwent elective surgical resection or debulking with complete histopathological clearance in 6 cases.

Conclusion This case series confirms previously described variation in presentation and management challenges associated with OSFT. STAT6 antibody can be a helpfu adjuvant in the diagnosis of OSFT
- 3646

MAP kinase and $\mathrm{pi3k} / \mathrm{mTOR}$ pathways involvement in tumor progression of conjunctival melanocytic proliferations

MOULINA (1), BUCHER M (2), SCHALENBOURG A (3), NICOLAS M (3)

(1) Jules Gonin Eye Hospital (JGEH),Lausanne University, Lausanne

(2) Dermatology, Lausanne University, Lausanne

(3) JGEH, Lausanne

Purpose As activating mutations of BRAF and NRAS have been identified in $47 \%$ of conjunctival melanoma, we evaluated the activation status of the MAP kinase and PI3K/ mTOR pathways in benign and malignant conjunctival melanocytic proliferations. As loss of PTEN locus has been reported in conjunctival melanoma, we also assessed the expression of PTEN in conjunctival nevi and melanoma.

Methods The phosphorylation status of MEK, ERK, AKT, S6 ribosomal protein and the expression of PTEN was evaluated by immunohistochemistry in 35 conjunctival naevi and 31 conjunctival melanoma. Statistical analysis was performed with JUMP 8,0 software. Immunohistochemistry was assessed independently by three observers.

Results There were 13 subepithelial nevi and 22 compound nevi. There were 14 females and 21 males with a mean age was 36.9 yo. P-MEK, p-ERK, p-S6, p-AKT and PTEN were respectively found in $59 \%, 49 \%, 49 \%, 83.9 \%$ and $100 \%$ of the nevi. The melanoma group was composed of 17 females and 14 males with a mean age of 67.1 yo. P-MEK, p-ERK, p-AKT, p-S6 and PTEN were respectively found in 87\%, 87\%, 94.3\%, $94 \%$ and $96.7 \%$ of the melanoma. The phosphorylation of MEK and ERK, AKT and S6 was significantly elevated in the melanoma compared to the nevi $(\mathrm{p}=0,0050$; $\mathrm{p}=0,0004$; $p=0,0031 ; p>0,0001$ respectively). There was also a significant correlation between the activation of MEK and ERK ( $\mathrm{p}=0,0045)$.

Conclusion Our results demonstrate ex vivo a significant increase in the activation of the MAP kinase pathway in malignant conjunctival melanocytic proliferations as well as a significant increase in the phosphorylation of pAKT and S6 ribosomal protein, suggesting a contribution of the PI3K/mTOR pathway to conjunctival melanoma development.

- 3648 / S121

Secondary Intraocular lymphoma of the iris following a succussefully treated diffuse large B-cell lymphoma of the orbit

MEYER P, GRAEFFE

Eye Clinic, Basel

Purpose To report clinical findings of a rare case of lymphoma with iris involvement and therapeutic approach with local application of rituximab

Methods Case report

Results $\mathrm{A} \neg \mathrm{n}$ 80-year old patient reported blurred vision in the right eye for one week. After dacryodacryocystorhinostomy he suffured from decreased vision, chemosis and double vision on downgaze. MRI of the head revealed a right orbital tumor mass, arising from a distinct tumor mass of the right maxillary and ethmoid sinuses. Biopsy revealed CD5-positive, diffuse large B-cell lymphoma. The patient was managed with tumor debulking and systemic chemotherapv. At completion of therapy he remained in remission until presentation of a cutaneous relapse on the lower leg with simultaneous eye discomfort.On ocular examination the visual acuity was hand motion in the right eye with normal Intraocular pressure.The right eye showed diffuse keratic precipitates and moderate cells and flare in the anterior chamber.From 8 to 1 oclock the iris showed diffuse thickening of the stroma and irregular pupil.Examination with ultrasound biomicroscopy showed involvement of the anterior part of the cilliary body. Posterior segment examination was unremarkable. Aspiration of the anterior chamber showed CD20 positive, atypical lymphocytes, compatible with the previously diagnosed non-Hodgkin's lymphoma. After local application of rituximab three weeks later the thickening of the iris tissue had resolved.

Conclusion Lymphomatous involvement oft he iris should be considered in the differential diagnosis of a severe anterior uveitis in elderly patients with known aggressive systemic lymphoma. An additional local application of rituximab may be suggested in isolated iris lymphoma. 


\section{- 3651}

Early diagnosis and treatment changes disease phenotype in birdshot retinochoroiditis

HERBORT C $(1,2)$

(1) Centre for Ophthalmic Specialised Care (COS), Lausanne

(2) University of Lausanne, Lausanne

Background and Aim: The diagnosis of birdshot retinochoroiditis (BRC) relies mainly on the presence of oval-shaped "birdshot lesions" (BL) part of the currently used inappropriate diagnostic criteria that do not use diagnostic tools for choroidal disease such as ICG angiography (ICGA) and do not allow to make an early diagnosis. It is now well-known that when BL become apparent, the disease has already been evolving since months or years.Patients and methods: Using appropriate diagnostic criteria, including vitritis and retinal vasculitis in at least one eye, presence of ICGA detected choroiditis in both eyes and the presence of the HLA-A29 antigen, we compared a group of patients treated early ( $<10$ months of symptoms) with a group of patients treated after more than 10 months of symptoms. Mean follow-up was 10 years in bothe groups.Results: Patients treated early almost never developed the characteristic BL, whereas patients treated late all presented BL at start of therapy that almost never disappeared after treatment.Conclusion: Early diagnosis and treatment modifies the phenotype of BRC by preventing the apparition of BL clearly indicating that the presently used diagnostic criteria are completely inappropriate

- 3653

When the clinician has to rely on disease phenotype alone for diagnosis: the example of Behçet's uveitis

TUGAL-TUTKUN I

Istanbul

Behçet's disease (BD) is a complex inflammatory disorder characterized by recurrent inflammatory attacks in multiple organ systems There is no specific diagnostic test. Clinical diagnostic criteria have been developed based on the presence of characteristic ocular and systemic inflammatory manifestations. However, ocular lesions defined in the current sets of diagnostic or classification criteria are nonspecific. It is important to recognize characteristic features of $\mathrm{BD}$ uveitis because there are $\mathrm{BD}$ patients with other forms of uveitis by coincidence, and patients with typical ocular involvement but without systemic manifestations of the disease. The course of the disease is characterized by recurrent nongranulomatous uveitis attacks of variable severity. Transient nature of the acute inflammatory signs is the most important diagnostic clue. Transient retinal infiltrates and inferior peripheral pearl-like precipitates are the pathognomonic findings that help differentiating BD uveitis from other causes of panuveitis and retinal vasculitis.
- 3652

The outcomes of Mycophenolate Mofetil therapy combined with systemic corticosteroids in acute uveitis associated with VogtKoyanagi-Harada Disease

ABU EL ASRARA

Riyadh

To study the effectiveness of mycophenolate mofetil (MMF) combined with systemic corticosteroids in acute uveitis associated with Vogt-Koyanagi-Harada (VKH) disease. The outcomes in this group were compared with those of another group of patients with VKH disease who were treated with corticosteroid monotherapy. Nineteen

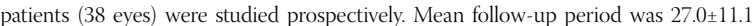
months. Corticosteroid-sparing effect was achieved in all patients. Mean interval between starting treatment and tapering prednisone to $10 \mathrm{mg}$ or less daily was $5.1 \pm 1.2$ months. Ten (53\%) patients discontinued treatment without relapse of inflammation. Mean time observed off of treatment was $17.3 \pm 11.9$ months. Visual acuity of 20/20 was achieved by $38 \%$ of the eves in the corticosteroid group and by $74 \%$ in the corticosteroid + MMF group $(\mathrm{p}<0.001)$. Recurrent inflammation of ${ }^{3} 3$ times was reduced significantly $\mathrm{p}=0.0383$ ) in the corticosteroid $+\mathrm{MMF}$ group (3\%) as compared to corticosteroid group $(18 \%)$. Development of all complications was significantly higher in the corticosteroid group (43\%) compared to the corticosteroid + MMF group ( $8 \%)(\mathrm{p}<0.001)$. None of the eyes in the corticosteroid + MMF group developed "sunset glow fundus".

- 3654

When disease mechanism is more important than disease phenotype: the example of multifocal choroiditis of the choriocapillaritis type

NERI P (1), ARAPI I (2), PIRANI V (2), GRESTI G (2)

(1) Agugliano

(2) The Eye Clinic, Polytechnic University of Marche, Ancona

Inflammatory choriocapillaropathies (ICCP) is the name given to a group of diseases affecting the choroicapillaris. Primary inflammation of the choriocapillaris was suspected long before indocyanine green angiography (ICGA) became routinely available. In fact, fluorescein angiography (FA) is not tool capable to detect choroidal ischemia and does not give imaging access to the rest of the choroid. One of the main characteristics described in such group of diseases is represented by their eclectic attitude. The reason why the disease behaviours are so different from one condition to the other is still unknown. Among this group it is recognized a sub-group of diseases known as multifocal choroiditis. When seen by the clinician most of the cases already show chorioretinal scars, as if preceding silent episodes occur before the disease becomes symptomatic at the time of a recurrence. Multifocal choroiditis occurs in the same age groups as other ICCPs, namely in young to middle aged adults with women being predominantly affected. Lesions tend to leave scars and are not spontaneously reversible but seem to respond corticosteroid therapy. Subretinal neovascular membranes occur in one third of patients. 
118

SIS: The precarious notion of disease phenotype in inflammatory eye conditions

- 3655

Disease phenotypes in emerging infectious ocular conditions: how strongly disease defining are they?

KHAIRALLAHM (1), KAHLOUNR (2), ZAOUALIS (2)

(1) Monastir

(2) Ophthalmology, Monastir

Emergent and resurgent infectious diseases are major causes of systemic morbidity and death that are expanding worldwide mainly because of climate changes and globalization. An array of ocular manifestations, involving mainly the posterior segment, have been recently described in association with specific arthropod vector-borne diseases including rickettsioses, West Nile virus, Rift Valley fever, Dengue fever, and Chikungunva. They include anterior uveitis, retinitis, chorioretinitis, retinal vasculitis and optic nerve involvement. Proper clinical diagnosis of any of these infectious diseases is based on epidemiological data, history, systemic symptoms and signs, and the pattern of ocular involvement. Several fairly typical fundus findings may help establish the diagnosis in specific emergent infectious diseases while serologic testing is pending. 
- 3661

One year results of the follow-up of patients with asymptomatic idiopathic epiretinal membrane

FERRERO A, BONNABEL A, GASCARD L, BRON AM, CREUZOT-GARCHER C Department of Ophthalmology, University Hospital, Dijon, Dijon

Purpose To determine anatomical and functional retinal modifications in patients with idiopathic epiretinal membrane (ERM) without objective symptoms.

Methods This prospective monocentric study included 28 eyes of 28 patients with ERM having a best corrected visual acuity greater than $20 / 25$. All patients underwent a complete ophthalmic examination, including the measurement of the visual acuity on the ETDRS scale, a macular SD-OCT and a microperimetry at 0,6 and 12 months. We assessed macular abnormalities on SD-OCT namely: retinal folds, retrofoveolar deposits, cystoid cavities and external retinal layers alterations.

Results At baseline the mean visual acuity was $85 \pm 4$ letters, the mean macular sensitivity was $14.3 \pm 1.3$ decibels, the mean macular thickness was $357 \pm 32$ micrometers and the score of the SD-OCT retinal abnormalities per patient was $2.5 \pm 0.8$. At one year the score of the SD-OCT retinal abnormalities per patient was significantly higher: $3.3 \pm 1.3(\mathrm{p}=0.007)$. At one year, the mean visual acuity, the mean macular sensitivity and the mean macular thickness were stable at $84 \pm 5$ letters $(\mathrm{p}=0.8), 14.4 \pm 1.7$ decibels $(\mathrm{p}=0.8)$ and $356 \pm 43$ micrometers $(\mathrm{p}=0.3)$, respectively.

Conclusion This study showed an increased number of retinal lesions observed on SD-OCT with a stable visual acuity and a stable retinal sensitivity measured with the microperimetry after one year of follow-up.

- 3663

Automatic identification of eyes at risk of developing idiopathic macular hole

C. SILVA AS (1), FIGUEIRA J (2, 3), LOBO C (2), SIMÃOS (2), MEIRELES A (4) GOMESN (5), BERNARDESR (6)

(1) IBILI, Faculty of Medicine, University of Coimbra, Coimbra

(2) AIBILI, Coimbra

(3) Ophthalmology Service, Coimbra University Hospital Center, Coimbra

(4) Hospital St. António, Porto

(5) Hospital de Braga, Braga

(6) IBILI, Coimbr

Purpose To automatically identify eyes with increased risk of developing idiopathic macular hole (IMH). This is proposed based on the fact that fellow eyes from patients with unilateral IMH have a risk increased by up to $29 \%$ of developing IMH. Our hypothesis is that there are changes in the topography of the macula that can allow the identification of these eyes prior to the development of IMH

Methods High-resolution OCT scans from eyes at risk and control patients are acquired using the Cirrus HD-OCT (Carl Zeiss Meditec, Dubin, CA, USA), Topcon 3D OCT (Topcon Medical Systems, Inc., Oakland, NJ, USA), and Spectralis HRA+OCT (Heidelberg Engineering GmbH, Heidelberg, Germany). From the volumetric scans, topographic maps are built from the segmentation of the inner limiting membrane and the retinal pigment epithelium. Parameters are extracted by fitting the maps to a set of bidimensional functions and from radial profiles of the region of interest. These are used to train a support vector machine (SVM), an automatic classification system. Optimizations to the system are ran in order to achieve the highest accuracy possible.

Results Forty-nine eyes with increased risk of IMH development (age: 67.4+-5.5 yrs) and 44 control eyes (age: $58.5+-10.2$ ) were imaged. The accuracy of the optimized SVM was $82.8 \%$, sensitivity $85.7 \%$, specificity $79.5 \%$, assessed by 10 -fold cross-validation.

Conclusion The achieved results support our working hypothesis - that structura changes are present well before being visible at a regular consultation.

\section{- 3662}

Inner retinal defects exist preoperatively in patients with epiretinal membrane

THEOCHARIS IP

Primevision.gr, Athen

Purpose To investigate the etiology of the inner retinal defects (IRD) that have been detected postoperatively in patients after membrane (ERM) and ILM peeling

Methods A retrospective comparative study based on enface and b scan OCT findings. Twenty healthy individuals with normal maculas served as control group. Twenty patients with unilateral or bilateral ERM that were not operated comprise one group and served as a tool to study the progress of the defects and the bilaterality of the findings. Twenty patients with preoperative and postoperative OCTs to study the changes of the inner retinal layers postoperatively.

Results No defects were found in the posterior pole of the healthy eyes. 16 out 20 patients with unilateral clinically significant ERM present with IRD at the diseased eye in contrast with the absence of IRD at the normal eye or at eyes with early stage of ERM. In operated eyes, preoperatively the IRDs were detected in 19 out of 20 eyes. Postoperatively the defects seem to faint and disappear when significant diffuse retinal edema is present and reappear when the edema is resorbed. IRD may expand postoperatively by time or new areas of defects may appear. A healing process of the inner layers may not be excluded.

Conclusion The IRDs do not result exclusively by the surgical manipulations but the tractional forces during the development of the membrane seem to cause loss of inner retinal layers tissue. Postoperative edema of the posterior pole in early postoperative period may obscure the presence of IRDs

- 3664

Predicting visual outcome after macular hole surgery using fundus - controlled microperimetry

SURGUCH V (1,2), ASATRYANS (3)

1) Vitreoretinal surgery, NIIGB RAMN, Moscow

(2) Chisinau

(3) NII GB RAMN Moscow

Purpose to explore the value of fundus-controlled (micro)perimetry in determining the functional state of macular retina in order to predict the functional results of vitrectomy surgery in patients with idiopathic macular hole (MH).

Methods Design: prospective study of 16 eyes with idiopathic MH (recruited regardless of the size of the holes and visual acuity). Pre- and postoperative examination additionally included microperimetry and fixation assessment performed with Maia microperimeter (CenterVue). Average retinal sensitivity was measured in the area of 1 (foveal) and 3 (parafoveal) degrees from the foveal center

Results Before surgery foveal retinal sensitivity fluctuated overall on group from 7.6 to $26.76 \mathrm{~dB}$ (average $19.04 \mathrm{~dB}$ ), a parafoveal sensitivity - from $16.33 \mathrm{~dB}$ to $29.79 \mathrm{~dB}$ (average $24.83 \mathrm{~dB}$ ). After surgery average foveal sensitivity imroved by $4 \mathrm{~dB}$ (up to $23.09 \mathrm{~dB}$ ), while parafoveal remained nearly unchanged (average - $25.43 \mathrm{~dB}$ ). Based on microperimetric results patients were divided into two groups: patient with rather high initial foveal sensitivity (more than $21 \mathrm{~dB}$, Group A - 9 eyes) and patient with reduced sensitivity (up to $21 \mathrm{~dB}$, Group B - 7 eves). Although both groups were comparable by initial VA $(0,18$ and 0,11$), B C V A$ in the group A improved after surgery in average up to $0.61(0,4-1,0)$, whereas in the group B improvement of BCVA was less significant - in average up to $0.31(0,2-0,5)$. Preoperative fixation stability was significantly higher in the first group ( $78 \%$ compared to $55 \%, \mathrm{P}<0,05)$. Baseline $\mathrm{MH}$ size was slightly larger in group B (min $416 \mathrm{mu}$ vs 342mu; max 984vu vs 650mu in Group A).

Conclusion Preoperative foveal sensitivity correlates with visual outcome in $\mathrm{MH}$ surgery and could be used as a predictive factor. 
- 3665 / F105

Differences in optical coherence tomography findings in early intervention vs late intervention surgically treated submacular hemorrhages

CANASTROM (1), FARIA M (1), MARQUES-NEVES C (2)

MONTEIRO-GRILLO M (2), NETO E (1), PERPETUA C (1), PROENCA H (1)

(1) Ophthalmology Department of Hospital de Santa Maria, Lisbon

(2) Ophthalmology Department of Hospital de Santa Maria, Faculdade de Medicina, Lisbon

Purpose The purpose of this retrospective observational study is to compare Spectral-Domain OCT findings in early-intervention vs late-intervention submacular hemorrages treated surgically with vitrectomy, recombinant tissue Plasminogen Activator (rtPA) subretinal injection and gas injection.

Methods Retrospective analysis of pre and post operative OCT findings of patients with submacular hemorrhages treated with $23 \mathrm{G}$ vitrectomy, rtPA subretinal injection and gas (SF6, C3F8) injection. 2 patients were treated after 6 and 10 days of diagnosis establishment and the other 2 were treated after 21 and 28 days of diagnosis establishment. Subfoveal thickness was measured in both pre and post operative exams. Analysis of the retinal structure and submacular hemorrhage characteristics were performed.

Results The early intervention group presented with higher pre-operative subfoveal thickness (716 and 973 microns compared with 405 and 554 microns). Post operative OCT showed an important decrease in subfoveal thickness in the early intervention group (716 to 260 microns and 973 to 433 microns), not seen in the late intervention group (405 to 593 and 554 to 548 microns). Images analysis shows recent submacular hemorrhages as low density, thick, homogeneous, subretinal tumefactions that do not severely distort retina internal anatomy. Older submacular hemorrhages appear as more dense, thinner, heterogeneous masses, with retinal distortion, cysts formation and separation of the inner and outer layers of the retina.

Conclusion OCT is an important exam in the evaluation of submacular hemorrhage, which may differentiate recent and late hemorrhages. Early intervention is crucial for good post operative anatomical result.
- 3666

Retinal detachment in congenital glaucoma

ZHAOL, WEIB

Beijing Tong Ren Eye Center, Beijing Tong Ren Hospital, Capital Medical University, Beijing

Purpose To investigate the clinical characteristics of retinal detachment in congenital glaucoma and related surgical treatments.

Methods 28 patients(30eyes)with congenital glaucoma suffering from retinal detachment were reviewed retrospectively.

Results 26 of 28 eyes which undergone retinal detachment surgical treatments were anatomic recovered. The postoperative rise of intraocular pressure took place in all eyes. Follow-up was made on 20 patients(21 eyes), retinal detachment was recurrent in 5 eyes. The final visual acuity was increased in 9 eyes, and the intraocular pressure of 14 eyes were normal.

Conclusion The area of retinal detachment and degree of proliferative vitreoretinopathy (PVR) are identified as the two most significant factors affecting functional prognoses. To stabilize the intraocular pressure, a regular examination is mandatory. 
- 3671

Progressive visual loss in Retinitis Pigmentosa leads to cortical and subcortical reorganization of the human brain

PEREIRA AC (1), FERREIRA S (1), QUENDERA B (1), MATEUS C (1), SILVA E (2), CASTELO-BRANCOM(1)

(1) IBILI, Coimbra

(2) CHUC, Coimbra

Purpose Retinitis Pigmentosa (RP) is a retinal disease characterized by photoreceptor degeneration. Usually, symptoms are early onset night blindness followed by progressive peripheral vision loss, with central vision being later affected. Some patients become blind after several years of disease. We studied the consequences of this progressive loss of visual input in the human brain using Magnetic Resonance Imaging.

Methods 13 RP patients (8M, 5F; mean age 47 \pm 13 yrs; range:23-66yrs) and 24 agematched controls (10M, 14F; mean age 44 $\pm 13 \mathrm{yrs}$; range 22-69yrs) were scanned in a $3 \mathrm{~T}$ Siemens scanner. Cortical thickness (CT), surface area (SA) and subcortical volumes were obtained with Freesurfer.

Results Patients' visual field ranged from 22deg to almost blindness. Self-reported symptoms' onset ranged from birth to 45 years of age (mean symptoms' duration: $30 \mathrm{yrs}$; range 6-51). Patients showed decreased $C T$ in pericalcarine $(\mathrm{p}=0.002)$; cuneus $(\mathrm{p}<0.001)$; lingual $(\mathrm{p}=0.02)$ and lateral occipital $(\mathrm{p}=0.007)$ regions and increased $C T$ in $\operatorname{supramarginal}(\mathrm{p}=0.02)$, superior frontal $(\mathrm{p}=0.02)$ and the parahippocampal $(\mathrm{p}=<0.02)$ gyri. Also, cortical SA was decreased only in superior parietal $\operatorname{cortex}(\mathrm{p}<0.009$, $)$ and increased in inferior temporal gyrus $(\mathrm{p}=0.03)$, isthmus cingulate $(\mathrm{p}=0.02)$, banks of superior temporal $\operatorname{sulcus}(\mathrm{p}=0.007)$, pars orbitalis $(\mathrm{p}=0.02)$ and transverse temporal sulcus $(\mathrm{p}=0.002)$. Also, the caudate nucleus was increased in $\mathrm{RP}(\mathrm{p}=0.003)$.

Conclusion We conclude that visual input deprivation in RP leads to a large scale subcortical and cortical reorganization of brain resources, with loss in visual processing areas and changes in multimodal integration areas that may compensate for this sensory input loss.

\section{- 3673}

Compressive chiasmal lesions: optical coherence tomography, ganglion cell and inner plexiform layers and visual field tests

VICENTE A, ANJOS R, COSTA L, VIEIRA L, SANTOS A, FERREIRA J, AMADO D CUNHA JP

Centro Hospitalar Lisboa Central, Lisboa

Purpose The authors present an analysis of the automated macular ganglion cel complex measurements in patients with compressive chasmal lesions.

Methods Observational study of 20 eyes of 20 patients with compressive chiasma lesions followed at the neurophthalmology outpatient clinic. Optical Coherence Tomography (OCT SPECTRALIS - Heidelberg Engineering GmbH) was performed and high-resolution macular scans were obtained. Ganglion cell laver and inne plexiform layer (GCL+IPL) average thickness and retinal nerve fiber layer (RNFL) thickness were also determined. A functional evaluation was performed with Static Computerized Perimetry (Octopus Perimetry", Haag-Streit). Statistical analysis was done with SPSS Statistics. A p value $<0.05$ was considered statistically significant.

Results There was a significant decrease in the RNFL and GCL+IPL thickness when compared with average values from normal population $(\mathrm{p}<0,05)$. Average RNFL and GCL+IPL thickness reduction evaluated by OCT were positively correlated. We identified a positive structural and functional correlation between the tomographic and perimetric evaluations. Nevertheless, 4 of the 20 patients had normal visual fields.

Conclusion Although the changes determined by OCT and perimetry are different, both methods are useful in studying the relationship between structure and function in patients with chiasmal compressive lesions. Structural damage documented by OCT was associated in the majority of the patients with functional damage. This study also emphasizes the importance of the GCL+IPL thickness measurement with OCT Evaluation of RNFL and GCL+IPL with OCT appears to be accurate in predicting functional damage.
- 3672

Incidences of visual disorders on academic difficulties

KOVARSKI C (1), PORTALIER S (1), FAUCHER C (2), CARLU C (3), MIOTTI H (4) ORSSAUDO (3)

(1) Université Lumière Lyon2, Lyon

(2) Université de Montréal, Québec

(3) Hôpital Européen Georges Pompidou, Paris

(4) Boulogne, Boulogne

Purpose Many students underestimate their visual discomfort, although it may have an educational impact. We studied the prevalence of visual disorders among students and compared these results to their academic level.

Methods Between September 2012 and April 2013, four hundred students between fifteen and twenty two years of age responded to a questionnaire followed by a visual screening (refraction and binocular vision) in order to detect any visual anomalies they might be unaware of. Then academic performance from participants was appraised and subjects were reviewed to determine whether wearing appropriate optical correction or taking orthoptic care improved their grades.

Results Methods from multidimensional approaches (principal component analysis) and explanatory approaches (econometrics) were used for data analysis. Results indicate that the questionnaire score is very significant to predict probability of having academic difficulties $(79 \%)$ or vision problems $(89.42 \%)$. Refraction error $(+16.78 \%)$ and accommodation anomalies $(+16.21 \%)$ have effects on academic difficulties and binocular vision disorders are even more disadvantageous (+21.95\%). Moreover, not spontaneously expressing visual discomfort doesn't mean that there are no visual defects.

Conclusion Once controlled by variables known as impacting learning process, a significant proportion of participants academic difficulties are related to vision anomalies. Therefore, screening of vision anomalies among adolescents appears to be necessary, especially if there are academic difficulties. In addition, the questionnaire used in case history seems to be an effective tool in the detection of vision anomalies and should be validated on a larger sample.

- 3674

Macular and retinal degeneration in Alzheimer's disease

CUNHA IP (1), SANTOS A (1), FERREIRA J (1), AMADO D (1), LOURO C (2),

CASTANHEIRA-DINIS A (3), VICENTE A (1)

(1) Serviço de Oftalmologia CHLC, Lisbon

(2) Faculdade d Ciências da Universidade Nova, Lisbon

(3) Centro de Estudos das Ciências da Visao, Lisbon

Purpose To evaluate the differences between the thickness of the separate quadrants at the peripapillary RNFL, thickness and volume of the macula and choroidal thickness in Alzheimer disease ( $\mathrm{AD}$ ) patients compared with a control group.

Methods Transversal case control study in 50 eyes of AD patients and 150 eyes of heathy subjects, measuring the thickness of the separate quadrants at the peripapillary RNFL, thickness and volume of macula with SD OCT (spectral domain optical coherence tomography) and choroidal thickness (CT) with enhanced depth imaging OCT in Alzheimer disease patients compared with a control group.

Results The average thickness of temporal superior quadrants of RNFL in AD patients was $30,5 \pm 8,5 \mu \mathrm{m}$ thinner than control group ( $\mathrm{p}<0,05 \mathrm{t}$ student test). Macular thickness and volume had no statistical differences between $\mathrm{AD}$ patients and heathy subjects. Mean subfoveal choroidal thickness was higher in AD than in control group ( 246,31 $\pm 68,23$ versus $214,31 \pm 72,85 \mu \mathrm{m}(\mathrm{p}<0,05$ t student test $)$

Conclusion This findings suggest that the macular and retinal degeneration may be multifactorial such as retrograde degeneration in retinal changes and ischemic factor in choroidal changes. 
- 3675 / T108

Usefulness of ophthalmology psychophysical test for diagnosis and monitoring support in mild Alzheimer's disease

GARCIA MARTIN ES (1), DE HOZ R (1, 2), ROIAS B (1, 3), RAMIREZ AI (1, 2), SALAZARIJ (1, 2), GIL P (4), YUBERO R (4), TRIVINO A (1,3), RAMIREZIM (1,3)

(1) Instituto de Investigaciones Oftalmológicas Ramón Castroviejo. Universidad Complutense de Madrid, Madrid

(2) Facultad de Optica y Optometría. Universidad Complutense de Madrid, Madrid

(3) Facultad de Medicina. Universidad Complutense de Madrid, Madrid

(4) Servicio de Geriatría. Hospital Clinico San Carlos, Madrid

Purpose Alzheimer's disease (AD) is a neurodegenerative disorder of the centra nervous system characterized by cortical atrophy most pronounced in the media temporal and posterior temporoparietal regions. The goal of the present study was to examine the visual pathway in patients with mild AD (GDS 4) by means of a different psychophysical test, specifically — visual acuity (VA), contrast sensitivity (CS), colour perception and perception digital test (PDT) - and to compare them with an agematched control.

Methods Twenty-three patients with mild AD and 27 controls were examined at Hospital Clínico San Carlos in Madrid (Spain). Subjects underwent a complete ophthalmological examination (VA, refraction, colour test, biomicroscopy, IOP, dilated fundoscopy, and OCT)

Results In comparison with the control group, patients with mild AD presented a significant decrease in the VA, in the CS (for all the spatial frequencies), in the colour perception (unspecific errors blue axis) and PDT ( $<<0.05$ in all instances).

Conclusion In mild AD, there are alterations in the $\mathrm{M}, \mathrm{P}$, and $\mathrm{K}$ visual pathways. The psychophysical ophthalmological tests used in this study could serve as a tool for AD diagnosis and follow-up.(Support: OFTARED (Grant RD12/0034/0002, ISCIII, Spanish Ministry of Science and Innovation)

- 3677 / T104

Visual intelligence in action: A question of balance? Neuroplasticity: recent findings on the visual system for the practitioner

VANDERMEULEN

Clinical neuropsychology, Geleen/Sittard

Purpose In this presentation I will focus on new insights in the balance of neuroplasticity for brain injury and the effects on vision from a neuropsychological point of view. I will also emphasize on the organisation of the visual pathways ( e.g. ventral and dorsal processing stream and the subcortical processing).

Methods From a literature and integrative research point of view of the last ten years we have found that the vision has changed. I will explain that visual processing is not only taken place by the old reponsibility pathways of eyes and the occipital lobes as key regions. Insight on brain injury in recent has changed this meaning. A case will be presented to support this view

Results There is a new insight in processing information of the brain and od pathways will be abandoned. It may very well that it takes place by other sensoric areas such as the thalamo-cortical route. The brain is highly sensitive to adverse environments, such as the visual impairment after brain injury, but it shows also resilience to neurological deficits The visual tracts are no longer seen as the only pathways for sensory information (this is an essential element for ophthamologists and practitionars), because they contains pathways from other areas.

Conclusion It is important for ophthalmologists and practitionars that the old paradigma of the visual system should be abandoned. We have to realize that clinical management on the visual disorders is more complex and that the impact from the newly acquired knowledge affects the diagnosis and intervention: an unbeatable approach for visual processing and daily work.Werner, J.S. \& Chalupa . L. M. (2014). The
- 3676 / T103

Brain structural and functional reorganization due to long term retinal peripheral degeneration in retinitis pigmentosa

FERREIRA S (1), PEREIRA AC (1), QUENDERA B (1), MATEUS C (1), SILVA E (2), CASTELO-BRANCOM(1)

(1) IBILI, Coimbra

(2) CHUC, Coimbra

Purpose Retinitis Pigmentosa (RP) is a rare retinal disease with progressive degeneration of photoreceptors leading to early peripheral and later central visual loss. It provides a model to analyze visual cortex plasticity since onset age ranges from infancy to adulthood. Prior studies have mostly covered structural or functional alterations in the same cohort; hence we aimed to determine the impact of RP on brain function and structure using magnetic resonance imaging.

Methods Brain images of 5 RP subjects (age 37-66 years, RP duration 6-42 years) and 10 matched healthy controls were acquired. Retinotopy was used to delineate visual cortical areas. Participants also performed a 1-back visual task

Results Retinal thickness, visual field, and visual acuity were decreased in RP $(\mathrm{p}<0.01)$. Central visual cortex responses were shifted anteriorly into regions usually representing more peripheral locations ( $\mathrm{p}<0.03$ ). Also, $\mathrm{V} 1$ and $\mathrm{V} 3$ dorsal had higher activation during the task execution $(\mathrm{p}<0.02)$. Moreover, cortical thickness was increased in Brodmann areas 7 and 8 , and decreased in pericalcarine $(\mathrm{p}<0.04)$. Surface area was enlarged in isthmus cingulated, pars orbitalis, and transverse temporal $(\mathrm{p}<0.02)$. Caudate volume was also augmented $(\mathrm{p}=0.01)$.

Conclusion Results suggest brain functional and structural reorganization in RP which is supported by the absence of a silent cortical visual zone, due to unmasking of peripheral responses to central stimuli. Data also suggest top down modulation of attentional higher level regions, and enhanced recruitment of visuospatial orientation mechanisms due to peripheral vision loss. Results are relevant to the development of rehabilitation methods for RP

\section{- 3678 / T112}

Microcystic macular edema in optic nerve atrophy: case series

VOIDE N, BORRUAT F

Department of Ophthalmology, University of Lausanne, Jules Gonin Eye Hospital, Lausanne

Purpose Microcystic macular edema (MME) is a new entity defined as lacunar areas of hyporeflectivity in the retinal inner nuclear layer after severe optic neuropathy (ON). Initially, MME was fortuitously discovered in optic neuritis

Methods From July 2013, we imaged all patients with ON by SD OCT. Retinal nerve fiber layer thickness (RNFLT), macular cube thickness (MCT) and retinal ganglion cell layer thickness (RGCLT) were assessed. Studied parameters included also the delay between onset of $\mathrm{ON}$ and detection of MME and correlation of the visual field defect to the MME topography.

Results Sixteeen patients (19 eyes) exhibited MME. Etiologies of ON were compressive (7), degenerative (4), inflammatory (3), ischemic (1), and hereditary (1). There were 9 women and 7 men, mean age at diagnosis being 34.1 years. Visual acuity ranged from NLP to $12 / 10$ (median 4/10). Mean RNFLT was $61.6 \mu \mathrm{m}[97+/-10 \mu \mathrm{m}]$, mean MCT $271.37 \mu \mathrm{m}[261+/-18 \mu \mathrm{m}]$ and mean RGCLT $57.22 \mu \mathrm{m}[82+/-7 \mu \mathrm{m}]$. The time from diagnosis until MME detection ranged from 4 months to 23 years. A good correlation was found between the zone of retinal atrophy on infrared photography and MME distribution. However, only 4/16 visual field defects correlated to the MME topography. Conclusion MME can occur as early as 4 months after ON (ocular trauma in our series) and can persist as late as 23 years after the onset of $\mathrm{ON}$. MME is not only related to inflammatory $\mathrm{ON}$, as postulated initially, but is a nonspecific manifestation from $\mathrm{ON}$ of any etiology. The precise mechanism leading to MME is unknown but transsynaptic retrograde degeneration is likely. Why only $10 \%$ of patients with ON exhibit MME is unknown, but MME might be a transient phenomenon. Further clinical and experimental studies are needed to answer these questions. 
- 3711

Retinal Oxygen distribution in normal and pathological cases; animal models and humans

\section{POURNARASC}

Genève

The distribution of $\mathrm{PO} 2$ close to the vitreoretinal interface is heterogeneous, found higher near the arteriolar wall. Preretinal and transretinal $\mathrm{PO} 2$ profiles indicate that $\mathrm{O} 2$ diffusion from the arterioles affects the $\mathrm{PO} 2$ in the juxta-arteriolar preretinal region. Both the preretinal and inner retinal $\mathrm{PO} 2$ recorded far from the vessels remain constant in all retinal areas. The oxygen tension (PO2) in the inner half of the retina remains largely unaffected by moderate changes in perfusion pressure. In addition a constant $\mathrm{PO} 2$ is observed during systemic $\mathrm{PaO} 2$ changes induced either by hyperoxia or hypoxia. However, an increase of $\mathrm{PaCO} 2$ (hypercapnia), as well as an intravenous injection of acetazolamide (carbonic anhydrase inhibitor) can both lead to an increase of preretinal $\mathrm{PO} 2$ due to retinal vessels dilatation. In the case of eyes with experimental branch retinal vein occlusion, $\mathrm{PO} 2$ values, within the inner retinal lavers, are indicative of hypoxic conditions, whereas in adjacent unaffected retinal areas the PO2 remain normal. In diabetic patients undergoing vitrectomy, reports have shown a lowered vitreal POg in the affected retinal areas. Occlusion of the retinal circulation renders most of the inner retina anoxic.

\section{- 3713}

\section{Physiology of retinal oxygen extraction}

\section{SCHMIDL D}

Department of Clinical Pharmacology, Medical University of Vienna, Vienna

It has been hypothesized that several ocular diseases such as glaucoma or diabetic retinopathy are associated with impaired ocular blood flow and altered oxygen supply of the retinal tissue. The recent development of new and sophisticated instruments for the non-invasive measurement of retinal oxygen saturation allow now for the investigation of retinal oxygenation in both healthy and disease. In addition, combination of these technologies with non-invasive measurements of retinal blood flow can provide data on oxygen extraction, which in turn allows for the estimation of oxygen consumption of the tissue. This talk aims to summarize our current understanding of the physiological mechanisms regulating the oxygen supply of the ocular tissues. In addition, recently introduced methods for the estimation of oxygen extraction of the retinal tissue with be presented. Finally, the possible clinical applications for these emerging techniques will be discussed.

\section{- 3712}

Assessment of retinal blood oxygen saturation in patients with primary open angle glaucoma

SHAHIDIAM (1, 2), HUDSON C (1, 2), PATEL SR (1), TAYYARI F (1, 2), FLANAGANJG $(1,2)$

(1) Ophthalmology and Vision Sciences, Toronto Western Research Institute, Toronto

(2) School of Optometry and Vision Science, University of Waterloo, Waterloo

Purpose: To investigate retinal SO2 in POAG using a retinal hyperspectral camera (HRC). Method: 9 controls and 15 POAG patients were imaged using a prototype HRC, at wavelengths between 500-610nm. POAG was classified as mild (mean deviation $[M D]_{<-6)}$ or moderate to severe $(M D \geq-6)$. Arteriolar, venular and arterio-venous (AV) $\mathrm{SO} 2$ difference values were compared between the groups and the correlation with MD was assessed. Result: Mean SO2 values were $93 \pm 1.55$ and $55.37 \pm 2.57$ for arterioles and venules respectively in controls and $94.74 \pm 2.75$ and $58.10 \pm 8.85$ in the POAG group. No significant differences were found between the two groups $(\mathrm{p}=0.13$ arterioles, $\mathrm{p}=0.42$ venules). AV difference was also not significantly different $(\mathrm{p}=0.78)$. In patients with $\mathrm{MD} \geq-6$, significantly higher venular $\mathrm{SO} 2(\mathrm{p}=0.005)$ and significantly lower $\mathrm{AV}$ differences $(\mathrm{p}=0.01)$ were found compared to $\mathrm{MD}<-6$ and controls. No differences were found for arteriolar SO2 $(\mathrm{p}=0.31)$. Significant correlations were found between venular $\mathrm{SO} 2$ and $\mathrm{AV}$ differences with $\mathrm{MD}(\mathrm{p}=0.02$ and $\mathrm{p}=0.03)$. Conclusion: Patients with moderate to severe POAG have higher venular $\mathrm{SO} 2$ and lower AV differences indicating a potential association with decreased oxygen extraction from the retinal capillaries.

\section{- 3714}

\section{Retinal oximetry in glaucoma}

MCNAUGHT A

Gloucestershire Eye Unit, Cheltenham

Retinal oximetry is an emerging technology which holds promise across in range of eve disease. Intra-vascular retinal oximetry measurements have been obtained using both dual wavelength, and hyper-spectral techniques. Retinal oximetric abnormalities have been demonstrated in central retinal vein occlusion, branch retinal vein occlusion and diabetic retinopathy. Abnormalities have also been described in glaucoma patients:1) Elevated retinal venous saturation, especially in eyes with reduced visual function, when compared to normal control eyes, possibly secondary to reduced inner retinal oxygen consumption.2) Reduced arterial saturation at the optic nerve head (ONH) in animal models with acutely elevated intra-ocular pressure, possibly related to reduced $\mathrm{ONH}$ perfusion.The elevated venous oxygen saturation found in retinal vessels in eyes with glaucoma damage may represent an objective measure of inner retinal function, and may hold promise as an index of ganglion cell health/remaining functional cell mass, and may therefore allow insights into the impact of therapeutic intervention eg IOP reduction, or neuro-protective agents.The available evidence for a role for retinal oximetry in glaucoma will be presented. 
- 3715

Retinal oximetry in diabetic retinopathy

$B E K T$

Aarhus $C$

Background: Diabetic retinopathy is accompanied by disturbances in retinal blood flow but the consequent changes in oxygenation of the retinal tissue have not been studied in detail. The advent of retinal oximetry for assessing the oxygen saturation in retinal vessels has enabled the study of changes in retinal oxygen saturation during the progression and after treatment of the disease.Methods: 71 patients with proliferative diabetic retinopathy and 149 with diabetic maculopathy were subjected to retinal oximetry of the larger retinal arterioles and venules before, immediately after and three months after retinal photocoagulation.Results: Before treatment the oxygen saturation was significantly increased, both in patients with proliferative diabetic retinopathy and diabetic maculopathy. The oxygen saturation was unchanged immediately after treatment, but had further increased three months after treatment.Conclusion: Disturbances in retinal oxygen saturation in diabetic patients are complex and may be due to changes in blood flow, oxygen extraction and regional variations in blood flow. Retinal photocoagulation in diabetic retinopathy does not exert its effect by normalizing retinal oxygenation

- 3717

The effect of image quality on retinal oximetry

HARDARSONS

Reykjavik

Dual wavelength retinal oximetry has proven to be a useful tool for detecting abnormal retinal vessel oxygen saturation in various diseases. Oximetry images are acquired in a similar fashion as regular fundus images. Oximetry calculations use light intensity measured from oximetry images. A ratio is taken of light intensity at and beside the retinal vessels. This means that the overall illumination of the image has less effect on the result.Despite the ratiometric approach, it is clear from experience and studies on cataract patients that image quality does influence oxygen saturation calculations. The challenge is to estimate or quantify image quality in a reliable manner and to control for image quality in all comparisons between healthy groups and patient groups.

Commercial interest
- 3716

Retinal oximetry in AMD

HEITMAR R

Birmingham

ABSTRACT NOT PROVIDED

- 3718

Retinal oxgen metabolism under visual stimulation of neuronal activity in health and disease

HAMMER M, RAMM L, PETERS S, JENTSCHS, SAUER L, AUGSTENR Jena

Goal: To measure retinal vessel oxygen saturation in rest and under stimulation of neuronal activity by flicker light as well as vascular dilation upon flickerMethods: 18 patients with non-proliferative diabetic retinopathy (mean age: $62.2 \pm 8.3$ years), 41 patients with primary open angle glaucoma $(64.0+12.8$ years) and 40 control subjects $63.6+14$ years) were investigated. Vessel diameters (central retinal arterial and venous equivalents - CRAE and CRVE) and oxygen saturation (SO2, dual - wavelength optical oximetry) were determined before and during luminance flicker stimulation $(12.5 \mathrm{~Hz}$, modulation depth: 1:20) for $90 \mathrm{~s}$. Results: Flicker light increased CRAE, CRVE, and venous $\mathrm{SO} 2$ by $1.42 \% \pm 3.72 \%, 2.80 \%{ }^{*} \pm 2.70 \%$, and $2.03 \%{ }^{*} \pm 2.43 \%$ in diabetic patients as well as $4.98 \%{ }^{*} \pm 6.23 \%, 8.94 \%{ }^{*} \pm 5.26 \%$, and $4.20 \%{ }^{*} \pm 3.71 \%$ in the controls $(*: p<0.05)$. This increase was significantly higher in the controls vs. patients for all parameters (t-test, $\mathrm{p}<0.05$. In glaucoma, the increase of CRVE $(3.7+3.3 \%, \mathrm{p}=0.039)$ and venous $\mathrm{SO} 2(2.482+2.453 \%$, $\mathrm{p}=0.029)$ was significantly lower than in controls.Conclusions: Combining $\mathrm{SO} 2$ and vessel diameter measurements with visual stimulation reveals pathologic alterations in retinal oxygen supply or consumption.

Commercial interest 
- 3721

Vitamins and glaucoma

CORDEIROM

London

Oxidative stress is strongly implicated in glaucoma in different tissues including trabecular meshwork, human Tenon's fibroblasts, and retinal ganglion cells and their axons. An established, naturally occurring anti-oxidant is Vitamin E. Vitamin E is found in phospholipid cell membranes where it prevents lipid peroxidation and membrane damage by acting as a peroxyl radical scavenger. It is able to move through bilipid membranes and can help in enzyme activity modulation. Likewise, it is involved in vesicle transport and cellular trafficking. Alpha tocopherol is perhaps the best known form of Vitamin E, although d- tocopheryl polyethylene glycol 1000 succinate (TPGS), a water soluble form of vitamin $E$, is also well-described being used as a drug delivery vehicle. Vitamin $\mathrm{E}$ has recently been shown to have other roles including regulating signal transduction, gene expression, and acting as redox sensor and is being investigated in neurodegeneration. This talk will discuss the various applications of Vitamin E in glaucoma.

- 3723

\section{Cholesterol and glaucoma}

BRETILLONL

Dijon

The association between cholesterol and glaucoma is still under debate. No real consensus can be drawn based on the effects of statins in the progression of glaucoma. Nevertheless cholesterol must not be restricted to its circulating levels. Indeed, cholesterol is a prominent component of the the retina. One would thus think on how cholesterol is regulated in the retina. Special emphasis would be given on retinal ganglion cells and glial cells, accounting their key roles in the metabolism of cholesterol in the retina and in the course of glaucoma.
- 3722

Fatty acids and glaucoma

ACARN (1), BRONAM (2, 1), CREUZOT-GARCHER C (2, 1), BRETILLONL (1)

(1) Eye and Nutrition Research Group, INRA, UMR CSGA, Dijon

(2) Department of Ophthalmology, University Hospital, Dijon

Retinal cell membranes are known to be rich in ether-linked phospholipids also termed as "plasmalogens". Although their functions remain enigmatic, plasmalogens are considered as reservoirs of polyunsaturated fatty acids (PUFAs). These PUFAs were shown to be released from plasmalogens by specific enzymes under specific conditions in order to play several key functions within cells. Data from our laboratory suggest that the metabolism of plasmalogens and PUFAs may be implicated in the pathophysiology of glaucoma, the leading cause of blindness worldwide. Through several experiments on human patients and rodent models of ocular hypertony, we have shown that the metabolism of plasmalogens and PUFAs is affected in patients with primary open-angle glaucoma (POAG) in a stage-dependent manner, that the modifications in plasmalogen metabolism may start years prior the first clinical symptoms of POAG, and that several combinations of dietary PUFAs could be efficient in preventing retinal glaucomatous damage in rodents.

3724

Possible pros- and cons- of the effects of visual light on the progression of glaucoma

OSBORNENN $(1,2)$

(1) Nuffield Dept.Clinical Neurosciences, Oxford University, Oxford

(2) Fundación de Investigación Oftalmológica, Oviedo

The visual spectrum of light reaching the retina ranges from $400-800 \mathrm{~nm}$. Certain photosensitizers associated with photoreceptors and the retinal pigment epithelium are able to absorb short wave blue light $(400-450 \mathrm{~nm})$ to cause oxidative stress and irreversible damage. Photosensitizers of specific types also exist in mitochondria that are abundant in intraocular ganglion cell axons. Recent studies show that blue light is detrimental to mitochondria while long wave red light $(700-800 \mathrm{~nm})$ has positive effects. Moreover, in vitro studies show blue light to kill cells while red light has beneficial effects on cell survival. We therefore hypothesis that when ganglion cells are stressed, as in glaucoma subjects, then reducing the blue light (with filters) and enhancing the red light components impinging on ganglion cell axons will attenuate the rate of progression. Experimental studies on rats supporting such a notion will be reported. 
- 3731

Current and future use of contact lenses

GRUENERT A

Duesseldorf

This talk gives an overview of different types of contact lenses and their present applications and future perspectives. The advantages and disadvantages of contact lenses will be discussed. Furthermore, we will present our own data regarding correction of infant aphakia and irregular astigmatism following PKP with rigid gaspermeable contact lenses and the use of soft silicone contact lenses as a drug delivery tool.
- 3733

Topical antiangiogenic therapy with Aganirsen eye drops reduces need for transplantation in herpetic keratitis

CURSIEFENC

Köln, Erlangen

This first Phase III study on a topical inhibitor of corneal angiogenesis showed aganirsen eve drops to significantly inhibit corneal neovascularization in patients with keratitis The need for transplantation was significantly reduced in patients with viral keratitis and central neovascularization. Topical application of aganirsen was safe and well-tolerated.
- 3732

Can we predict corneal graft rejection based on proangiogenic cytokines level at the time of transplantation?

DEKARIS I

Special Eye Hospital "Svjetlost", Institute Ruder Bošković, Zagreb

PURPUSE: To evaluate the quantity of vascular endothelial growth factor (VEGF) and its receptors (VEGFR) in the recipient cornea at the time of penetrating keratoplasty (PK).METHODS: Study included 20 eyes scheduled for PK. Quantity of angiogenic growth factors and their receptors: VEGF, sVEGFR1 and sVEGFR3 were analysed using an enzyme-linked immunosorbent assay. Postoperatively patients were followed-up for 3 years. Frequency of corneal graft reaction and degree of corneal neovascularisation were recorded. Donor corneas obtained from cadaver eves were controls $(\mathrm{n}=20)$. RESULTS: Overall quantity of VEGF-A was $287.74 \mathrm{pg} / \mathrm{ml}(\mathrm{SD}=129.181)$ in the inflammatory group, $115.37 \mathrm{pg} / \mathrm{ml}(\mathrm{SD}=105.93)$ in non-inflammatory group and 142.28 $\mathrm{pg} / \mathrm{ml}(\mathrm{SD}=93.081)$ in controls. sVEGFR1 and sVEGFR3 were significantly higher in non-inflammatory as compared to inflammatory group. Eyes with graft reaction had significantly higher VEGF-A (307.4 pg/ml, SD=100.058) as compared to those without graft reaction $(182.8 \mathrm{pg} / \mathrm{ml}, \mathrm{SD}=124.987)$. Statistical analysis was done by $\mathrm{t}$-test. CONCLUSION: Graft reaction occurs more often in corneas with increased VEGF-A. Elevated sVEGFR may act as a sink for VEGF and thus as suppressors of angiogenesis and graft reaction.

3734

Ensuring advanced therapy treatments for ocular surface patients

PONZIND

Venice

Ensuring Advanced Therapy treatments for ocular surface patients.Diego PonzinRecent advances in the fields of eye banking, ophthalmology and regenerative medicine are challenging the traditional activities of eve banks. Autologous serum eye-drops and amniotic membrane transplantation are being offered as complementary or alternative remedies for ocular surface disorders. While penetrating keratoplasty remains the most common procedure for cornea transplantation, advances in corneal surgery have led to the development of lamellar keratoplasty (LK) as a way to replace only the anterior stroma or the posterior stromal/endothelial layers. Progress in femtosecond lasers and microkeratome-based techniques in creating precut endothelial donor tissues are at the basis of the success of lamellar procedures, most of which are performed with tissues pre-cut, pre-stripped or pre-bubbled in the eye banks.Autologous cultured limbal stem cells have been used to restore damaged corneal surfaces in hundreds of patients with limbal stem cell deficiency. Strategies aimed at the regeneration of conjunctival epithelium and corneal endothelium through cell therapy and genetic engineering of $\mathrm{c}$ 
- 3735

Emerging clinical concepts in ocular surface reconstruction

DUA H

Nottingham

ABSTRACT NOT PROVIDED 
- 3741

Physiopathology of neovascular glauoma and preventive Anti VEGF injections

ZOGRAFOS L

Lausanne

Neovascular glaucoma is a major complication after proton therapy of uveal melanomas, and is provoked by vasoproliferative agents produced by the tumor or ischemic retina. Risk factors for the presence of an ischemic retina at presentation are largest tumor diameter (LTD) tumor height and the extent of retinal detachment.In a prospective study the efficacy of preventive anti VEGF treatment was estimated following Proton Beam radiotherapy of uveal Melanomas associated with an ischemic retinal detachment. None of the treated cases and 32\% of the controlled group developped an neovascular glaucoma. The anti VEGF treatment was completed with and extensive laser photocoagulation of the ischemic retina.Anti VEGF treatment associated to retina photocoagulation appears as valuable therapeutic modality in selected cases of uveal Melanomas following Protom Beam radiotherapy.

- 3743

Prevention of neovascular glaucoma by transcleral resection of the uveal melanoma

BECHRAKISN

Department of Ophthalmology, Innsbruck Medical University, Innsbruck

Radiation treatment of large uveal melanomas especially when they are infiltrating the ciliary body is often followed by the development of neovascular glaucoma. The reason for this is associated with the development of large hypoxic retinal areas and also necrosis of the irradiated tumor, that has been defined as "toxic tumor syndrome". Resection of the tumour after successful irradiation is a very helpful way of treating this potential post irradiation complication. Prevention of the development of secondary glaucoma is even better for log-term preservation of visual function. Especially when the tumor is located anteriorly where tumor endoresection is hazardous due to ciliary body hemorrhage, transscleral resection is a very helpful alternative. On the long term patients that have undergone this combined treatment regiment have a larger probability of eye and visual function preservation.
- 3742

Prevention of neovascular glaucoma by endoresection of the uveal melanoma scar after proton beam therapy

CASSOUXN

Paris

The conservative treatment of uveal melanoma by proton beau or plaque irradiation can induce severe side effects such as exudative retinal detachment, hard exudates, neovascular glaucoma. Part of these complications is not due to the irradiation itself but to a tumor necrosis. The scar releases factors not fully discovered (VGEF, TNF...) producing these complications called "toxic tumor syndrome." The goal of the surgery in this case is to remove the necrotic scar and to prevent long term somplications leading to secondary enucleation. The risk of "tumor toxic syndrome" is correlated to initial tumor volume. This surgical technique could be proposed in tumors of more than 8 $\mathrm{mm}$ thick but with a diameter of less than $16 \mathrm{~mm}$, without ciliary body or optic nerve invasion. The surgical technique and the results will be described in this paper

- 3744

Treatment of neovascular glaucoma by Anti VEGF injections and laser

MASCHIC

Nice

Since the 90s, most of the choroidal melanomas have been treated by radiotherapy either with brachytherapy or external beam. Unfortunately, post radiation complications may occur and neovascular glaucoma (GNV) is one the most feared. GNV happens in almost $23 \%$ of the cases at 5 years after irradiation, mostly for large melanomas but also for small ones. It complicates radiation retinopathy and toxic tumoral syndrome.As for diabetic retinopathy, laser photocoagulation can prevent the iris neovascularisation and glaucoma. If high pressure and secondary corneal edema are present, several authors have shown that intra-vitreal or intra cameral injections of anti-VEGF make iris neovessels regress and help the control of intra ocular pressure.In case of toxic tumoral syndrome, laser is ineffective and enucleation has often been necessary for intolerable pain. There is no recognized treatment for toxic tumoral syndrome: anti VEGF agents have shown good results for some patients but recent publications suggest that corticoid injections and surgical treatments may more efficient.GNV is a frequent and feared complication of ocular irradiation and its management requires several tools including aser and anti VEGF agents. 
- 3745

Surgical treatment of glaucoma following irradiation of uveal melanoma

SHARKAWIE

Jules Gonin Eye Hospital, Lausanne

Uveal melanoma treated by proton beam radiotherapy (PBRT) is associated with uncontrolled glaucoma arising from different aetiological mechanisms and often leads to enucleation. This presentation will demonstrate novel surgical approaches aimed at increasing ocular and visual preservation. Glaucoma subtypes include neovascular glaucoma in addition to concurrent pigmentary and uveitic disease. The role of aqueous shunt insertion, glaucoma filtration surgery, anti-VEGF and the limited role of cyclodestructive procedures are outlined. Prevention and treatment of surgical complications are discussed. Results from a large cohort of eyes with glaucoma and melanoma, from a tertiary referral center, are analyzed and the importance of a multidisciplinary approach is examined. 
- 3751

Stem cells in ocular diseases - implications to physiological and pathological angiogenesis

PETROVSKIG

Szeged

ABSTRACT NOT PROVIDED

- 3753

Endogenous production of anti-inflammatory molecules maintaining ocular immune privilege

WALLACE G

Birmingham

ABSTRACT NOT PROVIDED
- 3752

Genetics of keratoconus

WILLOUGHBYC

Liverpool

Keratoconus (MIM 148300), a common bilateral, progressive corneal thinning disorder, is the leading indication for corneal transplantation in the developed world. Despite the visual and social impact of keratoconus, the underlying biochemical processes and pathobiology remain poorly understood. There is strong evidence for a heritable component in the development of keratoconus, but few if any genes had been identified. Recently, the identification of two new keratoconus genes has given insight into the biological processes disrupted in the development of keratoconus. Our group identified mutations in a microRNA (miR184) responsible for keratoconus. MicroRNAs (miRNAs) are small, non-coding RNAs which are important regulators of eukaryotic gene expression. Subsequently, we have identified a significant enrichment of potentially pathologic heterozygous alleles in ZNF469 associated with the development of keratoconus in $12.5 \%$ of keratoconus patients, and highlights ZNF469 as the most significant genetic factor responsible for keratoconus identified to date.

- 3754

New insights into uveal melanoma

KALIRAIH

Liverpool

ABSTRACT NOT PROVIDED 


\section{- 3755}

Update on adenoid cystic carcinomas

VON HOLSTEINS

Copenhagen

Adenoid Cystic Carcinoma of the Lacrimal Gland: MYB Gene Activation, Genomic Imbalances, and Clinical CharacteristicsPurpose: To investigate genetic alterations in lacrimal gland adenoid cystic carcinomas (ACC) in relation to clinical data and survival. Methods: The expression and identity of MYB-NFIB fusion transcripts were studied using RT-PCR and nucleotide sequence analyses. Q-PCR and immunohistochemistry were used to evaluate the expression of MYB and MYB-NFIB target genes. Highresolution array-based comparative genomic hybridization (arrayCGH) and FISH were used to study copy number alterations and MYB rearrangements. Results: The median age of the patients were 43 years and the median time of survival was 8.6 years. The MYB-NFIB fusion was expressed in 7/14 ACCs. All non-ACC tumors were fusionnegative. All 13 ACCs tested stained positive for the MYB protein and for the MYB targets KIT and BCL2, 12 were positive for MYC and CCNE1, and nine for CCNB1 Rearrangements of MYB were detected in 8/13 cases. ArrayCGH analysis revealed recurrent copy number alterations with losses involving 6q23-q27, 12q12-q14.1, 17p13.3-p12 and gains involving 19q12, 19q13.31-qter, 8q24.13-q24.21, 11q12.3-q14.1 and 6q23.3. Neither MYB-NFIB fusion nor any copy number alteration correlated with survival.Conclusions: Lacrimal gland ACCs are genetically and clinically similar to their salivary gland counterparts and MYB-NFIB is a useful biomarker for ACC. Our data also suggests that MYB and its downstream targets are potential therapeutic targets for these tumors. 
- 3761

Viral vectors

BARNARD A

Nuffield Laboratory of Ophthalmology, Oxford

There are a number of ongoing clinical trials that utilise viral vectors to deliver potentially therapeutic genetic material to retinal cells. This talk will look at the major consideration of turning a wildtype virus into a clinical trial vector. These include the packaging capacity, tropism (preference for targeting specific cells/tissue) and immunogenicity of the viral vector. The talk will also discuss strategies for increasing transduction efficiency (e.g. by optimisation of the transgene expression construct) to potentially reduce the amount of viral particles that need to be used.The talk will look at ongoing laboratory research that may lead to improvements in the viral vectors that are available for gene therapy trials and potential ways in which the limitation imposed by the starting virus may be overcome. For example, a dual vector, split transgene strategy may be used to effectively double the packaging capacity of adeno-associated viral (AAV) vectors. As the majority of retinal gene therapy trials so far have used AAV vectors, there will be a bias toward the discussion of this type of virus.

- 3763

\section{Gene therapy for choroideremia}

BARNARD A

Nuffield Laboratory of Ophthalmology, Oxford

PurposeThis talk will cover the development and instigation of a clinical gene therapy trial for choroideremia. It will also outline some early results of the trial, which is currently still ongoing.MethodsDevelopment of the gene therapy vector involved a range of in vitro and in vivo studies, from cell-based protein assays to ERGs in laboratory mice. The trial is an open label dose escalation study, involving two groups treated with different doses of adeno-associated viral vector (AAV2) encoding Rab-escort Protein 1 (REP1). A range of clinical tests are used to assess the safety and efficacy of treatment. ResultsPreclinical results show AAV can successfully produce functional REP1 protein in patient fibroblasts. The vector shows targeting of retinal cells and has good safety and efficacy in animal models. So far in the clinical trial, 6 patients have been treated with the low dose AAV.REP1 vector. Early results show good safety and some encouraging functional effects. ConclusionsA gene therapy trial for choroideremia is ongoing, with some promising early results. This is the first time gene therapy has been tested in people with normal visual acuity and may have implications for other inherited retinal diseases.
- 3762

Gene therapy for RPE65-related Leber congenital amaurosis

LEROY BP

Ghent

PurposeThe talk will focus on an overview of gene therapy trials for RPE65-related Leber congenital amaurosis (LCA), which are currently ongoing or have been finalised. MethodsSystematic review of trials.ResultsCurrently there are 6 trials around the World focusing on gene therapy for RPE65-related Leber congenital amaurosis, with good safety outcomes and considerable success in restoring some visual function. So far, no safety issues have been encountered in these trials, whereas some improvement and/ or stabilization of retinal function is seen. However, progression of disease is not halted entirely.ConclusionsGene therapy trials for RPE65-related Leber congenital amaurosis are safe and somewhat successful in restoring and/or stabilizing retinal function, despite early indications of further progression of disease.
- 3764

\section{Gene therapy for Stargardt disease}

IIDO I (1, 2) MOHAND-SAID S (3), ZEITZ C (3), BARALE PO (3), J-A SAHEL (3)

(1) Centre de Recherche Institut de la Vision, UMR S 968 Inserm, Pari.

(2) Université Pierre et Marie Curie/CHNO des Quinze-Vingts, Paris

(3) Paris

Stargardt disease is the most common cause of macular degeneration and central visual loss in young people. Currently, there is no known effective treatment that can prevent or reverse the vision loss in this disease. The disease is caused by mutations in the photoreceptor-specific $\mathrm{ABCA} 4$ gene cording for a protein involved in the active transport of retinoids from the photoreceptors to the underlying retinal pigment epithelium to initiate the visual cycle. Pre-clinical studies on animal model of Stargardt disease have shown effects on subretinal administration of viral vectors containing the human $\mathrm{ABCA} 4$ gene upon liposfuscin accumulation, the hallmark of the disease, thus providing a proof-of-concept for gene therapy. Indeed, the first-ever gene-based therapy Phase I/II clinical trial for treatment of Stargardt disease, StarGen"', is currently underway (NCT01367444) at the Casey Eye Institute, Oregon Health \& Science University, US and the Centre Hospitalier Nationale d'Ophthalmologie des QuinzeVingts, Paris, France. We will discuss the principle and issues raised by the current trial as well as potential alternative therapies. 
- 3771

Hermenegildo Arruga (1886-1972): an example that the improvement in retinal surgery goes on

$\operatorname{ASCASOFJ}(1,2)$

(1) Department of Ophthalmology, University Clinic Hospital., Zaragoza

(2) Aragon Health Sciences Institute, Zaragoza

Hermenegildo Arruga (1886-1972) was a versatile Spanish ophthalmologist who specialized in retinal surgery. He designed numerous surgical instruments and introduced significant improvements in ophthalmic surgical techniques. Thus, he was among the first ophthalmologists to advocate the intracapsular cataract extraction. Furthermore, he refined several surgical procedures such as dacryocystorhinostomy corneal transplantation, evisceroenucleation and pterygium excision. But, above all, Arruga was fascinated with the retinal detachment surgery. He perfected Gonin's operation and contributed significantly for retinal detachment surgery to be put in place. Later, he adopted the injection of air at the end of retinal surgery. In the 1950s, Arruga simplified the technique of equatorial cerclage using a suture to encircle the equator of the eye. Even though ocular surgery has also developed significantly after Arrugas time, his achievements played a significant role when they were presented and they formed a good foundation for subsequent developments.

- 3773

The invention of the contact lenses $(1888)$

HEITZR

Strasbourg

In March 1888 appeared in the Archiv für Augenheilkunde an article entitled "Eine Contactbrille" submitted by Adolf Eugen Fick, lecturer at the University of Zurich This paper and his English translation, published the same month in the Archives of Ophthalmology as "A Contact-lens", described the first clinical application of a contact lenses.The analyze of Fick's article, of a memo on June 1887 send in a sealed envelope of the Saxon Academy of Science, and of five letters send to Professor Ernst Karl Abbe in Jena between June and October 1887, permit to describe this innovative step in the history of both corneal refractive neutralization and contact lenses.Key-words: Contact lenses, Invention, Adolf Eugen Fick, Year 1888
- 3772

A journey to Berlin, Utrecht, Paris and London in the footsteps of a cosmopolitan ophthalmologist of the second half of the 19th century

KESTELYNP

Gent

Richard Liebreich was born in Prussia in 1830. He studied medicine and graduated at Halle in 1853. He spent several months with Donders in Utrecht to refine his skills in optics and refraction. As a student of Helmholtz he witnessed the invention of the ophthalmoscope and took one of the first instruments to von Graefe's clinic in Berlin. From Berlin Liebreich moved to Paris, where he achieved great succes. After the fall of Napoleon in 1870, Liebreich moved to London, where he became the head of ophthalmology at St. Thomas hospital and medical school. Following several personal attacks in themedical literature, Liebreich returned to Paris, where he gradually retired from medical practice,yet continued a creative life as an artist.Richard Liebreich improved the ophthalmocope,created the first ophthalmoscope utilized for demonstration purposes, devised a binocular corneal microscope which was a forerunner of the slitlamp, was a pioneer in fundus photography, and wrote the first paper on ophthalmic genetics. However, his lasting claim to fame was the creation of the first atlas of ophthalmoscopy

- 3774

Nicolas Claude Fabri de Peiresc (1580-1637) and the lost humanism

GODARD G

Malakoff

Peiresc [1580-1637], councillor in the parliament of Aix-en-Provence (France), exchanged voluminous correspondence with many scholars in Europe, including Galileo and the Lincei. He carried out, with his friend Gassendi, researches on astronomy, geology and anatomy. Peiresc's writings on eye and vision are preserved in an unpublished manuscript of 980 pages, kept in the library of Carpentras (France). They include letters to Luilier, Bourdelot, Gassendi and Schikard, and contain several minutes of dissection of eyes from various animals (eagle, whale, cat, owl, seal and chameleons) carried out in 1634, as well as Peiresc's observations on his own view. Peiresc also undertook experiments that consisted in removing the eve lens or replacing it with glass lenses, so as to establish the course of light rays in the eye under observation. As the images formed on the retina are upside down compared to the original object, Peiresc magined that the retina was not the sensitive organ of vision but acted as a mirror, the image being captured on the back side of the lens. These studies precede Descartes' Dioptrique (1637) and postdate Plempius' Ophthalmographia (1632); they were not published, so they remain until today almost ignored. 
134

SIS: The hidden history of ophthalmology from the 16 to the 20 century

- 3775

Photocoagulation: early pioneers, pitfalls and progress

$\operatorname{GRZYBOWSKIA}(1,2)$

(1) Department of Ophthalmology. Poznan City Hospital, Poznan

(2) Chair of Ophthalmology, University of Warmia and Mazury, Olsztyn

Laser photocoagulation is currently a crucial therapy for numerous retinal diseases Although the effect of solar light on the retina has been known for centuries, the earliest thermal burns in the animals' retinas by using a concave mirror and a convex lens to focus sunlight through dilated pupils of rabbits, were described by Czerny (1867) and Deutschmann (1882). The first experimental photocoagulation of the human retina was performed in 1927 by Maggiore. It has been nearly completely forgotten that in 1945, the Spanish ophthalmologist José Morón had the idea to close retinal tears using a light source through pupil to produce a retinal burn similar to that of diathermy. Finally, the German ophthalmologist Gerd Meyer-Schwickerath conceived the idea of using photocoagulation to make burns in the retina to seal holes and prevent retinal detachment and developed in 1947 a sunlight photocoagulator, an instrument of mirrors and lenses that used sunlight. 


\section{SATURDAY \\ OCTOBER 4 \\ 2014}




\section{- 4211}

Natural history and epidemiology of myopic macular disease

KOURENTIS CHR

Department of Ophthalmology, Vitreoretinal Unit, Red Cross Hospital, Athens

Pathological myopia (PM) is characterized by a refractive error of -6 D and above and/or an axial length of $26.5 \mathrm{~mm}$ and above with concomitant degenerativechanges in the posterior segment of the eye. The prevalence of PM differs between ethnicities. Myopic retinopathy includes a choroidal pallor pattern in the macular area, a posterior pole ectasia, lacquer cracks in Bruch's membrane and posterior staphyloma, focal areas of chorioretinal thinning or atrophy and geographic areas of atrophy and choroidal neovascularisation (CNV).PM is the second commonest cause of choroidal neovascularization $(\mathrm{CNV})$ after age related macular degeneration $(\mathrm{AMD})$ and the most common cause of severe visual impairment in myopic patients younger than 50 years old. CNV occurs in $4 \%$ to $11 \%$ of patients with high myopia, and is the most common vision threatening complication of high myopia. The natural history of untreated myopic CNV is poor with a visual acuity of 20/200 or less. Apart from the macular degeneration there are other causes of visual dysfunction in PM, such as macular hole, retinal detachment associated with macular hole, posterior staphyloma and retinoschisis.

- 4213

Photodynamic therapy outcomes in myopic CNV

XIROU T

Ophthalmic Department, Glyfada

Choroidal Neovascularization (CNV) is a vision threatening complication of pathological myopia. Treatment modalities include thermal laser photocoagulation, surgical management, photodynamic therapy with verteporfin (vPDT) and Vascular endothelial growth factor (VEGF) inhibitors. The Verteporfin in Photodynamic Therapy (VIP) Study Group recommended verteporfin therapy for subfoveal CNV resulting from pathologic myopia based on 2-year results of this randomized clinical trial, although the primary outcome was not statistically significantly in favor of verteporfin therapy at 2 years follow-up. Since anti -VEGFs became available they have been used successfully in the treatment of $\mathrm{CNV}$ and promising results on their short-term efficacy have been reported. Furthermore clinical trials comparing anti-VEGFs with verteporfin therapy demonstrated superior efficacy of anti-VEGFs. The reason to concern an antiVEGFs treatment is arising over their long-term safety and efficacy. Recent reports on long-Term inhibitory effects of PDT on myopic CNV arising at the edge of chorioretinal atrophy, in eves with juxtafoveal neovascular membranes show that PDT remains to play a role in the management of myopic CNV.
- 4212

Diagnostic evaluation for myopic macular disease

KABANAROU S, XIROU T

Retina Department, Red Cross Hospital, Athens

Myopic macular disease (MMD) characterises a spectrum of structural changes of the macula such as epiretinal membranes, posterior tractional detachment, foveoschisis, macular holes, myopic choroidal neovascularisation (mCNV) and chorioretinal atrophy. Review of current literature regarding diagnosis of MMD and an appraisal of older (fluorescein angiographgy and indocyanine green angiography) and modern imaging tools (optical coherence tomography) are being presented.Fundus fluorescein angiography is the gold standard for diagnosis of patients with mCNV and remains an important imaging technique for their follow up. However in the era of spectral domain OCT macular pathology, such as epiretinal membranes, macular holes, foveoschisis, or pathology of the vitreoretinal interface, is better documented and evaluated. The additional use of enhanced depth imaging OCT (EDI-OCT) and recently developed swept-source OCT (SS-OCT) are also employed to evaluate macular pathology further. The diagnostic evaluation of MMD has evolved as the diagnostic tools have become increasingly more sophisticated allowing better visualization and documentation of macular pathology and any alterations occurring after medical and surgical intervention.

- 4214

Surgical management and treatment outcomes of myopic foveoschisis

POURNARASJA

Lausanne

Myopic foveoschisis is relatively uncommon complication of pathological myopia and has been nicely described using OCT examination.Pathophysiology of myopic foveoschisis still remains controversial.It is thought that tractions due to posterior vitreous cortex and epiretinal membrane may lead to foveoschisis formation.In some cases, macular hole or retinal detachment may occur.Surgical indications and timing are still controversial and will be discussed.Pars plana vitrectomy is reported to give good anatomical and functional outcomes. 
$-4215$

Surgical management and treatment outcomes of myopic macular hole retinal detachment

ZAMBARAKJI H

Eye Treatment Centre, Barts Health, London

Pathological myopia (PM) complicated by macular hole retinal detachment (MHRD) may lend itself to surgical correction, however severe axial elongation of the globe, the presence of a posterior staphyloma and atrophy of the RPE and choroid make these cases challenging. Whilst vitrectomy has been the mainstay of treatment, macular buckling (MB) was also developed in order to treat the posterior staphyloma. Macular hole closure rates using OCT monitoring have been reportedly low at 10-72\% and retinal reattachment rates have varied from 40-93\%. The reports of surgical outcomes of $\mathrm{MB}$ for MHRD are limited, but reported retinal reattachment rates are as high as $93 \%$ after primary surgery and $100 \%$ after secondary surgery. Surgical and visual outcomes appear better than those seen with PPV surgery but visual acuity improvements were modest in a long term series with a mean log MAR improvement of 1.80 to 1.00. We carried out a survey of the British and Eire Association of Vitreoretinal Surgeons (BEAVRS) to research the UK practice patterns for the management of MHRD secondary to PM. Anatomical and visual outcomes showed variable success rates. A review of the current literature and results of the BEAVRS will be presented.
- 4216

Anti VEGF treatment in myopic CNV

SOUBRANEG

Paris

ABSTRACT NOT PROVIDED 
- 4221

What is good pressure control

STEVENS A

Gent

The diagnosis of glaucoma is based on morphological and functional parameters and not on IOP level, whereas treatment is centered on adequate IOP control. Good IOP control implies that IOP is brought down to values at which no further damage occurs or to values that slow the rate of progression in order to maintain acceptable visual function during the patient's lifetime. Target pressure for an individual patients will depend on the age of onset of the disease, the amount of damage at diagnosis, the level of untreated IOP, and the rate of progression. The longer the life expectancy, the more severe the functional damage, the lower the untreated IOP and the faster the rate of progression, the lower the target pressure should be and vice versa. An additional number of systemic (blood pressure, family history) and ocular risk factors (disc hemorrhages, PEX, myopia, corneal thickness) should be taken into account. Further follow up will indicate whether the initially chosen target pressure needs adjustment. The determination of target pressure is a dynamic process and from the available evidence it seems that there is a wide gap between preferred practice patterns and real life.

- 4223

Sleep apnoe and perfusion pressure

KIEKENS S, DE GROOT V

Edegem

Glaucomatous disease may progress despite adequate intra ocular pressure (IOP) control. Large population based studies have shown that low ocular perfusion pressure (OPP) is a risk factor for glaucoma prevalence, incidence and progression. OPP can be calculated as the difference between arterial pressure and IOP. Obstructive sleep apnea (OSA) is a systemic condition associated with many eye conditions such as glaucoma. Its systemic implications are modifiable with Continuous positive airway pressure therapy (CPAP). In a population of OSA patients the increase in overnight IOP raise was significantly higher during CPAP therapy. The accompanied dip in nocturnal blood pressure results in a decreased ocular perfusion pressure. Moreover the auto regulatory mechanisms that need to compensate for perfusion pressure changes are affected in OSA by metabolic stresses, hypoxia and hypercapnea. Thus a higher glaucoma prevalence in OSA can be explained by the combination of an increased overnight IOP, decreased OPP and abnormal auto regulatory mechanisms that make the optic nerve more vulnerable for ischemic events.
- 4222

IOP fluctuation and its measurements

POURJAVANS

Zaventem

This SIS is focusing on the different factors which could cause progression of glaucoma despite correct/good IOP control. One of these factors could be the fluctuations in IOP and its measurements.The variations in IOP can be unpredictable and its behavior over the short and long term is still not fully known. These questions are still unanswered: How does IOP fluctuate over 24 hours? Does the fluctuation follow a repeating pattern? how can we assess this variability? And does the fluctuation affect the course of glaucoma progression? During this session we will discuss different theories and expert's opinions in light of our current understanding

4224

Influence of cerebrospinal fluid pressure and its composition around the optic nerve on the development of glaucoma. Overview

DE GROOT V (1), WOSTIYN P (2), VANDAM D (2), AUDENAERT K (3), DE DEYNPP (2)

(1) Ophthalmology, University of Antwerp, Antwerp

(2) Neurochemistry and Behavior, Institute Born-Bunge, University of Antwerp,

Antwerp

(3) Psychiatry, Ghent University Hospital, Ghent

Growing evidence in the literature provides strong support for the concept that cerebrospinal fluid pressure (CSFP) plays a role in the pathogenesis of glaucoma. The CSFP forms the counterbalance to the intraocular pressure (IOP) across the optic disc. The pressure difference across the lamina cribrosa seems to be more important than the IOP on its own. This trans-lamina cribrosa pressure difference depends on the imbalance between the IOP and the CSFP, and their fluctuations. Several studies have demonstrated that this pressure difference is higher in patients with normal tension glaucoma (NTG), providing an explanation for the mystery of NTG. Secondly, the CSF around the optic nerve may have a deficient clearance. Possible compartmentalization of this subarachnoidal space around the optic nerve has been documented. Also changes in general CSF circulatory physiology result in decreased CSF turnover, decreased CSFP and CSF stasis. Given the central role of CSF turnover in the clearance of potentially toxic metabolites from the brain, one might expect that CSF circulatory dysfunction could ultimately result in reduced neurotoxin clearance in the subarachnoid space surrounding the optic nerve and lead to glaucomatous damage. 
- 4231

S.Fyodorov Eye Microsurgery Complex: Past, present and future

MALYUGINB, CHUKRAYOVA

S. Fyodorov Eye Microsurgery Complex, Moscow

Prof. S. Fyodorov is known for his multiple innovations in refractive and cataract surgery fields. He founded Eve Microsurgery Institute in 1980. The main concept was to combine clinical, research, educational and manufacturing activities under one roof. In 1986, Eye Microsurgery Complex was created, headquartered in Moscow with satellite clinics installed in 11 cities. This allowed spreading modern eye surgery technologies, covering all Russian territories and providing high quality eye care. In 2000, after Fyodorov passed away, the Complex started to bear his name. Currently the number of employees is exceeding 4,500 employees. In 2013 all clinics jointly performed over 225,000 eye surgeries. Along with the clinical activities; extensive scientific and educational work is being performed. Wet Labs for teaching ophthalmic surgeries are established in 6 locations including Moscow. Wide range of research activities is focused on the new surgical technologies in cataract and implant surgery, corneal transplantation, laser refractive surgery, glaucoma, pediatric eye care, stem cell research, and many others. Overview of the historical and current activities of the S. Fyodorov Eye Microsurgery Complex will be presented.
- 4233

Local immunocorrection with MMSC-like limbal cells in highrisk keratoplasty patients

TONAEVA H, BORZENOK SA, ONISHENKO NA

Background. Local immunosuppressive therapy resistance in over that $45 \%$ of recipients in high-risk keratoplasty determines search for the new methods of immune reaction suppression by activation of physiological tolerance mechanisms based on the cell technologies. With this in view mesenchymal stem/progenitor limbal cells with phenotype and properties of bone marrow MMSCs are of great interest as they form an outer cell-tissue niche of the eyeball, accomplish local immunity regulation, physiological and reparative corneal regeneration. Purpose. To evaluate cadaveric donor graft survival rate in high-risk recipients by co-transplantation of pre-cultured allogenic limbal transplants. Materials and methods. As a first step limbal transplants taken from the cadaver eye were cultured for 25,0 $\pm 3,0$ days in the medium (BorzenokMoroz). Functional activity of the cells was monitored by the content of pro- and antiinflammatory cytokines in the culture medium by ELISA. Phenotype of the cells after culturing was determined by flow cytometry with a panel of antibodies to MMSCs.A the second (clinical) step penetrating keratoplasties were carried out in 69 high-risk patients. Experimental group (1st) comprised of 36 patients whom cultured limba cells and cadaver corneas were transplanted simultaneously. In the 2nd (control) group $(\mathrm{n}=33)$ standard penetrating keratoplasty was performed. Results. The results of observation during the first 12 months after the surgery showed that in the 1st group transplant survival rate was much higher than in the 2nd group, 31 recipient $(86,1 \%)$ and 23 recipient (69,7\%), respectively. Endothelial cell loss was also much higher in the control group as opposed to the experimental group, $23,8 \pm 1,4 \%$ and $14,1 \pm 0,57 \%$, respectively. Conclusion. Limbal transplants preserve their viability, demonstrate immune-regulatory activity, and contribute to reprogramming of pro-inflammatory to anti-inflammatory local immune response after keratoplasty. Inhibition of local immune reactions by limbal cells co-transplantation contributes to long-term survival rate of the corneal grafts and preserved endothelial cells.
- 4232

Russian KPro - UV crosslinked cornea complex implanation: Techniques and clinical results

MOROZZ, KOVSHUNE, GOLOVINA

Moscow

Penetrating keratoprosthetics of thin irregular corneal leukomas requires several preliminary surgical procedures. To reduce the period of visual rehabilitation and number of operations for this group of patients a new type of surgical treatment, combining keratoplasty and keratoprosthetics, was developed in 1996. This technique was named "K-Pro - cornea" complex transplantation.Methods: At first collagencrosslinking procedure was performed for allogeneic preserved donor cornea according to the Zurich protocol with the use of UV-X-1000 (IROC Innocross, Switzerland) and 1\% riboflavin solution (Dextralink). Further Fedorov-Zuev keratoprothesis was implanted into the modified cornea. Resulting complex was cut out of the corneal-scleral rim with $9,0 \mathrm{~mm}$ trephination blade. Recipients corneal disk of the same diameter was removed and all procedures, necessary for the lens and anterior chamber, were performed before placing "K-Pro - cornea" complex into the bed and fixing it with interrupted sutures. All in all 64 patients with vascularized leukomas were treated with the use of this technique. Observation period was up to 10 vears. Results: Best spectacle-corrected visual acuity (BSCVA) rose from pr. l. certae before surgery to 0,01-1,0 after. Following complications were met in the postoperative period: retroprothesis membrane (99,9\%), aseptic necrosis (39,6\%), K-Pro protrusion (11\%).Conclusions: Developed technique of transplantation of "K-Pro - cornea" complex enabled to reduce the number of main and preliminary surgical procedures and the period of patients visual rehabilitation.Key words: K-Pro, keratoplasty, collagen-crosslinking.

- 4234

Simultaneous penetrating keratoplasty and artificial iris-lens diaphragm implantation

POZDEYEVA N, PASHTAYEVNP

Fyodorov MNTK Eye Microsurgery, Cheboksary

Materials and methods: Forty four eyes of 44 patients with aniridia after penetrating corneal injuries and traumatic post radial keratotomy wound dehiscences have undergone penetrating keratoplasty (PKP) and simultaneous artificial iris-lens diaphragm (ILD) implantation. The average age was 36 years (8-60 years). Time since trauma ranged from 3 months to 17 years. In 27 eves intraocular pressure (IOP) was normal preoperatively, while 17 eyes were diagnosed with glaucoma. IOP was managed medically in 9 of these eyes, and 8 eyes had been operated for glaucoma before PKP + ILD. PKP was performed with the assistance of femtosecond laser in 10 eyes, and 34 eves were operated with the use of Barron vacuum trephine. Five eves had previous successful retinal detachment surgery, and 8 eyes had subtotal vitrectomy for vitreous hemorrhage. Results: Preoperatively mean non-corrected distance visual acuity (NCDVA) was $0.03 \pm 0.04(\mathrm{~m} \pm \mathrm{SD})$, corrected distance visual acuity (CDVA) was 0.04 \pm 0.07 , preoperative corneal astigmatism was $8.3 \pm 5.8$ diopters (D). Twelve months after the surgery mean NCDVA after traditional PKP was $0.08 \pm 0.09$, CDVA was $0.2 \pm 0.19$, corneal astigmatism was $4.7 \pm 3.9 \mathrm{D}$; and after femtolaser-assisted PKP NCDVA was 0.1 \pm 0.12 , CDVA $0.3 \pm 0.28$, corneal astigmatism was $3.9 \pm 3.1$ D.In the group of 8 eves previously operated for glaucoma only 1 eye experienced worsening of IOP control, requiring 2 extra filtering procedures including drainage device implantation. In the glaucoma medical management group of 9 eyes 1 eye required filtering surgery 1 month after PKP + ILD, while four more had to be operated for glaucoma as late as 9 to 24 months.Due to transplant failure cornea had to be regrafted in 6 of 9 patients in the glaucoma medical management group, and only in 2 of 8 patients who were previously operated for glaucoma.Conclusion: In patients after simultaneous penetrating keratoplasty and artificial iris-lens diaphragm implantation surgery corneal transplant failure rate increases with IOP decompensation. Previous glaucoma surgery improves IOP control. 
- 4235

Femtosecond laser-assisted anterior lamellar keratoplasty for keratoconus

PASHTAEVA (1), MALYUGINB (2)

(1) Dept. of the Anterior Segment Optico-Reconstructive and Transplant Surgery, Moscow (2) S. Fyodorov Eye Microsurgery Complex, Moscow

We report the results of several femtosecond (Fs) laser-assisted anterior lamellar keratoplasty modifications including Fs-ALK (11 eyes) and Fs-DALK (34 eyes). The control group consisted of the manual air-viscobubble DALK (35 eves). Optimal Fslaser parameters for corneas trephination and lamellar cuts were studied in the donor cadaver eyes. The quality of lamellar interface after laser dissection was assessed with atomic microscopy. Clinical data collected included the numbers of intra- and postop complications, VA with and without correction, postop astigmatism, corneal hysteresis and corneal resistance factors (ORA), EC loss and graft long-tern survival rates Mean follow-up was $18.6 \pm 5.1$ months. Fs-DALK with optimized cutting algorithm resulted in fastest visual recovery, lesser postop astigmatism and better BCVA. The outcome difference in between the Fs-DALK and manual DALK was borderline. FsALK provided the lowest visual outcomes (BCVA was $0.2 \pm 0.07 @ 12$ months) due to irregularities and light scattering at the donor-recipient interface. 


\section{- 4241}

Eye neoplasms: a bibliometric analysis from 1966 to 2012

MOURIAUX F (1, 2), BOUDRY C (3)

(1) Ophthalmology, Caen

(2) Québec

(3) Normandie University, Caen

Purpose To calculate the growth rate of biomedical literature on eye neoplasms and to assess key journals, authors, and country affiliations.

Methods PubMed was used to search for papers published from 1966 to 2012. Total number of articles per year was fitted to a linear equation as well as an exponential curve. To identify the core journals and predict the number of journals containing articles related to eye neoplasms, Bradford's law was applied. The mean number of publications per year and per author were calculated. For each country, the Gross Domestic Product (GDP) index (publications per 1 billion US dollars of GPD) and the population index (publications per million inhabitants) were calculated.

Results A total of 27943 references were retrieved. The growth in the number of publications showed a linear increase with a yearly average growth rate of $2.08 \%$. Using Bradford's law, 17 core journals were identified. Only 9 authors published more than 5 papers per year. The United States was by far the predominant country in number of publications, followed by Germany and the United Kingdom. However, population and Gross Domestic Product indexes showed that absolute production did not reflect the production per capita nor the economic efficiency

Conclusion This bibliometric study provides data contributing to a better understanding of the eye neoplasm research field.
- 4243

Multiple malignancies in uveal melanoma patients - the LOOC experience

HEUSSEN FM (1), DAMATO B (2), KALIRAI H (3), COUPLAND SE (3), HEIMANNH(1)

(1) Liverpool Ocular Oncology Center, Liverpool

(2) Ocular Oncology Service, University of California, San Francisco

(3) Pathology, Department of Molecular and Clinical Cancer Medicine, University of Liverpool, Liverpool

Purpose To identify the prevalence of multiple primary tumors in a patient cohort with uveal melanoma and describe the cohort's characteristics.

Methods A data query for all cases of uveal melanoma seen at the Liverpool Ocular Oncology Center (LOOC) since 1993 was performed. Based on systemic information only cases with multiple primary tumors were included. Demographic and clinical information data were analyzed.

Results A total of 5140 (2615 males, 50.9\%) patients with uveal melanoma were found, of which $228(4.4 \%)$ were noted to have at least one other primary malignancy. 15 case $(0.6 \%)$ had at least 3 primary tumors recorded. The gender breakdown is: 125 males (54.8\%) and 103 females. Of 125 men, 45 (36\%) had been diagnosed with prostate cancer; 30 with unspecified skin tumors; 8 with systemic lymphoma; 7 with bladder carcinoma; 6 with colon carcinoma; and the remaining patients with less common tumors. Of 103 females, 45 (44\%) had suffered breast carcinoma; 19 had unspecified skin tumours; 7 renal cell carcinoma; 5 lung carcinoma; and the remaining patients, other less common tumor types. 43 of the 125 (34.4\%) males and 39 of the 103 females (37.9\%) were deceased by time of data analysis. The incidence of the commonest tumors in males (e.g. prostate cancer: 45/2615, 1.7\%) and females (e.g. breast cancer: $45 / 2525$, $1.8 \%)$ in this cohort of patients with uveal melanoma did not differ from the incidence of these tumors in the general UK population

Conclusion Although rare, additional malignancies can occur in association with uveal melanoma; therefore, the patient history taking should include this aspect. Aside from demographic and clinical data, genetic testing in these cases with multiple malignancies may yet reveal unique common patterns.
- 4242

BAP1 germline mutations in Finnish uveal melanoma patients

TURUNENJ (1, 2), MARKKINENS (2), WILSKA R (1), MUONA M (2, 3), RAIVIO V (1), TÄLLM (1), LINDHS (2), LEHESJOKI AE (2, 4), KIVELÄ T (1)

(1) Department of Ophthalmology, Helsinki University Central Hospital, Helsinki

(2) Folkhälsan Institute of Genetics, Helsinki

(3) Institute for Molecular Medicine Finland, University of Helsinki, Helsinki

(4) Neuroscience Center and Research Programs Unit, Molecular Neurology, University of Helsinki, Helsinki

Purpose To estimate the frequency of germline mutations in the BRCA-1 associated protein-1 (BAP1) gene that predisposes to a range of cancer types, including uveal melanoma.

Methods In Finland, all uveal melanoma patients are referred to the Ocular Oncology Service, Helsinki University Central Hospital. We collected clinical data and genomic DNA from 146 patients treated between 2011 and 2013. In addition, we identified 12 families each with two uveal melanoma patients. We were able to collect genomic DNA from 14 members of uveal melanoma families. All 17 exons of the BAP1 gene were sequenced in a total of 160 patients.

Results We found two possible dominant mutations: a sporadic mutation in a highly conserved donor splice site after exon 2 and a frameshift insertion mutation in exon 14 in three patients. The latter mutation was found in a mother and son both diagnosed with uveal melanoma. The third patient, who shared the same mutation, is potentially a distant relative of this family. These mutations were not present in 3,325 Finnish controls from the Sisu project (www.sisuproject.fi).

Conclusion BAP1 germline mutations in Finland contribute to uveal melanoma in only $1.4 \%$ of patients ( 2 mutations, 147 patients; $95 \%$ CI 0.2 - 4.8) based on our nonselected sample, excluding second cases in families. The BAP1 syndrome exists but does not seem to be particularly prevalent in Finland in spite that Northern Europe is a high risk region for this cancer

4244

NRAS, BRAF and KIT expression/mutational status, miRNA related profile and clinico-pathologic parameters in pigmented lesions of the conjunctiva

PARROZZANI R (1), FRIZZIERO L (2), PERRINI P (2), ALESSANDRINI L (3), BLANDAMURA S(3) MIDENAE (2 I)

(1) G.B. Bietti Foundation, IRCCS, Roma

(2) Department of Ophthalmology, University of Padova, Padova

(3) Department of Medicine, Surgical Pathology \& Cytopathology Unit; University of Padova, Padova

Purpose To investigate NRAS and BRAF mutations and BRAF immunohistochemical expression in a group of melanocytic conjunctival tumors previously tested for KIT status, and to verify the correlation between KIT/BRAF mutational statuses with miRNAs involved in the RAS-RAF-MAPK pathway.

Methods 85 non-consecutive cases of conjunctival melanocytic proliferation whit no remarkable KIT gene mutations were included. BRAF was analyzed for the V600E mutation, whereas NRAS molecular analysis was conduced for exons 1 and 2. Expression profiling of mature miRNAs was performed with the NCode miRNA qRTPCR method.

Results The nucleic acids amount was suitable for the analyses in 25 cases (29\%). Of these, two melanomas (15\%) presented a NRAS point mutation (Q61K) and other two melanomas (15\%) and one nevus (10\%) resulted V600E BRAF-mutated. The sensitivity and specificity of VE1 antibody in detecting BRAF V600E mutation were, respectively, $100 \%$ and $82 \%$. Expression levels of miR-15b and miR-16 detected in BRAF mutated cases were higher than in wild type cases $(\mathrm{p}=0.05)$. MiR-222 was associated with tumor thickness $(\mathrm{p}=0.01)$ and tumor stage $(\mathrm{p}=0.02)$ whereas miR-15b was related to tumor stage $(\mathrm{p}=0.04)$. Let-7a, miR-15a/b and miR-16 were significantly down-regulated in melanomas and PAMs compared with nevi.

Conclusion VE1 immunohistochemical staining is an accurate, rapid, and costeffective method in selecting cases that should undergo molecular testing. Specific miRNAs have been identified as a potential diagnostic tool to differentiate between benign and malignant melanocytic lesions of the conjunctiva, and as prognostic markers among malignant ones. 
$-4245$

Can we trust the biopsy for prognostication in patients with choroidal malignant melanomas?

KIILGAARD J (1), BAGGERM (2), TOLSTRUP ANDERSENM (2), HEEGAARDS $(3,1)$ KL ARSKOV ANDERSENM (2)

(1) Copenhagen University Eye clinic, Copenhagen

(2) Dept of Clinical Genetics, Copenhagen

(3) Copenhagen University, Eyepathology Inst., Copenhagen

Purpose The purpose was to validate the minimal invasive TransVitreal-RetinoChoridal (TVRC) biopsy as prognostic tool for uveal melanoma.

Methods Thirty-six patients treated for choroidal melanoma at Rigshospitalet, Copenhagen from 1st January 2009 to 31st December 2011 were included. All patients had a minimal invasive TVRC biopsy performed to confirm the diagnosis before the eve was enucleated. Fluorescence in situ hybridization (FISH) for chromosome 1 , 3, 6 and 8 as well as multiplex ligation-dependent probe amplification (MLPA) were performed on all biopsy samples.Paraffin embedded sections of the enucleated eye were then divided into $3 \mathrm{~mm}$ hexagons. Each hexagon were then individually analyzed with MLPA in order to elucidate eventual heterogeneity of chromosomal status of the tumor: The MLPA results from the tumor were then compared to the results from the biopsy.

Results This is an ongoing study and so far results from 6 eves have been obtained. All tumors showed heterogeneity of their chromosomal status. In 5 of 6 tumors more chromosomal abnormalities were found in the vicinity of the optic nerve compared to the periphery. In 4 of 6 tumors there was a complete correspondence between biopsy result and worst chromosomal status of the tumor. The remaining 2 biopsies underestimated the chromosomal changes, but it did not affect the prognostic value.

Conclusion Minimal invasive TVRC biopsy technique for choroidal melanoma seems to be useful for prognostic purposes.
- 4246

SF3B1 and EIF1AX mutations in uveal melanoma: a protective factor, or not?

KILIC E (1), KOOPMANS AE (1,2), YAVUZYIGITOGLUS (1,2), VAARWATERJ (1, 2). VANIJCKEN WWEI(3) PARIDAENS D (4) DE KLEINJEMM (2)

(1) Ophthalmology, Rotterdam

(2) Clinical Genetics, Rotterdam

(3) Centre for Biomics, Rotterdam

(4) Rotterdam Eye Hospital, Rotterdam

Purpose The recent identification of mutations in the splicing factor SF3B1 and translation initiation factor EIF1AX prompted us to investigate the occurrence of these mutations in our uveal melanoma (UM) cohort.

Methods Nineteen tumours were subjected to whole exome sequencing. In addition, the SF3B1 (exon 14) and EIF1AX (exon 1 and 2) mutation status was determined in 95 UM by Sanger sequencing.

Results Mutations in SF3B1 were identified in 26\% of UM and 19\% harboured mutation in either exon 1 or 2 of the EIF1AX gene. SF3B1 and EIF1AX mutations correlated significantly with good prognostic features such as spindle cell type, absence of closed vascular loops, positive BAP1 immunohistochemistry staining and disomy 3. Univariate analyses of EIF1AX mutated cases compared to those without EIF1AX mutations showed a significantly increase in disease-free survival (DFS). Overall, the SF3B1 mutated cases did not show a significant difference in DFS compared to SF3B1 wild types. After stratifying for chromosome 3 status, patients with a disomy $3 \mathrm{UM}$ and a SF3B1 mutation had a worse DFS compared to those with a disomy $3 \mathrm{UM}$ and a wild type SF3B1 $(\mathrm{P}<0.01)$

Conclusion SF3B1 and EIF1AX mutations occur in approximately a quarter of UM. Mutations in these genes associate with favourable prognostic features. In our study, we also reveal that patients with a normal chromosome $3 \mathrm{UM}$ and a SF3B1 mutation have higher risk of developing late metastasis.
- 4247 / S106

Identification and characterization of melanin in choroidal melanoma by electron paramagnetic resonance spectroscopy (EPR)

ROMANOWSKA DIXON B (1), KLONOWSKA A (1), PLONKA P (2), SARNA T (2) (1) Ophthalmological Department of Jagiellonian University, Krakow

(2) Faculty of Biochemistry, Biophysics and Biotechnology, Krakow

Purpose The purpose of this study was to determine the quantity and type of melanin in choroidal melanomas in correlation with patient's clinical characteristics.

Methods Melanoma-containing samples were obtained after enucleation surgery, performed because advanced choroidal melanoma was diagnosed, not qualified to preserving eye therapy.Frozen samples were examined at liquid nitrogen temperature by EPR spectroscopy

Results Examination showed that choroidal melanoma cells had a low eumelanin to pheomelanin index. In most of investigated melanoma-containing samples the presence of pheomelanin was determined. Also strict correlation between patient's clinical characteristics such as skin phototype, iris and hair color and melanin type in tumor were observed. No correlation however were found between histopathological type and quality and quantity of melanin in choroidal melanoma. Macroscopic analysing cases of choroidal melanoma were characterized by wide pigment variations. The presence of tide correlation between tumor color and type of melanin was observed.

Conclusion Pheomelanin fenotype occurred in tumors with low content of pigment Tumors intensively pigmented (black, dark brown) belong to eumelanin tumors.

\section{- 4248 / S104 \\ Optico-reconstructive surgery after blockexcision of iridociliar tumors}

SAAKYANS (1), ANDREEVA T (1), CHENTSOVA E (2)

(1) Ocular Oncology and Radiology, Moscow

(2) Oculoplastic and reconstructive surgery, Moscow

Purpose To estimate the possibilities and indication for optico-reconstructive surgery after blockexcision of iridociliar tumors

Methods 68 patients with iridociliary tumors and complicated cataract were treated. 41 patients underwent combined surgery - block excision with cataract extraction (IOL implantation performed in 37 cases). In 14 of these patients iridectomy was performed using a tunnel corneal incision (in cases of small tumors of iris pupillary margin) followed by simultaneous phacoemulsification $+\mathrm{IOL}$. The other patients underwent cataract extracapsular extraction. Simultaneous operations were carried out only in benign tumors.27 patients underwent delayed cataract extraction after blockexcision ( 9 patients with malignant tumors, 18 patients - benign). In 5 cases surgery were performed with implantation of artificial iris, in 17 cases - with IOL implantation.

Results In all cases we detected increase the visual acuity, depending on the type of surgery and the tumor's sizes.

Conclusion Indications for simultaneous optico-reconstructive surgery are benign tumors, for delayed - even malignant ones. In cases of small benign tumors of iris papillary margin simultaneous surgery could be done using FEC + IOL, for large benign tumors (no more than $1 / 3$ of iris) - with simultaneous cataract extracapsular extraction, if more than $1 / 3$ of iris - delayed surgery with implantation of artificial iris. 
- 4251

How protein aggregations and lens fiber disorder result in nuclear and cortical cataracts

MICHAEL R, BARRAQUER R

Institut Universitari Barraquer, Barcelon

Commonly, lens opacities and cataract are considered protein aggregation disorders Increased light scattering is attributed to larger high-molecular-weight fractions of proteins. The impact on transparency of fiber membranes and their age- and diseaserelated changes is addressed to a lesser extent in the literature In the lens cortex of transparent human lenses, the lens fiber membranes have high refractive indexes compared with the cytoplasm, whereas there is almost no difference in the refractive indexes of membranes and cytoplasm in the lens nucleus. Therefore, in the lens cortex, transparency is obtained by a high spatial order of the lens fiber lattice to compensate for light scattering caused by differences of the refractive indexes. Any disturbance of the ordered lens fiber architecture in the cortex results in light scattering. In typical nuclear cataract there is no major fiber disruption or extracellular debris to explain the opacity. The strong implication is that local fluctuations in crystallin density, due to the formation of insoluble aggregates, are the major cause of nuclear opacification. These fluctuations in density and refractive index, are considered to produce significant light scattering.

$-4253$

Intraocular stray light compared with subjective lens grading scores in normal and cataractous eyes

BARRAQUER R, ALLENDE-MUNIOZ MJ, PINILLA-CORTÉ L, MONTENEGROG, MICHAELR

Barcelona

A European project studying the prevalence of visual function impairment in European drivers (2422 subjects) showed that intraocular stray light correlates with cataract grading scores. Measured intraocular stray light increases continuously with cataract severity, as estimated by the mean of the LOCS score (average of nuclear colour, nuclear opacity, cortical opacity and posterior subcapsular scores divided by four). Mean intraocular stray light for the lowest LOCS score (0.025) is about $1.00 \log [\mathrm{s}]$ and with a LOCS score for severe cataract $(>0.75)$ about $1.44 \log [\mathrm{s}]$. This corresponds to a more than threefold increase in stray light. The lowest levels of intraocular stray light are found in nuclear cataracts $(1.19 \log [\mathrm{s}])$, followed by cortical $(1.20 \log [\mathrm{s}])$ and posterior subcapsular cataract $(1.23 \log [\mathrm{s}])$. Mixed nuclear and cortical cataracts have a mean of $1.30 \log [\mathrm{s}]$ and mixed nuclear, cortical and posterior subcapsular cataract a mean of $1.35 \log [\mathrm{s}]$. A study in patients before lens surgery at the Centro de Oftalmologia Barraquer (218 eyes) did not showed a significant relation between lens grading scores and intraocular stray light values. About $90 \%$ had nuclear cataracts with low to medium severity (LOCS score between 0.2 and 0.6) with a mean stray light of $1.46 \log [\mathrm{s}]$. A few cases had cortical cataracts reaching inside the pupillary area or very minor posterior subcapsular cataracts. All studies showed a large variation of intraocular stray light values for similar cataract scores, ranging from 1.00 to $2.00 \mathrm{log}[\mathrm{s}]$. This probably reflects the theoretical expected important discrepancy between the backscattered light (lens grading) and forward scattered light (measured intraocular stray light).
- 4252

Refractive error changes in cortical, nuclear, and posterior subcapsular cataracts

ELLIOTT D

Bradford School of Optometry \& Vision Science, Bradford

In addition to the well known myopic shift in nuclear cataracts, astigmatic shifts have been reported in cortical cataracts, although posterior subcapsular cataracts typically show no significant refractive error change. These refractive shifts are thought to be due to changes in refractive index that are either diffuse (nuclear) or in isolated sections (cortical). Correction of these refractive changes can be troublesome for the patient as updated spectacles provide magnification changes as well as improved vision and older patients can find it difficult to adapt. Both spherical and astigmatic spectacle magnification changes can have significant effects on the vestibulo-ocular reflex gain and can lead to potentially unsafe adaptive gait on steps and stairs in older patients. Indeed, fully correcting large refractive changes in older, frail patients has been shown to increase the likelihood of falls and a conservative approach to correcting these refractive changes is recommended.

\section{- 4254}

\section{Optics of light scattering in the human eye lens}

VANDENBERG T

Amsterdam

The visual phenomenon of radiation of light around a bright light source ("ciliary corona", glare, quantified as straylight) is known as result of forward light scattering in the eve. The slitlamp image (or the Scheimpflug image) of the human eve lens, results from backward light scattering. Solid physical theory exists for the relation between characteristics of the scattering elements, and the angular and wavelength dependence of the scattered light. Reversely from angular and wavelength dependence predictions for the scattering elements can be derived. Optical bench study of human eve lenses will be presented. Backward scatter is found to contain two components; a wavelength independent and strongly localized component identified as "Wasserspalten", and a Rayleigh type component diffusely localized throughout the lens, originating from the crystalline molecules. The degree of ordering of the crystallines is critical for the size of this component. Forward scatter is dominated by particles of around 1 micrometer size. So, different processes govern forward and backward scatter, explaining why the slit image is not an accurate predictor of forward scatter or straylight.

Commercial interest 
144

SIS: Optical effects of cataracts

$\bullet 4255$

Is it possible to see through a cataractous lens?

ARTAL P

Murcia

Cataract increases light scatter in the lens and reduces the contrast in the retinal images severely degrading vision. The current solution is to perform surgery to remove the natural lens that is substituted by an intraocular lens. This is a successful procedure restoring good quality of vision in most patients. However, in many situations it would be incredible advantageous to actually "see" through a cataractous eye. I would address several options based in the use of spatial and temporal photonics techniques for imaging through the turbid media of the cataractous eye. There are two possible applications: a fundus camera to register images of the retina in patients affected by cataracts and opto-electronics spectacles to restore some vision in cataract patients. 
- 4261

Intravitreal aflibercept for macular oedema due to branch retinal vein occlusion

CLARK WL (1, 2), BROWND (3), STEMPER B (4)

(1) Ophthalmology, University of South Carolina School of Medicine, Columbia

(2) Palmetto Retina Center, West Columbia

(3) Retina Consultants of Houston, Houston

(4) Bayer HealthCare Pharmaceuticals, Berlin

Purpose To evaluate the efficacy and safety of intravitreal aflibercept (IVT-AFL) injection compared with macular grid laser photocoagulation for the treatment of macular oedema secondary to Branch Retinal Vein Occlusion (BRVO).

Methods VIBRANT was a double-masked, Phase 3 trial with a duration of 52 weeks in which patients with macular oedema secondary to BRVO were randomised 1:1 to receive IVT-AFL $2 \mathrm{mg}$ every 4 weeks or grid laser from baseline to Week 20. The primary efficacy endpoint was the proportion of eyes that gained $\geq 15$ letters in bestcorrected visual acuity (BCVA) from baseline at Week 24.

Results The proportion of eyes that gained $\geq 15$ letters from baseline to Week 24 was $52.7 \%$ and $26.7 \%(\mathrm{P}<0.001)$ in the IVT-AFL and laser groups, respectively. The mean improvement in BCVA from baseline to Week 24 was 17.0 and 6.9 letters $(\mathrm{P}<0.0001)$ respectively. The most common ocular adverse events (AEs) in the IVT-AFL and laser group were subconjunctival haemorrhage (19.8\%) and eye pain (5.4\%), respectively There were no cases of intraocular inflammation in either treatment group. One death due to pneumonia and one Anti-Platelet Trialists' Collaboration (APTC)-defined event of nonfatal stroke occurred during the first 24 weeks of study, both in the laser group. Week 52 data will be presented.

Conclusion In this study, monthly IVT-AFL provided statistically significant and clinically meaningful visual benefits which were superior to macular grid laser photocoagulation in eyes with macular oedema due to BRVO over 24 weeks. Compared to IVT-AFL's known profile no new safety signals were observed

Commercial interest
- 4262

Vision-related function in the COPERNICUS and GALILEO trials

PEARCE I (1), LORENZ K (2), WITTRUP-JENSEN K (3)

(1) Royal Liverpool Universtiy Hospital, Liverpool

(2) Johannes Gutenberg-University Mainz, Mainz

(3) Bayer, Berlin

Purpose To examine the impact of intravitreal aflibercept (IVT-AFL) injection on the 25-item National Eye Institute Visual Function Questionnaire (NEI VFQ) in the COPERNICUS and GALILEO trials.

Methods Patients (pts) with macular oedema secondary to central retinal vein occlusion received IVT-AFL 2 mg (IVT-AFL 2q4) or sham monthly for 24 weeks. After Week 24 (W24) in COPERNICUS all pts were eligible to receive IVT-AFL based on visual and anatomical outcomes (IVT-AFL 2q4+PRN, sham+IVT-AFL PRN). In GALILEO, sham-treated pts received sham tx until W52. The NEI VFQ was administered at baseline (BL), W24 and W52. Total and subscale scores were compared between groups. W24 data were integrated and W52 data were analysed by study.

Results Mean change from BL to W24 in total score was greater in the IVT-AFL $2 \mathrm{q} 4+\mathrm{PRN}$ than the sham group (7.3 vs $2.2, \mathrm{p}<0.0001$ ). Mean changes from BL to W24 were greater in the IVT-AFL 2q4+PRN group vs sham for near activities, distance activities, and dependency. In both studies, the mean change from BL to W52 in total score was greater in the IVT-AFL 2q4+PRN than in the sham+IVT-AFL PRN or sham groups (COPERNICUS: 7.9 vs 4.5, $\mathrm{p}=0.0049$, GALILEO: 7.5 vs 5.1, $\mathrm{p}=0.2164$ ). In both studies, mean changes from BL to W52 were greater in the IVT-AFL 2q4+PRN than the sham and sham+IVT-AFL PRN groups for near activities, distance activities, and dependency. The most common ocular SAEs in IVT-AFL2q4+PRN pts were cataract (COPERNICUS) and macular oedema (GALILEO).

Conclusion Pts in the IVT-AFL 2q4+PRN group experienced clinically meaningful mprovements (typically defined as an increase of $\geq 4$ points) from BL to W24 and BL to W52 in total and subscale NEI VFQ scores. Changes with IVT-AFL 2q4+PRN were greater than those in the sham and sham+IVT-AFL PRN groups

Commercial interest

- 4264

A post-market surveillance study on the processing and followup of patients with BRVO and CRVO prescribed Ozurdex

LARSEN L (1), MERKOUDIS N (2), LEABACKR (3)

(1) Department of Ophthalmology, Glostrup

2) Department of Neuroscience/Ophthalmology, Uppsala

(3) Allergan Holdings Ltd, Marlow

Purpose Examine the use in clinical practice of a dexamethasone intravitreal implant (Ozurdex", Allergan, Inc., CA) in treating macular oedema (ME).

Methods Prospective observational study (20 centres in Sweden, Denmark, Norway and Finland). Patients were enrolled if prescribed Ozurdex" $700 \mu \mathrm{g}$ for ME after branch or central retinal vein occlusion (BRVO/CRVO). Baseline examinations included visual acuity (VA), central retinal thickness (CRT) and intraocular pressure (IOP). Efficacy measures were time to retreatment, VA (ETDRS) and CRT (OCT) at weeks 6, 12, 24, 36 and 48. Adverse events (AEs) and discontinuations were recorded.

Results 99 patients (51\% CRVO, 49\% BRVO) were enrolled, mean age 72.9 years. Median follow-up was 403 days (36-609 days), with $45.5 \%, 24.2 \%$ and $24.2 \%$ of participants receiving 1,2 , and 3 injections, respectively $(<10 \%$ received $\geq 4)$. Among retreated patients, $25.3 \%$ received the first retreatment at week 24 . Mean time to first retreatment was 185 days. Median VA and CRT were similar between BRVO and CRVO groups throughout the study. At baseline, median VA was 54.9 letters, median CRT $499 \mu \mathrm{m}$ and median IOP $15 \mathrm{mmHg}$. At week 12, VA increased to 67.5 letters and CRT decreased to $297 \mu \mathrm{m}$. At week 36, these were 60.2 letters for VA and $318 \mu \mathrm{m}$ for CRT. Increased IOP (26.3\%) and cataracts (4.1\%) were the only AEs recorded in $>1 \%$ of patients; endophthalmitis, eye complication, eye pain and vitreous haemorrhage were each reported once.

Conclusion In this non-interventional, open-label study of routine clinical practice, the major short-term outcomes of improved mean VA and reduced mean CRT were comparable to those in clinical trials. Implementation of Ozurdex" was not associated with new or unexpected events.

Commercial interes 
146

EVER Keynote Lecture 3 - Jay NEITZ

- 4311

Lessons learned from gene therapy for color blindness in primates

NEITZJ

Department of Ophthalmology, University of Washington Medical School, Seattle

Color blindness is the most common genetic disorder. The possibility of curing color blindness using gene therapy was explored by adding a third type of cone pigment to dichromatic retinas of squirrel monkeys. This opened a new avenue to explore the requirements for establishing the neural circuits for a new dimension of color sensation. The addition of a third opsin in adults was sufficient to produce trichromatic color vision. Thus, trichromacy can arise from a single addition of a third cone class in a primate and it does not require an early developmental process. This has implications for understanding how our brain processes conscious visual information and it illuminates the opportunities and limits of gene therapy for treating human vision disorders. 
- 4411

Pathophysiology of vitreoretinal interface disorders

POURNARASC

Genève

Pathologic changes at the vitreoretinal interface and vitreomacular tractions lead to phenotypic lesions such as epiretinal membranes (ERMs), idiopathic macular holes, vitreomacular traction syndrome (VMTS) and myopic foveoschisis.In the presence of clinically evident posterior vitreous (PVD), remnants of vitreous collagen fibers and/or cellular proliferations of fibrous astrocytes or glial cells, migrate outward from the retina. Migration occuring on the inner vitreo-retinal interface through the internal limiting membrane(ILM), in the presence of growth factors such as laminin or fibronectin, may contribute to the fibrocellular proliferation at the retinal surface.Most studies regarding idiopathic or diabetic epiretinal membranes or late stages of macular holes, described the presence of fibrocytes or myofibroblasts. More recent studies confirmed those observations by using antibodies against alpha smooth muscle actine (a-SMA) Based on the mechanistic association between a-SMA expression and a tractional force generation, there is little doubt that these cells represented the source of traction during the evolution of those pathologies

- 4413

Enzymatic vitreolysis

LEMER Y

Paris

One year after the official approval of ocriplasmin in the indication of symptomatic vitreo-macular adhesion, the results and potential complications of enzymatic vitreolysis in "real life » are better understood.The best candidates for intravitreal injections seem to be the younger phakic patients with a symptomatic, focal and isolated (i.e. without associated epiretinal membrane) vitreous traction. The vast majority of traction release occurs before the end of the first week after injection. Ocriplasmin allow to close $40 \%$ of the small macular holes with vitreous traction, thus avoiding surgery with intraocular tamponade.The rate of complications is low but a specific one raises some concerns. A few cases of visual impairment, most of the time transitory even if long lasting, have been reported with modifications on OCT and ERG. An enzymatic cleavage of intraretinal laminin is a biologically plausible mechanism. Although rare, the potential complications of ocriplasmin have to be well known to avoid any unjustified risk in its clinical use. The better indications are now defined and to keep a good benefit/ risk ratio, it would not be justified to take any chance by using enzymatic vitreolysis in the less favorable cases.

Commercial interest
- 4412

Assessment and novel classification of vitreoretinal interface disorders

GUALINO $V$

Montauban

Optical coherence tomography has nowdays become essential investigation device in patient evaluation. It has changed our understanding of vitreomacular interface disorders. Principal pathologies include epiretinal membrane, vitreomacular traction syndrome, macular holes, and less commonly myopic foveoschisis. They characteristics will be detailled.Recently, new international classification of vitreomacular adhesion, traction, and macular hole has been proposed considering anatomical oct considerations. According to panel consensus definitions, this classification may help clinician in predicting therapeutical outcomes.

4414

What's new for the surgical management of vitreoretinal interface disorders?

POURNARASJA

Lausanne

The main vitreomacular interface disorders include epiretinal membranes, macular holes, vitreomacular traction syndrome, and less commonly myopic foveoschisis. They are characterized by abnormal mechanical and biochemical interactions between the posterior hyaloid and retinal surface. Based on patient visual impairment and on OCT findings, surgical approach may be considered. Depending on pathophvisiological process, ILM peeling may be discussed. Latest developments of surgical technology for the management of vitreomacular interface disorders will be detailled based on most recent data. 
148

SIS: Vitreoretinal interface disorders

- 4415

Case study

POURNARAS JA

Jules Gonin Eye Hospital, Lausanne

Case Study will be proposed and therapeutical options will be discussed with the panel. 
$-4421$

Orphan drug for acanthamoeba keratitis (ODAK) project: validation of an acanthamoeba polyphaga keratitis model

GUEUDRY J (1), RAZAKANDRAINIBE R (2), HUMBERT C (2), AIFA A (1), FRANCOIS A (3), LE GOFF L (2), FAVENNEC L (2), MURAINE M (1)

(1) Ophthalmology, Rouen

(2) Parasitology, Rouen

(3) Pathology, Rouen

Purpose Acanthamoeba keratitis is a sight-threatening infectious disease.The ODAK project (Orphan Drug for Acanthamoeba Keratitis) is a European research project, the objective of which is to assess safety and efficacy of PHMB (polyhexamethylene biguanide) eyedrops to provide the basis for marketing authorisation. The aim of our study is to develop a rat model of Acanthamoeba polyphaga keratitis suitable for this project.

Methods Sprague-Dawley rats were injected in the left cornea stromal laver with Acanthamoeba polyphaga. A subconjunctival injection of $0.57 \mathrm{mg}$ long-acting betamethasone was administred and clinical examination was performed weekly. One rat was injected with culture media as sham. Corneal scrapings were performed for bacterial and parasitological cultures and real-time PCR analyses. Paraffin-embedded corneas were analysed after hematoxylin-eosin and Schiff periodic acid staining.

Results Clinical infections were observed in $7 / 9$ rats $(78 \%)$. Trophozoites and/or cysts were detected in axenic culture after 1 month in $7 / 9$ rats $(78 \%)$. Acanthamoeba polyphaga DNA was detected in 7/9 rats (78\%) by real-time PCR. No clinical or microbiologic infection was detected in sham. Histological studies detected trophozoites and/or cysts only in $3 / 9$ rats.

Conclusion In this study, a rat model of Acanthamoeba polyphaga keratitis was obtained with good correlation between clinical findings, culture and/or PCR findings. This model was found suitable for assessment of PHMB in animals. The research leading to these results has received funding from the European Union Seventh Framework Programme ([FP7/2007-2013] under grant agreement $n^{\circ} 305661$

- 4423

The use of amniotic membrane homogenate is improved by a retinoid pre-treatment in healing corneal alkali burns, in a mouse model

DANIELE (1, 2), BONNINN (3, 2), BELVILLE C (2, 4), COMPTOUR A (2), BLANCHONL (2) SAPINV V(2) CHIAMBARETTA F $(1,2)$

(1) Ophthalmology, CHU Gabriel Montpied, Clermont-Ferrand

(2) R2D2 EA7281, Medical Biochemistry Laboratory, School of Medicine,Auvergne University, Clermont-Ferrand.

(3) Ophthalmology, Clermont-Ferrand

(4) GreD, Faculté de Médecine, Clermont-Ferrand

Purpose The purpose of this study is to establish the effect of a human amniotic membrane (HAM) homogenate previously cultivated with all-trans retinoic acid (ATRA) on corneal alkali burns,in a mouse model.

Methods 50 CD1 mice with alkali burn were divided in 5 groups: no treatment, saline, amniotic membrane homogenate, DMSO cultivated HAM homogenate or ATRA cultivated HAM homogenate (10-6mol/L for 24 hours). Before instillation of homogenate, the HAM retinoid induction was assessed by PCR quantification of a wellknown retinoid induced gene: RAR $\beta$. Wound healing was first evaluated through slit lamp photographs at days 1,2 and 7 (for corneal re-epithelialization), then histological analysis of ulcer depth was conducted. Finally, immuno-histochemical analysis of MMP9 and VEGF expression were done.

Results We demonstrated that there was no difference at day 2 for our main criterion (corneal re-epithelialization). At day 7 , we found an overall difference (ulcer surface) in the corneal healing between the groups $(p<0,001)$. Significant differences were also found by histological analysis between the untreated group and the HAM+ATRA group, between saline group and the HAM+ATRA group and between the HAM group and HAM+ATRA group. Finally, expressions of MMP9 and VEGF were visually weaker for the groups treated with HAM homogenate.

Conclusion Our results support the hypothesis that the effect of HAM seems to be more biological than mechanical. The use of HAM homogenate potentiated by ATRA already appears as a promising therapeutic alternative without the constraints of the more classical HAM graft. $\bullet 4422$

Topical treatment with a new matrix therapy agent (RGTA, CACICOL-20) improves epithelial wound healing after penetrating keratoplasty

JULIENNE R (1, 2), HAOUASM (1, 2), TRONE MC (1, 2), HEZ (1), CROUZETE (1) THURET G $(1,2,3)$, GAIN P $(1,2)$

(1) Corneal Graft Biology, Engineering and Imaging Laboratory, EA2521, Federative

Institute of Research in Sciences and Health Engineering, Faculty of Medicine, Jean Monnet University, Saint-Etienne

(2) Department of Ophthalmology, University Hospital, Saint-Etienne

(3) Institut Universitaire de France, Bd St Michel, Paris

Purpose Complete epithelial wound healing is a milestone in the early post-operative care after penetrating keratoplasty (PKP). It reduces the infectious risk, allows safe instillation of steroids, and conditions discharge from hospitalization. We assessed a new matrix therapy agent, mimicking heparan sulfates, for the management of post PKP epithelial defects

Methods Prospective, open-label, uncontrolled, single-centre study on 33 consecutive patients (33 eves) who benefited from a $8.25 \mathrm{~mm}$ PKP for all-comers indications. Patients were treated with RGTA eye drops (THEA): one drop in the operating theater immediately after graft, then on alternate days. Follow-up was performed by slit-lamp examination and photography with fluorescein-dye testing repeated every day at a fixed time. Dye area was measured using the freeware ImageJ. Main outcome: time of corneal surface healing

Results All corneas displayed complete healing after a mean 2.7 \pm 1.1 days (median 3, range; 1 to 6). Complete wound healing was obtained at D1 for 15\%, D2 for 33\%, D3 for $88 \%$, D4 for $94 \%$, and D6 for $100 \%$. Area of epithelial defect decreased by a mean $75 \pm 22 \%$ between D1 and D2 ( $n=28)$ corresponding to a wound healing speed of $11.5 \pm 6.5$ $\mathrm{mm} 2 / \mathrm{D}$. There were no systemic or local side effects. For comparison, in the series of allcomers indications of 1003 PKP without RGTA of Borderie, mean wound healing was $4.6+/-13.2$ days (median 2.5 , range 1-210)

Conclusion RGTA seems a potentially useful, noninvasive therapeutic approach in PKP management. A controlled double blind clinical trial vs placebo will be soon conducted to answer this question conclusively.

- 4424

Rho-associated kinase inhibition prevents pathological neovascularization after corneal trauma

SINNAVE D (1), VAN BERGEN T (1), VANDEWALLEE (2), STALMANS I $(2,1)$

(1) Department of Ophthalmology, KU Leuven, Leuven

(2) UZ Leuven, Opthalmology, Leuven

Purpose The aim of this study was to investigate the effect of AMA0526, a selective and locally acting ROCK inhibitor on endothelial cells in vitro and in vivo by using a mouse corneal neovascularization (NV) model.

Methods In vitro, the effect of AMA0526 $(0.1 ; 1$ and $10 \mu \mathrm{M})$ on endothelial cell proliferation and FBS-stimulated migration was investigated. A mouse corneal micropocket (MCM) model was used to study the in vivo effect of the ROCK inhibitor on corneal NV. A bFGF pellet was implanted in both eyes and topical administration was applied once daily using AMA0526 (0.1\%) in one eye and vehicle (PEG/H2O) in the contralateral eye. Outcome was investigated by analysing vessel length, clock hours and NV area 7 days post implantation. Histological outcome was evaluated by immunohistochemical staining for inflammation (CD45) and angiogenesis (CD31).

Results HBMEC and HUVEC proliferation was significantly inhibited in a dosedependent manner by ROCK inhibition. AMA0526 $(1$ and $10 \mu \mathrm{M})$ also induced significant reduction of FBS-stimulated endothelial cell migration, respectively by $29 \%$ and $40 \%$. In the MCM model, AMA0526 treatment significantly reduced NV area by $28 \%$ and vessel length by $28 \%$, as compared to vehicle treated. These effects were associated with a decreased infiltration of inflammatory cells ( $\mathrm{P}<0.05 ; 32 \%$ reduction) and a reduced blood vessel density ( $\mathrm{P}<0.05 ; 40 \%$ reduction) at day 7 .

Conclusion AMA0526 inhibits endothelial cell proliferation and migration in vitro and is efficacious in reducing corneal NV after bFGF micropocket implantation. These results indicate that ROCK inhibition by AMA0526 may have therapeutic value for the treatment of pathological corneal $\mathrm{NV}$. 
- 4425 / S065

Cytological features of inflammatory cells infiltration indicates the etiology of infectious keratitis

SMEDOWSKIA (1), TARNAWSKA D (1,2), WYLEGALA E (1)

(1) Clinical Department of Ophthalmology, Faculty of Medicine and Department of Dentistry in Zabrze, Medical University of Silesia, Katowice

(2) Department of Biophysics and Molecular Physics, Institute of Physics, University of Silesia, Katowice

Purpose To analyze histological findings of infectious keratitis in corneal epithelium including inflammatory cells infiltrations.

Methods We performed in vivo confocal microscopy (HRT III, Rostock Corne Module) in 190 patients with diagnosed infectious keratitis of various etiology - 72 viral, 66 bacterial, 34 fungal, 18 amoebal keratitis. For corneal scans we used 400x lens and 63x magnification. Microscopic analysis included inflammatory cells infiltration - number size and shape of cells; superficial corneal nerve plexus (number and characteristics of nerve fibers) and cytology of epithelial cells. Described features were compared between groups.

Results Inflammatory cells densities showed no differences between groups, however there was clearly different ratio of round/dendritic inflammatory cells, respectively 0.07 . 8.0, 1.0, 1.2 for viral, bacterial, fungal and amoebal keratitis (U-Mann Whitney test, $\mathrm{P}<0.05)$. Additionally inflammatory cells presented various stage of polimorphism and polimegatism depended on keratitis type. Differences were observed also in nerve fiber numbers (U-Mann Whitney test, $\mathrm{P}<0.05$ ) and epithelial layer architecture.

Conclusion In vivo analysis of corneal epithelial histology shows high specificity and sensitivity in infectious keratitis diagnosis. Correct diagnosis of infectious keratitis is often difficult. In vivo microscopic analysis of both epithelial layer and cytology of inflammatory infiltration might provide fast and highly specific differentiation of keratitis etiology
- 4426 / S066

Anti-glaucoma drugs and their additives, testing the influence of excipients on the corneal healing process in the EX-Vivo Eye Irritation Test (EVEIT) system

DUTESCU M, PINHEIRO R, PANFIL C, SCHRAGEN ACTO e.V. RWTH University, Aachen

Purpose This study examined the impact of anti-glaucoma medications and their additives on the corneal healing process and corneal toxicity.

Methods BAK-free PG analogues (Lumigan UD, Monoprost, Taflotan Sine), soft preserved Travatan BAK free, as well as polyquaternium-1 (0.001\%) and Macrogolglycerolhydroxystearate 40 (5\%) were tested regarding corneal irritability and effect on corneal healing against HYLO COMOD $(1 \mathrm{mg} / \mathrm{mL}$ sodium hyaluronate) as positive and $0.02 \%$ benzalkonium chloride (BAK) as negative control. Formulas were applied over three days, six times daily on rabbit corneas cultured on an artificial anterior chamber the Ex Vivo Eye Irritation Test (EVEIT) system. Initially, four corneal abrasions $(2.5-4.5 \mathrm{~mm} 2)$ were induced. All defects were monitored by fluorescein stains and photographs.

Results For BAK, the corneal erosion size increased from $14.16 \mathrm{~mm}^{2}$ to 88.89 $\mathrm{mm}^{2}$. A delay of corneal healing is evident for Monoprost $\left(14.84 \mathrm{~mm}^{2}\right.$ to $\left.9.45 \mathrm{~mm}^{2}\right)$ and Macrogolglycerolhydroxystearate $40\left(11.58 \mathrm{~mm}^{2}\right.$ to $\left.1.56 \mathrm{~mm}^{2}\right)$ in comparison to a complete healing for HYLO COMOD, Lumigan, Taflotan Sine, Travatan and polyquaternium-1. For both Monoprost and macrogolglycerolhydroxystearate 40, a bright opaque halo around the remaining erosion area could be noticed. Histology revealed severe alterations of the superficia stroma. Cornea tested without erosions show no corneal toxicity of macrogol 4000

Conclusion Macrogolglycerolhydroxystearate 40, an additive found in Monoprost" shows corneal toxicity solely in case of corneal erosions. This excipient is a known skin irritant. Therefore, the use of Monoprost" in patients with epithelial defects should be questioned.

Commercial interest

\section{- 4428 / S068}

- 4427 / S067

$3 \mathrm{D}$ reconstruction of corneal endothelial cell shape: new insight in structure-function relationships

HEZ (1), BERNARD A (1), DEFOE D (2), PISELLIS (1), ACQUART S (3) FOREST F (1, 4), DUMOLLARD JM $(1,4), \operatorname{PEOCHM}(1,4), \operatorname{GAIN} P(1)$, THURET G $(1,5)$

(1) Corneal Graft Biology, Engineering and Imaging Laboratory, EA2521, Federative Institute of Research in Sciences and Health Engineering, Faculty of Medicine, Jean Monnet University, Saint-Etienne

(2) Department of Biomedical Sciences, James H Quillen College of Medicine, East Tennessee State University, Johnson City

(3) Eye Bank, French Blood Centre, Saint-Etienne

(4) Department of Pathology, University Hospital, Saint-Etienne

(5) Institut Universitaire de France, Bd St Michel, Paris

Purpose Corneal endothelial cells (CECs) support the pump-leak theory explaining the maintenance of corneal transparency. CEC are highly polarized with a pole facing aqueous humor having an hexagonal shape bordered by apical tight-junctions that control permeability. The interdigitated shape of basolateral membranes, described in the late 70's, are much less known although they bear most of the pumping sites. Aim: to revisit the shape of $\mathrm{CEC}$ with modern microscopy and 3D reconstruction.

Methods Optimized methods of immunolocalization (fixation and antigen retrieval) were applied on flat mounted whole cornea (fresh or after organ culture). The subcellular structures were distinguished by visualizing apical pole with actin, myosin and ZO 1; lateral membranes with NCAM (CD56), connexion 43, vimentin and tubulin, and the basal pole with integrin. Stacks of high magnification images were acquired with a confocal microscope with a $150 \mathrm{~nm}$ pitch. 3D reconstruction was performed with imageJ using convolve and Gaussian blur filters, and a model was defined.

Results A 3D map of all markers was obtained. In particular, CD56 perfectly outlined the basolateral membranes of $C E C$. The $3 \mathrm{D}$ model highlighted the dramatic increase in membrane surface due to basolateral expansions.

Conclusion CD56 staining pattern and especially the presence of numerous interdigitations should be considered as a hallmark for functional CEC and their study should therefore be integrated in quality controls of CEC production for regenerative medicine processes. Modifications of this 3D structure should also be studied in endothelial diseases. Grant: IFRESIS, St-ETIENNE METROPOLE

\section{Terrien's marginal degeneration: Nordic collaborative study}

KURKINENM (1), DROLSUM L (2), FAGERHOLM P (3), HJORTDAL J (4) HOLOPAINENJ (5)

(1) Helsinki University Hospital, Helsink

(2) Ullevål University Hospital, Oslo

(3) Linköping University, Department of Clinical and Experimental MedicineOphtalmology, Linköping

(4) Aarhus University Hospital, Aarhus Universitet, Aarhus

(5) Helsinki Eye Lab, University of Helsinki, Helsinki

Purpose The objective of our study is to describe the clinical andimaging features of Terrien's marginal degeneration (TMD) andsubsequently to define the clinical diagnostic criteria for TMD.Lastly we aim to detect the natural course of this disease.

Methods Our study design is cross-sectional and includes TMD patientsdiagnosed in Helsinki University Eye Hospital, Aarhus UniversityHospital, Sahlgrenska University Hospital and Oslo University Hospitalby corneal specialists. Analysis include gender distribution, age,symptoms, as well as clinical, topographical and imaging findings. Theanalysis is based on descriptive variables due the limited sample size

Results We present here clinical findings of Finnish TMD patients. Allpatients were asymptomatic and did not suffer from any systemicautoimmune diseases. Topographical analysis showed high astigmatismdue to severe corneal thinning as evidenced by optical coherencetomography imaging.

Conclusion TMD is a bilateral corneal disease. Patients are commonlyasymptomatic and visit an ophthalmologist because of deterioratedvision due to increasing astigmatism. TMD is thus diagnosed bycoincidence. TMD does not seem to be associated with any systemicautoimmune diseases and it is commonly non-progressing. 
- 4431

Keratoprosthesis. Personal experience

BARRAQUERJ

Barraquer Eye Center, Barcelona

Purpose Comment the Barcelona experience with different types of keratoprostheses Methods Six cases of different keratoprostheses implanted at the Barraquer Eye Center in Barcelona from 1955 to 2014 are discussed.

Results The first keratoprosthesis implanted in Spain was a Dorzee acrylic keratoprosthesis in a case of bilateral chemical burn in 1955 with good functional and anatomic result for five years, when extrusion ocurred with simultaneous superior retinal detachment. The second case refers implantation of a Dorzee-BarraquerCardona acrylic keratoprosthesis in 1958 in a patient with terminal glaucoma. There was good anatomical retention until the patient's death in 1970. In the third case a Cardona keratoprosthesis was implanted in 1960 after severe failure of two corneal transplants. Acute endophthalmitis ocurred after a satisfactory anatomical and functional result for 8 years. The fourth case shows extrusion of a Teflon-supported keratoprosthesis (Girard). The fifth case discusses implantation of an osteo-odonto-keratoprosthesis (Strampelli)after blast injury with good anatomical and functional result for 10 years The last case refers to the experience with the Boston keratoprosthesis in our Center since 2006.

Conclusion Clinical experience for more than 50 years at the Barraquer Eye Center confirms evolution of the original Dorzee-Keratoprosthesis to the nowadays widely used Boston keratoprosthesis. In spite of progress in surgical technique, design and postoperative treatment, anatomical and functional results continue being a challenge for KPro surgeons due to the relatively high frequency of unresolvable serious sightdestructive complications

- 4433

Indian MOOKP results

IYER G

Cornea Services, Chennai

MOOKP is indicated for bilateral end stage ocular surface disorders. Initiated in 2003, for the first time in India at Sankara Nethralaya, Chennai, the center has so far performed 90 cases of MOOKP. Results of 85 eyes of 82 patients will be described. The two most common indications were chemical injuries and SIS. The anatomical and functional results of MOOKP over a 10 year period will be discussed. Specific focus on lamina resorption and means to combat the same will be the highlight of the talk The nuances and surgical specifics of combined glaucoma and vitreoretinal procedures in eyes undergoing the MOOKP procedure will be focused upon. Modifications in the technique, issues in eyes with SJS - a more common indication in our series and complications of the procedure will be discussed.
- 4432

UK results for the OOKP

LIU CSC (1, 2, 3), AVADHANAM VS (1), HEROLD J (4), BUSUTTIL A (1), THORP TAS (1)

(1) Sussex Eye Hospital, Brighton

(2) Brighton and Sussex Medical School, Brighton

(3) Tongdean Eye Clinic, Hove

(4) Royal Sussex County Hospital, Brighton

The Osteo-odonto-keratoprosthesis (OOKP) is a time tested procedure. It is the only KPro with a proven long term track record. There has been a steady improvement in patient selection, surgical techniques, optical cylinder design, and perioperative management protocol, which have resulted in improved outcomes. We aim to present the visual, retention, and complications results from the UK national cohort of OOKP patients.

4434

Singapore results for OOKP and superfunctional OOKP implants

MEHTA J (1), TAN X (2), TAND (1)

(1) Cornea, Singapore National Eye Centre, Singapore

(2) Tissue Engineering and Stem Cell, Singapore Eye Research Institute, Singapore

Purpose: To describe the outcome of OOKP in Singapore and improvements in outcomes

Method: Retrospective Case Review and comparison to the literatureResults: We have performed 40 cases over the last decade. The median visual acuity postop was $6 / 18$. The most common complications postop were glaucoma, mucosal ulceration and bone resorption. Titanium is a possible replacement for the tooth in OOKP. Functionalisation of the surface with antimicrobial peptide has been shown to reduce infective rates both in vitro and in vivoConclusion: Our OOKP program has promising results, however, development of an artificial haptic instead of a tooth that can be functionalized may improve outcome even further. 
$-4435$

The Boston type 1 keratoprosthesis and endophthalmitis: a systematic review

COLEST (1), AVADHANAM VS (2, 1), LIU CSC (2, 1, 3)

(1) Brighton and Sussex Medical School, Brighton

(2) Sussex Eye Hospital, Brighton

(3) Tongdean Eye Clinic, Hove

Substituting the natural cornea is no easy task. Researchers have shaped and honed methods for over 200 years, seeking the ultimate surrogate. Today the Boston type 1 keratoprosthesis is the world's most commonly used artificial cornea. Advances in both surgical technique and postoperative management have drastically reduced the prevalence of endophthalmitis.The authors conducted a systematic review of endophthalmitis arising from the Boston type 1 technique. Medline, Embase, Google Scholar and The Cochrane Library were systematically searched alongside grey literature sources for relevant studies. Many methods are currently employed to reduce complications including vancomvcin prophylaxis and the use of bandage contact lenses. The results will be presented with a view to collate the research conducted to date
- 4436

Challenges to globalizing keratoprothesis surgery

CHODOSHJ

Mass. Eye and Ear Infirmary - Harvard Medical School, Boston

This presentation will address the current status of keratoprosthesis globalization, world-wide, with special attention to use in less economically developed countries. The author will review the existing literature, and discuss obstacles and opportunities encountered in implementation of keratoprosthesis surgery in blind, indigent patients, world-wide. Implantation of keratoprostheses, including the modified osteo-odonto keratoprosthesis and the Boston keratoprosthesis, is increasing internationally, and in particular, in less economically developed nations with large proportions of indigent, corneal blind patients. Based on the published literature, specific complications, such as device extrusion and infectious endophthalmitis, may be more common in these settings. Increased implantation of keratoprosthesis use in less economically developed countries will bring new challenges. We can expect increased reports of severe complications after keratoprosthesis implantation, particularly in the poorest patients. New designs and approaches are needed before keratoprosthesis implantation can be fully globalized. 


\section{- 4441}

Incidense of iris melanomas in Denmark from 1942-2013

JØRGENSENA (1, 2), WADT K (1), HEEGAARD S (3), PRAUSE J (3), KIILGAARD J (2)

(1) Department of clinical genetics, Copenhagen

(2) Department of Ophthalmology, Copenhagen

(3) Eye Pathology Institute, Copenhagen

Purpose To estimate the incidence of iris melanoma in Denmark from 1942-2013 as well as the age distribution of the patients and specific location of the tumor.

Methods Retrospective, nonrandomized clinical case series including all patients with a clincal diagnosis of iris melanoma over 71 years. For all patients age, sex and specific location were registered.

Results From 1942-2013 a total of 389 cases were registered and 353 cases were treated (173 males, 116 females). This means an average of 5.6 cases/year in an average population of 5 million. A significant increase in the number of cases was found during the study period. The mean age at presentation of the surgically removed iris melanoma was 56 years (range 7 - 92). There were $11(3,12 \%)$ tumours in children and teenagers ( $\leq 20$ years), $58(16,43 \%)$ in young adults (21-40 years), 117 (33,14\%) in mid adults (4160 years) and $167(47,30 \%)$ in seniors ( $>61$ years). Location was described in 167 cases with 143 cases $(85 \%)$ inferior and $24(15 \%)$ superior in the iris.

Conclusion An increase in the incidence of iris melanomas in Denmark has been observed. In $85 \%$ of all cases the iris melanoma was located in the inferior part of the iris.

\section{- 4443}

Tumour regression pattern according to tumour shape after brachyther-apy for $\mathbf{3 3 0}$ uveal melanomas

MAMUNUR R, KIVELÄ T

Helsinki University Central Hospital, Department of Ophthalmology, Helsinki

Purpose To describe regression patterns and their relationship with tumour shape of uveal melanoma by thickness and cross-sectional area.

Methods A retrospective study of 330 consecutive ciliochoroidal melanomas treated with I or Ru plaques in 2000-2008 and imaged with Innovative Imaging I3-ultrasound. Tumours were measured with Olympus DP-Soft from original digital images. The COMS shape groups were partially collapsed: group 1: flat and crescent; group 2: oval and dome; group 3: mushroom; group 4: lobulated; and group 5: other or undetermined. Regression patterns were adapted from Abramson et al. (1990) into 4 main types: D (net decrease), $\mathrm{S}$ (stable), I (net increase), and M (for miscellaneous). We further subdivided $M$ into 5 subtypes: $\mathrm{DI}(\mathrm{D}>\mathrm{I}), \mathrm{DS}(\mathrm{D}>\mathrm{S}), \mathrm{ID}(\mathrm{I}>\mathrm{D}), \mathrm{SD}(\mathrm{S}>\mathrm{D})$, and $\mathrm{Z}$ (zigzag; no trend).

Results The regression pattern D was always the most common (31-63\% of tumors by thickness and 33-72\% by cross-sectional area, for shape groups 1-4); and the DS pattern was the second most common one (22-24\% and $16-26 \%$, respectively), except for group 2. Of the DI and Z patterns with later tumor increase, the former was fairly evenly spread across groups (5-6\% and 8-10\%, respectively) whereas the latter was observed mainly in shape groups 1 and 2 (15\% and 17-22\%, respectively). The SD and ID patterns late tumor decrease were most common in shape groups 1 and 2, respectively, and most tumors representing the $\mathrm{S}$ pattern were shape group 1

Conclusion Heterogeneity in regression patterns and tumour shapes must be taken into account when using regression as a statistical variable in outcome analysis. Until now, all tumours have been pooled together despite significant differences in regression patterns, except in the study by Abramson et al. (1990).
- 4442

Intraretinal hyperreflective spots in eyes with radiation induced maculopathy

MIDENA E (1, 2), PARROZZANI R (2), MIDENA G (3), FRIZZIERO L (1),

VUIOSEVIC S (1), PILOTTO E (1)

(1) Department of Ophthalmology, University of Padova, Padova

(2) G.B. Bietti Foundation, IRCCS, Roma

(3) University Campus Bio-Medico, Roma

Purpose To evaluate in vivo the presence of intraretinal hyperreflective spots in eyes with radiation induced maculopathy.

Methods 25 subjects affected by maculopathy secondary to eye irradiation for a primary uveal melanoma were enrolled. All eyes previously underwent I-125 brachytherapy with a standard apex dose $(85 \mathrm{~Gy})$. Full ophthalmic examination, including spectral domain-OCT (SD-OCT) and fluorescein angiography, was performed in all eyes. After segmentation of retinal layers, retinal images were analyzed for reflectivity changes (hyperreflectivity spots) at the level of: internal limiting membrane plus retinal nerve fiber layer (ILM+RNFL), inner nuclear layer plus outer plexiform layer (INL+OPL) and outer nuclear layer (ONL). Hyperreflective spots were analyzed in a full retinal section both in the foveal area, and between 500 and $1500 \mu \mathrm{m}$ from the foveal center. All examinations were performed twice, by two independent masked graders.

Results The inter-grader agreement was at least substantial for all measurements. In all studied eyes hyperreflective spots were detected by SD-OCT. Hyperreflective spots were mainly located in the inner retina (ILM+RNFL and INL+OPL) compared to the outer retina $(\mathrm{ONL})(\mathrm{p}<0.005)$, and their expression significantly progressed with the progression of macular (radiation induced) edema ( $\mathrm{p}=0.002)$.

Conclusion The presence of hyperreflective spots may represent an in vivo biomarker of retinal microglia activation in radiation maculopathy, as previously shown in other macular disorders. This aspect may be related to the so-called senescence process induced by irradiation of solid tumors. This phaenomenon may offer more tailored approaches for the treatment of radiation-induced maculopathy.

\section{- 4444}

Reasons for secondary enucleation after eye preventing treatment of uveal melanoma

SAAKYANS, AMIRYAN A, MIRONOVA I

Ocular Oncology and Radiology, Moscow

Purpose To determine the reasons for secondary enucleation after eye preventing treatment of uveal melanoma (UM).

Methods A retrospective analysis of cases of enucleation after previously treated UM. During 200765 patients underwent secondary enucleation - $39(60 \%)$ women and 26 (40\%) men, mean age before enucleation was 54 years (range, 25-75). The period of time between UM local treatment and enucleation planning composed $32 \pm 26,7$ months (range, 2-108).

Results Tumor thickness prior the enucleation was 4,36 $\pm 3,5 \mathrm{~mm}$ and base diameter $9,8 \pm 5,6 \mathrm{~mm}$, before local therapy-5,2 $\pm 2,7 \mathrm{~mm}$ and $11,7 \pm 4,3 \mathrm{~mm}$, respectively. UM was localized in the choroid (43 patients, $66.1 \%$ ), ciliochoroidal localization was observed in $12(18.5 \%)$, in the iris - $1(1.5 \%)$, iridociliary - $5(7,8 \%)$, iridociliochoroidal - $4(6.1 \%)$ patients. In most cases, the patients had previously been undergone brachytherapy (45 patients, 69.2\%), much less - other methods: combined treatment - $6(9.2 \%)$, laser treatment $-4(6.2 \%)$, local excision $-8(12.3 \%)$, proton irradiation $-2(3.1 \%)$ patients. The reasons for enucleation were: continued growth - 41 patients $(63.1 \%)$, secondary incurable hypertension - 23 (35.4\%), vitreos body hemorrhage - 13 (20\%), extraocular extension - $19(29.2 \%)$, sclera necrosis - 8 (12.3\%), the residual tumor - $23(35.4 \%)$, Histologically, spindle cell tumor type was confirmed in 17 patients (26.1\%), epitelioid cell UM - 15 (23.1\%), mixed cell UM - 19 (29.2\%) patients and total tumor necrosis- 14 (21.6\%).

Conclusion The most frequent causes of enucleation after previously local treatment were tumor continued growth and the presence of incompletely tumor tissue resorption. $\mathrm{UM}$ any location and with different cell types can be the cause of secondary enucleation. 


\section{- 4445 \\ Retrospective study of combined treatments for conjunctival melanoma}

DESIARDINS L (1), YIN Y (1), CASSOUX N (1), LUMBROSO LE ROUIC L (1), DENDALE R (2) SASTRE X (3), ASSELAIN B (4), PLANCHER C (4), LEVYC (1)

(1) Ophthalmology, Paris

(2) radiotherapy, Paris

(3) pathology, Paris

(4) biostatistics, Paris

Purpose Since 2OOO, we have been using proton beam therapy after surgery for all invasive conjunctival melanomas. Patients with associated primary acquired melanosis also receive additional treatment with mitmycin 0, 04\% drops at least two cycles. We have made a retrospective study to evaluate the results.

Methods Patients were sent to Curie Institute for initial treatment, after the initial surgery or for recurrence. Surgery was performed under general anesthesia; proton beam was applied only on the area of invasive melanoma with a dose of 60 grays in 8 fractions. Results concerning the initial findings, the treatment and the follow up were prospectively registered

Results 62 patients were treated, 36 for initial treatment and 26 after surgery performed elsewhere. The median follow up is 24 months.(range 7 to 146 months)Median tumor diameter was $8 \mathrm{~mm}$, with a thickness $<2 \mathrm{~mm}$ for $33,>2 \mathrm{~mm}$ for 22 patients. TNM and histology will be described.Surgical excision was considered complete in 21 cases. All patients received proton beam irradiation and 25 received mitomycin drops. 10 patients (16\%) presented local recurrence and 4 patients (6\%) developed metastatic disease

Conclusion : Because of direct contact with the sclera, invasive conjunctival melanoma cannot be removed with sufficient margin if the eye is not removed. The use of proton beam therapy immediately after surgery allows good ocular preservation with very low rate of local recurrence .The prognostic of patients with primary acquired melanosis with atypia has been improved by the use of preventive treatment of the conjunctiva by mitomycin before invasive melanoma occurs.

\section{- 4447 / S115}

HSP90 inhibitors and conjunctival melanoma

MADIGANM (1,2), QUAHX (2), MCALPINES (3), CONWAY RM (4)

(1) Optometry \& Vision Science, UNSW, Sydney

(2) Save Sight Institute Uni Sydney, Sydney

(3) Chemistry UNSW, Sydney

(4) Save Sight Insittute Uni Sydney, Sydney

Purpose To investigate the expression of HSP90 in human conjunctival melanoma (CM) cell lines and primary human melanocytes, and to assess the effects of HSP90 inhibitors on cell growth and proliferation.

Methods CM cell lines (CRMM1 and 2; CM2005.1) and primary human melanocytes ( $\mathrm{n}=3$, P2 to P4) were immunolabelled for HSP90, and visualised using mmunofluorescence and confocal microscopv. HSP90 expression was also examined in cell lysates from CM cell lines (CRMM1 and 2; CM2005.1) and primary melanocytes $(\mathrm{n}=3)$. The effect of several HSP90 inhibitors (17DMAG, SM252 and SM122) on cell growth and viability was assessed at $24 \mathrm{hrs}$

Results CRMM1 and 2, and CM2005.1 cells showed strong cytoplasmic expression of HSP90, with low levels of cytoplasmic expression in primary melanocytes. This was confirmed using western blot. The HSP90 inhibitors induced dose-dependent cell death in CM cells, 17DMAG (water soluble) being most effective (LD50 at 24 hrs $\sim 30 \mathrm{uM}$ compared with 50um for SM122 and SM122).Primary human melanocytes viability was not significantly reduced by the HSP90 inhibitors.

Conclusion HSP90 is a widely expressed chaperone protein that can regulate cell growth, survival and angiogenesis pathways. CM cell lines expressed high levels of HSP90 protein compared to melanocytes, consistent with a recent study of primary conjunctival melanoma specimens, that found over-expressed HSP90 in CM particularly recurrent tumours. HSP90 inhibitors may be useful in inhibiting CM growth alone or in combination therapies. Funding: National Foundation for Medical
- 4446 / S116

Gene expression in conjunctival melanoma cell lines treated with the histone deacetylase (HDAC) inhibitor valproic acid (VPA)

MUNOZ-ERAZO L (1, 2), MADIGANM (3), CONWAYRM (2)

(1) Department of Pathology, Charles Perkins Centre, University of Sydney., Sydney

(2) Save Sight Institute, University of Sydney, Sydney

(3) Optometry \& Vision Science, UNSW, Sydney

Purpose To investigate the differential regulation of genes in conjunctival melanoma cell lines (CRMM1 and CM2005.1) treated with valproic acid (VPA), a class I and II histone deacetylase (HDAC) inhibitor

Methods Based on previous viability assays, cell lines were treated with VPA (CRMM1: 3mM, CM2005.1:5mM). At 24 and $72 \mathrm{~h}$ post-treatment, RNA was extracted and processed for RT-PCR using defined gene-array microfluidic cards. Gene functions chosen for investigation related to HDACs, cell cycle (cyclins e.g. CCND2, CCNA1) cell death (caspases), melanocyte function (PAX6), increased susceptibility to malignant transformation (BAP1, GNAQ) and DNA damage (GADD45A)

Results Upregulation of HDACs 1,2,3,4 6, SIRT2 and GADD45A occurred in CRMM1 cells by 24h but only after 24h for CM2005.1. Both CRMM1 and CM2005.1 showed increased CCND2 after VPA treatment, albeit higher in CM2005.1 ( $>700$-fold by 72h, compared to 22-fold in CRMM1). CCNA1 was increased at 24 and $72 \mathrm{~h}$ in CRMM1 (>200 fold 24h). For CM2005.1 cells, CCNA1 was initially downregulated and upregulated by $72 \mathrm{~h}$ ( $\sim 30$-fold).

Conclusion The delayed increase in GADD45A (DNA damage gene) in CM2005.1 cells compared with CRMM1 cells, is consistent with our toxicity assays showing CRMM1 more responsive to VPA than CM2005.1. The differences of CCND2 and CCNA1 fold-changes (which are partially regulated by HDACs) between the two cell lines, may explain their dissimilar responses to VPA.Funding - NFMRI

- 4448 / S119

Periorbital necrotizing fasciitis in a 69 year old patient. Medical management and use of hyperbaric oxygen as further adjunctive therapy

PORTALIOU DM (1), KOUROUPAKIA (1), KABANAROU S (1), KARVELA I (2), FERETIS E (1) XIROU T(1)

(1) Ophthalmology Department, Red Cross Hospital, Athens

(2) Ophthalmology Department, 'Agios Savvas' Hospital, Athens

Purpose To report a case of periorbital necrotizing fasciitis in a 69 year old patient that was successfully managed with the use of intravenous antibiotics and surgical debridement. In addition to the usual treatment modalities, Hyperbaric Oxygen (HBO) was used as further adjunctive therapy

Methods A 69 year old male presented with profound swelling over the right eye and right side of forehead. On admission the physicians made the diagnosis of septic shock. Patient had no history of alcohol abuse, insect bite, infection, recent penetrating injury, blunt trauma, face surgery or known immunosuppression conditions such as diabetes mellitus. Orbital CT showed a predominantly preseptal soft-tissue swelling consistent with the diagnosis of periorbital necrotizing fasciitis

Results Patient started on empiric treatment with broad-spectrum intravenous antibiotics that modified according to blood culture results few days later. Surgical debridement and microbiologic evaluation of the necrotic tissue were performed. Streptococcus pyogenes and Staphylococcus aureus were isolated from tissue samples. Patient was further treated with twenty sessions of Hyperbaric Oxygen Therapy. Complete resolution of the infection occurred six weeks after initial diagnosis.

Conclusion Treatment of periorbital necrotizing fasciitis with intravenous antibiotics and surgical debridement can be further enhanced with Hyperbaric Oxygen sessions with promising results. 


\section{- 4449 / S118}

Aneurysmal bone cyst: an exceptional entity in the orbit

LEMAITRE S (1), ZMUDA M (2), JACOMET PV (2), BERGES O (2),

GALATOIRE O (2)

(1) Institut Curie, Paris

(2) Fondation ophtalmologique Rothschild, Paris

Purpose Aneurysmal bone cyst is a rare benign bone neoplasm. The most common affected anatomical sites are vertebral column and long bones. We report two uncommon cases of orbital aneurysmal bone cyst.

Methods Two patients presented with a sudden left proptosis and ocular motility restriction.Case 1 is a 74-year-old female whose visual acuity is perception of light in the left eye due to severe papilledema. Imaging studies of the orbit reveal a large cystic frontal bone tumor associated with a subperiosteal hematoma causing eyeball and optic nerve compression. A preoperative arteriography shows a moderate vascular blush Drainage of the haematoma is performed. A local recurrence with hematoma formation occurred two years after the first surgery.Case 2 is a 45 -year-old female. Imaging studies reveal a smaller cystic frontal bone tumour associated with a subperiosteal hematoma. The patient achieved full visual recovery after the drainage of the hematoma with no recurrence after treatment.

Results In both cases the aneurysmal bone cyst was revealed by an acute orbital compartment syndrome due to a subperiosteal hemorrhage. A biopsy of the tumor within the frontal bone was performed during surgery and submitted to pathology for examination. Aneurysmal bone cyst secondary to another primary bone lesion was therefore excluded.

Conclusion Aneurysmal bone cysts are tumors of unknown cause which occur more often in the first 2 decades of life. Complete surgical resection is not always possible and the local recurrence rate is $20 \%$. 


\section{- 4451}

Role of Azithromycin in the treatment of children meibomian gland disease

LAZREG S (1), BENALI ABDELLAHM (2)

(1) Cabinet lazreg, Dar el Beida

(2) Cabinet lazreg, Blida

Purpose To determine the usefulness of azitromycin eye drops in the treatment of moderate to severe meibomian gland disease (MGD) in children;

Methods Prospective study conducted on 78 children with MGD. At the first examination, all patients already experienced various ophthalmological treatments without any success leading us to test azithromycin eye drops, a macrolide family with anti-inflammatory properties. The treatment consisted on azithromycin twice a day during 5 days repeated each month for 3 months associated with Dexamethasone eye drops ( 6 drops a day during one week, the firs month) and daily eyelid hygiene (manual or warm glasses) during the all the treatment course.The follow-up visits were performed on D7, D30, D60 and D90 with slit lamp examination and photography

Results 78 children were included, $76.4 \%$ had severe corneal complications (neovascularization, ulcers, phlyctenular keratitis). After the first week of treatment, a marked improvement in symptoms was observed in all children probably due to the effect of dexametasone drops, At D60; we observed improvement in corneal signs in $76.8 \%$ of cases with a significant decrease of corneal neovessels and phlyctenular Associated with azithromycin eye drops, warm glasses showed a better efficacy than manual technic. In 5 cases, in spite of more than 3 months treatment with azithromycin, the results were no conclusive;

Conclusion Azithromycin associated with eyelid hygiene is a very effective in treatment in children with MGD; furthermore, its ease of use, its very good efficacy and the absence of side effects, makes this therapy a good alternative and choice in this disease,

\section{- 4452}

Prospective evaluation of intense pulsed light (IPL) therapy for meibomian gland dysfunction (MGD)

CRAIG JP (1), TURNBULL PR (1), CHEN A $(1,2)$

(1) Department of Ophthalmology, Auckland

(2) Department of Optometry and Vision Science, Auckland

Purpose To evaluate the effect of intense pulsed light (IPL) applied to the periocular area for meibomian gland dysfunction (MGD) in a prospective, double-masked, placebo-controlled, paired-eye study.

Methods Twenty-eight participants (aged $45 \pm 16$ years, $68 \%$ female) underwent IPL treatment (E>Eye, E-Swin, France), with homogeneously sequenced light pulses delivered to one eye (treated eye, randomised, masked), while placebo treatment was performed on the partner eye (control eye) on 3 occasions following baseline (BL) evaluation; on Day 1, Day 15, and Day 45. Lipid layer grade (LLG), non-invasive tear breakup time (NIBUT), tear evaporation rate (TER), tear meniscus height (TMH) and subjective symptom score (SPEED) were compared to BL and control values at each visit.

Results LLG improved significantly from BL to Day 45 in the treated eye $(\mathrm{p}=0.0002)$, but not control eye ( $\mathrm{p}=0.714$ ) with an increase of 1.5 grades exhibited by $75 \%$ of treated eyes by Day 45. NIBUT also improved significantly from BL to Day 45 in the treated $(\mathrm{p}<0.0001)$, but not control eye $(\mathrm{p}=0.056)$. NIBUT in the treated eye was significantly longer than that in the control eye at Day $45(14.1 \pm 9.8 \mathrm{~s}$ vs $8.6 \pm 8.2 \mathrm{~s}, \mathrm{p}<0.0001)$. TER was not significantly lower at Day 45 compared to $\mathrm{BL}$ in the control eye ( $\mathrm{p}=0.165)$, but approached significance in the treated eye $(\mathrm{p}=0.080)$. No change in TMH was noted from BL in either eye at Day $45(\mathrm{p}>0.05)$. SPEED scores improved at each visit in both the treated $(\mathrm{p}<0.0001)$ and control eye $(\mathrm{p}=0.002)$, with $86 \%$ of participants noting reduced symptoms in the treated eye by Day 45 .

Conclusion IPL with multiple sculpted pulses shows therapeutic potential for MGD, statistically and clinically improving tear film quality and reducing symptoms of dry eye. Commercial interest
- 4453

Novel method for the isolation and measurement of microRNA and microRNA related targeted genes in conjunctival epithelial cells in primary Sjogren's syndrome patients

PILSONQ (1, 2, 3), NÍ GABHANNJ (3, 1), MURPHY C (3, 2)

(1) Molecular and Cellular Therapeutics department, Royal College of Surgeons in Ireland, Dublin

(2) Ophthalmology department, The Royal Victoria Eye and Ear hospital, Dubiln

(3) Ophthalmology department, Royal College of Surgeons in Ireland, Dublin

Purpose Siogren's syndrome (SS) is a systemic autoimmune disorder characterized by inflammation that affects exocrine glands notably the salivary and lacrimal glands leading to sicca complex. Dry eye disease in primary SS (pSS) is a combination of reduced lacrimal flow from gland destruction and tear hyperosmolarity leading to inflammatory damage on the ocular surface. Several studies have shown differential expression of certain miRs in PBMCs and salivary glands from SS patients compared to healthy controls, however no functional role has been shown. This study aims to isolate miRs and mRNA from conjunctival epithelial cells by impression cytology to determine if dysregulated miR expression contributes disease pathogenesis in PSS.

Methods Impression cytology (IC) was optimized using different membranes. miR and mRNAs were isolated from conjunctival epithelial cells (CEC) obtained from pSS patients and healthy controls by IC and sent for screening to Ocean Ridge Biosciences. qPCR was performed to evaluate expression of miRs from CEC

Results Milicell biopore was the best membrane with significant yield of miRs (47.5ng $\pm 0.6)$ and mRNA (158.0 ng +21.6) as well as patient comfort. Appreciable levels of miRs and targeted genes were detected from the CEC from healthy controls, confirming that it is possible to isolate miRs and miRs targeted genes from CEC. Screening studies in CECs demonstrated differential expression of miRs and mRNA between pSS patients and healthy controls. future diagnostics, prognostics and therapeutics of pSS patients as well as other ocular surface conditions. 


\section{- 4455 / 5061}

Anti-evaporative mechanism of wax esters: implications for the function of tear fluid

PAANANEN RO, RANTAMÄKI AH, HOLOPAINENJ

Helsinki Eye Lab, Department of Ophthalmology, University of Helsinki, Helsinki

Purpose Our aim was to explain the molecular level basis of the ability of wax ester (WE) films to retard evaporation close to their bulk melting temperature. We studied the surface behavior of behenyl palmitoleate (BP), a WE representing the most abundant meibum WEs.

Methods Isotherm and isochor measurements coupled with imaging by Brewste angle microscopy (BAM) were used to study BP films at the air-water interface. In addition, evaporation rates through BP films were measured.

Results Close to room temperature, BP formed solid, non-spreading islands when applied to the air-water interface. Between 35 and $38^{\circ} \mathrm{C}$, a fluid, effectively spreading monolayer phase coexisted with solid domains of BP. At higher temperatures, BP formed completely fluid films. Mean molecular areas of the fluid and solid films were determined to be approximately $50 \AA 2$ and $18 \AA 2$, respectively. The thickness of a solid BP monolayer was determined to be $42 \pm 6 \AA$ by BAM measurements. A fluid BP monolayer only had minimal evaporation resistance, while a solid monolayer retarded evaporation by $>50 \%$

Conclusion Below $35{ }^{\circ} \mathrm{C}$, BP assumes an extended conformation at the air-wate interface, similar to the bulk crystal structure of WEs. The extended conformation allows tight packing of hydrocarbon chains, which hinders the permeation of water molecules and leads to the evaporation retarding effect. Although the solid islands effectively retard evaporation, they do not spread at low temperatures and water evaporation proceeds through the uncovered areas of the surface. Coexistence of fluid and solid monolaver phases between 35 and $38^{\circ} \mathrm{C}$ allows the film to spread, leading to a complete coverage of the interface by the solid phase and effective evaporation retardation.

\section{- 4457 / S063 \\ Changes of inflammatory cytokines and tear osmolarity in systemic sclerosis after treatment with liposomes sprayed}

GAGLIANOC (1, 2), AMATOR (1,2), FALLICOM (2), TOROM (2), AVITABILE T (2), FOTIR (3)

(1) NEST (Neurovisual Science Technology), Catania

(2) Ophthalmic Clinic, Catania

(3) Rheumatology Unit, Catania

Purpose To investigate the effect of treatment with a liposomal spray solution in patients with systemic sclerosis $(\mathrm{SSc})$ and severe dry eye by measuring the levels of inflammatory cytokines and tear osmolarity.

Methods Twenty-two subjects (12M, 10F) aged 45.1+/-7.1 years participated in this prospective, randomised, double-masked investigation. DES was identified in patients with SSc according to the classification of Dry Eye Disease of International Dry Eye Workshop (2007) and tear osmolarity values (TearLab system) according to recommendations of the manufacturer with a cutoff value of $308 \mathrm{mOsm} / \mathrm{L}(3)$. Liposomal spray (nebulized, two sprays each, on the eyelids, 3 times per day for 3 weeks) with vitamin A and $\mathrm{E}$ was applied to one eye, and an equal volume of saline spray (control) applied to the contralateral eye. Non-invasive tear film stability (NIBUT), osmolarity and inflammatory cytokines (IL-1 $\beta$, IL6, TNF- $\alpha$,IFN- $\gamma$ ) were evaluated at baseline, and at 5, 10, 15 days after spray solution application.

Results A highly significant reduction $(\mathrm{p}<0.01-0.001)$ was detected in the inflammatory cytokine levels and osmolarity in tears of eye treated with the spray liposomes solution in comparison with fellow eye treated with sprayed saline solution. There were significant differences among the 2 eyes concerning tear osmolarity $(\mathrm{p}<0.001)$ and TBUT $(\mathrm{p}<$ $0.05)$ scores

Conclusion This study showed the ability of liposomal spray application to modify the pro-inflammatory cytokine profile and to increase stability characteristics of the preocular tear film in severe dry eye of SSc patients.This effect could be due to the antiinflammatory mechanism of the vitamins and also to the barrier effect of liposomes.
- 4456 / S062

Systematic allergy investigation of patients with red eyes

MUNKSJ (1, 2), GARVEY LH (2), HEEGAARD S (3, 1), HANSEN KS (2, 4), SKOV L (5), ZACHARIAE C (5), MENNÉ T (5), JULIAN HO (1), HALKJARR LB (2), JOHANSENJD (6)

(1) Department of Ophthalmology, Glostrup Hospital, University of Copenhagen, Copenhagen

(2) Allergy Clinic, Gentofte Hospital, University of Copenhagen, Copenhagen

(3) Eye Pathology Institute, Department of Neuroscience and Pharmacology, University of Copenhagen, Copenhagen

(4) Department of Pediatrics, Herlev Hospital, University of Copenhagen, Copenhagen

(5) Department of Dermato-Allergology, Gentofte Hospital, University of Copenhagen, Copenhagen

(6) National Allergy Research Centre, Department of Dermato-Allergology, Gentofte Hospital, University of Copenhagen, Copenhagen

Purpose "Red eyes" is a common patient complaint. Determining the underlying cause is often difficult as differential diagnoses are numerous. Allergic causes may be easily overlooked as the diagnosis relies on specialized investigation. We wanted to determine the prevalence of relevant allergy in patients with "red eyes", where initial ophthalmological consultation did not result in a definite diagnosis.

Methods A multidisciplinary cooperation was established between ophthalmologists, allergologists, dermatologists and pediatricians. The investigation algorithm was based on clinical presentation. Patients with eye symptoms only, or eye and skin symptoms, underwent dermatological investigation comprising patch testing with European baseline series, eye drops and skin prick tests with inhalant allergens and the patients' own ophthalmological products. Patients with eye and airway symptoms were reviewed by allergologists and underwent skin prick tests and specific IgE analysis for relevant allergens and lung function tests. Children were reviewed by pediatric allergologists. Multidisciplinary conferences were held regularly and patients were reviewed and cross-referred if relevant.

Results In total 58 patients ( 21 males and 37 females) with "red eyes" were included over a one year period. In 21 patients (36\%) a relevant allergy was found, diagnosing contact allergy in 15 patients ( 9 perfume, 3 phenylephrine, 3 others) and IgE-mediated inhalant allergies in 6 patients. In 37 patients (64\%) no allergy was found, and of these 10 patients were diagnosed with rosacea.

Conclusion Multidisciplinary cooperation and systematic investigation resulted in identifying relevant allergy in 36\% of patients presenting with "red eyes".

\section{- 4458 / S064 \\ Morphogenesis of eyelid meibomian gland}

GUEDIRA TARIGHT G(1), TARIGHTN (1), SEVESTRE H (2), COPIN H (3), BREMOND-GIGNACD $(1,4)$

(1) Ophthalmology, University Hospital of Amiens, Saint Victor Center, Amiens

(2) Pathology Department, University Hospital of Amiens, Amiens

(3) Biology Reproduction and cytogenetic department, Amiens

(4) CNRS FR 3636, University Paris V, Paris

Purpose Dysfunction of Meibomian gland is the primary cause of dry eye. The purpose of our study is to understand embryogenesis for a better understanding of the pathophysiology of these diseases.

Methods Eyelids were obtained from 22 fetuses formalin fixated of 10-34 weeks gestational age (GA) with approval of Ethics Committee. These 88 evelids were processed with sections $5 \mu \mathrm{m}$ thick and analyzed by light microscopy after staining with hematoxylin-phloxine-saffron and PAS reaction. For each sample, different morphological criteria were investigated: evaluation of glandular bud, sebaceous gland differentiation, arborization of the gland, opening of excretory duct.

Results Histological analysis revealed that up to 13 weeks of GA, the fetal lid consists of fine vascular structures in a loose mesenchyme, under a very thin epithelium. At 14 weeks of GA, a first process of the excretory duct is individualized as a cell invagination extending from the eyelid margin. Sebaceous cell differentiation is identified at 15 weeks of GA. The excretory canal keeps its maturation to appear as a small cellular cord, unique and full around 16 weeks of GA, glands begin its buds arborization at 19 weeks of GA. This channel gradually widens and expands, appearing always blocked by a plug of squamous cells and keratin cuticle at 22 weeks of GA. The plugging lasts until 34 weeks of GA.

Conclusion Glandular structures involved in tear secretion appear in fetus around fourteen weeks of gestation. The Meibomian glands extend its maturation after birth. Further studies will allow a better understanding of the physiopathology of dry eye. 
- 4461

Eye movement examination

BORRUAT F

Lausanne

The eyes move in order either to keep track of an object of interest or to change visual attention from an object to another. An adequate comprehension of the anatomy and physiology of the different types of eye movements is necessary when examining a patient with oculomotility disorder, be it peripheral, central or supranuclear.This presentation will summarize the infranuclear, nuclear and supranuclear pathways of oculomotility with an emphasis on fixation, saccades, pursuit, convergence and basic examination of the vestibulo-ocular reflex. Guidelines for an examination of eye movements in clinical practice will be provided with video presentations of both normal and abnormal situations. At the end of the session, participants should be able to examine eye movements adequately and recognize abnormal fixation, defective pursuit, and inappropriate saccades.
- 4462

Third, fourth and sixth cranial nerves palsy

$\operatorname{KAESER~PF~}(1,2)$

(1) Department of ophthalmology, University of Lausanne, Lausanne

(2) Jules Gonin Eye Hospital, Lausanne

Acquired binocular diplopia commonly results from dysfunction of the third, fourth or sixth cranial nerve. Some cases need an urgent work-up and it is mandatory to identify them early.This presentation will summarize the clinical findings and common causes of III, IV and VI cranial nerve palsy, and highlight the necessary investigations. At the end of the session, participants should be able to recognize, provide a differential diagnosis and propose appropriate investigations for III, IV, and VI cranial nerve palsy.

- 4464

Interactive case presentations

KAESER PF (1, 2), BORRUAT F (1,2)

(1) Department of ophthalmology, University of Lausanne, Lausanne

(2) Jules Gonin Eye Hospital, Lausanne

Various cases illustrating the supranuclear and infranuclear oculomotility disorders will be presented. Attendees will actively participate to solve these cases with the use of an electronic audience response system. 
- 4471

Impact of Baseline (BL) Visual Acuity (VA) in the VIVID-DME and VISTA-DME trials

MASSIN P (1), KOROBELNIKJ (2), METZIG C (3)

(1) APHP-Hôpital Lariboisière, Paris

(2) Hôpital Pellegrin CHU de Bordeaux, Bordeaux

(3) Bayer Pharma AG, Berlin

Purpose To evaluate the impact of BL VA on changes in best-corrected VA (BCVA) and central retinal thickness (CRT) in patients (pts) with diabetic macular edema (DME) in the VIVID-DME and VISTA-DME trials.

Methods Pts received intravitreal aflibercept (IVT-AFL) $2 \mathrm{mg}$ plus sham laser every 4 wks (2q4) or every 8 wks (2q8) (after 5 monthly doses), or laser photocoagulation plus sham IVT treatment. Subgroup analyses assessed the impact of BL BCVA on changes in BCVA and CRT at Week 52 (W52). BL BCVA subgroups were $<40, \geq 40$ ) $<55, \geq 55-<65$, and $\geq 65$ ETDRS letters.

Results VIVID-DME: Mean BCVA changes from BL to W52 for laser, 2q4, and 2q8, respectively were $2.8,153$, and $20.6(<40) \cdot-0.1,120$ and $10.2(>40-<55) ; 24,116$, and $11.5(\geq 55-<65)$ and $0.5,8.6$, and $8.1(\geq 65)$. Mean CRT changes $(\mu \mathrm{m})$ for laser, $2 q 4$, and $2 \mathrm{q} 8$ were $-29.8,-423.3$, and $-355.3(<40) ;-80.2,-256.2$, and $-204.9(\geq 40-<55) ;-111.2$ -2091 , and $-215.1(255-<65)$ and $-347,-135.7$ and $-1326(\geq 65)$. VISTA-DME: Mean BCVA changes from BL to W52 for laser, $2 \mathrm{q} 4$, and $2 \mathrm{q} 8$, respectively were $-4.7,19.9$, and $14.5(<40) ; 5.2,14.1$, and $9.4(\geq 40-<55) ; 1.6,12.8$, and $11.7(\geq 55-<65)$; and $-1.8,9.8$, and 9.4 ( $\geq 65)$. Mean CRT changes $(\mu \mathrm{m})$ for laser, $2 \mathrm{q} 4$, and $2 \mathrm{q} 8$ were $-124.9,-358.7$, and -401.9 $(<40) \cdot-145.9,-2092$, and $-2094(>40-<55) \cdot-726,-194.6$, and $-179.5(\geq 55-<65)$ : and $-33.7,-131.2$, and $-128.4(\geq 65)$. IVT-AFL was generally well tolerated. Incidence of AEs and serious AEs was similar across all groups, with no significant differences in the incidence of APTC events.

Conclusion Outcomes based on BL VA were consistent with those in the overall study population, with greater improvements seen in the IVT-AFL groups vs laser. There was a trend towards greater improvements in pts with worse vs better BL VA.

Commercial interest

\section{- 4473}

Incidence of macular edema following single versus 4 session pattern scanning laser panretinal photocoagulation in diabetic retinopathy

PALLOT C (1), KOEHRER P (1), ISAICO R (1), KODJIKIAN L (2), MASSIN P (3), JONVAL L (4), BERTAUX AC (4), D'ATHIS P (4), BRONAM (1), CREUZOT-GARCHER C (1)

(1) Department of Ophthalmology, University Hospital, Dijon, France, Dijon

(2) Department of Ophthalmology, University Hospital Croix-Rousse, Lyon, France, Lyon (3) Department of ophthalmology, University Hospital Lariboisiere, Paris, France., Paris (4) Department of Clinical Research, University Hospital, Dijon, France, Dijon

Purpose The purpose of this study was to investigate a change in macular thickness 9 months after single versus 4 sessions of panretinal photocoagulation (PRP) performed with Pattern Scanning Laser (PSL) in diabetic patients and to make a medico-economic assessment

Methods This prospective, multicenter, randomized trial enrolled patients in 2 arms single session PRP (group A) and 4 session PRP (group B) between October 2011 and October 2013. Patients had non proliferative severe diabetic retinopathy or proliferative non complicated diabetic retinopathy. The primary endpoint was the difference in macular central retinal thickness (CRT) using spectral domain optical coherence tomography (SD-OCT) at 9 months. CRT with SD-OCT, ETDRS visual acuity (VA), pain with Visual Analogic Scale (VAS), were recorded at baseline, 7 days, 1, 3, 6, and 9 months. A medico-economic assessment was also performed

Results 26 eyes of 26 patients were included. On inclusion, the median CRT was 258 [240-294] $\mu \mathrm{m}$ in group $\mathrm{A}$ and $282[261-292] \mu \mathrm{m}$ in group $\mathrm{B}(\mathrm{p}=0.06)$, the median VA was 84 [76-88] letters in group A and 83 [75-90] letters in group B ( $p=0.94)$. At M9, the median CRT was 281 [245-322] $\mu \mathrm{m}$ in group A and 301[278-313] $\mu \mathrm{m}$ in group B $(\mathrm{p}=0.34)$. At M9, the median VA was 84 [70-91] letters in group $A$ and 85 [74-85] letters in group B (0.58). The median VAS value was 3.5 [2.0-5.0] in in group A and 3.5 [1.0-7.0] for all sessions in group B. The medico-economic assessment was in favor of the single session protocol

Conclusion Single session PSL-PRP seems to be a possible, well tolerated protocol for the treatment of diabetic retinopathy.
- 4472 / F106

Multicenter OZurdex long term assessment for diabetic macular edema: MOZART 2 study

MATONTI F (1, 2), HAJIAR C (3), PARRAT E (3), MERITE PY (4), POMMIERS (5), MEYER F (4), PROST-MAGNINO (6), GUIGOUS (7)

(1) P1.5 Network, Marseille

(2) Hôpital Nord - Ophtalmologie, Marseille

(3) P1.5 Network, Guadeloupe

(4) P1.5 Network, Aix-en-Provence

(5) P1.5 Network, L'Isle-sur-la-Sorgue

(6) P1.5 Network, Dijon

(7) P1.5 Network, Avignon

Purpose To evaluate the long-term efficacy and safety of intravitreal implant of $0,7 \mathrm{mg}$ dexamathasone (ozurdex ${ }^{\circ}$ ) in diabetic macular edema(DME)

Methods This was a retrospective, multicenter, study. 19 patients, with mean age of 67 years, followed for at least 8 months (mean 10,7 months) were included in 5 French eye clinics (P1,5 network). The mean systolic blood pressure was $138 \mathrm{mmHg}$ and the mean $\mathrm{HbAlc}$ was $7,2 \%$. We monitored 2 systemic parameters: blood pressure and glycemic balance. Best-corrected visual acuity (BCVA), central retinal thickness by spectralis OCT, intraocular pressure and cataract progression are studied at baseline and then at 1,6 and 12 Months.

Results Visual acuity significantly increased compared to baseline: at M1, M6 and M12, mean value of 8,7, 9,1 and 5,8 ETDRSletters, respectively. The improvement was $\geq 10$ ETDRS letters in 46,7\%,57,9\% and 58,3\% of patients at M1, M6 and M12, respectively. The average CRT decrease was: $332 \mu \mathrm{m}$ at M1, 259 $\mu \mathrm{m}$ at M6 and $296 \mu \mathrm{m}$ at M12. The average interval between two injections was of $5.6 \mathrm{months}$. Ocular hypertension greater than $25 \mathrm{mmHg}$, managed by topical treatment, was observed in 13,4\% of patients. None glaucoma surgery was necessary

Conclusion Ozurdex has an anatomical and functional effectiveness in the treatment of DME. Outcomes for naive patients and lower CRT suggest that the duration of diabetes mellitus and previous treatments are negative factors for recovery. Side effects are rare and manageable. Ozurdex seems to be an effective treatment for visual impairment due to DME with a favorable safety profile. Patient follow-up must be adapted to half-life of the product with a control before M1 (intraocular pressure) and before M5 (DME recurrence)

Commercial interest

\section{- 4474}

Serous retinal detachment associated with diabetic macular edema over the course of Ranibizumab treatment

QUL (1), FAINKUCHEN F (1), CHAINE G (2), GIOCANTI-AUREGANA (3)

(1) Paris

(2) Bobigny

(3) Ophthalmology, Avicenne hospita, Bobigny

Purpose Diabetic macular edema (DME) is frequently associated with subretinal detachment (SRD). SRD does not seem associated with a change of spontaneous evolution of DME. But we do not know wether SRD has an impact on DME resolution under intravitreal injections (IVT) of Ranibizumab. The purpose of our study was to describe the course of DME treated by Ranibizumab with or without SRD over 6 months.

Methods We retrospectively enrolled patients treated by ranibizumab for DME with central foveal thickness $($ CFT) $>300 \mu \mathrm{m}$ between November 2012 and November 2013. Group 1 was DME with SRD and group 2 was DME without SRD. We measured manually SRD on a SD-OCT (OPKO). We studied best corrected visual acuity (BCVA), $B C V A$ variation from baseline, CFT with or without SRD on OCT and the total number of IVT over 6 months.

Results 48 eyes with DME of 40 patients were included. 23 eyes (47,9\%) had SRD at baseline (group 1) and 25 eyes (41 \%) did not (group 2). At baseline, BCVA in decimal was 0,19 (40,3 letters in ETDRS) in group 1 and 0,25 (51,3 letters ETDRS) in group 2 $(\mathrm{p}<0,05)$. CFT was $666 \mu \mathrm{m}$ and SRD was $215 \mu \mathrm{m}$ in group 1 and $528 \mu \mathrm{m}$ in group 2 . After 6 months, mean change of BCVA was $+0,21$ in decimal (+ 20,9 letters in ETDRS) in group 1 , and $+0,17$ in decimal $(+7,8$ letters ETDRS $)$ in group $2(p<0,05)$. The final CFT was $327 \mu \mathrm{m}$ in group $1(-339 \mu \mathrm{m})$ and $379 \mu \mathrm{m}(-147 \mu \mathrm{m})$ in group 2. Group 1 received 4,3 IVT over 6 months against 4,2 IVT in group 2.

Conclusion DME with SRD had a thicker CFT and a lower BCVA at baseline than DME without SRD in our case series. DME with SRD seemed to have the best results under ranibizumab regarding BCVA, and CFT after treatment.SRD associated with DME seems to be a good functiona 


\section{- 4475}

Spectral domain ocular coherence tomography finding post vitrectomy and membrane delamination for proliferative diabetic retinopathy

DOOLEY I, LAVIERS H, PAPAVASILEIOU E, MCKECHNIE C, ZAMBARAKII H Barts Health, London

Purpose To describe the intra-retinal microstructure using serial spectral domainoptical coherence tomography (SD-OCT) following pars plana vitrectomy (PPV) and delamination of fibrovascular membranes for severe proliferative diabetic retinopathy (PDR)

Methods This retrospective, interventional case series included 26 eves. Outcome measures included LogMAR best-corrected visual acuity (BCVA) and SD-OCT findings, including automated central $1-\mathrm{mm}$ subfield retinal thickness, integrity of photoreceptor inner and outer segments junction (IS/OS) and integrity of external limiting membrane (ELM). Two vitreoretinal-trained observers graded SD-OCT images.

Results The eyes were into 3 groups based on post-op IS/OS integrity (group 1: IS/ OS intact; group 2: IS/OS irregular but not completely disrupted; group 3: IS/OS completely disrupted. Mean BCVA improved significantly in group $1(n=9)$ from $1.64 \pm$ 0.71 preoperatively to $0.33 \pm 0.21$ (student's t-test: $\mathrm{p}=0.01)$, in group $2(\mathrm{n}=8)$ the BCVA improved from $1.30 \pm 0.75$ to $0.63 \pm 0.30$ (student's t-test: $\mathrm{p}=0.44$ ) and in group $3(\mathrm{n}=$ 9) the BCVA improved from $1.65 \pm 0.53$ to $1.53 \pm 0.75$ (student's t-test: $\mathrm{p}=0.47$ ). IS/ OS integrity and ELM integrity were positively correlated with final BCVA (Pearson's coefficient: $\mathrm{r}=0.61, \mathrm{p}<0.001$ and $\mathrm{r}=0.68, \mathrm{p}<0.001$, respectively).

Conclusion The postoperative intra-retinal structure at the level of the IS/OS and ELM based on SD-OCT imaging are positively correlated with final postoperative visual acuity. The IS/OS boundary and ELM continuity appear to be indicators of macular function. These findings may provide prognostic factors for patients with this challenging and blinding disease

\section{- 4477 / F112}

Pronostic factors and complications of vitrectomy in patients with advanced diabetic retinopathy

AJAMIL RODANES S, MANZANAS LEAL L, LOPEZ GALVEZ MI

Ophthalmology, University Hospital, Valladolid

Purpose The aim of this study is to present the results of pars plana vitrectomy in patients with advanced disease and to identify preoperative risk factors and postoperative visual outcome

Methods The study was carried out at the University Hospital in Valladolid (Spain) with data from patients throughout two years.

Results Retrospective chart review of patients who underwent posterior plas plana vitrectomy (PPV) during 2010-2011 for non-clearing vitreous haemorrhage (NCVH), tractional retinal detachment (TDR) or diabetic edema with vitreomacular traction (TME) and followed for at least 6 months.The primary outcome measure was the best corrected visual acuity (BCVA) at the end of the follow-up period and its correlation with preoperative risk factors. The secondary outcomes were postoperative complications and the influence of the status of the external retina in the ocular coherence tomography (OCT).58 patients underwent PPV for advanced retinal disease $\mathrm{NCVH}$ was the most frequent surgical indication in 32 patients and 21 of them had gone through laser previously. Mean preoparative BCVA was $1.96 \pm 1.13$ logarithm of minimum angle of resolution (logMAR) units and it improved.TDR was the indication in 10 patients. Only $50 \%$ of them had laser and all of them suffered from long term disease. There was not significant improvement in the BCVA and 3 patients developed neovascular glaucoma. Ind

Conclusion Preoperative BCVA, long term disease, the lack of laser treatment and the external retina seem to be some of the most important risk factors in these patients
- 4476

Improved outcome after primary vitrectomy in diabetic patients treated with simvastatin

LOUKOVAARA S (1), SAHANNE S (2), TUUMINEN R (1)

(1) Ophthalmology, Helsinki

(2) Anesthesiology and Intensive Care Medicine, Helsinki

Purpose Simvastatin treatment may reduce intravitreal levels of potent vasoactive, angiogenic, and fibroproliferative factors. Here, we evaluated the effect of preoperative simvastatin treatment on the outcome of primary vitrectomy in diabetic patients.

Methods Prospective, observational case control study of type 1 and 2 adult diabetic patients admitted for primary vitrectomy in management of sight-threatening forms of diabetic retinopathy (DR). A 1-month best corrected visual acuity (BCVA) gain and cumulative 12-month re-vitrectomy frequency of 192 diabetic patients, 79 on simvastatin treatment and 113 without any statin medication.

Results Diabetic patients with simvastatin treatment had a better 1-month BCVA mprovement than did those without statin treatment $(\mathrm{p}=0.009)$. Subgroup analysis revealed that diabetics on simvastatin had better post-operative BCVA improvement when preoperative status included partial or panretinal laser photocoagulation $(\mathrm{p}=0.042$ and $\mathrm{p}=0.049)$ and anti-VEGF therapy $(\mathrm{p}=0.011)$. Moreover, diabetics preoperatively with macular edema $(\mathrm{p}=0.009)$, vitreous hemorrhage $(\mathrm{p}<0.001)$, proliferative retinopathy $(\mathrm{p}<0.001)$, or tractional retinal detachment $(\mathrm{p}=0.010)$ had better $\mathrm{BCVA}$ recovery, if receiving simvastatin. Our 12-month follow-up revealed that the frequency of revitrectomies $(\mathrm{p}<0.001)$ and re-revitrectomies $(\mathrm{p}=0.016)$ were lower in diabetics on simvastatin treatment.

Conclusion These data provide novel insight into the potential clinical benefit for patients with sight-threatening DR undergoing vitrectomy treated with simvastatin. 
- 4531

The role of inflammation in chronic DME

SOUBRANEG

Paris

ABSTRACT NOT PROVIDED

- 4533

Understanding and managing steroid induced elevation of IOP

GONIF

Barcelona

ABSTRACT NOT PROVIDED
4532

The long-term benefit of new sustained delivery micro-implant in patients with chronic DME

CUNHA-VAZJ

Coimbra

ABSTRACT NOT PROVIDED

- 4534

European early experience

AUGUSTINA

Karlsrube

ABSTRACT NOT PROVIDED 
- 4621

Visual deprivation: mechanism and practical applications

BREMOND-GIGNACD $(1,2)$

(1) Ophthalmology, University Hospital, Amiens

(2) CNRS, FR3636, Paris $V$

Maturation of the visual system is incomplete at birth. Visual deprivation mechanism, amblyopia, or ocular misalignment, leads to abnormalities in visual development, including abnormal eyeball growth and neurological changes. The current knowledge on ocular conditions related to abnormal visual development in infants, includes prevalence, risk factors, causes, and mechanisms involved. The role of eyeball growth with pathologic mechanism of visual deprivation and development of amblyopia in infants appears essential to be analyzed. Particular developmental issues in preterm neonates, methods of visual assessment and screening, diagnosis, treatment, and nutritional issues will be discussed. Visual deprivation mechanism, amblyopia, or ocular misalignment, leads to abnormalities in visual development, including abnormal eyeball growth and neurological changes. Abnormal visual input can lead to abnormalities in visual development, which can become permanent visual impairment if left untreated. Reduce the risk of abnormal visual development andprevent long-term or permanent visual deficits needs an early screening and a better understanding of the complex impairment mechanisms.

- 4623

Postural control and binocular anomalies

KAPOULA Z, MORIZE A, DANIEL F, ORSSAUD C, IONOUA F

BREMOND GIGNACD

CNRS FR3636, Paris V University, Paris

Vergence insufficiency is an extraocular dysfunction screened regularly by ophthalmologists and orthoptists. Incidence increases with screen and computer work, resulting in visual quality and quality of life loss. Clinical diagnosis is based on associated well-defined criteria (Alvarez 2010, Horwood 2014). Quantification of symptoms reported (Echelle CISS), evaluation of response to disparity stimuli and accommodation in natural spaces, calculation of ratio accommodation/vergence and measure of static performance. These criteria are essentially subjective and break the natural bond of two synergistic systems: convergence and accommodation. In the literature very few studies objectify dysfunction with video-oculography because most authors induce either angular disparities by artificial haploscopic devices or defocusing. However restricting the visual examination to each component, the vergence of the one part, and on the other hand accommodation, this analytical fragmentation is not representative of the daily behavior of patients. Indeed, in the natural space simultaneous variations of angles of disparity and focus stimulate and maintain synergy vergence-accommodation. Therefore, we propose an ecological study of the vergence system by stimulating real space. In the meantime our daily actions seek simultaneously vergence and saccadic eye movements, we also extend the study to the combined movement.
- 4622

Lens and visual development

LÖFGRENS

St. Erik's Eye Hospital/Karolinska Institutet, Stockholm

The visual development is dependent on a number of factors, such as eye media transparency, refraction, eye growth, environmental light input and visual image input. The lens plays an important role for the visual development.The lecture will cover lens growth and development, in connection to visual development in children. Form changing lens disease and light absorbing disease in or around the lens can be amblyogenic, although in many cases the situation can be resolved with refractive treatment or lens removal. Unfortunately the postoperative or treatment efficiency on visual development is not always perfect. Besides the above diseases there is also the question about indirect effects of aphakia or intraocular lens on eve and visual development. Further, some words on emmetropization related to lens structure and function. Pillar-stone papers will be presented to serve as backbone for newer data. 


\section{$-4631$}

Electrospun nanofibrous scaffolds and corneal tissue engineering

SALEHIS (1), BAHNERS T (2), GUTMANN J (2), FUCHSLUGER T (1)

(1) Eye clinic, Universitätsklinikum Düsseldorf, Düsseldorf

(2) Deutsches Textil forschungzentrum Nord-West gGmbH, Krefeld

Generation of a tissue-engineered cornea composed of biomaterials and cells will reduce dependency on the short supply of donor corneal tissue. In the last decade, the supply of viable donor tissue for corneal transplants is relatively less than the demand for donor corneal tissue. A tissue- engineered cornea that incorporates cells with a biocompatible and aligned nanofibrous scaffolds that replicates the stromal extracellular matrix will be an optimal alternative to synthetic replacements and donor tissue. Biodegradable aligned electrospun substitutes of a tissue- engineered cornea would also serve as an in- vivo and ex-vivo platform for investigating the mechanisms of corneal diseases, regeneration of corneal cell layers and human cellular responses to new ophthalmic drugs

- 4633

\section{Hydrogel based keratoprosthesis}

STORSBERGJ

Potsdam

Keratoprosthesis (KPs) are made from biomaterials which can differ significantly in their physical, chemical and biological properties. There are hydrophobic matteials as well as hydrophilic materials, each material has a limited spectra of use. An complex implantant, such as a KPs needs different surfaces to be integrated stable and for a long term without causing side reactions such as inflammation or rejection. This, there a different pre requirements to be fullfilled in order to create a long tetm stable KP. Articial hydrogels differ in many kinds from the natural hydrogel, the collagen, of the cornea.In this presentation, the fundamental mechanisms of understanding hydrogels and there limits of use as well the modification of artifical hydrogels to adopt the requirements to be be used for long-term stable KP are discussed. Furthermore it will be shown, how the perfomrkance of a KP can be predicted in vivo and the results can be used to use this expermental results and translate them into clinical application. Different kinds of KPs based an hydgrogel materials, native based and artifical materials, their limits, use andtheir performanance will be presented in order to contribute to the medical advances in corneal science.

\section{2}

Functional properties of nerves regenerating within bioengineered corneas

GALLARJ

Instituto de Neurociencias, San Juan de Alicante

Bioengineered substitutes for corneal extracellular matrix based on crosslinked collagen promote nerve regeneration. Electrophysiological recording of corneal nerves at different time points after implant showed no electrical activity inside the implant 1 day-2 months after surgery. Active mechano- and polymodal nerve terminals showing increased spontaneous activity in absence of intended stimulation were recorded inside the implants at 3 months. At 8 months after the implant, active mechano- and polymodal nociceptors, and cold thermoreceptors with normal response to natural stimulation were recorded at the implant area. Spontaneous firing of nociceptive units was higher and spontaneous activity of cold thermoreceptive units was slightly lower than in intact eyes. Corneal nerves regenerate into collagen-based materials, starting to be functional at 3 months post surgery. This is on the basis of the recovery of corneal sensitivity with time in transplanted corneas. The altered activity observed in regenerating corneal nerves is probably due to an increased expression of ion channels induced after nerve lesion, and may explain the presence of abnormal sensations experienced by patients after keratoplasty.(SAF2011-22500 MINECO)

\section{- 4634}

Next generation corneal implants as alternative to high risk donor transplantation

GRIFFITH M (1, 2), ISLAM M (2, 1), IAKYMENKOS (3), PASYECHNIKOVA N (3), BUZNYKO (3)

(1) IGEN Centre, Dept. of Clinical and Experimental Medicine, Linköping University, Linköping

(2) Swedish Medical Nanoscience Centre, Dept. of Neuroscience, Karolinska Inst., Stockholm

(3) Filatov Institute of Eye Diseases \& Tissue Therapy of the NAMS of Ukraine, Odessa

We have previously shown that recombinant human collagen (RHC)-based hydrogels fabricated into cell-free corneal implants are able to induce regeneration of the corneas of 10 patients in a Phase 1 clinical study. At four years post-operative, all implants were stably integrated without the use of sustained immunosuppression. Corneal tissue and nerves were regenerated. For use in high risk corneal transplantation such as burns and cases of persistent, non-infectious ulcers, we had reinforced the collagen implants with a second network of svnthetic phosphorylcholine to form a hydogel comprising of interpenetratig networks of collagen-phosphorylcholine (RHC-MPC). In a pilot study within a hospital setting, in accordance to the Declaration of Helsinki, with ethical permission and informed consent, four patients were grafted with cell-free RHC-MPC implants after manual excision of the ulcerated areas. After six to 12 months postgrafting, all implanted corneas had a stable epithelium and visual acuity improved 1-2 lines compared to pre-operative levels. Our pilot study shows RHC-MPC implants are safe in patients and appeared to withstand the adverse microenvironment within the corneas with severe pathology and high risk of rejection. 
164

- 4635

Biocompatibility and functionality of a tissue-engineered stroma transplanted in the living animal

BRUNETTE I, BOULZE PANKERT M, GOYER B, ZAGUIA F, BAREILLEM. PERRONMC, LIUX, DOUGLAS CAMERON I, PROULX S

Montréal

Corneal tissue shortage has become a major concern worldwide, which has motivated the search for alternative solutions to eye bank human eyes for corneal transplantation. Biocompatibility and functionality of stromal lamellar grafts tissue-engineered in vitro from corneal stromal cells using self-assembly approach, without the addition of exogenous material or scaffold, and transplanted in vivo in the cornea of an experimental model open the way to a new therapeutic modality for the rehabilitation of diseased corneas.
- 4636

KPro Biomaterials

AVADHANAM VS (1, 2), INGLAVE G (3), SANDEMANS (4), LIU CSC (1, 2)

(1) Sussex Eye Hospital, Brighton

(2) Brighton and Sussex Medical School, Brighton

(3) Brighton

(4) University of Brighton, Brighton

Research into keratoprosthessis(KPro) is at least 200 years old. In the early days, when corneal pathology was recognised as a specific cause for blindness, its treatment involved a replacement of the diseased cornea with artificial materials. Afterwards cadaveric corneal transplantation has proven to be more successful than prosthetic corneal substitutes. However, the need for keratoprostheses is still present. Scores of

KPro devices have been proposed and many biomaterials have been investigated. Only 2 devices, the osteo-odonto-keratoprosthesis (OOKP) and Boston type1 KPro, have proven to be viable in the longer term although complications do occur. A true artificial cornea in form and function is still a fantasy and may not be clinically feasible. Since corneal involvement can be secondary to ocular surface disease, a universal KPro that cures every type of corneal blindness may not be a pragmatic solution. Development of biomaterials may be tailored towards KPros designed specifically for different ocular pathology rather than towards a one size fit for all' device. Translation of laboratory work into clinical practice for KPro development will require better concordance between clinicians and scientists. 
- 4641 / S100

Vascularized vitreous seeding regression following systemic and intravitrealchemotherapy for advanced "late" retinoblastoma

HADIISTILIANOU T (1), DE FRANCESCOS (1), BORRI M (1), MICHELI L (1), GALIMBERTID (2), CAINIM (2), GALLUZZIP (3), MENICACCIF (4)

(1) Ocular Oncology, Ophthalmology unit, Siena

(2) Paediatrics, Siena

(3) Neuroradiology, Siena

(5) Ophthalmology unit, Siena

Purpose To report the regression of vascularized vitreous seeding in advanced "late" retinoblastoma

Methods Case report8 year old girl with bilateral disease. The affected eye was the left eye (group D - ABC classification- and group VB -Reese Classification-) while the right eye presented two peripheral retinomas.

Results Complete remission of the massive vascularized vitreous seeding was achieved and multiple retinal tumors regressed following six cycles of systemic chemotherapy (three drugs-carboplatin etoposide and vincristine) and four intravitreal injections with Melphalan (20 micrograms).Retinal tumors presented type I regression (calcified tumors) while massive vitreous seeding was replaced by spidernet fibrotic formations.

Conclusion This case highlights the successful combination of three-drugs systemic chemotherapy with intravitreal melphalan in advanced "late" retinoblastoma presenting with vascularized vitreous seeding and multiple retinal tumors.

\section{- 4643}

Cavitary retinoblastoma: clinical features and treatment outcome

HADIISTILIANOU T (1), DE FRANCESCOS (1), BORRIM (1), GALLUZZI P (2), DELUCA MC (3), BRACCOS (2)

(1) Ocular Oncology, Ophthalmology unit, Siena

(2) Neuroradiology, Siena

(3) Opthalmology, Reggio Emilia

Purpose To describe the ophthalmoscopic features of seven cavitary retinoblastomas and treatment outcome

Methods Patient demographics, tumor characteristics, ultrasounds, OCT and MRI findings and response to treatment (systemic chemotherapy, intrarterial chemotherapy, focal therapy, external beam radiotherapy)were recorded.

Results Two cases were bilateral, three cases were unilateral. All cases were unifocal. The median age at first observation was 18 months. No vitreous or subretinal seeds were present. Two tumors were treated with systemic $\mathrm{CH}$ followed by $\mathrm{IA}-\mathrm{CH}$; one tumor with systemic $\mathrm{CH}$ and radiotherapy; four tumors with IA-CH.All cases received adjuvant photocoagulation. Globe salvage was achieved in six out of seven cases (85.7\%) Three months after treatment the mean reduction in tumor thickness was 19\%. During a mean follow-up of 18 months one case recurred and one gave rise to subretinal seeds

Conclusion Cavitary retinoblastomas do not show a substantial decrease in size after treatment and do not display dramatic ophthalmoscopic changes.
- 4642

Anterior ischemic optic neuropathy following selective intraarterial chemotherapy for unilateral retinoblastoma

DE FRANCESCOS (1), MENICACCI F (2), BORRI M (1), HADIISTILIANOU T (1)

(1) Referral Center for Retinoblastoma, Siena

(2) Ophthalmology, Siena

Purpose To describe anterior ischemic optic neuropathy (AION) observed in a child treated with selective intra-arterial chemotherapy (SIAC) for relapse in unilateral retinoblastoma.

Methods Charts and retcam images of patient were reviewed, and procedures data were collected. The ophthalmoscopic features at diagnosis suggested for a malignant transformation of a retinoma/retinocytoma. Fluorangiographic and MRI findings were evaluated.

Results AION spontaneously resolved within 4 weeks.

Conclusion AION could be a possible transient side effect of repeated intra-arterial chemotherapy.
- 4644

Proteomic analysis of aqueous humor in retinoblastoma patients: a focus on intra-arterial chemotherapy effects

MICHELI L (1, 2), LANDI G (2), MORIGGIM (3), VANNONID (2), VASSO M (3), MENICACCI F (1), GELFI C (3), LEONCINI R (2), HADIISTILIANOU T (1)

(1) Neurosciences and medical-surgical sciences, Siena

(2) Medical Biotechnologies, Siena

(3) Bioimagine institute and molecular physiology-CNR, Milan

Purpose Proteomics can be a useful tool to understand how therapies act and affect on the molecular composition of ocular fluids. In this retrospective study we focus on how the aqueous humor (AH) proteic pattern from retinoblastoma (RTB) patients is modified by intra-arterial(IA)treatment.

Methods We enrolled 22 RTB patients(Reese stage VB or ABC group E) undergoing enucleation and 20 normal subjects undergoing cataract surgery(CTR). Five of 22 patients presented with associated secondary glaucoma (SG) whereas 17 had no secondary glaucoma (NSG);8 of 17 NSG received IA Melphalan (CHM). Pools were firstly investigated with Bradford method and SDS-PAGE and then,analyzed using 2D-DIGE coupled to mass spectrometer.DIA and BVA were fully automated with 2D DeCyder" and statistical analysis was performed with EDA ${ }^{\circ}$ module.Significant spots were In-Gel digested and identified with Maldi-TOF/TOF.

Results According to Bradford method,CTR protein content was not significantly different from CHM.After melphalan treatment, total protein contents decreased about $70 \%$ while in respect to all non-treated patients were significantly different(Tamhane $\mathrm{p}<0.01) . P C A$ analysis underlines high distance either between CTR pool and SG/NSG pool or CHM and RTB.In particular the analysis of single protein shows that $\mathrm{CHM}$ pool is characterized by a high level of Crystallin and pro-inflammatory proteins and CTR pool is characterized by a high level of Epithelium Growth Factor.

Conclusion IA chemotherapy with Melphalan modifies the AH proteic pattern revealing the presence of Crystallin family and pro-inflammatory proteins. This opens new horizons to further explore local pharmacological effects of new drugs. 


\section{- 4645 \\ Intraocular Metastasis as presentation of unknown systemic neoplasia}

SAORNIL ALVAREZM (1), RAMOA R (1), GARCIA-ALVAREZ C (1) MUNOZM (2) LOPEZ-LARA F (3, 4)

(1) Intraocular Tumors unit, Hospital Clinico Universitario, Valladolid

(2) Reasearch unit. Hospital Clinico Universitario, Valladolid

(3) Intraocular Tumors unit. Hospital Clinico universitario, Valladolid

(4) Oncology \& Radiotheraphy. Hospital Clinico Universitario, Valladolid

Purpose To assess the frequency of metastases as first presentation of systemic disease in a referral clinic, and to identify clinical characteristics.

Methods Retrsopective study of consecutive csees diagnosed of intraocular metastasis at the referral intraocular tumors Unit. General and clinical characteristics were registered in a database design in Noraybanks (software approved by the research committe. Descriptive analysis was performed with SPSS 20.0.

Results Between 1990 and 2013,21 patients were diagnosed of intraocular metastasis with a mean age of 62.7 years (31-89). Most frequent primary tumors were breast (47.6\%) and lung (23.8\%).Bilateral tumor was observed in 4 cases.The intraocular tumor was the first manifestation of the neoplasia in 13 cases (61.9\%).Primary tumor was breast in $46.2 \%$ and lung in $38.5 \%$, eshophagus in $7.7 \%$ and stomach in $7.7 \%$. Reason for consultation was decreased vision in all cases. All 13 cases were unilateral and $75 \%$ as a solitary mass. Tumor was located at posterior pole in $69.2 \%$ and in all cases behind the equator.Diagnosis of primary tumor was performed by systemic studies and only one patient required intraocular biopsy. Ragrading the treatment, 7 cases were controlled with systemic chemotherapy, 4 cases required additional external beam radiotherapy and only one underwent to enucleation due to associated neovascular glaucoma

Conclusion When intraocular metastases are the first presentation of unknown neoplasia used to be solitary non pigmented mass with retinal detachment.Early diagnosis is essential to settle prompt treatment to preserve visual function and improve the patient's prognosis.
- 4647

Genetic analysis of an orbital metastasis from a primary hepatic neuroendocrine carcinoma

HEEGAARD S (1, 2), VON HOLSTEIN S (2), PRAUSEJ (1), STENMAN G (3) (1) Eye Pathology Institute, Department of Neuroscience and Pharmacology, University of Copenhagen, Copenhagen

(2) Department of Ophthalmology, Glostrup Hospital, University of Copenhagen,, Copenhagen

(3) Sablgrenska Cancer Center, Department of Pathology, University of Gothenburg, Gothenburg

Purpose To give an overview of neuroendocrine carcinoma metastases to the eye region

Methods Critical review of the present literature demonstrated by a patient case

Results A 71-year-old female with a known history of primary hepatic neuroendocrine carcinoma, presented with a visual defect, proptosis and restricted eye movements of the right eye. Biopsies from the orbit and from the primary hepatic neuroendocrine carcinoma showed similar morphological and immunohistochemical features and high-resolution, array-based comparative genomic hybridization demonstrated loss of one copy each of chromosomes 3 and 18, and gain of 1q both in the primary hepatic neuroendocrine carcinoma and in the orbital tumour. The orbital mass was diagnosed as a metastasis from the primary hepatic neuroendocrine carcinoma.

Conclusion Primary hepatic neuroendocrine tumours are very rare, and the orbit is an extremely rare location for a neuroendocrine carcinoma metastasis. This is the first reported case of an orbital metastasis with origin from a primary hepatic neuroendocrine carcinoma.

\section{6}

Unusual lesion of the orbit in a larynx cancer patient

GRISHINA E

Ophtalmology, Moscow

Purpose Orbital metastasis is uncommon accounting for $15 \%$ of all orbital malignant umors.Aim. To present an unusual orbital lesion in a larynx cancer patient.

Methods 66-year-old male had laryngectomy due to larynx cancer 3 years ago. Histological study revealed squamous cell non-keratinous carcinoma. Following 3 years, an infiltrate under the orbital roof that provoked recurrent upper eyelid edema, 3-mm exophthalmos and mild limitation of upward movements was revealed. No larynx cancer recurrence or other organ lesions were detected.

Results Transcutaneous orbitotomy was performed. Intra-op, living helminth Dirofilaria and its granuloma were removed.

Conclusion In a suspected orbital metastasis, histological verification of the lesion is required.

- 4648 / S110

Suspicious choroidal mass - an interdisciplinary challenge

GRAEFFE, GRIESHABER $M$, MEYER P

Eye Clinic, Basel

Purpose To highlight the importance of interdisciplinary work up based on a patient with presumed choroidal metastatic of unknown primary tumor.

Methods Clinical-pathological case report

Results A 57 year old woman was referred to our clinic because of a progressive vision loss in her right eye for seven days. Loss of vision field and light flashes followed 3 days later. General medical history was inconspicuous and last general check-up including gynaecologic examination performed by general practitioner was apparently normal. At first presentation, a yellowish choroidal mass in the inferior part of fundus was found clinically. Further diagnostic exams like ultrasound, OCT, fluorescence angiography and MRI of the orbit were suspicious for a choroidal metastasis of unknown primary tumor. An immediate interdisciplinary work-up with gynaecologists and oncologist was performed; histology of a punch biopsy of the left mammae confirmed the suspected finding of a multiple low differentiated invasive growing ductal carcinoma. Furthermore, metastasis of the lung and axillary lymph nodes were found. Because of the multiple metastases, a combined chemotherapy and radiation therapy was initiated. Conclusion Choroidal mass is indicative for metastasis which requires immediate interdisciplinary work-up even in the absence of a positive medical history. Since biopsy of the choroidal mass should not be performed, the diagnosis was made by the biopsy of the primary tumor, showing a metastasized aggressive breast tumor. Thanks to efficient interdisciplinary work up, local and systemic therapy saved vision in her right eye and best palliative care was offered. 
- 4651

Mechanical performance of the lens capsule in accommodation

BURD H

Department of Engineering Science, Oxford University., Oxford

The human lens consists of a relatively stiff capsule surrounding a soft lens substance. It is generally understood that an intralenticular pressure exists within the lens.A simple mechanical model, in which the capsule is represented as a fluid-filled membrane, suggests that any intralenticular pressure will cause bi-axial tensile stresses to occur in the capsule near the optical axis. In the equatorial region, however, the circumferentia stresses in the capsule are found to be compressive. This indicates that wrinkles may occur in this region.Images of isolated human lenses (e.g. Rosen et al. 2006) typically indicate a smooth outline when viewed in the sagittal plane (suggesting that meridional stresses are tensile at all locations). When viewed in the frontal plane, however, wrinkles are typically visible around the equator. These observations are consistent with the simple capsule model.To investigate the influence that capsule wrinkling might have on the accommodation mechanism, a finite element analysis is conducted, of a 29-year lens, in which equatorial wrinkles are included in the model. The results indicate that equatorial wrinkles have a modest, but significant, influence on the accommodation amplitude.

- 4653

Mechatronic system to restore accommodation

GUTHOFF RF

Rostock

The Artificial Accommodation system is a mechatronic approach to restoring accommodation. It consists of an autonomous device that will be implanted into the capsular bag of the eve similar to implantation of intraocular lenses today. The device measures accommodation demand and accordingly adjusts its refractive power by means of an integrated active optical element. Realization of this far reaching vision requires development of the respective subsystems, their integration into a small hermetic and biocompatible housing and adapted surgical procedures. Development of subsystems is well under way and first integration steps are being taken starting from macro scale down to target size. In this talk, the concept of the overall system is described and the results obtained for the different subsystems are discussed.
- 4652

Brillouin spectroscopy and lens refilling

STACHSO

Department of Ophthalmology, University of Rostock, Rostock

The confocal Brillouin spectroscopy is an innovative measurement method that allows the non-invasive determination of the rheological properties of materials. Its application in ophthalmology can offer the possibility to determine in-vivo the biomechanical properties of transparent biological tissue such as the crystalline lens. This seems to be a promising approach concerning current presbyopia research. Due to the spatially resolved detection of the viscoelastic lens properties, a better understanding of the natural aging process of the lens and the influences of different lens opacities on the stiffness is expected. From the obtained spectral data the relative protein levels, the relative refractive index profile and the relative density profile within the lens tissue can be derived. A measurement set-up for confocal Brillouin microscopy based on spectral analysis of spontaneous Brillouin scattering signals by using a high-resolution dispersive device is presented. In-vitro and in-vivo test results on native and artificial lenses are presented and evaluated concerning their rheological significance.

4654

The AkkoLens Lumina accommodative intraocular lens

ROMBACHM

Breda

The AkkoLens Lumina accommodating intraocular lens is located within the ciliary muscle which drives the lens directly; it is not in the capsular bag which has no further role after the implantation. The Lumina is designed to provide $\sim 4 \mathrm{D}$ of accommodative power using small movements requiring little force. The Lumina is implanted with a standard butterfly cartridge through a $2.8 \mathrm{~mm}$ incision making the surgery suture-free. In ongoing clinical trials, the Lumina has been placed in $~ 65$ eyes with phenomenal results; patients see near intermediate and far with all the data confirming this. The visual acuity and contrast sensitivity of the Lumina are comparable to the high-quality monofocal IOL used as the control lens. 
168

SIS: Accommodation

- 4655

Summary and discussion

BARRAQUER R, MICHAEL R

Barcelona

We will conclude our SIS with an overview of additional technologies to restore accommodation. This should open a short a discussion between our speakers and the audience about the most promising future approach to resolve presbyopia. 
$-4661$

FRO 2014: Exploring the role of cis-acting pre-mRNA splicing dynamics in the pathogenesis and treatment of Stargardt disease

BAUWENS M (1), DE BAERE E (1), LEROY BP (2)

(1) Center for Medical Genetics Ghent, Ghent University Hospital, Ghent

(2) Department of Ophthalmology, Ghent University Hospital, Ghent

To functionally study the effect of cis-acting splice site mutations of ABCA4 found in Stargardt patients in vitro and in vivo. To increase our insights into the role of pre-mRNA splicing in the pathogenesis of Stargardt disease. To explore antisense oligonucleotidemediated treatment in patient-derived cells. To uncover novel treatment strategies for Stargardt disease
- 4662

FRO 2014: Shine a light on glaucoma: an optogenetic approach towards retinal ganglion cell protection

DEKEYSTER E, GEERAERTS E, MOONS L

Neural Circuit Development and Regeneration Research Group, Leuven

Our overall objective is to investigate whether modulation of neuronal activity in the superior colliculus (SC), the principal retinal target structure and main extraretinal neurotrophic factor source in mice, can protect against chronic RGCloss, experimentally induced using the well-establised optic nerve crush (ONC) mouse model of glaucoma. For activity modulation, we will make use of the novel revolutionary technology of optogenetics. More precisely, in this project we aim to: 1) validate the genetic labeling of SC neurons with AAV vectors, carrying an stable-step function opsin (SSFO) under control of a neuronal (CMV) promoter; 2) test the ability to chronically stimulate the transduced cells; and 3) evaluate the potential of chronic optogenetic modulation of SC neuronal activity to promote RGC survival in the optic nerve crush model.

$-4664$

FRO 2014: ROCK as a potential target in the treatment of different retinopathies

HOLLANDERS K, SPILEERS W Catholic University of Leuven, Leuven

The aim of this project is to investigate the therapeutic effect of ROCK-inhibition in two mouse models for ischemia-related retinopathy, diabetic retinopathy (DR) and retinopathy of prematurity (ROP).1) The first objective of this project is to elucidate the in vivo role of ROCK on the process of inflammation and angiogenesis in ischemic retinopathies. Therefore, the efficacy of a potent ROCK inhibitor will be compared to anti-VEGF therapy (DC101), using two different mouse models.2) The second objective is to evaluate the possible neuroprotective effect of ROCK inhibition. 
- 4665

FRO 2014: MMP-2 as a potential benefactor in zebrafish optic nerve regeneration

LEMMENS K, BOLLAERTS I, MOONS L

Neural Circuit Development and Regeneration Research Group, Leuven

The zebrafish retinotectal system proves to be a valuable research model to investigate regenerative strategies of damaged retinal ganglion cells (RGCs) and thus holds great promise towards identifying new regenerative molecules. Recent research performed in our lab shows that retinal broad-spectrum MMP inhibition seriously reduces retinotectal regeneration after optic nerve crush $(\mathrm{ONC})$ in the adult zebrafish. Based on preliminary results, we now intend to determine the exact role of MMP-2 in zebrafish RGC axonal regrowth and identify underlving molecules, which might ultimately serve as new therapeutic targets for the injured mammalian CNS. Hereto, we will pursue the following goals:(1) Determination of the regenerative potential of RGCs after retinal MMP-2 inhibition/knockdown in zebrafish subjected to ONC.(2) Proteomic identification of MMP-2 regulated pathways underlying a successful regeneration in the zebrafish visual system.

$-4667$

FRO 2014: Potential implications of epigenetic changes in human retinal pigmented epithelium during diabetic retinopathy

SALIK D

Bruxelles

Diabetic retinopathy (DR) is a major cause of blindness. Prevention of DR is mainly based on control glycaemia however in vivo and in vitro evidences demonstrate that vascular complications can occur despite adequate glycemic control due to a process called "metabolic memory". One of the very new hypothesis to explain the metabolic memory involves epigenetic modifications that prevent retinal cells from returning to their physiological status even when the exposure to stimuli has ceased. Our goal is to study in vitro the egigenic modifications of human retinal pigment epithelium ( hRPE) in response to glucose in order to identify new therapeutic targets for diabetic retinopathy. Studies of the epigenetic field, represents an original new approach with consistent and applicable methodology to investigate these mechanisms.
- 4666

FRO 2014: Automated detection, classification and notification of pathological corneas using the Oculus Pentacam

RUIZ HIDALGO I $(1,2$

(1) Ophthalmology department, Antwerp University Hospital, Edegem

(2) Faculty of Medicine, Antwerp

To develop an automated classification system for normal and abnormal corneal topography based on data obtained from the Pentacam, which can be used for the following purposes:1. To investigate the variability of corneal topography.2. To automatically detect pathological corneas such as keratoconus, high astigmatism, postrefractive surgery and early onset of edema or corneal dystrophies (e.g. Fuchs dystrophy) before it is visible with the slit-lamp.3. To create an add-on program to the Pentacam software that gives a warning message with a probability of belonging to a disease group when a new patient is measured. 
- 4671

Analysis of the vitreomacular interface in a French populationbased study (The MONTRACHET study: Maculopathy, Optic Nerve, nuTRition, neurovAsCular and HEarT diseases)

BOUCHE-PILLON J (1), KOEHRER P (1), BINQUET C (2), TZOURIO C (3), BRONAM (1), CREUZOT-GARCHER C (1)

(1) Ophthalmology, Dijon

(2) Epidemiology, Dijon

(3) Epidemiology, Bordeaux

Purpose To analyse prevalence and distribution of vitreomacular adhesion (VMA) in a french population over 75 years old within MONTRACHET study.

Methods Three city study (3C) included 9294 subjects aged 65 years or more from three cities (Bordeaux, Dijon and Montpellier) in 1999. After a 10 year follow up Dijon's cohort had a complete ophthalmic examination in the MONTRACHET study. Vitreomacular interface (VMI) was analysed with a spectral-domain OCT. VMA of each patient was classified according to the IVTS (International Vitreomacular Traction Study) criteria, defining the presence of vitreomacular adhesion (VMA), vitreomacular traction (VMT) isolated or associated with a foveal distorsion

Results One thousand one hundred fifty three subjects were included in the study, the median age was 84 [79-99] years. Prevalence of vitreomacular adhesion (VMA) was $12.2 \%$. VMA affected both eyes in $28.5 \%$ of cases ( 80 patients). The median size of the diameter of vitreous attachment to the macular surface was 1377 [50-5639] um. Attachment was focal ( $<1500$ um) for $523 \%$ of cases (147 eyes) and broad $(>1500$ um) for $47.7 \%$ of cases (134 eves). Prevalence of isolated VMT was $0.9 \%$ (22 eves) and was focal $(<1500 \mu \mathrm{m})$ for all cases. Prevalence of lamellar holes was $0.6 \%$ (13 eyes) and macular pseudoholes was $0.2 \%$ ( 4 eves). Prevalence of full-thickness macular hole was $0.01 \%$ ( 2 eyes). ARMD (Age Related Macular Degeneration) was associated with VMA in 4 eyes $(2.49 \%)$

Conclusion The analysis of vitreomacular interface (VMI) is interesting due to the emergence of enzymatic vitreolysis. These results provide new data in old population over 75 .

\section{- 4673 / F098 \\ Comparison of ocriplasmin efficacy and treatment guidance as determined by European regulatory agencies}

KOROBELNIK

Chef du Service d'Ophtalmologie Hôpital Pellegrin, Bordeaux

Purpose Ocriplasmin, a truncated form of human plasmin, has proteolytic activity against components of the vitreous body and the vitreoretinal interface.

Methods Results from placebo-controlled trials designed to determine efficacy and safety of ocriplasmin for the treatment of VMT were used in the determination by the French National Health Authority (Haute Autorité de Santé, or HAS), the National Institute for Health and Care Excellence (NICE), and the Isntitute for Quality and Efficiency in Healthcare (Institut fur Qualitat und Wirtschaftlichkeit in Gesundheitswesen, or IQWiG). Different subgroups according to presence or absence of anatomic features and visual acuity (VA) were analysed.

Results All 3 regulatory agencies recommend ocriplasmin for the treatment of VMT including when associated with $\mathrm{MH}$ less than or equal to 400 microns. The HAS recommends ocriplasmin for the treatment of adult patients with VMT for whom symptomatology does not require immediately a vitrectomy, at the earlier stage of disease. IQWiG reported indication of an added benefit for patients with mild (VA $>60$ ETDRS letters) to moderate visual impairment (VA 35-60 ETDRS letters). According to the NICE guidance, ocriplasmin is recommended as an option for treating VMT in adults only if an epiretinal membrane is absent, with or without severe symptoms.

Conclusion The benefit of ocriplasmin for the treatment of VMT including when associated with FTMH of 400 microns or less has been evaluated and confirmed by Europe's leading regulatory agencies. Minor differences exist in the recommendations from each country, based on anatomic features (absence of ERM), visual acuity, and severity of symptoms
- 4672 / F108

Posterior hyaloid findings as visualized by SD OCT

KRASTEL H (1), HOFMANN F (2), BAIER D (2), KAHLERT CHR (3), KUZNIAR A (4), HARDER B (4), JONAS J (5)

(1) Dept. of Ophthalmology, University Eye Center, Mannheim

(2) Oculus Optik Geräte GmbH, Wetzlar

(3) Dept. of Ophthalmology, Klinikum Chemnitz, Chemnitz

4) Dept. of Ophthalmology, University Medcal Center, Mannheim

(5) Mannheim Medical Faculty, University of Heidelberg, Mannheim

Purpose To direct attention to the structures of the posterior hyaloid as visualized by SD OCT, and to improve understanding of vitreo-retinal biomechanics.

Methods Findings were collected from a Wetzlar study cohort of subjects who gave their informed consent to gather experience in vitreal SD OCT. In addition, OCT images were worked up retrospectively from the Mannheim pool of recordings. Exams were performed by the RS 3000 SD OCT (Nidek) or by the Spectralis SD OCT (Heidelberg engineering). Images underwent a modified processing to favour the visualization of vitreous structures

Results Observations range from a completely attached and homogeneous hyaloid with regular foveal profile at the age of 75 , to premacular vitreal bursae and intravitreal acunae, regions of low and high density within an apparently disintegrating hyaloid, intravitreal condensates and supposed cell aggregations. Various patterns of holes within the posterior limitating vitreal membrane appeared in connection with vitreoretinal and vitreo-papillar traction of a partially detached vitreous

Conclusion OCT visualization of vitreous structures contributes to our understanding of vitreal degradation and vitreo - retinal interaction.

Commercial interest

4674

Cystoid macular edema after epiretinal membrane surgery: Incidence, risk factors and evolution with treatments

LEROY B, CONART JB, SELTON J, HUBERT I, ANGIOI K, BERROD JP CHU Nancy - Hôpital Brabois, Nancy

Purpose To report the incidence, the risk factors and the evolution after treatment of cystoid macular edema (CME) complicating epiretinal membrane (ERM) surgery.

Methods This study included 647 consecutive patients with ERM operated by 23-gauge parsplana vitrectomy between January 2011 and July 2013. Combined phacoemulsification of the lens was performed in $71 \%$ of cases. Patients were considered as having a CME if they presented augmentation of central macular thickness with presence of cysts on OCT scans in the first year after surgery. Patients with history of retinal detachment, vein occlusion, uveitis, diabetic macular edema or neovascular AMD were excluded

Results The incidence of CME was $26 \%(16 / 605)$ occurring within an average time of $129 \pm 101$ days. Predictive factors were the presence of preoperative CME on OCT scans $(\mathrm{p}=0.04)$ and preoperative prostaglandin use $(\mathrm{p}=0.02)$. Combined phacoemulsification was not identified as a risk factor Treatment consisted in topical applications of nonsteroidal anti-inflammatory drugs (NSAIDs) associated with dexamethasone and/ or acetazolamide for a mean duration of 4 months. Intravitreal or subconjonctival corticoids injections were performed in 6 patients. A two-line or more improvement in visual acuity was achieved respectively in 6 of $14(43 \%)$ and 4 of 6 patients (67\%). A dissipation of cysts on OCT scans was observed respectively in 4 of $14(29 \%)$ and in 5 of 6 patients $(83 \%)$.

Conclusion The incidence of CME after ERM surgery was 2.6\%. Preoperative prostaglandin use and preoperative CME were associated with an increased risk of postoperative CME. Treatment with NSAIDs was effective in about one third of patients, corticoids injections in 5 of 6 patients. 
$\bullet 4675$

Cytologic analysis in vitreo-retinal surgery

ARNDT C (1), DUCASSE A (1), DIEBOLD MC (2), BOULAGNON C (2)

(1) Ophtalmologie, Reims

(2) Pathology, Reims

Purpose Proliferative vitreoretinopathy (PVR) is the leading cause of failure in retinal detachment (RD)surgery. The migration of retinal pigment epithelial cells and the proliferation of the extracellular matrix has been associated with PVR. The purpose of this prospective study was to compare the cellular contents of the vitreous of patients undergoing RD surgery with patients scheduled for epiretinal membrane surgery (ERM).

Methods The vitreous samples of patients with ERM and RD were obtained at the initial phase of surgery. A immunocytochemical labeling was performed in all cases with AE1/AE3, CD68, Vimentin and GFAP antibodies. The cytological identification was performed by a trained pathologist unaware of immunocytochemical labeling. The concentration of vitreous cells were analyzed semi-quantitatively.

Results 14 vitreous samples were analysed, 9 patients with RD and 5 undergoing ERM. In the RD group, the cytological findings correlated with the results of immunolabeling. Hyalocytes (labeled with vimentin only)were present in all cases, more abundant in RD cases. Macrophages (labeled with vimentin and CD68) were found in 10/14 cases Macrophages were more abundant in RD cases $(\mathrm{p}<0,05)$. Only in one case of RD, GFAP positive cells were found, in which PVR was particularly severe. In 6/14 samples, glial cells were identified with AE1/AE3 without difference between RD and epiretinal membrane cases.

Conclusion Vitreous changes observed in patients with retinal detachment appear to involve particularly hyalocytes and macrophages, which could be identified with immunolabeling. Interestingly glial cells (GFAP positive)were only present in severe PVR. A link between this cell population in the vitreous and the clinical outcome remains to evaluated in a prospective study.
- 4676

Treatment of vitreomacular traction with ocriplasmin in a nonreimbursed setting

WILLEKENS K (1), PINTO LA (2), VANDEWALLE E (3), STALMANS I (1), STALMANS P (1)

(1) Ophthalmology - UZ Leuven, Leuven

(2) a. Prof. Dr. Luís Abegão Pinto: Department of Pharmacology and Neurosciences,

Faculty of Medicine of Lisbon, Lisbon

(3) Ophthalmology - UZ Leven, Leuven

Purpose Vitreomacular adhesion can lead to vitreomacular traction (VMT) and macular hole $(\mathrm{MH})$ formation. Intravitreal ocriplasmin provides a non-surgical solution to release VMT with or without macular hole. A series of 37 patients treated with ocriplasmin is presented. Since ocriplasmin was used in a non-reimbursed setting, the injection was only recommended when a high success chance was anticipated (e.g. excluding patients with a concomitant epiretinal membrane).

Methods An observational trial was conducted on 37 patients with VMT +/- MH treated with ocriplasmin with a minimal follow up time of 28 days. Patients had OCT scans of the macular and papillary region prior to the treatment and regularly in the following month. Data on papillary changes were also recorded by Heidelberg Retinal Tomography.

Results Overall, 38 eyes of 37 patients were treated (27 with VMT and 11 with VMT+MH). VMT resolved in 28 cases $(74 \%)$ and macular holes closed in 4 cases (36\%). The cup/disc ratio transiently became smaller, associated with a small increase in retinal nerve fiber laver thickness. Interestingly, formation of transient subretinal fluid was observed in the subfoveal and peripapillar region in some patients and a subfoveal drusenoid deposit disappeared in two patients after injection.

Conclusion A high success rate of VMT release can be obtained after injection of ocriplasmin when patients are carefully selected. Further research on observed transient changes in the appearance of subretinal fluid and the peripapillary nerve fiber layer thickness is needed. 
- 4721

Emerging evidence for the role of mitochondria in glaucoma

GARWAY-HEATHD

London

\section{ABSTRACT NOT PROVIDED}

$\bullet 4723$

Does normal-pressure glaucoma and high-pressure glaucoma differ in the optic nerve head appearance?

BRON AM

Dijon

Many studies have been published trying to find out some differences in the phenotype of normal-pressure glaucoma (NPG) and high-pressure glaucoma (HPG). The authors of these studies mainly focused on the "vascular signs", the visual field and the optic nerve head $(\mathrm{ONH})$ appearance of these 2 clinical presentations. However the debate is very controversial and several papers have reported that after all there is no difference in clinical presentation between NPG and HPG. In this review we will detail the pros and cons of each theory and we will provide the physician with useful practical tips leaving him/her with the following questions:Is the limit between NPG and HPG so tight?Can we find specific clinical characteristics of the $\mathrm{ONH}$ according to the clinical presentation?Should not we concentrate on the erroneous NPG diagnoses, i.e. the nonglaucomatous optic neuropathies?
- 4722

Vascular optic neuropathies versus glaucomatous optic neuropathy

SCHMETTERER L $(1,2)$

(1) Clinical Pharmacology, Vienna

(2) Center of Medical Physics and Biomedical Engineering, Vienna

Glaucoma is a group of progressive optic neuropathies characterized by excavation of the optic nerve head and associated visual field changes. Elevated intraocular pressure (IOP) is the most important risk factor for glaucoma. The pathophysiology of glaucoma is not well understood, but mechanical compression of the optic nerve head (ONH) appears to play a role. Anterior ischemic optic neuropathy (AION) is the second most common cause of optic nerve-related loss of vision and comprises non-arteritic AION (NAION) and arteritic AION (AAION). Factors that help in separating AION cases from glaucoma cases are: IOP is typically in the normal range or reduced, ONH cupping does not involve the peripapillary zones, visual field loss usually occurs in the central or cecocentral visual field and disk pallor. Whereas AION is ischemic in nature, the vascular involvement in glaucoma is still a matter of debate. The difference in appearance of the two diseases does, however, not argue against the vascular component in glaucoma.

4724

Brain pressure and glaucomatous and non-glaucomatous parameters

JONASJ

Mannheim

Eyes with normal-pressure glaucoma and eyes with high-pressure glaucoma show a similar optic disc appearance with marked differences to eyes with vascular optic neuropathies. Non-vascular, potentially barotraumatic, factors in addition to intraocular pressure (IOP) may thus play a role in glaucoma. Recent studies showed that (1) cerebrospinal fluid pressure (CSFP), arterial blood pressure and IOP are correlated with each other; (2) higher CSFP is associated with younger age, higher blood pressure and higher body mass index; (3) some patients with normal-(ÍOP)-pressure glaucoma have abnormally low CSFP, and thus an abnormally high trans-lamina cribrosa pressure difference, and a small orbital CSF-space; (4) the orbital CSF-space width is associated with CSFP; and (5) the estimated CSFP as compared to IOP correlated better with openangle glaucoma-related parameters. The orbital CSFP as counter-pressure against IOP may play a role in pathogenesis of glaucoma. 
Workshop: EEBA symposium: Innovative approaches in the treatment of corneal \& ocular surface diseases: potential new frontiers for eye, cell and tissue banking

- 4731

Penetrating keratoplasty - long-term follow-up data in low and high risk keratoplasties

FUCHSLUGERT

Düsseldorf

The talk will present long-term follow up data from the Dusseldorf Graft Registry on graft survival and outcome. Both low and high risk keratoplasty cases will be presented.

- 4733

Ultrathin DSAEK vs. Conventional DSAEK: Results from a Dutch multicentre randomised clinical trial

NUIJTS R (1), DICKMANMM (1), VANDEN BIGGELAAR FJHM (1),

BERENDSCHOT TTJM (1), VANROOIJ (2), REMEIJER L (2)

VAN DER LELIJ G (3), WIIDH RIH (4), VANMARION FW (5)

(1) University Eye Clinic Maastricht, Maastricht University Medical Center, Maastrich

(2) Rotterdam Eye Hospital, Rotterdam

(3) University Medical Center, Utrech

(4) University Medical Center, Groningen

(5) Euro Cornea Bank, Beverwijk

The Dutch Ultra-Thin DSAEK study is a multicenter randomized controlled study comparing the effectiveness and cost-effectiveness of precut ultra-thin $(<100 \mathrm{~m})$ DSAEK with standard thickness DSAEK. Fifty-eight adult patients with Fuchs endothelial dystrophy and no other visual limiting comorbidities were randomized to either ultra-thin or standard DSAEK in four tertiary medical centers in The Netherlands. Preliminary (6 months follow-up) outcomes, including: ETDRS best corrected visual acuity, refractive and topographic astigmatism, endothelial cell density, contrast sensitivity, straylight, complication profile, quality of life and vision related quality of life will be presented. $\bullet 4732$

Small "Big Bubble" Technique to optimize DALK results

BUSINM

Forli

The small "big bubble" technique limits descemetic dissection to the central $6 \mathrm{~mm}$ optical zone but uses a $9 \mathrm{~mm}$ graft with both graft and recipient wound edges cut by machine.Visual outcomes after DALK utilizing the small "big bubble" technique were evaluated in 88 eyes. After complete suture removal, BSCVA was $\geq 20 / 25$ and refractive astigmatism was $\leq 4.5$ diopters in in 21 of 27 eves $(30.7 \%)$. This variation of DALK technique can provide good visual results while limiting to a minimum the area of descemetic dissection

- 4734

Human limbal epithelial cells cultured on dense fibrillated collagen I transparent matrices

BORDERIE V, GHOUBAY D, MOSSER G Paris

Transparent ( 78 to $84 \%$ at $580 \mathrm{~nm}$ ) collagen I fibrillated matrices have been synthesized at different concentrations making use of liquid crystal properties of collagen. The $90 \mathrm{mg} /$ $\mathrm{mL}$ scaffold presents an organization that resembles the ones found in corneal stroma. Although matrices present low mechanical properties (with Young's modulus of $2 \mathrm{kPa}$ at most) they allow development of an epithelium presenting the main characteristics of the corneal epithelial cells (expression of cytokertin 3 and presence of desmosomes) and maintenance of stemness during culture (i.e. expression of Delta N p63 $\alpha$ and formation of holoclones in colony formation assay). Matrices with cultured cells feature higher transparency compared with matrices with no cells. 
Workshop: EEBA symposium: Innovative approaches in the treatment of corneal \& ocular surface diseases: potential new frontiers for eye, cell and tissue banking

- 4735

Growth factor and interleukin concentration in amniotic membrane suspension (AMS) and amniotic tissue homogenates (AMTH)

SZENTMARYN (1), STACHON T (1), BISCHOFFM (1), HUBER M (2), ZAWADA M (2), LANGENBUCHER A (3), SEITZ B (1)

(1) Department of Ophthalmology, Saarland University Medical Center, Homburg/Saar

(2) LIONS Cornea Bank Saar-Lor-Lux, Trier/Westpfalz at the Department of Ophthalmology, Saarland University Medical Center, Homburg/Saar (3) Experimental Ophthalmology, Saarland University, Homburg/Saar

Application of AMS or AMTH eye drops was suggested as a potential non-surgical treatment alternative of therapy resistant corneal epithelial defects. We determined the concentration of growth factors EGF and FGFb and interleukins IL-6 and IL-8 in AMS and AMTH following different preparation methods. In AMTH EGF concentration was about 1.7-17x and FGFb concentration about 1.2-8.5x higher than in AMS. With higher EGF and FGFb concentrations and lack of IL-6 and IL-8 in AMTH, its use may be more appropriate in treatment of corneal epithelial defects. However, the most effective preparation method and the harvesting timepoint are yet to be determined. $\bullet 4736$

What's new about contact lens complications?

KUHND

Riedlingen

The results of a study about 2260 contact lens related complications reported in Germany between 2006 to 2013 shall be presented. The analysis was done with a questionnaire including the following topics:- For how many years were the lenses worn before the damage appeared?- Which kind of contact lenses is related to the highest incidence of complications?- Where had the contact lenses been fitted and bought?Which was the wearing modality?- What kind of cleaning solutions were used?- How was the hygienic behavior of the wearer?- Which were the ophthalmological findings?Which therapy was given?- Was the contact lens wearer unable to work? The results are the following: contact lens complications occur rather in the first year of contact lens wearing and with monthly replacement lenses. Most of them are cheap lenses bought without prescription or any control through a contact lens specialist. Poor hygiene is another important risk factor as well as the kind of wearing modality. The most frequent ophthalmological finding was a contact lens associated red eye. Antibiotic drops are the most frequently prescribed therapy.The results will be discussed and recommendations will be given. 


\section{$-4741$}

The proinflammatory cytokine high-mobility group box-1 mediates retinal neuropathy induced by diabetes

ABUEL ASRAR A (1), SIDDIQUEIM (1), NAWAZM (1), GEBOES K (2) MOHAMMADG(1)

(1) Ophthalmology, Riyadh

(2) Laboratory of Histochemistry and Cytochemistry, Leuven

Purpose To test the hypothesis that increased expression of proinflammatory cytokine high-mobility group box-1 (HMGB1) in epiretinal membranes and vitreous fluid from patients with proliferative diabetic retinopathy and in retinas of diabetic rats plays a pathogenetic role in mediating diabetes-induced retinal neuropathy.

Methods Retinas of 1-month diabetic rats and HMGB1 intravitreally injected normal rats were studied using Western blot analysis, RT-PCR and glutamate assay. In addition we studied the effect of the HMGB1 inhibitor glycyrrhizin on diabetes-induced biochemical changes in the retina.

Results Diabetes and intravitreal injection of HMGB1 in normal rats induced significant upregulation of HMGB1 protein and mRNA, activated extracellular signalregulated kinase 1 and 2 (ERK1/2) cleaved caspase-3 and glutamate and significant downregulation of synaptophysin, tyrosine hydroxylase, glutamine synthetase and glyoxalase 1. Constant glycyrrhizin intake from the onset of diabetes did not affect the metabolic status of the diabetic rats, but it significantly attenuated diabetes-induced upregulation of HMGB1 protein and mRNA, activated ERK1/2 , cleaved caspase-3 and glutamate. In the glycyrrhizin-fed diabetic rats, the decrease in synaptophysin, tyrosine hydoxylase and glyoxalase 1 caused by diabetes was significantly attenuated.

Conclusion These findings suggest that early retinal neuropathy of diabetes involves upregulated expression of HMGB1 and can be ameliorated by inhibition of HMGB1.

\section{- 4742}

Apoptosis and proliferation in diabetic eyes

STEWART EA, SAKER S, AMOAKU WM

Academic Ophthalmology, University of Nottingham, Nottingham

Purpose Diabetic retinopathy (DR) is the most common cause of visual loss in the working population and the incidence of diabetes is increasing. In addition, 80\% of diabetic patients will show some form of retinopathy after 15 years, even with good glycaemic control. The mechanism of diabetic retinopathy is still not fully understood although hyperglycaemia is the main determinant. Diabetes appears to have contradictory effects on vasculature, causing reduced angiogenesis or increased angiogenesis at different vascular sites

Methods The effect of high glucose (HG) on in vitro proliferation, apoptosis and angiogenesis of retinal endothelial cells was investigated using relevant assays. Real time PCR was used to quantify signalling responses to high glucose through gene expression of key signalling molecules such as HIF and expression of VEGF.

Results HG conditions reduced the proliferation of retinal endothelial cells but no increase in apoptosis was detected, and increased VEGF prevented this response. Gene expression of HIF was increased with HG and that of VEGF decreased dependent on glucose concentration and exposure time

Conclusion Contrary to other research in this area we found that apoptosis may not play the major role in the early phase of DR, and that reduced endothelial cell proliferation precedes apoptosis. Angiogenic signalling induced by hyperglycaemia is complex and requires further elucidation.

Commercial interest
- 4744

PGE2 and PGF2a stimulation of porcine retinal arterioles in vitro is associated with $\mathrm{Ca} 2+$ activity in retinal perivascular cells

KUDRYAVTSEVA O, BEK T

Opthalmology, Aarhus University Hospital, Aarhus

Purpose Disturbances in the regulation of retinal blood flow are a hallmark of vision threatening diseases. Retinal blood flow is controlled by autoregulation, which involves $\mathrm{Ca} 2+$ activity in a recently identified population of perivascular cells with pericyte characteristics (PVCs). The purpose of the study was to identify the sources of Ca2+ recruitment in these PVCs during stimulation of porcine retinal arterioles in vitro with PGE2 and PGF2a.

Methods Porcine retinal arterioles with preserved perivascular retinal tissue were mounted in a confocal myograph, placed in a confocal microscope and loaded with Oregon Green. After addition of PGE2 (10-5 M) or PGF2a (10-5 M) arteriolar tone and fluorescence from PVCs were recorded simultaneously in the absence and in the presence of $\mathrm{Ca} 2+$ channel blockers (ryanodine, nifedipine, LOE908, CPA, 2-APB).

Results PGE2 induced significant relaxation of preconstricted retinal arterioles and $\mathrm{Ca} 2+$ activity in PVCs. The percentage of active PVCs, and the number but not the amplitude of $\mathrm{Ca} 2+$ oscillations were significantly reduced by $\mathrm{CPA}$ and 2-APB, but not by ryanodine, nifedipine and LOE908. PGF2a caused arteriolar contraction and the percentage of active PVCs, the number and the amplitude of $\mathrm{Ca} 2+$ oscillations were significantly reduced by $\mathrm{CPA}$ and $2-\mathrm{APB}$

Conclusion Ca2+ activity in PVCs accompanies porcine retinal arterioles responses to PGE2 and PGF2a Sarcoplasmic reticulum and inositol triphosphate receptors are important sources of this $\mathrm{Ca} 2+$ activity. The amplitude of $\mathrm{Ca} 2+$ oscillations seems to play a particular role in contraction of retinal arterioles. 
- 4745 / F111

Semi-automated quantification of the parafoveal capillary network in diabetic subjects

KAPSALA Z (1), PALLIKARIS A (2), MOSCHANDREAS J (3), TSILIMBARIS MK $(4,2)$

(1) Ophthalmology Department, Medical School, University of Crete, Heraklion (2) Institute of Vision and Optics, University of Crete, Heraklion

(3) Department of Social Medicine, Medical School, University of Crete, Heraklion (4) Ophthalmology-Research Acct, University of Crete, Heraklion

Purpose The detection and quantification of the parafoveal capillary network in diabetic subjects using a novel semi-automated computerized method.

Methods Using the MatLab R2011a we developed an algorithm that detects automatically the parafoveal capillary bed in fluorescein angiography (FA) images. The detection starts after delineating manually the foveal avascular zone (FAZ) in a cropped $1500 \mu \mathrm{m}$ subimage and hence excluding this area from the process. The algorithm calculates the capillary density in a $1000 \mu \mathrm{m}$ area measured from the centroid of the FAZ. The method was applied on high resolution FA images from 49 subjects (age $=43 \pm 19$ years); 13 controls, 13 subjects with diabetes mellitus (type I or II) without diabetic retinopathy (DR) findings (no DR), 15 subjects with non-proliferative DR (NPDR) and 8 subjects with proliferative DR (PDR). Using the statistical software SPSS (version 20) we assessed the mean capillary density for the mentioned subject groups. Results Capillary density in the mentioned area was found $0.86 \pm 0.17,1.01 \pm 0.22$, $0.67 \pm 0.25$ and $0.76 \pm 0.30$ degrees -1 for the studied groups, respectively $(\mathrm{p}=0.003)$. The no DR group did not differ significantly from the controls and the only statistically significant differences were those between the following three groups; control-NPDR, no DR-NPDR and no DR-PDR.

Conclusion This semi-automated algorithm could serve as a potential tool for detecting, classifying and monitoring automatically capillary abnormalities in this area. Our preliminary results indicate that the capillary density in the central 1000um area varies among different DR stages. However, a larger sample is required to confirm these initial data.

- 4747

Diabetic retinopathy and estimated cerebrospinal fluid pressure. The Beijing eye study 2011

JONAS J (1), WANG N(2), XUL L (3), XU JIE (2), WANG Y (3), YOU Q (3) (1) Department of Ophthalmology Mannheim of the University of Heidelberg, Mannheim

(2) Tongren Hospital, Beijing

(3) Beijing Institute of Ophthalmology, Beijing

Purpose We tested the hypothesis whether prevalence and severity of diabetic retinopathy are associated with cerebrospinal fluid pressure (CSFP)

Methods The population-based Beijing Eye Study 2011 included 3468 individuals with a mean age of $64.6+/-9.8$ years. Based on a previous study with lumbar cerebrospinal fluid pressure (CSFP) measurements, CSFP was calculated as CSFP $[\mathrm{mmHg}]=0.44 x$ Body Mass Index $[\mathrm{kg} / \mathrm{m} 2]+0.16$ Diastolic Blood Pressure $[\mathrm{mmHg}]-0.18 x$ Age[Years] $]-1.91$.

Results In binary regression analysis, presence of diabetic retinopathy was significantly associated with higher levels of $\mathrm{HbA1c}(\mathrm{P}<0.001)$, higher blood concentration of glucose $(\mathrm{P}<0.001)$, longer known duration of diabetes mellitus $(\mathrm{P}<0.001)$, higher systolic blood pressure $(\mathrm{P}<0.001$; ), lower diastolic blood pressure $(\mathrm{P}<0.001)$, and higher CSFP $(\mathrm{P}=0.002)$. Severity of diabetic retinopathy was significantly associated with higher $\mathrm{HbA1c}$ value $(\mathrm{P}<0.001)$, higher blood concentration of glucose () , longer known duration of diabetes mellitus $(\mathrm{P}<0.001)$, lower level of education $(\mathrm{P}=0.001)$, lower diastolic blood pressure $(\mathrm{P}=0.002)$, higher systolic blood pressure $(\mathrm{P}=0.006)$, and higher $\operatorname{CSFP}(\mathrm{P}=0.006)$.

Conclusion Higher prevalence and severity of diabetic retinopathy were associated with higher estimated CSFP after adjusting for systemic parameters. Higher CSFP through a higher retinal vein pressure may lead to more marked retinal venous congestion and vascular leakage in diabetic retinae.
- 4746 / F113

Study of endothelial progenitor cells in young diabetic patients with type 1 diabetes mellitus

TSILIMBARIS M (1), SIMANTIRAKID (1), KAPSALA Z (1), TSIKA C (1), PONTIKOGLOUC(1) MAMOULAKISD (2) PAPADAKIH(1)

(1) University of Crete Medical School, Heraklion

(2) University Hospital of Heraklion, Heraklion

Purpose Purpose: To evaluate the presence of Endothelial Progenitor Cells (EPCs) in the peripheral blood of young patients with Diabetes Mellitus Type I (T1DM).

Methods Blood samples were collected and analyzed from 23 individuals: 15 patients with DM (mean DM duration-years: 13 (range:3-31)), mean age-years: 26(9-45) and 8 controls without DM mean age-years: 32 (range:10-41). The presence of Diabetic Retinopathy (DR) was documented with slit lamp examination and fluorescence angiography, according to the Early Treatment of Diabetic Retinopathy Study (ETDRS) criteria. The EPCs were detected with flow cytometry using the following primary antibodies: anti-CD34, anti-CD45, anti-CD133 and anti-hVEGFR2.

Results Progenitor EPCs, defined as CD45-/CD34+/CD133+/VEGFR2+ cells, were detected in 10/23 samples (mean\%: 0.00069, SEM:0.000261). 7/15 patients had EPCs detected in the periphery blood (mean\%: 0.000899, SEM:0.000384). EPCs were detected in all 5 patients with PDR (mean\%: 0.000998, SEM:0.000206). Also, 1/5 patients with $\operatorname{NPDR}(0.0035 \%)$ and $1 / 5$ patients without clinically proven DR $(0.005 \%)$ had detectable EPCs. Finally, circulating EPCs were identified in $3 / 8$ individuals of the control group (mean\%: 0.000299, SEM:0.000169).

Conclusion CD45-/CD34+/CD133+/hVEGFR2+ EPCs were detected in the peripheral blood of non diabetic persons and diabetic patients with or without evidence of DR. The clarification of the presence of EPCs in the peripheral blood early in the disease course could potentially offer significant information about the pathophysiology of diabetic microangiopathy.

\section{- 4748 / F110}

Hyperosmolar stress induces TonEBP activation in human retinal pigmented epithelial cells

LIBERT S (1, 2), WILLERMAIN F (2, 3), SALIK D (1, 2), DELPORTE C (1)

(1) Laboratory of pathophysiological and nutritional biochemistry, Anderlecht

(2) CHU Saint-Pierre-Brugmann, Brussels

(3) I.R.I.B.H.M., Anderlecht

Purpose Macular edema, a frequently encountered complication of diabetic retinopathy (DR), results from alterations of the blood retinal barrier (BRB) and leads to modifications of the retinal pigmented epithelium (RPE) functions. Osmolar changes of the surrounding medium could be responsible for some modifications of the RPE functions leading to a disturbance of the retinal homeostasis.

Methods To study the modification and stability of the key hyperosmolar response factor Tonicity Enhancer Binding Protein's (TonEBP) expression in ARPE-19 cells, derived from human RPE, were submitted to hyperosmolar stress of increasing concentration and for various times.

Results In response to hyperosmolar stimulation of ARPE-19 cells, a dose-dependent increase in TonEBP mRNA and protein levels, as well as TonEBP nuclear translocation, were observed. Moreover, the mRNA levels of Aldose Reductase (AR) and Sodiumdependent Taurine Transporter (TauT), two TonEBP target genes required for increasing the intracellular ionic force, increased in a delayed manner following hyperosmolar stimulation. Additionally, SB203580, a p38 protein kinase inhibitor, but not its inactive analogue SB202474, inhibited TonEBP nuclear translocation in ARPE-19 cells submitted to hyperosmolar stimulation.

Conclusion In ARPE-19 cells submitted to hyperosmolar stress, TonEBP expression is induced, and its nuclear translocation stimulated through a mechanism involving p38 protein kinase activation. It is likely that TonEBP nuclear translocation mediates the increase of AR and TauT expression induced by hyperosmolar stress and plays a role in RPE cells osmoadaptation. 
$\bullet 4751$

Postoperative endophthalmitis

BARRAQUERR

Barcelona

\section{ABSTRACT NOT PROVIDED}

- 4753

The evidence: the intraoperative antibiotic

BARRYP

Dublin

The evidence on which the "ESCRS Guidelines on Endophthalmitis prophylaxis following cataract surgery (2013) " is based will be presented. This comprises studies from Sweden, Spain, France, South Africa, Asia, North America and Japan showing a reduction in infection rates reducing by factors of $3-22$ fold when the intracameral route was introduced.Risks and relative contraindications will also be discussed.
- 4752

The problem of bacterial resistance in medicine and in ophthalmology

GRZYBOWSKIA $(1,2)$

(1) Department of Ophthalmology. Poznan City Hospital, Poznan

(2) Chair of Ophthalmology, University of Warmia and Mazury, Olsztyn

In 2013 US Centers for Disease Control and Prevention released its first ever report on antibiotic resistance and pointed that antimicrobial resistance is one of the most serious health threats nowdays. The same year report on global risks by the World Economic Forum argued that "the greatest risk ... to human health comes in the form of antibioticresistant bacteria". Although it is mainly caused by agricultural use of antibiotics, the other actors include overuse of antibiotics for systemic infections, inappropriate durations of treatment, prophylactic use of antibiotics, use of subtherapeutic dosages of antibiotics by non-compliant patients, and misuse of antibiotics for non-bacterial infections. The most common use of antibiotic in ophthalmology include conjunctivities, intravitreal njections and post-cataract endophthalmitis prophylaxis. There is increasing number of evidance that their use should be re-considered and often limited or eliminated. Finally, limiting the use of antibiotics is consistent with both ethics of rationing and ethics of waste avoidance.

4754

The evidence: pre-operative and post-operative antibiotics

GRZYBOWSKIA $(1,2)$

(1) Department of Ophthalmology. Poznan City Hospital, Poznan

(2) Chair of Ophthalmology, University of Warmia and Mazury, Olsztyn

Postoperative endophthalmitis is an ophthalmic surgeon's most serious complication, as it is related with a high risk of visual loss. As such, many strategies are employed to minimize this possibility including preoperative, intraoperative and postoperative strategies. Although most surgeons can justify their approach, there is a paucity of prospective studies, which can serve as true evidence. The purpose of this paper is to review the literature on the subject of pre-operative and post-operative antibiotics used for prevention of postoperative endophthalmitis and to determine the effectiveness of these measures. 
$-4755$

The evidence: the role of antibiotics in intravitreal injections

$L A M W$

Department of Ophthalmology and Vision Sciences, University of Toronto, Toronto,

Ontario

The current management of many retinal conditions, including age related macular degeneration, retinal vascular occlusion, and diabetic macular edema is not curative and requires prolonged monthly intravitreal injections. The most severe complication of this procedure is endophthalmitis and accordingly, more than two thirds of retinal specialists reported the use of topical antibiotics post- injection in a recent survey. However, there is a lack of evidence to demonstrate the effectiveness of topical antibiotics in reducing the risk of post-injection endophthalmitis.We reported a large multicentre, retrospective consecutive case series, the incidence of endophthalmitis following intravitreal injections in association with different post-injection antibiotic practices. We found that the omission of routine topical antibiotic post-injection did not produce a pronounced increase in endophthalmitis rates, suggesting that the use of topical antibiotic post-injection might be unnecessary. In fact, the use of antibiotic prophylaxis, both immediately after the injection and for five days post-injection is associated with greater rates of endophthalmitis, than without antibiotics. 
- 4761

FRO 2014: Development of a new animal model for corneal endothelial research purposes

SIJNAVE D, SPILEERS W

Catholic University of Leuven, Leuven

1. To develop a new rabbit model for corneal endothelial dystrophy using an intracameral injection (ICI) of Mitomycin C (MMC)2. To investigate new pharmacological therapies in the new optimized rabbit model.
$-4763$

FRO 2014: VEGF-Trap as a novel antifibrotic strategy to prevent filtration failure after glaucoma surgery

VAN BERGEN T, STALMANS I

Catholic University Leuven, Leuven

To explore the combined inhibition of VEGF and PlGF, by the use of VEGF-Trap, as a new therapeutic strategy to prevent filtration failure:1) To investigate the most optimal administration route for VEGF-Trap2) To compare the effects of VEGF-Trap to those of the gold standard in clinical practice, mitomycin-C (MMC)3) To investigate the potential complementary effects of VEGF-Trap and MMC4) To compare the efficacy of VEGF-Trap to bevacizumab (known to be effective in improving surgical outcome in a prospective randomized clinical trial)
- 4762

FRO 2014: using targeted next-generation sequencing to identify genes underlying keratoconus

VALGAERENH

Center of Medical Genetics, Edegem

This project is part of a larger $\mathrm{PhD}$ project which aims to unravel the molecular pathology of keratoconus (KC). During the first part of my $\mathrm{PhD}$, the focus was placed on the identification of genes that underlie $\mathrm{KC}$. Using whole exome sequencing of large $\mathrm{KC}$ milies, 8 genes were identified that might be implicated in the disease pathogenicity. In this project, we will further establish the (causative) role of these genes in KC. We will develop a gene panel that will be screened in a population of approximately 750 KC patients and 750 ethnically-matched healthy individuals using next-generation sequencing (NGS). The comparison between both groups will allow identification of genes that are frequently mutated in $\mathrm{KC}$ patients. As these genes most likely play a role in the pathogenesis, identification of causative genes and their functional role in biochemical pathways will allow us to unravel the biochemical background of the disease, and form the basis for potential new forms of therapy.

$\bullet 4764$

FRO 2014: Can Xiaflex ${ }^{\oplus}$ improve glaucoma surgery outcome?

VANDE VELDE S, STALMANS I

Catholic University Leuven, Leuven

Failure of glaucoma filtration surgery due to excessive post operative wound healing is still a major problem in the treatment of glaucoma. With this project we want to examine if Xiaflex" is able to reduce fibrosis after glaucoma filtration surgery and in this way may improve surgical outcome. 
- 4765

FRO 2014: Proteomic analysis of posterior capsular plaques and capsules in developmental cataracts

VAN LOOVERENJ

Edegem

When operating children with developmental cataract surgeons are often confronted with an opaque plaque adhering to the inside of the posterior capsule. In a previous study using immunohistochemical analysis we were able to demonstrate that these posterior capsular plaques can contain collagen type II, a type of collagen which was never demonstrated in a human lens before. We also exposed the strong adherence of the anterior hyaloid membrane (containing collagen type II) to the center of the posterior lens capsule indicating a dysgenesis of Berger's space in these specific cataract cases. The purpose of this study is to increase knowledge of the biochemical composition of these posterior capsular plaques and capsules in developmental cataract by using proteomic approaches. We believe these insights will be beneficial to understand cataract formation in these children and ultimately will improve the surgical outcome of cataract surgery in these particular cases with generally worse prognosis.

- 4767

FRO 2014: Inflammatory tear cytokine profile in acute HSV epithelial keratitis

ZAKARIA N

Ophthalmology, Edegem

Herpetic eye disease presents a wide spectrum of pathology, from superficial ulceration to deep stromal scarring and vascularisation. The biomolecular changes that occur as the disease progresses are poorly understood. Therefore the aim of the project is to better characterise the acute cytokine changes in the tear film of patients with herpetic eve disease.
- 4766

FRO 2014: Exploring the role of a novel disease gene EML4 in autosomal recessive retinitis pigmentosa

VAN SCHIL K (1), LEROY BP (1, 2), DE BAERE E (1)

(1) Center for Medical Genetics Ghent, Ghent University, Ghent

(2) Department of Ophthalmology, Ghent University Hospital, Ghent

Whole exome sequencing (WES) in a patient with autosomal recessive retinitis pigmentosa (arRP) revealed a missense mutation in the Echinoderm Microtubule associated protein Like 4 (EML4) gene, affecting a highly conserved amino acid residue. The protein has been shown to be essential for microtubule formation and stabilization, and to interact with ARL6, which has been previously linked to recessive Bardet-Biedl syndrome. The major aim of this project is to assess the contribution of the EML4 gene to the arRP phenotype observed in this patient and in additional patients with overlapping retinal dystrophies (RDs) 
- 4771

Validation of a novel device to easy the preparation of endothelial graft for DMEK

MURAINEM (1), HEZ (2), TOUBEAUD (1), GUEUDRY J (1), THURET G (2)

LEFEVRES (1), GAIN P (2)

(1) Ophthalmology, Rouen

(2) Ophthalmology, Saint-Etienne

Purpose The DMEK is the only technique for endothelial graft without interface. It is however difficult to perform, especially the preparation of the graft. We present in this study a new simple device facilitating the preparation of grafts for DMEK.

Methods The device, named "Muraine Punch" is a circular trephine with a blade which was open in two places and a guard to 300 micronmeters deep. The blade was pressed against the endothelial surface so as to sever Descemet's membrane and cut part of stroma. The preparation of descemetic graft was then continued on an artificial anterior chamber using a 27 gauge cannula , reversed upwardly corneal endothelium. Three methods of assessing injury of endothelial cells (ECs) were then performed: 1 / conventional measurement of the endothelial cell density (ECD). 2 / laboratory measurement of the viable ECD by image analysis after staining of ECs by alizarin red followed an counterstaining using Dioc 6 (cytoplasm) and Hoechst (nucleus). 3/ post operatory ECD of 47 patients.

Results Histological analysis confirmed this "Muraine technique" offered a pure Descemet membrane grafts for DMEK. The ECD analysis showed consistent with that of the grafts for transfixant keratoplasty.

Conclusion The new technique with "Muraine Punch" facilitates the preparation of grafts for DMEK and causes only a minimal cell loss.

Commercial interes
- 4772

Predictive value of color Doppler imaging of central retinal artery in outcome of corneal graft

AMAOUCHEN (1), KOSKASP (2), FADLALLAHA (3), COCHEREAUI (1), BERGES O (2), GABISONE(1)

(1) Department of Ophthalmology, Fondation Rothschild, Paris

(2) Department of Medical Imaging, Fondation Rothschild, Paris

(3) Department of Ophthalmology, Hopital Hotel Dieu, Paris

Purpose To assess the value of color Doppler imaging (CDI) of the Central Retinal Artery (CRA) in predicting visual acuity post corneal graft procedures.

Methods Forty-seven eyes of 47 patients with severe corneal disease that require corneal graft were included in a retrospective cohort study. At baseline, CDI were performed for all eyes and hemodynamic parameters of the CRA were measured: Resistive Index (RI) and peak systolic velocity (PSV). All eyes underwent B mode Echography: Analysis of vitreo- retinal morphology, macula, and papilla. Inclusion criteria were the following: Corneal graft for any etiology, Best Corrected visual acuity (BCVA) less than 0.8 Log MAR, and no correct visualization of the fundus at preoperative clinical examination.

Results 2 DALK (Depth Anterior Lamellar Keratoplasty), 2 DSEAK (Descemet stripping automated endothelial keratoplasty) and 43 PK (Penetrating Keratoplasty) were performed. After 12 mouths of follow-up: 41.5\% of eyes (Group A) had visual acuity improvement (BCVA equal or superior to 2 lines Log MAR scale compared to baseline) and $58.5 \%$ of eyes (Group B)had no improvement. The mean (SD) PSV was $15.00 \pm 8.72$ in group $A$ and $11.04 \pm 5.39$ in the group B with significative difference $(\mathrm{p}=0.032)$. The mean $(\mathrm{SD}) \mathrm{RI}$ was $0.68 \pm 0.11$ in group $\mathrm{A}$ and $0.67 \pm 0.06$ in the group $\mathrm{B}$ with no significative statistical difference $(\mathrm{p}=0.864)$. However a Pearson correlation test showed a significative negative correlation between RI and BCVA ( $\mathrm{p}=0.046)$.

Conclusion In eyes with opaque cornea, the RI and PSV of the CRA may reliably predict prognosis in corneal graft. Discovering bad CDI parameters in advance allows avoiding post-operative disappointments.

- 4774

Big bubble deep anterior lamellar keratoplasty from the perspective of the novel predescemetic corneal layer

GOWEIDA $M$

Ophthalmology Department, Faculty of Medicine, Alexandria University, Alexandria

Purpose To evaluate the intraoperative findings and complications during big bubble deep anterior lamellar keratoplasty (DALK) based on the newly described predescemetic (Dua layer).

Methods A retrospective review of medical records and surgical videos of a series of patients who underwent DALK using the big bubble technique from September 2009 to March 2014.

Results One hundred and thirty four eyes were enrolled in this study. Type 1 bubble was achieved in 56 eyes (41.8\%) while in 14 eyes (10.4\%) type 2 bubble was formed, 2 eyes $(1.5 \%)$ had mixed bubble. Failure of formation of the big bubble occurred in 62 eyes (46.3\%). All eyes with type 1 bubble were completed as DALK with microperforation in 4 eyes. All eyes with type 2 bubble were converted to PKP due to large perforation except 2 eyes were successfully completed as DALK with microperforation in one eye and intact DM in the other

Conclusion Type 2 bubbles are very likely to perforate during removal of the overlying stroma, factors leading to the formation of the thin walled type 2 bubbles should be studied and better strategies to avoid perforation should be adapted. 


\section{Posters}

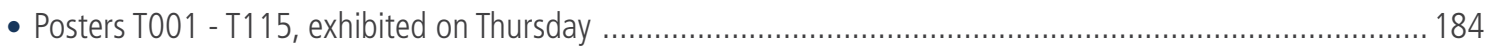

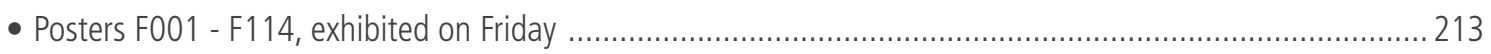

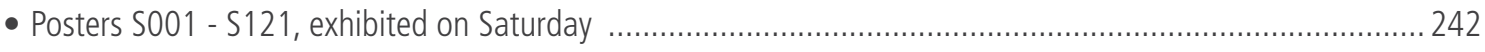


Poster session 1: Electrophysiology-physiological Optics-Vision Sciences/Glaucoma/Immunology-Microbiology/Molecular Biology-Genetics-Epidemiology/ Neuro-ophthalmology-Strabismology-Paediatric Ophthalmology-History of Ophthalmology

- T001

Association between ocular optical components and refractive errors in bank's Employees of Sari, Iran

YEKTA AA (1, 2), HASHEMI H (3), NAKHAEEMA (1), KHABAZKHOOB M (3), OSTADIMOGHADDAM H (1, 2), HERAVIANI (1, 2), AZIMIA (1, 2), YEKTA R $(4)$ (1) Optometry, Mashhad University of Medical Sciences, Mashhad

(2) Refractive Errors Research Center, Mashhad University of Medical Sciences, Mashhad (3) Noor Ophthalmology Research Center, Noor Eye Hospital, Tehran

(4) Fadak clinic, Mashhad

Purpose To investigate the association between ocular optical components and refractive errors in bank's employees of Sari, Iran

Methods In this cross sectional study 398 bank's employees of Sari, Iran were selected 232 of them used the eyes for close work (computers, etc...) for a minimum of 7 hours per day for the 5 past years. The other 166 bank's employees acted as a control group and did not did a close work more than one hour daily for the 5 past years. Age of the participants were between 25 to 40 years old. Axial length, anterior chamber depth, lens diameter and vitreous depth were measured with Nidek biometer. Corneal radius and non cycloplegic refraction performed with autorefracto-keratometer; refractive errors were checked by retinoscopy and subjective tests. There was a high correlation between equivalent sphere (ES) right and left eyes of the subjects (0.9) and data of the right eye was analyzed. Data were analyzed by using version 19 of SPSS software.

Results The results showed that the bank's employees who had excessive use of the eyes for near work have more axial length $(\mathrm{p}<0.001)$, more anterior chamber depth $(\mathrm{p}=0.003)$ and more vitreous chamber depth $(\mathrm{p}<0.001)$ than the control group. There was no significant difference between lens diameter $(\mathrm{p}=0.525)$ and cornel radius $(\mathrm{p}=0.666)$ of the two groups. There was correlation between axial length and SE by increasing in axial length $(\mathrm{p}<0.000)$. The least amount correlation was found between lens diameter and ES $(\mathrm{P}<0.000)$

Conclusion The results of this study indicated that ocular optical components changed toward myopiain people who had excessive close work.

\section{- T003}

Using statistical decomposition to improve the identification of the photopic negative response of the erg

SAROSSYM $(1,2)$, HADOUX X (3), TANG J (2)

(1) RMIT University, Melbourne

(2) Centre for Eye Research, University of Melbourne, Melbourne

(3) Irstea UMR ITAP, Montpellier

Purpose The Photopic Negative Response (PhNR) is known to be correlated with disease severity in animal models of glaucoma. The technique has found little utility in the clinical setting due to the difficulty involved in obtaining repeatable measures. . InterSession reliability has been poorly successful because of the difficulty to consistently identify and define the PhNR. In this study we propose a robust PhNR measure based on curve fit modelling of the three components of the electroretinogram (ERG) response The robustness of this measure comes from the use of a global 'shape' as a PhNR measure which is less sensitive to variations than measuring a single value on the ERG.

Methods 39 subjects with 'normal' eves were recruited. Each subject underwent 2 sessions of bilateral ERG tests at five brightness levels. Raw data obtained from the tests was exported to the R statistical program. A parametric function consisting of a linear combination of three Gaussian functions was fitted to each averaged response. For each of these components, the optimal parameters were estimated using non-linear least squares. The amplitude of the 3rd Gaussian was used as a measure of the PhNR.

Results Rapid convergence and successful fitting was achieved for 446 out of 457 traces. The amplitude of the third Gaussian correlated well with the manually measured PhNR $(\mathrm{r}=0.79)$. Bland Altman plots showed good agreement between modelled waveforms and raw data.

Conclusion By decomposing the ERG into three components the effect of variability of the baseline and the b wave can be decreased. Agreement between the 3rd Gaussian amplitude and manually estimated PhNR was reasonable. Inter-session reliability of this new measure will be further assessed.
- T002

Objective and subjective examinations for evaluation the degree of drusen-related optic nerve neuropathy

POIDA-WILCZEK D (1), WYCISEO-GAWRON P (2)

(1) Ophthalmology Clinic and Department of Ophthalmology, School of Medicine in Katowice, Medical University of Silesia in Katowice, Katowice

(2) University Center of Ophthalmology and Oncology, Clinical Hospital nr 5,Medical University of Silesia in Katowice, Katowice

Purpose Evaluation of influence of the drusen on optic nerve function. Comparison of various diagnostic methods.

Methods 27 patients (54 eyes) with optic nerve drusen (average age: 33 years) and 15 healthy patients ( 30 eyes; average age: 32 years) have been examined. By all patients full ophthalmological examination, static perimetry (Octopus 301, Zeiss), contrast sensitivity with the sine wave contrast test (SWCT) as well as pattern electroretinography (PERG) and pattern visual evoked potentials (PVEP; Reti-Port, Roland Consult) were conducted. Statistical analysis was based on Mann-Whitney U Test and Spearman Test. Results Best Corrected Visual Acuity in all eyes amounted to 0.0 logMAR. In PVEP examination, the eyes with optic nerve drusen have revealed a statistically significantly lower P100 wave amplitude at $1 \otimes$ check size $(\mathrm{p}=0,0004)$ and at $15^{\prime}(\mathrm{p}=0,046)$ as well as longer P100 wave latency at $15^{\prime}$ check size $(\mathrm{p}=0,046)$ than healthy eyes. In PERG the amplitude of N95 wave was statistically significantly lower for eyes with optic nerve drusen than for healthy eyes. Statistically significant, negative correlation between the amplitude of N95 wave PERG and the value of MD factor of static visual field $(r=$ 0,1003 ) has been found. No significant differences in degrees of contrast sensitivity have been identified.

Conclusion Presence of drusen disturbs optic nerve function. Electrophysiological examinations are a sensitive test in identifying visual neuropathy.

- T004

Follow-up of patients under therapy with hydroxichloroquine via merg, sd-oct, af and automated perimetry

MOGAVERO V, RUBERTO G, RAIMONDI M, LANTERIS, PICCININI P, BIANCHI PE IRCC Policlinico San Matteo, Pavia

Purpose To analyze alterations in patients in therapy with hydroxychloroquine via multiple functional tests.

Methods We examined 55 patients (mean age $56.1 \pm 1.9)$ in long term therapy $(93.8 \pm 95.6$ months) with hydroxichloroquine The patients were divided into 4 patological groups (RA,LES,connectivitis and others) and assuming therapy from less than 5 years (47 eyes), 5 to 10 ( 28 eyes) and more than 10 ( 36 eyes). They had a complete ophthalmologic visit, including $\log M A R$ visual acuity, multifocal ERG(61 hexagons program), frequency doubling technology, automated perimetry program 10-2, spectral domain optical coherence tomography and autofluorescence at time 0 and at after 1 year. For the statistical analysis we considered the latencies and amplitudes of the $5 \mathrm{mfERG}$ rings, the mean and pattern deviation (MD, PSD) results of the FDT and SD-OCT.

Results The patients were treated for 78 months in median and the median total dose was $115 \mathrm{gr}$. We found a reduction of the mfERG rings statistically significant (N1nV1, P1nV1, P1nVdeg1, N1nV2, P1nV2, P1nVdeg2, P1nV3, P1nVdeg3, P1nV4, P1nVdeg4, P1nV5, P1nVdeg5) while PSD and the MD did't show a loss of sensitivity in the follow up. We found a statistically significant thicker in 4 sector of retina(foveal, superior, nasal and periferical inferior)with SD-OCT. The AF was positive in 9 eyes and negative in 58 eyes and did not change at the follow up.

Conclusion In our study mfERG has been the first exam to show early damage in absence of manifest retinal changes. The meaning of thickening at SD-OCT remains uncertain. Our aim now is to estabilish the eventual relationship between the thickened areas in SD-OCT and the mfERG reduction lasting the follow up. 
Poster session 1: Electrophysiology-physiological Optics-Vision Sciences/Glaucoma/Immunology-Microbiology/Molecular Biology-Genetics-Epidemiology/ Neuro-ophthalmology-Strabismology-Paediatric Ophthalmology-History of Ophthalmology

\section{- T005}

Clinical application of 3D monitor in ophthalmologic field: Measurement of metamorphopsia

KIM YT, KIM JW

Department of Ophthalmology, Ewha Mokdong Hospital, School of Medicine, Ewha Womans University, Seoul

Purpose To develop a new tool of measuring metamorphopsia

Methods Fifty-eight patients diagnosed with macular disease were examined. Images for three-dimensional monitor were prepared. Macular optical coherence tomography and the Amsler grid test were also performed.

Results The sensitivity of metamorphopsia was $66.7 \%$ with Amsler grid and 100\% with three-dimensional monitor. The specificity of metamorphopsia was $97.7 \%$ with Amsler grid and $90.7 \%$ with three-dimensional monitor

Conclusion The detection rate of metamorphopsia in macular disease with threedimensional monitor was significantly higher than with Amsler grid. The threedimensional monitor may be used for the detection of metamorphopsia, moreover, they can be used even at home for the self-assessment.

\section{- T007}

Prevalence of refractive error in school childrens with color vision deficiencies

OSTADIMOGHADDAM H, YEKTA AA, HERAVIAN J, AHMADI HOSSEINIM, VATANDOUST S, ABOLBASHARI F, SHARIFI F

Optometry, Mashhad

Purpose To assess the refractive error status in subjects with color vision deficiency (CVD) and color normal (CN) in order to make a contribution to a better understanding of emmetropization process.

Methods A total of 4400 students were screened using a Ishihara pseudoisochromatic color vision plate set. One hundred and sixty students were detected to have CVD. Four hundred age and sex match were set as the control group. Objective refraction was performed to determine the refractive status of the subjects.

Results The prevalence of myopia in CVD group was $7.7 \%$ that was significantly lower than CN subjects (13.9\%). Furthermore, CVD group presented with lower prevalence of hyperopia (41\%) in comparison with $\mathrm{CN}$ group (57.4\%). In addition, CVD subjects were presented with lower magnitude of refractive error in comparison with $\mathrm{NC}$.

Conclusion Although lower prevalence of myopia support the theory of the role of Longitudinal chromatic aberration in refractive error development, lower prevalence of hyperopia in CVD group is in contrary with that. This Results indicated that longitudinal chromatic aberration is not the only factor that could influences the emmetropization process.

\section{- T006}

Reproducibility of retinal oximetry measurements in healthy and diseased retinas

TÜRKSEVER C, ORGÜL S, TODOROVA MG

Department of Ophthalmology, University of Basel, Basel

Purpose Retinal oximetry ( $\mathrm{RO}$ ) has been established as a non-invasive method to analyse oxygen saturation in retinal vessels. The aim of our study was to determine the reproducibility of $\mathrm{RO}$ images in healthy and in diseased retinas.

Methods A total of 46 right eyes (184 oximetry images) in 46 subjects (21区, 25 区) were examined: 16 controls, 10 glaucoma patients and 20 patients with inherited retinal diseases. Four test-retest RO images were obtained in each subject. Oxygen saturation was measured with the oxygen saturation measurement tool of the Retinal Vessel Analyser (RVA, IMEDOS Systems UG, Jena, Germany). The test-retest standard deviation within the subject $( \pm \mathrm{SDw})$ of the mean vessel oxygen saturation in retinal veins and in arteries, its coefficient of variation $(\mathrm{CoV})$ and the interclass correlation coefficients (ICC) were analysed.

Results The average test re-test SDw in veins was $\pm 2.52 \%(\mathrm{CoV}=4.30 \%)$ and in arteries $\pm 1.62 \%$ ( $\mathrm{CoV}=1.72 \%)$. Among controls, glaucoma eyes and eyes with inherited retinal diseases, the test re-test $\mathrm{SDw}$ in veins were: $\pm 2.17 \%(\mathrm{CoV}=4.11 \%), \pm 2.81 \%(\mathrm{CoV}=4.64 \%)$ and $\pm 2.66 \%(\mathrm{CoV}=4.28 \%)(\mathrm{p}=0.361$; one-way ANOVA $)$ respectively. The test-retest $\mathrm{SDw}$ in arteries were: $\pm 1.53 \%(\mathrm{CoV}=1.65 \%), \pm 1.44 \%(\mathrm{CoV}=1.05 \%)$ and $\pm 1.83 \%(\mathrm{CoV}=1.91 \%)$, respectively $(\mathrm{p}=0.633)$. The ICCs in veins were 0.77 in controls, 0.76 in glaucoma patients and 0.83 in patients with inherited retinal disease. The ICCs in arteries were respectively: $0.92,0.77$, and 0.93 .

Conclusion Reproducibility of retinal oxygen saturation in healthy, as well as in diseased retinas is excellent. Measurements in arteries seem more reliable when compared to veins.

\section{- T008}

Changes in ocular factors according to motion-in-depth of 3D display and viewer age

YUM HR (1), SHIN SY (1), KANG HB (2), PARK SH (1)

(1) Ophthalmology \& Visual Science, Seoul

(2) Digital Media, Bucheon-si

Purpose To compare changes in ocular factors according to the motion-in-depth of three-dimensional(3D) images and viewer age after watching a 3D display.

Methods 30 healthy subjects were enrolled(17 in their 20-30s, 13 in their 40$50 \mathrm{~s})$. Subjects watched a $3 \mathrm{D}$ display with a $1^{\circ}$ and $3^{\circ}$ binocular disparity with two defined velocities(slow, $105 \mathrm{~mm} / \mathrm{s}$; fast, $257 \mathrm{~mm} / \mathrm{s}$ )for $30 \mathrm{~min}$. The near point of accommodation(NPA) and convergence(NPC), and tear break-up time(tBUT) were measured before and after watching the display. All measures were remeasured until it returned to the baseline. Questionnaires on subjective symptoms were collected before and after watching the display.

Results NPA increased more in the fast motion-in-depth than slow motion-in-depth after watching 3D video. NPC increased more in the fast motion-in-depth than slow motion-in-depth after watching 3D video with $3^{\circ}$ disparity only. According to the age, NPA increased more in the 40-50s group than in the 20-30s group after watching with $3^{\circ}$ disparity/fast motion-in-depth only. NPC increased more in the 40-50s group than in the 20-30s group after watching with $3^{\circ}$ disparity/fast motion-in-depth and slow motion-indepth. The recovery time of NPA and NPC were same results as the change of NPA and NPC. However, the changes of tBUT were not significantly different between the two age groups and between the two motion-in-depths. Changes of subjective symptoms were different according to the motion-in-depth of 3D display and viewer age.

Conclusion We quantitiatively investigate the effect of motion-in-depth of 3D display and viewers' age on the viewers' visual function. The results indicate that motion-indepth might be considered in determining visual comfort of 3D display, especially in older people. 
Poster session 1: Electrophysiology-physiological Optics-Vision Sciences/Glaucoma/Immunology-Microbiology/Molecular Biology-Genetics-Epidemiology/ Neuro-ophthalmology-Strabismology-Paediatric Ophthalmology-History of Ophthalmology

- T009

Effects of long duration dive (8, 10 and 12 hours) with hyperbaric hyperoxia on Navy divers' eye and visual function

VALERO B (1), CASTAGNA O (2), BRUGIER A (1), VIGNAL R (1), DE FARIA A (1) BOURNIQUEL M (1) SEKFALIR (1) BL ATTEAUIE (2) GIRAUD IM (1)

(1) Sainte-Anne Military Hospital, Toulon

(2) Underwater research team (ERSSO), Military biomedical research (IRBA), Toulon

Purpose To assess anatomical and functional effects of long duration dive ( 8 to 12 hours) on the eye, by reproducing all the constraints undergone by professional divers, so as to prevent potential hazard to diver visual function.

Methods 18 male Navy divers performed prolonged dive sessions ( 8,10 and 12 hours) breathing $50 \%$ Nitrox or $100 \%$ O2, according to immersion depth (7 to $20 \mathrm{msw}$ ), and amounting to 1.54 to 1.7 bar inhaled PO2.for each diver, we studied several parameters 24 hours before and 15 hours after immersion: visual acuity, low spatial contrast sensitivity, color vision, eye refraction, ocular examination, visual field, full-field clinical electroretinography and multifocal electroretinography

Results None of the 18 divers had any loss of visual acuity. No change occurred following dive in spatial contrast sensitivity, color vision, eye refraction, visual field parameters (corrected mean deviation, foveolar threshold). Ocular examination was normal for all the divers, before and after immersion. Regarding full-field clinical electroretinography, b-wave was significantly decreased after immersion $(286,64 \mu \mathrm{V}$ $\pm 60,34$ vs $270,07 \mu \mathrm{V} \pm 57,64, \mathrm{p}=0,015)$ on scotopic response with white attenuated stimulation. No significant modification occurred in multifocal electroretinography.

Conclusion Thus long duration hyperoxic dive didn't cause any clinically significant eye modification. No immediate harm to diver eye and visual function appeared. The slight modifications on electroretinography could be due to intra or inter-individual variations, but they raise question about subclinical functional effects of hyperoxia that could affect rod-cell function.

\section{- T011}

\section{Color vision evaluation test}

IONICA $V$

06480, La Colle sur Loup

Purpose Color Vision Evaluation Test (CVET) is used to test sensitivity to color and makes it possible to identify and evaluate all color vision disabilities.

Methods CVET is made up of a set of 72 pseudoisochromatic images, a lamp that provides daylight illumination, and software to acquire a graphic representation of the results of the investigation. The test makes it possible to diagnose all deficiencies in seeing color, both hereditary and acquired, and measure the deficiencies on a ten level scale.Color vision has been calibrated in the same way as vision acuity. Normal color vision is considered 1, and an anopia (complete absence of a color from the chromatic spectrum) is considered 0 . Between these two values there are 9 levels of an abnomal trichromacy marked from 0.9 to 0.1 .The lamp provides a consistent and adequate illumination of the text charts so that the test can be replicated identically.

Results The software suggests a diagnostic of the deficiency, provides 3 graphic representations, and offers a simulation of the vision of the patient on pictures chosen by the person who administers the test to better evaluate the professional abilities of the person whose color vision is tested.

Conclusion The test provides accurate measurements that make it possible to evaluate the professional abilities in cases of hereditary dyschromatopsia as well as following the progress of vision disabilities or other illnesses that cause vision diasbilities.
- T010

A novel technique for measuring dark adaptation using a dual stimulus method: Effects of aging and AMD on dark adaptation

RODRIGO E (1), TAHIR HJ (1), PARRY NRA (2), KELLY JMF (1), ASLAM T (3), CARDEND (1), MARRAY IJ (1)

(1) Faculty of Life Sciences University of Manchester, Manchester

(2) Centre for Hearing and Vision Research, Institute of Human Development, University of Manchester, UK., Manchester

(3) Vision Science Centre, Manchester Royal Eye Hospital, Central Manchester University Hospitals NHS Foundation Trust, Manchester Academic Health Science Centre, Manchester

Purpose To study abnormal dark adaptation (DA) in aging and macular degeneration (AMD) using a new technique that measures DA at two retinal locations by using a dual stimulus. To establish whether there are localized regions of impaired rod function, especially the rate of the rod-mediated recovery $(\mathrm{S} 2)$ as it has been previously suggested Methods Dual arc-shaped white stimuli were presented on a black CRT monitor at locations $6^{\circ}$ and $11^{\circ}$ of eccentricity in the inferior visual fiel. Recovery of sensitivity to the two stimuli was measured concurrently using the method of adjustment, following a bleaching exposure of at least $30 \%$. The dynamic range of the CRT was expanded using ND filters. DA curves were obtained after fitting the data by non-linear regression to a seven-parameter model. Three groups of observers (young, older and AMD) were tested to detrmine any differences between them and the effect of the testing location, focusing on parameters $\alpha$ (rod-cone break) and S2 (slope of the post- $\alpha$ phase of rod recovery

Results Both stimuli produced matched cone recovery curves within each group. Comparing groups (young vs. older) and (older vs. AMD), we found significant differences in the two DA parameters studied here. Regarding the AMD group, we found a trend in both parameters to be more affected at $6^{\circ}$ than at $11^{\circ}$, however, these differences were not significant

Conclusion This technique has shown to be sensitive to detect changes in rod recovery in AMD patients compared to healthy subjects and allows the measurement of DA in two retinal locations simultaneously. It may help in the early diagnosis and monitoring of degenerative diseases of the macula, which are increasingly common

\section{- T012}

Color perception levels in patients with hyperopia

BOGATYREVA E, KOVALEVSKAYA $M$ ophthalmology, Voronezh

Purpose Evaluation of color perception levels in different types of hyperopia.

Methods 4 groups of patients: 1 ( $\mathrm{n}=35)$ - low hyperopia; $2(\mathrm{n}=29)$ - moderate hyperopia; 3 ( $\mathrm{n}=26$ ) - high hyperopia; 4 (control, $\mathrm{n}=39$ ) - emmetropia. The supplement - Farnsworth-Munsell 100 Hue Test (FM 100). Measures of assessing were: total error score (TES), the level of recognition of colors (LRC), color vision deficiency (CVD), the type of color deficiency (TCD). Color vision defects were studied using a special computer program for FM 100.

Results Group 1: TES=67 \pm 25 (p<0,05), LRC 16-100 TES, CVD 41-100 TES, TCD individual abnormalities in blue color. Group 2: TES $=93 \pm 25$ ( $p<0,05)$, LRC 16 and more TES, CVD 41-200 TES, TCD cian, blue. Group 3: TES=180 25 ( $p<0,05)$, LRC 101 and more TES, CVD 101 and more TES, TCD cian, blue, violet Group 4: TES=53+25 $(\mathrm{p}<0,05)$, LRC 16-100 TES, CVD no more 100 TES, TCD individual abnormalities in blue color.

Conclusion We revealed correlation between stages of hyperopia and color level perception: in the 1st group LRC average, CVD slight; 2nd group: LRC average/low, CVD slight/moderate; 3rd group: LRC low, CVD moderate/severe; 4th group: LRC average, CVD none/slight. Also, hyperopic refractive errors are associated with defects of blue color. Quantitative analysis of color vision defects with FM100 provides the possibility for detecting condition of cone photoreceptor cells, which mostly damaged in the 3rd group. 
Poster session 1: Electrophysiology-physiological Optics-Vision Sciences/Glaucoma/Immunology-Microbiology/Molecular Biology-Genetics-Epidemiology/ Neuro-ophthalmology-Strabismology-Paediatric Ophthalmology-History of Ophthalmology

- T013

Comparing short-duration electro-oculogram with and without mydriasis in controls

TÜRKSEVER C, ORGÜL S, TODOROVA MG

Department of Ophthalmology, University of Basel, Basel

Purpose In order to obtain artefact-free EOG recordings the subject's cooperation is necessary. The aim of our study is to evaluate recording characteristics of the shortduration EOG and to compare the effect of mydriasis on EOG recordings in a cohort of controls.

Methods EOG recordings were performed on a LED stimulus screen using a RETIport gamma plus2 system (RETIscanTM, Roland Consult). Dark phase included: preadaptation (6min), alternate fixation (4min), fixation-rest (20sek), 100 sweeps. Light phase included: light adaptation (4min), alternate fixation (10min), fixation-rest (20sek), 250 sweeps. Amplifier was band pass filtered at $0.1-50 \mathrm{~Hz}$. Background illumination in mydriasis was $100 \mathrm{~cd} / \mathrm{m} 2$ and in miosis $450 \mathrm{~cd} / \mathrm{m} 2$. Fast oscillations were set at $1.5 \mathrm{sek}(6$ cycles; total duration $75 \mathrm{sek}$ ).

Results A total of 55 controls divided in three age-groups participated (number mean (years, y); \pm SD): group 1: 18- 20y (19; 19.49y; \pm 0.89$)$; group 2: 20-40y $(18 ; 27.91 \mathrm{y}$ \pm 5.39 ) and group $3: 40-60 \mathrm{y}(18 ; 48.66 \mathrm{y} ; \pm 4.00)$. Arden ratio (AR), dark-trough (DT) and light-peak (LP) did not differ between recordings with or without mydriasis $(\mathrm{p}=0.885$; $\mathrm{p}=0.860 ; \mathrm{p}=0.776$, linear mixed-effects model). Age did not influence the AR, DT, LP ( $\mathrm{p}=$ $0.206 ; \mathrm{p}=0.112 ; \mathrm{p}=0.155)$. AR, DT, LP were comparable between eyes tested $(\mathrm{p}=0.998$; $\mathrm{p}=0.196 ; \mathrm{p}=0.324$ )

Conclusion Short-duration EOG allows successful recording, where the application of mydriasis does not influence the quality of the recording.

\section{T014}

Topical treatment- are we adhering to guidelines?

$\operatorname{ANSARIE}(1,2)$, FAGJENBAUM M (1)

(1) Eye Ear and Mouth Unit, Maidstone

(2) Physical Sciences, University of Kent, Canterbury

Purpose To determine if established guidelines for medical treatment are being used in glaucoma clinics

Methods Retrospective case note review of 120 consecutive patients in a glaucoma clinic who had been started on topical treatment within the last 5 years. The prescribing trends were compared to EGS guidelines and local guidelines. first line, second line and combination regimens, was analysed. The persistence of individual classes of drops and combination regimens was studied.

Results First line: Prostaglandin analogues (PGA) were prescribed in 70\%, Betablockers (BB) in 20\% and Carbonic anhydrase inhibitors (CAI) and combination preparations in $10 \%$

Persistence: 25\% of PGA group, effect of one PGA lasted > 3 years; $75 \%$ of PGA group, effect of one PGA lasted < 3 years. $36 \%$ of BB group, effect of one BB lasted > 3years; in $64 \%$, effect of single BB lasted < 3 years.Second line: PGA prescribed in $25 \%$, BB in $9 \%$ and combination preparations in 66\%.Persistence: $30 \%$ of BB+PGA lasted > 3 years; $43 \%$ of PGA+BB lasted > 3

Conclusion First line drop prescription was in line with EGS and local guidelines. Persistence of effect of BB at > 3 years was better than PGA (36\% v 25\%). Combination drops were the most popular 2nd line, with Cosopt being the most popular (38\%) 2nd line drop. The combination of PGA+combination drop had the best persistence (39\%). There was a surprisingly large number of cases with inappropriate combination regimes. Commercial interest
- T015

Treatment satisfaction: Can we trust our glaucoma patients?

TISSIER P (1), LEMIJ H (2), BAUDOUIN C (3)

(1) Laboratoires Théa, Clermont Ferrand

(2) Rotterdam Eye Hospital, Rotterdam

(3) Quinze-vingts National Hospital, Paris

Purpose To determine the characteristics and satisfaction regarding tolerance of glaucoma patients currently treated by prostaglandin analogues, in general ophthalmological consultations.

Methods Multi-centre, European and epidemiological survey among glaucoma patients receiving prostaglandin analogues. In a two page questionnaire, the participating ophthalmologists recorded: glaucoma history, previous treatments and reasons for change, tolerability and patient satisfaction, ocular surface disease (OSD) prevalence and other ocular signs.

Results The data from the first country, The Netherlands, (164 patients) were analyzed. At the time of the study, $94 \%$ of patients were treated for glaucoma with preserved products. Of all patients, $89 \%$ declared to be satisfied or very satisfied. The mean score of tolerability evaluated on VAS (Visual Analog Scale) was $76.4 \pm 17.5 \mathrm{~mm}$. Signs of OSD were evident in $44 \%$ of patients. Of all patients, $32 \%$ reported symptoms upon instillation and $47 \%$ between instillations. Conjunctival Hyperemia was present in $47 \%$ of patients. The prevalences of both blepharitis/Meibomian gland dysfunction and dry eye were more than twice as high compared to the time of onset of therapy, with a change from 8 to $19 \%$ of patients for blepharitis and 14\% to 33\% for dry eye. Artificial tears were used in $38 \%$ of patients. Patient dissatisfaction was significantly associated with the presence of OSD, hyperaemia, ocular signs, symptoms upon and between instillation and the use of artificial tears.

Conclusion These first results highlight the disconnection between the patients reported satisfaction and their ocular signs and symptoms. Ocular surface dysfunction needs to be investigated thoroughly in those who use preserved prostaglandin analogues. Commercial interest

\section{- T016}

Patient satisfaction after preservative free latanoprost; the PASSY survey in Germany

$E R B C$

Augenklinik, Berlin

Purpose To determine the characteristics and satisfaction regarding tolerance of glaucoma patients treated by Monoprost, preservative-free (PF) latanoprost, in ophthalmology consultations

Methods Multicenter, epidemiological, retrospective survey in 6 European countries. OHT/Glaucoma Patients treated by PF latanoprost for more than 2 months were included.A 2 page questionnaire recorded: ocular history, data on previous treatment, reason for change, tolerance of the treatments, OSD and tear substitutes use, eye examination.

Results 248 patients from Germany were analyzed. Mean age was $67.89 .5 \%$ of the patients had received another previous treatment and the reasons for previous changes were mainly local intolerance (66\%) and insufficient efficacy (46\%).96\% of patients declared to be satisfied or very satisfied with their PF latanoprost treatment. Tolerance was declared as better or much better than their previous treatment by $79 \%$ of patients and the same by $17 \%$.The use of Monoprost ${ }^{\circ}$ UD in comparison to the previous treatment was easier for $40 \%$ of the patients or the same for $52 \%$.Within the $38 \%$ of patients who use tear substitutes, this use decreased for $28 \%$ and did not change for $66 \%$ of patients.

Conclusion After 2 months of Monoprost" treatment, almost all the patients were satisfied of their treatment and more than three-fourth considered that the tolerance was better than the previous treatment.

Commercial interest 
Poster session 1: Electrophysiology-physiological Optics-Vision Sciences/Glaucoma/Immunology-Microbiology/Molecular Biology-Genetics-Epidemiology/ Neuro-ophthalmology-Strabismology-Paediatric Ophthalmology-History of Ophthalmology

\section{- T017}

Two simple animal models of intraocular pressure elevation for testing therapeutic drugs in glaucoma

GRILLO-ANTONELLIS, CIMBOLININ, FERAILLE L, ELENA P

Alpes Maritimes, La Gaude

Purpose Glaucoma is a progressive optic neuropathy and elevated intraocular pressure (IOP) is one of the most important risk factors. Here we present two experimental models of high IOP in rats and rabbits. One is related to the side effects of corticosteroids and the other one on the hypersaline stress.

Methods In the rat model, elevation of IOP was induced by instillations of $0.1 \%$ dexamethasone twice daily. One group received only saline instillations. The three dexamethasone induced groups received either $0.9 \% \mathrm{NaCl}$ or brimonidine/timolol $(0.2 \% / 0.25 \%)$ or latanoprost $(0.002 \%)$. The IOP was measured using a TonoLab" tonometer.In the rabbit model, elevation of IOP was induced by intravitreal (IVT) injection of $5 \%$ saline solution. The first received isotonic saline solution $(0.9 \%)$ by IVT injection, the other two groups received hypertonic saline solution (5\%) by IVT injection, one treated with an instillation of $0.9 \% \mathrm{NaCl}$ and the other with an instillation of brimonidine-timolol $(0.2 \% / 0.25 \%)$. IOP were recorded with a pneumatonometer.

Results In the rat model, $0.1 \%$ dexamethasone instillations induced a significant and chronic increase of the IOP over 35 days. The high IOP significantly decreased after treatment with the IOP-lowering agents.In the rabbit model, the treatment showed a significant and acute increase of the IOP over $30 \mathrm{~min}$ to $120 \mathrm{~min}$, after treatment the IOP significantly decreased.

Conclusion These two models of high intraocular pressure with simple experimental handling and low cost, can be used as a tool for discovering therapeutic drugs in glaucoma targeting the outflow

\section{- T019}

Intravenous hypertonic saline - the effect of splitting the dose

TORVINEN P, HARJU M, KIVELÄ T

Department of Ophthalmology, Helsinki

Purpose To compare the effect of a single vs. splitted dose of intravenous hypertonic saline (IVHTS) on intraocular pressure (IOP) among patients with open-angle glaucoma (OAG).

Methods In a prospective, randomised, interventional trial, we enrolled OAG patients with an IOP of 22-34 mmHg. We excluded patients using oral acetazolamide, those with heart or kidney failure, dementia or another systemic condition that notably decreased physical performance. Participants received 23.4\% IVHTS trough an antecubital vein at a rate of $1 \mathrm{ml} / \mathrm{s}$. Group 1 received a single dose of $1 \mathrm{mmol} / \mathrm{kg}$, and Group 2 two doses of $0.5 \mathrm{mmol} / \mathrm{kg}$ sodium separated by 10 minutes. We measured IOP before IVHTS and repeatedly for 20 minutes after infusion.

Results A total of 32 participants (median age 69 years, range 35-78); 20 patients in Group 1 and 12 in Group 2. The mean baseline IOP was 26.2 (SD, 2.7) mmHg in Group 1, and 25.4 (3.5) $\mathrm{mmHg}$ in Group $2(\mathrm{P}=0.15)$. In both groups, mean IOP was significantly reduced after IVHTS; mean reduction in Group 1 was 7.0 (2.8) $\mathrm{mmHg} 10$ minutes after IVHTS $(\mathrm{P}<0.001)$, and $7.6(2.6) \mathrm{mmHg}(\mathrm{P}<0.001)$ at 20 minutes. In group 2 , the corresponding mean reductions after the 2 nd dose were $8.1(2.0) \mathrm{mmHg}(\mathrm{P}<0.001)$ and $8.2(1.8) \mathrm{mmHg}(\mathrm{P}<0.001)$, respectively. The mean IOP reductions 10 and 20 minutes after IVHTS were comparable for Group 1 and $2(\mathrm{P}=0.24$ and $\mathrm{P}=0.47$, respectively). Within 1-3 minutes of infusion, 16 patients in Group 1 felt pain at the infusion site as compared to 7 patients in Group 2. The 2nd dose in Group 2 induced more pain than the 1st dose according to 8 patients.

Conclusion A single dose of $1.0 \mathrm{mmol} / \mathrm{kg}$ and two sequential splitted doses of 0.5 $\mathrm{mmol} / \mathrm{kg}$ of IVHTS reduce IOP comparably. Splitting the dose did not eliminate the pain felt at the infusion site.

\section{- T018}

Impact of preservatives' elimination on tolerance and efficacy of latanoprost

MISIUK HOJLO M (1), MULAKM (1), POMORSKA M (1), REKASM (2) WIERZBOWSKA I (2), PROST M (3), WASYLUK I (3), LUBINSKI W (4), PODBORACZYNSKA-JODKOK (4), ROMANIUK W (5), KINASZ R (6), ORTYL-MARKIEWICZR (7), MOCKO L (8), ZALESKA-ZMIJEWSKA A (9), ROKICKID (9)

(1) Department of Ophthalmology, Wroclaw Medical University, Wroclaw (2) Department of Ophthalmology, Military Institute of Medicine in Warsaw, Warsaw (3) Department of Ophthalmology, Military Institute of Aviation Medicine, Warsaw

(4) Department of Ophthalmology, Pomeranian Medical University, Szczecin (5) Department of Ophthalmology, Medical University of Silesia, Katowice (6) Department and Clinic of Ophthalmology, Medical University of Silesia, Katowice (7) Glaucoma Outpatient Clinic, Regional Specialist Hospital, Legnica (8) Ophthalmology Outpatient Clinic, ZWPS in Katowice, Katowice

(9) Department of Ophthalmology, Medical University of Warsaw and SPKSO Hospital, Warsaw

Purpose To compare the tolerance and efficacy of preservative-free latanoprost (latanoprost-PF) after switching from benzalkonium chloride containing agent (latanoprost-BAK)

Methods 140 patients with glaucoma or OHT controlled by latanoprost-BAK were included into the study. At a baseline visit (D0) a new formulation of latanoprost-FP (Monoprost ; Laboratoires Thea) was introduced. An observation was conducted, including three visits after 15, 45 and 90 days from the baseline (D15, D45, D90 respectively). The following outcomes were evaluated: BCVA, IOP, TBUT, corneal fluorescein staining and subjective assessment of tolerance by patient using 10- point VAS scale (0 - very good, 10 - very poor tolerance)

Results Mean IOP during all subsequent visits was reduced compared to the baseline ( $\mathrm{p}<0.006 \mathrm{vs} \mathrm{D} 0$ ) and measured $15.95 \mathrm{mmHg}, 15.37 \mathrm{mmHg}, 15.33 \mathrm{mmHg}$ and 15.27 mmHg at D0, D15, D45 and D90, respectively. TBUT was improved in $23.4 \%$ of patients at D45 and in $29.7 \%$ of patients at D90, but deteriorated in $8 \%$ of cases at both time points. It remained unchanged in $68.6 \%$ and $62 \%$ of patients at D 45 and D 90 , respectively. A conjunctival hypaeremia was present in $56.8 \%$ and in $13,7 \%, 2,2 \%$ and $1,6 \%$ of patients at D0, D15, D45 and D90 visits, respectively. Subjective assessment of tolerance showed an improvement from 5.3 at baseline to 1.91 at $\mathrm{D} 90(\mathrm{p}<0.0001)$.

Conclusion Preservative-free latanoprost has at least the same IOP-lowering efficacy in comparison to BAK-preserved latanoprost. Latanoprost-PF has a better tolerability profile which may translate into better control of the treatment and better quality of life for the patient.

- T020

IOP-lowering effect and ocular toxicity of ONO-0476, a prodrug of a novel prostanoid EP2 receptor agonist, in normotensive cynomolgus monkeys

GOTO Y, MORIYUKI K, YAMAMOTO H, YAMANE S, SUGITANI M, HIROTA Y Ono Pharmaceutical Co., Ltd., Osaka

Purpose ONO-0476 is a prodrug of ONO-AE9-078, a potent and selective prostanoid EP2 receptor agonist manufactured by ONO Pharmaceutical Co., Ltd. The purpose of this study is to evaluate the IOP-lowering effect and ocular toxicity of ONO-0476 in cynomolgus monkeys.

Methods The binding affinity and agonistic activity of ONO-0476 and ONO-AE9-078 for the prostanoid receptors were evaluated. Male cynomolgus monkeys received single or repeated topical dosing of ONO-0476 or current prostaglandin analogues (latanoprost, travoprost, tafluprost, bimatoprost) at a volume of $30 \mathrm{uL}$. The IOP was evaluated by using applanation pneumatonometer. Ocular toxicity was evaluated by using portable slit lamp, laser flare photometry, and ultrasound pachymetry in a 4-week repeated dosing study in monkeys.

Results ONO-AE9-078 had high selectivity and high affinity for the prostanoid EP2 receptor (EC50 value was $2.3 \mathrm{nmol} / \mathrm{L}$ ). Single dosing of ONO- $0476(0.3 \mathrm{ug} / \mathrm{mL}$ or more) reduced IOP in a dose-dependent manner. IOP-lowering effects were sustained through $24 \mathrm{~h}$ following a single dosing. Repeated dosing (once daily for one week) of ONO-0476 $(0.3 \mathrm{ug} / \mathrm{mL})$ reduced IOP more potently than the current prostaglandin analogues. During 4-week repeated dosing of ONO-0476 $(1 \mathrm{ug} / \mathrm{mL})$, there were no evidence of significant toxicity.

Conclusion ONO-0476 causes potent and sustained IOP reduction, and has good tolerability following topical dosing. ONO-0476 is an attractive candidate for treatment of glaucoma and ocular hypertension

Commercial interest 
Poster session 1: Electrophysiology-physiological Optics-Vision Sciences/Glaucoma/Immunology-Microbiology/Molecular Biology-Genetics-Epidemiology/ Neuro-ophthalmology-Strabismology-Paediatric Ophthalmology-History of Ophthalmology

- T021

The estimate prevalence of glaucoma \& trend of glaucoma care in south korea based on national claim database

SEOSJ (1), BAE HW (1), LEE YH (1), LEESY (1), HONG S (1), SEONG GJ (2), KIM CY (1) (1) Department of Ophthalmology,Severance Hospital, Institute of Vision Research, Yonsei University College of Medicine, Seoul

(2) Department of Ophthalmology, Gangnam Severance Hospital, Institute of Vision Research, Yonsei University College of Medicine, Seoul

Purpose To estimate the prevalence and to analyze the trend of glaucoma care in South Korea from 2008 to 2012

Methods Patients who diagnosed with glaucoma from 2008 to 2012 were retrospectively identified using the diagnostic code for glaucoma and the surgical codes for glaucoma surgeries in National Health Insurance (NHI) \& Health Insurance Review and Assessment service(HIRA) database. The prevalence of glaucoma, glaucoma care cost, cost for glaucoma procedure and percentage of cost for glaucoma procedure were estimated.

Results The number of glaucoma patients was increased by 60\% from 2008 to 2012 The estimated prevalence of glaucoma in the population aged $\geq 40$ years was increase from $1.30 \%$ in 2008 to $1.90 \%$ in 2012 ( $\mathrm{P}<0.001$, test for linear trend). The prevalence of glaucoma increased with age and was higher in men than in women(OR 0.9 (95\% confidential interval $0.89 \sim 0.90$ ) in 2012). Although the cost for glaucoma procedure has increased from 2008 to 2012, the percentage of cost for glaucoma procedure among total glaucoma care cost showed a decreasing trend annually(from $9.07 \%$ in 2008 to $5.21 \%$ in 2012).

Conclusion The number of glaucoma patients and the prevalence of glaucoma increased annually in South Korea. However, in this study, the prevalence of glaucoma was underestimated comparing with other population based study. This result mean glaucoma screening test was not enough to finding glaucoma patients in South Korea. In conclusion, to find glaucoma patients, it is necessary to develop glaucoma screening system in countries having similar health care circumstance with South Korea.

\section{- T023}

The effect of preservative free tafluprost on intraocular pressure in patients with ocular hypertension or primary open angle glaucoma

HOMMER A (1), KROMUSM (1), SCHMIDL D (2), GARHOFER G (2),

SCHMETTERER L $(2,3)$

(1) Sanatorium Hera, Vienna

(2) Department of Clinical Pharmacology, Medical University of Vienna, Vienna

(3) Center for Medical Physics and Biomedical Engineering, Medical University of Vienna, Vienna

Purpose Since increased intraocular pressure (IOP) is the most important modifiable risk factor for the development and progression of glaucoma, potent and save IOP lowering therapy is of special importance. Preservative free prostaglandins offer a new treatment option using a potent topical drug without the disadvantages of a coadministered preservatives. The aim of the present study was to investigate the effect of 8 weeks treatment with preservative free tafluprost on IOP in patients with glaucoma or ocular hypertension.

Methods 16 patients with either primary open angle glaucoma with an uncontrolled intraocular pressure $\geq 30 \mathrm{mmHg}$ or patients with ocular hypertension with an IOP $\geq$ $30 \mathrm{mmHg}$ were included in the present open pilot study. For patients who were already on antiglaucoma therapy, a washout period with a duration of 4 weeks was scheduled. IOP was measured at $8 \mathrm{am}$ and $6 \mathrm{pm}$ after the washout period as well as 4 and 8 weeks of after start of topical drug treatment.

Results A total of 16 patients (age range 31-76 years) were included in the study After the 4 week washout period, mean IOP was $35.6 \pm 4.5 \mathrm{mmHg}$ at the morning and $32.9 \pm 5.4 \mathrm{mmHg}$ at the evening measurement. 4 weeks after treatment start, a decrease in IOP by $-31 \% \pm 12 \%$ to $24.4 \pm 4.6 \mathrm{mmHg}(\mathrm{p}<0.01)$ was observed at the morning measurement and by $-34 \% \pm 14 \%$ to $21.6+4.6 \mathrm{mmHg}(\mathrm{p}<0.01)$ at the evening measurement. 8 weeks after treatment start IOP remained low, on average $24.4 \pm 4.6 \mathrm{mmHg}(-31 \% \pm 13 \%$ compared to baseline, $\mathrm{p}<0.01)$ in the morning and $21.9 \pm 3.9 \mathrm{mmHg}(-32 \% \pm 16 \%$ compared to baseline, $\mathrm{p}<0.01)$ in the evening.

Conclusion The present data indicate that treatment with preservative free saflutan is safe and has the capacity to significantly decrease IOP in patients with primary open angle glaucoma and ocular hypertension. Further studies are needed to investigate the effect of preservative free formulations for long term treatment.

\section{T022}

Cannabinoids: a novel treatment for glaucoma

SAMUDRE S (1), HOSSEINI A (2), LATTANZIO F (2)

(1) Experimental Therapeutics, Chesapeake

(2) Physiological Sciences, Norfolk

Purpose Synthetic cannabinoids are emerging novel agents for the treatment of glaucoma. Although increased intraocular pressure (IOP) is a risk factor, associated retinal damage is of prime concern. This study determines the ability of cannabinoids to decrease IOP and confer neuroprotection.

Methods Ocular hypertension was created in Sprague-Dawley (SD) rats by ligating 3 of 4 episcleral veins. IOP increased by at least $5 \mathrm{~mm} \mathrm{Hg}$ in the operated eye. Cannabinoids, O-1812 (1\%) or O-2545 (1\%), were administered topically. IOP was measured by Goldmann tonometry at baseline (-30), 0, 30, 60 and $120 \mathrm{~min}$. For neuroprotection experiments: excitotoxic retinal damage was induced by injecting NMDA ( $2 \mathrm{ul}$ of 10 $\mathrm{mM}$ ) intravitreally in SD rats. Cannabinoids were injected following NMDA induction. Full-field Electroretinograms were recorded at baseline, 1 wk and 2 wk. ERG a-wave amplitudes are expressed as a percentage of baseline.

Results After treatment with O-2545, IOP was significantly reduced to $11 \pm 0.9 \mathrm{mmHg}$ after $60 \mathrm{~min}$ and to $12 \pm 1.1 \mathrm{mmHg}$ after $120 \mathrm{~min}$ compared to baseline of $14 \pm 0.6 \mathrm{mmHg}$ $(\mathrm{p}<0.03, \mathrm{n}=6)$. Similarly, O-1812 treatment reduced IOP to $8.4 \pm 1.8 \mathrm{mmHg}$ after $60 \mathrm{~min}$ and maintained for up to $120 \mathrm{~min}(\mathrm{p}<0.001, \mathrm{n}=6)$. After $1 \mathrm{wk}, \mathrm{NMDA}$ (control) reduced a-wave amplitude by $38 \pm 0.1 \%(\mathrm{p}<0.001, \mathrm{n}=6)$ from baseline, $\mathrm{O}-2545$ by $46 \pm 0.02 \%$ $(\mathrm{p}<0.001, \mathrm{n}=6)$ and $\mathrm{O}-1812$ by $23 \pm 0.1 \%(\mathrm{p}=0.004, \mathrm{n}=6)$. After 2 wks, NMDA further decreased a-wave amplitude by $48 \pm 0.1 \%(\mathrm{p}<0.001, \mathrm{n}=6), \mathrm{O}-2545$ by $39 \pm 0.03 \%(\mathrm{p}<0.05$, $\mathrm{n}=6)$ and $\mathrm{O}-1812$ by $8 \pm 0.1 \%(\mathrm{p}=0.08, \mathrm{n}=6)$.

Conclusion Topically applied cannabinoids are effective agents that reduce IOP and confer neuroprotection and are prime candidates for potential glaucoma treatment.

\section{- T024}

Dynamic dark-light changes in anterior chamber and iris parameters in angle closure patients with novel automatic algorithm using anterior segment optical coherence tomography (AS-OCT)

LEE TL, ANG L, TIANI, YIP L

Ophthalmology, Tan Tock Seng Hospital, Singapore

Purpose To describe the dynamic dark-light changes in anterior chamber (AC) measurements and iris parameters in angle closure patients compared to normal angle patients using a fully automatic algorithm developed for anterior segment optical coherence tomography (AS-OCT).

Methods 10 patients ( 17 eyes with closed angles) and 8 patients (15 eyes with normal angles) on gonioscopic grading were recruited. We acquired images in both dark and light conditions using Visante OCT.Custom software analyzed the images using the iris rim as the landmark instead of the sclera spur. The parameters analyzed included angle opening distance and angle recess area, at 750 $\mu \mathrm{m}$ from the iris rim (AOD 750 \& ARA 750), AC depth and width, lens vault and iris curvature, thickness, length and area. We analyzed the amount of change from dark to light in both groups of patients.

Results In both conditions, angle closure patients had significantly smaller AOD and ARA, but there was no difference in iris parameters between the 2 groups.In both groups of patients, there was significant change in iris length, area, thickness and ARA when moving from dark to light conditions. The AOD changed significantly in normal patients but not in angle closure patients.There was no difference in the magnitude of change in the above parameters, when changing from dark to light conditions, in both groups of patients.

Conclusion The automated algorithm can identify differences between the $\mathrm{AC}$ measurements and iris parameters in both groups of patients. The lighting condition affects the measurements taken regardless of diagnosis. As such, lighting control should be consistent. 
Poster session 1: Electrophysiology-physiological Optics-Vision Sciences/Glaucoma/Immunology-Microbiology/Molecular Biology-Genetics-Epidemiology/ Neuro-ophthalmology-Strabismology-Paediatric Ophthalmology-History of Ophthalmology

- T025

Test-retest reliability on measuring anterior chamber parameters with a novel automatic algorithm using anterior segment optical coherence tomography

ANG L, LEE T, NG I, YIP L

Department of Ophthalmology, Tan Tock Seng Hospital, Singapore

Purpose To describe the test-retest variability in anterior chamber and iris parameters obtained on anterior segment optical coherence tomography (AS-OCT) when measured with a novel automatic Location-Based Graph Search segmentation algorithm.

Methods Patients with open angles and primary angle closure on gonioscopic finding were invited for anterior chamber (AC) angle imaging with the Visante AS-OCT. 3 scans of each eye were acquired in darkness with the patients repositioning their heads after each scan. The images were analysed with a custom software which used the iris rim as the landmark instead of the sclera spur. The parameters analyzed included angle opening distance and angle recess area at $750 \mu \mathrm{m}$ from the angle (AOD750 \& ARA750), AC depth and width, lens vault, iris curvature, thickness, length and angle recess area. The test-retest intrasession variability, coefficient of variation (COV) and intraclass correlation coefficient (ICC) were calculated to evaluate repeatability and reproducibility.

Results 17 eyes with closed angles and 15 eyes with open angles were recruited. The ICC was $>0.75$ for most parameters of eyes with open angles and COV was $<15 \%$. For narrow angled eyes, the ICC was $>0.75$ for AC depth and width, lens vault, iris length and area, left AOD750 and ARA750 and angle recess, and $<0.75$ for other parameters The COV for AC depth and width, iris length and area was $<10 \%$ and $>10 \%$ for other parameters.

Conclusion There is test-retest variability in AC parameters measured with the Visante AS-OCT. The variability is greater in eyes with narrow angles but reliable in eyes with open angles. Caution must be exercised when using the AS-OCT to follow up patients with narrow angles.

- T027

Changes in ganglion cell complex thickness in patients treated with intravitreal anti-vascular endothelial growth factor therapy

CHO B, LEE IY, YOO RM

Department of Ophthalmology, College of Medicine, Konkuk University, Seoul

Purpose To evaluate the effect of repeated intravitreal injections of anti-vascular endothelial growth factor(VEGF) on the thickness of the ganglion cell complex (GCC) in patients with retinal diseases.

Methods This is a retrospective study of 91 patient with injections for agerelated macular degeneration(AMD), diabetic retinopathy(DMR), or retinal vein occlusion(RVO), who received more than three anti-VEGF injections. For this study, Ganglion cell complex thickness was measured by SD-OCT. The measurements of GCC thickness were made at the fovea and at 3 points nasal and 3 points temporal to the fovea, each separated by a distance of 500 microns. We also evaluated the correlation between changes in ganglion cell complex and other factors including intraocular pressure (IOP), injection times, and the type of disease.

Results After multiple intravitreal anti-VEGF treatments, ganglion cell complex thickness decreased from the initial GCC thickness. Changes of GCC thickness(final GCC thickness - initial GCC thickness) were $-7.47 \pm 22.81,-4.93 \pm 21.93,-3.60 \pm 33.39$ $-1.19 \pm 9.38,-9.10 \pm 32.89,-0.93 \pm 24.44$ and $-2.57 \pm 24.78$ at T 1500 , T 1000, T 500, the fovea, N 500, N 1000 and N 1500, respectively. And mean GCC thickness was changed from $98.56+17.04$ to $94.30+14.49(\mathrm{p}=0.15)$. When classified by disease, the decrease in GCC thickness was statistically significant in RVO patients. The number of injections was found to have no correlation with changes in GCC thickness.

Conclusion Multiple intravitreal injections of anti-VEGF lead to a significant change in GCC thickness. But, the number of injections did not appear to adversely affect GCC thickness.

\section{T026}

Comparison of prelaminar thickness between primary open angle glaucoma and normal tension glaucoma patients

IUNG Y, PARK H, PARK C

Ophthalmology, Seoul

Purpose To investigate the difference in prelaminar tissue thickness between patients with primary open-angle glaucoma (POAG) and normal-tension glaucoma (NTG) and to determine the factors related to prelaminar thickness.

Methods Complete ophthalmic examination including standard automatic perimetry was performed in all patients. The prelaminar tissue thickness was measured in all patients by performing enhanced depth imaging with a Heidelberg Spectralis Optical Coherence Tomography. The prelaminar thickness was compared between patients with POAG and those with NTG. The retinal nerve fiber layer and optic nerve head parameters were obtained using the Heidelberg Retina Tomography II and Cirrus Optical Coherence Tomography and analyzed to verify factors related to prelaminar thickness

Results The mean prelaminar tissue thickness was significantly thinner in patients with POAG than in those with NTG. The difference in the prelaminar thickness between patients with POAG and those with NTG was greater in the early field defect group than in the moderate and severe field groups. In multivariate analysis, the mean prelaminar thickness was related to the intraocular pressure, cup volume, and mean cup depth.

Conclusion The prelaminar tissue was thinner in patients with POAG than in patients with NTG, and the intraocular pressure had a strong influence on the prelaminar thickness in both patients with POAG and those with NTG.

- T028

Hemifield patter electroretinogram and macular thickness asymmetry parameters in ocular hypertension patients

FINZI A, TASSI F, GIZZI C, CELLINI M

Departement of Specialized, Diagnostic and Experimental Medicine, Bologna

Purpose To assess which is the most sensitive and specific exam for detecting an early retinal ganglion cells (RGC) damage between hemifield test pattern electroretinogram (PERG HF) and the posterior pole asymmetry analysis with optical coherence tomography (OCT-PPAA) in ocular hypertensive patients $(\mathrm{OH})$

Methods Forty-two $\mathrm{OH}$ patients (mean age of $56.0 \pm 5.6$ yrs.) with an intraocular pressure (IOP) $>21 \mathrm{mmHg}$ and forty healthy controls (mean age of $55.2 \pm 6.4$ yrs.) with $\mathrm{IOP}<21 \mathrm{mmHg}$ were assessed. All patients had normal visual acuity, normal optic disc appearance and normal standard achromatic perimetric (SAP) indices. All subjects underwent an OCT-PPAA with the OCT-Spectralis and PERG HF examination. Data were analyzed with Wilcoxon test and Receiver Operating Characteristic (ROC) curve analysis taking $\mathrm{p}<0.05$ as significant.

Results We found that $\mathrm{OH}$ patients compared to healthy controls with full field PERG had a not significant decrease of the N95 wave amplitude $(\mathrm{p}<0.097)$ with a significant increase of its peak-time $(103.88 \pm 11.11$ vs $92.78 \pm 4.30 \mathrm{msec}$; $\mathrm{p}<0.027)$.With PERG HF we found a significant intraeye asymmetry of the N95 wave ( $-1.99 \pm 1.58$ vs $-0.67 \pm$ $0.35 \mu$ Volt: $\mathrm{p}<0.023$. With OCT-PPAA in $\mathrm{OH}$ patients we found a decrease in macular thickness but not a statistical significant intraeye hemispheric asymmetry $(3.94 \pm 2.89 \mathrm{vs}$ $5.98 \pm 4.74 \mu$; $<<0.116)$. ROC curve analysis revealed a sensitivity of $80 \%$ and specificity of $87.6 \%$ for PERG HF whereas by using OCT-PPAA we found a sensitivity of $70 \%$ and a specificity of $74 \%$

Conclusion Our study shows that at the moment the PERG HF is the most sensitive and specific technique than OCT-PPAA for detecting early RGC damages in eyes with ocular hypertension. 
Poster session 1: Electrophysiology-physiological Optics-Vision Sciences/Glaucoma/Immunology-Microbiology/Molecular Biology-Genetics-Epidemiology/ Neuro-ophthalmology-Strabismology-Paediatric Ophthalmology-History of Ophthalmology

- T029

Swept source optical coherence tomography artifacts in glaucoma patients

LEE SY, SEO SJ, LEE YH, BAE HY, KIM CY

Department of Ophthalmology, Severance Hospital, Institute of Vision Research, Yonsei University College of Medicine, Seoul

Purpose To demonstrate the frequency and the type of swept-source optical coherence tomography (SS -OCT) imaging artifacts in patients being evaluated glaucoma and to present common artifacts examples

Methods The SS-OCT images of 101 glaucoma suspect and glaucoma patients were used to evaluate image artifacts. We used a 3 dimensional imaging data set of $6 \times 6 \mathrm{~mm}$ raster scan centered on the optic disc (3D disc scan) and a 3 dimensional imaging data set of $12 \mathrm{~mm}$ horizontal line scan centered between optic disc and the fovea (3D side scan). For each scan, both printout report and the source image of printout report were examined.

Results In 101 patients, 101 3D disc scans and 97 3D wide scans were obtained. In 3D disc scan, there were 30 artifacts in source image and 23 artifacts in final report. In $3 \mathrm{D}$ wide scan, there were 24 artifacts in source image and 20 artifacts in final report. The most common type of artifacts for 3D disc scan source data was boundary misidentification (66.7\%). That was also the most common type of artifacts of 3D wide scan source data. Ocular pathology accounted for $25 \%$ of the cause in 3D disc scan source data with artifacts and $38.9 \%$ in 3D wide disc scan source data with artifacts. Epiretinal membrane (ERM) was the most common cause of ocular pathologic artifacts Conclusion The artifacts in SS OCT images can be made by ocular pathology or software error. Operator also can be the cause of SS OCT artifact. Those factors can make errors in interpreting OCT result. Although it is known that SS OCT has an advantage in image detection efficiency, we should consider possibility of artifacts in source data and final report. Especially, we should pay attention in patient who has ocular lesions such as ERM or vitreous traction.

- T031

Evaluation of peripapillary choroidal thickness in patients with glaucoma

MUMCUOGLU T(1), OZGE G (1), OZGONUL C (2)

(1) GATA Medical Academy Department of Ophthalmology, Ankara

(2) Anittepe Military Dispensary Department of Ophthalmology, Ankara

Purpose We aimed to compare peripapillary choroidal thickness measurements between normal and glaucoma eyes.

Methods Prospective, cross-sectional comparative study. 46 eyes of 23 normal and 86 eyes of 45 glaucoma subjects were enrolled in the study. Peripapillary choroidal thickness was measured with spectral-domain optical coherence tomography and enhanced depth imaging. After obtaining circular B-scans around the disc, choroidal thicknesses were calculated for all quadrants. Difference in peripapillary choroida thickness, retinal nerve fiber layer (RNFL), central macula thickness and macula volume between the normal subjects and the patients with glaucoma was analyzed.

Results There were no significant differences in age, gender or refraction between the two groups. RNFL in all quadrants and overall average thickness significantly thinner in the glaucoma group as compared to normal subjects $(\mathrm{p}<0.01)$. Peripapillary choroidal thickness of inferior (130 vs 112,6 $\mu \mathrm{m}, \mathrm{P}<0.01)$, temporal $(180,1$ vs $152,9 \mu \mathrm{m}, \mathrm{P}<0.03)$ regions were significantly thinner and macular volume $(8,42$ vs $7,95 \mathrm{~mm} 3, \mathrm{P}<0.015)$ were significantly lower in the glaucoma group as compared to normal subjects.

Conclusion As compared to normal subjects, peripapillary choroidal thickness was significantly thinner and macula volume decreased in the glaucoma patients. The structural features of the choroid may be associated with pathophysiology of glaucoma.

\section{T030}

Position of the central retinal vessel trunk and location of visual field and parapapillary nerve fibers damage in early to moderate glaucoma

BESOMBES G, GRUNEWALD F, ROULANDJ OPHTALMOLOGIE, Lille

Purpose To address the question of whether the position of the central retinal vessel trunk (CRVT) exit in the optic disc has a spatial relationship with glaucomatous damage Methods This clinical observational study included patients with early to moderate primary open-angle glaucoma. The position of the CRVT exit on the lamina cribrosa was evaluated on optic disc photographs. Visual field tests and retinal nerve fibers laver (RNFL) thicknesses, as measured by optical coherence tomography, were evaluated.

Results The study included 65 eyes with a CRVT mostly decentered into the nasal region followed by the temporal half of the optic disc. Comparing measurements between the opposite disc quadrants indicated that the RNFL thickness was significantly thinner in the inferotemporal quadrant when the CRVT was located in the superonasal quadrant compared with an exit in the inferonasal $(\mathrm{p}=0.0183)$ or temporal $(\mathrm{p}=0.0039)$ quadrant. The distance from the CRVT to the nasal disc border was significantly smaller and correlated positively with the occurrence of RNFL abnormality in the temporal region ( $\mathrm{p}=0.0334)$. Visual field defect incidence was higher in the superonasal quadrant of the pattern deviation plot when the CRVT was located in the superonasal quadrant compared with an exit in the inferonasal quadrant $(\mathrm{p}<0.0003)$. Temporal visual field abnormalities occurred more often when the CRVT exit was located in the temporal part of the optic disc than when the CRVT exit was located in the nasal half $(\mathrm{p}=0.008)$.

Conclusion Local susceptibility for development of glaucomatous damage correlates with the distance to the CRVT, which may act as a stabilizing element against the deformation of the lamina cribrosa.

- T032

Progression rate in glaucoma using spectral-domain optical coherence tomography

LEE Y

Yonsei University College of Medicine, Seoul

Purpose To evaluate the thining rates of retinal nerve fiber layer (RNFL), macular and ganclion cell-inner plexiform layer (GC-IPL) thickness in eyes with POAG, NTG and ACG.

Methods Retrospective cohort study included 145 eyes of 90 glaucoma patient(POAG 70; NTG 60; ACG 15). RNFL, macular and GI-IPL, thickness were measured by spectraldomain optical coherence tomography (Cirrus SD-OCT). Also all the patient was reclassified as 3 groups according to visual field severity (early, moderate and advanced) and as 3 groups according to spherical equivalent (myopia, mild myopia and hyperopia). The meaningful thining was defined on exceed the age-related physiological loss.

Results The mean $( \pm \mathrm{SD})$ follow-up period was $3.86 \pm 0.16$ years.ACG eyes showed highest progression rate of RNFLT in all of quadrant and clock hour. NTG eyes showed no significant different in average RNFLT but significantly higher RNFL thining rates than POAG in inferior quadrant. In ACG eves, RNFLT thining rate is highest in clockhour-6.In NTG eyes showed highest in clock-hour-7 while similar rate in clock-hour-7 and clock-hour-12 in POAG eyes. Thining of macular thickness is highest at outer inferior sector and thining of GC-IPL is highest in inferior sector and inferior hemifield. Thining rate of RNFLT is slower in myopic eyes than mild myopic and hyperopic eyes. In the advanced group, thining rate of RNFLT is slower than early and moderate groups. Conclusion Reduction rate of RNFL, macular and GC-IPL thickness calculated by Ciruss SD-OCT is differ according to the types of glaucoma, spherical equivalent and severity of glaucoma. 
Poster session 1: Electrophysiology-physiological Optics-Vision Sciences/Glaucoma/Immunology-Microbiology/Molecular Biology-Genetics-Epidemiology/ Neuro-ophthalmology-Strabismology-Paediatric Ophthalmology-History of Ophthalmology

- T033

HRT versus OCT in diagnosing preperimetric glaucoma

BOUHACINA BENMANSOUR A, ROULANDJ

Claude Huriez Hospital, Lille

Purpose To compare the final classification of Glaucoma Probability Score (GPS and analyze RNFL of OCT and to evaluate their capacities to detect precociously the glaucomatous defects

Methods Retrospective study, led in the regional center of glaucoma, in CHUR of Lille, including 122 eyes of 71 patientsAll the eyes included had, to the maximum, a light visual field defects and having profited all of them of a complete ophthalmic examination including vision, measurement of the PIO, standard automated perimetry, optic nerve head analysis was performed by using a confocal scanning laser ophthalmoscopy type Heidelberg retinal tomography (HRT III) and retinal nerve fiber layer (RNFL) by Optical Coherence Tomography (Cirrus OCT) The patients were divided into 3 groups according to the defect of the visual field

- Group 1 : normal visual field

- Group 2: MD between $[0,-2]$ decibels

- Group 3 : MD between $[-2.1,-6]$ decibelsThe results of analysis RNFL on OCT and analysis GPS on the HRT were compared in each group.

Results The discordance is important in group 2. The sensitivity of the two apparatuses increases with the depth of the defect but remains higher with the HRT.

Conclusion There is not a difference between the two analyzers from - 2,1 Decibel The GPS appears to be an algorithm more sensitive than analysis RNFL on OCT in detecting preperimetric glaucoma.

- T035

Color Doppler imaging and glaucoma: comparison of Doppler

Waveform and velocities of retrobulbar vessels between a glaucomatous patient and a healthy subject

VERTICCHIO VERCELLIN A (1), CUTOLO C (1), DELLAFIORE C (2), LAVA M (2), TINELLIC (3) CALLIADA F(2) MILANOG(1)

(1) Eye Clinic, University of Pavia, Pavia

(2) Institute of Radiology, Fondazione I.R.C.C.S. Policlinico San Matteo, Pavia

(3) Fondazione I.R.C.C.S. Policlinico San Matteo, Pavia

Purpose To compare the colour Doppler imaging (CDI) waveform and velocities of the ophthalmic artery $(\mathrm{OA})$, central retinal artery (CRA) and short posterior ciliary arteries (SPCA) of a patient with normal tension glaucoma (NTG) compared to a healthy subject.

Methods The right eye of two subjects (a patient with NTG and a healthy subject) were investigated. The same examiner performed CDI examination of the OA, CRA and SPCA using the CDI device ESAOTE MYLAB" (probe LA533 13-6 MHz small partsvascular linear array). The peak systolic velocity (PSV), the end-diastolic velocity (EDV), the resulting resistivity index (RI) were evaluated. Two aspects of the Doppler waveform were considered the Early Systolic Acceleration (the slope of the fastest moving portion of the systolic component, ESA) and the ratio between the mean velocities of the systolic and diastolic components ( $\mathrm{Sm} / \mathrm{Dm})$.

Results All the measured velocities, except for the PSV in the ophthalmic artery, are reduced and the RIs are increased in the glaucomatous patient compared to the healty subject. Moreover, ESA is reduced and Sm/Dm increased if compared to the healthy volunteer.

Conclusion The values obtained are in agreement with the literature. Further studies are needed to evaluate the degree of the pathological alterations and their role in the progression of the optic nerve injury.

\section{- T034}

Structure-function relationship: Ganglion cell-inner plexiform layer thickness by SD-OCT and central visual field sensitivity

CHAPRON T, MARECHAL M, EL CHEHAB H, FENOLLAND JR, MARILL AF, ROSENBERG R, DELBARREM, MOUINGA A, RENARD IP Military Hospital Val de Grace, Paris

Purpose To evaluate relationships between central 10-2 visual field (VF) sensitivity by standard automatic perimetry (SAP) and the ganglion cell inner plexiform layer (GCIPL) thickness by Cirrus HD-OCT.

Methods 64 eyes: 29 control and 34 glaucomatous eyes with an isolated 10-2 central VF defect were included. Each of them underwent analyze of GCIPL thickness by Cirrus HD-OCT and a reproducible central VF testing (Humphrey Field Analyser, SITA standard 10-2, Carl Zeiss Meditec). Relationship between VF sensitivity and GCIPL thickness was analysed globally and in the six sectors of the HD-OCT-Cirrus using Spearman rank order correlations.

Results Macular VF sensitivity and GCIPL thickness relationships were statistically significant in each sector $(\mathrm{R}=0.455-0.576$, all $\mathrm{p}<0.001)$. Correlation for mean macular VF sensivity and mean GCIPL thickness was higher than sectorial correlations $(\mathrm{R}=0.645, \mathrm{p}<0.0001)$. For sectors analysed, the highest correlation was in inferotemporal sector $(\mathrm{R}=0.576)$

Conclusion GCIPL thickness by Cirrus-HD OCT showed statistically significant structure-function associations with central VF sensitivity. Mean central VF sensitivity had the strongest correlation

\section{- T036}

Evaluation of CANON SD HS 100 OCT : Reproducibility of macular ganglion cell complex (GCC) thickness measurement in normal, hypertensive and glaucomatous eyes

ROSENBERG R, MARILL AF, FENOLLAND JR, EL CHEHAB H, DELBARRE M, MARECHAL M, MOUINGA A, RENARD JP

Military Hospital Val-De-Grâce, Paris

Purpose To evaluate intra- and interobserver reproducibility of macular GCC thickness measurement by automated segmentation on CANON SD HS 100 OCT (Japan) in normal $(\mathrm{N})$, hypertensive $(\mathrm{OHT})$ and glaucomatous eyes.

Methods A total of 179 eyes of 93 patients were included: 88 N, 26 OHT and 65 glaucoma. All patients underwent a complete ophthalmologic exam, pachymetry and 24-2 automated perimetry (HFA SITA Standard). Each of two observers performed three times macular imaging with SD HS 100 OCT. Acquisitions were analyzed with Glaucoma 3D mode which estimated the macular GCC thickness in global, upper and lower macular parts and in eight separate areas. Reproducibility was assessed by intraclasss correlation coefficient (ICC), coefficient of variation (CV) and test-retest variability (TRTV) calculated as 1.96 times the standard deviation.

Results Mean GCC thickness was respectively $92.6 \mu \mathrm{m}, 88.7 \mu \mathrm{m}, 77.4 \mu \mathrm{m}$ in N, OHT and glaucomatous eyes. In all groups, intra- and interobserver reproducibility was respectively ranged for ICC from 96 to $99.7 \%$ and from 84.5 to $99.7 \%$, for $\mathrm{CV}$ from 0.44 to $2 \%$ and from 0.37 to $1.1 \%$ and for TRTV from 0.75 to $2.62 \mu \mathrm{m}$ and from 0.59 to $1.47 \mu \mathrm{m}$. Reproducibility is significantly better in $\mathrm{N}$ and $\mathrm{OHT}$ group than in glaucoma group $(\mathrm{p}<0.001)$.

Conclusion Reproducibility of GCC thickness measurements using the new OCT SD HS 100 is high. These results might be related to a high axial resolution $(3 \mu \mathrm{m})$. They underline reliability data for analyze of GCC. 
Poster session 1: Electrophysiology-physiological Optics-Vision Sciences/Glaucoma/Immunology-Microbiology/Molecular Biology-Genetics-Epidemiology/ Neuro-ophthalmology-Strabismology-Paediatric Ophthalmology-History of Ophthalmology

- T037

Visual field defects in pre-perimetric glaucoma identified by customized oct - guided white-on-white automated perimetry

MAGOURITSAS G, GEORGIADIS O, PORTALIOU DM, MOSCHONAS K Ophthalmology Department, Red Cross Hospital, Athens

Purpose We studied cases of "preperimetric" glaucoma using detailed strategies of the AP in order to detect any functional disturbance in visual field areas corresponding to the localized structural damage.

Methods We enrolled 5 patients with inter-ocular optic disc cupping asymmetry, ocular hypertension asymmetry, optic disc hemorrhage and retinal nerve fiber layer (RNFL) outside normal limits, as measured by means of optical coherence tomography (OCT). All of the 5 patients fulfilled the diagnostic criteria for "preperimetric" glaucoma, showing a normal standard visual field analysis. They were submitted in a more specific white-on-white AP using customized programs with a 3 degrees grid, instead of the standard 6 degrees used in routine 24-2 and 30-2 programs of the Humphrey AP. The examination of the visual field test points in a customized pattern has been guided by the corresponding abnormal region of the OCT, according to the structure-function map proposed by Garway-Heath et al (Ophthalmology. 2000;107:1809)

Results Using the denser grid of 3 degrees spot, we detected reduced threshold sensitivity in 3 study eves, in specific areas of the visual fields. The topography of these areas corresponded to the morphology of the localized RNFL lesion. In 2 eyes the 3 degrees customized, OCT guided, AP could not identify any functional disturbance.

Conclusion Early defects of visual fields may coexist with morphological damage of optic nerve and RNFL, even in "preperimetric" glaucoma, but remain undetected by the currently available routine 6 degrees grid programs of AP. Customized OCT guided AP may establish the diagnosis of glaucoma and put controversy in the term of "preperimetric" glaucoma.

- T039

Interest of central 10-2 visual field in glaucomatous and glaucoma suspects patients with normal central 24-2 visual field

MARECHAL M, CHAPRON T, EL CHEHAB H, FENOLLAND IR, MARILL AF, ROSENBERG R, DELBARRE M, MOUINGA A, RENARD IP

Military Hospital Val-de-Grâce, Paris

Purpose To evaluate benefit of performing central 10-2 visual field (VF) in association with 24-2 VF in glaucomatous or glaucoma suspect patients.

Methods 20 glaucoma suspect (Mean Deviation $\mathrm{MD}=0.19 \pm 0.8 \mathrm{~dB}$ ) and 48 glaucomatous eyes $(\mathrm{MD}=-2.33 \pm 1.9 \mathrm{~dB})$ with normal central $24-2 \mathrm{VF}$ were included. Patients without macular pathology underwent a complete ophthalmological examination and a reproducible VF testing (Humphrey Field Analyser, SITA standard 24-2 and 10-2, Carl Zeiss Meditec) and analyze of ganglion cell inner plexiform layer and of retinal fiber layer thickness by Cirrus HD-OCT.

Results Central 10-2 VF detect a central defect in 15 eves (22\%). In glaucoma suspect group, a defect was detected by 10-2 VF whereas 24-2 VF was normal for 2 patient $(10 \%)$. In glaucomatous group a central 10-2 VF defect missed by $24-2 \mathrm{VF}$ was detected in 7 patients (15\%). An extended central 10-2 VF defect associated with a suspect or a small paracentral 24-2 VF defect was reported for 6 glaucomatous patients (13\%).

Conclusion Central VF defect may be missed by 24-2 VF analyze. Central 10-2 VF seems to be useful in glaucoma suspect and in glaucomatous eyes for a better detection of early central functional defect
- T038

Analyze of visual field indices in ocular hypertension: PROG-F3 study

DELBARREM (1), GIRAUD IM (1), APTEL F (2), EL CHEHAB H (1), RENARD JP (1) (1) Hôpital d'Instruction des armées du Val de Grâce, Paris (2) Centre Hospitalier Universitaire Grenoble, Grenoble

Purpose To analyze visual field (VF) indices and their progression of patients treated for ocular hypertension (OHT)

Methods This retrospective study concerned $27.4 \%$ of a bicentric cohort (121 eyes of 441). These eyes with normal VF and without clinical structural defect at inclusion were monitored and treated for OHT at least 9.7+/-1.65yrs. Patients underwent every 6 months an eye examination and a reproducible VF testing (Humphrey Field Analyser, SITA Standard 24-2, Carl Zeiss Meditec). At the end of follow-up, patients who developed POAG were included in evolutive group and patients without functional defects were considered stable. MD, VFI, and their rate of progression were analyzed. Statistical analyse compared populations by a Student t-test and diagnostic performance was assessed by sensitivity (Se), specificity (Sp) and area under receiver operating characteristic curve (AUROC).

Results Evolutive group included 46 eyes and stable group included 58 eyes. Rate of progression of $\mathrm{MD}(\mathrm{dB} / \mathrm{yr})$ for stable and evolutive groups were respectively -0.007 and 0.285 ( $\mathrm{p}<0.001)$. Rate of progression of VFI (\%/yr) for stable and evolutive groups were respectively -0.026 and $-0.407(\mathrm{p}=0.001)$. Initial $\mathrm{MD}(\mathrm{dB})$ at beginning of follow up for stable and evolutive groups were 1.139 and $0.616(\mathrm{p}<0.001)$. Initial VFI (\%) were 99.793 and $99.304(\mathrm{p}<0.001)$. AUC were, respectively, for MD and VFI rate of progression : 0.967 and 0.827 . The best cut off value of AUROC was for MD rate of progression: -0.100 $\mathrm{dB} / \mathrm{yr}(\mathrm{Se}=93.5 \%, \mathrm{Sp}=93.1 \%)$.

Conclusion Rate of progression of MD and VFI in OHT evolutive group were higher than in OHT stable group. This study reports a higher diagnostic power for rate of progression of MD. It could be used to screen OHT patients at risk of POAG.

\section{- T040}

Risk factors for an initial central scotoma compared with an initial peripheral scotoma

CHO B, KANGJW, PARK BJ

Department of Ophthalmology, Konkuk University Medical Center, Konkuk University School of Medicine, Seoul

Purpose To investigate the risk factors for an initial central scotoma (ICS) compared with an initial peripheral scotoma (IPS) in normal tension glaucoma (NTG).

Methods Sixty NTG patients (60 eves) with an ICS and 106 NTG patients (106 eves) with an IPS were included. Retrospectively, the differences were assessed with baseline characteristics, ocular factors, systemic factors, and life style factors between two groups. Also, the parameters of visual field were compared between two groups. Clinical characteristics and various systemic factors were analyzed between the 2 groups

Results Patients from both ICS and IPS groups were of similar age, gender, family history of glaucoma, and follow-up periods. Frequency of disc hemorrhage was significantly higher among patients with an ICS than in patients with an IPS ( $<<0.001)$. Moreover, systemic risk factors such as hypotension, migraine, Raynaud's phenomenon, and snoring were more prevalent in the ICS group than in the IPS group $(\mathrm{p}<0.001$, $\mathrm{p}=0.002, \mathrm{p}=0.007, \mathrm{p}=0.009$, respectively). There were no statistically differences in lifestyle risk factor such as smoking or body mass index. Pattern standard deviation was significantly greater in the ICS group than in the IPS group $(\mathrm{p}=0.005)$, but the mean deviation was similar between the 2 groups ( $\mathrm{p}=0.096$ ).

Conclusion NTG patients with ICS and IPS have different profiles of risk factors and clinical characteristics. This suggests that the pattern of initial VF loss may be useful to identify patients at higher risk of central field loss. 
Poster session 1: Electrophysiology-physiological Optics-Vision Sciences/Glaucoma/Immunology-Microbiology/Molecular Biology-Genetics-Epidemiology/ Neuro-ophthalmology-Strabismology-Paediatric Ophthalmology-History of Ophthalmology

- T041

The association of nailfold capillaroscopy with systemic matrix metalloproteinase- 9 concentration in normal-tension glaucoma

$\operatorname{LEEN}(1), \operatorname{PARKC}(2)$

(1) Ophthalmology, Incheon St.Mary's hospital, Seoul

(2) Ophthalmology, Seoul St.Mary's hospital, Seoul

Purpose To investigate the association of nailfold capillaroscopy, heart rate variability (HRV), and clinical characteristics of glaucoma with the plasma matrix metalloproteinase-9 (MMP-9) level in normal-tension glaucoma (NTG)

Methods We conducted a prospective, cross-sectional study on 25 patients with NTG. Subjects with systemic diseases were excluded. The patients underwent a complete ophthalmic examination and were referred to the Rheumatology Department, where nailfold capillaroscopy and HRV assessment were performed. The patients were assigned to the lowest and highest HRV groups according to the standard deviation value of the qualified normal-to-normal intervals of the HRV assessment. Blood samples from all of the subjects were assayed for MMP-9 concentrations.

Results The systemic MMP-9 level was significantly associated with the nailfold capillaroscopy result $(\rho=0.439, \mathrm{p}=0.032$ ). However, there was no significant association of HRV parameters, disc hemorrhage, or visual field progression with the systemic MMP-9 level.

Conclusion The systemic MMP-9 level was associated with the nailfold capillaroscopy results in patients with NTG but had no direct association with optic disc hemorrhage.

- T043

Comparison of two methods for location of the ciliary body

before glaucoma treatment using High Intensity Focused Ultrasound (HIFU)

APTEL F (1), ROULAND J (2), CHAVEROT D (3), DUPUY C (1), COPPIN J (2)

(1) University Hospital of Grenoble, Grenoble

(2) University Hospital of Lille, Lille

(3) Eyetechcare, Rillieux-la-Pape

Purpose To evaluate the ability of the white-to-white (WTW) and axial length (AL) measurements to determine the location of the ciliary body before High Intensity Focused Ultrasound (HIFU) glaucoma treatment.

Methods 189 eyes (104 phakic, 85 pseudophakic) of 161 patients were included. WTW and $\mathrm{AL}$ measurements were measured with the IOLMaster. The HIFU size probe selection was done based using ultrasound biomicroscopy (UBM) and a computer assisted overlay drawing method performed by the manufacturer of the HIFU device Three probe models with different ring diameters $(11,12$ and $13 \mathrm{~mm})$ are available for HIFU treatment. Eyes were divided into 3 groups: "11" ( $\mathrm{n}=11)$, "12" ( $\mathrm{n}=83)$ and "13" $(\mathrm{n}=95)$ corresponding to the size of the probe recommended based on UBM images. The agreement between the group and the WTW and AL were evaluated.

Results For groups "11", "12" and "13", mean WTW and AL measurements were respectively $11.44 \pm 0.11 \mathrm{~mm}$ and $22.04 \pm 0.28 \mathrm{~mm}, 11.84 \pm 0.04 \mathrm{~mm}$ and $23.22 \pm 0.20$ $\mathrm{mm}$, and $12.36 \pm 0.06 \mathrm{~mm}$ and $25.43 \pm 0.3 \mathrm{~mm}$. Mann\&Whitney and ANOVA tests showed a significant difference between the 3 groups $(\mathrm{p}<05)$. By placing all the data in a coordinate system WTW-AL, we can distinguish 3 areas (11,12 and 13) allowing to choose the diameter with a $93 \%$ agreement with the UBM-based determination.

Conclusion It seems possible to determine the probe model with a nomogram based on easily measurable ocular parameters.

\section{- T042}

Refractory open angle glaucoma treated by high intensity focused ultrasound (HIFU). Results at one year of a prospective series

ROULANDJ (1), APTEL F (2)

(1) University of Lille 2, Lille

(2) University of Grenoble, Grenoble

Purpose To assess the safety and efficacy of Ultrasound Circular Cyclo-Coagulation (UC3 procedure) using HIFU (high intensity focused ultrasound) in patients with refractory open angle glaucoma.

Methods Prospective clinical series performed in two centers, on twenty-eight eyes of twenty-eight patients with primary open-angle glaucoma, treated with the EyeOP1 medical device equipped with six miniaturized cylindrical piezoelectric transducers. All eyes were treated with a 6 -second exposure time from each transducer. The main assessment criteria were safety and efficacy as indicated by the incidence of complications and by the IOP reduction. Ophthalmic, ultrasound biomicroscopy and flare examinations were performed before treatment and during clinical follow-up at D1, D7, M1, M2, M3 M6 and M12

Results The mean intraocular pressure was significantly reduced from $29.0 \pm 7.2$ $\mathrm{mmHg}$ before treatment to $21.6 \pm 9.4 \mathrm{mmHg}$ at last follow-up. Eight patients needed to be re-treated. Complete success rate, as defined by an IOP reduction $>20 \%$ and IOP> $5 \mathrm{mmHg}$ after one UC3 procedure was 50\%, and $68 \%$ after re-treatments. The mean IOP reduction achieved in responding patients was $45 \%$. No major intra- or postoperative complications occurred. Clinical examination showed no lesions of the ocular structures other than the ciliary body and no or few signs of intraocular inflammation after treatment. Ocular inflammation evidenced by flare was very limited and nonsignificant.

Conclusion Coagulation of the ciliary body using high intensity focused ultrasound delivered by miniaturized transducers is a simple, well-tolerated procedure which enables to significantly reduce the intraocular pressure in patients with refractory Open Angle Glaucoma.

Commercial interest

\section{- T044}

Multicenter clinical trial of ultrasonic circular cyclo coagulation in glaucoma patients naive of filtering surgery. Preliminary results at 6 months

APTEL F (1), DENIS P (2), ROULAND J (3), RENARD JP (4), BRONAM (5)

(1) University Hospital of Grenoble, Grenoble

(2) University Hospital of Lyon, Lyon

(3) University Hospital of Lille, Lille

(4) Hôpital d'Instruction des Armées du Val de Grâce, Paris

(5) University Hospital of Dijon, Dijon

Purpose To evaluate the efficacy and safety of the Ultrasonic Circular Cyclo Coagulation (UC3) procedure in patients naive of previous filtering glaucoma surgery

Methods Prospective non comparative interventional clinical study performed in 5 French glaucoma centers. Thirty eyes of 30 patients (24 primary open-angle glaucoma and 6 secondary glaucoma), with intraocular pressure (IOP) $>21 \mathrm{mmHg}$, naive of previous filtering glaucoma surgeries, were insonified with a therapy probe comprising 6 piezoelectric transducers. The 6 transducers were activated with a 6 seconds exposure time. Complete ophthalmic examinations were performed before the procedure, and at 1 dav, 1 week, 1, 2, 3, 6 and 12 months after. Primary outcomes were surgical success (defined as IOP reduction from baseline $\geq 20 \%$ and IOP $>5 \mathrm{mmHg}$ ) at the last followup visit, and vision-threatening complications. Secondary outcomes were mean IOP at each follow-up visits compared to baseline, medication use, and complications.

Results IOP was significantly reduced ( $\mathrm{p}<0.05$ ), from a mean preoperative value of 28.3 $\pm 7.0 \mathrm{mmHg}$ to a mean value of $19.5 \pm 7.2 \mathrm{mmHg}$ at 6 months. Success (IOP reduction $>20 \%$ ) was achieved in $63 \%$ of eyes at 6 months after one HIFU procedure, with a mean IOP decrease of $39 \%$. No major intra- or post-operative complications occurred.

Conclusion Ultrasonic Circular Cyclo Coagulation seems to be an effective and well tolerated method to reduce intraocular pressure in glaucoma patients naive of previous glaucoma surgery.

Commercial interest 
Poster session 1: Electrophysiology-physiological Optics-Vision Sciences/Glaucoma/Immunology-Microbiology/Molecular Biology-Genetics-Epidemiology/ Neuro-ophthalmology-Strabismology-Paediatric Ophthalmology-History of Ophthalmology

- T045

Intraocular pressure dynamics during one year followup after phacotrabeculectomy versus trabeculectomy for primary open angle glaucoma

POPA CHERECHEANU A (1, 2), COMAN C (2), PIRVULESCUR (2)

DASCALUAM (2), GEAMANUA (2), STANA D (1), DUTA S (1)

GRADINARUS (2), IANCUR (2)

(1) Department of Ophthalmology, Emergency University Hospital, Bucharest

(2) "Carol Davila" University of Medicine and Pharmacy, Bucharest

Purpose Maintaining intraocular pressure in range of target values interval is one of the desiderates of antiglaucoma surgery. Intraocular pressure (IOP) variations occur after trabeculectomy or phacotrabeculectomy. The aim of our study is to provide data regarding the magnitude of these variations during one year following the surgical interventions mentioned above

Methods This is a study assessing two groups, one comprising of 28 patients that underwent trabeculectomy with 5 -fluorouracil and the other comprising of 26 patients that underwent phacotrabeculectomy with 5-fluorouracil. All patients had surgery for uncontrolled primary open angle glaucoma. The IOP was monitored using Goldmann aplanotonometry 1 day, 1 week, 1 month, 3 months, 6 months and 1 year postoperative. Results The difference between the mean IOP after 1 year and the preoperative IOP was statistically significant in both groups $(9.41+/-4,32 \mathrm{mmHg}, 42 \%$ reduction in trabeculectomy group, p value 4.45x10-19 and 8.03+/-5,17 mmHg, 34\% reduction in phacotrabeculectomy group, $\mathrm{p}$ value $1.46 \times 10-9)$ and the mean IOP was significantly reduced at all time points in both groups

Conclusion IOP was statistically significant reduced after surgery in both groups. The maximal variation occurred after phacotrabeculectomy, with the highest magnitude in the first 6 months after surgery. In the following 6 months, IOP did not change statistically significant after either phacotrabeculectomy or trabeculectomy.

- T047

Bleb needling outcomes for failed trabeculectomy blebs in Asian eyes: a 2 year follow up

TSAI A, BOEY PY, HTOON HM, WONG TT

Singapore National Eye Centre, Singapore

Purpose To describe the outcomes and success rates of bleb needling in primary angle closure glaucoma (PACG), primary open angle glaucoma (POAG) and secondary glaucoma.

Methods Retrospective review of 270 patients who underwent bleb needling over a 2 year period. Complete success was defined as intraocular pressure $(\mathrm{IOP})>=6 \mathrm{mmHg}$ and $=<21 \mathrm{mmHg}$, in absence of further surgery or use of antiglaucoma medication. Qualified success met the above criteria with or without use of antiglaucoma medications.

Results 207 eyes completed the two year follow up. 84\% was primary glaucomas and $16 \%$ was secondary glaucomas. The mean interval between filtering surgery and bleb needling was 8.4(SD 17.2) months. The mean IOP pre-needling was 21 (SD7.2) and there was a statistically significant reduction of IOP of at least $22 \%$ from month 1 through to month 24. The complete success rates at month 6 for POAG, PACG and secondary glaucoma are $70 \%, 66 \%$ and $49 \%$ respectively, $62 \%, 62 \%$ and $51 \%$ at month 12 and $58 \%, 63 \%$ and $38 \%$ at month 24 . The qualified success rates at month 6 for POAG, PACG and secondary glaucoma $24 \%, 30 \%$ and $29 \%$ respectively, 32\%, $29 \%$ and $36 \%$ at month 12 , and $35 \%, 30 \%$ and $53 \%$ at month 24 . Logistic regression analysis showed that at month 6, POAG and PACG had a greater needling success compared to secondary glaucoma (OR 4.3.and 6.0 respectively; $\mathrm{p}<0.05$ ). However, at month 12 and 24 the difference in success rates was not statistically significant.

Conclusion Bleb needling within one year of trabeculectomy in Asian eyes can provide clinically significant IOP lowering of $>22 \%$ for 2 years. Eyes with primary glaucomas had a greater success. Asian eyes have a greater propensity for scarring but timely bleb needling can rescue bleb function.

\section{T046}

Choroidal detachment in post trabeculectomy patients in an asian tertiary eye centre

XU YP, YIP WLL, YONG KYV, LIM BA, WONG HT, GANN

Tan Tock Seng Hospital Opthalmology, Singapore

Purpose The aim of this study is to identify risk factors that lead to choroidal effusion in post trabeculectomy patients.

Methods The clinical notes of all patients who developed choroidal detachment after trabeculectomy for glaucoma between 2000 to 2011 in a tertiary institution were reviewed. Potential risk factors including glaucoma subtype, intraoperative complications, ischemic risk factors, use of anti-metabolites and post-operative procedures were analysed as potential risk factors.

Results Twenty-three subjects developed post-trabeculectomy choroidal detachments. The mean age was $70.8+/-12$ years (range 38 to 86 years old). 17 out of the 23 choroidal detachments were male patients. The most common subtype of glaucoma associated with choroidal detachment was primary open angle glaucoma. 2 patients $(8.7 \%)$ developed choroidal detachments within 1 week post-operatively. The hypotony lasted 2 weeks to 48 months with 4 patients (17.4\%) requiring revision of the bleb. All patients had adjunctive anti-metabolites used, with MMC used in 22 cases and 5FU used in 1. 4 patients (17.4\%) had diabetes, 6 (26.0\%) had hyperlipidemia, 11 (47.8\%) had hypertension and $2(8.7 \%)$ were smokers. The mean final visual outcome was 0.30 on the $\log$ MAR scale.

Conclusion All patients had good visual outcomes. Of the factors analysed, use of antimetabolites was the only one found to be associated with development of choroidal detachment post-trabeculectomy. Vast majority of choroidal detachments occur beyond 2 weeks after surgery and clinicians should continue to monitor these patients carefully.

- T048

Serial changes in the bleb wall after glaucoma drainage implant surgery: Characteristics during the hypertensive phase

JUNG KI

Department of Ophthalmology and Visual Science, Seoul St. Mary's Hospital,

The Catholic University of Korea, Seoul

Purpose To investigate serial changes in the bleb wall using anterior segment-optical coherence tomography (AS-OCT) in patients who had undergone Ahmed glaucoma valve (AGV) implantation

Methods A total of 52 patients who had undergone AGV implantation were enrolled. Reflectivity and mean thickness of the bleb wall were examined using AS-OCT at 1 day, 1 week, 1 month, 3 months, and 6 months postoperatively. Intraocular pressure (IOP) and the number of glaucoma medications was evaluated. The hypertensive phase was defined as IOP $>21 \mathrm{mmHg}$ during the first 3 months after surgery. Patients were divided into two groups: hypertensive and non-hypertensive groups.

Results Following AGV implantation, IOP decreased at each time point during postoperative follow-up (P value $<0.001$, all) with a peak in the first month. Mean bleb wall thickness showed a U-shaped curve postoperatively, being thinnest 1 month after the operation. Reflectivity showed an inverted U-shaped curve postoperatively, being brightest 1 month after the operation. A hypertensive phase was observed in 44 patients (84.6\%). The bleb wall was significantly more reflective $(130.67 \pm 27.00$ vs. $106.57 \pm$ $10.35 ; \mathrm{P}=0.044$ ) in the hypertensive group than in non-hypertensive group 1 month postoperatively.

Conclusion Hypertensive phase peaked at 1 month after aqueous shunt surgery. At that time, the bleb wall had the highest reflectivity. The unique IOP pattern after tube surgery is related to fibrosis of the bleb wall qualitatively. Specific modulation of wound healing may be the target for attempts to decrease the development of hypertensive phase. 
Poster session 1: Electrophysiology-physiological Optics-Vision Sciences/Glaucoma/Immunology-Microbiology/Molecular Biology-Genetics-Epidemiology/ Neuro-ophthalmology-Strabismology-Paediatric Ophthalmology-History of Ophthalmology

\section{- T049}

Management of secondary glaucoma in patients with uveitis

MUMCUOGLU T, OZGEG

GATA Medical Academy Department of Ophthalmology, Ankara

Purpose To report the clinical course of patients with uveitic glaucoma.

Methods The medical records of the patients diagnosed with uveitic glaucoma at the Glaucoma Service of the Department of Ophthalmology were reviewed retrospectively

Results Twenty eight patients ( 38 eyes) presented, of whom 20 were male and 8 were female. The mean age was $50.4 \pm 16.5$. Eighteen patients had disease unilaterally and 10 bilaterally. Uveitis was associated Behçet's disease in 6 patients, and idiopathic in 22 patients. All patients initially received topical medical therapy. Sixteen eyes were treated with monotherapy and 22 eyes with multitherapy. Surgical procedures (trabeculectomy or combined phacotabeculectomy) were performed in 20 eyes in which medical therapy failed. Secondary surgical procedures such as bleb revision or drainage implantation were needed in 6 eyes.

Conclusion Secondary glaucoma associated with uveitis is an underappreciated vision-threatening complication and a challenging condition to manage.
- T050 / 1736

Self-tonometry is useful to detect IOP elevations in DALK patients with apparently normal intraocular pressures

SMEDOWSKIA (1), TARNAWSKA D (1,2), WYLEGALA E (1)

(1) Clinical Department of Ophthalmology, Faculty of Medicine and Department of Dentistry in Zabrze, Medical University of Silesia, Katowice

(2) Department of Biophysics and Molecular Physics, Institute of Physics, University of Silesia, Katowice

Purpose To perform increased intraocular pressure (IOP) screening in nonglaucomatous patients after deep anterior lamellar keratoplasty (DALK) due to keratoconus with normal IOP measured during control visits.

Methods Ten non-glaucomatous patients, who underwent DALK procedure due to keratoconus were included into study. Patients were measuring IOP using self-tonometer Icare One (Icare, Finland) continuously for 30 days, 3 times per each day. Additionally patients were checked by ophthalmologist 3-times during follow-up time in Outpatient Clinic, where applanation tonometry and central corneal thickness measurements were performed. After 30-days, self-measured IOP was evaluated for rises above $21 \mathrm{mmHg}$ as well as rises above mean GAT values measured during control visits.

Results Mean IOP measured with GAT and adjusted according to CCT was $15 \mathrm{mmHg}$ and ranged between 11 and $19 \mathrm{mmHg}$. There were no values above $21 \mathrm{mmHg}$ reported during follow-up time. In Icare One measurements, all patients showed incidents of IOP values higher than measured with GAT, however mean values ranged between 10$18 \mathrm{mmHg}$. Six out of 10 patients revealed to have IOP elevations higher than $21 \mathrm{mmHg}$ between control visits (with maximum values up to $46 \mathrm{mmHg}$ ), and this elevated values constituted from 3.3 up to $20.0 \%$ of all self-measurements.

Conclusion Self-tonometry with Icare One might be useful tool to identify patients who develop undetectable IOP increases between control visits. This can help in prevention of vision loss and transplant procedure failure in DALK patients.

- T052 / 1737

Different aspects of IOP measurements with GAT versus DCT in glaucoma patients

MEIER-GIBBONS F

Private office, Rapperswil

Purpose 1. To compare the intraocular pressure (IOP) measurements with Goldmann Applanation Tonometry (GAT) versus Dynamic Contour Tonometry (DCT) in patients with glaucoma or ocular hypertension (OHT).2. To evaluate whether the measurements of GAT versus DCT are influenced firstly by the type of glaucoma (Primary Open Angle Glaucoma, Pseudoexfoliative Glaucoma, Angle Closure Glaucoma or OHT), secondly by the type of glaucoma therapy and thirdly by the Central Corneal Thickness (CCT).

Methods Office based clinical study of 282 consecutive patients with glaucoma or OHT. Consecutive IOP measurements with GAT and DCT and CCT measurements (with ultrasound pachymetry) by one examiner.A statistical analysis evaluates whether the type of glaucoma, the type of glaucoma therapy or the CCT influence the difference between the IOP measurements with GAT and DCT.

Results Ongoing study, preliminary results show a difference between GAT and DCT in the range of other studies (DCT slightly higher in most of the patients). The type of glaucoma therapy does not seem to influence the difference between GAT and DCT, nor does the CCT or the type of glaucoma.

Conclusion The GAT is still the gold standard, but the IOP measurements with the CCT independent DCT become more and more important. Preliminary results show that in this study, unlike in another study with much less patients, the glaucoma therapy did not influence the difference between GAT and DCT.

Commercial interest 
Poster session 1: Electrophysiology-physiological Optics-Vision Sciences/Glaucoma/Immunology-Microbiology/Molecular Biology-Genetics-Epidemiology/ Neuro-ophthalmology-Strabismology-Paediatric Ophthalmology-History of Ophthalmology

\section{- T053 \\ Long-term efficacy of interferon in severe uveitis asssociated with Behçet disease}

DIWO E (1), GUEUDRY J (2), WESCHLER B (3), SAADOUND (3), LEHOANG P (1), BODAGHIB $(4)$

(1) Department of Ophthalmology, Salpêtrière Hospital, Paris

(2) Department of Ophthalmology, Rouen

(3) Department of Internal Medicine, Salpêtrière Hospital, Paris

(4) Department of Ophthalmology, Salpêtrière Hospital, Paris

Purpose We aimed to evaluate long-term efficacy of interferon alpha 2a (IFNo2a) in severe Behçet disease(BD)-related uveitis.

Methods Our retrospective study includes all patients with severe uveitis treated with IFN 2 a for at least 18 consecutive months at Pitié Salpêtrière Hospital between 05/1995 and $07 / 2013$. Before, during and after treatment, best corrected visual acuity (VA), ophthalmologic examination and fluorescein angiography were performed to evaluate the number of acute uveitis. The main evaluation criteria was the number of uveitis relapses per person per year before, during and after treatment.

Results 33 patients, $12 \mathrm{~W} / 21 \mathrm{M}$, with a mean age of 30,5 years and with mainly panuveitis $(74.2 \%)$ were included. The mean observation period was 8,82 years. We followed 33, 25, 20 and 18 patients for 2, 4, 7 and 9 years respectively. The relapse rate decreased from 1,36 before treatment to 0,045 relapses/patient/year during IFNa treatment. After IFNo discontinuation (possible for $64 \%$ of all patients), it increased to 0,057 relapses/patient/year and the mean period between discontinuation and relapse was 24.2 months. Average VA improved from 0,49 to 0,27 and from 0,55 to 0,39 LogMar for the patients with a followed-up of 4 and 9 years respectively. During 4 years following IFN 2 2a initiation, we observed rapid decrease of anterior inflammation, hyalitis, vasculitis, macular and papillary edema. Mean oral prednisone consumption decreased from $22,8 \mathrm{mg}$ at IFN initiation to $5,5 \mathrm{mg}$ per day at 9 years.

Conclusion IFN 22 a is efficient to treat severe BD-related uveitis. For initial responders allowing discontinuation of IFN $\alpha 2 a$, the long-term remission was confirmed with our study.

- T055 / 2645

FGF $\beta$ and TGF $\beta$ contribute to tissue remodeling, fibrosis and inflammation in the orbital tissue of severe Grav

PAWLOWSKIP (1, 2), RESZECJ (1), ECKSTEINA (3), JOHNSON K (3), GRZYBOWSKIA (4, 5), CHYCZEWSKI L (6), MYSLIWIEC J (7)

(1) Department of Medical Pathomorphology, Cathedral of Biostructure, Medical University of Bialystok, Bialystok

(2) Department of Paediatric Ophthalmology with Strabismus Treatment Unit, Medical University of Bialystok, Bialystok

(3) Department of Ophthalmology, University Hospital Essen, University of DuisburgEssen, Essen

(4) Department of Ophthalmology, Poznań City Hospital, Poznan

(5) Department of Ophthalmology, University of Warmia and Mazury, Olsztyn

(6) Department of Medical Pathomorphology, Cathedral of Biostructure, Medical

University of Biatystok, Bialystok

(7) Department of Nuclear Medicine, Medical University of Bialystok, Bialystok

Purpose To assess FGF- $\beta$, TGF- $\beta$, COX2 expression and immunocompetent cells in the orbital fat/connective tissue of patients with severe and mild Graves' orbitopathy as possible prognosting factors of the course of disease.

Methods Orbital tissue from 27 patients with GO undergoing orbital decompression (26 females and 1 male): 1) patients with severe $\mathrm{GO}(\mathrm{n}=18)$, the mean clinical activity score (CAS) was 8,5 (SD 2.5); 2) Patients with mild GO (n= 9), CAS was 2.2 (SD 0.8). 10 individuals undergoing blepharoplasty with no history of orbital inflammation served as controls. We evaluated the expression of CD4+, CD8+. CD20+, CD68 and FGF- $\beta$, TGF- $\beta$, COX 2 in the orbital tissue by immunohistochemical staining

Results The robust intraorbital CD4 + T cells infiltration in severe GO more than with mild GO with absence of $\mathrm{CD} 20+\mathrm{B}$ lymphocytes and less CD8 infiltration were seen. CD68 expression (fibroblasts and macrophages staining) observed in the fibrous connective area of the adipose tissue of the mild GO and robust in severe GO. Increased FGE $\beta$ expression was observed in the fibroblasts and adipocytes in the connective tissue of the severe GO. The prominent TGF $\beta$ expression was observed in the fibrous connective tissue of the severe and mild GO. No expression of COX2 in the orbital tissue specimen was found in patients with $\mathrm{GO}$.

Conclusion Macrophages and CD4 T lymphocytes are both engaged in the active/ severe and long stage of inflammation in the orbital tissue. FGF $\beta$ and TGF $\beta$ expression may contribute to tissue remodeling, fibrosis and perpetuation of inflammation in the orbital tissue of GO especially in severe GO. Their high expression may suggest lack of effect of anti-inflammatory treatment

\section{T054}

Efficacy and safety of anti TNFo in non-infectious uveitis: a multi-center retrospective study

BIDAUT GARNIER M (1, 2), VALLET H (3), SEVE P (4), HERON E (5), PERLAT A (6) TIEULIEN (7), PERARD L (8), LEHOANG P (2, 9)

SAADOUND (10, 9), BODAGHI B (2, 9)

(1) Ophthalmology - University Hospital of Besançon, Besançon

(2) Ophthalmology, DHU ViewMaintain, Pitie Salpetriere Hospital, Paris

(3) Internal Medicine - University Hospital of Pitié Salpêtrière, Pari

(4) Internal Medicine - University Hospital of Lyon, Groupement Hospitalier Nord, Croix-Rousse Hospital, Lyon

(5) Internal Medicine, Quinze-Vingts National Ophthalmology Hospital, Paris

(6) Internal Medicine, University Hospital of Rennes, Southern Hospital, Rennes

(7) Internal Medicine, University Hospital of Nice, Archet Hospital, Nice

(8) Internal Medicine, University Hospital of Lyon, Groupe Hospitalier Edouard Herriot, Lyon

(9) Vision and Handicaps, ViewMaintain, University of Pierre et Marie Curie, University Department of Hospital, Paris

(10) Internal medicine, University Hospital of Pitié Salpêtrière, Pari

Purpose TNF- $\alpha$ inhibitors have been used over the last few years in noninfectious uveitis refractory to traditional immunosuppressive agents, with promising results. However, in the literature, TNF- $\alpha$ blockers have mostly been studied in uncontrolled trials, or case-series with small effectives. The purpose was to evaluate the efficacy and safety of anti TNF- $\alpha$ in a larger effective of patients.

Methods Evolution of noninfectious uveitis in patients treated with anti TNF- $\alpha$ agents was retrospectively reviewed, for subjects managed of between July 2001 and June 2013 in ten French departments of ophthalmology. Ocular inflammation, dosage of prednisone were the main criteria for efficacy.

Results We included 162 patients. Uveitis was bilateral in $82 \%$, panuveitis in $62 \%$, with retinal vasculitis in $35 \%$ and macular edema in $54 \%$ of cases. Anti TNF $\alpha$ (infliximab $57 \%$, adalimumab $40 \%$, etanercept $3 \%$ ) were introduced in case of non-response to immunosuppressives in $88 \%$. Median duration of treatment was 18 months [8-33] and median follow up, 27 months [8-53]. Complete or partial response was reported in $93.2 \%$ of subjects at 1 month. Mean dose of prednisone was significantly reduced after 6 and 12 months of treatment $(\mathrm{n}=101 ; 15 \mathrm{mg}[6-47,5]$ at introduction vs $10 \mathrm{mg}[8$ 15] at 6 months and 9mg [5-15] at 12 months; $\mathrm{p}<0,0001)$. Side effects were observed in 32 patients $(\mathrm{n}=130 ; 25 \%)$; they were severe in 3 cases ( 1 lymph node tuberculosis and 2 angioedema). Side effects came from infliximab rather than adalimumab $(\mathrm{p}<0.001)$.

Conclusion TNF- $\alpha$ blockers seem to be safe and effective in reducing inflammation with further tapering of corticosteroids, in severe cases of noninfectious uveitis.

\section{- T056}

MHC Class II expression in the retina during experimental autoimmune uveitis

IPSKID (1, 2), DEWISPELAERE R (3, 2), FOUCART V (2), CASPERS L (3), BRUYNSC (2) WILLERMAINF $(3,2)$

(1) Erasme Hospital, Brussels

(2) IRIBHM, Brussels

(3) CHU Saint-PIerre, Brussels

Purpose Autoimmune posterior non infectious uveitis induction requires intraocular MHCII-dependent recognition of a retinal antigen by autoreactive T cells. MHCII basal retinal expression is weak, but possibly induced during uveitis. In this work, we aim to identify the retinal cell types susceptible to express MHCII during EAU.

Methods C57Bl/6 mice were immunized with IRBP1-20. At day 12, T cells were semi-purified from lymph nodes and spleens, re-stimulated in vitro and injected to naive $\mathrm{C} 57 \mathrm{Bl} / 6$ mice. Fundoscopy was performed after 3 weeks. MHCII expression was analyzed by immunofluorescence on eye cryosections and co-labellings for GFAP, CD31, endoglin and IBA-1 realized to identify cells expressing MHCII.

Results No expression of MHCII is detected in naive retinas, while strong induction is found during EAU. The majority of cells infiltrating the vitreous co-stain for MHCII and IBA-1, a marker of macrophages and microglial cells. Extravasated cells around vasculitis lesions also show MHCII staining, partially co-localized with IBA-1. At the vascular level, marginal expression of MHCII appears on GFAP+ (glial and Müller cells) and CD31+ (endothelial cells, platelets and macrophages) cells, while no co-staining is observed with endoglin. MHCII is expressed on the ciliary body epithelium and lightly on the RPE. Microglia displays an intense diffuse MHCII expression.

Conclusion Our results support a strong MHCII induction during EAU, on both retinal and infiltrating cells. At the level of the BRB, although RPE cells express some MHCII, our data do not demonstrate expression of MHCII by endothelial cells. The question of how antigen presentation to activated $\mathrm{T}$ cells occurs at the site of the inner $\mathrm{BRB}$ remains to be addressed. 
Poster session 1: Electrophysiology-physiological Optics-Vision Sciences/Glaucoma/Immunology-Microbiology/Molecular Biology-Genetics-Epidemiology/ Neuro-ophthalmology-Strabismology-Paediatric Ophthalmology-History of Ophthalmology

- T057

Staphylococcal Panton-Valentine leucotoxin targets retina through the ganglion cell layer in a rabbit toxin-induced endophthalmitis model

HEITZP (1), TAWKM (2), PRÉVOST G (2), GAUCHER D (1)

(1) Ophthalmology, Strasbourg

(2) Institut de bactériologie, Strasbourg

Purpose Staphylococcal leucotoxins form a cluster of bi-component leucotoxins that are associated with tissue necrosis pathologies, such as necrotising pneumonia, osteomyelitis and endophtalmitis. Among them, Panton-Valentine leucotoxine (PVL) is often associated with strains isolated in such diseases and seems to possess acute capabilities to promote necrosis. When injected into the rabbit vitreous, these toxin were proven to induce a huge inflammatory reaction that might concern the full eyeball. The purpose of this study was to identify the PVL target cells into the retina, using a rabbit model of endophtalmitis.

Methods Three $\mu \mathrm{g}$ of LukS-PV were injected in a series of rabbit eyes. After 1 hour, the eyes were enucleated. Frozen sections were prepared for immunofluorescence detection tests. PVL was immunolabelled using a specific antibody and a secondary fluorescent antibody. Co-labelling with GFAP (Muller cells), RBPMS (ganglion cells), ChAT (starbust displaced amacrine cells) antibodies were realized. Left eyes were used as negative controls.

Results LukS-PV was present at the ganglion cells layer. A few dendrites in the inner plexiform laver were also noted. Co-labeling experiments with the specific marker ChAT (acetylcholin transferase) segregated starbust displaced amacrine cells as a retinal target of LukS-PV. $65 \%$ of the infected cells were ChAT positive

Conclusion At least, one retinal cell type, i.e. Starbust amacrine cells, can now be designated as a target of PVL and it will be important to explore how this cell fitness is sensitive to such a toxin compared with the activation of polymorphonuclear cells, and neurons that also were reported as possible targets.

- T059 / 2646

Study of TOXO KO vaccine efficiency on the ophtalmological damage bound to Toxoplasmaa gondii, on a mouse model of ocular toxoplasmosis

LEMEE G (1), MORISSE S (2), TARFAOUIN (1), DIMIER-POISSONI (2) SECHE E (2) PISELLA P(1)

(1) ophtalmology, Tours

(2) immunology, Tours

Purpose The toxoplasmosis is one of the most wild-spread parasitoses, affecting almost a third of the world population, and as the main svmptomatic demonstration : eye infringement.The aim of this study is to test, on a murin model, the efficiency of a vaccine candidate named Toxo $\mathrm{KO}$, developped by genetic engineering against this pathology.

Methods Ninety Swiss Webster mice (OF1), were filled up with fifty cysts of the 76K strain of Toxoplasmaa gondii (type II). A part of these mice received the TOXO KO vaccine intraperitoneally before. Ophtalmological examination and immunological investigation were performed on aqueous humor samples. The intra retinal cysts were also counted after dissection of the entire retinal tissue.

Results After vaccination, we observed a decrease of $69 \%$ of ophtalmological damage and $90 \%$ of intra-retinal cysts. Besides, this group presented no secretion of IFN-y in aqueous humor, testifying of an absence of deleterious inflammation in these eyes unlike those of the control group.

Conclusion Our results are highly significant. This proof of concept lets think that the forward use of such a tool of prevention is possible, to fight more effectively and prematurely against ocular toxoplasmosis. 
Poster session 1: Electrophysiology-physiological Optics-Vision Sciences/Glaucoma/Immunology-Microbiology/Molecular Biology-Genetics-Epidemiology/ Neuro-ophthalmology-Strabismology-Paediatric Ophthalmology-History of Ophthalmology

- T061

Ocular manifestations among hiv children at the regional hospital of bafoussam - cameroon

DOMNGANG NOCHE C (1), KAGMENI G (2), KEMAYOUD (1), BELLA A (2) (1) Higher Institute of Health Sciences / Université des Montagnes, Bangangte (2) FMSB/ Université de Yaounde 1, Yaounde

Purpose to determine the frequency and types of ocular manifestations in HIV children, as well as search for determinants of these ocular manifestations

Methods Our study was cross-sectional and descriptive, and was held in the Regional Hospital of Bafoussam (Cameroon) from March to June 2013. The sampling was consecutive.

Results Forty (21 boys and 19 girls) children were included in the study (mean age= 7.5 years) which $97.5 \%$ were under treatment. The overall frequency of participants with ocular manifestations was $27.5 \%(\mathrm{n}=11 / 40)$ with a predominance of adnexal disorders $(\mathrm{n}=7)$. Adnexal pathologies found were infectious conjunctivitis $(\mathrm{n}=3)$, allergic conjunctivitis $(\mathrm{n}=1)$, blepharitis $(\mathrm{n}=1)$, molluscum contagiosum $(\mathrm{n}=1)$ and nystagmus $(\mathrm{n}=1)$. The only anterior segment disease found was a cataract with posterior synechiae $(\mathrm{n}=1)$ and the posterior segment pathologies were: a retinitis with vasculitis $(\mathrm{n}=1)$ and a papillary atrophy $(\mathrm{n}=2)$. The frequency of eye diseases was $33,3 \%(\mathrm{n}=7 / 21)$ among boys and $22,0 \%(4 / 19)$ among girls $(\mathrm{p}=0,38)$. They were more prevalent in the $<5$ years $(33,3 \%$, $\mathrm{n}=4 / 12)$ and $11-15$ years $(38,5 \%, \mathrm{n}=5 / 13)$ and were less in the $6-10$ years $(13,3 \%, \mathrm{n}=2$ /15) $(\mathrm{p}=0,52)$. They were also more frequent among participants with CD4 between 201 and 400 cells $/ \mathrm{mm} 3(\mathrm{p}=0,36)$.

Conclusion The frequency of ocular manifestations of HIV children (victims of mother to child transmission) was $27.5 \%$ in our study. The most frequent pathologies were not specific to the HIV infection.

- T063

Impact of herpes zoster on quality of life and age-related functional decline

LABETOULLEM $(1,2)$

(1) on behalf of the QUALIPZO multidisciplinary group, France

(2) Ophthalmology, Bicetre Hospital, South Paris University, Le Kremlin Bicêtre

Purpose Herpes zoster (HZ) is a painful and debilitating disease, which incidence and severity increase with age. Whereas the burden of post-herpetic neuralgia (PHN), its most common complication, has been evaluated in several studies, the specific impact of $\mathrm{HZ}$ ophthalmicus $(\mathrm{HZO})$ on health-related quality of life (HR-QoL) and functional decline has been poorly studied. The objective of our study was to describe the rationale and design of the QUALIPZO (QUAlity of LIfe in Patients with herpes ZOster) study.

Methods Our multidisciplinary group (pharmacologist, general practitioner geriatrician, neurologist, ophthalmologist, pain specialist, psychiatrist, and virologist) performed a narrative analysis of the literature on the relationship between HZ (HZO incl.) and functional decline.

Results Functional decline, defined by reduced ability to perform everyday activities is enhanced with age, comorbidities, and infections. $\mathrm{HZO}$ represents about $10 \%$ of $\mathrm{HZ}$ cases, with direct ocular impairment occurring in $>=50 \%$ of cases and vision decrease in about $7 \%$ of cases. PHN is more frequent after HZO than non-ophthalmic HZ. Several studies have clearly shown that HZ dramatically impairs HR-QoL and functional status; however, no large-scale study has focused on $\mathrm{HZO}$.

Conclusion The impact of $\mathrm{HZO}$ needs to be assessed, as proposed in the incoming QUALIPZO study.
- T062 / 2647

Eswab $^{\circ}$ transport media and $16 \mathrm{~S}$ RNA PCR in the microbiological diagnosis of infectious keratitis

BAUDONNET R (1, 2, 3), GARNIER F (4, 2, 3), ROBERT PY (1, 2,3)

(1) Limoges University Hospital, Departement of Ophtalmology, Limoges

(2) Inserm, UMR 1092, Limoges

(3) Limoges University, UMR-S1092, Limoges

(4) Limoges University Hospital, Departement of Bacteriology and Virology, Limoges

Purpose The microbiological diagnosis of infectious keratitis based on a corneal scrapping is necessary in order to adapt the treatment. Our study aimed to determinate if the use of Eswab" transport media and 16S RNA PCR increase the sensitivity of this microbiological diagnosis

Methods Retrospective cohort study including all patients who benefited from a corneal scrapping for an infectious keratitis suspicion from June 1, 2007 to June 1 , 2011. They were divided into two groups according to the methods of microbiological analysis. In non-Eswab" (NES) group, four samples per patient were collected, directed to laboratories: bacteriology (direct exam and culture); virology (PCR and culture); mycology and parasitology (direct exam and Acanthamoeba PCR). In the Eswab" (ES) group, three samples per patient were collected. The first one, on Eswab" transport media, was directed to PCR analysis, including 16S RNA, viral and Acanthamoeba research and bacteriological culture. The two others samples were analysed in mycology and parasitology. Infectious gravity factors and the use of antibiotics before corneal scraping, were reported. The main criteria of judgement was the number of patients with a microbiological diagnosis

Results 190 patients were included, 84 in the NES group and 106 in the ES group. Infectious keratitis gravity factors $(47,6 \%$ vs $56,6 \% \mathrm{p}=0,356)$ and use of antibiotics before the corneal scrapings ( $23,8 \%$ vs $29,2 \% \mathrm{p}=0,5)$ was not significantly different between two groups. The diagnosis sensibility in the NES group was $36,9 \%$ versus $60,4 \%$ in the ES group $(\mathrm{p}=0,002)$

Conclusion The usage of a transport media such as Eswab and 16S RNA PCR research on corneal scrapping significantly improves the sensibility of microbiological diagnosis

\section{- T064}

Antiviral effects of HSV1-specific meganucleases in a mouse model of relapsing herpes keratitis

LABETOULLEM (1), GABISONE (2), ROUSSEAUA (1), ARNOULDS (3),

GALETTOR (3), PASDELOUP D (4)

(1) South Paris University, Kremlin Bicêtre

(2) Institut de la Vision, Paris

(3) Cellectis, Paris

4) Virology CNRS, GiflYvette

Purpose Conventional treatments of infections by Herpes simplex virus type 1 (HSV1) do not reduce the burden of latent virus and the resulting risk of viral reactivation. Specific endonucleases (such as meganucleases) could be a tool to reduce relapsing herpetic keratitis in pre-treated tissues.

Methods Three weeks after subconjunctival inoculation of rAAV (recombinant adenovirus-associated vector) encoding either a meganuclease targeting the HSV1 genome or the Green Fluorescent Protein (GFP) as a negative control, mice were infected with HSV1 strain SC16 to induce a latent infection in the trigeminal ganglia (TGs). 28 days later, mice were subjected to a reactivation stimulus (heatshock). Corneas and TGs were analyzed for the presence of HSV-1 genome and of several viral transcripts (LAT, TK and UL18).

Results In the corneas, as in TG, meganuclease-treated mice had fewer copies of viral genomes (viral load) and LAT, TK and UL18 transcripts (signs of viral replication) than control mice, ie treated with rAAV encoding GFP ( $\mathrm{p}=0.002$ to $\mathrm{p}=0.0008$ ), suggesting a significant reduction of viral replication after herpes reactivation stimulus.

Conclusion The production of a specific HSV1 meganuclease seems to reduce the importance of HSV1 reactivation in TGs (the site of herpetic latency) and corneas, suggesting that specific HSV1 meganuclease could be tested in therapeutic protocols with the aim of reducing herpetic corneal relapses. 
Poster session 1: Electrophysiology-physiological Optics-Vision Sciences/Glaucoma/Immunology-Microbiology/Molecular Biology-Genetics-Epidemiology/ Neuro-ophthalmology-Strabismology-Paediatric Ophthalmology-History of Ophthalmology

- T065

Comparative immunological study of of lamellar and penetrating keratoplasty in murine model

LEE HS, CHOI MINY, JOO CHOUN

Department of Ophthalmology and Catholic Institute for Visual Science, Seoul

Purpose Lamellar keratoplasty (LK) has been considered an acceptable alternative surgery for penetrating keratoplasty (PKP), because LK has the advantage of lower risk of graft rejection and intraocular complications, according to recent clinical outcomes But the relative immunological mechanism of graft rejections for the two procedures is uncertain. We have developed a murine model of lamellar keratoplasty to try to understand the immunological mechanisms of those two procedures.

Methods We have developed a murine model of lamellar keratoplasty to try to understand the immunological mechanisms of those two procedures through real-time PCR, immunohistochenistry, and flow cytometric analysis.

Results LK mice showed less graft rejection than PK mice and LK led to less DTH response and IFN-ysecretion in vitro recall assay of $\mathrm{T}$ cells from drainage lymph nodes, as compared to PK (acceptance; acc). Corneal expression of IL-1 $\beta$ and IFN- $\gamma$ in PKacc were significantly increased later. In addition, LK showed less angiogenesis and lymphangiogenesis in grafted cornea than PK, and LK led to decreased number of MHCIlhighCD11 c+ antigen presenting cells(APCs) in the draining LNs.

Conclusion In conclusion, these results show that LK presents less graft rejection than PK through lower angiogenesis and lymphangiogenesis, which could make pathologic T cells and APC less migration into drainage LNs and grafted corneas, respectively.

- T067

Comparative analysis of two ocular endothelial cells

MAMMADZADA P, GUDMUNDSSON J, KVANTA A, ANDRE H Vitreo-Retinal Clinic, STE/KI, Stockholm

Purpose In the eye, angiogenesis is associated with many pathological conditions. Retinal and choroidal endothelial cells (RE and CE cells) are the main cellular components of ocular angiogenesis. It is widely accepted that endothelial cells (EC) from different vascular beds show heterogeneity. Comparative study of RE and $\mathrm{CE}$ cells can lead to deeper understanding of the mechanisms through which EC become activated to undergo pathologic behaviors. Therefore, the goal of the study was isolation, cell culturing, and characterization of human CE and RE cells, and comparative analysis of these two cell lines.

Methods Human consented-donor eyes were used for preparation of CE and RE cell cultures. The posterior segment of these eyes was microdissected into isolated tissues (retina and choroid). Isolation of RE and CE cells from these tissues was achieved by positive selection of endothelium by targeting CD31. Characterization of RE and CE cells was performed by immunostaining for EC specific markers (CD31, isolectin, and acetylated-LDL uptake). Further analysis is achieved by qPCR

Results Endothelial cells from the retina and the choroid have been specifically isolated and displayed high endothelial cell-type purity. Both RE and CE cell cultures displayed positive staining for CD31 and isolectin, as well as acetylated-LDL uptake positive staining was confirmed to $100 \%$ of the cultured cells. Differences between RE and CE cells was not clear upon IF microscopy. Initial qPCR analysis has shown discreet variations between $\mathrm{RE}$ and $\mathrm{CE}$ cells

Conclusion RE and CE cells exhibit similar morphologies and staining patterns for the specific endothelial cell markers studied. Extensive comparative analysis of these two ocular EC-lines is undergoing by a series of qPCR arrays.

\section{T066}

Funcionality characterization of RS2229094 $(\mathrm{T}>\mathrm{C})$ polymorphism and LTA expression in human retinas

DELGADO TIRADO S (1), PASTOR IDOATE S $(1,2)$,

RODRIGLEZ HERNANDEZ I (2 3) ROIAS I (1) GONZALEZ BUENDIA L (1)

GONZALEZ SARMIENTOR (2, 3), PASTOR JC (1)

(1) Instituto de Oftalmobiologia Aplicada (IOBA-Retina Group). University of Valladolid, Valladolid

(2) Unidad de Medicina Molecular. Departamento de Medicina. University of Salamanca, Salamanca

(3) Instituto de Biología Molecular y Celular del Cáncer (IBMCC). Consejo Superior de Investigaciones Cientificas (CSIC). Instituto de Investigación Biomédica de Salamanca (IBSAL). University of Sala, Salamanca

Purpose To determine the expression and localization of lymphotoxin alpha (LT $\alpha$ ) in human retinas, and investigate the functionality of the LTo rs2229094 (T>C) polymorphism, previously associated to proliferative vitreoretinopathy (PVR) development.

Methods Phase I: RNA from 3 healthy human retinas and 2 peripheral blood samples was subjected to reverse transcription polymerase chain reaction (RT-PCR) analysis. In addition, 3 human eyes with chronic retinal detachment (RD) and one healthy control were subjected to immunohistochemistry (IHC) with specific antibody against LT $\alpha$. Phase II: Functionality of $\mathrm{T}$ and $\mathrm{C}$ alleles was assessed by using pCEFL-Flag expression vector, and transient transfection assays. Besides, expression analysis by RT-PCR, Western-blot and subcellular localization of both alleles, by immunofluorescence (IF) assay were performed

Results Phase I: Signals of mRNA LT $\alpha$ below significance levels were detected in healthy human retinas. Peripheral blood analysis showed different LT $\alpha$ transcripts with premature stop codons. IHC staining revealed no differences between human healthy and RD retinas.Phase II: No differences in mRNA and protein expression levels and subcellular localization between both alleles were found. Both alelles showed cytoplasmic LTa localization

Conclusion Although results suggest lack of functionality, and it appears to be no differences regarding LTo expression and localization, this polymorphism could remain as a valid biomarker to identify patients at high-risk for developing PVR after RD, due to the strong association between PVR and LTa rs2229094 polymorphism previously identified by our group.

- T068

Exogenous regulation of the HIF pathway in RPE cells

ANDRE H, EKSTROM M, TAKEI A, MA Y, KVANTA A

Vitreo-Retinal Clinic, STE/KI, Stockholm

Purpose To investigate the effects of a series of hypoxia-inducible factors (HIF)regulating molecules (HRM) on the hypoxia pathway in retinal pigment epithelial (RPE) cells, critically involved in neovascular age-related macular degeneration (nAMD) pathogenesis.

Methods ARPE-19 cells were transfected with plasmids encoding FLAG-tagged HRM as well as HIF-1 $\alpha$ and HIF- $2 \alpha$. Effects of HMR on ARPE-19 were analyzed by a luciferase reporter assay (DLR), Western blot, and soluble vascular endothelial factor (VEGF)capture assay. Effects on endothelial cells (EC) were studied by exposing HUVEC cells to ARPE-19 pre-conditioned media after transfection with particular HRM.

Results The DLR denoted a marked negative regulation of HIF-1 $\alpha$ activity in ARPE-19 in the presence of all PHDs. Analysis of HIF-1 $\alpha$ protein showed a very considerable decrease in the presence of PHD2, together with the most dramatic reduction of HIF$1 \alpha$ protein life-time.The immunoprecipitated level of VEGF from the media of ARPE-19 cells transfected with PHD2 was considerably lower than that of an empty control. HUVEC proliferation was decreased in conditioned media from ARPE-19 transfected with PHD2.

Conclusion PHD2 seems to be the most potent negative-regulator of the HIF pathway. Furthermore, the negative effects of PHD2 were clearly associated with decrease in secreted VEGF and reduced EC proliferation. These results may have implications for the clinical treatment of patients with nAMD, particularly regarding the use of gene therapy to negatively regulate the neoangiogenesis present in these patients. 
Poster session 1: Electrophysiology-physiological Optics-Vision Sciences/Glaucoma/Immunology-Microbiology/Molecular Biology-Genetics-Epidemiology/ Neuro-ophthalmology-Strabismology-Paediatric Ophthalmology-History of Ophthalmology

\section{- T069 / 1767}

Visual dysfunction and pupillary responses are dissociated in the Opa3 mutant mouse with retinal degeneration

VOTRUBA M (1, 2), DAVIES JR (1), DOUGLAS RH (3), DAVIES VJ (1)

(1) Cardiff Centre for Vision Sciences, Cardiff University, Cardiff

(2) University Hospital of Wales Eye Department, Cardiff

(3) Department of Optometry \& Visual Sciences, City University, London

Purpose To assess the affect of the missense mutation p.L122P in a mouse model of 3-methylglutaconic aciduria (MGA-III), a neuro-metabolic syndrome which presents with retinal and optic atrophy and neurological impairment.

Methods Visual acuity was quantified in an optokinetic nystagmus drum by increasing the spatial frequency of the grating until an optomotor response could not be elicited. Pupillary light responsiveness was assessed by video pupillometry. Time course and phenotype of retinal degeneration was examined using haematoxylin and eosin and terminal dUTP transferase nick end labelling.

Results Opa3+/+ and Opa3+/- mice showed normal visual acuity by tracking a $2^{\circ}$ grating. However, Opa3-/- mice displayed no optomotor response at any grating frequency. Despite this, their pupil response was little affected. Pupil response/intensity curves of Opa3-/- and Opa3+/+ mice diverged somewhat at lower intensities although they only differed significantly at two irradiance levels. At 50\% constriction Opa3-/mice were only $0.61 \log$ units less sensitive than wildtypes. Histology showed panretinal degeneration in adult Opa3 $/$ - mice and TUNEL revealed increased cell death in postnatal and adult Opa3-/- retinae.

Conclusion The Opa3-/- mouse is a useful model of the human disease. Retinal atrophy in Opa3-/- animals is associated with widespread retinal thinning. However, dissociation between visual perception and pupillary function implies that the intrinsically photosensitive retinal ganglion cells (ipRGCs) subserving pupillary function may be less susceptible to damage by mutations of Opa3 than the retinogeniculate fibres underlying visual perception.

\section{- T071}

The functional role of RPGR protein complex in retinitis pigmentosa

SHU X, PATNAIK S, CRAFT J, MCCULLOCHD

Department of Life Sciences / Glasgow Caledonian University, Glasgow

Purpose Retinitis pigmentosa (RP) is a progressive outer retinal dystrophy, affecting $1 / 3500$ individuals in most populations. Mutations in the retinitis pigmentosa GTPase regulator (RPGR) gene are the most common single cause of RP, accounting for up to $20 \%$ of all cases. RPGR forms a protein complex by interacting with the RPGRinteracting protein 1 (RPGRIP1) and/or RPGRIP1 like protein (RPGRIP1L). We aim to investigate the functional role of RPGR protein complex in the pathogenesis of RP.

Methods Small interfering RNA (siRNA) was used to knock-down RPGR, RPGRIP1 or RPGRIP1L in human retinal pigment epithelium (RPE) 1 cell line. The efficiency of siRNA knock-down was assessed by qualitative RT-PCR and western blotting. The knock-down effects of RPGR, RPGRIP1, or RPGRIP1L were characterized by immnocytochemistry, enzyme linked immunosorbent assay (ELISA), and western blotting.

Results Knock-down of RPGR, RPGRIP1 or RPGRIP1L caused defects in ciliogenesis, remodelling of the actin cytoskeleton, and centrosome positioning. Since planar cell polarity (PCP) pathway regulates actin cytoskeleton rearrangement through activation of small RhoA GTPases. So, we performed ELISA and found that RhoA-GTPase activity were upregulated in the absence of RPGR, RPGRIP1, or RPGRIP1L protein. We also found knock-down of RPGR, RPGRIP1 or RPGRIP1L decreased the stability of DVl3 proteins, key components of polar cell polarity (PCP) pathway.

Conclusion We provide compelling evidence that the RPGR protein complex is required for the stability of DVl3, and regulates RhoA for apical centrosome positioning required for cilia formation. We show a novel functional role of RPGR complex in cilia formation by regulating actin cytoskeleton through alteration of PCP pathway.

\section{- T070}

Severe retinal degeneration in females with c.2543del mutation in the RPGR gene

KOUSAL B (1), SKALICKA P (1), VALESOVA L (1), COLCLOUGH T (2), HART-HOLDENN (2), O'GRADY A (2), HARDCASTLE AJ (3), LISKOVA P (4)

(1) Charles University in Prague, Praha

(2) St Mary's Hospital, Manchester

(3) UCL Institute of Ophthalmology, London

(4) Charles University in Prague, Prague

Purpose To describe the genotype-phenotype correlation and serial observations in a five-generation Czech family with retinitis pigmentosa (RP) associated with total blindness in females.

Methods Comprehensive ophthalmological examination was performed in sixteen family members (nine females and seven males). Autosomal dominant inheritance was suspected and screening for known mutations by genotyping microarray was performed. Next, direct sequencing of ORF15 RPGR exon was undertaken.

Results The c.2543delA, in ORF15 of the RPGR gene was detected. Three females became bilaterally totally blind in the sixth decade. Four examined females and five males showed pigmentary deposits, progressive visual field constriction, moderate to high myopia and myopic astigmatism. Three other females had moderate to high myopia and myopic astigmatism but without the presence of bone spicule formation. Only one 34 year old female carrier had clinically normal fundus findings.

Conclusion The c.2543delA in RPGR is associated with severe phenotype in females in the studied family. The presence of refractive errors, especially high myopia and astigmatism in the absence of male to male transmission may be indicative of an X-linked inheritance.

\section{- T072 / 1765}

Biomarkers of Age-Related Macular Degeneration (AMD): Four new obesity-related genetic loci are associated with advanced AMD

PATERNO JJ (1), HELISALMIS (2), TOKARZ P (3), BLASIAKJ (3), HILTUNENM (2), UUSITUPA M (4), KAARNIRANTA K(1)

(1) Department of Ophthalmology, University of Eastern Finland (UEF), Kuopio (2) Department of Neurology, University of Eastern Finland (UEF), Kuopio (3) Department of Molecular Genetics, University of Lodz, Lodz

(4) Department of Clinical Nutrition, University of Eastern Finland (UEF), Kuopio

Purpose AMD is the leading cause of central blindness in the elderly in Western countries. It has a complex multifactorial etiology, including aging, genetic factors, smoking, hypertension and atherosclerosis. Moreover, obesity has been considered to increase the risk for AMD. We hypothesize that the genetic variations associated with obesity might also be involved in the pathogenesis of AMD.

Methods Cross-sectional clinical data from 348 advanced AMD cases and 722 controls were collected. All subjects were over 65 years old, and without diabetes mellitus. We genotyped 40 newly associated obesity related loci using the Sequenom iPlex platform. Results Of the 40 SNPs analysed, PTBP2 (rs1555543), GNPDA2 (rs10938397), HOXC13 (rs1443512) and MAP2K5 ( $r$ 2241423) showed a nominally $(\mathrm{P}<0.05)$ significant association with advanced AMD in this local Finnish population.

Conclusion These new findings suggest that the link between AMD and obesity could be partly explained by genetic factors. Besides providing new insights into pathogenic mechanisms of AMD, these findings may also help to reveal possible targets for therapeutic intervention and useful diagnostic biomarkers in this disease. 
Poster session 1: Electrophysiology-physiological Optics-Vision Sciences/Glaucoma/Immunology-Microbiology/Molecular Biology-Genetics-Epidemiology/ Neuro-ophthalmology-Strabismology-Paediatric Ophthalmology-History of Ophthalmology

- T073

Epidemiology of age-related-macular-degeneration in north of France

DURIEUX P (1), DELEMOTTE A (2), LABREUCHEJ (3), ROULAND J (1),

LABALETTEP(1)

(1) Ophthalmology, Lille

(2) Association Diabhainaut, Anzin

(3) Biostatistics, Lille

Purpose As the leading cause of blindness in industrialized countries. AMD is an increasing public health problem. An early detection is important to provide prevention or treatment. The aim of our study was to estimate the prevalence of AMD in the North of France by a mass screening facility with a non-mydriatic retinal camera, and to identify risk factors of the disease.

Methods This is an epidemiological study performed in the North French department for a period of 6 months. Fundus photographs of both eyes were taken in a population aged 65 years and older with no known history of AMD. Images were analyzed by two independent readers in our Department for grading pictures along to the AREDS classification. Each participant answered a questionnaire about their medical and family history, and alcohol and tobacco, fruit, vegetables, and fish consumption respectively.

Results Photographs from 2,240 eves of 1,130 participants were analyzed. The average age in the cohort was 75 years. $13 \%$ of pictures were ungradable. $68.88 \%$ of patients were assessed in category $1,9.56 \%$ in category $2,1.44 \%$ in category 3 , and $5.56 \%$ in category 4 (exudative or atrophic), respectively. Age was significantly associated with the disease $(\mathrm{p}<0.0001)$, fruits and vegetables consumption was a significant protective factor $(\mathrm{p}=0.002)$.

Conclusion The prevalence of AMD is found similar than in the EUREYE epidemiological study ( $8 \%$ of AMD after 50 years old, more than $30 \%$ after 75 years old). In our cohort, the overall prevalence of AMD is $24 \%$ in the population over 65 years old. Nonmydriatic fundus photography is a simple, fast, non-invasive and reliable AMD detecting technique that can be performed by nonphysicians then teletransfered to an ophthalmologist for reading.

- T075

Lysyl Oxidase-Like 1 gene haplotypes and their association with pseudoexfoliation glaucoma in the Spanish population

ALVAREZL (1), GARCIA M (1), GONZALEZ-IGLESIAS H (1), ESCRIBANOJ (2), RODRIGUEZ-CALVO PP (1), FERNANDEZ-VEGA L (1), COCA-PRADOS M $(1,4)$

(1) Fundación de Investigación Oftalmológica, Instituto Oftalmológico Fernández-Vega, Oviedo

(2) Laboratorio de Genética Molecular Humana, Universidad de Castilla-La Mancha, Albacete

(4) Department of Ophthalmology and Visual Science, Yale University School of Medicine, New Haven

Purpose To investigate the potential association of genetic variants across the lysy oxidase-like 1 (LOXL1) gene in Spanish patients with Pseudoexfoliation Glaucoma (XFG).

Methods A case-control study of 105 unrelated individuals from Spain with XFG and 200 unrelated healthy controls matched by age and ethnicity were enrolled. A region of 39,230 bp-long of genomic DNA covering the entire LOXL1 gene and regulatory regions were sequenced in 99 patients using next generation sequencing. Variant frequencies were compared with those reported from the HapMap CEU study. Restriction fragment-length polymorphism (RFLP) analysis was conducted to genotype five SNPs in the LOXL1 gene (rs16958477, rs1048661, rs3825942, rs2165241 and rs3522) in all controls and in 6 XFG patients. A case-control association study was performed using the HaploView 4.0 software.

Results The allelic frequency analysis of the five selected LOXL1 SNPs revealed that (with the exception of rs3522) they were significantly associated with an increased risk for XFG. The haplotypes CGGT and AGGT, which carry the risk alleles of rs1048661, rs3825942, and rs2165241 were significantly associated with XFG. In contrast, the haplotypes ATGC and AGAC were found to be protective.
- T074 / 1768

BAX and BCL-2 genes in patients with Retinal Detachment with and without Proliferative Vitreoretinopathy. The Retina 4 project

GONZALEZ-BUENDIA L (1), PASTOR-IDOATE S $(1,2)$

RODRIGUEZ-HERNANDEZI(2 3), ROIASI(1)

GONZALEZ-SARMIENTOR (2, 3), PASTORJC (1)

(1) Instituto de Oftalmobiologia Aplicada (IOBA), University of Valladolid, Valladolid

(2) Unidad de Medicina Molecular, University of Salamanca, Salamanca

(3) Instituto de Biologia Molecular y Celular del Cancer (IBMCC). Consejo Superior de Investigaciones Cientificas (CSIC). Instituto de Investigacion Biomedica de

Salamanca (IBSAL), University of Salamanca, Salamanca

Purpose To compare the distribution of BAX G(-248)A and BCL-2 C(-938)A genotypes among European subjects undergoing rhegmatogenous retinal detachment surgery in relation to further development of proliferative vitreoretinopathy (PVR).

Methods A case-control gene association study as part of the Retina 4 Project was designed. Two promoter single nucleotide polymorphisms (rs2279115 and rs4645878) were analysed by TaqMan 5'exonuclease allelic discrimination assay, using a StepOne system in 134 samples from patients with PVR and 421 without PVR. Proportions of genotypes and AA homozygote groups of these polymorphisms were analysed. Genotypic and allelic frequencies were compared in global sample and in sub-samples. Results BAX gene: In the comparison of proportions of genotypes in Spain, Spain+Portugal and in the global sample, significant differences were found. The odds ratio (OR) for A carriers in Spain and Spain+Portugal was 1.8, and 1.7 in the global sample. BCL-2 gene: Significant differences were observed regarding proportions of genotypes in Spain+Portugal. Furthermore, a protective effect was found in the analysis of A carriers from Spain+Portugal with an OR of 0.6.

Conclusion The A allele of BAX is associated with a higher risk of developing PVR, suggesting that a down-regulation in the apoptosis pathway could be an important key in PVR pathogenesis. Additionally, the role of BCL-2 gene (inhibitor of necroptosis pathway) is proposed as a possible new target in PVR prophylaxis.

- T076

Analysis of the polymorphic variants of genes involved in miRNA pathway and its impact on matrix metalloproteinases (MMPs) in pathogenesis of primary open angle galucoma

MAISTEREK I (1), WALCZAKA (1), MARKIEWICZL (1), PRZYBYLOWSKA-SYGUTK (1) SZYMANEKK (2) GACEKM (2) SZAFLIKJ(2) SZAFLIK JP (2)

(1) Department of Clinical Chemistry and Biochemistry, Medical University of Lodz, Lodz

(2) Department of Ophthalmology, Medical University of Warsaw, Warsaw

Purpose Elevated intraocular pressure is concerned as the major risk factor of primary open angle glaucoma (POAG). Matrix metalloproteinases (MMPs) are involved in extracellular matrix (ECM) remodeling resulting in impaired outflow of aqueous humor from the eye. Their expression is crucial for a normal ECM structure synthesis and organization. It is estimated that about $30 \%$ of all human genes are regulated by microRNA (miRNA) pathway.

Methods Blood samples of 250 patients and 250 age matched controls were enrolled in the study. We evaluated effects of 24 functional single nucleotide polymorphisms (SNPs) of miRNA pathway genes including DROSHA, DGCR8, XPO5, RAN, DICER1, TARBP2, AGO1, AGO2, GEMIN3, GEMIN4, HIWI by TaqMan SNP Genotyping Assay. We also performed a polygenic approach to assess the cumulative effects of these SNPs on MMPs expression level in POAG patients by Real-Time PCR.

Results The data statistical analysis showed that SNPs of miRNA might be associated with risk of POAG in a Polish population. Data also showed a correlation between disturbances in expression of MMPs genes connected with glaucoma pathophysiology and diversity of miRNA levels in eye tissues. Thus, the results may contribute to a better understanding of the molecular mechanisms underlying this disease.

Conclusion Furthermore, the results may be useful for direct application of specific types of miRNAs in the expression regulation of MMPs involved in pathogenesis of primary open angle glaucoma. The work was supported by the grant of National Science Center Poland no. 2012/05/B/NZ7/02502. 
Poster session 1: Electrophysiology-physiological Optics-Vision Sciences/Glaucoma/Immunology-Microbiology/Molecular Biology-Genetics-Epidemiology/ Neuro-ophthalmology-Strabismology-Paediatric Ophthalmology-History of Ophthalmology

\section{- T077}

A prospective study of ocular manifestations in transthyretinrelated familial amyloid polyneuropathy

MENEY J (1), BARREAUE (1), MINCHEVA Z (2), CAUQUIL C (2), LABETOULLEM (1), ADAMSD (2), ROUSSEAUA (1)

(1) Departement of Ophtalmology, CHU bicêtre, Le Kremlin-Bicêtre

(2) Departement of Neurology, CHU bicêtre, Le Kremlin-Bicêtre

Purpose To study the prevalence and the clinical characteristics of ocular manifestations of transthyretin-related familial amyloid polyneuropathy (TTR-FAP).

Methods This prospective monocentric observational study was conducted at the french national reference center for TTR-FAP. Genetically confirmed TTR-FAP patients had a complete neurologic and ophthalmologic evaluation. Sensorimotor polyneuropathy (SPN)and vegetative neuropathy were staged. Ophthalmic examination included best corrected visual acuity (BCVA), Schirmer test, intraocular pressure (IOP), slit lamp photographs, gonioscopy, fundus examination, ultrasound pachymetry, and RNFL-OCT. Automated perimetry was performed when necessary. Medical and surgical treatments were analysed for all patients.

Results Fifty seven patients ( 31 males and 26 females, aged 26-83 years, mean $53.6 \pm 13.6$ years, were included between 2011 and 2013. Patients were mainly of Portuguese origin $(\mathrm{N}=28 ; 49 \%)$. Val30Met mutation was present in 42 patients $(73.7 \%)$. Ocular Hypertension ( $\mathrm{IOP}>21 \mathrm{mmHg}$ ) and glaucoma occurred in 11 patients $(19.3 \%)$ and were associated with amyloid deposits in the anterior chamber with a "clamshell" pupil in 91.3\% of cases. Amyloid vitreous deposits were present in 12 patients (21\%) and had already required vitrectomy in 9 eyes of 5 patients. A BCVA of 20/200 or worse in one eye was found in 9 patients (15.8\%) and was caused by secondary glaucoma in $66 \%$ of cases.

Conclusion In our series, amyloid glaucoma was exclusively found in carriers of the Val30Met mutation. OHT and glaucoma occurred more frequently in patients with autonomic neuropathy. Severe visual impairment was mainly caused by secondary glaucoma, and was associated with severe SPN.

\section{- T079 \\ Impact of age of myopia onset and parental myopia severity on the eventual myopia status in an individual \\ LIM SJ \\ University of Edinburgh, Edinburgh}

Purpose To examine whether parental myopia and the age of myopia onset can predict the eventual severity of myopia for an individual. This will help decide whether a child needs treatment to slow myopia progression and the likely duration of treatment.

Methods All myopic Chinese students aged 17 to 19 years old from a school in Singapore, and their parents were invited to participate in this study. Each eligible student and both parents had a comprehensive ocular examination (Retinomax K-Plus2; Nikon, Tokyo, Japan). Participants also completed a questionnaire on their age, sex, age of onset and stabilisation of myopia. Statistical analysis was performed using SPSS.

Results 59 female and 41 male eligible students and their biological parents were assessed. The individual's age of myopia onset was moderately correlated $(\mathrm{r}=0.600)$ with his post-stabilisation myopia status. Parental myopia severity was mildly associated with the individual's myopia status (Pearson's correlation $(r)=0.337$ ). The daughters' myopia status correlated better with their mothers' than fathers', while that of sons' better with their fathers.' Despite a general trend of myopia severity being worse in females than males, a Z-test showed that the means of myopia severity of the 2 groups were not significantly different, and a Chi-square test showed that the individual's myopia status is independent of parental myopia.

Conclusion The results suggest that for a Chinese myopic child in Singapore, a young age of myopia onset would be associated with a more severe eventual myopia status. Decision to prescribe drugs to slow myopia progression should be based on a young age of onset rather than family history, as this has a greater impact on eventual myopia status than parental myopia.
- T078 / 1766

Analysis of Keratoconus genetic factors within Keratoconus Loci and mtDNA

NOWAKDM (1,2), KAROLAKJA (2, 1), KUBICKA M (2), KULINSKA K $(2,3)$ POLAKOWSKI P (4) SZAFLIKJP (4) GAJECKA M $(2,1)$

(1) Institute of Human Genetics, Polish Academy of Sciences, Poznan

(2) Department of Genetics and Pharmaceutical Microbiology, Faculty of Pharmacy, Poznan University of Medical Sciences, Poznan

(3) Department of Experimental Anaesthesiology, Poznan University of Medical Sciences, Poznan

(4) Department of Ophthalmology II, Medical Faculty, Medical University of Warsaw, Warsaw

Purpose Keratoconus (KTCN) is a multifactorial disorder in which both environmental and genetic factors are involved. Genetic studies have led to the identification of several loci on different chromosomes, linked to KTCN. However, only few reports indicated causative genes in these loci. The aim of this project was to analyze the DNA sequence information available in the databases of SNVs located within known KTCN loci. We extended the analyzes to include miRNA genes located. Additionally, sequencing of mitochondrial genome in KTCN patients from Polish population was performed.

Methods The list of SNVs, which are located in KTCN loci were obtained on the basis of Ensemble. For analyzed SNVs allele frequencies were obtained from 1,000 Genomes Project. The list of miRNA genes was obtained from the miRBase. The potential impact of nonsynonymous amino acid substitutions on protein structure and function was assessed with PolyPhen and SIFT. Additionally, mtDNA was analyzed in samples from 93 people with the Polish population (42 - KTCN, 51 - controls)

Results In this study 94 miRNA-encoding genes and over 2 million SNVs were identified within known KTCN loci. From these SNVs, over 4,500 were located within exons and almost $600 \mathrm{SNV}$ s were indicated as deleterious. Sequencing analysis of fragments of the mitochondrial genome revealed a number of changes, including several new polymorphisms.

Conclusion The KTCN development does not depend on a single change in the gene, but on the accumulation of numerous sequence variants. The complexity of KTCN etiology causes the need to find appropriate approach to investigate this disease. Support: National Science Centre, Poland, grant no. 2011/03/N/NZ5/01470

\section{- T080}

\section{ADVISE (ADenoVirus Initiative Study in Epidemiology)}

DUQUESROIX B (1), TUIL E (2), MESSMER EM (3)

(1) Nicox, Sophia-Antipolis, Valbonne

(2) CHNO des Quinze-Vingts, Paris

(3) Ludwig-Maximilians-University, Munich

Purpose ADVISE is an epidemiological, prospective, multicentric, open study to assess the characteristics and frequency of adenoviral conjunctivitis (AC) as diagnosed with the point of care AdenoPlus test in patients suffering from acute conjunctivitis in $5 \mathrm{EU}$ countries (F, It, Ger, UK, Sp).

Methods Bacterial or viral conjunctivitis are usually self-limiting but some viral cases may cause persistent disease and significant morbidity. AC causes more severe disease, is extremely contagious (transmission rate 10-50\%), of longer duration than bacterial (shed for 14-16 days, signs and symptoms last 2-3 weeks) and may lead to decreased visual acuity or light sensitivity from persistent subepithelial infiltrates, chronic dry eye, and visual loss from conjunctival scarring.

Results AdenoPlus is a single use, rapid (10 min.) immunoassay test for the qualitative in-vitro detection of Adenoviral hexon protein antigens directly from human eye fluid. It is a CE marked, point-of-care medical device intended for health professional use as an aid in the rapid differential diagnosis of acute conjunctivitis.Study objectives are to assess the $\%$ of patients with acute conjunctivitis who have $\mathrm{AC}$, the clinical profiles associated with positive and negative tests, the correlation between the initial clinical diagnosis and the final diagnosis (post-test), the AC seasonality and geographic distribution, and to collect pharmacoeconomic data (before/after test prescribed treatments, work/school absenteeism...).

Conclusion Enrollment started in June 2013 in France and in January 2014 in Germany. Italian, Spanish and UK sites will start recruiting Q2-Q3/2014. Approximately 2500 patients are expected across EU, 500 patients from 15-20 sites per country (20-30 patients per site). The first interim study results will be presented with data analyses from at least 400 patients recruited in EU. 
Poster session 1: Electrophysiology-physiological Optics-Vision Sciences/Glaucoma/Immunology-Microbiology/Molecular Biology-Genetics-Epidemiology/ Neuro-ophthalmology-Strabismology-Paediatric Ophthalmology-History of Ophthalmology

- T081

Novel and known FRMD7 mutations and copy number variation in Belgian patients with $\mathrm{X}$-linked idiopathic infantile nystagmus

ALMOALLEM B (1), BAUWENSM (1), WALRAEDT S (2), DELBEKE P (2), VERDINH(1), VAN CAUWENBERGHC (1), DE LEENEER K (1), HOOGHES (1), KESTELYN P (2), AL-OBEIDANS, DE ZAEYTIJD J (2), LEROY BP (1, 2), DE BAERE E (1)

(1) Center for Medical Genetics, Ghent University Hospital, Ghent (2) Department of Ophthalmology, Ghent University Hospital, Ghent

Purpose Idiopathic Infantile Nystagmus (IIN [MIM\# 310700]) is one of the commonest forms of infantile nystagmus characterized by bilateral uncontrollable eye movements that does not manifest until the child starts to fixate objects at the age of three months leading to low visual acuity scores. Genetically IIN is a heterogeneous disorder which is mostly inherited in an X-linked fashion. There are three known loci and two identified genes. Approximately 50\% of families with X-linked IIN have been shown to be linked to mutations in the FERM domain-containing protein 7 (FRMD7) gene. Thus far 45 unique mutations have been reported in FRMD7 related patients with IIN. We investigate the role of FRMD7 mutations and copy number variations in Belgian cohort of 49 unrelated families with a clinical diagnosis of IIN

Methods Forty-nine families underwent detailed ophthalmological examinations and DNA extraction. We set up a comprehensive molecular genetic test based on Sanger sequencing, targeted next generation sequencing (NGS) and copy number variation (CNV) analysis. The reference sequence used was NM_194277.2.

Results In eleven unrelated Belgian families with IIN, seven unique FRMD7 changes were found, five of which are novel: frameshift mutation c.2036del p.(Leu679Argfs"8), missense mutations c.801C >A p.(Phe267Leu) and c.875T>C p.( Leu292Pro), splice site mutation c.497+5G>A and one $\mathrm{CNV}(1.4 \mathrm{Mb}$ deletion). .Additionally, four known mutations were found: missense mutations c.70G $>A$ p.(Gly24Arg) and c.886G $>C$ p.(Gly296Arg), nonsense mutation c.910C $>\mathrm{T}$ p.(Arg303*); frameshift mutations c.2036del p.(Leu679Argfs"8) and c.660del p.(Asn221Ilefs"11) which were found in a three independent families. Haplotype reconstruction suggests a potential founder effect in Belgian IIN patients. Segregation testing of these mutations was performed and supports their pathogenic effect.

Conclusion Overall, we found both coding FRMD7 mutations and a CNV in 11/49 Belgian families with IIN (22\%) and expand the mutational spectrum of FRMD7 as a common cause of IIN. Finally, our study generates a discovery cohort of IIN patients harboring either undetected mutations in non-coding region of FRMD7 or in genes at known or novel loci sustaining the genetic heterogeneity of the disease.

\section{- T083}

Fluorescein angiography in the management of ROP occurred at a late postmenstrual age: our experience on seven premature infants

GUAGLIANOR, BARILLA'D, BERTONE C, SPALLONE L, MAFFIA A, BIANCHI PE Eye Clinic IRCCS Policlinico San Matteo, Pavia

Purpose To report author's management with digital camera fluorescein angiography (FA) of Type 1 retinopathy of prematurity (ROP) developed after 45 weeks of postmenstrual age (PMA)

Methods Retrospective non comparative case series consisting of seven prematurely born infants with Type 1 ROP that falls outside of usual time line for the disease (PMA $>45$ weeks)

Results 212 premature infants with gestational age $\leq 30$ weeks underwent ROP screening between January 2009 and December 2013 at Neonatal Pathology Department at San Matteo Hospital in Pavia. ROP was found in 86 babies (172 eyes, $40 \%$ ) and 28 of these (56 eyes, 20.6\%) were treated with laser photocoagulation. 14 eyes of 7 children developed temporal peripheral ROP (stage 2, zone 3) between 45 and 50 weeks of PMA and were included in our study. The mean gestational age for this subgroup was 27,8 weeks and mean birth weight was $864 \mathrm{~g}$. Diagnosis and management were performed with indirect ophthalmoscopy, RetCam 3 and digital video FA. In 7 eves ROP was treated with laser fotocoagulation at 47-48 weeks PMA, while in 7 eyes regressed spontaneously with full retinal vascularisation between 48-54 weeks PMA

Conclusion Although rare, Type 1 ROP may occur lately, after 45 weeks PMA It is insidious because unexpected and located in extreme retinal periphery. In our experience, FA images were very useful to study the clinical features of ROP and to reveal us whether retinopathy was progressing towards threshold disease or regressing Fluorescein Angiography lead our decisional choose: to perform laser treatment or go on with closely observation over time until the complete retinal peripheral vascularisation.

\section{- T082}

Change of ocular components after cycloplegia in children using the IOLMaster

CHANG HR, JANG HJ

Department of Ophthalmology, Kangbuk Samsung Hospital, Sungkyunkwan University School of Medicine, Seoul

Purpose Ocular components are traditionally measured using A-scan ultrasound. With ultrasound, the probe makes contact with the cornea, so there is some problem in children. The IOLMaster is non-contact device that can measure ocular components, so it can be used in children with poor cooperation. We evaluated change of ocular components after cycloplegia in children using the IOLMaster.

Methods One hundred and sixty eight children aged from 3 to 15 years were recruited from the Kangbuk Samsung Hospital, Sungkyunkwan University, School of Medicine. Children with ocular problem except refractive error were excluded. Pre- and postcycloplegic corneal curvature $(\mathrm{CC})$, anterior chamber depth $(\mathrm{ACD})$ and axial length (AL) on the right eye were measured with the IOLMaster. We evaluated the change of ocular components after cycloplegia by age and refractive error.

Results ACD was $0.134 \pm 0.134 \mathrm{~mm}$ deeper after cycloplegia $(\mathrm{p}=.000)$. The amount of change had statistically significant negative correlation with age (Pearson correlation $-0.332, \mathrm{p}=.006)$, but it was not significantly different between myopic and hyperopic group after age correction ( $\mathrm{p}=.398)$. AL was also significantly elongated after cycloplegia $(\mathrm{p}=.000)$, but the amount of change was very small $(0.011 \pm 0.022 \mathrm{~mm})$. There was no change in CC after cycloplegia ( $\mathrm{p}=.428)$.

Conclusion Effect of cycloplegia on ACD in children measured with the IOLMaster was significant. We suppose that the negative correlation between the change of ACD and age may be associated with decreasing accommodation with age.

\section{- T084}

Interest of an ophthalmological consultation dedicated to the children presenting autism spectrum disorders

JANY B (1), MONTANARIS (2), BASSON W (1), JONQUA F (3), MILLE C (2) MILAZZOS (1)

(1) Ophtalmology, Amiens

(2) Pedopsychiatry, Amiens

(3) Orthoptie, Amiens

Purpose The children presenting Autism spectrum disorders (ASD) often present phthalmological disorders. This is why, we plan an ophthalmological consultation dedicated to the children ASD with a partnership between the ophthalmology's department and the "Centre Ressource Autisme" (CRA)

Methods The conditions of set up the consultation were: - Training took place at the orthoptist's formation and with the residents in ophthalmology to educate - Some modifications in the ophthalmological consultation were performed :- An adaptation of the consultation's site-Schedules of dedicated consultations - An increased time of consultation with 2 examinations: first orthoptic and after, ophthalmologic. Every step of the exam is announced, the used equipments are gradually brought to the children who can watch and also touch them before the examination.- A preparation for the consultation with the child may take place through the use of specific visual medias.The second or the third examination could be proposed if necessary

Results Fifteen children were examined in one year (year 2013) for whom an orthoptic examination, measurement of the objective refraction, the slit lamp examination and fundus were performed. Refractive errors and strabismus were detected or confirmed and a bilateral cataract was diagnosed and treated.

Conclusion This medical network allows to dedramatize of the ophthalmological examination of the children with ASD both the families and the medical professionals. It also has an interest in the training of orthoptists and residents.This type of care could develop and allow a better pathology's detection. 
Poster session 1: Electrophysiology-physiological Optics-Vision Sciences/Glaucoma/Immunology-Microbiology/Molecular Biology-Genetics-Epidemiology/ Neuro-ophthalmology-Strabismology-Paediatric Ophthalmology-History of Ophthalmology

- T085

Susac syndrome revealed by retinal arterial occlusions in a 12 year old girl

BEAUIEUX P, CLOCHE V, TRECHOT F, ANGIOI K

Ophtalmology, Nancy

Purpose Susac's syndrome (SS) is a rare immune disorder. It combines encephalopathy, retinopathy and hearing loss. We report a new pediatric case, revealed by an occlusive retinal arteritis.

Methods A 12-year old girl came to emergency room due to sudden onset of photopsia in her right temporal visual field. One year before, she had developed an acute disseminated encephalomyelitis, resolved after steroids. Visual acuity was 10/10 P2. Fundus examination revealed a thin right inferior nasal artery associated with an ischemic oedema. Fluorescein angiography evidenced a right nasal artery occlusion and multiple peripheral arterial occlusive vasculitis. Steroid treatment was dispended. Brain MRI showed high signal-intensity abnormalities in corpus callosum. The triad was completed by a bilateral hearing loss. Intravenous immunoglobulins were initiated. Occurrence of contralateral occlusive retinal arteritis two months later led to introduce cyclophosphamide. After 6 infusions, visual acuity is stable but visual field defects are still present and regression of vasculitis remains incomplete.

Results 300 cases of SS have been reported with predominance in young women Pediatric cases are extremely rare. Diagnosis is difficult, because the typical clinical triad is often incomplete. Occurrence of relapses is unpredictable. Visual prognosis depends on localisation of occluded retinal territories. Treatment is based on a combination of steroids, immunosuppressive and antiplatelet agents, which is not always able to prevent the relapse as in our young patient.

Conclusion Unpredictable evolution of SS requires an early diagnosis and treatment as well as a close monitoring. It must always be considered in case of occlusive retinal arteritis even in children.

\section{- T087}

Comparative study of RNFL,GCA (macular ganglionnar cells) OCT alteration and MRI lesions in a population of multiple sclerosis patients (MS) with a recent history (less than 4 years)

BOURNIQUEL M, VALERO B, DE FARIA A, GIRAUD JM Ophtalmology, Toulon

Purpose Spectral domain OCT allows measuring peripapillary retinal nerve fiber layer and macular ganglionar cells and inner plexiform layers (GCA). Previous studies established alterations of these layers in ON.The aim of this study was to evaluate eyes without $\mathrm{ON}$ - and to compare GCA and RNFL lesions.

Methods We studied 48 eyes from 24 patients with authentified MS.On 48 eyes,8 eyes had clinically proven optic neuritis in previous years. Patients with $\mathrm{ON}$ in the last 6 months, or with elevated intra ocular pressure,high myopia,or macular pathology were excluded.All had clinical exam and OCT measurement (Zeiss Cirrus)

Results The 8 eyes with history of ON showed clear thinning of both GCA and RNFL $(\mathrm{p}<<0,05$ with both).With the 40 other eyes, 11 showed abnormalities with OCT,29 had normal OCT.In those 11 eyes,both OCT measurements ( RNFL and GCA) showed a thickness loss, but higher with GCA with a better statistical correlation ( $p=0,037$ versus $\mathrm{p}=0,062$ ).Futhermore, more dials were involved in GCA versus RNFL in the thinning topography for 7 eyes out this 11 eyes group.We ran a screening for IRM abnormalities for all 24 patients, categorizing them in three groups (high, medium and low lesion load) without any clear association to the OCT alterations.

Conclusion This study confirmed acquired ganglion cells alterations,remaining after $\mathrm{ON}$,in patients who had a complete visual function recovery.It showed GCA or RNFL abnormalities in one quarter of patient without ON history.GCA seems to have greater sensitivity than RNFL, but further studies on biggest population are necessary to precise specificity an sensitivity of these two measures.
- T086

Subfoveal choroidal thickness in myopic and non myopic children

FONTAINEM, SAUER A, GAUCHER D, SPEEG-SCHATZC

Ophthalmology, Strasbourg

Purpose Since the recent development of Enhanced-Depth-Imaging program (Spectral domain OCT), most of the studies concerning choroidal thickness and myopia were performed on adults. The purpose of our study is to demonstrate a link between subfoveal choroidal thickness and ametropia in children.

Methods We included 115 healthy children aged between 3 and 15 years. We measured refraction with cycloplegia, axial length and subfoveal choroidal thickness with EDI program (Spectral domain OCT). We compared mean subfoveal choroidal thickness in the myopic population of children, to non myopic population of children, using t-test. We correlated axial length and subfoveal choroidal thickness using Spearmann test.

Results We included 115 right eyes. The mean age was 7.8 years. The mean spherical equivalent refraction was 0.0 diopters,the mean subfoveal choroidal thickness was $314.82 \mu \mathrm{m} .78$ children had a positive spherical equivalent refraction (emmetropic and hyperopic group), 35 had a negative spherical equivalent refraction (myopic group). In the emmetropic/hyperopic group, the mean subfoveal choroidal thickness was 332.3 $\mu \mathrm{m}$. In the myopic group, it was $275.9 \mu \mathrm{m}$. The mean sufoveal choroidal thickness was significantly thinner in myopic children compared to the other group, with a difference of $53.4 \mu \mathrm{m}, \mathrm{p}=0.032$, IC $95 \%$ [18.29; 88.5]. The subfoveal choroidal thickness was significantly correlated to axial length $(r=-0.4343, \mathrm{p}<0.0001$, IC $95 \%[-0.5768 ;-0.2662]$.

Conclusion This study shows that subfoveal choroid is thinner in myopic children. This has been demonstrated in adult, but there are only a few studies about children. Our results confirm the recent literature. It leads to new projects of study: a thin choroid could be a predictive factor of myopia.

\section{- T088}

Multifocal and toric implantation in a paediatric population: a retrospective study

HEITZ A, RITZ M, SAUER A, SPEEG-SCHATZ C

Ophthalmology, Strasbourg

Purpose The management of pediatric cataract is a therapeutic challenge. The aim of our study was to assess the potential benefits of the multifocal and toric lens implantation in the children depending on the age.The previous results of multifocal IOL in the child are encouraging but are derived from analyzes of children over 4 years. The toric implantation in the childhood was never studied.

Methods In this retrospective non-randomized monocentric study, children with bilateral and unilateral cataract between 0 and 9 years with a visual acuity less than $0,3 \log M A R$ were implanted with multifocal IOL (or with a toric IOL when the corneal astigmatism was higher than 2 dioptries). Outcome measures of best corrected visual acuity (BCVA) for distance, distance-corrected near visual acuity (DCNVA), contrast sensitivity, stereopsis and total astigmatism were analysed with the Student test in 2 age groups : less than 4 years (group 1) and more than 4 years (group 2).

Results 21 eves of 16 children (mean age 5,05 years, from 9 months to 9 years) were included in the multifocal study, 3 eyes of 2 children were included in the toric study. Group 1 included 5 eyes (4 children), group 2 included 13 eyes (10 children). Both groups showed significant improvement in BCVA at one year follow-up. Stereopsis was better in group 2 with unilateral implantation. The improvement of the DCNVA was significantly better in group 2 with unilateral implantation $(0,38 \log M A R, p<0,05)$. The total astigmatism, the BCVA and DCNVA were improved with a toric implantation, the stereopsis was similar

Conclusion Multifocal IOL implantation is a viable option for the children more than four years with unilateral cataract. The toric implantation analysis needs further results. 
Poster session 1: Electrophysiology-physiological Optics-Vision Sciences/Glaucoma/Immunology-Microbiology/Molecular Biology-Genetics-Epidemiology/ Neuro-ophthalmology-Strabismology-Paediatric Ophthalmology-History of Ophthalmology

- T089

Strabismus and nystagmus in the congenital cataracts

LEES (1), PARKJ (2)

(1) Haeundae Paik Hospital, Busan

(2) Maryknoll Medical Center, Busan

Purpose To evaluate the clinical manifestations and factors affecting onset of strabismus and nystagmus in the congenital cataracts.

Methods 148 eyes of the 88 patients who had undergone cataract removal for congenital cataracts between January 1996 year and January 2011 year were reviewed. The age at the surgery was from 6 weeks to 21 years. The factors related with the development of strabismus and nystagmus were analyzed.

Results The prevalence rate of strabismus was $28.8 \%$ in the bilateral cataracts, and $45 \%$ the unilateral cataracts at the last follow-up. Exotropia was the most common type of strabismus both preoperatively and postoperatively regardless of bilaterality of the cataracts. The same type of strabismus persisted after surgery. Nystagmus was noted in the 18 patients (30.5\%) only with bilateral cataracts. Nystagmus was accompanied significantly by strabismus in the 10 patients (55.6\%). In the patients with nystagmus, esotropia:exotropia was 4:6 and poor visual prognosis was noted. Strabismus developed more significantly in the patients without primary IOL implantation.

Conclusion The factors related with development of strabismus were unilateral cataracts, nystagmus, and aphakic state. Exotropia was the most common type and nystagmus was shown only in the bilateral cataracts.
- T091

Clinical features of oculomotor disorders and nystagmus among children presenting congenital glaucoma

AZIZ A (1), MATONTI F(1), SAUVANL (1), FAKHOURY O (1), PIERIE (2), DENIS D (2) (1) Ophthalmology CHU Nord, Marseille

(2) CHU Nord, Marseille

Purpose Strabismus and nystagmus are often reported in congenital glaucoma ( CG) but few data are available. This study aims at describing clinical features of oculomotor disorders associated with congenital glaucoma.

Methods A retrospective study was led in a service of Pediatric Ophthalmology of the South of France taking for reference the registers of the children operated for primitive CG between 2008 and 2013. We registered the caracteristics of cross-eyed infringement, the nystagmus, refractive data and amblyopia. Tests of chi ${ }^{2}$ or exact tests of Fisher were used for the category-specific variables and the tests of Mann-Whitney for the quantitative variables.

Results 47 children were included. 2 children presented an unilateral CG. 25\%(12 children) presented a strabismus ; 4 had a convergent deviation $(33 \%)$ and 8 an exotropia(66 \%).4 children ( $8 \%$ ) had a nystagmus, and all of them had a low best corrected visual acuteness(BCVA) (BCVA lower than 0,6 LogMAR). We find an average spherical equivalent (SE) more important in myopia in the cross-eyed population (average SE -1.75 D) with regard to the non strabismic population (average SE -0.35 D) Regarding nystagmic patients, we find a much more important average SE in myopia (average SE -7.8 D) with regard to the non-strabismic population (average SE - 0.35 D) ( p: 0.016). A statistically significant link exists between amblyopia and nystagmus occurency (p Fischer: 0.013).

Conclusion Most of the children taken in care for primitive CG and presenting a deviation had a visual acuteness decreased in our study. The nystagmus seems to appear for BCVA lower than 0,6 LogMAR. This amblyopia seems to have both organic origin but also refractive origin.

\section{- T090}

Results of the surgical treatment of concomitant strabismus with preliminary modeling of operation by using three-dimensional biomechanical eye's model

BUSHUYEVA NN, ROMANENKODV

lab. of binocular vision, Odessa

Purpose Analysation of the surgical treatment results of concomitant strabismus in patients based on volume of operation preliminary modeling using three-dimensional biomechanical eye model (TBEM "SEE-KID")

Methods We examined the angel of strabismus by Hirschberg, with Frenel's prisms on table Maddox,binocular vision. There were analysed surgical treatment results of concomitant strabismus in 106 patients, wich were opereted after investigation preliminary modeling using three-dimensional biomechanical eye model (TBEM "SEEKID").

Results Orthotropy was obtained in 54 from 106 patients (50,9\%)at the first day after operation.52from 106 patients (49,1\%) had residual angle of 5-10 hypocorrection was in 44. patients, and hypocorrection was in 8 patients. 18 patients were lost to follow-up. At 3-12 months folliw-ups ortothrop has been preserved in 53 from 88 patients $(60,2 \%)$, and 35 from 88patients residual angle of 5-10 remained - hypocorrection was in 31 patients, and hypocorrection was in 4 patients.

Conclusion TBEM "SEE-KID" use in complex examination and results of surgical treatment in patients with strabismus allows prognosing surgical result in $60 \%$ of cases and accelerates diagnostic process. This method needs further investigation.Particularly extraocular muscles biomechanics needs precisely estimated (degree hyper or hypofunction, movement amlitude).
- T092 / 2655

A new pediatric vision screener employing polarizationmodulated, retinal-birefringence-scanning-based strabismus detection and bull's eye focus detection

IRSCH K (1, 2), GAMATIKOV B (1), WU YK (1), GUYTOND (1)

(1) The Wilmer Eye Institute, The Johns Hopkins University School of Medicine Baltimore

(2) Centre d'Investigation Clinique, CHNO des Quinze-Vingts, Paris

Purpose Commercially available pediatric vision screening devices that test for amblyopiás primary causes (strabismus and defocus) can detect strabismus only indirectly and inaccurately via assessment of the positions of external light reflections from the cornea, but they cannot detect the anatomical feature of the eyes where fixation actually occurs (the fovea).

Methods Our laboratory has been developing technology to detect true foveal fixation, by exploiting the birefringence of the uniquely arranged Henle fibers delineating the fovea, using retinal birefringence scanning (RBS), and we recently described a new, polarization-modulated approach to RBS that enables entirely direct and reliable detection of true foveal fixation, with greatly enhanced signal-to-noise ratio and essentially independent of corneal birefringence Here we present a new pediatric vision screener (PVS) that employs polarization-modulated RBS for strabismus detection, and double-pass focus detection using a bull's eye photodetector and improved target system with accommodative control.

Results Performance tests in adult volunteers and pediatric patients demonstrate that our new PVS 1) is capable of detecting even small angles of eye misalignment (at least $0.75^{\circ}$ or $\left.1.3 \mathrm{PD}\right), 2$ ) has the potential to detect spherical focus within $\pm 1.00 \mathrm{D}$, and 3 ) has the potential to detect the misalignment in patients with accommodative esotropia who only develop a crossed eye when focusing intently on a near target.

Conclusion In conclusion, the new PVS shows real promise in supplying the demand for a reliable, automated strabismus/defocus screening tool for children at risk for amblyopia. 
Poster session 1: Electrophysiology-physiological Optics-Vision Sciences/Glaucoma/Immunology-Microbiology/Molecular Biology-Genetics-Epidemiology/ Neuro-ophthalmology-Strabismology-Paediatric Ophthalmology-History of Ophthalmology

- T093 / 2656

Neosynephrine and tropicamide eyedrops for pupil dilation in premature infants is almost twice as efficient as tropicamide eyedrops alone

LUX AL (1), GUILLOIS B (2), DEGOUMOIS A (1), MOURIAUX F (1), DENIONE (1) (1) Ophthalmology, Caen

(2) Neonatology, Caen

Purpose Retinopathy of prematurity screening requires pupil dilation of premature infants. While essential for reliable fundus examination, proper dilatation may sometimes be difficult to achieve in this population. The aim of our study was to compare the efficacy of two mydriatic regimens: one consisting in three drops of tropicamide $0.5 \%$ and the other one consisting in two drops of tropicamide $0.5 \%$ with one drop of phenylephrine $5 \%$.

Methods Thirty premature infants were enrolled and each infant was dilated with one regimen in one eye and the other regimen in the other eye. The side of each regimen was determined by randomization. A photograph of each eye was taken. The photographs were anonymised and randomly presented to two independant readers. The effect of the two regimens was determined by calculating the ratio of pupil diameter divided by cornea diameter. Assuming that pupil and cornea are circle-shaped, we calculated the ratio of pupil surface area relative to cornea surface area for both regimens.

Results The pupil to cornea diameters ratio was 46.7\% (+/-8.8) with tropicamide alone and $65.2 \%(+/-6.0)$ with tropicamide-phenylephrine combination. The ratio of pupil to cornea surface areas was $21.8 \%$ with tropicamide alone and $42.6 \%$ with tropicamidephenylephrine combination. In other words, the pupil surface area was 1.95 fold greater with tropicamide-phenylephrine combination than with tropicamide alone.

Conclusion The use of a combination of two drops of tropicamide $0.5 \%$ with one drop of phenylephrine $5 \%$ is far more efficient than tropicamide alone to achieve proper pupil in premature infants.

\section{- T094 / 2657}

3D shape perception in strabismus subjects

SAWAMURA H (1), GILLEBERT C (2), TODD JT (3), VANDENBERGHE R (4), ORBANGA (5)

(1) Department of Ophthalmology, Tokyo University, Tokyo

(2) Department of Experimental Psychology, Oxford University, Oxford

(3) Department of Psychology, The Ohio State University, Columbus

(4) Laboratory for cognitive neurology, Department of. Neuroscience, KU Leuven, Leuven

(5) Department of Neruoscience, Parma University, Parma

Purpose To evaluate 3D shape perception in strabismus subjects, psychophysical experiment was performed using 3D shapes extracted from four different visual cues (shading, texture, motion and disparity), comparing normal and strabismus subjects.

Methods The 11 randomly generated complex 3D shape visual stimuli were created and rendered in 4 modalities (3D shape from shading, texture, motion and disparity) and displayed on a PC monitor. The subjects were required to indicate the global maximum of the 3D shape as accurately as possible by displacing a red cross using the computer mouse. The distance in depth $(\mathrm{cm})$ between the global maximum indicated by the subject and the true global maximum was calculated. Strabismus subjects ( $\mathrm{n}=43$ : 6-65 years of age) and normal subjects ( $\mathrm{n}=18: 6-57$ years of age) participated in the task. All the subjects have normal or corrected normal visual acuity. Stereo acuity was also evaluated by titmus stereo test.

Results 3D shapes extracted from disparity were more difficult to perceive than those extracted from texture (ANOVA, $\mathrm{p}<0.03)$, motion $(\mathrm{p}<0.01)$ and shading $(\mathrm{p}<0.01)$ in both normal and strabismus subjects. The 3D shape perception in normal and strabismus subjects differed only for the binocular stereopsis cue, not the other visual cues of motion, shading and texture. In addition, 3D shape perception of motion, shading and texture was not affected by the stereo acuity in either normal or strabismus subjects.

Conclusion Even the strabismus patients with weak or no stereopsis can use other visual cues, such as motion, texture and shading to perceive 3D shape to the same degree as normal subjects.

\section{- T095}

\section{Microglial activation in a rat model of NAION}

REMOND A (1), FEL A (1), SIMONUTTIM (2), IVKOVICI (2), FROGERN (2), SAHEL J (2, 3, 4), LE HOANG P (1), BODAGHI B (1), PICAUD S (2), TOUITOU V (1) (1) Pitié Salpêtrière, Paris

(2) Institut de la Vision, Paris

(3) Centre Hospitalier National d'Ophtalmologie, Paris

(4) Fondation Rothschild, Paris

Purpose Nonarteritic anterior ischemic optic neuropathy (NAION) is the most common optic neuropathy over 50 years and there is no treatment for this condition. Activation of retinal microglial cells (RMC) is known to contribute to retinal ganglion cell (RCG) death after optic nerve injury. The purpose of this study was to examine early microglial activation and investigate the effect of minocycline on this activation in a rat model of NAION ( $\mathrm{rNAION}$ )

Methods We used a rNAION model to generate axonal ischemic infarct by rose Bengal dye laser photoactivation. NAION was induced in the right eye of each animals. 1 group received minocycline $(22 \mathrm{mg} / \mathrm{kg})$ intraperitoneally and the other had a sham injection of saline, 3 days before induction till sacrifice. Animals were euthanazied at dayl, 3 and 7. Immunohistochemistry and quantitative stereology of RCG and RMC were performed on flat-mounted retina. Expression levels of apoptosis-related genes (Bax, caspase 3), inflammation related genes (IL-1 $13, \mathrm{TNF} \alpha$ ), and vascular growth factor related genes (VEGF-a, VEGF-R1, VEGF-R2) were measured by qPCR.

Results Microglia was activated early in the course of NAION. Quantitative analyses showed that the RMC number was increased in the right eye at day 1,3 , and 7 in both groups. Animals untreated tend to have increased activated RMC compared to controls $(\mathrm{p}>0.05)$. Minocycline also modulates proinflammatory cytokines expression, and caspase 3 activation.

Conclusion Our results suggest that microglial activation contribute to retina ganglion cell death after NAION. Minocycline may promote RGC survival by microglial inhibition.

\section{- T096}

Metastasis of breast cancer presenting as one and half syndrome

HYUNKYUNG KIM, TAEYOONLA

Department of ophthalmolgy and Visual science St. Vincents Hospital, The Catholic University Of Korea Medical College, Suwon

Purpose To report a case of one-and-a-half syndrome caused by pontine metastasis of breast cancer.

Methods A 46-year-old woman with breast cancer presented with binocular diplopia for 2 month. She had previously been diagnosed with invasive ductal carcinoma in her left breast and had known metastases to the lung. She had undergone a modified radical mastectomy and chemotherapy. Her binocular horizontal diplopia worsened progressively and accompanied ipsilateral facial parethesia.

Results Her visual acuity was $10 / 20$ in both eyes and no abnormal findings were detected on anterior segment exam. Her both eyes were fixed in the midline on attempted right gaze. On attempted left gaze, only left eye abduction was preserved and vertical eye movements were normal. Brain MRI revealed that variable sized nodular enhancing lesions at right pons, both cerebral hemispheres, left globus pallidus, right temporal lobe and upper portion of cerebellar vermis.

Conclusion Breast cancer is known to be the second most common cause of brain metastasis. We should pay attention to the patient's medical history who presenting diplopia 
Poster session 1: Electrophysiology-physiological Optics-Vision Sciences/Glaucoma/Immunology-Microbiology/Molecular Biology-Genetics-Epidemiology/ Neuro-ophthalmology-Strabismology-Paediatric Ophthalmology-History of Ophthalmology

- T097

The use of statistical modeling to predict temporal artery biopsy outcome from presenting symptoms and laboratory results

LEEM (1), DE SMIT E (2), WONG TEN YUENA (2), SAROSSY M (1) (1) Ocular Diagnostic Clinic, Royal Victorian Eye and Ear Hospital, Melbourne (2) Centre for Eye Research Australia, Melbourne

Purpose Giant cell arteritis (GCA) is a vasculitis affecting the elderly that can cause blindness if untreated. The current diagnostic gold standard is a temporal artery biopsy (TAB) which requires theatre time and histopathology analysis, causing a delay in formal diagnosis. In this study we investigated whether various predictive statistical models could accurately predict the TAB result from presenting symptoms and baseline laboratory tests.

Methods Clinical characteristics of 182 patients who underwent TABs performed at the Roval Victorian Eve \& Ear Hospital (RVEEH) were analysed. Clinical data was extracted from patient files including demographics (age, gender), presence of typical GCA symptoms (headache, scalp tenderness, jaw claudication, anorexia, fatigue malaise, fever, weight loss, visual disturbance and previous diagnosis of polymyalgia rheumatica), and laboratory tests (CRP, ESR and platelet count). Various predictive statistical models were fitted in R - Random Forest (RF), Adaboost, Regression Tree test and train parts for cross-validation. We used a binary classification where equivocal results were considered positive.

Results Of the TABs, 65 were negative, 117 were "positive" (94 positive, 23 equivocal). SVM achieved the best classification accuracy at 100\% (40/40 predicted correctly). Rpart, Adaboost and RF achieved 65, 65 and 67.5\% respectively.

Conclusion Our statistical model using open source software has shown a high accuracy in predicting the TAB outcome based on presenting features. This could inform the clinician of the likelihood of TAB positive GCA and hence guide immediate management. (rpart) and Support Vector Machine (SVM) to fit the dataset which was segregated into

\section{- T098}

The role of cerebrospinal fluid in optic disc pit maculopathy: a case report studied by enhanced depth imaging optical coherence tomography (EDI-OCT)

MARTINEZ VELEZM (1), ASCASO FJ (1), HUERVA V (2), CRISTOBAL J (1) ZABADANI K (1), ALMENARA C (1), HERRERA L (1), PEREZ NAVARRO I (1), CARAMELLOC (1)

(1) Ophthalmology, Hospital Clinico Universitario 'Lozano Blesa", Zaragoza (2) Ophthalmology, Hospital Arnau Vilanova, Zaragoza

Purpose Optic disc pit(ODP) is a rare congenital optic disc abnormality characterized by a localized grayish-white depression usually in the center or inferotemporal part of the optic nerve head. It is generally unilateral $(85-90 \%)$ and its prevalence is estimated to be 1 in 11,000. Unless the ODP develops maculopathy, patients remain asymptomatic. Serous macular detachment may occur in up to $50 \%$ of cases, leading to significant decrease in visual acuity. Although the pathogenesis of ODP maculopathy is unclear, various theories about its onset have implicated fluid entry either from the vitreous cavity or from leakage of cerebrospinal fluid through the peripapillary subarachnoid space. The aim of this study was to evaluate the source of subretinal fluid under the macula in a patient with ODP

Methods Enhanced depth imaging optical coherence tomography(EDI-OCT) using the stars scan pattern of the Spectralis HRA + OCT (version 1.5.12.0; Heidelberg Engineering) was used

Results Horizontal SD-OCT section revealed a small amount of intraretinal fluid in the outer nuclear layer and a prominent serous macular detachment at the same time but with no relationship with either the vitreous cavity or the subarachnoid space. However, a direct communication between the subretinal space and the peripapillary subarachnoid space through the ODP was demonstrated for the first time by using EDIOCT.

Conclusion This case supports the theory that the source of serous macular detachment in some ODP cases could be cerebrospinal fluid passing into the retina through the ODP due to an incomplete closure of the embryonic fissure

\section{- T100}

The choroid profiles at the posterior pole measured by enhanced depth imaging optical coherence tomography in healthy Korean children

\section{enhanced depth imaging optical coherence tomography}

HERRERA L (1), PEREZ NAVARRO I (1), CARAMELLO C (1), ZABADANI K (1), ALMENARA C (1), PÉREZ D (1), MARTINEZM (1), SANCHEZA (2), PINILLA I (1), ESTEBANO(1)

(1) Department of Ophthalmology, Lozano Blesa University Hospital, Zaragoza

(2) Science Faculty, University of Zaragoza, Zaragoza

Purpose To evaluate choroidal thickness during childhood using enhanced depth imaging (EDI) spectral-domain optical coherence tomography (SD-OCT), and its correlation with age, sex, axial length $(\mathrm{AL})$ and refractive error $(\mathrm{RE})$

Methods Cross-sectional study in which 93 eyes of 93 healthy children were studied using a 25 A-scans-EDI SD-OCT protocol. Choroidal thickness was segmented manually, and an ETDRS grid was applied to analyze choroidal thickness and volume in each of its nine sectors. Two observers independently studied 30 children to assess repeatability and reproducibility, using intraclass coefficient (ICC).

Results We included 50 males and 43 females, with mean age of $9.62 \pm 2.89$ years mean refractive error (spherical equivalent) $+0.03 \pm 2.22 \mathrm{D}$ and mean axial length $(\mathrm{AL})$ of $23.19 \pm 13 \mathrm{~mm}$. Mean subfoveal choroidal thickness (SFCT) and volume was 314.22 \pm 55.48 , and $0.25 \pm 0.04 \mathrm{~m} 3$, respectively. SFCT was significantly thicker in the hyperopic group (AL 22.39 $\pm 0.83 \mathrm{~mm}$ ). There were no statistically significant differences between myopic (AL 24.33 $\pm 0.76 \mathrm{~mm}$ ) and emmetropic (AL 23.27 $0.54 \mathrm{~mm}$ ) children, as well as between males and females. There was a weak, positive correlation between SFCT and age $(r=0.259, p=0.012)$. Inter and intra-observer ICC of the nine sector of the ETDRS grid ranged from 0.929-0.991 and 0.992-0.998, respectively.

Conclusion Pediatric SFCT is greater in hyperopic children. The choroid appears to become thicker with age. Manual choroidal thickness segmentation by EDI SD-OCT
LEE J, KIM J

Cheil Eye Hospital, Daegu

Purpose To assess the changes of the choroidal thickness in healthy Korean Children using enhanced depth imaging (EDI) optical coherence tomography (OCT) and to evaluate the association of choroidal thickness and axial length.

Methods Seventy-nine eyes ( 79 children) with cycloplegic refractive errors within \pm 1 diopter spherical equivalent underwent horizontal scan using EDI OCT. The choroidal thickness was the vertical distance between the posterior edge of the retinal pigment epithelium and the inner scleral border at $1 \mathrm{~mm}$ intervals from $3 \mathrm{~mm}$ nasal and $4 \mathrm{~mm}$ temporal to the fovea using the manual caliper provided by the software of the device. Axial length was examined by partial coherence interferometry. Statistical analysis was performed to evaluate variations of choroidal thickness at each location and to correlate choroidal thickness and axial length

Results The mean age was 7.67 years. The mean \pm SD subfoveal choroidal thickness was $296.13 \pm 61.36 \mu \mathrm{m}$. And the mean choroidal thickness was $160.57 \mu \mathrm{m}, 209.19 \mu \mathrm{m}$, and $256.90 \mu \mathrm{m}$ at $3 \mathrm{~mm}, 2 \mathrm{~mm}, 1 \mathrm{~mm}$ nasal to the fovea and $302.77 \mu \mathrm{m}, 307.68 \mu \mathrm{m}, 314.33 \mu \mathrm{m}$, $319.49 \mu \mathrm{m}$ at $1 \mathrm{~mm}, 2 \mathrm{~mm}, 3 \mathrm{~mm}$, and $4 \mathrm{~mm}$ temporal to the fovea respectively. The mean axial length was $22.96 \pm 0.77 \mathrm{~mm}$. The mean choroidal thickness was $28.69 \mu \mathrm{m}$ and $28.36 \mu \mathrm{m}$ thicker at $3 \mathrm{~mm}$ and $2 \mathrm{~mm}$ nasal to the fovea respectively in eyes with shorter than mean axial length $(\mathrm{p}=0.011, \mathrm{p}=0.033)$.

Conclusion The choroidal thickness increased nasal to the temporal direction at posterior pole and eyes with shorter axial length tend to present thicker choroids at nasal area to the fovea in Korean children with no refractive errors. 
Poster session 1: Electrophysiology-physiological Optics-Vision Sciences/Glaucoma/Immunology-Microbiology/Molecular Biology-Genetics-Epidemiology/ Neuro-ophthalmology-Strabismology-Paediatric Ophthalmology-History of Ophthalmology

- T101

Comparative difference of the choroidal thickness according to the refractive errors measured by enhanced depth imaging optical coherence tomography

LEE J, YUS

Cheil Eye Hospital, Daegu

Purpose To assess the differences of the choroidal thickness(CT) according to the refractive errors using enhanced depth imaging(EDI) optical coherence tomography $(\mathrm{OCT})$

Methods Ninety-seven eyes in healthy Korean children are categorized into three groups according to the refractive errors: hyperopia(27 eyes), emmetropia(39 eyes), and myopia(31 eyes). They underwent horizontal and vertical EDI OCT scan. The CT was measured at 9 locations: fovea $(\mathrm{F})$ and $1 \mathrm{~mm} \& 3 \mathrm{~mm}$ nasal(N1,N3), temporal(T1,T3), superior $(\mathrm{S} 1, \mathrm{~S} 3)$ and inferior $(\mathrm{I} 1, \mathrm{I} 3)$ area to the fovea. Statistical analysis was performed to evaluate the comparative difference of the CT at each location.

Results The overall mean age was 8.2 years and there was no difference in three groups $(\mathrm{p}=0.320)$. The foveal CT in hyperopia was $359.41 \mu \mathrm{m}$ which was thicker than those in emmetropia $(301.97 \mu \mathrm{m})$ and myopia $(263.94 \mu \mathrm{m})$ and $\mathrm{CT}$ at the other 8 locations in hyperopia was thicker than in emmtropia and myopia(p<0.05). The CT at $\mathrm{S} 1, \mathrm{~T} 1$, $\mathrm{N} 1$ and N3 in hyperopia was thicker than those of emmetropia(p<0.05). The CT of hyperopia was thicker than those of myopia except T3 location $(\mathrm{p}<0.05)$. The thickest location of three groups was fovea in hyperopia, and T3 in emmetropia and myopia which was $320.79 \mu \mathrm{m}$ and $291.74 \mu \mathrm{m}$, respectively. The thinnest location was N3 in three groups $(220.22 \mu \mathrm{m}$ in hyperopia, $163.92 \mu \mathrm{m}$ in emmetropia and $151.29 \mu \mathrm{m}$ in myopia)

Conclusion The CT of hyperopia was thicker in comparison with emmetropia and myopia, especially at nasal area between hyperopia and emmetropia and at all locations except T3 between hyperopia and myopia. The temporal choroid was thicker than the nasal choroid regardless of refractive errors, but the thickest location in hyperopia was the fovea.

- T103 / 3676

Brain structural and functional reorganization due to long term retinal peripheral degeneration in retinitis pigmentosa

FERREIRA S (1), PEREIRA AC (1), QUENDERA B (1), MATEUS C (1), SILVA E (2), CASTELO-BRANCOM (1)

(1) IBILI, Coimbra

(2) CHUC, Coimbra

Purpose Retinitis Pigmentosa (RP) is a rare retinal disease with progressive degeneration of photoreceptors leading to early peripheral and later central visual loss. It provides a model to analyze visual cortex plasticity since onset age ranges from infancy to adulthood. Prior studies have mostly covered structural or functional alterations in the same cohort; hence we aimed to determine the impact of RP on brain function and structure using magnetic resonance imaging.

Methods Brain images of 5 RP subjects (age 37-66 years, RP duration 6-42 years) and 10 matched healthy controls were acquired. Retinotopy was used to delineate visual cortical areas. Participants also performed a 1-back visual task.

Results Retinal thickness, visual field, and visual acuity were decreased in $R P(p<0.01)$ Central visual cortex responses were shifted anteriorly into regions usually representing more peripheral locations $(\mathrm{p}<0.03)$. Also, $\mathrm{V} 1$ and $\mathrm{V} 3$ dorsal had higher activation during the task execution $(\mathrm{p}<0.02)$. Moreover, cortical thickness was increased in Brodmann areas 7 and 8 , and decreased in pericalcarine $(\mathrm{p}<0.04)$. Surface area was enlarged in isthmus cingulated, pars orbitalis, and transverse temporal $(\mathrm{p}<0.02)$. Caudate volume was also augmented $(\mathrm{p}=0.01)$

Conclusion Results suggest brain functional and structural reorganization in RP which is supported by the absence of a silent cortical visual zone, due to unmasking of peripheral responses to central stimuli. Data also suggest top down modulation of attentional higher level regions, and enhanced recruitment of visuospatial orientation mechanisms due to peripheral vision loss. Results are relevant to the development of rehabilitation methods for RP
- T102

The application of Colour Contrast Sensitivity test to the early prediction of chiasm damage in cases of pituitary adenoma

KRIAUCIUNIENE L (1, 2), SLATKEVICIENE G (1), LIUTKEVICIENE R (1, 2), ZALIUNIENE D (1), BERNOTAS G(1), GLEBAUSKIENE B (1), TAMASAUSKAS $A(2,1)$

(1) Lithuanian University of Health Sciences, Medical Academy, Department of Ophthalmology, Lithuania, Kaunas

(2) Neuroscience Institute, Lithuanian University of Health Sciences, Medicine Academy, Kaunas

Purpose Our objective was to estimate the maximum colour contrast sensitivity (MCCS) thresholds in persons with damage of chiasma opticum.

Methods Forty one persons with pituitary adenoma (PA) and 100 persons as age and gender matched controls were examined. Patients were divided into groups: according to who had chiasmal compression on imaging and who had not. A new computerized MCSS test program was used for colour discrimination.

Results The mean of error score (ES) of MCCS in the group without chiasmal compression was 1.8 [SD, 0.38], in the group with chiasmal compression it was 3.5 [SD, $0.96]$, and in the control group it was 1.4 [SD, 0.31], $(\mathrm{P}<0.001)$. When visual acuity and visual field and fundus were normal in patients with PA, MCCS ES were 3.3 [SD, 1.8], and when VA was less than 1.0 the results were - 4.6 [SD, 2.9]

Conclusion Results of MCSS test ES were 1.9 times better in patients who had not chiasmal compression compared to patients who had chiasmal compression $(\mathrm{P}<0.001)$. Even when VA was normal in PA patients group, their ES was 2.35 times worse compared to healthy persons, $\mathrm{P}<0.001$. Financial support: Lithuanian Science Council (grant no. MIP-008/2014).

- T104 / 3677

Visual intelligence in action: A question of balance?

Neuroplasticity: recent findings on the visual system for the practitioner

VANDERMEULEN J

Clinical neuropsychology, Geleen/ Sittard

Purpose In this presentation I will focus on new insights in the balance of neuroplasticity for brain injury and the effects on vision from a neuropsychological point of view. I will also emphasize on the organisation of the visual pathways ( e.g. ventral and dorsal processing stream and the subcortical processing).

Methods From a literature and integrative research point of view of the last ten years we have found that the vision has changed. I will explain that visual processing is not only taken place by the old reponsibility pathways of eyes and the occipital lobes as key regions. Insight on brain injury in recent has changed this meaning. A case will be presented to support this view

Results There is a new insight in processing information of the brain and od pathways will be abandoned. It may very well that it takes place by other sensoric areas such as the thalamo-cortical route. The brain is highly sensitive to adverse environments, such as the visual impairment after brain injury, but it shows also resilience to neurological deficits. The visual tracts are no longer seen as the only pathways for sensory information (this is an essential element for ophthamologists and practitionars), because they contains pathways from other areas.

Conclusion It is important for ophthalmologists and practitionars that the old paradigma of the visual system should be abandoned. We have to realize that clinical management on the visual disorders is more complex and that the impact from the newly acquired knowledge affects the diagnosis and intervention: an unbeatable approach for visual processing and daily work.Werner, J.S. \& Chalupa . L. M. (2014). The new visual neurosciences. London: MIT Press 
Poster session 1: Electrophysiology-physiological Optics-Vision Sciences/Glaucoma/Immunology-Microbiology/Molecular Biology-Genetics-Epidemiology/ Neuro-ophthalmology-Strabismology-Paediatric Ophthalmology-History of Ophthalmology

- T105

Sudden bilateral vision loss and binasal campimetric involvement in an unusual case of lateral geniculate myelinolysis

IIMENEZ DEL RIO B (1), PALACINM (2), PÉREZ D (1), ASCASO FJ (1)

(1) Ophthalmology, University Clinical Hospital Lozano Blesa, Zaragoza

(2) Neurología, Ernest Lluch Hospital, Calatayud

Purpose Central pontine myelinolysis, a devastating demyelinating disease, is thought to be caused by an aggressive correction of a hyper- or hypo-osmolar state. Lateral geniculate nucleus (LGN) has been incidentally found to be affected by an extrapontine demyelination on magnetic resonance imaging (MRI), although visual disturbances implicating both LGNs are unusual. We describe a campimetric involvement secondary to an intrageniculate damage.

Methods A 40-year-old man, who was diagnosed with pancreatitis following cholecystectomy, complained of bilateral acute vision loss. His best corrected visua acuity (BCVA) was counting fingers in the right eye and 20/200 in the left eye. Optical coherence tomography (OCT) showed a slightly increased macular thickness in the nasal region of both eyes as well as an altered reflectivity of the retinal layers. Automated perimetry revealed a binasal hemianopsia and brain MRI confirmed symmetrical lesions within both LGNs. BCVA was gradually recovered, reaching 20/20 within 6 weeks.

Results Lateral geniculate myelinolysis is an uncommon cause of acute bilateral vision loss which might cause retinal alterations by a retrograde way. Although MRI is the optimal imaging technique to confirm the diagnosis, OCT and visual field examination are able to detect subtle changes.

Conclusion Binasal hemianopsia from intrageniculate myelinolysis has been described after rapid changes in serum sodium, following an emergency caesarean section or secondary to anaphylactic shock. To the best of our knowledge, this is the first case reported where it was induced by fluid and electrolyte imbalance in an acute pancreatitis

- T107

Ganglion cell complex analysis in Parkinson's disease by optical coherence tomography

VIEIRA L (1), ANJOS R (1), SOUSA A (2), SILVA N (1), VICENTE A (1) BORGES B (1), COSTA L (1), FERREIRA J (1), CUNHA JP (1)

(1) Centro Hospitalar Lisboa Central - Ophthalmology, Lisboa

(2) Centro Hospitalar Lisboa Central - Neurology, Lisboa

Purpose To analyze the ganglion cell complex (GCC) by optical coherence tomography $(\mathrm{OCT})$ in Parkinson's disease and to correlate it with disease's severity and duration.

Methods We conducted a prospective, case-control study, in healthy subjects and in patients with Parkinson's disease (PD). Full ophthalmologic examination, OCT (Spectralis ${ }^{\circ}$ ) and neurological evaluation were performed. Macular thickness of GCC (retinal ganglion cell layer and inner plexiform layer) were measured after manual segmentation

Results The study included 25 healthy subjects ( 25 eyes) and 25 patients ( 25 eves) with PD. Age and sex did not differ between the two groups. A statistically significant decrease in the thickness of GCC was detected in the PD group compared to control ( $<<0,05)$. GCC thickness was inversely correlated with Parkinson's disease severity and duration.

Conclusion The decreased GCC thickness detected in this study goes in favor to the characteristic neurodegeneration of this disease. GCC analysis may contribute to the diagnosis and management of this pathology
- T106

Prediction of Parkinson's disease severity based on RNFL thickness evaluated by OCT

JIMENEZ DEL RIO B (1), ASCASO FJ (1), CRISTOBAL J (1), PÉREZ D (1) IBANEZ J (1), LOPEZ DEL VAL J (2)

(1) Ophthalmology, University Clinical Hospital Lozano Blesa, Zaragoza

(2) Neurology, University Clinical Hospital Lozano Blesa, Zaragoza

Purpose To determine the relationship between peripapillary retinal nerve fiber aver (RNFL) thickness in Parkinson disease (PD) with the severity of this neurological disorder and to define a simple biomarker to predict clinical severity.

Methods Peripapillary RNFL thickness was measured in 102 eyes from 52 PD patients by using optical coherence tomography (OCT) and compared with 97 eyes from 50 agematched healthy controls. The Unified Parkinson's Disease Rating Scale (UPDRS) total score was used and responses in the $\mathrm{ON}$ medication state were measured.

Results PD patients showed a statistically significant decrease in average peripapillary RNFL thickness compared with control eyes $(\mathrm{p}<0.001)$. This reduction was observed in every quadrant: inferior $(\mathrm{p}<0.001)$, superior $(\mathrm{p}<0.001)$, nasal $(\mathrm{p}<0.001)$ and temporal $(\mathrm{p}=0.017)$ in $\mathrm{PD}$ patients. Furthermore, a strong inverse correlation was found between PD severity measured according to the UPDRS score with either the average peripapillary RNFL thickness $(\mathrm{r}=-0.615 ; \mathrm{p}<0.001)$ or PD duration $(\mathrm{r}=-0.303 ; \mathrm{p}=0.002)$. From these results, we defined the following regression equation, which is able to predict the UPDRS score from the mentioned variables: UPDRS $=81.6+29.6 * \log$ PD duration $(\mathrm{yr})-0.6$ * RFNL thickness $(\mu \mathrm{m})$

Conclusion As the evolution and severity of PD progress, the peripapillary RNFL thickness gradually diminishes. These results suggest that the average peripapillary RNFL thickness measured by OCT might be useful as a biomarker to detect the early onset and progression of $\mathrm{PD}$.

\section{- T108 / 3675}

Usefulness of ophthalmology psychophysical test for diagnosis and monitoring support in mild Alzheimer's disease

GARCIA MARTINES (1), DEHOZR $(1,2)$, ROJAS B $(1,3), \operatorname{RAMIREZ~AI~}(1,2)$ SALAZARJJ (1, 2), GIL P (4), YUBEROR (4), TRIVINO A (1,3), RAMIREZ IM (1,3)

(1) Instituto de Investigaciones Oftalmológicas Ramón Castroviejo. Universidad Complutense de Madrid, Madrid

(2) Facultad de Óptica y Optometría. Universidad Complutense de Madrid, Madrid

(3) Facultad de Medicina. Universidad Complutense de Madrid, Madrid

(4) Servicio de Geriatría. Hospital Clinico San Carlos, Madrid

Purpose Alzheimer's disease (AD) is a neurodegenerative disorder of the central nervous system characterized by cortical atrophy most pronounced in the medial temporal and posterior temporoparietal regions. The goal of the present study was to examine the visual pathway in patients with mild AD (GDS 4) by means of a different psychophysical test, specifically - visual acuity (VA), contrast sensitivity (CS), colour perception and perception digital test (PDT) - and to compare them with an agematched control.

Methods Twenty-three patients with mild AD and 27 controls were examined at Hospital Clínico San Carlos in Madrid (Spain). Subjects underwent a complete ophthalmological examination (VA, refraction, colour test, biomicroscopy, IOP, dilated fundoscopy, and OCT).

Results In comparison with the control group, patients with mild AD presented a significant decrease in the VA, in the CS (for all the spatial frequencies), in the colour perception (unspecific errors blue axis) and PDT ( $\mathrm{p}<0.05$ in all instances).

Conclusion In mild $\mathrm{AD}$, there are alterations in the $\mathrm{M}$, $\mathrm{P}$, and $\mathrm{K}$ visual pathways. The psychophysical ophthalmological tests used in this study could serve as a tool for $\mathrm{AD}$ diagnosis and follow-up. (Support: OFTARED (Grant RD12/0034/0002, ISCIII, Spanish Ministry of Science and Innovation) 
Poster session 1: Electrophysiology-physiological Optics-Vision Sciences/Glaucoma/Immunology-Microbiology/Molecular Biology-Genetics-Epidemiology/ Neuro-ophthalmology-Strabismology-Paediatric Ophthalmology-History of Ophthalmology

\section{- T109}

Optic neuropathy caused by type A botulinic toxin

MORREALE BUBELLA R, VADALA M, MORREALE BUBELLA D

Department of Experimental Biomedicine and Clinical Neuroscience (BioNeC), Palermo

Purpose To report the development of an optical neuropathy after injection of type A botulinic toxin in a subject with blepharospasm.

Methods Optical neuropathy was evaluated through a complete ophthalmologic examination consisting of bio-microscopy, tonometry and study of visual sharpness, colour test, visual field, OCT, fluorangiography and determination of VEPs.

Results Intravenous therapy was begun with Metilprednisolone $(20 \mathrm{mg} / \mathrm{Kg} / \mathrm{dav})$ and Vitamin B6 associated with oral Citicoline, and there was a clear improvement down to normalization of the symptoms. After 6 months the Humphrey computerized visual field with central 30-2 program threshold test emphasised in ODx an upper half-field arc-shaped defect starting from the optical papilla and also affecting the fixation zone, while for the left eye the perimeter picture was always normal. Optical coherence tomography (OCT) examination of the peripapillary nervous fibres and of the head of the optical nerve highlighted a reduction in thickness of the papillary fibres with a rim area of 0.011 compared to the contralateral side, which presented a normal thickness of the fibres and a rim area of 0.026 . The colour test was normal and the speed of execution of the test proved to be equal in the two eyes.

Conclusion The authors draw attention to the need for correct administration of the botulinic toxin in the treatment of blepharospasm.

\section{- T111 / 2658}

Clinical experience with Raxone ${ }^{\oplus}$ (Idebenone) in the treatment of patients with Leber's hereditary optic neuropathy (LHON)

METZ G (1), GALLENMÜLLER C (2), VONLIVONIUS B (2), LOB F (2), COPPARD N (1), MEIER T (1), KLOPSTOCK T (2)

(1) Santhera Pharmaceuticals, Liestal

(2) Ludwig-Maximilians-University, Munich

Purpose $\mathrm{LHON}$ is an orphan mitochondrial disorder affecting the retinal ganglion cells leading to permanent blindness from which recovery is rare. Here we report visual acuity outcomes for patients with recent onset who received Raxone" (idebenone) under an ongoing global Expanded Access Program (EAP).

Methods Visual acuity was measured in 3-monthly intervals. Clinically relevant recovery was defined as (i) improvement from nadir by at least 10 letters on the ETDRS chart or (ii) improvement from "off-chart" at nadir to being able to read at least 5 letters on-chart.

Results Currently there are $61 \mathrm{LHON}$ patients enrolled, of which 48 patients have been treated for an average of 11 months. So far, 24 of 48 patients (50\%) have experienced a clinically relevant and stable recovery in VA (89\% for T14484C, 70\% for G3460A and $31 \%$ for G11778A). The average treatment effect size in patients with recovery was 29 letters and $84 \%$ recovered within 12 months of the initiation of the EAP.

Conclusion A high proportion of LHON patients treated with idebenone under a global EAP experienced a clinically meaningful recovery of vision, further demonstrating the therapeutic potential of idebenone in the treatment of LHON

Commercial interest

\section{- T110}

A case report survey (CRS) on the natural history of visual acuity in patients with Leber's hereditary optic neuropathy (LHON)

METZ G (1), COPPARD N (1), PETRAKI D (1), MEIER T (1), KLOPSTOCK T (2), SAHELJ (3)

(1) Santhera Pharmaceuticals, Liestal

(2) Ludwig-Maximilians-University, Munich

(3) Institut de la Vision, Paris

Purpose $\mathrm{LHON}$ is an inherited mitochondrial orphan disease leading to rapid, irreversible vision loss in the majority patients. This survey reports the clinical course of vision loss and recovery in patients with LHON based on a large retrospective data collection.

Methods Visual acuity (VA) data from 383 individual patients were collected by chart review conducted in 11 clinical centres under local ethics approval using a standardized VA report form. Change of VA with time since onset of symptoms was analysed using cross sectional and longitudinal data analyses. Mutation dependent rates for spontaneous clinically relevant recovery (off-to-on-chart or at least 2 lines on chart) of VA were established from 774 individual observations available from 74 patients for whom relevant longitudinal VA data were available for up to 31 months.

Results The data confirm the expected rapid loss of VA with $73 \%$ of eyes deteriorating beyond the threshold for legal blindness within 3 months of onset. The VA nadir was typically reached within less than 6 months from onset and for observations available between 12 and 24 months from onset, $78 \%$ of eyes remained legally blind.The time to VA recovery and proportion of patients with recovery from nadir varied with the LHON-associated mtDNA mutation carried. A total of $31.1 \%$ patients presented with spontaneous clinically relevant recovery G11778A: 25\%, G3460A: 50\%, T14484C: 43\%). Conclusion These data demonstrate that in patients with LHON vision loss is rapidly progressive and severe. The great majority of patients do not regain VA but remain legally blind.

Commercial interest

\section{- T112 / 3678}

Microcystic macular edema in optic nerve atrophy: case series

VOIDE N, BORRUAT F

Department of Ophthalmology, University of Lausanne, Jules Gonin Eye Hospital, Lausanne

Purpose Microcystic macular edema (MME) is a new entity defined as lacunar areas of hyporeflectivity in the retinal inner nuclear layer after severe optic neuropathy $(\mathrm{ON})$. Initially, MME was fortuitously discovered in optic neuritis.

Methods From July 2013, we imaged all patients with ON by SD OCT. Retinal nerve fiber layer thickness (RNFLT), macular cube thickness (MCT) and retinal ganglion cell layer thickness (RGCLT) were assessed. Studied parameters included also the delay between onset of $\mathrm{ON}$ and detection of MME and correlation of the visual field defect to the MME topography.

Results Sixteeen patients (19 eyes) exhibited MME. Etiologies of ON were compressive (7), degenerative (4), inflammatory (3), ischemic (1), and hereditary (1). There were 9 women and 7 men, mean age at diagnosis being 34.1 years. Visual acuity ranged from NLP to $12 / 10$ (median 4/10). Mean RNFLT was $61.6 \mu \mathrm{m}[97+/-10 \mu \mathrm{m}]$, mean MCT $271.37 \mu \mathrm{m}[261+/-18 \mu \mathrm{m}]$ and mean RGCLT $57.22 \mu \mathrm{m}[82+/-7 \mu \mathrm{m}]$. The time from diagnosis until MME detection ranged from 4 months to 23 years. A good correlation was found between the zone of retinal atrophy on infrared photography and MME distribution. However, only $4 / 16$ visual field defects correlated to the MME topography. Conclusion MME can occur as early as 4 months after ON (ocular trauma in our series) and can persist as late as 23 years after the onset of ON. MME is not only related to inflammatory $\mathrm{ON}$, as postulated initially, but is a nonspecific manifestation from $\mathrm{ON}$ of any etiology. The precise mechanism leading to MME is unknown but transsynaptic retrograde degeneration is likely. Why only $10 \%$ of patients with ON exhibit MME is unknown, but MME might be a transient phenomenon. Further clinical and experimental studies are needed to answer these questions. 
Poster session 1: Electrophysiology-physiological Optics-Vision Sciences/Glaucoma/Immunology-Microbiology/Molecular Biology-Genetics-Epidemiology/ Neuro-ophthalmology-Strabismology-Paediatric Ophthalmology-History of Ophthalmology

- T113

Mutational and clinical spectrum in Korean with Leber's hereditary optic neuropathy

YUM HR (1), KIM MS (2), SHIN SY (1), PARK SH (1)

(1) Ophthalmology and Visual Science, Seoul

(2) Laboratory Medicine, Seoul

Purpose To investigate the spectrum of mutations of mitochondrial DNA (mtDNA) and clinical features in Korean with Leber's hereditary optic neuropathy (LHON).

Methods Total genomic DNA was isolated from the peripheral blood leukocytes of the patients who were suspicious of LHON. The presence of mutations were screened by direct sequencing were analyzed against mitochondrial reference sequence on genetic analyzer. The ophthalmologic examinations of patients were conducted, including visual acuity(VA), color vision, retinal nerve fiber layer(RNFL) thickness and visual field(VF).

Results Two primary mutations (G1778A, T14484C) and five secondary mutations (T3394C, C3497T, G11696A, A11392C, T14502C) were detected in 34 of 164 unrelated patients. Among the 34 patients, primary mutation was detected in 21 patients, secondary mutation in 11 patients, and combined primary and secondary mutation in 2 patients. Compared the patients with primary mutation, patients with secondary mutation had increased proportion of woman. The VA was significantly better in secondary mutation than primary mutation. Also, the severity of the color vision deficiency was significantly mild in secondary mutation relative to primary mutation. No significant difference was found in the OCT parameters between the groups including temporal area. However, the visual field index and mean deviation of the visual field test were different significantly between groups.

Conclusion Our results emphasize the possible heterogeneity of LHON-associated mtDNA mutations and clinical phenotypes in Korean LHON patients. Candidate pathogenic mtDNA mutations including major primary mutations should be rigorously investigated.

\section{- T115}

Prevalence of optic neuropathy in alcoholic patients and predictive value of optic nerve alterations for its diagnosis

MICHAU S (1), RIGOLE H (2), PERNEY P (3), VILLAIN M (1), DAIEN V (1)

(1) Universitary Hospital, Ophtalmology, Montpellier

(2) Universitary Hospital, Addictology, Montpellier

(3) Universitary Hospital, Addictology, Nimes

Purpose Optic neuropathy $(\mathrm{ON})$ is one of the complications of alcohol. The aim of this study was to assess the prevalence of alcoholic $\mathrm{ON}$ in a cohort of patients hospitalized for alcohol withdrawal. Secondary objective was to explore the predictive value of optic nerve changes for diagnosis of $\mathrm{ON}$.

Methods This was a single-center prospective study. A complete eye examination was performed during the patient's alcohol withdrawal. The definition of alcoholic ON used was an assocation of alterations of visual field with impaired color vision. Optic nerve function and morphology were assessed using visual evoked potentials, optical coherence tomography (OCT), Nerve Fiber Indicator from polarimetry (GDx) and Moorfields Regression Analysis of the Heidelberg Retina Tomograph II (MRA-HRT II).

Results Two hundred patients were included prospectively from January 2010 to June 2013 (145 men and 55 women) with a mean age of $47 \pm 12$ year. The average alcohol consumption was $220 \pm 122 \mathrm{~g} /$ day. 46 patients (23\%) responded to alcoholic ON defintion (13.5\% in both eyes and 9.5\% in only one eye). Diagnostic sensitivity and specificity for visual evoked potentials were of $55.5 \%$ and $54.5 \%$ respectively; for GDx $18 \%$ and $97 \%$, respectively; for OCT $38 \%$ and $92 \%$, respectively; and for MRA-HRT II $19 \%$ and $79 \%$, respectively.

Conclusion A high prevalence of alcoholic ON observed in this study should prompt screening and early treatment. From predictive value of objective optic nerve changes we discuss the best couple of exams to complete the screening of alcoholic $\mathrm{ON}$.
- T114

Leber hereditary optic neuropathy and a new MT-ND1 pathologic mutation

ESTEBANO (1), MARTINEZ-ROMERO I (2), HERRERO-MARTINMD (2), LLOBET L (2), EMPERADOR S (2), MARTIN-NAVARROA (2). NARBERHAUS B (2), ASCASO FJ (1), LOPEZ-GALLARDO E (2), MONTOYA J (2), RUIZ-PESINIE (2)

(1) Ophthalmology, HCU Lozano Blesa, Zaragoza

(2) Biochemistry and molecular biology, University, Zaragoze

Purpose Over $90 \%$ of Leber hereditary optic neuropathy (LHON) is caused by one of three mitochondrial DNA (mtDNA) mutations (m.11778A $>\mathrm{G}, \mathrm{m} .3460 \mathrm{G}>\mathrm{A}$, m.14484T $>C$ ) in genes for respiratory complex I $(\mathrm{CI})$ subunits. These mutations are homoplasmic and characterized by incomplete penetrance, and a large percentage of patients harboring these mutations have no family history of disease. However, the pathogenicity of these mutations has not been confirmed. We report a patient who exhibited typical clinical features of LHON and presented a new mutation in the MTND1 gene and lacked all three of the most common mtDNA mutations.

Methods The diagnosis of LHON in our patient was based on clinical studies. The mtDNA was completely sequenced and the candidate mutation was analyzed in more than 18,000 individuals around the world, its conservation index was estimated in more than 3,100 species from protists to mammals, its position was modeled in the crystal structure of a bacteria ortholog subunit and its functional consequences were studied in a cybrid model.

Results Genetic analysis revealed an m.3472T>C transition in the MT-ND1 gene that changes a phenylalanine to leucine at position 56 . Bioinformatics, molecular-genetic analysis and functional studies suggest that this transition is the etiologic factor for the disorder.

Conclusion The evidence presented strongly suggest that this new mutation expands the spectrum of deleterious changes in mtDNA-encoded complex I polypeptides associated with this pathology and highlights the difficulties in assigning pathogenicity to new homoplasmic mutations that show incomplete penetrance in sporadic LHON patients. 


\section{- F001}

Functional presence of $\mathrm{Na}+-\mathrm{K}+-2 \mathrm{Cl}$ - cotransporter in rabbit lacrimal gland ductal epithelial cells

TÓTH-MOLNARE (1), KATONA M (2), VIZVARIE (1), FACSKO A (1), VENGLOVECZ V (3), RAKONCZAYZ (2), HEGYIP (2)

(1) Dept. of Ophthalmology, Univ. of Szeged, Szeged

(2) 1st Dept. of Internal Medicine, Univ. of Szeged, Szeged

(3) Dept. of Pharmacology, Univ. of Szeged, Szeged

Purpose Capability of fluid secretion of rabbit lacrimal gland duct epithelial cells was recently demonstrated by the authors using isolated duct segment model developed earlier. Inhibition of basolateral $\mathrm{Na}+-\mathrm{K}+-2 \mathrm{Cl}$ - cotransporter (NKCC1) with bumetanide resulted in a complete stoppage of fluid secretion. Aim of the present work was to determine the activity and the regulation of NKCC1.

Methods Experiments were performed on isolated rabbit lacrimal gland interlobular duct segments. Intracellular fluorophotometry with ammonium pulse technique was used to measure cotransporter activity. The rate of bumetanide sensitive cytosolic acidification after addition of NH4- can be used to quantify the activity of the cotransporter. Role of various potentially stimulatory effects were investigated.

Results Unstimulated activity of NKCC1 was determined. Elevated intracellular cAMP level evoked by forskolin stimulation resulted in marked increase in NKCC1 activity. Beside increased intracellular cAMP levels, low cytosolic $\mathrm{Cl}$ - concentration and cell shrinkage evoked by hyperosmotic environment were significant determinants of increased cotransporter activity.

Conclusion Our earlier results suggested the dominant role of $\mathrm{Cl}$ - dependent mechanisms in lacrimal gland ductal fluid secretion. The presented experimental work proves the substantial role of $\mathrm{NKCC1}$ in basolateral $\mathrm{Cl}$ - uptake. Intracellular regulation of $\mathrm{Cl}$ - handling and luminal $\mathrm{Cl}$ - secretion needs further investigation.

\section{- F002}

Pharmacological matrix metalloproteinase (MMP) inhibition blocks axonal regeneration in the damaged retinotectal system of the adult zebrafish

LEMMENS K (1), BOLLAERTS I (1), BHUMIKA S (2), VANHOUCKEJ (1),

VANHOVE I (1), MOONS L (1)

(1) Neural Circuit Development and Regeneration Research Group, Leuven

(2) Comparative Endocrinology Research Group, Leuven

Purpose Complete restoration of the injured mammalian central nervous system (CNS) remains a challenge, making the search for regenerative molecules essential. Matrix metalloproteinases (MMPs), (non)-matrix protein cleaving endopeptidases, are upregulated during CNS repair, reduce glial scar formation and potentially promote axonal regrowth. As such MMPs or their underlying molecules likely form potent regenerative molecules. One study already reported upregulated mRNA levels of specific MMPs in spontaneously regenerating eyes of adult zebrafish subjected to an optic nerve crush (ONC). Here, we intend to elucidate the role of these MMPs in zebrafish retinotectal regeneration.

Methods Immunohistochemistry and Western blotting were used to determine the protein expression pattern of MMP-2,-9,-13a and -14 after ONC in the regenerating zebrafish retina. To investigate the role of MMPs in retinal ganglion cell (RGC) axonal regeneration, a broad-spectrum (GM6001) inhibitor was intravitreally injected at specific time points after ONC. Biocytin labeling was used to study tectal reinnervation. Results Our expression data show a spatiotemporal expression of these MMPs in the regenerating zebrafish retina and suggest an individual role in RGC survival, axonal regrowth and dendritic/synaptic remodeling. Moreover, broad-spectrum MMP inhibition during the first week after ONC significantly reduces retinotectal regeneration without influencing RGC survival.

Conclusion Our study reveals that MMPs are associated with zebrafish retinotectal regeneration and that these enzymes or their downstream targets might be of therapeutic value for the injured mammalian CNS.

\section{- F003}

\section{Inhaled nitric oxide dilates retinal arterioles in minipigs}

PETROPOULOS I (1), MARTINAL (2), MANGIORIS G (1), MENDRINOS E (1), RIMENSBERGER PC (2), POURNARAS C (1)

(1) Laboratory of Neurobiology and Physiology of the Retinal Circulation, Geneva University Hospitals, Geneva

(2) Department of Pediatrics, Geneva University Hospitals, Geneva

Purpose To investigate the effect of inhaled nitric oxide (NO) on the retinal arteriolar diameter in minipigs.

Methods Seven minipigs were anesthetized, intubated and mechanically ventilated. A Swan-Ganz catheter was inserted for measuring cardiac output by the thermodilution technique. Fundus image (one eye per animal) was recorded in a digital videotape under stable hemodynamic and ventilatory conditions. After baseline recordings, $\mathrm{NO}$ was added to the breathing circuit first at $20 \mathrm{ppm}$ for 30 minutes and then at 40 ppm for another 30 minutes. NO was then weaned off. Retinal arteriolar diameter was measured in arbitrary units (AU) at baseline and 30 minutes after each manipulation, using Retinal Vessel Analyzer. Systemic and pulmonary hemodynamic parameters were also measured.

Results Mean baseline retinal arteriolar diameter was $231.0 \pm 38.1 \mathrm{AU}$. Thirty minutes after initiation of 20 -ppm NO inhalation, this diameter had increased only by $3.2 \pm 3.5 \%$ compared to baseline ( $p>0.05$ ), to $237.9 \pm 36.5$ AU. Thirty minutes after initiation of 40ppm NO inhalation, this diameter had increased by $7.6 \pm 4.7 \%$ compared to baseline $(\mathrm{p}<0.05)$, to $248.3 \pm 39.8 \mathrm{AU}$. Thirty minutes after weaning off the inhaled NO, this diameter had decreased to $232.9 \pm 39.1 \mathrm{AU}$, having returned practically to baseline ( $>0.1$ compared to baseline). Hemodynamic parameters (mainly cardiac output, blood pressure, and systemic vascular resistance) did not change significantly.

Conclusion In minipigs, NO inhalation at a concentration increased stepwise from 20 to $40 \mathrm{ppm}$ induces a retinal arteriolar vasodilation which is reversed 30 minutes after weaning off the inhaled NO. A potential clinical application of this treatment modality in vascular diseases of the retina needs to be evaluated.

\section{- F004 \\ Interaction between leukocytes and erythrocytes in the human retina: Effects of pentoxifylline on hyperoxia-induced vasoconstriction during increased neutrophil counts}

FUCHSJAGER-MAYRL G (1), TOLD R (2), WOLZT M (2), SCHMETTERER L (2, 3), SCHMIDT-ERFURTHU (1), GARHOFER G (2)

(1) Opthalmology and Optometry, Medical University of Vienna, Vienna

(2) Clinical Pharmacology, Medical University of Vienna, Vienna

(3) Medical Physics and Biomedical Engineering

Purpose We have previously shown that under states of vasoconstriction an interaction between circulating erythrocytes and leukocytes may play a role in the control of blood flow. In the present study we hypothesized that pentoxifylline may alter this interaction during oxygen-induced vasoconstriction.

Methods 24 healthy male subjects participated in this double masked, randomized, placebo-controlled 2 way cross over trail. In order to increase white blood cell count (WBC) count, $300 \mu \mathrm{g}$ of G-CSF was administered intravenously. Vasoconstriction of retinal vessels was induced by oxygen inhalation. $400 \mathrm{mg}$ of pentoxifylline or placebo was infused at two different study days. White blood cell flux was assessed with the blue-field entoptic technique. Vessel calibers were measured with a dynamic vessel analyzer (DVA) and red blood cell velocity (RBCV) was determined with laser Doppler velocimetry (LDV). Retinal blood flow was calculated based on retinal vessel diameters and RBCV.

Results Retinal vessel diameter, RBCV, calculated retinal blood flow and white blood cell flow were not altered by administration of pentoxifylline. Hyperoxia induced a pronounced decrease in retinal blood flow parameters. No difference was observed between groups during oxygen breathing in vessel diameters $(\mathrm{p}=0.54), \mathrm{RBCV}(\mathrm{p}=0.34)$, calculated retinal blood flow ( $\mathrm{p}=0.3$ ) and white blood cell flow $(\mathrm{p}=0.26)$.

Conclusion Our data indicate that short time administration of pentoxifylline does not alter the oxygen-induced effect on ocular blood flow parameters during leukocytosis. 
- F005

Genetic deletion of $\beta 1$ - and $\beta 2$-adrenergic receptors rescues the retina from hypoxia-induced pathologic angiogenesis

DAL MONTE M, CAMMALLERI M, FORNACIARI I, CASINI G, BAGNOLIP Department of Biology, Pisa

Purpose To investigate the effects of $\beta 1$ - and $\beta 2$-adrenergic receptor (AR) deletion in the vascular retinal response to hypoxia, to confirm and expand the role of $\beta 1$ - and $\beta 2$ ARs in regulating retinal angiogenesis and to get insights into the role of $\beta 3$-ARs.

Methods We used wild type (WT) and $\beta 1 / \beta 2$-AR knockout (KO) mice. The retinal angiogenic response to hypoxia was studied in a mouse model of oxygeninduced retinopathy (OIR) using real time RT-PCR, Western blot, ELISA and immunohistochemistry.

Results Our results show that $\beta 1$ - and $\beta 2$-AR deletion did not affect $\beta 3$-AR expression or norepinephrine (NE) levels. In $\mathrm{KO}$ mice, $\mathrm{NE}$ was less upregulated in response to hypoxia indicating a reduced stimulation of the $\beta$-adrenergic system. In $\mathrm{KO}$, the angiogenic response to hypoxia, including the formation of both a central avascular area and neovascular tufts, was nearly abolished while no improvements were observed in blood-retinal barrier dysfunction typical of OIR. Unexpectedly, VEGF levels did not differ between WT and $\mathrm{KO}$ in spite of a recover of the angiogenic response, but a reduced activation of VEGF signaling was observed in $\mathrm{KO}$, which may explain the reduced response to hypoxia. To get insights into the role of $\beta 3$-ARs, we treated OIR mice with a 33 -AR agonist, BRL37344 (2 mg/ $/ \mathrm{gg}$, s.c. injected), which resulted in an increase in VEGF levels and the formation of neovascular tufts in both WT and KO, indicating that 33 -AR stimulation may function as a proangiogenic switch.

Conclusion Taken together, our results confirm the notion that $\beta 1$ - and/or $\beta 2$ ARs play a role in retinal angiogenesis and indicate that $\beta 3$-ARs may potentiate the angiogenic response in WT and sustain the angiogenic drive in $\mathrm{KO}$.

\section{- F007 \\ Development of new anti-VEGF drug by SRPK1 inhibitor screening}

MOROOKA S (1), OKUNO Y (2), HOSOYA T (3), HAGIWARA M (2), YOSHIMURA N (1)

(1) Department of Ophthalmology and visual sciences, Kyoto university, Kyoto

(2) Anatomy and development Biology, Kyoto university, Kyoto

(3) Chemical Biosciences, Institute of Biomaterials and Bioengineering, Tokyo Medical and Dental university, Tokyo

Purpose AMD is a major cause of blindness. Current therapy aims to suppress VEGF levels in order to prevent CNV, which causes AMD. Recently we found that SRPIN340, a specific inhibitor of SR protein kinase 1 (SRPK1) which phosphorylates Serine-argininerich (SR) proteins and regulates splicing and transcription, suppressed the expression of VEGF and CNV. Here we screened for more active SRPK1 inhibitors which suppressed CNV effectively than SRPIN340.

Methods In-silico screening; Structure-based virtual screening (SBVS) was applied against the general chemical library, which include 71955 compounds. In vitro kinase assay; The reaction mixture was incubated at $30^{\circ} \mathrm{C}$ for $15 \mathrm{~min}$ and detected the residual ATP was measured by Kinase Glow following the manufacturer's instruction. VEGF ELISA: We added 10uM compounds or control to ARPE-19 cells. The medium was collected 72 hours after the addition of the compounds. We evaluated the VEGF protein level in the culture medium by ELISA with manufacturer's instruction.In vivo examination; The compound was intravitreally injected to laser CNV model mice immediately after laser photocoagulation. Seven days after laser injury, the mice were perfused with $1 \mathrm{~mL}$ of $0.5 \%$ FITC-labeled dextran. Flat mounts of RPE-choroid complex were obtained and the area of $\mathrm{CNV}$ was measured by fluorescence microscopy.

Results 11994 compounds were selected by SBVS, and we were able to obtain 9535 compounds among them. Next we screened by in vitro kinase assay and selected fou compunds, which were named SRPIN801-804. SRPIN(03 decreased the VEGF protein levels most effectively as measured by VEGF ELISA, and suppressed CNV growth in a dose-dependent manner

Conclusion We obtained a new anti-VEGF drug, which inhibits SRPK1 kinase acitivity

\section{- F006}

Aquaporin 4 is required to induce retinal angiogenesis in a mouse model of oxygen-induced retinopathy

DALMONTEM (1), NICCHIA GP (2), CAMMALLERIM (1), FRIGERI IA (2) PORNACIARII (1) PISANIF (2) BAGNOLIP(1) SVELTOM (2)

(1) Department of Biology, Pisa

(2) Department of Biosciences, Biotechnologies and Biopharmaceutics, Bari

Purpose Aquaporin 4 (AQP4) is the most abundant water channel in the retina and participates in the formation of blood-retinal barrier (BRB). In several retinal pathologies accompanied by BRB dysfunction the expression of AQP4 is altered. In the present study, we investigated the effects of AQP4 deletion in the vascular retinal response to hypoxia.

Methods We used wild type (WT) and AQP4 knockout (KO) mice. The retinal angiogenic response to hypoxia was studied in a mouse model of oxygeninduced retinopathy (OIR) using real time RT-PCR, Western blot, ELISA and immunohistochemistry.

Results In WT mice, retinal levels of AQP4 were increased in response to hypoxia. In OIR mice, the BRB dysfunction was more pronounced in KO than in WT mice. BRB tight junction proteins, occludin, ZO-1 and JAM-C, were analyzed and significant alteration was found for IAM-C in $\mathrm{KO}$ mice, indicating a role for this protein in the observed dysfunctions. The formation of neovascular tufts, which are characteristic of OIR, was completely prevented in KO mice, possibly as a result of the decrease in hypoxia-induced up-regulation of VEGF mRNA and protein. Unexpectedly, this reduced increase in VEGF levels was paralleled by a dramatic increase in the activity of transcription factors which regulate VEGF expression indicating that in $\mathrm{KO}$ retinas mechanisms of VEGF transcription, such as promoter methylation, may be altered.

Conclusion Together, the present results indicate a role for AQP4 in the formation of angiogenic processes in the retina and suggest that inhibiting AQP4 function may be a strategy to reduce pathologic angiogenesis in proliferative retinopathies.

\section{- F008}

Rapamycin down-regulates REDD1 to blunt cell death: a potential way to maintain retinal ganglion cell function as in glaucoma

DEL OLMO AGUADO S, NÚNEZZ ÄLVAREZ C, OSBORNE NN

Fundación de Investigación Oftalmológica, Oviedo

Purpose Determine whether REDD1 is present the retina and located to retinal ganglion cells (RGCs). Moreover, to deduce whether siRNA silencing of REDD1 synthesis and/or rapamycin counteract insults to cells in culture.

Methods Western blot, quantitative PCR and immunohistochemistry were used to show the presence and localisation of REDD1 in the rat retina and RGC-5 cells (a cell line derived from the retina). REDD1 was down-regulated in RGC-5 cells by gene silencing (siRNA) methodology. RGC-5 cells where REDD1 was silenced or not were subjected to insults of either cobalt chloride $(\mathrm{CoCl} 2)$ or blue light, for defined times.

Results REDD1 is present in the rat retina. Moreover, REDD1-immunoreactivity is located to different retinal cell-types but intensely concentrated to a many but not all RGCs. REDD1 is also present in the cytoplasm of RGC-5 cells and appears not to particularly locate in mitochondria. $\mathrm{CoCl} 2$ or blue light insults to RGC-5 cells resulted in cell death and an up-regulation of REDD1. Loss of cell viability was significantly nullified in RGC-5 cells exposed to rapamycin or where REDD1 was silenced. Moreover, rapamycin was not toxic to $\mathrm{RGC}-5$ cells even at high concentrations.

Conclusion Evidence is provided to suggest that rapamycin can down regulate REDD1, part of the mTOR complex receptor mechanism. It is concluded that REDD1, present in RGCs, is activated when insulted as in glaucoma and that this process can be attenuated by use of rapamycin. 


\section{- F009}

Ocular hypotensive properties of Cornus mas extract and loganic acid

SZUMNY D (1, 2, 3), KUCHARSKA AZ (4), PIORECKIN (5, 6, 3), SZUMNY A (7), SOZANSKI T (1), DZIEWISZEK W (1), CHLEBDA E (1), SZELAG A (1)

(1) Department of Pharmacology, Wroclaw Medical University, Wroctaw

(2) Ophthalmology Clinic, Uniwersytecki Szpital Kliniczny, Wroctaw

(3) Wroctaw

(4) Department of Fruit, Vegetable and Cereals Technology, Wroctaw University of Environmental and Life Sciences, Wroctaw

(5) Arboretum and Institute of Physiography in Bolestraszyce, Bolestraszyce

(6) Department of Turism \& Recreation, University of Rzeszow, Rzeszów

(7) Department of Chemistry, Wroctaw University of Environmental and Life Sciences, Wroctaw

Purpose Ocular hypertension became a serious problem in a population with increasingly longer life expectancy. Although there are available effective and well established drugs each group possess their disadvantages. In European medicine according to Pharmacopoeia (EP) herbal drugs affected on the eye condition are on the margin of a purely chemical treatment. A few of them possess as an active ingredients biologically active group of terpenoids called iridoids. The loganic acid is an active iridoid in Cornelian cherry (Cornus mas) fruits as a main compound of a polar fraction. Methods We checked on the animal model (New Zealand rabbits) ocular properties of C. cherry polar fraction and loganic acid. The ultra high pressure liquid chromatography (coupled with mass spectrometers) analysis proved the presence of iridoids: loganic acid, cornuside, and pelaronidin, delfinidin galacto- and rhamnosides. The intraocular pressure was measured by ICare vet tonometer after 1, 2, 3 and 5 hours of applanation of one drop $50 \mu$ l. The intraocular pressure measurements were performed in conscious rabbits, standing calmly on the table, held delicately without head immobilization.

Results A single dose of $0.7 \%$ loganic acid extract in vehiculum containing 0,15\% sodium hyaluronate administered directly into the conjunctival sac decreased after 60 min the mean value of intraocular pressure (IOP) about $15 \%$. The fraction's main compound - loganic acid decreased the IOP for about $23 \%$. This effect was compared to timolol, used similarly to the investigated compounds

Conclusion Although the Cornelian cherry as well as loganic acid are not mentioned in EP we proved it's possible utility in future ocular hypertension therapy.

\section{- F011}

New non-invasive technique for dosing eye drops concentration in tears

GAUTHIER AS (1), FRIOTM (1), PRONGUÉ A (1), ROYER B (2), DELBOSC B (1) (1) Ophthalmology, Besançon

(2) Pharmacology, Besançon

Purpose IntroductionThe efficacy of tacrolimus ointment and eye drops has been proven in the treatment of immune corneal pathologies and in prevention of high risk corneal graft rejection. We recently developed a new formulation of tacrolimus $0.06 \%$ eye drops with a better tolerance than classical formulation. In such context, the concentration of tacrolimus in tears was investigated in the absence of data in human We thus developed a non-invasive technique for dosing eye drops concentration in tears. The objective of the study was to assess the feasibility of this assay.

Methods The tears sampling was performed using Schirmer's paper strips. After collection, the strips were transferred into a solution of acetonitril with internal standard. This solution allows both the preservation of the samples and the extraction of tacrolimus. The tacrolimus was then measured with a LC/MS/MS. The results were normalized with the length of wetting of the paper

Results Detectable concentrations of tacrolimus were easily observed until 4 hours after instillation. The main issue was ocular irritation in few patients.

Conclusion This method allows to measure tacrolimus concentration in tears using a non-invasive method for at least 4 hours. The use of Schirmer's paper strips will facilitate such studies with children. This convenient method will allow us to investigate the behavior of tacrolimus concentrations with time in tears.

\section{- F010}

The role of $\mathrm{Cl}$ - channels in ion transport across the apical and basolateral sides of the mouse retinal pigment epithelium

SKARPHEDINSDOTTIR S, EYSTEINSSON T, ARNASONS

Department of Physiology, University of Iceland, Reykjavik

Purpose The retinal pigment epithelium (RPE) is important in transporting water from the subretinal space to the choroid of the eye. The water transport across the RPE is supposed to be mediated by $\mathrm{Cl}$ - channels situated at the basolateral side of the RPE tissue. The purpose of this study was to assess the function of $\mathrm{Cl}$ - channels at the apical and basolateral side of mouse RPE.

Methods RPE of healthy mice (C57BL/6J) was mounted in Ussing chambers. The short-circuit current (Isc) was measured in $\mu \mathrm{Amp} / \mathrm{cm} 2$. The non-specific $\mathrm{Cl}$ - channel blocker NPPB ( $4 \mathrm{mM}$ ) was applied to both sides. The CFTR blocker CFTRinh-172 $(0.8 \mathrm{mM})$ was added to the apical side. The $\mathrm{Ca}++$ dependent $\mathrm{Cl}$ - channel blocker CaCCinh-A01 (1.15 mM ) was added to the apical side. Each dose of the blockers was tested for 30 minutes. The results are presented as mean \pm SEM, $n=6$ in all experiments. Results NPPB ( $4 \mathrm{mM})$ on the basolateral side produced no significant change $(\mathrm{p}=0.36$; $30 \mathrm{~min})$, but $4 \mathrm{mM} \mathrm{NPPB}$ on the apical side reversed the Isc $(\mu \mathrm{Amp} / \mathrm{cm} 2)$ from $-4,8 \pm$ 5,9 to $+19.9 \pm 7,3(\mathrm{p}=0.004)$ at $5 \mathrm{~min}$ which stabilised at $+2.9 \pm 4,9(\mathrm{p}=0.03)$ at $30 \mathrm{~min}$. CFTRinh- $172(0.8 \mathrm{mM})$ caused a transient increase, $-13 \pm 3,5$ to $-15,1 \pm 3,3(\mathrm{p}=0.02)$ at 5 min. CaCCinh-A01 (1.15 mM) reversed the Isc from $-10,6 \pm 2,1$ to $+5,5 \pm 3,9(\mathrm{p}=0.0002)$ at $10 \mathrm{~min}$, which stabilized at $-0.10 \pm 3,0(\mathrm{p}=0.002)$ at $30 \mathrm{~min}$.

Conclusion The results indicate that $\mathrm{Cl}$ - channels are situated on the apical side of the RPE and drive a part of the ion transport across the mouse RPE. The channels may play a lesser role in ion transport across the basolateral side.

\section{- F012}

Lymphocytic microparticles modulate macrophages function in experimental choroidal neovascularization

TAHIRI H (1), OMRI S (2), CHUN Y (3), DUHAMEL F (1), CHEMTOB S (4) HARDY P (4)

(1) Pharmacology, Université de Montréal, Montréal

(2) Ophtalmology, Université de Montréal, Montréal

(3) Ste-Justine Hospital Research Center, Montréal

(4) Pediatrics \& Pharmacology/Ste-Justine Hospital Research Center, Montréal

Purpose Pathological choroidal neovascularization (CNV) is the major cause of severe vision loss in patients with age-related macular degeneration (AMD). Inflammation is a key component in AMD, and macrophages play an important role in CNV generation. We have demonstrated that human T-lymphocyte-derived microparticles (LMPs) significantly inhibit angiogenesis in several models of ocular neovascularization. In this study, we investigated whether LMPs modulate angiogenic microenvironment by altering macrophages activities

Methods LMPs were produced from apoptotic human T lymphocytes after treated with actinomycin D. The effects of LMPs on cell viability and cell migration were studied in apoptosis assay and migration assay respectively. Cell growth of human retinal microvascular endothelial cells was assessed after cells were co-cultured with LMPs pre-treated macrophages. A laser-induced CNV model was used to determine labelled choroidal flat-mounts.

Results LMPs dose-dependently inhibited macrophages cell growth without altering cell death. In addition, LMPs dramatically abrogated VEGF-induced macrophages migration. LMPs-pretreated macrophages exhibited strong inhibitory effect on endothelial cell growth and this effect was associated with the increased expression of IL-12, CD36 and HIF- $1 \alpha$. In vivo, intravitreal injection of LMPs significantly suppressed laser-induced $\mathrm{CNV}$ and reduced macrophages infiltration at the lesion sites.

Conclusion These results suggest that LMPs are potent antiangiogenic therapeutic agent. In addition to the direct effects on endothelial cells, LMPs may interfere the proangiogenic environment through modulation of macrophages function during pathophysiological conditions. 
- F013

Rabbit cornea epithelization after the antlerogenic stem cells of Cervus elaphus homogenate

SZUMNY D (1, 2), CEGIELSKIM (3), GROSMAN-DZIEWISZEK P (3), SZELAG A (1) BOCHNIA M (4), DZIEWISZEKW

(1) Department of Pharmacology, Wroclaw Medical University, Wroctaw

(2) Ophthalmology Clinic, Uniwersytecki Szpital Kliniczny, Wroctaw

(3) Departament of Histology, Wroclaw Medical University, Wroctaw

(4) Departament of Otolaryngology, Wroclaw Medical University, Wroctaw

Purpose There are many possibly cause of disturbances of the injured cornea epithelization, like for example corneal limbal stem-cell deficiency, diabetes. Growing antlers constitute a model organ for examining regeneration processes of tissues because they are the only mammalian appendages capable of regeneration.

Methods New Zealand White rabbits were used, one eye of each animal was treated, and the fellow eye was the control. Erosion of the corneal epithelium was performed: after local anesthesia a $3 \mathrm{~mm}$ diameter disc soaked in $\mathrm{n}$-heptanol was placed on the cornea for 30 seconds. Then the eye was washed with a saline. Formulation was made of antlerogenic stem cells of Cervus elaphus cultured under standard conditions. After homogenization of 500000 cells we received $1 \mathrm{ml}$ of drops or gel. Rabbits received the homogenate - drops or gel - 3 times a day. The eyes were examined using slit lamp, fluorescein staining and photography of the cornea - test and 10 hours after injury. The surface of the damaged cornea was measured in pixels, analyzing the extent of damage using Adobe Photoshop CS4 Extende

Results The results were averaged - assessing the reduction in the surface of the wound over time. The experiment was terminated to obtain $100 \%$ of the closing of each corneal wound - test and control groups. Corneal healing treated by the investigated preparations followed on the second day and on the 3rd day in the control group. There was observed no magnification the lesion of the injury in case of gel application in the first 10 hours.

Conclusion Further experimental and clinical studies of Cervus elaphus stem cells are recommended. They are necessary for evaluation of usefulness to treat corneal injures.

\section{- F015}

Ocular toxicity of voriconazole: a case report and review of litterature

MOUNIER A (1), DOUMA I (1), EL CHEHAB H (1), VIE A (1), AGARD E (2), RUSSO A (1), DOT C (1)

(1) HIA Desgenettes, Lyon

(2) HIA, Lyon

Purpose Voriconazole is an antifungal therapy widely used for invasive fungal diseases, particularly in aspergillosis.

Methods We report a 77 year-old man treated by voriconazole (100mg twice a day) for a pulmonary aspergillosis. Three days after voriconazole initiation, he complained of visual disorders such as dyschromatopsia and visual hallucinations. Visual acuity was measured at 10/10 in both eyes. Goldman visual field found a bilateral enlarged blind spot. Color vision was assessed using the Lanthony desaturate panel D 15 test, and demonstrates disorders along the tritan confusion axe for the left eye. The multifocal electroretinogram (mfERG) found a global decrease of the foveal peak on both eyes Visual Evoked Potentiel (VEP) showed asymetric data and lower amplitudes of the $\mathrm{P}(100)$ wave on the left eye

Results In a first time, according to the dose dependant toxicity, it was decided to reduce the voriconazole dose to $50 \mathrm{mg}$ twice a day. In spite of this, visual acuity decreased at $8 / 10$ in the left eye and visual field worsed. Voriconazole was then switched with itraconazole (SPORANOX). The outcome was improved one month after, visual field and mfERG were better, visual hallucinations disappeared.

Conclusion Voriconazole ocular toxicity is not well known and few publication exist on this topic. Visual hallucinations, dose dependant macular toxicity, reversible disorders were described. On our knowledge we report the first case of combined macular and optical nerve dysfonction. This case suggests that voriconazole can induce
- F014

Acquired trichomegaly and hypertrichosis after treatment with epidermal growth factor receptor inhibitors

LAUWERS N, AERTS L, SMETS RME

Ophthalmology UZA, Edegem

Purpose To describe a case of acquired trichomegaly and hypertrichosis after treatment with epidermal growth factor receptor inhibitors (EGFR TKI's).

Methods A case-report.

Results We describe the case of an 88-year old patient treated for metastatic lung arcinoma with EGFR TKI's. A few weeks after treatment with Afatinib, she presented with a painful squamous blepharitis. Her eyelashes were abnormally long and abundant. After trimming of the eyelashes, lid hygiene and topical lubricants the symptoms disappeared.

Conclusion EGFR TKI's are a new type of oral anti-tumoral agents with ocular side effects such as keratitis, conjunctivitis, blepharitis and trichomegaly. Symptomatic topical treatment can be successful. This case report has already be published. "Aerts L, Lauwers N, Smets RME. Trichomégalie et hypertrichose acquise. Journal francais d'Ophtalmologie 2014; 37: 339-341:

- F016

Characterisation of RPGRIP1 and its interacting partners in Zebrafish

KOTAPATI RAGHUPATHY R (1), MCCULLOCH D (1), CRAFT J (2), SHU X (2)

(1) Vision Sciences, Glasgow Caledonian University, Glasgow

(2) Biological Sciences, Glasgow Caledonian University, Glasgow

Purpose Mutations in the gene RPGRIP1 causes LCA, most severe form of retinal dystrophy. Several studies using animal models demonstrate RPGRIP1 show species specific expression and different isoforms of the gene have subcellular specific localisation. RPGRIP1 is mainly localised in the connecting cilium and interacts with RPGR, RPGRIP1L, but the exact function of the RPGRIP1 is still unclear

Methods We have carried out gene expression pattern analysis at various stages of development and different adult tissues of zebrafish using PCR and SYBR green based QPCR. We have also carried promoter analysis of human and zebrafish RPGRIP1 to understand mechanism regulating the transcription of these genes in retina using dual luciferase reporter assay

Results The expression pattern analysis of ZRPGR demonstrates ZRPGR2ORF15 and ZRPGR2Ex1-17 have different expression pattern in the tissues and the developmental stages. ZRPGR2ORF15 is highly expressed in the eye and brain compared to the expression of ZRPGR2Ex1-17. Both ZRPGRIP1 and ZRPGRIP1L show higher expression in the eye and ZRPGRIP1Like is highly expressed during the early stages of the development. The binding sites of key transcription binding factors such as CRX, NRL, PAX6 were identified in the 3Kb region upstream of the human RPGRIP1startcoding site, and a 288bp fragment upstream of transcription start site directed higher luciferase activity in the RPE-1 cells.

Conclusion In this study we found that ZRPGRIP1 is highly expressed in the eye and in the early stages of development than that of its interacting partners ZRPGR and ZRPGRIP1L. 288bp promoter region upstream of human RPGRIP1 transcription start site showed high luciferase activity and supposed to be the core promoter of RPGRIP1. 
- F017

The embryonic stem cell derived retinal pigment epithelial cell trial for Stargardt macular dystrophy: preliminary phase 1 results in Asian

KWONHJ (1), SONG W (1), CHOIJ (2), CHUNGSY (3), KIM HJ (4), LEEJH (4)

(1) Ophthalmology, Gyunggido

(2) Rheumatology, Gyunggido

(3) Hemato-oncology, Gyunggido

(4) Clinical Research/Development Division, Seoul

Purpose To report interim results of Stargardt macular dystrophy(SMD) patients who underwent human embryonic stem cell derived retinal pigment epithelial cells(hES RPE) transplantation

Methods hESC-RPE cells were manufactured in a fully validated good-manufacturingpractice facility. Total pars plana vitrectomy with induction of posterior vitreous detachment was performed in the eyes with the worst vision. A volume of $150 \mu \mathrm{L}$ of RPE reconstituted in BSS Plus with the target dose of 50000 RPE cells was injected into the subretinal space. Immunosuppression was started at lower dosages of tacrolimus and mycophenolate mofetil. Ophthalmologic examinations; ETDRS visual acuity(VA), visual field examination(VF), fluorescein angiography, spectral-domain OCT, and electroretinography were performed.

Results A 45-year-old male SMD patient with initial VA of 1 letter(Counting fingers) had improved to 13 letters $(20 / 640)$ and smaller central scotoma observed on goldman VF at 52 weeks. A 40-year-old male SMD patient with initial VA of 13 letters $(20 / 640$ improved to 30 letters $(20 / 200)$ at 26 weeks. Subretinal pigmentations with multiple increased autofluorescence spots were noted. A 40-year-old male SMD patient with initial VA of 41 letters $(20 / 160)$ has stable VA of 41 letters $(20 / 160)$ at 4 weeks. Subretinal pigmentations around injection site were noted at 4 weeks.

Conclusion Two of three SMD patients who received the target dose and relevant immunosuppression showed gradual improvements of visual acuity only in the study eye. No serious systemic or ocular adverse events occurred. Longer follow up and further randomized studies with a control group are needed to determine the safety, tolerability, efficacy.

\section{- F019}

\section{Tamoxifen retinopathy}

HALFELD FURTADO DE MENDONCA R (1), LUZ LEITÃO GUERRA R (2),

DE OLIVEIRA MAIA JR O (2), YUKIHIKO TAKAHASHI W (3)

(1) University of Juiz de Fora, Juiz de Fora

(2) Monte Tabor Foundation. São Rafael Hospital, Salvador

(3) University of Sáo Paulo, São Paulo

Purpose To report a rare and interesting case of retinopathy secondary to tamoxifen therapy. Setting: Tamoxifen is a selective estrogen receptor modulator widely used in the treatment of hormone-responsive breast cancer.

Methods A 38-year-old white woman underwent right breast cancer quadrantectomy with axillary lymph node dissection. She was started on long term tamoxifen $(20 \mathrm{mg}$ daily).

Results After a total dose of $29.2 \mathrm{~g}$ of Tamoxifen over a period of 48 months, she complained of blurred vision. Her corrected visual acuity was 0.3 in the right eye and 0.1 in the left eye. The retinography showed yellowish-white deposits, more heavily concentrated in the inferior and temporal macular area. A greyish alteration following the vascular arcade with some pigment changes were detected. Fundus autofluorescence showed bilateral paramacular rings of hypofluorescence and a central increased autofluorescence in the macula (punctate spots with bright autofluorescence signal) which was not clearly visible on fundus photography. Many hypofluorescence areas following the vascular arcade were detected. Retinopathy was diagnosed and tamoxifen was discontinued.

Conclusion Patients treated with tamoxifen who complain of visual problems should be immediately referred to an ophthalmological examination. In some cases, the retinopathy is not limited to the macula.
- F018

SD OCT and fundus auto fluorescence findings in Stargardt disease

EL MATRI L, CHEBIL A, KORT F, CHARFI H, MAMOURI R, HSAIRI A

Institute of Ophthalmology, Tunis

Purpose To analyze macular morphology with SD OCT and Fundus Auto Fluorescence (FAF) in patients with Stargardt disease (STGD) and to correlate between visual acuity (VA) and macular patterns.

Methods Forty eyes with STGD phenotypes, underwent FAF and SD-OCT imaging. The levels and the distribution of FAF and atrophic lesions were evaluated.

Results Patients were separated in 2 groups: $\mathrm{BCVA} \geq 0.05$ and $\mathrm{BCVA}<0.05$.We found 5 patterns in FAF: Homogeneous high AF of the entire retina; Heterogeneous high and low retinal AF with low central AF; Numerous small areas of atrophy in heterogeneous low AF; Large an isolated central retinal atrophy; Central retinal atrophy without foveolar involvement. Six patterns were found in SD-OCT: Normal foveal depression, regular photoreceptor IS/OS junction with hyper reflectivity of outer nuclear laver; subnormal foveal depression, foveal disorganization and/or loss of IS/OS junction; Absence of foveal depression, loss of the IS/OS junction and the photoreceptors layer; Macular hole aspect; large retinal atrophy with macular layers disorganization; Pseudocoloboma image. Foveal thickness was significantly reduced in all patients (Mean $=107.92 \mu \mathrm{m}$ ) excepted in group I with subclinical STGD. There was a statistically significant correlation between FAF pattern and SD-OCT pattern $(\mathrm{p}=0.03)$ and between BCVA and SD-OCT pattern $(\mathrm{p}=0.04)$

Conclusion Both SD-OCT and FAF are useful in the management of STGD. Correlation between structure and function should be useful in guiding selection of patients for future therapeutic.

\section{- F020}

Ocular manifestations in a patient with osteopetrosis

KABANAROUS (1), MOSCHONASK (1), PAPAPANOS P (2), KOUROUPAKIA (1) TSAKIRIS K (1), XIROU T(1)

(1) Retina Department, Red Cross Hospital, Athens

(2) Ophthalmology Department, General Hospital, Corinth

Purpose To report a case of osteopetrosis, a rare genetic disorder, with ocular manifestations

Methods A 45 year old male was presented with a history of progressive visual loss. There was a past medical history of osteopetrosis. He underwent a full ophthalmic examination and best corrected visual acuity (BCVA) was recorded. Subsequently, visual field test, OCT and international-standard electrophysiological (ISCEV) evaluation including pattern and full-field electroretinography (PERG and FFERG), visual evoked potentials (PVEP), were performed. Genetic evaluation and counselling were scheduled.

Results BCVA was 0.8 in the right and hand movements in the left eye. Anterior segment examination was normal. Fundoscopy revealed disc pallor in the right eye and optic disc atrophy in the left eve. There was a visual field defect in both eyes, mainly in the left one. OCT-RNFL thickness was found borderline in the right and reduced in the left eye. The P100 component of PVEP demonstrated latency delay in the right eye and reduced amplitude in the left eye, indicative of optic nerve dysfunction. The N95 component of PERG was also affected. Full field ERG for both scotopic and photopic responses was borderline in the right eye and subnormal in the left eye, indicating mild retinal dysfunction. Genetic testing showed mutations in the CLCN7 gene.

Conclusion In osteopetrosis there is a dysfunction in or lack of osteoclasts resulting in an increase in bone mass. Visual loss occurs because of optic nerve compression, and more rarely retinal dysfunction. 


\section{- F021}

Bilateral macular coloboma of sorsby: contribution of the oct

BELAHDA A, MATONTI F, RENDU I, DENIS D

Hopital Nord, Marseille

Purpose To assess the contribution of the Optical Coherence Tomography (OCT) for the diagnosis of macular coloboma.

Methods We report the case of a 33-year-old patient affected by a bilateral macular coloboma of Sorsby. The medical check-up was negative. Eye fundus imaging, OCT and fluorescein angiography were performed.

Results Eye fundus imaging found a deep macular defect putting the sclera to naked, pigmented, well bounded, with size about one papilla diameter in both eyes The fluorescein retinal angiography revealed a hypofluorescent macular lesion with a bigger size than the one visible at the eye fundus. The lesion was fulfilled with fluorescein in a heterogenous way during the angiographic sequence because of atrophic damage of the pigmentary epithelium and the infringement of the surrounding choroid layer area. The OCT showed in the crater shape a depression regarding the lesion with atrophy of the retinas neurosensory the major damage of the pigmentary epithelium, the choroï and scleral infringement regarding the lesion. We observed more marked deficits in the scleral and the choroïdal layers than in the neurosensory retina.However, changes within the retina were also visible in the form of microcysts and of disintegration of the retinal stratification.

Conclusion In front of a crater macular pigmented lesion, the first diagnosis to think about is toxoplasmosis chorioretinitis, however you should not neglect a large number of other pathologies whithin the Sorsby coloboma because the scleral defect found in OCT is strongly suggestive.The OCT's aspect of the macular lesion allows us to diagnose with more precision a macular infringement and to confirm the diagnostic of macular coloboma.

\section{- F022}

Clinical presentation of a Roma family with autosomal recessive Usher Syndrome

KOEV K (1), CHERNINKOVA S (2), KAMENAROVA K (3), GEORGIEVR (4), KANEVA R (3)

(1) Department of Ophthalmology, Medical University - Sofia (Sofia),, Sofia (2) Department of Neurology, University Alexandrovska Hospital Sofia, Sofia (3) Molecular Medicine Center, Medical University - Sofia, Sofia

(4) Clinical Centre of Emergency Medicine, Medical University - Sofia, Sofia

Purpose Usher Syndrome is a genetic condition involving sensory neural hearing loss and retinitis pigmentosa (RP).RP is a heritable group of genetic diseases that is characterized by degeneration of the retinal pigment epithelium due to loss of photoreceptors, primary rods and secondary cones.

Methods We present 2 familial cases, brother (48 years old) and sister (45 years old), with Usher syndrome. According to family history data their grandmother (the mother of their father) had the same disease.

Results The visual acuity of the brother was pplc of both eyes, while the visual acuity of the sister was 0,1 of both eyes.Fundoscopically we observed osteoclast-like pigmentations in the posterior pole of the retina and in and around the macula lutea of both cases. In both cases we found narrowed arterioles and diffuse bilateral wax pallor of the papilla.The ERG of the both had micropotentials with lack of flicker response. The FA of both siblings - revealed dense osteoclast-like pigment in the posterior pole and the macula area. In the case of the sister through Kugel perimetry was established unnel vision to $10 \mathrm{o}$. Humphrey perimetry showed reduced retinal sensitivity in bilateral central and peripheral zone; concentric narrowing of the visual field only for the sister.In both of the cases audiogram showed high rates of sensory neural bilateral hearing decrease.

Conclusion The presented two cases of Usher syndrome are a significantly rarer form of RP with probable autosomal-recessive form of inheritance. Blood for DNA analysis was taken.

\section{- F024}

Comparison of efficacy between focal laser photocoagulation and photodynamic therapy in CSCR

POFAL Study: «Protection Oculaire Face aux Agressions Lasers", Phase I

EL CHEHAB H (1, 2, 3), BEMELMANS A (2), NOUVEL-JAILLARD C (3, 2 NIEPON ML (3), RENARD JP (1)

(1) Military Hospital Val de Grâce, Paris

(2) MIRCen Laboratory, Fontenay-Aux-Roses

(3) Vision Institute, Paris

Purpose Risk of laser beam exposure, particularly to laser "pointers" easily available on open sale or on internet increases every year. Many incidents and accidents have been reported over the last three years in France. The purpose of this study is to try to determine a retinal lesion threshold secondary to such exposures.

Methods A laser system $(532 \mathrm{~nm})$ with a power and duration control has been developed specifically for this study to achieve exposure. A first phase was performed in pigmented rodents with different combinations of time - power beam exposure Powers ranged from 0.5 to $4.5 \mathrm{~mW}$ and durations from 0.1 to $0.35 \mathrm{sec}$. A second phase was performed in non-human primates (NPH) with blink reflex time $(0.25 \mathrm{sec})$ and 1,2 , 3.5 and $4.5 \mathrm{~mW}$. Histological analysis of outer layers and immunoassay to assess tissular damage (GFAP) were performed to each phase.

Results A significant correlation between energy and photoreceptor thickness is found in rodents. Histological analysis did not find a structural alteration or combination threshold. Immunoassay confirm tissular damage in inner lavers.Photoreceptors alterations are located at foveal zone in NHP. Outer layers thickness is statistically lower when exposure power is greater than $1 \mathrm{~mW}$. A significant correlation between energy and photoreceptor thickness is also found only in foveal zone.

Conclusion This study reports systematic tissue damage when power exposures correspond to upper limits of the class II lasers $(1 \mathrm{~mW})$. This is the first in-vivo study analyzing exposure effects to low power laser beam or easily accessible. These initial results are used to guide and adapt research on protection systems for people exposed.
STRUPAITE R, KUOLIENE K, STRELKAUSKAITE E, ASOKLIS R

Vilnius University Centre of Eye diseases, Vilnius

Purpose To evaluate and compare clinical outcomes for patients with acute central serous chorioretinopathy (CSCR) undergoing either focal laser photocoagulation (LPK), photodynamic therapy (PDT) or just observation

Methods In a retrospective, comparative case series study 73 patients (73 eyes) with acute central serous chorioretinopathy of at least 3 months' duration were recruited. Out of 73 patients 51 were treated with LPK, 10 with half dose PDT and 12 patients have been in the observation group. Patients follow-ups were conducted every one to two months until complete subretinal fluid resorbtion on OCT was achieved. The main outcome measures were change in BCVA by Snellen and subretinal fluid (SRF) assessed by OCT (Zeiss Stratus OCT)

Results The mean age of the patients was $47.76 \pm 8.43$ years (range 24-65). 55 of 73 patients were male, 16 were female. The mean BCVA (by Snellen) at baseline was $0.81 \pm 0.23$ in LPK group, $0.69 \pm 0.24$ in PDT group and $0.88 \pm 0.15$ in the non-treated group. At the last visit, comparing BCVA between LPK and PDT, LPK and non-treated group and PDT and non-treated group - there was no statistically significant differences between these groups, $\mathrm{p}<0.289, \mathrm{p}<0.089$ and $\mathrm{p}<0.894$ respectively. Comparing the central retinal thickness outcomes by OCT, there was also no statistically significant difference between these groups: LPK and PDT ( $<<0.387$ ), LPK and non-treated group $(\mathrm{p}<0.371)$, and PDT and non-treated group $(\mathrm{p}<0.246)$.

Conclusion Our study did not comfirm that any of the used therapy options is superior to another. At the final visit after treatment no statistically significant difference in anatomical and functional recovery was noted between the two modalities of treatment and observation. 


\section{- F025}

Intravitreal Bevacizumab in treatment of chronic central serous chorioretinopathy: the efficacy according to indocyanine green angiography findings

KU HC, RHIM WI, LEE MK, LEE EK

Kong Eye Hospital, Seoul

Purpose To evaluate the efficacy of bebacizumab according to baseline indocyanine green angiography (ICGA) findings for treatment of chronic central serous chorioretinopathy (CSC).

Methods Twenty-two eyes of 22 patients with chronic CSC and symptoms for at least 3 months were treated with intravitreal injections of $1.25 \mathrm{mg}(0.05 \mathrm{ml})$ bevacizumab. The mean follow-up was 12.5 months. The degrees of hyperfluorescence of ICGA included intense, weak, or no hyperfluorescence. Main outcomes measures were the resolution of the subretinal fluid and visual acuity.

Results On the basis of ICGA, intense hyperfluorescence was found in 5 eyes (23\%), diffusely weak hyperfluorescence in 6 eyes (27\%), focally weak hyperfluorescence in 7 eyes $(32 \%)$ and no hyperfluorescence in 4 eyes $(18 \%)$. The complete resolution rate of the subretinal fluid was higher in eyes with focally weak and no hyperfluorescence (4 eyes $(57 \%)$ and 2 eyes $(50 \%))$ than in eyes with intense and diffuse weak hyperfluorescence ( 1 eye $(20 \%)$ and 2 eyes $(33 \%))$.Visual acuty was increased in eyes with complete ( $\mathrm{n}=9$, $0.20)$ and partial $(n=5,0.11)$ resolution of the subretinal fluid. However, in eyes without response, visual acuity was rather decreased $(n=8,-0.11)$.

Conclusion The efficacy of bebacizumab in eyes with chronic CSC was heterogeneous according to baseline ICGA findings and seemed to be higher in eyes with lower choroidal permeability on ICGA.

\section{- F027 \\ Retinal nerve fibre layer thickness measurements in myopia by optical coherence tomography}

MORREALE BUBELLA R, MORREALE BUBELLA D

Department of Experimental Biomedicine and Clinical Neuroscience (BioNeC)

University of Palermo, Palermo

Purpose Our study aimed to evaluate through OCT, in a homogeneous group of subjects with various degrees of myopia, the macular thickness and that of the RNFL with the purpose of highlighting the possible presence of a correlation between their impairment and the degree of myopia.

Methods 83 students of the Faculty of Medicine and Surgery of the University of Palermo were considered, distinguished into 4 groups, 3 of them according to the degree of myopia and the last group (control group) was made of emmetropic subjects. Each subject was submitted to a determination of visual acuity and refractive defect and evaluation of the thickness of the macular fibres and of the retinal nerve fibre layer (RNFL) through OCT (Stratus Oct 3000 - Carl Zeiss Meditec inc.).

Results With a growth in axial length there was a reduction in the average macular thickness in all quadrants of the parafovea with the exception of the superior one. By contrast, in the foveola and fovea there was an increase in thickness with a rise in the refractive defect. The lowest thickness was found in the emmetropic subject.

Conclusion The data found therefore allow us to conclude, in agreement with others that the progressive increase in axial length in myopic subjects involves reshaping of the arrangement of the nerve fibres which needs to be taken into account, especially in subjects with glaucoma that are also very myopic.

\section{- F026}

Improved analysis of the outer foveal microstructure - OCT imaging of healthy and abnormal retina

SJÖSTRAND J (1), NILSSONM (2), ROSÉN R (2), POPOVIC Z (1)

(1) Department of Ophthalmology, University of Gothenburg, Gothenburg

(2) Unit of Optometry, Department of Clinical Neuroscience, Karolinska Institutet, Stockholm

Purpose To improve visualization and segmentation of the foveal photoreceptors from the level of outer segments to the synapse layer in the inner portion of the outer plexiform layerFurthermore, to create a normative model of the topography of the outer nuclear layer (ONL) with cone and rod cell bodies and the Henle fiber layer (HFL) with the axons radiating out from the foveal center (FC).An improved separation of ONL and HFL is clinically relevant for the understanding of microstructural changes in abnormal retina.

Methods Selected eyes from 14 normals and 5 young adults with a history of prematurity were imaged using a commercial spectral domain optical coherence tomography (SD-OCT) system. Centered and displaced SD-OCT entrance beam positions were used to obtain straight and tilted scans, respectively. Horizontal scans through FC with a distinct light reflex were selected for analysis.Straight and tilted SDOCT images were flattened to the RPE layer prior to registration and averaging. Retinal layer thickness was measured manually at $\mathrm{FC}$ and three lateral positions along the temporal and nasal hemi-meridians.

Results We found a close correspondence between layer thickness measurements at the same lateral positions for both straight and tilted scans. However, a discrepancy was found for the tilt-up paracentral position for tilted scans, where the foveal slope appeared flatter and the thickness of inner retinal layers was lower than in the straight and tilt-down positions.

Conclusion Improved visualization of HFL/ONL was confirmed in tilted images. Image flattening and registration simplifies measurement of outer foveal layers in normal and abnormal retina.

\section{- F028}

\section{Macular Bruch's membrane defects in $\mathrm{Hi}$}

JONAS J (1), YOU Q (2), PENG XY (2), XU L (2), WEI WB (3), WANG Y (2), CHENCX (2)

(1) Department of Ophthalmology, Medical Faculty Mannheim of the Ruprecht-KarlsUniversity of Heidelberg, Mannheim

(2) Beijing Institute of Ophthalmology, Beijing

(3) Tongren Hospital, Beijing

Purpose To examine prevalence and associations of macular Bruch's membrane defects (MBMDs) in a population-based setting.

Methods The Beijing Eye Study 2011 included 3468 subjects with an age of 50+ years. MBMDs were defined as an interruption of Bruch's membrane on the OCT images in the macular region.

Results MBMDs could be detected only in highly myopic eyes ( $\leq-6$ diopters or axial length of $\geq 26.5 \mathrm{~mm}$ ). Prevalence of MBMDs in the highly myopic group was $17 / 164$ $(10.4 \pm 2.4 \%)$. Mean axial length of eyes with MBMD was $29.6 \pm 0.3 \mathrm{~mm}$. Best corrected visual acuity of $<0.05$ was found in $5(29 \%)$ out of 17 eyes with MBMDs, and in $7(41 \%)$ eyes, visual acuity was $<0.3$ and $\geq 0.05$. In the MBMD region, RPE and choriocapillaris were completely lost, and the deep and middle layers of the retina and the choroid were almost completely absent. In the MBMD region, mean retinal thickness was $153 \pm 57 \mu \mathrm{m}$

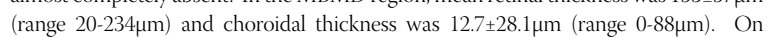
the fundus photographs, MBMDs appeared as whitish areas with round borders and without signs of RPE and choroid except of large choroidal vessels. In multivariate regression analysis, presence of MBMD was significantly associated only with longer axial length ( $\mathrm{P}=0.002$; Odds ratio: $3.87 ; 95 \%$ confidence interval: $1.64,9.14)$.

Conclusion MBMDs with a prevalence of about $10 \%$ in highly myopic eyes are associated with a complete loss of RPE and choriocapillaris, an almost complete loss of photoreceptors and choroid, and marked reduction in visual acuity. Characterized by a whitish fundus lesion with round borders, MBMDs can be differentiated from a chorioretinal atrophic lesion with Bruch's membrane present. MBMDs may be added to the panoply of features of myopic maculopathy. 
- F029

The relation between peripapillary and macular thickness with refractive errors

OSTADIMOGHADDAM H (1), AHMADI HOSSEINI M (1), YEKTA AA (1), SEDAGHAT M (2), AZIMI A (1), MOHAMMADIANM (1) NAKHIANPOURN (1) FAZILATI F (1)

(1) Optometry, Mashhad

(2) Ophthalmologhy, Mashhad

Purpose To determine the correlation of the peripapillary retinal nerve fiber layer thickness (RNFLT) and macular thickness (MT) with type and degree of refractive errors.

Methods Seventy-four (37 myopic and 37 hyperopic) subjects with the mean age of $26.47 \pm 0.75$ years were participated in this study. Visual acuity (VA), Refractive errors $(\mathrm{RE})$, axial length $(\mathrm{AL})$, Intra ocular pressure (IOP) and corneal power $(\mathrm{CP})$ were determined. RNFLT were measured by cirrus OCT using circular scan with diameter $3.4 \mathrm{~mm}$ around optic disc, and MT was captured using radial scan in a $200 \times 200 \mathrm{~mm}$ area, Independent student T-test was performed to compare the CP, RNFLT, MT and AL between groups. The correlation of the AL with RNFLT and MT were assessed using the Pearson correlation.

Results There was a significant difference in AL between myopic and hyperopic eyes $(\mathrm{p}<0.05)$. In myopic eyes, the AL significantly correlated with RNFLT $(\mathrm{p}<0.001)$ and MT $(\mathrm{p}<0.05)$. Notwithstanding, axial length had no significant correlations with RNFLT and MT in hyperopic eyes. Also there is statistically significant relationship between MT and RNFLT with degree of the refractive errors in myopic eyes $(\mathrm{p}<0.05)$.

Conclusion The results of this investigation indicated that myopes have thinner RNFLT and MT than hyperopes. These results could help clinicians in diagnosis and management of the patients with glaucoma.

- F031

Age-related changes of ocular parameters in an emmetropic Spanish healthy population

ZABADANI K, ASCASO FJ, HERRERA L, ESTEBANO, MARTINEZ M CARAMELLO C, PEREZ NAVARRO I, ALMENARA C, CASAS P, CRUZ N CABEZON L, CRISTOBALJ

Ophthalmology, Zaragoza

Purpose To evaluate the age-related variations of ocular parameters in an emmetropic Spanish healthy population.

Methods We investigated 117 eyes of 117 healthy and emmetropic subjects. All patients were evaluated in the Department of Ophthalmology at the "Lozano Blesa" University Clinic Hospital, Zaragoza, Spain, between January and October 2013. Axial length (AL), refractive error (RE), subfoveal choroidal thickness (SCT), foveal thickness (FT) and foveal volume (FV) were measured by using auto-refractive keratometer optical biometer and spectral domain optical coherence tomography (SD-OCT), respectively

Results The mean RE was $0.09 \pm 0.97$ diopters (range, from -1.5 to +1.5 diopters); the mean AL was $23.08+0.90 \mathrm{~mm}$ (range, 20.9-24.5 mm); the mean SCT was $278.9 \pm 113.2$ $\mu \mathrm{m}$ (range, $57-527 \mu \mathrm{m}$ ), the mean FT was $266.6 \pm 21.1 \mu \mathrm{m}$ (range, $210-342 \mu \mathrm{m}$ ) and the mean FV was $0.20 \pm 0.01 \mathrm{~mm} 3$ (range, $0.17-0.27 \mathrm{~mm} 3$ ). In correlation analysis, from 20 to 89 years, subjects showed a positive and statistically significant correlation between age and $\operatorname{RE}(\mathrm{r}=0.262, \mathrm{p}=0.004)$, a negative and statistically significant correlation between age an AL $(r=-0.198, p=0.045)$ and a negative correlation between age and SCT $(r=-$ $0.468, \mathrm{p}<0.001)$. There were no significant correlations between age and FT $(\mathrm{r}=0.006$ $\mathrm{p}=0.972)$, and between age and $\mathrm{FV}(\mathrm{r}=0.046, \mathrm{p}=0.773)$

Conclusion SCT and AL decreased with aging RE increased with age. No correlation was found between age and either FT or FV.

\section{- F030 / 1666}

Macular response to a carotenoid supplement in healthy eyes

MARQUINA I, BRAUTASET R, NILSSON M

Karolinska Institutet, Unit of Optometry, Stockholm

Purpose Reduced macular pigment (MP) density is related to an increased risk of developing age-related macular degeneration (AMD). The aim of this study was to measure the macular response to a MP supplement by measuring macular pigment optical density (MPOD) before and after consumption.

Methods Fifty-one healthy subjects (age 18-62 years) participated in the study and were divided in two groups, one control group and one supplementary group. MPOD values were measured using heterochromatic flicker photometry (HFP) at baseline and after 90 days of supplementation with the dietary supplement MacuShield ${ }^{\circ}(10 \mathrm{mg}$ lutein, $10 \mathrm{mg}$ meso-zeaxanthin and $2 \mathrm{mg}$ zeaxanthin).

Results Mean MPOD values did not change significantly in any of the groups. A correlation between low MPOD values at baseline and a larger increase of MPOD values after supplementation was found in the supplementary group $(r=-0.52, p=$ 0.008). Increased MPOD values also correlated with increasing age $(r=0.40, p=0.0454)$.

Conclusion The results of the present study indicate that older subjects with low MPOD values increase their MPOD values after supplementation. As increased age and low MPOD values increase the risk of developing AMD, it might be beneficial for these individuals to consume macular pigments to reduce the risk of developing AMD.

\section{- F032}

Unilateral peripapillary intrachoroidal cavitation and optic disc rotation

DAI Y (1), JONAS J (2), LINGZ (1), SUN X (1)

(1) Ophthalmology, Eye \& ENT Hospital, Fudan University, Shanghai

(2) Medical Faculty Mannheim of the Ruprecht-Karls-University, Heidelberg

Purpose To examine the morphology of the optic nerve head in patients with unilateral peripapillary intrachoroidal cavitations (PICCs).

Methods The hospital-based observational study included patients with unilateral PICCs. Tomographic images of the parapapillary fundus were taken by enhanced depth imaging mode of optical coherent tomography. The ocular biometric parameters were compared between the affected eyes and the contralateral unaffected eyes.

Results The study population consisted of 30 patients with a mean age of $42.7 \pm 13.8$ years (range:22-72 years), mean axial length of $26.7 \pm 2.4 \mathrm{~mm}$ (range: $22.00-32.30 \mathrm{~mm}$ ) and mean refractive error of $-8.71+5.21$ diopters (range: -20.50 diopters to +0.50 diopters). In the eyes affected by PICC as compared with the contralateral eyes, the vertical optic disc diameter $(\mathrm{P}=0.001)$ and the minimal disc diameter $(\mathrm{P}=0.03)$ were significantly shorter, the ratio of minimal to maximal disc diameter was significantly lower $(\mathrm{P}=0.02)$, and the angle of disc rotation was significantly higher $(\mathrm{P}<0.001)$.

Conclusion In patients with unilateral PICCS, the eyes with PICCs as compared with the contralateral eyes have optic discs which are more spindle-like configured due to a disc rotation around the vertical axis and around the sagittal axis. In association with the myopic axial elongation in PICC eyes, the findings may suggest that PICCs are associated with an external traction of the optic nerve at its adhesion at the optic nerve head, leading to a rotation of the optic disc into the temporal inferior direction and simultaneously leading to an increased distance between the parapapillary sclera and parapapillary Bruch’s membrane. 
- F033

Performance characteristics of multicolor versus blue light and infrared imaging in the identification of reticular pseudodrusen

AL MUHTASEB H (1, 2), BADAL J (1), BIARNÉS M (1), MONÉS J (1, 2)

(1) Institut de la Màcula i de la Retina, Barcelona

(2) Barcelona Macula Foundation: Research for Vision, Barcelona

Purpose To describe the appearance of reticular pseudodrusen (RPD) on multicolor imaging $(\mathrm{MC})$ and to evaluate its diagnostic accuracy as compared with the two modalities considered the current reference standard, blue light (BL) and infrared (IR).

Methods A retrospective study reviewing all multicolor images of a series of consecutive patients. Inclusion criteria involved the presence of $>1$ RPD on a $30^{\circ} \times 30^{\circ}$ image centered on the fovea as seen with the BL channel derived from the multicolor imaging of the Spectralis HRA+OCT * The diagnosis of RPD was confirmed by the identification of subretinal debris on a tracking-assisted SD OCT Heidelberg. A 3.0 mm diameter circle divided in 4 fields ( $, \mathrm{N}, \mathrm{I}$ and $\mathrm{T}$ ) was overlaid on each image and manually centered on the foveola. Three experienced observers, masked to their own results with other imaging modalities, independently classified the number of RPD in each field in each image with each modality, according to this classification: Category 0 0-5 RPD ; $1: 6-20 ; 2: 21-35 ; 3: 36-49$; and 4:>50. Friedman and Wilcoxon tests were used for the comparison between different imaging modalities per field by the same observer. The kappa (K) coefficient was used to measure interobserver agreement

Results MC and IR modalities showed higher sensitivity and interobserver agreement in RPD detection than BL. No clinically significant differences were found between multicolor and IR.

Conclusion Multicolor imaging modality (the new index test) can play an important role in the identification, quantification and categorization of RPD when compared to the reference standard test (images of BL and IR), a result which is still the subject of much debate.

\section{- F035 \\ The comparison of OCT findings and retinal artery diameter in good and poor prognosis RAO patients}

PARKJ (1), JOS (1), LEES (2)

(1) Department of Ophthalmology, Maryknoll medical center, Busan

(2) Department of Ophthalmology, Haeundae Paik Hospital, Inje University College of Medicine, Busan

Purpose To determine the different clinical findings between good and poor prognosis group which is diagnosed with branch retinal artery obstruction(BRAO) and treated with conservative care. We evaluate the average macular thickness, foveal thickness, outer nuclear layer $(\mathrm{ONL})$ on optical coherence tomography $(\mathrm{OCT})$ and retinal artery diameter in both groups

Methods 9 eyes ( 9 patients) in patients with non-complicated BRAO with good prognosis and a poor prognosis control group with non-complicated BRAO of 11 eyes(11 patients) were used in this study. The average macular thickness, foveal thickness and ONL thickness at the center of fovea on OCT were measured. And branch retinal artery widths were measured by a semi-automated retinal vessel width measurement system retrospectively.

Results The average age of the patients was $67.3 \pm 11.5$ years. The average ONL thickness at the central fovea of the good prognosis group was significantly thicker than that of the control group $(\mathrm{p}=0.016)$. There were no statistically significant result at average macular thickness and foveal thickness. In good prognosis group, Ischemic retinal artery diameter and Central retinal artery equivalent(CRAE) were wider than those of poor prognosis group and they were statistically significant $(\mathrm{p}=0.028, \mathrm{p}=0.01)$.

Conclusion In the patients diagnosed with BRAO and treated with conservative care, foveal thickness, ischemic retinal artery diameter and CRAE were statistically significant between good and poor prognosis groups. In the patients diagnosed with BRAO, foveal thickness and retinal artery diameter could be prognostic factors that predict visual prognosis.
- F034

Retinal vessel course and retinal nerve fiber bundle trajectories in the human eye

JANSONIUS NM (1, 2), QIU K (1), SCHIEFERJ (3), NEVALAINEN J (4), PAETZOLD I (5), SCHIEFER U (5)

(1) Ophthalmology, University of Groningen, University Medical Center Groningen, Groningen

(2) Epidemiology, ErasmusMC, Rotterdam

(3) University Eye Hospital, Ludwig Maximilians University, Munich

(4) University Eye Hospital, Oulu

(5) Centre for Ophthalmology, University of Tuebingen, Tuebingen

Purpose To determine the relationship between the retinal vessel course and the retinal nerve fiber bundle (RNFB) trajectories in the human retina.

Methods High-quality fundus photographs were used, comprising 28 eyes of 28 subjects (Exp Eve Res 2012). Mean (range) refraction was $-0.4(-8.75$ to +6.25$)$ D. In total 625 RNFB trajectories were traced. For all individual trajectories, the departure from the previously published RNFB trajectory model (Vision Res 2009) was calculated. Subsequently, we calculated, per subject, a 'mean departure' for the superior-temporal and inferior-temporal regions. To quantify the retinal vessel course, we measured the angles between a line connecting the centers of the fovea and the $\mathrm{ONH}$ and a line connecting the center of the $\mathrm{ONH}$ and the crossing of the superior or inferior temporal artery or vene with a circle around the $\mathrm{ONH}$; circle radius was half the fovea-ONH distance. This yielded four angles: AAS, AVS, AAI, and AVI. Multiple linear regression analysis was performed for the superior-temporal and inferior-temporal regions separately, with mean departure as dependent variable and refraction, $\mathrm{ONH}$ inclination, and the vessel angles as independent variables.

Results In the superior-temporal region, refraction $(\mathrm{P}=0.017)$. $\mathrm{ONH}$ inclination $(\mathrm{P}=0.021)$, and $\mathrm{AAS}(\mathrm{P}<0.001)$ were significantly associated with mean departure. The explained variance was 0.54 . In the inferior-temporal region, AAI $(\mathrm{P}=0.037)$ and AVI $(\mathrm{P}=0.021)$ were significantly associated with mean departure. The explained variance was 0.30

Conclusion The wiring of the human retina displays a considerable variability. The angles at which the vessels leave the $\mathrm{ONH}$ explain an important part of this variability.

\section{- F036}

Cilioretinal artery occlusion and protein $\mathrm{S}$ deficiency in pregnancy

CARAMELLOC (1), MATEO OROBIA AJ (2), HERRERA L (1),

ZABADANI K (1), PINILLA I (1), PEREZ NAVARRO I (1),

ALMENARA C (1), ESTEBANO (1), ASCASOJ (1), CASAS P (1)

(1) HCU Lozano Blesa, Zaragoza

(2) Hospital Universitario Miguel Servet, Zaragoza

Purpose Protein S (PS) is a vitamin K-dependent plasma protein that has antithrombotic effects. Congenital deficiency of PS is a relatively rare disorder characterized clinically by thrombosis in young people. Cilioretinal arteries are present in about $20 \%$ of individuals, supplying a variable territory of the inner retina and macula. To our knowledge, only three cases of branch retinal artery occlusion associated with PS deficiency have been reported.

Methods We report the case of a 25 year-old woman, 38 weeks pregnant, who developed cilioretinal artery branch occlusion in her left eye and who was found to have a PS deficiency. Her best corrected visual acuity (BCVA) was 20/20 in the right eye and 20/30 in the left one. Fundoscopic examination showed an area of retinal pallor located on the posterior pole. Visual field study demonstrated a paracentral scotoma. Spectral-domain optical coherence tomography (SD-OCT) showed retinal atrophy in the cilioretinal artery irrigation area. Retinal Nerve Fiber Layer thickness was decrease in the papillomacular bundle.

Results Blood analysis demonstrated that PS values recovered after childbirth. The BCVA was 20/20 in both eyes and the funduscopic exploration returned to normal

Conclusion When cilioretinal occlusion occurs, the extent of visual loss depends on the precise area of macular supply. Protein S defiency is another factor that should be considered in young patients with unexplained retinal vascular occlusion, particularly if it occurs during pregnancy. 
- F037

Comparison of subfoveal choroidal thickness changes following two different doses of intravitreal bevacizumab therapy for branch retinal vein occlusion

LEES

ophthalmology, Dongguk University, Gyeong-Ju

Purpose To investigate the effects of two different doses of intravitreal bevacizumab on subfoveal choroidal thickness(SFChT) in patients with branched retinal vein occlusion.

Methods An interventional, retrospective study of patients that were treated with intravitreal bevacizumab injection and had completed 12 months of follow-up. The study included 16 eyes (16 patients) in the group $1(1.25 \mathrm{mg}$ dose bevacizumb) and 15 eyes (15 patients) in the group 2 ( $2.5 \mathrm{mg}$ dose bevacizumab). Complete ophthalmic examination, fluorescein angiography, and enhanced depth imaging from spectral domain optical coherence tomography(SD-OCT) were performed at baseline, and during the follow-up visits.

Results In the group 1, the mean central retinal thickness(CRT) decreased from $553.0 \pm 199.8 \mu \mathrm{m}$ before, $277.0 \pm 56.5 \mu \mathrm{m}$ at 6 months, and $287.1 \pm 40.3 \mu \mathrm{m}$ at 12 months $(\mathrm{P}<0.05)$. The average BCVA were $0.90 \pm 0.66,0.23 \pm 0.13$, and $0.20 \pm 0.16$ $\log M A R$, respectively. In the group 2, the mean CRT decreased from $515.0 \pm 129.3 \mu \mathrm{m}$ before, and $286.5+85.2 \mu \mathrm{m}$ at 6 months, and $309.6+69.3 \mu \mathrm{m}$ at 12 months $(\mathrm{P}<0.05)$. The average BCVA were $0.50 \pm 0.22,0.30 \pm 0.15$, and $0.26 \pm 0.14 \log \mathrm{MAR}$, respectively. The SFChT decreased from $269.0 \mu \mathrm{m}$ (165-431) to $259.5 \mu \mathrm{m}$ (149-413) in the group 1 and from $322.6 \mu \mathrm{m}$ (212-483) to $315.1 \mu \mathrm{m}$ (199-514) in the group 2. There was no significant difference regarding the CRT change and SFChT between groups $(\mathrm{p}=0.48, \mathrm{p}=0.22)$.

Conclusion The SFChT did not change significantly after intravitreal bevacizumab injection in two groups. There were no statistically significant differences between the two dose groups with regard to CRT, SFChT, and change in visual acuity.

- F039

Follow-up of IOP and central macular thickness after repeated dexamethasone implants in retinal vein occlusion, about 29 cases

VIE A, DOTC

Rhones, Lyon

Purpose To investigate the impact of intravitreal dexamethasone implant (ozurdex) on intraocular pressure and on central macular thickness in eyes with macular oedema secondary to retinal vein occlusion.

Methods Twenty-nine patients diagnosed with macular oedema due to retinal vein occlusion (11/29 branch RVO-18/29 central RVO) and treated with Ozurdex were enrolled in this prospective uncontrolled study. Patients were followed up at 1, 2, 3, 4 and 6 months for IOP (air pulsed tonometer) and central macular thickness (CMT, spectralis $\mathrm{SD}-\mathrm{OCT})$.

Results The mean ozurdex injections was 2,4 (1-5). Most of the patients increased their IOP less than $6 \mathrm{mmHg}$ from baseline and were considered as low responder. $27 \%$ of patients were « medium responder » and increased their IOP from +6 to $15 \mathrm{mmHg}$ Only one was a high responder $(+19 \mathrm{mmHg})$. OHT occurred during the first $(12,5 \%)$ or the second month (24\%). Two patients has developed OHT only at the second injection $(24 \mathrm{mmHg})$. thirty-one \% of patients need a topical hypotensive therapy.The mean initial CMT was $579 \mu \mathrm{m}(+/-162 \mu \mathrm{m})$. The mean CMT reduction between M0 and M2 was $282 \mu \mathrm{m}+/-204 \mu \mathrm{m}$ for the first injection. The anatomic effect was reproducible for the second and the third ozurdex but decreased at the fourth injection $(-196 \mu \mathrm{m})$. Only two patients had a chronic retinal oedema after two injections.

Conclusion $\mathrm{OHT}$ has been observed in some patients since M1, so visit at M1 and M2 must be keept to check this point for the first injection. We confirm that very few new patients present OHT during the following injections.Repeated injections of ozurdex
- F038

Correlation between hiperreflective foci in patients with macular edema secondary to retinal vein occlusion, and visual outcomes after an intravitreal dexamethasone implant injection

ALMENARA MICHELENA C, ASCASO FJ, LAVILLA L, CRISTOBAL J, ZABADANI K, CARAMELLO C, MARTINEZ M, HERRERA L, ESTEBANO Ophthalmology, Zaragozd

Purpose To investigate the relationship between the presence of macular hyperreflective dots (HRD) detected by spectral-domain optical coherence tomography (SD-OCT) and visual outcomes in eyes with macular edema secondary to retinal vein occlusion (RVO), following treatment with intravitreal dexamethasone implant.

Methods Retrospective observational study of 16 consecutive cases of RVO-associated macular edema treated with Ozurdex". The correlation between best corrected visual acuity (BCVA) (logMAR), central macular thickness (CMT) and the presence of HRD in SD-OCT before the treatment and two months postoperatively was determined.

Results CMT decreased significantly after treatment $(553 \pm 131 \mu \mathrm{m}$ vs $300 \pm 67 \mu \mathrm{m}$, $\mathrm{p}<0.0001$ ). Although there was a visual acuity improvement in seven of the 16 eyes $(43.7 \%)$, there were no significant differences in BCVA between before and following reatment $(0.61 \pm 0.28$ vs $0,59 \pm 0.31, \mathrm{p}>0.05)$. Numerous HRD were detected in 11 eves (68.8\%), disappearing in eight of them (72.7\%) after corticosteroid intravitreal therapy. Two months following treatment, $\log$ MAR BCVA was significantly worse in the eyes with numerous HRD $(0.90 \pm 0.17)$ than in those with few or absent HRD $(0.52 \pm 0.29)$ (p $=0.045$, independent Student $t$ test .

Conclusion Disappearance of HRD following intravitreal dexamethasone therapy and the consequent improvement in visual function would support the hypothesis that hiperreflective foci could represent inflammatory cells. Nevertheless, the presence of HRD in SD-OCT was not able to predict BCVA after intravitreal Ozurdex.

\section{- F040}

REMIDO 2 Study: Retrospective Multicentric study of the dexamethasone drug delivery system, OZURDEX, in the treatment of macular edema following retinal vein occlusion; 2 years follow-up

POMMIERS

COPS, Lisle sur la Sorgu

Purpose To evaluate the long-term visual prognosis and complications of patients who received intravitreal Ozurdex injections for the treatment of macular edema (ME) due to retinal vein occlusion $(\mathrm{RVO})$.

Methods A total of 94 patients who received Ozurdex injections on an 'as-needed' basis in our center network (P 1,5 network) as part of the REMIDO study were followed for examination and retreatment. Main outcome measures included changes in final visual acuity (VA), final central macular thickness (CMT), retreatment interval, number of injection, and incidence of side effects.

Results Mean follow-up time was 26.3 months. A total of 94 eyes were included for studyt. Retreatment with Ozurdex was judged necessary after $5.3+0.8$ months. No tachyphylaxis effect was observed. Initial mean VA was 42 letters. It significantly improved to 62 letters after 2 years of treatment : the improvement was $\geq 15$ ETDRS letters in $50 \%$ of patients $51.2 \%$ of patients were cured at 2 years with a mean injection of 2.15 . The profile of cured patients presented $64 \%$ of naive and $60 \%$ of branch RVO. Mean CMT decreased from $688 \pm 117 \mu \mathrm{m}$ (baseline) to $325 \pm 54 \mu \mathrm{m}$. No serious adverse events were observed; Ocular hypertension greater than $25 \mathrm{mmHg}$, managed by topical treatment, is observed in $15 \%$ of patients. Switch was necessary for $18 \%$ of patients. Cataracts were extracted in $31 \%$ of patients at 2 years.

Conclusion This is the first reported 2 years long-term evaluation of patients treated with Ozurdex. Repeated intravitreal Ozurdex on an 'as-needed' basis, with a retreatment interval $<6$ months, produce long-term clinically meaningful benefits in the treatment of ME due to RVO. 


\section{- F041}

Can Ozurdex ${ }^{\oplus}$ make the difference in a retinal vein occlusion population with longstanding macular edema and multiple prior treatments?

VANCALSTERJ

Dept. of Ophthalmology, University Hospitals Leuven, Leuven

Purpose To evaluate the efficacy and safety of Ozurdex in the treatment of macular edema (ME) due to retinal vein occlusion (RVO) in a difficult-to-treat population with multiple prior treatments and long-standing $\mathrm{ME}$

Methods In this multicentric, anonymised, retrospective data collection, 77 patients were included during the early access program in Belgium. 46 patients had CRVO (59.7\%) and 31 had BRVO (40.3\%)

Results The mean time between the diagnosis of RVO and the first Ozurdex injection was $1.91 \pm 2.13$ years, with a median time of $1.26(0.03-10.57)$ years. In $55.8 \%$, this interval was more than one year. Concerning prior treatments before the first Ozurdex injection, 55 patients (71\%) did receive one or more anti-VEGF injections, 24 patients (31\%) did receive one or more Triamcinolone injections and 33 patients (43\%) underwent laser treatment (panretinal/focal). A total of 40 (52\%) patients showed 2 line improvement and $29(38 \%)$ showed 3 line improvement in BCVA (ETDRS letters). Nine $(11.6 \%)$ patients underwent cataract surgery. Post-injection, 14 (18\%) patients had an IOP $>25 \mathrm{mmHg}$. There was no need for glaucoma surgery

Conclusion In this difficult-to-treat population with multiple prior treatments and long-standing ME, Ozurdex has shown to be efficacious. The safety profile is in line with previously reported trials. Larger prospective observational studies are currently underway to clarify the usage patterns of Ozurdex" in clinical practice.

- F043

Inhibition of NO and COX products modifies the hypoxiainduced dilatation of retinal vessels in vivo

KAYA M, PEDERSEN L, BEK T

Ophtalmology, Aarhus

Purpose Retinal hypoxia with consequent changes in blood perfusion is a central feature in common vision threatening diseases. The aim of this study was to examine the effects of inhibiting COX and NO-synthesis on hypoxia-induced relaxation of retinal vessels in humans

Methods Twenty healthy persons aged 20-55 years were examined on two days separated by 4-7 days. The resting diameter and the diameter response to isometric exercise and flicker stimulation of retinal vessels were studied using the Dynamic Vesse Analyzer before and during breathing a hypoxic gas mixture and before and during intravenous infusion with the NOS inhibitor L-NMMA and were repeated on a second day after topical administration of the COX-inhibitor diclofenac

Results The resting diameter of arterioles increased significantly during hypoxia and decreased significantly during L-NMMA infusion $(\mathrm{p}<0.0001)$ whereas no change in diameter was observed with the two applied together. When hypoxia and L-NMMA were applied simultaneously, the gain factor was significantly lower than $1 \quad(\mathrm{p}=0.002)$ indicating that when diameter changes were corrected for changes in arterial blood pressure the blood flow increased, whereas this was not the case for either of the two interventions alone.Diclofenac significantly reduced contraction of retinal arterioles induced by isometric exercise $(\mathrm{p}=0.04)$ but not by the other interventions.Flickerinduced dilatation of retinal arterioles was increased during L-NMMA infusion $(\mathrm{p}<0.0001)$ but not during the other interventions

Conclusion Diameter changes of retinal vessels during hypoxia are influenced by $\mathrm{NO}$ and COX products. This may point to new treatment strategies for diseases characterized by retinal hypoxia and disturbances in retinal perfusion
- F042

Why should we systematically screen sleep apnea syndrome in RVO patients?

RUSSO A, DOT C

Military Desgenettes hospital, Lyon

Purpose Sleep apnea syndrome (SAS ) has recently been identified as an independent cardiovascular risk factor, involved in numerous diseases The aim of our study is to evaluate the prevalence and physiopathologic links of SAS in patients with retinal vein occlusion (RVO)

Methods Prospective study including 35 patients. Each patient had a simplified screening questionary, and an ambulatory respiratory nocturnal events measurement using the device RU-sleeping@ (Philips Respironics,Inc.).Ventilatory polysomnography (VPS), reference method, is associated in all cases.

Results Mean age of patients was 73.6 year, the sex ratio of 1.4 and CRVO as many as BRVO. Glaucoma was found in $31 \%$ of patients, hypertension in $52 \%$. Signs of SAS were noted in $95 \%$ of patients. The prevalence of severe SAS detected by RU-sleeping@ is $84 \%$, it is significantly higher than that of the population over 60 years $(25 \%)(\mathrm{p}<0.001)$. Preliminary results of VPS are very interesting : they found a strong linear correlation between initial macular thickness evalued by SD OCT $(590+/-170 \mu \mathrm{m})$ and results of Apnea-Hypopnea Index $(\mathrm{r}=0.88, \mathrm{p}=0.004)$, Arousal Index $(\mathrm{r}=0.74, \mathrm{p}=0.035)$ and Oxygen Desaturation Index $(r=0.58, p=0.027)$.Side effects of SAS could explain the occurrence of RVO. RVO could be a consequence of a slow-down of blood flow circulation secondary to hypoxemia, elevated nocturnal intracranial pressure and rheologic variations in SAS. Arousals cause an additional acute increase in arterial blood pressure and heart rate.

Conclusion The results of this preliminary study show a high prevalence of severe SAS in the RVO population. The benefit of early ventilation on prognosis post-RVO is still unknown. RU-sleeping@ is a simple medical device, which seems interesting for screening SAS ambulatory.

\section{- F044}

A new model for studying diameter regulation of porcine retinal arterioles and capillaries in vitro

IENSEN PS, BEK T

Department of Ophthalmology, Aarhus University Hospital, Aarhus

Purpose Studies of diameter regulation in retinal arterioles in vitro have mostly been performed on larger vessels, but evidence suggests that perturbations in the retinal microcirculation may also play a role in the development of vision threatening retinal diseases. Therefore, the purpose of the present study was to develop an in vitro technique for studying diameter regulation in both larger and smaller vessels.

Methods A special tissue chamber was developed for mounting, cannulating and perfusing porcine hemiretinas while controlling temperature, $\mathrm{pH}$ and oxygen saturation. The chamber was mounted in a flourescens microscope, and the effect on the diameter of larger arterioles, pre-capillary arterioles and capillaries was studied after intravascular and extravascular addition of the thromboxane analogue U46619 and lactate ( $\mathrm{n}=6$ for each variable) and NMDA (preliminary).

Results In all vessel calibers the thromboxane analogue U46619 induced a significant contraction after extraluminal application ( $\mathrm{p}<0.02)$, but not after intraluminal $(\mathrm{p}>0.13)$. Lactate had no effect on the diameter of non-precontracted vessels $(\mathrm{p}>0.21)$, but in precontracted vessels lactate relaxed the vessel diameter.

Conclusion The response to vasoactive compounds is different after intraluminal and extraluminal application, and the diameter response of vasoactive compounds is different in larger and smaller retinal vessels. The dilating effect of lactate depends on the state of contraction of the retinal vessel. 
- F045

Diabetic retinopathy: A descriptive study of a method of investigation: BOA's project

$B A Z I N L$

CHRU, Brest

Purpose The purpose of this study is to report the results of 208 patients observation comparing two different interfaces of delayed reading retinophotographies and thus two different logistics managements of diabetic patients at the University Hospital of Brest

Methods Prospective study of 208 diabetic patients followed. Retinophotographies were automatically and randomly assigned to one of two consoles for delayed reading by an ophthalmologist.The " examination time " and " writing report time " were identified. The settings « diagnosis », « image quality » and «follow-up » were graduated Results 208 patients were included.The examination time did not differ between the two consoles (Student $\mathrm{t}$ test: $\mathrm{t}=0.623, \mathrm{p}=0.534$ ). « Writing report time » differ a statistically significant manner $(\mathrm{p}<<10-4)$.There is a positive correlation between examination time » and « writing report time » $(\mathrm{r}=0.382, \mathrm{p}<10-4)$ for both consoles There are no statistically significant differences in the « image quality » setting between the two systems $(p=0.623)$. There was no statistically significant difference between the two consoles on the variables « follow-up » $(\mathrm{p}=0.067)$

Conclusion There is a positive correlation between « examination time » and « writing report time » $(r=0.382, p<10-4)$ for both consoles. There are no statistically significant differences in the « image quality » setting between the two systems $(\mathrm{p}=0.623)$. There was no statistically significant difference between the two consoles on the variables follow-up " $(\mathrm{p}=0.067)$

- F047

Severity of type 2 diabetes in Seine St Denis among patients with diabetic macular edema treated by antiVEGF

STEPHAN S, FAINKUCHEN F, GIOCANTI-AUREGANA

Department of ophtalmology, 125 rue de Stalingrad

Purpose Diabetic macular edema (DME) is the main cause of loss of vision over the course of diabetes. In this study we sought to know whether DME was more severe in Seine st Denis, a french area, where the poverty is higher than in other french plac

Methods We enrolled all patients suffering from DME and treated by ranibizumab intravitreal injections between November 2012 and April 2013. In order to evaluate the severity of DME and the medical management of diabetes of these patients, we collected the following parameters: central macular thickness measured by SD-OCT the best corrected visual acuity, diabetic retinopathy severity, $\mathrm{HbAlc}$, diabetes duration, and associated diseases.

Results We included 25 type 2 diabetic patients ( 8 women and 17 men), the mean age was $64 \pm 8,1$ years. Mean central macular thickness was $523 \pm 145 \mu \mathrm{m}$. The best corrected visual acuity was 51 letters at baseline (counting fingers- 70 letters). 22 patients (88\%) had a severe non proliferative diabetic retinopathy or a proliferative diabetic retinopathy. Mean $\mathrm{HbAlc}$ was $7,8 \%(+/-2,3 \%)$. For 23 cases $(92 \%)$, diabetes was associated with high blood pressure, and the average was 14,5 $\pm 2 / 8,5 \pm 1,3 \mathrm{mmHg}$. Diabetes lasted for 13,1 years at baseline.

Conclusion Diabetic patients, in this case series, had a more severe DME regarding macular thickness and visual acuity than patients from large randomized studies found in the literature. This severity could be due to a sub optimal management of their diabetes.DME may become a tool to witness a limited access to good medical cares.
- F046

Retinal vessel oxygen saturation in diabetic patients with and without cardiovascular disease and its association with renal function

HEITMAR R (1),BLANNA (2)

(1) School of Life and Health Sciences, Birmingham

(2) University Department of Medicine, City Hospital, Birmingham

Purpose To explore the association of renal function and retinal vessel oxygen saturation in patients suffering from diabetes and cardiovascular disease.

Methods We examined three groups of patients (1: Diabetics, 2: Diabetics with cardiovascular disease and 3: patients suffering from cardiovascular disease without diabetes). All patients Intraocular Pressure, Systemic Blood Pressure and Dual Wavelength Retinal Vessel Oxygen Saturation (arterial and venous vessel saturation nd a-v saturation difference) and Retinal Vessel Calibers were measured following phlebotomy to determine their HbAlc levels. Markers of renal function (ceratinine, eGfr and $\mathrm{uACR}$ ) were measured in all patients.

Results All three groups were age matched (1: 63 (10)yrs; 2: 64 (9)yrs and 3:65 (10) yrs). Unsurprisingly HbA1c was significantly different between groups $(\mathrm{p}<0.001)$, while Intraocular Pressure and Systemic Blood Pressure variables were comparable. While arterial and venous oxygen saturation were comparable between groups, the cardiovascular group had the lowest venous saturation, resulting in the highest $\mathrm{a}-\mathrm{v}$ values but this was not statistically significant.Markers of renal function showed a statistically significant correlation with a-v saturation values in patients suffering from both: diabetes and cardiovascular disease (eGfR and a- $-\mathrm{v}$-saturation: $\mathrm{r}=0.65, \mathrm{p}=0.001$ ) but not in those suffering from cardiovascular disease alone $(\mathrm{r}=0.36, \mathrm{p}=0.063)$

Conclusion Retinal vessel oxygen saturation parameters appear to be associated with renal function in patients suffering from both diabetes and cardiovascular disease.

\section{- F048}

Comparison of the time required for panretinal photocoagulation and associated pain between Navilas ${ }^{\circ}$ and conventional laser therapy in diabetic retinopathy

KIM MS, KIM JS

Inje University Sanggye Paik Hospital, Seoul

Purpose To compare the pain scale and time necessary for panretinal photocoagulation (PRP) between Navilas" (OD-OS, Teltow, Germany) and conventional laser in diabetic retinopathy.

Methods Fifteen patients who required PRP for diabetic retinopathy were enrolled in the present study. PRP was performed using Navilas ( 5 X 5 array patterned system) in the superior, nasal and inferior areas, and using conventional laser at the temporal area 1 week later. Total time of laser application and number of laser shots were counted for calculating required time per 100 spots of each laser system. Immediately after the laser photocoagulation, patients were asked to quantify their pain on a visual analog pain scale $(0=$ no pain; 10 = worst pain $)$

Results PRP using Navilas required shorter time per 100 laser spots (27.7 sec vs. 102.0 sec, $\mathrm{p}<0.001$ ) and subjects had lower treatment-related pain than with the conventional laser system (3.3 vs. 6.9, $\mathrm{p}<0.001)$.

Conclusion PRP using Navilas" can be considered as an efficient method for improving patient and operator's comfort with faster laser application and lower treatment-related pain. 
- F049

Prognostic factors for neovascular glaucoma after vitrectomy in eyes with proliferative diabetic retinopathy: comparative study in the same person

LEES (1), YEOM M (2), PARKJ (2)

(1) Department of Ophthalmology, Haeundae Paik Hospital, Inje University College of Medicine, Busan

(2) Department of Ophthalmology, Maryknoll medical center, Busan

Purpose To investigate the prognostic factors for neovascularg after vitrectomy in eyes with proliferative diabetic retinopathy.

Methods To evaluate intraindividual, interocular differences, eleven patients(22 eyes) who had undergone pars planavitrectomy for PDR and had only one eye neovascular glaucoma were retrospectively reviewed. They were underwent vitrectomy for PDR between March 2008 and July 2013 at Maryknol hospital.The clinical data on preoperative, intraoperative, and postoperative factors for these patients's eyes were compared each other. Statistical analysis was performed using the Wilcoxon matchedpairs signed-rank (Mann-Whitney) test and Chi-square test in order to evaluate the significance of differences within the groups of patients.

Results Of the 22 eyes(11 patients), the mean age was $50.64 \pm 11.66$ years(range $27-69$ ) and the mean follow-up period was $37 \pm 17$ months. The decrease of photopic b-waves amplitude on preoperative electroretinogram was significantly correlated with the development of neovascular glaucoma after vitrectomy for PDR

Conclusion Our findings suggested that the decrease of photopic b-waves amplitude on preoperative electroretinogram is considered an effective prognostic factorsfor the development of neovascular glaucoma after vitrectomy for PDR

\section{- F051 \\ Use of bromfenac eye drops in the treatment of diabetic macular edema: a pilot study}

BLASETTI F, CASU G, D'AMICO RICCI G, BOSCIA F, PINNA A

Department of Surgical, Microsurgical and Medical Sciences, Sassari

Purpose To evaluate the effect of bromfenac eye drops on diabetic macular edema (DME).

Methods In this pilot study, 20 eyes of 20 patients with monocular DME were analyzed. Topical bromfenac was administered in the affected eye twice daily for 30 days. Visual acuity (VA) and macular thickness, determined by optical coherence tomography (OCT), were evaluated initially and after 30 days' therapy. Statistical analysis was performed by Student's t test.

Results Pre-treatment mean VA was $0.38 \pm 0.2 \log$ MAR and final mean VA was $0.41 \pm 0.18 \log M A R$, a not statistically significant result $(\mathrm{p}=0.21)$. Initial mean macular thickness was $476.15 \pm 130.16 \mu \mathrm{m}$ and post-treatment macular thickness was $413.8 \pm 161.13 \mu \mathrm{m}$, a statistically significant difference $(\mathrm{p}=0.04)$

Conclusion The results of this pilot study suggest that topical bromfenac twice daily may play a role in the reduction of DME. This preliminary finding is interesting; however, further larger multi-centre studies are necessary to establish whether, or not, topical bromfenac may be beneficial in the treatment of DME.

\section{- F050}

Effects of combination of antioxidants and omega 3 fatty acids in diabetic retinopathy

SANTANDER-TRENTINI F (1), VIVAR-LLOPIS B, CHAQUES-ALEPUZ V, ALONSO L, ZANON-MORENO V, GALLEGO-PINAZOR, DOLZ-MARCO R, SHOAIE-NIA K, PINAZO-DURANMD (2)

(1) Valencia

(2) Valencia Study Group on Diabetic Retinopathy (VSGDR)

Purpose Oxidative stress is a relevant pathogenic factor in the development of diabetic retinopathy (DR). We evaluated whether the combination of antioxidants and omega 3 fatty acids (A-O3FA) would reduce the biochemical parameters of risk for diabetic complications in patients with type 1 (T1) and 2 (T2) diabetes mellitus (DM) who were vulnerable to vitreoretinal disorders.

Methods A prospective case-control study was carried out in 400 initial participants of both sexes aged 15 to 80 years that were distributed into three groups: 1) T1DMG $(\mathrm{n}=80), 2)$ T2DMG $(\mathrm{n}=220)$, and 3$)$ healthy controls $(C G ; \mathrm{n}=100)$. We randomly assigned half participants of each group to be orally supplemented (+OS) with 1 pill/day of openlabel formulation containing A-O3FA, during one year of follow-up. Demographics, nutrition, life style, ocular and blood biochemical data were recorded and statistically processed by the SPSS 15.0 program.

Results Best-corrected visual acuity did not vary during the follow-up, irrespective of supplementation. Diabetics showed severer glycosilated haemoglobin, lipid profile, homocysteine and C-reactive protein levels than the CG. Plasmatic malondialdehyde (MDA) $(\mathrm{p}<0,01)$, total antioxidant activity (TAA) $(\mathrm{p}<0,001)$ and glutathione $(\mathrm{GSH})$ $(p<0,01)$ levels were significantly higher in T1DMG/T2DMG than in the CG. All these parameters noticeably changed in the supplemented groups. No significant variations were detected in the ocular coherence tomography examinations regarding three macula parameters, and no correlation with the biochemical data was found.

Conclusion The biochemical background in DM creates the optimal conditions for the increased oxidative stress that can be minimized by the appropriate combination of A-O3FA. Plasmatic homocysteine, C-reactive protein, MDA, TAA and GSH can be presumptive biomarkers for DR.

\section{- F052}

The efficacy of dexamethasone implant (Ozurdex $700 \mu \mathrm{g})$ in refractory diabetic macular oedema

KARTTUNEN T, KAARNIRANTA K, KINNUNEN K

Department of ophthalmology, Kuopio

Purpose The efficacy of dexamethasone implant in refractory diabetic macular oedema (DMO).

Methods 24 eyes of 22 patients having single dexamethasone implant for refractory DMO were included in the study. Patients with another retinal disease causing macular oedema were excluded. The patient data was collected and analyzed retrospectively. As a demographic data, age, gender and the type of diabetes and duration of DMO were collected. Morphology of the retina and changes in foveal thickness were analyzed with Heidelberg SD-OCT. Furthermore improvement in the best-corrected visual acuity (BCVA) and changes in the intraocular pressure (IOP) were observed.

Results The mean age of patients was $65,6( \pm 10,6)$ years. $36,4 \%$ were female and $63,6 \%$ were male. $22,7 \%$ had type 1 (DM1) and $77,3 \%$ had type 2 diabetes (DM2). The mean duration of DMO was $26,1( \pm 23,8)$ months. $90,9 \%$ of the eyes had had previous argon laser treatment to macula and/or periphery. Panphotocoagulation was made in $29,2 \%$ of the eyes, $87,5 \%$ of the eyes were treated with intravitreal bevacizumab $(4,3 \pm 3,0$ injections) and $37,5 \%$ were treated with triamcinolone before. The mean BCVA at baseline was $0,34( \pm 0,17)$ in Snellen. Best improvement in visual acuity was seen 120,5 $( \pm 78,8)$ days after dexamethasone implant. Best achieved VA was $0,53( \pm 0,19)$. Baseline foveal thickness was $413,4( \pm 120,2) \mu \mathrm{m}$ and at the point of best achieved VA 333,5 $( \pm 46,5) \mu \mathrm{m}$. Vitrectomy was performed in $20,8 \%$ before. $45,8 \%$ of the eyes were phakic and cataract developed in $72,7 \%$ of these eyes. The highest increase in IOP was $4,9( \pm 6,5)$ mmHg. 8,3\% of the eyes needed IOP lowering medication.

Conclusion We suggest that dexamethasone implant is a useful treatment in DMO even in relatively poor eyes.

Commercial interest 


\section{- F053}

Complete retinal fluid resorption with low visual acuity in patients with diabetic macular edema (DME) over the course of ranibizumab treatment: $\mathrm{OCT}$ analysis

PENAUD B, GIOCANTI-AUREGAN A, LEVYO, CHAINE G, FAINKUCHENF Ophthalmology, Bobigny

Purpose Intravitreal ranibizumab injections in DME often leads to resorption of edema. Nevertheless, complete resorption of DME is weakly correlated with good visual acuity (VA). The purpose of our study was to investigate the anatomical OCT features of patients treated with ranibizumab for DME with low VA despite of complete dry retina.

Methods We included in a retrospective fashion all patients over the course of ranibizumab treatment for DME at Avicenne Hospital (Bobigny, France) with a dry retina after treatment (central foveal thickness (CFT) $\leq 250 \mu \mathrm{m}$ and presence of foveal pit). We analyzed the characteristics of outer retinal lavers in spectral-domain OCT (Optos OCT SLO) in case of low final VA (best-corrected VA (BCVA) with dry retina $<5 / 10$ after at least 3 injections).

Results We included 40 patients. Complete retinal fluid resorption was achieved for 24 patients $(60 \%)$. Among these patients, half of them (12 patients ie $30 \%$ of our cohort) had low VA (group 1) and 12 patients had good VA (group 2). Mean CFT was $713 \mu \mathrm{m}$ in group 1 (G1) and 666 $\mathrm{mm}(\mathrm{p}=0.64)$ in group 2 (G2). After treatment, mean CFT in G1 was $165 \mu \mathrm{m}$ against $174 \mu \mathrm{m}(\mathrm{p}=0.6)$ in $\mathrm{G} 2$. For all patients in $\mathrm{G} 1$, anatomical changes were noted on OCT pictures after treatment: loss or thinning of ellipsoid band $(n=6)$ atrophic cyst $(n=6)$, exudates $(n=3)$, epiretinal membrane $(n=2)$.

Conclusion Insufficient improvement of BCVA after complete retinal fluid resorption happened in about 30\% of patients over the course of ranibizumab treatment for DME, and was often associated in our study with alterations of outer retinal layers in SD-OCT imaging

\section{F054}

The efficacy and safety of intravitreal bevacizumab in the treatment of diabetic macular oedema

MALYSKO K (1,2), KUOLIENE K (1, 2), CIMBALAS A (1,2), ASOKLIS R (1,2)

(1) Vilnius University, Faculty of Medicine, Vilnius

(2) Vilnius University Hospital Santariskiu Klinikos, Centre of Eye Diseases, Vilnius

Purpose to evaluate the efficacy and safety of $1.25 \mathrm{mg}$ intravitreal bevacizumab (IVB) in the treatment of diabetic macular oedema at Vilnius University Hospital Santariskiu Klinikos, Centre of Eye Diseases from July, 2012 to May, 2014.

Methods Clinical data (best corrected visual acuity (BCVA) and OCT findings at baseline and at the end of the follow-up) of 52 patients (76 eyes) were reviewed retrospectively. Patients who received only one intravitreal bevacizumab injection or whose medical data were missing were excluded.

Results 76 eves were treated with $1.25 \mathrm{mg}$ intravitreal bevacizumab every 4 weeks at least with two injections for one eye and then continued if needed. The mean BCVA in patients with diabetic macular oedema at baseline (Snellen) was 0.31 (SD \pm 0.24$)$. The mean number of intravitreal bevacizumab injections per eve was - 3 (range 2-8). Patients were observed for an average of 36 weeks. At the end of the follow-up the mean BCVA increased to $0.4(\mathrm{SD} \pm 0.31)$ and the central macular thickness detected on OCT decreased significantly (mean $325(\mathrm{SD} \pm 228 \mu \mathrm{m})$ ). Four patients with resistant diabetic macular oedema underwent combined IVB and laser treatment. One case of endophthalmitis was observed.

Conclusion Intravitreal bevacizumab seems to be effective and safe in the management of diabetic macular oedema in this short term retrospective study.

- F056

Ocular distribution and pharmacokinetics of 125I-OPT302 and 125I-Aflibercept (EYLEA) following intravitreal administration to pigmented rabbits

STRUBLEC(1), TESTER A (2), GEROMETTA M (2), KREUGER M (1)

PRUSAKIEWICZ J(1), BALDIKINM (2)

(1) Covance Laboratories, Madison, Wisconsin

(2) Opthea Pty Ltd, Circadian Technologies Limited, Victoria

Purpose OPT-302 is a soluble receptor that specifically and potently blocks VEGF-C and VEGF-D activity which are involved in the progression of retinal and corneal diseases. OPT-302 is currently under development for the treatment of wet AMD. The purpose of this study was to assess ocular distribution and pharmacokinetics of 125I-OPT-302 and 125I-EYLEA following intravitreal (IVT)dosing in Dutch Belted rabbits.

Methods 125I-OPT-302 and 125I-EYLEA were prepared at $\sim 10 \mathrm{mg} / \mathrm{mL}$ and $50 \mu \mathrm{Ci} /$ $\mathrm{mg}$. Eight animals/group were dosed with $0.5 \mathrm{mg}$ test article/eve. Serum and ocular tissues were collected at eight time points between 1 and 672 hours for radioanalysis. The formulations were homogeneous, stable, and active with their cognate ligands.

Results Following IVT dosing the test articles were well tolerated. There was low systemic exposure of 125I-OPT-302 with AUC $(0-\mathrm{t})$ of 110000 ng eq"hours $/ \mathrm{mL}$. Systemic exposure to 125I-EYLEA was $\sim 4$ fold greater at $420000 \mathrm{ng}$ eq"hours $/ \mathrm{mL}$. Concentrations of 125I-OPT-302 and 125I-EYLEA in the vitreous humor $(\mathrm{VH})$ at $\mathrm{C} 0$ were 368000 and $337000 \mathrm{ng}$ equivalents/g, respectively. Elimination $\mathrm{t} 1 / 2$ from $\mathrm{VH}$ were similar at 104 and 112 hours. For ocular tissues, the highest concentrations of radioactivity were found in retina, RPE and choroid at 1 and 12 hours postdose. Maximum concentrations were similar for each test article. Half-lives were 104 \& 102 hours in choroid and 109 \& 137 hours in RPE for 125I-OPT-302 and 125I-EYLEA,respectively.

Conclusion 125I-OPT-302 and 125I-EYLEA had prolonged exposure of posterior and anterior ocular tissues at similar concentrations for each compound, while systemic clearance of 125I-OP'T-302 was faster than 125I-Eylea. 


\section{- F057}

Antiangiogenic effect of ALS-L1023 on experimental choroidal neovascularization in mice

KANGS, RHO YJ

Department of Ophthalmology and Visual Science, College of Medicine, The Catholic University of Korea, Seoul

Purpose This study was conducted to evaluate the effect of ALS-L1023 on experimental choroidal neovascularization $(\mathrm{CNV})$ in mice.

Methods C57BL/6 mice were administered either vehicle or ALS-L1023 daily by oral gavage for three weeks (day 0-21). CNV was induced in each mouse by rupturing Bruch's membrane using laser photocoagulation (day 7). Two weeks after laser injury (day 21), the CNV lesions were evaluated by choroidal flat mounts using fluoresceinlabeled dextran, immunofluorescence staining with isolectin IB4, and fluorescence angiography. The effects of ALS-L1023 on endothelial cell tube formation and on the expression of phosphorylated extracellular signal-regulated kinase (p-ERK1/2) were evaluated using human umbilical vein endothelial cells (HUVECs).

Results ALS-L1023 reduced the extent of CNV. The groups treated with 100 and 200 $\mathrm{mg} / \mathrm{kg} /$ day showed 43.3 and $68.1 \%$ reduction of CNV lesions, respectively, compared to the vehicle group $(\mathrm{P}<0.001)$. The size of isolectin IB4 labeled area was also significantly decreased in the ALS-L1023-treated group $(\mathrm{P}<0.001)$. On fluorescein angiography ALS-L1023-treated mice had significantly less fluorescence leakage than vehicle-treated mice. ALS-L1023 decreased VEGF-stimulated tube formation of HUVECs in a dosedependent manner. The expression of p-ERK was suppressed by ALS-L1023.

Conclusion ALS-L1023 can inhibit laser-induced CNV in mice and may have therapeutic potential for treatment of choroidal neovascularization diseases.

\section{- F059}

Effect of a single intravitreal injection of anti-VEGF agents on retinal arteriolar caliber in mini pig eyes

MANGIORIS G, PETROPOULOS I, MENDRINOS E, POURNARASC Laboratory of Neurobiology and Physiology of the Retinal Circulation, Department of Ophthalmology, Geneva University Hospitals, Geneva

Purpose The aim is to investigate the short-term effect of a single intravitreal bevacizumab, ranibizumab or pegaptanib sodium injection on the retinal arteriolar caliber in minipigs.

Methods 15 eyes received an intravitreal injection: bevacizumab $1,25 \mathrm{mg}(\mathrm{n}=5)$ ranibizumab $0,5 \mathrm{mg}(\mathrm{n}=5)$ and pegaptanib sodium $0,3 \mathrm{mg}(\mathrm{n}=5)$. The diameter of the retinal arterioles was measured in vivo with a Retinal Vessel Analyzer (RVA) every 15 minutes for 2 hours.

Results After the injection of bevacizumab statistical significant vasoconstriction was reached $1 \mathrm{~h}$ after injection $(\mathrm{p}<0.01)$ and persisted until the end of the measurements (2h). Although there appears to be a trend towards vasoconstriction after ranibizumab injection, analysis of measurements indicate that statistical significance $(\mathrm{p}<0.05)$ is reached only $1 \mathrm{~h}$ 30min following injection. After the injection of pegaptanib sodium injection revealed no vasomotor effect of the substance. $(\mathrm{p}>0.05)$

Conclusion The results suggest that intravitreal injection of anti-VEGF that blocks all isoforms of VEGF, induces retinal vasoconstriction. Further studies with a larger number of subjects would be helpful in establishing more clearly the effect of intravitreal anti-vascular endothelial growth factor treatment on retinal vessel diameters.

\section{- F058}

Tissue plasminogen activator as an anti-angiogenic agent in experimental laser-induced choroidal neovascularization

OZONE D, NOZAKIM, OHBAYASHI M, HASEGAWA N, KATOA,

YASUKAWA T, OGURA Y

Department of Ophthalmology \& Visual Science, Nagoya City University Graduate School of Medical Sciences, Nagoya

Purpose Tissue plasminogen activator (tPA) is a fibrinolytic compound, utilized originally to treat embolic or thrombotic stroke and as an adjuvant for displacement of submacular hemorrhage. The purpose of this study is to investigate anti-angiogenic effects of tPA on experimental laser-induced choroidal neovascularization (CNV) in mice.

Methods $\mathrm{CNV}$ was induced by laser injury in $\mathrm{C} 57 \mathrm{BL} / 6 \mathrm{~J}$ mice, and intravitreal injection of tPA (4 or $40 \mathrm{IU} / \mu \mathrm{l}$ ) or PBS was performed immediately after laser injury. Fluorescein angiography was performed 7 days after laser treatment to grade fluorescein leakage. And CNV volumes were measured by confocal evaluation of Isolectin B4 staining of RPE-choroid flatmounts. The expression of fibrin on day 3 was observed by mmunostaining.

Results Fluorescein leakage was inhibited by tPA in a dose-dependent manner, and a significant difference was found with tPA (40 IU/ $\mu$ l) compared with PBS $(\mathrm{p}=0.02)$. A dose-dependent suppression of $\mathrm{CNV}$ volume was also observed by tPA, and there was a significant differences between tPA $(40 \mathrm{IU} / \mu \mathrm{l})(208988 \pm 52456 \mu \mathrm{m} 3)$ and PBS $(386902$ $\pm 103060 \mu \mathrm{m} 3, \mathrm{p}<0.01$ ). The expression of fibrin was reduced in eyes treated with tPA.

Conclusion Intravitreal injection of tPA reduced the expression of fibrin and significantly suppressed laser-induced CNV in mice. These findings suggested that tPA might be anti-angiogenic and have a potential as an adjuvant to anti-vascular endothelial growth factor therapy.

\section{- F060}

Evaluation of two methods for measurements of macular pigment optical density (MPOD)

BIRKELDH U, WAHLBERG RAMSAY M, NILSSON M, BRAUTASET R Unit of Optometry, Eye and Vision, Karolinska Institutet, Stockholm

Purpose To evaluate the outcomes of two methods for measurements of macular pigment optical density (MPOD).

Methods In 33 subjects (mean age 51.85 years \pm 17.79 ; 4 men and 29 women), without any signs of ocular pathologies, MPOD was measured in the right eve using singlewavelength reflectometry (Visucam 500) and heterochromatic flicker photometry (MacuLux ${ }^{\circ}$ ). In 13 subjects with discrete signs of age-related macuolopathy (ARM), i.e., soft drusen and/or hyper-/hypopigmentation of the retinal pigment epithelium (RPE), MPOD was measured after 6 and 12 methods of supplementation with MacuShield (lutein $10 \mathrm{mg}$ and zeaxanthin $2 \mathrm{mg}$ and the non-dietary carotenoid meso-zeaxanthin $10 \mathrm{mg}$ ).

Results There was a statistical significant difference $(\mathrm{p}<0.001)$ between the average maximum MPOD in the healthy subjects measured with the Visucam $(0.384 \pm 0.049)$ and with the Maculux $(0.559 \pm 0.133)$. No correlation ( $\mathrm{r} 2=0.089, \mathrm{p}=0.09)$ was found between the two techniques. In the 13 subjects, treated with Macushield, the Visucam measurements showed a statistically significant MPOD reduction $(\mathrm{p}<0.0005)$ while a significant increase $(\mathrm{p}<0.0045)$ in MPOD was found with the MacuLux".

Conclusion The results from this study indicate that measurements of MPOD with different techniques do not correlate and that the Visucam seems unable to detect the increase in macular pigment after intake of supplementation. 


\section{- F061}

Major American dietary patterns are related to age-related macular degeneration

CHIU CJ (1, 2), CHANG ML (1), ZHANG FF (3), LI T (4), GENSLER G (5), SCHLEICHER M(1) TAYLOR A (1)

(1) USDA Human Nutrition Research Center on Aging, Tufts University, Boston

(2) Department of Ophthalmology, School of Medicine, Tufts University, Boston

(3) Friedman School of Nutrition Science and Policy, Tufts University, Boston

(4) Channing Division of Network Medicine, Brigham and Women's Hospital, Harvard Medical School, Boston

(5) AREDS Coordinating Center, The EMMES Corporation, Rockville

Purpose We hypothesized that major American dietary patterns are associated with age-related macular degeneration (AMD) risk

Methods 8,103 eyes from 4,088 eligible participants in the baseline Age-Related Eve Disease Study (AREDS) were classified into control $(\mathrm{n}=2,739)$, early AMD $(\mathrm{n}=4,599)$, and advanced AMD ( $\mathrm{n}=765)$ by AREDS AMD Classification System. Food consumption data were collected by a 90-item food frequency questionnaire.

Results Two major dietary patterns were identified by factor (principle component) analysis based on 37 food groups and named Oriental and Western patterns. The Oriental pattern was characterized by higher intake of vegetables, legumes, fruit, whole grains, tomatoes, and seafood. The Western pattern was characterized by higher intake of red meat, processed meat, high-fat dairy products, French fries, refined grains, andeggs. We ranked our participants according to how closely their diets line up with the two patterns by calculating the two factor scores for each participant For early AMD, multivariate-adjusted odds ratio (OR) from generalized estimating equation logistic analysis comparing the highest to lowest quintile of the Oriental pattern score was ORE5O=0.74 (95\% confidence interval (CI): 0.59-0.91; Ptrend $=0.01$ ), and the OR comparing the highest to lowest quintile of the Western pattern score was ORE5W=1.56 (1.18-2.06; Ptrend=0.01). For advanced AMD, the ORA5O was 0.38 (0.27-0.54; Ptrend $<0.0001)$, and the ORA5W was 3.70 (2.31-5.92; Ptrend $<0.0001)$.

Conclusion Our data indicate that overall diet is significantly associated with the odds of AMD and that dietary management as an AMD prevention strategy warrants further study.

- F063

Relation between subfoveal choroidal thickness and visual acuity in patients with wet and dry age-related macular degeneration

ZABADANI K, HERRERA L, ASCASO FJ, CARAMELLO C, ALMENARA C, PEREZ NAVARRO I, MARTINEZ M, ESTEBAN O, PADGETT E, CABEZONL, CRUZ N, CRISTOBAL

Ophthalmology, Zaragoza

Purpose To evaluate the relationship between best-corrected visual acuity (BCVA) and subfoveal choroidal thickness (SCT) in patients with wet and dry age-related macular degeneration (AMD)

Methods We investigated 63 eyes of 58 patients who were diagnosed with wet AMD and 69 eyes of 56 patients who were diagnosed with dry AMD. All patients were evaluated in the Department of Ophthalmology at the "Lozano Blesa” University Clinic Hospital, Zaragoza, Spain, between January and October 2013. SCT was measured by using enhanced depth imaging (EDI) in spectral domain optical coherence tomography (SD-OCT)

Results The mean age of patients was $80.2 \pm 5.9$ year and $81.1 \pm 5.2$ years in patients with wet and dry AMD respectively ( $\mathrm{p}>0.05$, t student test). BCVA was 20/100 and 20/40, in patients with wet AMD and dry AMD, respectively ( $\mathrm{p}<0.001$, $\mathrm{t}$ student test). Mean SCT was $151.1 \pm 73.5 \mu \mathrm{m}$ and $155.6 \pm 55.1 \mu \mathrm{m}$, in wet AMD and dry AMD patients, respectively (No statistically significant differences, $\mathrm{p}>0.05$ ). Wet AMD patients showed a positive and statistically significant correlation between SCT and BCVA ( $r=0.298, p=0.018)$. Nevertheless, patients diagnosed with dry AMD showed no relationship between SCT and BCVA $(\mathrm{r}=0.085, \mathrm{p}=0.488)$.

Conclusion BCVA is positively correlated with SCT in patients diagnosed with wet AMD. No correlation was observed between both parameters in patients with dry AMD
- F062

Method for comparative evaluation of therapeutic efficacy in dry AMD patients using 3D-CTAG

MILYUTKINA S (1), FINK W (2, 3), KOVALEVSKAYA M (1)

(1) Voronezh State Medical Academy, named after N.N. Burdenko, Ministry of Health Russian Federation, Voronezh

(2) Visual and Autonomous Exploration Systems Research Laboratory, California Institute of Technology, Pasadena, California, USA, Pasadene

(3) Depts. of BME, ECE, and Ophthalmology \& Vision Science, University of Arizona, Tucson, Arizona, USA, Tucson

Purpose Evaluation of therapeutic efficacy in dry AMD patients using 3DComputerautomated Threshold Amsler Grid testing (3D-CTAG; Fink \&Sadun, 2004).

Methods Dry AMD patients ( $\mathrm{n}=87,174$ eyes). Group\#1 ( $\mathrm{n}=29)$ : $200 \mathrm{mg}$ Docosahexaenoic Acid (DHA) and $300 \mathrm{mg}$ Eicosapentaenoic Acid (EPA). Group \#2 $(\mathrm{n}=29)$ : Lutein $(12 \mathrm{mg})$ and Zeaxanthin $(0,5 \mathrm{mg})$. Controls $(\mathrm{n}=29)$. 3D-CTAG testing after 3 months of treatment.Indices: number of VF defects per eye (ND),lowest perceivablecontrast level (CL), absolute volume lost (AVL), VF volume lost relative to hill-of-vision (VLRH)

Results Group\#1: $\mathrm{ND}=0,31 \pm 0,54$ (before) down to $0,16 \pm 0,37$ (after treatment); $\mathrm{CL}=12,31 \pm 8,03 \%$ down to $10,9 \pm 7,21 \% ; \quad \mathrm{VLRH}=1,2 \pm 1,14 \%$ down to $0,26 \pm 0,52 \%$. Group\#2: $\mathrm{ND}=0,29 \pm 0,5$ down to $0,21 \pm 0,41 ; \mathrm{CL}=11,72 \pm 12,79 \%$ down to $9,66 \pm 10,2 \%$; VLRH $=1,01 \pm 0,88 \%$ down to $0,68 \pm 1,14 \%$. Controls: $N D=0,29 \pm 0,5$ up to $0,34 \pm 0,51$; $\mathrm{CL}=13,79 \pm 8,65 \%$ up to $14,38 \pm 10,38 \%$; V LRH $=0,93 \pm 0,9 \%$ up to $1,22 \pm 1,21 \%$.

Conclusion We demonstrated with 3D-CTAG a decrease of VLRH of $0,94 \%(\mathrm{p}=0,01)$ in group\#1 after 3 months DHA/EPA treatment compared to $0,33 \%(\mathrm{p}=0,05)$ in group\#2, and an increase of VLRH of $0,29 \%(\mathrm{p}=0,01)$ in the controls. This seems to indicate that DHA/EPA treatment of dry AMD may be more effective than LuteinZeaxanthin therapy.

Commercial interest

\section{- F064}

AMD Drusenoid deposits "Lipid Type" characteristics and evolution. Multimodal imaging evaluation

GONZALEZC

FUTUROPHTA, Toulouse

Purpose To study AMD drusenoid deposits "Lipid Type" evolution with multimodal imaging. To individualize their evolution and complication and to consider various etiopathogenic AMD options

Methods 98 eves of 49 patients, 18 men, 31 women, with AMD AMD drusenoid deposits, "Lipid type": soft Drusen, Drusenoid PED (lipid type), were evaluated by Autofluorescence, IR imaging, ETDRS visual acuity (VA), ocular Fundus examination, Ocular Confocal Tomography exam (OCT), OCT en Face software (Spectralis HRAOCT). Size, characteristics, number, topography of the lesions, growth were evaluated, as well as their environment above and below. With OCT, each element was studied, compared cut to cut, layer to layer and time to time to itself and to each other data. Follow-up was done every 4 months during 2 years

Results Soft drusen were larger, roughly homogenous, dark-grey, translucent, domeshaped mounds of deposit under the RPE. Drusenoid PED, lipid type, were homogeneous, as convergence of soft drusen, dark-grey. Multimodal imaging, especially OCT, OCT en Face features let individualize "lipid type" drusenoid deposits: morphologic types appearing homogeneous, dark, optically empty, fatty. Evolution change, depend on hose characteristics, rather to IS/OS impairment, photoreceptor disappearance, RPE involution, and so atrophy. They seem result from a specific metabolic defect outcome, peculiar etiopathogenic pathways: lipid metabolic pathway disorder with particular metabolic dysfunctions inrolled. Furthermore, lipid type drusenoid deposits' prognostic, predictive value allow to designate them for biomarker feature

Conclusion AMD Drusenoid deposits "lipid type" knowledge, evolution allow better AMD metabolism dysfunction understanding and etiopathogenic concept 
- F065

Predictive value of outer retina Enface OCT imaging in geographic atrophy progression

GIOCANTI-AUREGANA (1), TADAYONIR (2), DOURMADP P (3) MAGAZZINIS (4), COHENSY (3)

(1) Ophthalmology, Avicenne hospital, Bobigny

(2) Ophthalmology, Lariboisiere hospital, Pari

(3) Centre d'imagerie et de Laser, Paris

(4) Carl Zeiss meditec, Paris

Purpose To determine wether there was a correlation between hypo reflective area in Enface OCT of outer retina at baseline, and geographic atrophy (GA) area 1 year later in dry age-related macular degeneration (AMD)

Methods Retrospective study. We included all patients who underwent a clinical examination for dry AMD from July to November 2013 and who had macular captured Enface OCT. Eyes with previously treated wet AMD were excluded.We analysed hypo reflectivity on Enface SD-OCT extraction (Cirrus 2, Carl Zeiss Meditec,Dublin) of the outer retina $20 \mu \mathrm{m}$ above retinal pigmented epithelium (RPE), corresponding to the disruption of ellipsoid line (EZ), and extraction below RPE corresponding to GA, the speed of progression of these 2 parameters, the pattern of these areas, best corrected visual acuity (BCVA) on ETDRS scale, and foveal involvement in GA.Our endpoint was the correlation between the difference area EZ-GA and GA enlargement over 1 year on Enface OCT. Our secondary endpoint was the pattern similarities between both of these parameters.

Results We included 38 eyes of 31 patients with dry AMD, mean age was 84,9 years. At baseline, mean size of GA area was $7.1 \mathrm{~mm} 2$, mean size of hypo reflectivity was 9.28 $\mathrm{mm} 2$, fovea was involved in GA in 49\% of cases, BCVA was 64.2 letters. One year later mean size of GA area was $92 \mathrm{~mm} 2$, fovea was involved in $54 \%$ of cases, BCVA was 58.8 letters. The rate of progression of GA was $2.04 \mathrm{~mm} 2$ per year. The difference EZ-GA at baseline and GA enlargement were not strongly correlated.The pattern of EZ disruption on Enface OCT was correlated in more than 2/3rd of cases with the pattern of GA 1 year later.

Conclusion EZ disruption and GA 1 year later are not constantly correlated

\section{- F067}

Detecting recurrence of macular edema in patients with wet AMD after anti-VEGF treatment using 3D-CTAG test

MILYUTKINA S (1), FINK W (2, 3), KOVALEVSKAYA M (1)

(1) Voronezh State Medical Academy, named after N.N. Burdenko, Ministry of Health Russian Federation, Voronezh

(2) Visual and Autonomous Exploration Systems Research Laboratory, California Institute of Technology, Pasadena, California, USA, Pasadena

(3) Depts. of BME, ECE, and Ophthalmology \& Vision Science, University of Arizona, Tucson, Arizona, USA, Tucson

Purpose To detect recurrence of macular edema inwet AMD patients after anti-VEGF treatment using 3D-Computer-automated Threshold Amsler Grid testing (3D-CTAG; Fink \&Sadun, 2004).

Methods Wet AMD patients ( $\mathrm{n}=10,11$ eyes) 6 months after 3 injections of Ranibizumab (Lucentis, Novartis). Group \#1 (n=5, 6 eyes): norecurrence. Group \#2 (n=5, 5 eyes): recurrence confirmed by OCT. Indices: \# of VF defects per eye (ND); lowest perceivable contrast level (CL); VF volume lost relative to hill-of-vision (VLRH); lost area grade (LAG): [scotoma area at lowest tested contrast sensitivity level/scotoma area at highest tested contrast sensitivity level] $\times$ scotoma depth (\%).

Results Group \#1:Visus $=0,43 \pm 0,18 \log M A R ; \quad N D=0,67 \pm 1,2 ; \quad C L=10 \pm 7,04 \%$; VLRH $=2,1 \pm 3,97 \% ; \quad$ LAG $=150,92 \pm 341,35 \%$. Group \#2:Visus $=0,51 \pm 0,49 \log M A R$; $\mathrm{ND}=1,4 \pm 0,55 ; \mathrm{CL}=38,8 \pm 12,03 \% ; \mathrm{VLRH}=15,18 \pm 10,56 \% ; \mathrm{LAG}=32,13 \pm 20,13 \%$

Conclusion In group \#2 3D-CTAG revealed scotomas typical for macular edema: VLRH $=15,18 \pm 10,56 \%$ compared to $2,1 \pm 3,97 \%(\mathrm{p}=0,05)$ in group $\# 1$ and LAG $=32,13 \pm 20,13 \%$ compared to $150,92 \pm 341,35 \%(\mathrm{p}=0,05)$ in group \#1. Observed functional disorders show that 3 -fold injection of Ranibizumab, adopted as the standard, appears to be insufficient in $45,45 \%$ of the cases. 3D-CTAG may be an effective method for self-monitoring the recurrence of macular edema in wet AMD patients.

Commercial interest

\section{- F066}

Assessment of risk factors of age macular degeneration in general population with STARS 2.0 questionnaire

CREUZOT-GARCHER C (1, 2), CHIAMBARETTA F (3), COHEN SY (4), KOROBELNIKJ (5), SOUIED E (4), WEBER M (6), DELCOURT C (7)

(1) Ophthalmology, Dijon

(2) Eye and Nutrition Group, CSGA INRA, Dijon

(3) Ophthalmology, Clermont Ferrand

(4) Ophthalmology, Créteil

(5) Ophthalmology, Bordeaux

(6) Ophthalmology, Nantes

(7) Epidemiology of Nutrition INSERM U897, Bordeaux

Purpose Age-related Macular Degeneration (AMD) is the leading cause of blindness and visual impairment in industrialized countries.STARS questionnaire was developed as a fast and simple tool for detecting individuals at high risk of AMD. A study performed with first version included more than 12,000 people and confirmed the relevance of different items. Some limits were also noticed and corrected in the present questionnaire named STARS 2.0.

Methods All patients over 55 years examined by eighty French ophthalmologists were included during one week selected at clinicien disctretion between March 2013 and March 2014. AMD risks factors were collected with a statistical weight assigned to each risk factors. A composite score given by the sum of each risk factors was calculated. Fundus examination to detect macular disease was performed in the same time.

Results 6837 Patients with 60\% of female were included. 31\%, 29.6\%, 29.6\% and 9.9\% of the patients were respectively between [55 and 65], [65 and 74], [75 and 85], and over 85 . Fundus exam found that $71 \%$ of the patient did not suffer from AMD on both sides while $10.3 \%$ of the patients presented with an advanced AMD on at least one eye.

Conclusion STARS 2.0 questionnaire can help ophthalmologists to identify AMD risk factors to prevent $\mathrm{AMD}$ in clinical practice. It should be used also in general population to detect patients at high risk for AMD.

Commercial interest

\section{- F068}

Management of choroidal neovascularisation in late onset retinal degeneration

WIRYASAPUTRA S, YONG SO

Tan Tock Seng Hospital Department of Ophthalmology, Singapore

Purpose Late onset retinal degeneration is a hereditary condition characterized by progressive difficulty in dark adaptation and risk of choroidal neovascularisation. This in turn leads to a marked decline in visual acuity. We describe our experience treating a patient with a positive family history of late onset retinal degeneration who developed subfoveal choroidal neovascularisation.

Methods Case report. A 57 year old Caucasian lady with a positive family history of late onset retinal degeneration and progressive decline in dark adaptation presented to us with a 1-week history of blurring of vision. Fundus fluorescein and indocyanine green angiography revealed a subfoveal classic neovascular membrane.

Results Our patient was initially treated with intra-vitreal Ranibizumab and clinical activity of neovascularisation was monitored with serial spectral domain optical coherence tomography and visual acuity. Serial scans revealed recurrence of activity within a month of intra-vitreal Ranibizumab. With the advent of intra-vitreal Aflibercept, we offered our patient a change in treatment regime. Treatment with Aflibercept has thus far demonstrated a stabilization of disease activity on spectral domain optical coherence tomography and visual acuity for at least 8 weeks.

Conclusion Whilst Ranibizumab and Aflibercept remain off-label for the treatment of late onset retinal degeneration, this suggests that Aflibercept is possibly more efficacious in the management of choroidal neovascular membranes in late onset retinal degeneration. 
- F069

Intravitreal anti-VEGF in submacular hemorrhage due to age related macular degeneration

DESSERRE J, LABALETTE P

Ophtalmologie, Lille

Purpose To evaluate efficacy and prognostic factors of intravitreal anti-VEGF for large submacular hemorrhage (HSM) secondary to age-related macular degeneration (AMD).

Methods Eighteen eyes of 18 patients with HSM neovascular AMD were reviewed HSM had to respect a diameter greater than three papillary disk diameters and with fovea participation.Visual acuity was considered at diagnosed and when best final visua acuity was achieved. HSM outcomes were measured by SD-OCT. Statistical analysis was used to evaluate association between visual acuity and different outcomes: macular status of fellow eye, hemorrhagic surface, hemorrhage localization, central foveal thickness, highest macular thickness and macular volume.

Results Mean duration of symptoms was $25.94+/-32$ days. Mean anti-VEGF injection was $3.31+/-0.3$. Mean hemorrhagic surface was $8.77+/-6.52$ disc areas. $62 \%$ patients presented their first exudative episode. The mean $\log M A R$ visual acuity improved from $1.5+/-0.52$ to $1.0+/-0.40$ at final examination $(\mathrm{p}=0.04)$. A gain of three EDTRS lines or more was found in $50 \%$ patients. No association was evident between opposite side status, hemorrhagic surface and initial central fovea thickness with final visual acuity Although an association between highest macular thickness, macular volume ( $p 0.05)$ and final visual acuity was significant. Patients with predominant sub-retinal pigment epithelial hemorrhage seemed to have a worst visual prognosis.

Conclusion Anti-VEGF therapy is a useful treatment for large HSM. In our study, highest macular thickness and macular volume at diagnosis seem more predictive than central fovea thickness or hemorrhagic surface. Factor prognosis need to be clearly identify to select patients for surgical procedures.

- F071

Intravitreal ranibizumab for neovascular agerelated macular degeneration with a Treat and Extend protocol: anatomical and functional results

MEILLON C, KOEHRER P, ISAICO R, BONNABEL A, BRON AM, CREUZOTGARCHERC

Department Of Ophthalmology, university Hospital, Dijon

Purpose To evaluate the efficacy of the Treat andExtend regimen using intravitreal ranibizumab for neovascular age-related macular degeneration (nAMD)

Methods A retrospective single-center study including naive nAMD patients confirmed with a fluorescein and indocyanine green angiography and a macular SD-OCT. After 3 monthly intravitreal ranibizumab injections, the interval between two injections was sequentially extended or shortened by 2 weeks depending on the presence or the absence of exudative disease with a maximum of 12 weeks. At each visit, an ETDRS VA and a macular SD-OCT were performed. The functional outcomes were the gain in VA and the proportion of patients gaining at least three lines. The anatomical outcome was central retinal thickness (CRT) on OCT. The secondary outcomes were the number of injections.

Results 111 patients were followed for one year, and 66 of them for two years. At baseline, the median VA was 59 [45-70] letters and the median CRT was 326 [271-402] $\mu \mathrm{m}$. The median VA gain was 9 [2-17] letters after one year and 10 [2-23] letters after two years. Almost 30\% gained more than three lines after 1 year and $42 \%$ after 2 years The median decrease in CRT was 80 [29-161] $\mu \mathrm{m}$ and 100 [57-151] $\mu \mathrm{m}$ after 1 and 2 years, respectively. The median number of injections was $9[8-10]$ after one year and 10 [2-23] after 2 years.

Conclusion A Tret and Extend regimen with ranibizumab provides good visual outcomes after 1 and 2 years of follow-up and seems to be an efficient strategy for the

\section{- F070}

Five-year results of ICG-Guided Photodynamic Therapy combined with intravitreal Ranibizumab for juxtapapillary polypoidal choroidal vasculopathy with macular involvement

PETROPOULOS IK, MATTER MA, DESMANGLES PM Ophthalmological Center of Rive, Geneva

Purpose Long-term results of the treatment of 3 patients with juxtapapillary polypoidal choroidal vasculopathy complicated by a serous macular pigment epithelium detachment (PED) are presented.

Methods Two women and one man ( 70,77 , and 84 years of age, respectively) presented with recent vision decrease in one eye. Decimal visual acuity (VA) of the affected eye was $0.2,0.6$, and 0.3 , respectively. Fluorescein and ICG angiography as well as optical coherence tomography revealed juxtapapillary polypoidal choroidal vasculopathy and a large, dome-shaped, serous macular PED.

Results Selective ICG-guided photodynamic therapy (PDT) with verteporfin directed to the juxtapapillary polyps was performed, followed by 3 monthly intravitreal injections of ranibizumab (Lucentis'), resulting in complete regression of the serous macular PED and in sustained VA increase. One month after the third injection, VA of the affected eye was $0.6,0.9$, and 1.0, respectively. One year later, VA of the affected eye was 0.7 , 1.0 , and 0.9 , respectively. During 5 years of follow-up, no recurrence of exudation was observed and VA remained the same.

Conclusion The association of selective ICG-guided juxtapapillary PDT with intravitreal injections of ranibizumab apparently led to scarring of the source of subretinal fluid, to protection of the adjacent normal choriocapillary network, and to protection against the VEGF spike described after PDT, thus enabling significant vision improvement. Combined treatment with ICG-guided PDT and intravitreal ranibizumab is beneficial for juxtapapillary polypoidal choroidal vasculopathy with macular involvement, offering excellent long-term results.

\section{- F072}

Treatment of neovascular AMD by Aflibercept (Eylea) with proreactive protocol

GONZALEZC

FUTUROPHTA, Toulouse

Purpose To evaluate the functional, anatomical, vascular flow, effects of intravitreal Aflibercept injections(IVT) for subretinal neovascularisation complicating AMD, done with Proreactive protocol, and the recurrences frequency at 1 year follow-up

Methods 42 eyes of 38 patients, 12 men, 26 women, with subretinal neovascularisation complicating AMD. 38 eyes were switch cases, 4 naïve cases. Patients received intravitreous Eylea, 3 times, every 4 weeks in an inductive treatment. The next injections depended on the follow-up results, and were done by series of 3 IVT, IVT done every 1 $1 / 2$ months. First exam, 2 months, and then 3 months' interval follow-up exam included ETDRS visual acuity (VA), complete ophthalmic examination, optical coherence tomography (OCT), fluorescein and infracyanine (ICG) angiography.VA and OCT were done before each IVT. We want to evaluate the incidence of this protocol on the functional results and frequency of recurrences so on the number of IVT needed

Results VA improved in 44\% cases, more than 10 letters in 22\% cases. Diffuse edema was $30 \%$ reduced in $88 \%$, pigment epithelial detachment was flattened and less dense in all cases, by OCT. Angiographic leakage reduced about 70\% in 65\% cases, $30 \%$ in $96 \%$ cases. At ICG, vascular flow, vessel's diameter were $2 / 3$ time less in $68 \%$ cases, $1 / 3$ in $97 \%$ cases. Most of patients had good functional, anatomical, vascular flow, results, with less IVT needed. Inductive treatment was sufficient in 12 cases, needed added IVT in 30 cases. This protocol was compared, discussed

Conclusion The results, improved visual function, reduction of exudation, leakage, low neovascularisation's flow at OCT, AF and ICG, less recurrences, suggest Proreactive Eylea protocol seems effective, less restrictive and appears attractive 


\section{- F073}

Neovascular amd: multimodal criteria of neovascular activity

GONZALEZC

FUTUROPHTA, Toulouse

Purpose To evaluate the multimodal criteria and the best evaluation of neovascular activity to indicate recurrence and so treatment indication

Methods 218 eyes of 170 patients, 50 men, 120 women, with subretinal neovascularization complicating AMD. First and 2 months' interval follow-up exam included ETDRS visual acuity (VA), complete ophthalmic examination with fundus exam, optical coherence tomography (HRA spectral domain OCT), fluorescein (FA) and infracyanine (ICG) angiography. VA and OCT were done before each IVT

Results Each exam was analyzed, evaluation was given, and compared to previous one. A score was established for each data, so exam, using cross. Every score was added to each other, the value of final score determine the degree of activity. The cross grading scale was: for VA: impaired $=++$, same $=+$, improved $=0$; for OCT: increased (inflammation, diffuse edema, MCO, SRD, Neovascularization thickness) $=++$, same $=+$, reduced $=0$; for FA: increased (diffusion, occults, PED, MCO, Neovascularization density $)=++$ (according to increased intensity : ++++ (intense), +++ (moderate), ++ $($ mild $)$ ), same $=+$, decreased $=0$; for ICG: High Flow NeoVx $=++++$, Middle Flow $\mathrm{NeoV} x=+++$, Low Flow NeoVx $=++$. Score superior or equal to 8 mean Neovascular activity and so treatment indication. Using those criteria, Neovascular activity definition is more discriminating and efficient. Treatment indications are more precise and discerning. With those Neovascular activity and indication treatment criteria, this so called Proreactive protocol was defined and applied

Conclusion The multimodal criteria of Neovascular activity allow us to improve Neovascularization definition, then treatment indications, therefore to propose Proreactive protocol treatment, optimization of antiVEGF IVT treatment

- F075

The functional effects and expression of HGF and FGF in the choroid

STEWART EA, AMOAKU WM

Academic Ophthalmology, University of Nottingham, Nottingham

Purpose Age-related macular degeneration is the most common cause of irreversible visual loss in the increasingly ageing population of the western world. Most current treatments focus on sequestering excess vascular endothelial growth factor (VEGF) produced in response to inflammation and ischaemia. However, compensation by other angiogenic growth factors such as hepatocyte growth factor (HGF) and fibroblast growth factor (FGF) may occur

Methods Expression of HGF and FGF and their receptors MET and FGFR2 was investigated in vivo and in vitro in choroidal endothelial cells (CEC) using immunostaining, western blotting and ELISA. The in vitro response of CEC to these factors was assessed using proliferation and angiogenesis assays. Real time PCR was used to quantify signalling responses through gene expression of key signalling molecules such as HIF and expression of VEGF

Results Expression of HGE, FGF and their receptors was found in human choroid and cultured CEC. Both growth factors increased proliferation and angiogenesis independent of, and additive with VEGF. VEGF and HIF gene expression were altered dependent on growth factor concentration and time.

Conclusion Although much research and treatment focuses on VEGF, other growth factors which may compensate for the loss of VEGF signalling have significant stimulatory effects on angiogenesis. This study supports recent clinical advances into combination therapies.

Commercial interest
- F074

The impact of intravitreal aflibercept on pigment epithelial detachement morphology in eyes with persistent subretinal fluid despite a minimum of 14 previous treatments with ranibizumab in patients with neovascular age-related macular degeneration

PASU S, LUK S, MITRY D, KURUMTHOTTICAL M, YOUNIS S

Western Eye Unit, London

Purpose To assess the efficacy of intravitreal aflibercept $(2.0 \mathrm{mg})$ in patients with treatment-resistant neovascular age-related macular degeneration.

Methods Retrospective analysis of eyes treated with aflibercept with persistent subretinal and/or intraretinal fluid despite a minimum of 14 previous treatments with intravitreal ranibizumab $(0.5 \mathrm{mg})$. All patients were switched to intravitreal aflibercept $2.0 \mathrm{mg}$ ) and analyzed after 3 consecutive injections and at month 4 of treatment. Main outcome measures included change in visual acuity, central foveal thickness, and the height and diameter of the largest pigment epithelial detachment on spectral domain optical coherence tomography.

Results Fifteen eyes of fifteen patients were analysed. The median number of previous injections of ranibizumab was 19 (IQR 15-22). At month four, the mean visual acuity and central retinal thickness (CRT) did not change significantly from baseline: $\log M A R$ 0.64 (95\%CI- 0.45-0.83) and 0.65 (0.44-0.89; $\mathrm{p}=0.5)$; CRT $282 \mu \mathrm{m}(233-333 \mu \mathrm{m})$ and $247 \mu \mathrm{m}(201-293 \mu \mathrm{m} ; \mathrm{p}=0.06)$. However, the maximum pigment epithelial detachment height improved significantly from $279 \mu \mathrm{m}(95 \% \mathrm{CI} 124-434 \mu \mathrm{m})$ to $195 \mu \mathrm{m}(95 \% \mathrm{CI} 65-$ $326 \mu \mathrm{m})(\mathrm{p}=0.03)$ and pigment epithelial detachment diameter decreased significantly from $2,515 \mu \mathrm{m}(95 \% \mathrm{CI} 1,705-3,3324 \mu \mathrm{m})$ to $1,833 \mu \mathrm{m}(95 \% \mathrm{CI} 256-1,106 \mu \mathrm{m})(\mathrm{p}=0.002)$. Conclusion Intravitreal injections of aflibercept resulted in a significant improvement in the maximum height and width of the PED in eyes with a minimum of 14 previous injections of ranibizumab.

\section{- F076}

Cost of complications for patients with non-infectious nonanterior uveitis

TUNDIA N (1), SKUPM (1), OYE B (2), OKALIBE X (2), CHAO J (1), BAO Y (1), DICK A (3)

(1) AbbVie Inc., North Chicago

(2) University of Illinois, Chicago

(3) Bristol Eye Hospital, Bristol

Purpose To estimate costs associated with eye-related complications in non-anterior non-infectious uveitis (NIU).

Methods Cases were $\geq 18$ years old, had $\geq 2$ diagnoses for non-anterior (intermediate, posterior, or pan) NIU (Truven Health, 1/1/2000-12/31/2010), and developed complications (glaucoma, cataracts, visual disturbance, blindness, retinal detachment, cystoid macular degeneration). Cases and controls (NIU patients without complications) were matched 1:1 by uveitis type, sex, age, region, index date, and enrollment and followed for 1 year post complication. Annual mean total costs differences (medical and drugs) post complication were compared (ANCOVA).

Results Annual total cost difference for any complication was significantly greater in patients with non-anterior-NIU overall $(\$ 7,596, \mathrm{~N}=1,327)$, posterior-NIU $(\mathrm{N}=1,246$, $\$ 7,117)$, and pan-NIU $(\$ 24,595, \mathrm{~N}=27$ ) vs controls (all $\mathrm{P}<.05)$; medical costs contributed more than drug costs. Annual mean total costs for visual disturbances, cataracts, and glaucoma combined were greater for non-anterior-NIU overall, posterior-NIU, and panNIU vs controls by $\$ 23,915, \$ 23,182$, and $\$ 50,213$, respectively. Overall, the 1 -year postcomplication total cost burden was greater by $\$ 10,079,892$ in non-anterior NIU patients who developed complications vs controls.

Conclusion This medical claims analysis revealed a significant economic burden due to complications of non-anterior NIU.

Commercial interest 
- F077

Influence of lornoxicam and triamcinolone on the dynamics of eye remodeling in concanavalin model of inflammation

ERDIAKOVA, GAVRILOVA S

Physiology and General Pathology Department, Faculty of Medicine, M.V. Lomonosov Moscow State University, Moscow

Purpose To compare the effects of NSAID lornoxicam and corticosteroid triamcinolone on the dynamics of eye remodeling in concanavalin model of inflammation.

Methods Eye of Wistar rat was injected with $0,5 \mathrm{mcg}(2,5 \mathrm{mcl})$ concanavalin A (ConA) in vitreous cavity. After $20 \mathrm{~min}$ rats received a saline, $16 \mathrm{mcg}$ lornoxicam or $80 \mathrm{mcg}$ triamcinolone intravitreal injection $(2 \mathrm{mcl}$ ). On 1 and 2 day after that the medication was administered systemically. Histological evaluation of retinas was made on 7, 14, 28 and 56 days after inravitreal injection of ConA.

Results The total frequency of cararactogenesis after the injection of ConA was $72 \%$ Lornoxicam and triamcinolone suppressed the development of cataracts. Lornoxicam 6 times decreased the total frequency of eye hemorrhage. After 7 days following ConA injection lornoxicam decreased thickness of more retina layers than triamcinolone After 14 days triamcinolone increased the thickness of the outer plexiform, inner nuclear inner plexiform layers as compared with the control group. At day 14 lornoxicam but not triamcinolone reduced the thickness of ganglion cell layer (GCL). At day 28 both drugs reduced the thickness of GCL. Phospholipase A2 and cyclooxygenase (COX) blocking reduced structural abnormalities of the retina, but its thickness increased on 56 day after triamcinolone administration.

Conclusion Lornoxicam prevented the development of proliferative vitreoretinopathy (PVR), reduced the severity of symptoms typical for proliferative diseases of the eye. It also inhibited the proliferation of retinal cells. Triamcinolone impaired the overall health of animals, less prevented the development of PVR compared to lornoxicam. Its effect on the proliferation of retinal cells was ambiguous.

- F079

Ophthalmologists play a key role in the management of syphilis presenting with ocular involvement

BONNINN (1, 2), LAURICHESSE H (3), BEYTOUT J (3), LESENS O (3), ANDREM (4), AUMAITRE O (4), CHIAMBARETTA F (1)

(I) Ophthalmology, Clermont-Ferrand

(2) EA 7281 R2D2, Biochemistery Laboratory, Medicine Faculty, Auvergne University, Clermont-Ferrand

(3) Infectious diseases, Clermont-Ferrand

(4) Internal Medecine, Clermont-Ferrand

Purpose To provide the key role of ophthalmologists in the management of syphilis presenting with ocular involvement, describe the increase of patients diagnosed with syphilis infection presenting ophthalmological involvement and their clinical features.

Methods Retrospective, observational case series analysis: patients were selected retrospectively based on serological criteria: VDRL $>1$ and TPHA $>80$, with upper cut-off limit TPHA<320 and VDRL $<4$ (cases with VDRL $<4$ were retained if TPHA $>$ 1280). Among these patients, patients presenting ophthalmological involvement were analyzed further

Results Over the 10-year period, among 126 patients diagnosed with syphilis, 22 patients with various ocular involvements were treated. The resurgence of syphilis has also been registered in ophthalmology. Among the specialized departments, ophthalmology ranks second in number of cases of syphilis infection diagnosed: 77\% of patients with ocular involvement are diagnosed in ophthalmology.

Conclusion This underlines the central diagnostic role of ophthalmologists, as in cases of eye involvement, if ophthalmologists ignore the infection, diagnosis will rarely be picked up in other care units, especially given that visual symptoms are isolated in $80 \%$ of cases.
- F078

Risk of ocular complications in persistent non-infectious nonanterior uveitis

DICK A (1), TUNDIA N (2), SKUP M (2), SORG R (3), ZHAO C (3), BAO Y (2)

CHAOJ (2)

(1) Bristol Eye Hospital, Bristol

(2) AbbVie Inc., North Chicago

(3) Analysis Group, Inc, Boston

Purpose To assess risk of developing ocular complications in privately insured US patients with persistent non-anterior NIU compared to matched controls without uveitis

Methods Adults 18-64 years old with $\geq 2$ NIU ICD-9-CM claims for intermediateposterior- or pan-NIU were identified (OptumHealth, 01/01/1998-31/03/2012), and those with $\geq 90$ days of corticosteroids, immunosuppressants, and/or biologics use were defined as persistent cases. Cases were matched 1:1 by sex, age, region, company, employment status, and index date to controls. Risks of developing ocular complications were compared using unadjusted Kaplan-Meier survival (risk of and time to complications) and adjusted Cox regression (hazard ratios) analyses.

Results During the follow-up period, persistent cases $(\mathrm{N}=302)$ had significantly higher risk of any ocular complication vs their matched controls $(\mathrm{P}<001)$; the 1-, 5- and 10-year risks were $55 \%$ vs $5 \%, 83 \%$ vs $27 \%$, and $88 \%$ vs $49 \%$, respectively. Five-year risks of specific complications for cases vs controls were glaucoma (31\% vs $10 \%)$, cataract ( $57 \%$ vs $14 \%$ ), visual disturbance ( $41 \%$ vs $9 \%$ ), blindness/low vision ( $4 \%$ vs $2 \%$ ), retinal detachment $(13 \%$ vs $1 \%)$, and retinal disorder ( $42 \%$ vs $3 \%)$. Adjusted analysis showed persistent cases had hazard rates that were $8.9,8.1,6.2$, and 4.2 times higher than controls for any complication, visual disturbance, cataract, and glaucoma, respectively

Conclusion Patients with persistent non-anterior NIU are at high risk of ocular complications, which suggests high unmet need for an optimal treatment.

Commercial interest

- F080

Bilateral ocular involvement in a patient with tertiary syphilis

ZOZOLOUM (1), MINAKAKIS P (1), KANELLAS D (1), XIROU T (2),

KABANAROUS (2), VAIKOUSIS E (1)

(1) Eye Clinic, Nikaia General Hospital, Piraeus

(2) Eye Clinic, Hellenic Red Cross Hospital, Athens

Purpose To report a case of a 46-year-old man with bilateral neuroretinitis due to tertiary syphilis.

Methods The patient presented at the clinic with a history of blurred vision. He was agitated and was looking unwell. Snellen Visual acuity (VA) was 0.2 in the right eye and 0.1 in the left eye. Pupillary reflexes to light were sluggish but normally reactive to accommodation. Fundoscopy showed bilateral optic disc oedema and fluorescein angiography revealed active neuroretinitis Visual fields (VF) were abnormal in both eyes. Laboratory blood tests, cardiological investigations and a brain MRI were performed. Mantoux test was negative and IV treatment with corticosteroids was initiated.

Results Treatment with steroids was discontinued due to the appearance of a maculopapular rash on the head and neck. Laboratory results showed: RPR (Rapid Plasma Reagin)/FTA: positive, TPPA of serum and CSF (IgG, IgM): positive. Virological tests were negative. Based on these findings the diagnosis of neurosyphilis was made. Cardiological investigation was normal and brain MRI did not show any major abnormalities. Patient was started on IV crystalline penicillin, 3-4 million units q $4 \mathrm{~h}$ for two weeks. Three months after treatment VA was 0.6 in the right eye and 0.4 in the left. There was an improvement on the VF mainly in the right eye. Electroencephalogram showed no significant pathology. ISCEV standard full field and pattern electroretinography were affected bilaterally.

Conclusion Bilateral neuroretinitis due to tertiary syphilis affects vision and complete visual recovery mav not occur despite adequate treatment. 


\section{- F081}

A rare case of Crohn's disease with papilledema of the optic nerve

KOEVK

Department of Ophthalmology, Medical University, Sofia

Purpose A case of a thirty year old woman is described with Crohn's disease who presented with ocular symptoms. Crohn's disease is a type of inflammatory bowel disease, resulting in swelling and dysfunction of the intestinal tract.

Methods The patient was examined for visual acuity, Optical coherence tomography (OCT), automatic computerized perimetry (ACP) and fundoscopy. Except the intestinal manifestations peculiar for Crohn's Disease, in the patient were observed articular, dermatological manifestations (dermatitis and psoriasis of the forehead and the head).

Results The patient examined presented with ocular changes, which is very uncommon with the disease $\mathrm{VOD}=1.0+0,75 \mathrm{DS} V \mathrm{OS}=1.0+0,75 \mathrm{DS}$. We observed changes in the course of the conjunctival vessels, tortuosity and a light edema of the vessels. Similar changes were observed in the vessels of the posterior pole of the retina. We observed changes in the papilla of the optic nerve, which are unrecognized in literature. Under the OCT performed, we observed edema in the central areas of the optic nerves, more pronounced in the vascular funnel of the right eye - $534 \mu \mathrm{m}$, and less pronounced in the left eye - $356 \mu \mathrm{m}$. Under an ACP, we observed changes in the blind spot.

Conclusion Generally, in ophthalmologic aspect, Crohn's Disease affects episclera cornea, conjunctiva, iris and uvea. There is presented a rare case of Crohn's Disease with changes in the papilla of the optic nerve.
- F083

Cystoid macular edema and ocular toxocariasis in adult patients

DESPREAUX R (1), FARDEAU C (1), CHAMPIONE (1), BRASNU E (2),

BODAGHI B (1), LEHOANG P (1)

(1) Hôpital La Pitié-Salpétrière, Pari

(2) Centre National d'Ophtalmologie des Quinze-Vingts, Paris

Purpose To report clinical features in proven ocular Toxocariasis in adult patients.

Methods This retrospective study included 14 adults suffering from chronic uveitis whose clinical signs suggested an ocular toxocariasis, secondarely confirmed by semi-quantitative western blot (WB) in aqueous or vitreous humor. The clinical characteristics and signs of uveitis had been analysed, especially : anterior chamber flare meter measurement, vitreous aspect, presence of retinal granuloma, retinal haemorrhages or cystoid macular edema.

Results We had included 6 women and 8 men. The average age of patients at diagnosis was 45.6 years with an average time of diagnosis of 15.1 months. Uveitis was unilateral. All patients having a vitreous inflammation whose 11 also had a panuveitis. The main clinical signs observed were : one or more active focal deep whitish lesions in retinal periphery $(78 \%)$, retinal hemorrhages $(36 \%)$ or vitreoretinal tractions $(64 \%)$. The main posterior segment complication observed was a chronic macular edema (71\%). Patients had been followed during an average of 21.4 months.

Conclusion Ocular toxocariasis could induce chronic unilateral posterior uveitis in adults. The presence of a vitreous cellular inflammation is consistenly found. While whitish peripheral granuloma and vitreoretinal tractions appear very suggestive, a chronic cystoid macular edema is found as main complication. This diagnosis could be delayed in atypical forms so aqueous humor and vitreous sampling for testing toxocariasis by semi-quantitative WB remains still helpful.

\section{- F082}

Multifocal choroïditis: is it a granulomatosis close to sarcoidosis?

BENLAHBIB M, HAJIIZ, BENCHERIFA F, BOULANOUAR A, BERRAHOA

Ophtalmologie B, Hôpital des spécialités, Rabat

Purpose Multifocal choroiditis is an idiopathic posterior uveitis. It combines small white spots in fundus of eye and recurrent intraocular inflammation. Some biological or radiological manifestations of sarcoidosis may be found in these patients.

Methods We report a case of severe multifocal choroiditis associated with systemic granulomatosis. The aim of our study was to describe the clinical and therapeutic aspects, and discuss the etiopathogenic approach of this rare entity.

Results A 45 years old women who consults for blurred vision. Visual acuity is reduced at $1 / 20 \mathrm{P} 8$ in right eye and $3 / 10 \mathrm{P} 5$ in the left. There is a hyalite + bilaterally. Peri papillary atrophy, hemorrhage around the macula, multiple white and pigmentis small spots was found in fundus of eyes. Retinal thickening and involvements of the pigment epithelium and choroid are revealed by macular OCT in the right eye. The fluorescein angiography show hypofluorescence of chorioretinal spots in early time, a window effect scarring and bilateral papillitis. The ECA is 74 UECA. Chest scan reveal a non specific parenchymal micronodules. The patient received systemic corticosteroids. Control OCT show a subretinal fibrosis explaining the introduction of immunosuppressant treatment. The final visual acuity is $1 / 10$ right and $12 / 10$ left with a decline of 3 years.

Conclusion Although intraocular manifestations of multifocal choroiditis, it most correspond to a systemic granulomatosis near sarcoidosis.
- F084

Reactivation of oxoplasmic retinochoroiditis after macula hole surgery

HALFELD FURTADO DE MENDONCA R (1), DE OLIVEIRA MAIA JR O (2)

(1) University of Juiz de Fora, Juiz de Fora

(2) Monte Tabor Foundation. São Rafael Hospital, Salvador

Purpose To describe a very interesting case of reactivation of ocular toxoplasmosis after macula hole surgery.Setting : Toxoplasmic retinochoroiditis is the most frequently cause of posterior uveitis worldwide and reactivation is unpredictable.

Methods A 53 year old high myopic man complained of blurred vision in his left eye over the past 3 months. He underwent cataract surgery with IOL in both eyes one year ago with success. Visual acuity in the right eye was 20/20 and in the left eye 20/400. The intraocular pressure was $12 \mathrm{mmHg}$ in both eyes. Fundus examination of the right eye was normal. Fundus examination of the left eye showed a macular hole and a small inactive toxoplasmosis scar

Results The patient underwent vitrectomy to relieve traction on the edge of the hole. At 20-days follow-up, the visual acuity in the left eye was 20/100. Fundus examination showed a complete resolution of the macular hole. Two months later, the patient had a reactivation of the toxoplasmic retinochoroiditis and the visual acuity dropped to counting fingers at 2 meters. After a specific treatment for Toxoplasmosis, the visual acuity improved to $20 / 80$ (two months later).

Conclusion Toxoplasmosis reactivation may develop after vitrectomy. More studies are necessary to confirm if, in the presence of preoperative toxoplasmic chorioretinitis scars, the prophylactic treatment with antiparasitic drugs is necessary. 
- F085

Serous macular detachment of the neuro-epithelium in patient with tuberculous choroidal granuloma.

HALFELD FURTADO DE MENDONCA R (1), DE OLIVEIRA MAIA JR. O (2)

(1) University of Juiz de Fora, Juiz de Fora

(2) Monte Tabor Foundation. São Rafael Hospital, Salvador

Purpose To describe an interesting serous macular detachment of the neuroepithelium in a patient with tuberculous choroidal granuloma. Setting : Choroidal tubercles are a common manifestation of ocular tuberculosis and represent a direct mycobacterial infection of the choroid.

Methods A 41 year old man complained of blurred vision in his right eve over the past 5 days. His brother was recently treated for pulmonary tuberculosis. Visual acuity in the right eye was 20/40 and in the left eye 20/20. The intraocular pressure was 12 $\mathrm{mmHg}$ in RE and $10 \mathrm{mmHg}$ LE. Fundus examination of the right eye showed an active tuberculous choroidal granuloma. Fundus examination of the left eye was normal. The OCT of the right eye showed a serous macular detachment of the neuro-epithelium. The tests were positive for tuberculosis.

Results The patient underwent a specific treatment for tuberculosis. At 3-months follow-up, the visual acuity in both eyes was $20 / 20$. Fundus examination showed a complete resolution of the tuberculous choroidal granuloma and of the serous macular detachment of the neuro-epithelium.

Conclusion Ocular tuberculosis may occur in the absence of systemic clinical activity and may mimic several clinical entities. In some cases, the ophthalmologic exams are very important for the early diagnosis to avoid systemic complications.

- F087

Bilateral multifocal chorioretinitis and optic neuritis due to epstein-barr virus; a case report.

CHATZIRALLI I, PARIKAKIS E, PEPONIS V, DRAKOS E, MITROPOULOSP Ophthalmiatrion Athinon, Athens

Purpose Only few cases of Epstein-Barr virus (EBV) involving the retina or the optic nerve have been reported. Our purpose was to report a case of bilateral chorioretinitis and optic neuritis due to EBV.

Methods A 67-year-old man with no previous ocular history presented with decreased vision in both eyes since two months. The patient underwent a thorough ophthalmological examination, including visual acuity measurement by means of Snellen charts, slit-lamp examination, intraocular pressure (IOP) measurement and dilated fundoscopy. His past medical history included EBV encephalopathy, which was confirmed serologically, few months before.

Results At presentation, his best corrected visual acuity (BCVA) was 6/60 in the right eye and hand movement in the left eye. The anterior segment was totally normal and IOP was normal in both eyes as well. Dilated fundoscopy revealed multiple well-demarcated, gray to white areas of retinal atrophy involving predominantly the periphery in the right eye and the macula in the left eye. Of note, all lesions were on the pathway of retinal vessels. Bilateral chorioretinitis and optic neuritis were confirmed by fluorescein angiography as well as electrophysiological tests and presumed to be due to EBV.

Conclusion It is very important to include EBV in the differential diagnosis of chorioretinal atrophic lesions. Clinicians should be aware of ocular manifestations of $\mathrm{EBV}$, in order to suggest ophthalmological examination and start treatment promptly before irreversible damage of the optic nerve or retina.

\section{- F086}

Ocular manifestations in Korean patients with transbronchial lung biopsy-proven sarcoidosis

SHIN J, YUNG Y

Department of Ophthalmology \& Visual Science, Seoul

Purpose To evaluate the characteristic ocular signs and systemic examination findings in patients with transbronchial lung biopsy (TBLB)-proven sarcoidosis

Methods A total of 28 patients with TBLB-proven sarcoidosis were evaluated between January 2011 and December 2012. The medical records of patients were retrospectively reviewed. We evaluated the following five characteristic ocular signs that are suggestive for the diagnosis of sarcoidosis: (1) mutton-fat keratic precipitates and iris nodules; (2) nodules at the trabecular meshwork and tent-shaped peripheral anterior synechia; (3) snowball or string-of-pearls vitreous opacities; (4) nodular periphlebitis; (5) multiple chorioretinal lesions. The results of systemic examination, including serum angiotensin converting enzyme (ACE) assay, chest radiography or computed tomography of the chest, pulmonary function tests (PFT), tuberculin skin test and bronchoalveolar lavage (BAL), were considered for each patient.

Results Eighteen patients (64.2\%) showed ocular signs, which are suggestive of ocular sarcoidosis. Thirteen $(72.2 \%)$ of these 18 patients presented with ocular signs as the initial manifestation of the disease. The typical findings of chest CT, such as bilateral hilar lymphadenopathy (BHL), were observed in all of the 28 patients.

Conclusion It can be suggested that intraocular lesions could be the sole clinical manifestation of the disease. Therefore, it is important to perform pulmonary examination including chest $\mathrm{CT}$ in patients with suspicious ocular sarcoidosis.

\section{- F088}

\section{A review of acute posterior multifocal placoid epitheliopathy}

PEREZ NAVARRO I, ALMENARA MICHELENA C, CARAMELLO C, HERRERA L, PEREZ GARCIA D, MARTINEZ M, ESTEBANO

Ophthalmology.Hospital Clinico Lozano Blesa, Zaragoza

Purpose Acute posterior multifocal placoid pigment epitheliopathy (APMPPE) is a rare inflammatory disease, generally of unknown a etiology, affecting the choriocapillaris, the pigment epithelium and the outer retina. It predominantly affects young patients.

Methods Our patient was a 14-year-old female presented with rapid bilateral loss of vision She reported lu-like symptoms and taking antibiotics therapy. Fundoscopy revealed yellow-white subretinal lesions in the posterior pole with foveolar affectation of both eyes. Fluorescein angiography in acute stage of the disease demonstrated early hypofluorescence followed by later hyperfluorescence of the lesions. Indocyanine green angiography demonstrated hypofluorescence in all stages of the angiogram.

Results After a period of 7 days oral prednisone treatment she was completely recovered

Conclusion Acute posterior multifocal placoid pigment epitheliopathy (APMPPE) is characterized by rapid, but transient loss of visual acuity. Diagnosis is established in fluorescein angiography and prognosis for recovery is good, however in cases with poor visual acuity glicocorticosteroids might be beneficial. Usage of steroids is recommended for treating APMPPE in cases where macula is involved and in recurrent cases 


\section{- F089}

Eye AIDS patholgy evaluation by using ultrasound examination

KRIAUCIUNIENE L (1, 2), BARZDZIUKAS V (1, 2), LIUTKEVICIENE R (1, 2), GUMBELEVICIUS A (2)

(1) Lithuanian University of Health Sciences, Neuroscience Institute, Kaunas (2) Kaunas Eye Clinic, Kaunas

Purpose Ultrasound B scan is an important diagnostic method in eye pathology. We want to demonstrate the usefulness of ultrasound B scan in early diagnosis of AIDS symptoms and structural changes in the eyes. 30 years old female with bilateral uveitis was examined. Patient had suddenly unilateral loss of vision acuity.

Methods Patient undergo full ophthalmological examination and ultrasound B scan diagnostic examination.

Results During 2 weeks patient noticed sudden loss of vision acuity. Slit lamp examination revealed acute anterior uveitis. Fundus examination revealed hard changes in vitreous body. In right eye we diagnosed vitreitis and in left eye was more intensive changes of endophthalmitis. During systemic treatment of toxoplasmosis and AIDS in right eve we noticed full process stabilisation. Acute retinal necrosis and full loss vision we noticed in left eye. Ultrasound B scan revealed only structural changes of vitreous in right eye and vitreous hemorrhages and total retina detachment in left eye.

Conclusion We recommend early referral of cases of AIDS pathology in the eyes for baseline Ultrasound scan and follow up scan in 2 weeks, 4 weeks, 3 months and for later time to detect underlying pathology.

\section{- F091}

Immunosuppressive treatment in sympathetic ophthalmia longterm visual outcome

HRARAT L (1), FARDEAU C (2), LEHOANG P (2)

(1) 93, Stains

(2) 75 , Paris

Purpose Sympathetic ophthalmia(OS) is a chronic bilateral recurrent granulomatous pan uveitis following a traumatic or surgical transfixing wound.The aim of the study is to evaluate retrospectively the long-term visual outcome on various immunosuppressants(IS) used in the OS management

Methods Serial patients with fitting clinical and ancillary data were included in the study.On admission, all patients were treated with a methylprednisolone bolus followed by oral prednisone to $1 \mathrm{mg} / \mathrm{kg} /$ day,tapered before beginning IS therapy.Three phases have been identified for the collection of various parameters:Admission time,after one and three year under various IS treatments.The primary endpoint is a criterion combining cellular vitreous decrease of 2 marks with steroid dose sparing.

Results 10 eyes of 10 patients with OS were included.After admission time the administrated IS were: Azathioprine(1year $\mathrm{N}=6$ and 3years $\mathrm{N}=3$ ),Mycophenolate mophetyl(1year $\mathrm{N}=2$ ), Methotrexate(1year $\mathrm{N}=1$ ) Interferon alpha2a(1year $\mathrm{N}=6,3$ years $\mathrm{N}=1$ ). Evaluation of the primary endpoint is success at 1 year under IS:4 with Azathioprine $(66 \%)$,with Mycophenolate mophetyl(50\%),No success with Methotrexate and 6 with Interferon(100\%).The mean steroid sparing was $12 \mathrm{mg}$ under Interferon,18mg under Azathioprine,20mg under Mycophenolate mophetyl,20mg under Methotrexate.Evaluation of the primary endpoint is Success At 3 years under IS: 3 with Azathioprine(100\%)and 1 with Interferon(100\%).The mean steroid sparing was $7 \mathrm{mg}$ under Azathioprine and $5 \mathrm{mg}$ under Interferon.

Conclusion Interferon alpha2a and Azathioprine appeared the most effective in controlling the disease and in terms of steroid sparing effect.

\section{- F090}

Transient branch retinal artery occlusion as the first microvascular sing of systemic lupus erythematosus

PAWLOWSKI P (1, 2, 3), ZINCZUKM (4), PAWLOWSKA M (5), RESZECJ (2) (1) Department of Ophthalmology, Provincial Combined Hospital in Bialystok, Bialystok (2) Department of Medical Patomorphology, Cathedral of Biostructure, Medical University of Bialystok, Bialystok

(3) Laboratory of Electrophysiology of Vision, Department of Paediatric Ophthalmology and Strabismus, Medical University of Bialystok, Bialystok

(4) Department of Cardiology with Subdivision of Cardiac Intensive Care Unit, Marine Hospital in Gdynia,, Gdynia

(5) Department of Haematology, Medical University of Bialystok, Bialystok

Purpose To report a case of transient branch retinal artery occlusion (transient BRAO) in the course of lupus erythematosus preceding other systemic vascular complications. Methods Detailed ophthalmological examination including fluorescent angiography, SOCT and mfERG was performed. Laboratory test including morphology, antinuclear antibodies (ANA), antiphospholipid antibodies (APLA) were obtained. The patient was followed-up for six months and one and three years after

Results An 29-year-old woman was presented with the reduced best corrected visual acuity (BCVA) (Vos= 0.1), and the acute retinal ischaemic lesion. Laboratory tests and the history of thrombocytopenia indicated the systemic lupus erythematosus. 6 months later the mean BCVA improved from 0,1 to 0,9. The scotoma observed in her central visual field had been reduced. Multifocal ERG (Espion E2, Diagnosys LLC, USA) demonstrated the impaired retinal function and spectral domain optical tomography (Nidek RS-3000, Japan) revealed the macular thinning in the area of BRAO. Before the three-year-follow-up patient had been referred to kindney biopsy were the advanced lupus nephritis was diagnosed, and had been operated due to aortic aneurysm.

Conclusion The retinal vascular occlusion disorders in systemic lupus erythematosus may be the rare cause of transient $\mathrm{BRAO}$ in young patients and precede other systemic vascular complications.

\section{- F092}

Rare ocular involvement due to leukocidin "panton valentine" (pvl) positive staphylococcus aureus after bacterial endocarditis

ZOZOLOUM (1), MINAKAKIS P (1), PASCHALIDIS T (1), XIROU T (2),

KABANAROU S (2), VAIKOUSISE (1)

(1) Eye Clinic, Nikaia General Hospital, Piraeus

(2) Eye Clinic, Hellenic Red Cross Hospital, Athens

Purpose To present an unusual case of a 38-year-old man with pituitary apoplexy, bacterial endocarditis, and chorioretinitis due to leukocidin "PVL" positive staphylococcus aureus, not reported in the current literature.

Methods He presented with a history of sudden loss of vision, headache and high fever. Visual acuity (VA) was Hand Movements in both eyes with sluggish pupillary reflexes. Fundoscopy revealed bilateral yellowish focal retinal lesions and Roth's spots in right eye, indicative of active chorioretinitis. Fluorescein angiography (FA) showed active chorioretinal lesions. Visual fields and OCT were normal. Brain MRI showed a pituitary adenoma. The diagnosis of pituitary apoplexy was made based on clinical history, systemic findings and MRI imaging. He was treated with high doses of IV steroids. Snellen Visual acuity (VA) was improved to 0.8 in the right eye and 0.9 in the left eye. Three days later he presented Osler's nodes and septic emboli on palms and soles, characteristics of bacterial endocarditis. Blood culture showed leukocidin "PVL" positive staphylococcus aureus. Transesophageal echocardiogram demonstrated prolapse and degenerative alterations of the mitral valve. A quadruple IV antibiotic treatment was initiated for eight weeks. An operation to remove the pituitary adenoma in two months was also scheduled.

Results One year later his VA was 10/10 bilaterally. FA showed inactive chorioretinal scars. ISCEV standard full field ERG was affected bilaterally.

Conclusion Ocular complications may follow pituitary apoplexy and bacterial endocarditis due to "PVL" (+) Staphylococcus aureus. Early diagnosis is important for an effective treatment 


\section{- F093}

Mycophenolate mofetil and cyclophosphamide treatment suppress inflammation intensity in experimental model of autoimmune uveitis

KLIMOVA A (1), SEIDLER STANGOVA P (1), HEISSIGEROVA I(1),

SVOZILKOVA P (1), KUCERA T (2)

(1) Department of Ophthalmology, First Faculty of Medicine, Charles University in Prague and General University Hospital in Prague, Prague

(2) Institute of Histology and Embryology, First Faculty of Medicine, Charles University in Prague, Prague

Purpose The research in experimental models of autoimmune uveitis helps to find new therapeutical strategies. The aim of this study is to compare the effect of cyclophosphamide (CPA), mycophenolate mofetil (MMF) and golimumab on inflammation intensity in mice model of experimental autoimmune uveitis (EAU).

Methods EAU was induced in C57BL/6 mice by subcutaneous application of IRBP (interphotoreceptor retinoid binding protein) in complete Freund's adjuvant and pertussis toxin. CPA was injected intraperitoneally in a single dose of $100 \mathrm{mg} / \mathrm{kg}$ MMF intraperitoneally daily in a dose of 30 and $50 \mathrm{mg} / \mathrm{kg}$, golimumab subcutaneously weekly in a dose of $70 \mathrm{mg} / \mathrm{kg}$. Mice with EAU without treatment and group with EAU with sham treatment served as controls. Histological grading of uveitis at day 35 post induction of EAU was used.

Results The comparison of intensity of inflammation in group of treated and untreated mice shows that MMF significantly suppressed the inflammation $(\mathrm{p}<0,05)$. The absolute suppression of inflammation by CPA $(\mathrm{p}<0,0001)$ was shown. On the contrary, golimumab enhanced the ocular inflammation.

Conclusion In this study, we have confirmed the efficacy of CPA and MMF in treatment of posterior uveitis, which supports its use in human medicine.This project was supported by grant IGA MZ NT/14017-3/2013 and SVV-266505/2013.

- F095

Intravitreal ranibizumab for the treatment of irvine-gass syndrome

CHATZIRALLII, PARIKAKIS E, PEPONIS V, TSIOTRA V, MITROPOULOS P Ophthalmiatrion Athinon, Athen

Purpose The purpose of our study was to evaluate the potential efficacy and safety of intravitreal ranibizumab in patients with pseudophakic cystoid macular edema (CME).

Methods This retrospective study comprised seven eyes with CME treated with intravitreal ranibizumab. Patients were examined at one week one month after injection and monthly thereafter. Main outcome measures included changes in bestcorrected visual acuity (BCVA) and central retinal thickness (CRT). Safety was assessed by ophthalmic examination and report of systemic adverse effects.

Results There was a statistically significant difference on BCVA $(p<0.001)$ and CRT $(\mathrm{p}<0.001)$ before and after the ranibizumab injection. One injection appeared to be sufficient for the resolution of CME, while recurrence was observed in one patient, in a long-term follow-up. No observable ocular or systemic side-effects were found.

Conclusion Intravitreal ranibizumab seems to be effective and safe for the treatment of pseudophakic CME, demonstrating a statistically significant difference in BCVA as well as CRT.
- F094

Intravitreal dexamethasone implant in the treatment of postoperative cystoid macular edema (Irvine Gass syndrom): one year retrospective study

LANDRÉ C, GASTAUD P, BAILLIFS

Service d'Ophtalmologie CHU St Roch, Nice

Purpose To evaluate the efficacy of dexamethasone $0.7 \mathrm{mg}$ intravitreal implant in patients with chronic postoperative cystoid macular edema.

Methods Fourteen patients' charts were retrospectively reviewed. Inclusion criteria comprised patients with postoperative cystoid macular edema. The main outcome measures were best corrected visual acuity (BCVA) and mean central retinal thickness (CRT)

Results Patients mean age was 72.1 years. All patients were pseudophakic. Six patients were diabetic. Four patients had received a previous treatment with intravitreal ranimizumab. The mean BCVA before intravitreal dexamethasone was 0.75 logmar. It mproved to 0.56 at month 1 and was 0,45 at month 3 . The pre injection mean CRT was $584.6 \mu \mathrm{m}$. It improved to $309 \mu \mathrm{m}$ at month 1 and was 341,5 at month 3. Seven patients presented an elevated intraocular pressure during the follow up

Conclusion Intravitreal dexamethasone implant improved visual acuity and macular thickness at month 1 and month 3 in patients with postoperative cystoid macular edema. However its beneficial effect seemed to decrease at month 3 .

- F096

Efficacy of dexamethasone intravitreal implants in macular oedema excluding venous occlusions : results about 80 patients

DEGOUMOIS A (1, 2), AKESBIJ (2), LAURENS C (2), RODALLEC T (2), ADAM R (2), BLUMEN-OHANA E (2), LAPLACE O (2), VIREVIALLEC (2), LE DÛ B (2), GUYADER V, NORDMANN JP (2)

(1) CHU Caen, Caen

(2) CHNO des quinze-vingts, Paris

Purpose The dexamethasone intravitreal implant has shown efficacy in the treatment of macular edema (ME) of non-infectious uveitis and retinal venous occlusions. The aim was to evaluate the efficacy of this implant in the treatment of other diffuse macular edemas with an inflammatory mechanism.

Methods Retrospective cohort study over 2 years : from January 2012 to December 2013, including all patients who received at least one injection of intravitreal dexamethasone implant, excluding venous occlusions and non-infectious uveitis. The primary endpoint was the change in visual acuity.

Results 80 patients were included.The indications of treatment were : diabetic ME when anti-VEGF were ineffective (53\%), ME after retinal detachment(RD) (22\%), ME of Irvine Gass syndrome(16\%), ME after endophtalmitis(4\%), macular telangiectasia(4\%), ME of retinitis pigmentosa (1\%). The mean ETDRS visual acuity was 53.7 letters before injection, 62.3 letters after injection $(\mathrm{p}<0.001)$. The average gain in visual acuity was 6.7 letters [4.5:8.8] ( $<<0.001)$ in patients treated for diabetic ME, 9.6 letters [6.1;13.1] ( $<<0.001)$ for ME after RD, and 15.2 letters[10.2;20.2] $(\mathrm{p}<0.001)$ for Irvine Gass syndrome. The mean duration of efficiency was 4.6 months, with a median of 3.8 months.

Conclusion The intravitreal implant of dexamethasone appears to be an effective second-line treatment even in patients with diabetic ME after failure of anti-VEGF. It is also effective and well tolerated in patients with ME after RD, and Irvine Gass syndrome. 


\section{- F097}

Prognostic factors for epiretinal membrane surgery

PAPANDREOU I, FALLON T

Ophthalmology Department, Central Middlesex Hospital, London

Purpose To evaluate the outcome of epiretinal membrane surgery and to recognize potential prognostic factors

Methods Case notes and SD-OCT scans from all patients that were operated for epiretinal membranes between 01/2010 and 12/2013 were reviewed. All patients had received a standard 23G pars plana vitrectomy with epiretinal membrane peel by a single surgeon. Best corrected visual acuity (BCVA) on the logMAR chart was recorded before surgery and at the last visit. Central retina thickness (CRT) was measured manually on the corresponding OCT scans. OCT was performed with the Spectralis OCT device (Heidelberg Engineering, Heidelberg, Germany) using the standard protocol of the clinic.Statistical analysis involved the Wilcoxon signed rank test and Spearman correlation test.

Results 26 eyes of 26 patients were included in this study. The mean age was 76.2 years (range 36-87). The follow up time was up to 41 months with a mean of 15 . The mean BCVA was 0.89 before and 0.70 after surgery $(\mathrm{p}=0.01)$. The mean $\mathrm{BCVA}$ improvement of 2 lines remained among patients with idiopathic $(\mathrm{p}=0.02)$ and secondary membranes $(\mathrm{p}=0.29)$. Pre- and postoperative lens status, sex and the postoperative use of NSAIDs did not seem to affect the outcome. The postoperative BCVA correlated strongly with the preoperative one $(\rho=0,789, p<0.001)$ but not with the preoperative CRT. There was no statistically significant difference in the postoperative BCVA between patients with intact or disrupted ellipsoid zone, external limiting membrane (ELM) or fovea contour The preoperative existence of intraretinal cysts also did not seem to influence the BCVA after surgery.

Conclusion Preoperative BCVA seems to be the main prognostic factor for the outcome of epiretinal membrane surgery.

\section{- F099}

Severe inferior retinal folding after retinectomy: an uncommon form of posterior proliferative vitreoretinopathy

BARCATALIMG, DENIONE

Calvados, Caen

Purpose Relaxing retinectomy is a surgical procedure commonly used in the presence of a retinal shortening in retinal detachments with severe proliferative vitreoretinopathy (PVR. Severe inferior retinal folding (SIRF) is an uncommon form of posterior PVR observed after retinectomy. This rare complication has been described in a single publication so far.

Methods A 49-year-old woman presented with a $180^{\circ}$ giant retinal tear with diffuse posterior PVR.

Results She was treated with pars plana vitrectomy, retinectomy, internal limiting membrane peeling, endolaser and silicone oil tamponnade. A week after the retina was completely attached. Visual acuity was counting fingers. Twenty seven days after the intervention, a recurrent PVR detachment involving the lower hemi-retina was observed. The lower retinal edge had retracted upwards away from the retinopexy scar (SIRF). Six months after the patient underwent silicone oil removal, epi-retinal membrane peeling, repeat inferior retinectomy, endolaser and C2F6 tamponnade. The spared retina remained attached. Visual acuity was "hand motion"

Conclusion SIRF consists in an inferior widespread epi-retinal membrane whose massive retraction capacity overcomes retinopexy adhesion. The inferior retina ends up retracted upwards like a Venetian blind, away from the retinopexy scars. SIRF is rare. It has been described only in 5 of 254 patients after relaxing retinectomy over a 9 -year period in Gupta et al. study.
- F098 / 4673

Comparison of ocriplasmin efficacy and treatment guidance as determined by European regulatory agencies

KOROBELNIKJ

Chef du Service d'Ophtalmologie Hôpital Pellegrin, Bordeaux

Purpose Ocriplasmin, a truncated form of human plasmin, has proteolytic activity against components of the vitreous body and the vitreoretinal interface.

Methods Results from placebo-controlled trials designed to determine efficacy and safety of ocriplasmin for the treatment of VMT were used in the determination by the French National Health Authority (Haute Autorité de Santé, or HAS), the National Institute for Health and Care Excellence (NICE), and the Isntitute for Quality and Efficiency in Healthcare (Institut fur Qualitat und Wirtschaftlichkeit im Gesundheitswesen, or IQWiG). Different subgroups according to presence or absence of anatomic features and visual acuity (VA) were analysed

Results All 3 regulatory agencies recommend ocriplasmin for the treatment of VMT including when associated with $\mathrm{MH}$ less than or equal to 400 microns. The HAS recommends ocriplasmin for the treatment of adult patients with VMT for whom symptomatology does not require immediately a vitrectomy, at the earlier stage of disease. IQWiG reported indication of an added benefit for patients with mild (VA >60 ETDRS letters) to moderate visual impairment (VA 35-60 ETDRS letters). According to the NICE guidance, ocriplasmin is recommended as an option for treating VMT in adults only if an epiretinal membrane is absent, with or without severe symptoms.

Conclusion The benefit of ocriplasmin for the treatment of VMT including when associated with FTMH of 400 microns or less has been evaluated and confirmed by Europe's leading regulatory agencies. Minor differences exist in the recommendations from each country, based on anatomic features (absence of ERM), visual acuity, and severity of symptoms
- F100

Clinical review of rhegmatogenous retinal detachment in Korea - Does seasonal variation exist in rhegmatogenous retinal detachment?

IIN H, LIM HS, LEE KH

Ajou University School of medicine, Dept of Ophthalmology, Suwon

Purpose We investigated demographic and clinical features including seasonal variation of rhegmatogenous retinal detachment in Suwon, South Korea.

Methods We reviewed medical records of 778 patients who were diagnosed with rhegmatogenous retinal detachment at Ajou university hospital located in Suwon City (Gveuonggi-do, South Korea) from February 1995 to December 2013. Incidence of rhegmatogenous retinal detachment was counted by months and seasons. Demographic and clinical features were also analyzed.

Results Total 778 eyes of 778 patients ( 778 patients; 418 men and 360 women; the mean age, $46.6 \pm 17.3$ years; range, $10-85$ years) were included in the study. $86.1 \%$ (670 eyes) of eyes were phakic and high myopic eyes accounted for $11.6 \%$ (90 eyes) of the cases. The cases with single retinal break were $47.9 \%$ (373 eves) and the average count of retinal break was $1.46 .65 .8 \%$ (512 eyes) of the cases were treated successfully with sclera buckling. Incidence of retinal detachment was not different according to months $(\mathrm{p}=0.385)$ and seasons $(\mathrm{p}=0.090)$. Comparing the results of warm seasons (spring and summer) and cold seasons (autumn and winter), incidence of rhegmatogenous retinal detachment did not differ $(\mathrm{p}=0.071)$

Conclusion Unlike previous reports in Japan, Middle East and Europe, our study has no correlation with seasons. Previous studies suggested the higher incidence of rhegmatogenous retinal detachment in warm seasons is due to ambient temperature and increased outdoor activity, but summer in Korea is highly humid and hot. Also, it includes long rainy season, so it is not a good season for outdoor activity as other regions. These features may influence the result of our study 
- F101

Significant visual improvement after spontaneous resolution of longstanding macular detachment due to optic disc pit

PARIKAKIS E, CHATZIRALLII, STRATOS E, PEPONIS V, KARAGIANNIS D, TSIOTRA V MITROPOULOSP

Ophthalmiatreio Eye Hospital, Athens

Purpose To report a case of spontaneous resolution of a longstanding serous macular detachment associated with an optic disc pit, leading to significant visual improvement.

Methods A 63-year-old female presented with blurred vision and micropsia in her left eye since six months. Her best-corrected visual acuity was $6 / 24$ in the left eye and fundoscopy revealed serous macular detachment associated with optic disc pit, confirmed by optical coherence tomography.

Results The patient was offered the treatment alternative of vitrectomy, but she preferred to be reviewed conservatively. Three years after initial presentation, neither macular detachment nor subretinal fluid was evident in optical coherence tomography, while inner segment/outer segment (IS/OS) junction line was intact. Her visual acuity was improved from $6 / 24$ to $6 / 12$ in her left eye, remaining stable at the 6 -month followup after resolution.

Conclusion This is a case of spontaneous resolution of longstanding optic disc pit associated macular detachment with significant visual improvement, postulating that the integrity of IS/OS junction line may be a prognostic factor for final visual acuity and suggesting optical coherence tomography as an indicator of visual prognosis and probable necessity of surgical management.
- F102

Vitreoretinal complications in hematology patients after hematopoietic stem cell transplantation

PARK YH (1), YOO YS (1), SHIN IA (1), LEE J (2)

(1) Department of Ophthalmology and Visual Science, Seoul St Mary's Hospital, Seoul

(2) Department of Ophthalmology, Ilsan Paik Hospitla, Goyang

Purpose To identify vitreoretinal complications in patients after hematopoietic stem cell transplantation (HSCT)

Methods Patients with hematologic diagnosis after HSCT were enrolled this study at St. Mary's Hospital, Korea from 2009 to 2012. Among them, we included the patients who have survived more than 2 years at least since they transplanted. The frequency and subgroup analysis of patients with vitreoretinal complications were done. From the medical records, we confirmed the prognosis of them. As a factor associated with vitreoretinal complications, the occurrence of ocular graft-versus-host disease (GVHD) was also analyzed.

Results Total 635 patients were included in this study. There were 207 patients with CMV viremia, 16 (2.52\%) patients was diagnosed as CMV retinitis among them. 24 (3.78\%) patients was diagnosed as retinal hemorrhage. In addition, there were $5(0.79$ $\%)$ patients with uveitis, $2(0.31 \%)$ patients with non-proliferative diabetic retinopathy, $1(0.16 \%)$ patients with optic neuropathy, $1(0.16 \%)$ patients with retinal detachment, $1(0.16 \%)$ patient with central serous chorioretinopathy, $1(0.16 \%)$ patient with central retinal vein occlusion, $1(0.16 \%)$ patient with age-related macular degeneration, and $1(0.16 \%)$ patient with endophthalmitis. Most of them had regular follow-up without ocular treatments for retinal complications. In subgroup analysis, there was no significant difference in the incidence of ocular GVHD between groups which had vitreoretinal complications or not.

Conclusion The vitreoretinal complications is not usual event in patients who received HSCT. Most of them had good prognosis for retinal complications. The occurrence of vitreoretinal complications was not associated with ocular GVHD.

- F104

Optic nerve oximetry mapping using a novel metabolic hyperspectral retinal camera

DESJARDINSM (1), SYLVESTRE JP (2), TRLSSART R (3), ARBOUR JD (3), LESAGE F (1)

(1) Institut de génie biomédical, École Polytechnique de Montréal, Montréal

(2) Optina Diagnostics, Montréal

(3) Département d'ophtalmologie, Université de Montréal, Montréal

Purpose In retinal detachment surgery when primary vitrectomy is performed, macular atrophy limits visual recovery. The physiopathological mechanism of this macular impairment remains unknown. The purpose of the present study is to evaluate whether the vitreous composition could be a prognostic factor for visual outcome and macular atrophy.

Methods In a prospective study on 51 non diabetic patients undergoing primary vitrectomy for rhegmateous retinal detachment, a biochemical analysis of $\mathrm{Na}, \mathrm{K}, \mathrm{Cl}$ glucose was performed. Post-operative visual acuity and macular thickness assessed with OCT was correlated with the biochemical analysis.

Results In the group with low glucose levels, macular thickness was higher than in the patients with higher glucose levels ( $\mathrm{p}=0.0002 \mathrm{Mann}$-Whitney). No difference in terms of post-operative visual acuity was found between the 2 groups with high and low glucose levels in the vitreous. $\mathrm{Na}, \mathrm{K}, \mathrm{Cl}$ appeared to have no incidence on macular thickness or post operative visual acuity.

Conclusion Increased glucose levels appear to be assocated with macular atrophy. They may be reflect retinal glycation even in non diabetic patients.
Purpose Oximetry measurement of the principal retinal vessels represents a first step towards understanding retinal metabolic state. Spectral imaging is expected to significantly enhance metabolic state evaluation via local fundus oximetry determination. In this preliminary study, we focus on the oximetry of the entire optic nerve head $(\mathrm{ONH})$ microvasculature from datasets obtained with a novel metabolic hyperspectral retinal camera (MHRC).

Methods Five healthy volunteers had retinal images captured between 490-650 nm in steps of $5 \mathrm{~nm}$ using a prototype MHRC (Optina Diagnostics, Montreal, Canada) based on a tunable laser source that permits the selection of a specific wavelength $(2 \mathrm{~nm}$ bandwidth) from a supercontinuum source. Each subject's data was fit to a model where oxy- and deoxyhemoglobin are the main absorbers and scattering is modeled by a $\log (1 /$ wavelength) term. The fitted parameters were used to extract an estimation of oxygen saturation and total hemoglobin content

Results The local oximetry and hemoglobin content maps over the entire $\mathrm{ONH}$ microvasculature were extracted for all subjects from the spectral-rich information. Physiologically plausible oxygen saturation values were obtained for all subjects over this region (mean $58+/-6 \%$ ).

Conclusion The oximetry and hemoglobin content maps of the ONH microvasculature obtained with the MHRC could ultimately contribute to the diagnostic and optimal management of diseases affecting the $\mathrm{ONH}$ such as glaucoma. 
- F105 / 3665

Differences in optical coherence tomography findings in early intervention vs late intervention surgically treated submacular hemorrhages

CANASTRO M (1), FARIA M (1), MARQUES-NEVES C (2),

MONTEIRO-GRILLOM (2), NETO E (1), PERPETUA C (1), PROENCA H (1)

(1) Ophthalmology Department of Hospital de Santa Maria, Lisbon

(2) Ophthalmology Department of Hospital de Santa Maria, Faculdade de Medicina, Lisbon

Purpose The purpose of this retrospective observational study is to compare Spectral-Domain OCT findings in early-intervention vs late-intervention submacular hemorrages treated surgically with vitrectomy, recombinant tissue Plasminogen Activator (rtPA) subretinal injection and gas injection.

Methods Retrospective analysis of pre and post operative OCT findings of 4 patients with submacular hemorrhages treated with $23 \mathrm{G}$ vitrectomy, rtPA subretinal injection and gas (SF6, C3F8) injection. 2 patients were treated after 6 and 10 days of diagnosis establishment and the other 2 were treated after 21 and 28 days of diagnosis establishment. Subfoveal thickness was measured in both pre and post operative exams. Analysis of the retinal structure and submacular hemorrhage characteristics were performed.

Results The early intervention group presented with higher pre-operative subfoveal thickness (716 and 973 microns compared with 405 and 554 microns). Post operative OCT showed an important decrease in subfoveal thickness in the early intervention group (716 to 260 microns and 973 to 433 microns), not seen in the late intervention group ( 405 to 593 and 554 to 548 microns). Images analysis shows recent submacular hemorrhages as low density, thick, homogeneous, subretinal tumefactions that do not severely distort retina internal anatomy. Older submacular hemorrhages appear as more dense, thinner, heterogeneous masses, with retinal distortion, cysts formation and separation of the inner and outer layers of the retina.

Conclusion OCT is an important exam in the evaluation of submacular hemorrhage, which may differentiate recent and late hemorrhages. Early intervention is crucial for good post operative anatomical result.
- F106 / 4472

Multicenter OZurdex long term assessment for diabetic macular edema: MOZART 2 study

MATONTI F (1, 2), HAJJAR C (3), PARRAT E (3), MERITE PY (4), POMMIER S (5), MEYER F (4), PROST-MAGNIN O (6), GUIGOUS (7)

(1) P1.5 Network, Marseille

(2) Hôpital Nord - Ophtalmologie, Marseille

(3) P1.5 Network, Guadeloupe

(4) P1.5 Network, Aix-en-Provence

(5) P1.5 Network, L'Isle-sur-la-Sorgue

(6) P1.5 Network, Dijon

(7) P1.5 Network, Avignon

Purpose To evaluate the long-term efficacy and safety of intravitreal implant of $0,7 \mathrm{mg}$ dexamathasone (ozurdex*) in diabetic macular edema(DME)

Methods This was a retrospective, multicenter, study. 19 patients, with mean age of 67 years, followed for at least 8 months (mean 10,7 months) were included in 5 French eye clinics (P1,5 network). The mean systolic blood pressure was $138 \mathrm{mmHg}$ and the mean $\mathrm{HbAlc}$ was $7,2 \%$. We monitored 2 systemic parameters: blood pressure and glycemic balance. Best-corrected visual acuity (BCVA), central retinal thickness by spectralis OCT, intraocular pressure and cataract progression are studied at baseline and then at 1, 6 and 12 Months.

Results Visual acuity significantly increased compared to baseline: at M1, M6 and M12, mean value of 8,7, 9,1 and 5,8 ETDRSletters, respectively. The improvement was $\geq 10$ ETDRS letters in $46,7 \%, 57,9 \%$ and $58,3 \%$ of patients at M1, M6 and M12, respectively. The average CRT decrease was: $332 \mu \mathrm{m}$ at M1, $259 \mu \mathrm{m}$ at M6 and $296 \mu \mathrm{m}$ at M12. The average interval between two injections was of 5.6 months. Ocular hypertension greater than $25 \mathrm{mmHg}$, managed by topical treatment, was observed in 13,4\% of patients. None glaucoma surgery was necessary

Conclusion Ozurdex" has an anatomical and functional effectiveness in the treatment of DME. Outcomes for naive patients and lower CRT suggest that the duration of diabetes mellitus and previous treatments are negative factors for recovery. Side effects are rare and manageable. Ozurdex seems to be an effective treatment for visual impairment due to DME with a favorable safety profile. Patient follow-up must be adapted to half-life of the product with a control before M1 (intraocular pressure) and before M5 (DME recurrence)

Commercial interest

\section{- F108 / 4672}

Posterior hyaloid findings as visualized by SD OCT

KRASTEL H (1), HOFMANNF (2), BAIER D (2), KAHLERT CHR (3), KUZNIAR A (4), HARDER B (4), JONAS J (5)

(1) Dept. of Ophthalmology, University Eye Center, Mannheim

(2) Oculus Optik Geräte GmbH, Wetzlar

(3) Dept. of Ophthalmology, Klinikum Chemnitz, Chemnitz

(4) Dept. of Ophthalmology, University Medcal Center, Mannheim

(5) Mannheim Medical Faculty, University of Heidelberg, Mannheim

Purpose To direct attention to the structures of the posterior hyaloid as visualized by SD OCT, and to improve understanding of vitreo-retinal biomechanics.

Methods Findings were collected from a Wetzlar study cohort of subjects who gave their informed consent to gather experience in vitreal SD OCT. In addition, OCT images were worked up retrospectively from the Mannheim pool of recordings. Exams were performed by the RS 3000 SD OCT (Nidek) or by the Spectralis SD OCT (Heidelberg engineering). Images underwent a modified processing to favour the visualization of vitreous structures.

Results Observations range from a completely attached and homogeneous hyaloid with regular foveal profile at the age of 75 , to premacular vitreal bursae and intravitreal lacunae, regions of low and high density within an apparently disintegrating hyaloid, intravitreal condensates and supposed cell aggregations. Various patterns of holes within the posterior limitating vitreal membrane appeared in connection with vitreoretinal and vitreo-papillar traction of a partially detached vitreous

Conclusion OCT visualization of vitreous structures contributes to our understanding of vitreal degradation and vitreo - retinal interaction.

Commercial interest 
- F109 / 1663

Variations and repeatability of macular pigment and its spatial profiles in south Asian and white subjects

HUNTJENS B, CTORII

Applied Vision Research Centre, The Henry Wellcome Laboratories for Vision Sciences, City University London, London

Purpose To investigate variations in macular pigment optical density (MPOD) between south Asian and white subjects and explore the repeatability of the MPOD spatial profile

Methods MPOD was measured by heterochromatic flicker photometry in 77 south Asian and 60 white healthy volunteers aged 18 to 39 years, and repeated at a second visit in 24 subjects. The MPOD spatial profile was classified as typical exponential, or atypical ring-like or central dip (IOVS 2014;55:1440-1446).

Results We report excellent MPOD spatial profile repeatability (96\%) between visits Our findings showed $67 \%$ of all subjects with atypical ring-like or central dip profiles were south Asian. Average integrated MPOD up to 1.8 (MPODav0-1.8) was increased in subjects with atypical profiles $(\mathrm{P}<0.0005)$. Half peak MPOD value occurred at an average $1.8 \pm 0.7^{\circ}$ for the typical profile group, which was significantly broader than that for the atypical ring-like $\left(1.5 \pm 0.3^{\circ}\right)$ or central dip profiles $\left(1.4 \pm 0.3^{\circ}, \mathrm{P}=0.01\right)$. South Asian subjects had greater MPODav0-1.8 $(0.39+0.13)$ compared to whites $(0.32+0.13$ $\log$ units, $\mathrm{P}=0.01$ ) after controlling for age. White subjects with dark eyes had higher MPODav0-1.8 than those with light eyes $(\mathrm{P}=0.012)$.

Conclusion Classification of the MPOD spatial profile was highly repeatable. Since half peak MPOD value occurred within $1.8^{\circ}$ eccentricity, the spatial profile and MPODav0-1.8 together best describe the distribution of MPOD over the central area where macular pigment has its maximum effect. Further, atypical MPOD profiles offer increased MPOD over a more concentrated area centred on the fovea. Both parameters showed significant variations between the two ethnic groups.

\section{- F111 / 4745}

Semi-automated quantification of the parafoveal capillary network in diabetic subjects

KAPSALA Z (1), PALLIKARIS A (2), MOSCHANDREAS J (3), TSILIMBARIS MK $(4,2)$

(1) Ophthalmology Department, Medical School, University of Crete, Heraklion

(2) Institute of Vision and Optics, University of Crete, Heraklion

(3) Department of Social Medicine, Medical School, University of Crete, Heraklion

(4) Ophthalmology-Research Acct, University of Crete, Heraklion

Purpose The detection and quantification of the parafoveal capillary network in diabetic subjects using a novel semi-automated computerized method.

Methods Using the MatLab R2011a we developed an algorithm that detects automatically the parafoveal capillary bed in fluorescein angiography (FA) images The detection starts after delineating manually the foveal avascular zone (FAZ) in a cropped $1500 \mu \mathrm{m}$ subimage and hence excluding this area from the process. The algorithm calculates the capillary density in a $1000 \mu \mathrm{m}$ area measured from the centroid of the FAZ. The method was applied on high resolution FA images from 49 subjects (age $=43 \pm 19$ years); 13 controls, 13 subjects with diabetes mellitus (type I or II) without diabetic retinopathy (DR) findings (no DR), 15 subjects with non-proliferative DR (NPDR) and 8 subjects with proliferative DR (PDR). Using the statistical software SPSS (version 20) we assessed the mean capillary density for the mentioned subject groups.

Results Capillary density in the mentioned area was found $0.86 \pm 0.17,1.01 \pm 0.22$ $0.67 \pm 0.25$ and $0.76 \pm 0.30$ degrees- 1 for the studied groups, respectively $(\mathrm{p}=0.003)$. The no DR group did not differ significantly from the controls and the only statistically significant differences were those between the following three groups; control-NPDR, no DR-NPDR and no DR-PDR.

Conclusion This semi-automated algorithm could serve as a potential tool for detecting, classifying and monitoring automatically capillary abnormalities in this area Our preliminary results indicate that the capillary density in the central $1000 \mu \mathrm{m}$ area varies among different DR stages. However, a larger sample is required to confirm these initial data.
- F110 / 4748

Hyperosmolar stress induces TonEBP activation in human retinal pigmented epithelial cells

LIBERT S (1, 2), WILLERMAIN F (2, 3), SALIK D (1, 2), DELPORTE C (1)

(1) Laboratory of pathophysiological and nutritional biochemistry, Anderlech

(2) CHU Saint-Pierre-Brugmann, Brussels

(3) I.R.I.B.H.M., Anderlecht

Purpose Macular edema, a frequently encountered complication of diabetic retinopathy (DR), results from alterations of the blood retinal barrier (BRB) and leads to modifications of the retinal pigmented epithelium (RPE) functions. Osmolar changes of the surrounding medium could be responsible for some modifications of the RPE functions leading to a disturbance of the retinal homeostasis.

Methods To study the modification and stability of the key hyperosmolar response actor Tonicity Enhancer Binding Protein's (TonEBP) expression in ARPE-19 cells, derived from human RPE, were submitted to hyperosmolar stress of increasing concentration and for various times.

Results In response to hyperosmolar stimulation of ARPE-19 cells, a dose-dependent increase in TonEBP mRNA and protein levels, as well as TonEBP nuclear translocation, were observed. Moreover, the mRNA levels of Aldose Reductase (AR) and Sodiumdependent Taurine Transporter (TauT), two TonEBP target genes required for increasing the intracellular ionic force, increased in a delayed manner following hyperosmolar stimulation. Additionally, SB203580, a p38 protein kinase inhibitor, but not its inactive analogue SB202474, inhibited TonEBP nuclear translocation in ARPE-19 cells submitted to hyperosmolar stimulation.

Conclusion In ARPE-19 cells submitted to hyperosmolar stress, TonEBP expression is induced, and its nuclear translocation stimulated through a mechanism involving p38 protein kinase activation. It is likely that TonEBP nuclear translocation mediates the increase of AR and TauT expression induced by hyperosmolar stress and plays a role in RPE cells osmoadaptation

\section{- F112 / 4477}

Pronostic factors and complications of vitrectomy in patients with advanced diabetic retinopathy

AJAMIL RODANES S, MANZANAS LEAL L, LOPEZ GALVEZ MI

Ophthalmology, University Hospital, Valladolid

Purpose The aim of this study is to present the results of pars plana vitrectomy in patients with advanced disease and to identify preoperative risk factors and postoperative visual outcome

Methods The study was carried out at the University Hospital in Valladolid (Spain) with data from patients throughout two years.

Results Retrospective chart review of patients who underwent posterior plas plana vitrectomy (PPV) during 2010-2011 for non-clearing vitreous haemorrhage (NCVH), tractional retinal detachment (TDR) or diabetic edema with vitreomacular traction TME) and followed for at least 6 months.The primary outcome measure was the best corrected visual acuity (BCVA) at the end of the follow-up period and its correlation with preoperative risk factors. The secondary outcomes were postoperative complications and the influence of the status of the external retina in the ocular coherence tomography (OCT). 58 patients underwent PPV for advanced retinal disease. $\mathrm{NCVH}$ was the most frequent surgical indication in 32 patients and 21 of them had gone through laser previously. Mean preoparative BCVA was $1.96 \pm 1.13$ logarithm of minimum angle of resolution $(\log M A R)$ units and it improved.TDR was the indication in 10 patients. Only $50 \%$ of them had laser and all of them suffered from long term disease. There was not significant improvement in the BCVA and 3 patients developed neovascular glaucoma. Ind

Conclusion Preoperative BCVA, long term disease, the lack of laser treatment and the external retina seem to be some of the most important risk factors in these patients. 
- F113 / 4746

Study of endothelial progenitor cells in young diabetic patients with type 1 diabetes mellitus

TSILIMBARIS M (1), SIMANTIRAKI D (1), KAPSALA Z (1), TSIKA C (1), PONTIKOGLOU C (1), MAMOULAKIS D (2), PAPADAKI H(1)

(1) University of Crete Medical School, Heraklion

(2) University Hospital of Heraklion, Heraklion

Purpose Purpose: To evaluate the presence of Endothelial Progenitor Cells (EPCs) in the peripheral blood of young patients with Diabetes Mellitus Type I (T1DM)

Methods Blood samples were collected and analyzed from 23 individuals: 15 patients with DM (mean DM duration-vears: 13 (range:3-31)), mean age-vears: 26(9-45) and 8 controls without DM mean age-years: 32 (range:10-41). The presence of Diabetic Retinopathy (DR) was documented with slit lamp examination and fluorescence angiography, according to the Early Treatment of Diabetic Retinopathy Study (ETDRS) criteria. The EPCs were detected with flow cytometry using the following primary antibodies: anti-CD34, anti-CD45, anti-CD133 and anti-hVEGFR2

Results Progenitor EPCs, defined as CD45-/CD34+/CD133+/VEGFR2+ cells, were detected in 10/23 samples (mean\%: 0.00069, SEM:0.000261). 7/15 patients had EPCs detected in the periphery blood (mean\%: 0.000899, SEM:0.000384) . EPCs were detected in all 5 patients with PDR (mean\%: 0.000998, SEM:0.000206). Also, 1/5 patients with NPDR $(0.0035 \%)$ and $1 / 5$ patients without clinically proven DR $(0.005 \%)$ had detectable EPCs. Finally, circulating EPCs were identified in $3 / 8$ individuals of the control group (mean\%: 0.000299, SEM:0.000169).

Conclusion CD45-/CD34+/CD133+/hVEGFR2+ EPCs were detected in the peripheral blood of non diabetic persons and diabetic patients with or without evidence of DR. The clarification of the presence of EPCs in the peripheral blood early in the disease course could potentially offer significant information about the pathophysiology of diabetic microangiopathy.

\section{- F114}

Five-year incidence of branch retinal vein occlusion and its systemic and retinal risk associations: The Funagata study

KAWASAKI R (1), SUGANO A (2), KAWASAKI Y (1), OIZUMI T (3), DAIMONM (4), KATO T (3), KAYAMA T (5), YAMASHITA H (2)

(1) Department of Public Health, Yamagata University, Yamagata

(2) Department of Ophthalmology, Yamagata University, Yamagata

(3) Department of Neurology, Hematology, Metabolism, Endocrinology and Diabetology Yamagata University, Yamagata

(4) Department of Endocrinology and Metabolism, Hirosaki University, Hirosaki

(5) Department of Neurosurgery, Yamagata University, Yamagata

Purpose To determine retinal and systemic risk associations of branch retinal vein occlusion (BRVO) over 5 years in adult population-based cohort in Japan.

Methods The Funagata study is a population-based longitudinal cohort study in Yamagata, Japan. In 2000-2, we performed baseline survey including fundus photography and systemic examination. In 2005-7, 5-year follow-up survey was performed and newly developed BRVO cases were determined. There were 954 persons who attended both baseline and 5-year follow-up survey; 878 persons had good quality fundus photographs suitable for grading (right eye only). Using multiple logistic regression models, retinal and systemic risk characteristics were determined.

Results Mean age of study participants were 58.9 years old and $57.2 \%$ were women. Prevalence of diabetes and hypertension was $4.0 \%$ and $32.2 \%$, respectively. There were 12 newly developed BRVO over 5 - years (1.37\%, right eyes only). In multiple logistic regression models including those significantly associated systemic risks, older age (adjusted odds ratio [OR] 1.15 per 1 year, 95\% confidence interval [CI] 1.02-1.31, $\mathrm{p}=0.026$ ) and waist-to-hip ratio (adjusted OR 6.15 per $0.1,95 \% \mathrm{CI} 1.50-25.3, \mathrm{p}=0.012$ ) remained as significant risk characteristics. Retinal vessel opacification was significantly associated with incident BRVO after adjusting for systemic risk characteristics.

Conclusion In addition to older age, larger waist-to-hip ratio was identified as a modifiable systemic risk characteristic of 5-year incident BRVO. Retinal vessel opacification was also identified as a risk characteristic in the retina; there is a potential as a marker to predict BRVO.

Commercial interest 


\section{- S001}

Embryology of extraocular muscles : A new vision

TRECHOT F (1), MICARD E (2), CENDRE R (2), TONNELET R (3), ANGIOI K (1), BRAUNM (3)

(1) Department of Ophthalmology, Nancy

(2) Inserm U-947, Nancy

(3) Department of Anatomy, Nancy

Purpose To describe the embryology of extraocular muscles, through a 3D viewing algorithm, from Carnegie stage XVII to Carnegie stage XXIII (41st-57th day of development)

Methods The work consisted of a scanning and a data processing reconstruction of embryonic histological material. Material came from anatomical universities collections. 12 embryos were selected, and 7 were dedicated to muscle study (from $14.5 \mathrm{~mm}$ to $54.5 \mathrm{~mm}$ ). This data processing has allowed a study in three spatial planes.

Results From Carnegie stage XVII, all extraocular muscles are in place, including levator palpebrae. The study in 3 planes makes their individualization easier. Gilbert (Carnegie Institution) described the origin of inferior rectus and inferior oblique by a common primordium, however from the stage XVII the two muscles are clearly separated. The decussation of the superior oblique is seen from the stage XIX, the trochlea appears at stage XXII. Our work also demonstrates the nerves : optic, oculomotor, trochlear trigeminal and abducens. The junctions between nerve and muscle are put in evidence, especially for the lower branch of the oculomotor nerve.

Conclusion This new study tool has resulted in explicit images of the development of extraocular muscles in the embryonic period. It helped to provide details with respect to the work already established by the Carnegie Institution.
- S003

Changes in choroidal thickness and corneal parameters in diabetic eyes

NORVYDAITE D, LAUIRINAVICIUTE G, STECHS, SKVARCIANYI, GALGAUSKAS S, ASOKLIS R

Vilnius University, Center of Eye Diseases, Vilnius

Purpose To evaluate the effect of diabetes mellitus on the central corneal thickness (CCT), corneal endothelial parameters (endothelial cell density (ECD), their average size (Ave), hexagonality (A6), polymegathism (CV)) and the subfoveal choroidal thickness (SFCT). To determine whether these parameters depend on the duration of diabetes and haemoglobinA1c (HbA1c) level.

Methods 62 diabetic patients and 65 healthy subjects were examined using a noncontact specular microscope, A-scan ultrasound and spectral domain optical coherence tomography. The study parameters included medical history, age, axial length, SFCT CCT, ECD, A6, CV, Ave. The duration of diabetes and HbA1 level of 2 latest tests (6 months period) were noted.

Results There was equal proportion of men and women 31(50\%) in the diabetes group (62 patients, 124 eyes). The mean age was $45.5(+134)$ years, duration of diabetes $-9.3( \pm 6.6)$ years, HbAlc level $-9.8( \pm 2.3) \%$. Diabetic retinopathy (DR) was present in $22(17.9 \%)$ eyes, macular edema - $10(8.1 \%)$. There were no differences in age, gender or axial length between the groups ( $\mathrm{p}>0.05)$. SFCT $(258.0 \pm 74.4 \mu \mathrm{m})$ and ECD $(2721.8 \pm 264.1 \mathrm{cell} / \mathrm{mm} 2)$ were significantly lower, CCT $(566.7 \pm 35.7 \mu \mathrm{m})$ - higher in diabetic patients than in control group $(313.1 \pm 88.5 \mu \mathrm{m}, 2967.3 \pm 220.6 \mathrm{cell} / \mathrm{mm} 2$, $550.0 \pm 56.4 \mu \mathrm{m})(\mathrm{p}<0.05)$. DR and macular edema didn't influence SFCT significantly $(\mathrm{p}>0.05)$. No differences in endothelial parameters between groups and no correlation between $\mathrm{HbA1c}$, duration of diabetes and any of the examined parameters were found $(\mathrm{p}>0.05)$

\section{- S002}

Mapping angiogenesis on choroidal membranes of a murine laser-induced CNV model

TUNIK S (1, 2), ARONSSONM (1), KVANTA A (1), ANDRE H (1)

(1) Vitreo-Retinal Clinic, STE/Karolinska Institutet, Stockholm

(2) Histology and Embryology, Faculty of Medicine, University of Dicle, Diyarbakir

Purpose We investigated the expression and distribution of neoangiogenic molecules during the development of choroid neovascular (CNV) lesions in a laser-induced mouse model of neovascular age-related macular degeneration (AMD), with particular focus on the choroid of these lesions.

Methods Lesions were induced on C57BL/6 mice using laser photocoagulation. Animals were sacrificed and eye-cups were dissected from enucleated eyes, and the choroids were shortly fixed. Choroids were immunostained for NG2 (pericytes), VEGFR2 (vascular endothelial growth factor receptor 2; sprouting endothelial cells(ECs)), or VEGF with co-staining for CD31 (vascular ECs). Images of the choroidal membranes were obtained by microscopy. Vascular permeability was also determined.

Results CNV lesions displayed positive staining for the four angiogenic markers studied. The distributions of the angiogenic markers were determined in comparison to the area of neovascularization, as observed by increased CD31 staining. Both NG2 and VEGFR2 displayed a radial progression, while VEGF displaved a uniform distribution across the choroidal membranes of the lesions in the studied times. Vascular permeability showed an increase concomitant with the increase in vascularization observed by immunostaining throughout the time-course.

Conclusion Our data shows that the choroidal membranes of CNV lesions recover through a canonical sprouting angiogenesis pathway, in response to a VEGF-mediated signal. In addition, these membranes display an increasing radial endothelial sprouting with concomitant pericyte recruitment from the laser injury to the exterior of the lesions, in a time-dependent. These results may have implications for the clinical understanding of $\mathrm{CNV}$ in patients with wet AMD.

\section{- 5004 \\ Choroidal thickness in young myopic adults assessed by enhanced depth imaging optical coherence tomography}

KADZIAUSKIENE A (1, 2), AUGYTE A (1), GALGAUSKAS S (1, 2), STECHS (2), STRELKAUSKAITE E $(1,2), \operatorname{ASOKLISR}(1,2)$

(1) Vilnius University, Faculty of Medicine, Vilnius

(2) Vilnius University Hospital Santariškių Klinikos, Center of Eye Diseases, Vilnius

Purpose To compare choroidal thickness of emetropic eyes and ones with different degree of myopia in young adults.

Methods 40 participants (80 eyes) had choroidal measurements using enhanced depth imaging spectral domain optical coherence tomography at three locations: fovea, $3 \mathrm{~mm}$ nasally and $3 \mathrm{~mm}$ temporally to it. According to their refractive error patients were divided into 3 groups: emetropia (from $+0.5 \mathrm{D}$ to $-0.5 \mathrm{D}$ ) - 22 eyes, mild to moderate degree myopia (from -1.0 D to -6.0 D) - 34 eyes, high degree myopia (-6.0 D \& greater) 24 eyes. Axial length biometry and autorefractometry were also performed.

Results The mean age of the participants was 23.6 years (standard deviation \pm 1.5 ). The average choroidal thickness at fovea, $3 \mathrm{~mm}$ nasally and $3 \mathrm{~mm}$ temporally was estimated to be 352.5 (median 328.0, interquartile range 116.5) $\mu \mathrm{m}, 161.0(139.0,88.5) \mu \mathrm{m}$ and $311.6,(298.5,101.5) \mu \mathrm{m}$ respectively. Subfoveal and temporal choroid was significantly thinner in high degree myopic eyes than in the group of low to moderate myopia and emetropic eves $(\mathrm{p}<0.05)$. At the fovea the choroid was thicker than nasally and temporally to it $(\mathrm{p}<0.05)$. A moderate negative correlation was found between the thickness of the choroid and the degree of myopia $(p=-0.44 ; p<0.01)$. Axial length was significantly shorter in normal vision group than in the groups of myopia $(\mathrm{p}<0.05)$ and correlated negatively with the central choroidal thickness $(p=-0.47 ; p<0.01)$.

Conclusion Myopic young adults had significantly thinner central choroid layer compared to emetropic individuals. At all measured locations the choroidal thickness decreased with the higher degree of myopia and longer axial length. 
- S005

Comparison of ocular tissue weights (volumes) and tissue collection techniques in commonly used preclinical animal species.

STRUBLE C, HOWARD S, RELPHI

Covance Laboratories, Madison, Wisconsin

Purpose Ocular pharmacokinetic and distribution studies are key elements for the development of ophthalmic drugs and are used to correlate drug levels in preclinical species with human dosages and exposures. The purpose of this study was to compare ocular tissue weights for five commonly used preclinical species using two collection techniques.

Methods Standard ocular tissues were collected from five males and five females of each species; New Zealand white (NZW) and Dutch Belted (DB) rabbits, beagle dogs Gottingen minipigs, and Cynomolgus monkeys. Two collection techniques were used; a collection from fresh eyes (OS, immediately following enucleation) or a collection from frozen eyes (OD, following flash freezing). A specific procedure and collection sequence was followed for each technique.

Results Aqueous and vitreous humor, cornea, lens, iris-ciliary body, retina, choroid, and sclera were collected from each species; these tissues represented mean values of $70.0-82.0 \%$ and $84.3-88.9 \%$ of the total eye weight, respectively, for fresh and frozen collection. Tissue recovery levels were consistently higher using the frozen collection technique. Mean combined male \& female vitreous humor weights using the frozen collection were 1.41, 1.31, 2.22, 2.34, and $2.04 \mathrm{~g}$ for NZW \& DB rabbits, dogs, minipigs, and monkeys, respectively. Other tissue weights will be compared.

Conclusion Comparative ocular tissue weights are presented for common preclinical species using fresh or frozen collection techniques. Vitreous humor and other tissue weights collected by the frozen technique accurately represent the weight (and volume assuming a VH density of 1) of the total tissue for comparative purposes to human vitreous volumes.

\section{- 5007}

Temporal response of the phagocytic microglia in the axotomized rat retina: optic nerve crush vs. transection

NADAL-NICOLAS FM, JIMENEZ-LOPEZ M, SALINAS-NAVARRO M, NIETO-LOPEZ L, CANOVAS-MARTINEZ I, SOBRADO-CALVOP, VIDAL-SANZM, AGUDO-BARRIUSO M

Oftalmologia Experimental. Instituto Murciano de Investigación Biosanitaria-VIRGEN DE LA ARRIXACA, Murcia

Purpose To analyze the appearance of phagocytic microglial cells (PMC) in rat retinas after optic nerve crush or transection.

Methods Retinal ganglion cells (RGCs) from adult Sprague Dawley rats were traced with fluorogold applied to both superior colliculi. One week later, the left optic nerve was either crushed (ONC, $n=32$ ) or transected $(\mathrm{ONT}, \mathrm{n}=32)$. Rats were euthanized at 2, 5, 9 and 14 days after the axotomy ( $\mathrm{n}=8 /$ group). Retinas were dissected as flatmounts and Brn3a immunodetected to identify RGCs. Retinas were photographed for each signal. Brn3a+RGCs were automatically quantified and their distribution visualized by isodensity maps. The position of each PMC found in the ganglion cell layer (identified by their transcellularly labelling with fluorogold) was dotted on the retinal photomontages. These dots were counted and graphically represented with neighbour maps.

Results PMCs were observed at 2 days $(43 \pm 8$, ONT; $11 \pm 5$, ONC). Their number increased quicker after ONT than after ONC in opposition with the loss of RGCs which was slower after ONC. The peak of PMCs was observed at 9 days after ONT and at 14 days after $\mathrm{ONC}(19,842 \pm 2534$ and $19,757 \pm 1968$, respectively). The appearance of PMCs and the loss of RGCs was highly correlated ( $\mathrm{r} 2=0.8$ for both lesions). PMCs were observed across the retina and their distribution matched the retinal areas of RGC loss. Conclusion ONC induces a slower loss of RGCs than ONT, and this is followed by a slower response of microglial cells.

\section{- S006}

Microglia activation in mice retina contralateral to experimental glaucoma: increased cell number and retraction of processes beyond GCL

RAMIREZ AI (1, 2), GALLEGOB $(1,2), \operatorname{ROJAS} B(1,3), \operatorname{DEHOZ} R(1,2)$,

SALAZARJJ (1, 2), VALIENTE-SORIANO FJ (4), AVILES-TRIGUEROS M (4),

VILLEGAS PEREZ MP (4), VIDAL-SANZM (4), TRIVINO A (1,3),

RAMIREZIM $(1,3)$

(1) Instituto de Investigaciones Oftalmológicas Ramón Castroviejo. UCM, Madrid

(2) Fac. Óptica y Optometría. UCM, Madrid

(3) Fac. Medicina. UCM, Madrid

(4) Experimental Ophthalmology Laboratory, Dept. Ophthalmology, College of Medicine, University of Murcia, Murcia

Purpose To analyse quantitatively retinal microglia signs of activation after two weeks of laser-induced ocular hypertension (OHT) in OHT-eyes and their contralateral eyes

Methods Adult albino Swiss mice were assigned to two groups: naive $(\mathrm{n}=6)$ and lasered $(\mathrm{n}=9)$. Anti-Iba1 was used in retinal whole-mounts to analyse microglial number and the area covered by each microglial cell (arbor area)

Results In naïve, contralateral and OHT-eye microglia were distributed throughout the retina in the photoreceptor laver (PRL), outer plexiform layer (OPL), inner plexiform layer (IPL), nerve-fibre layer (NFL) and ganglion-cell layer (GCL). In comparison with naive, contralateral eyes and OHT-eyes showed a significant increase in the number of microglial ( $\mathrm{p}<0.05$ and $\mathrm{p}<0.001$, respectively) and in the area of the retina occupied by microglial cells in the NFL GCL ( $\mathrm{p}<0.01$ and $\mathrm{p}<0.001$ respectively). OHT eyes exhibited significantly higher values than did contralateral eyes for microglial number $(\mathrm{p}<0.001)$ and for the area of the retina occupied by microglial cells in the NFL-GCL ( $<<0.001)$. In comparison with naïve, the microglial arbor area in the OPL and IPL was decreased in both OHT eyes ( $\mathrm{p}<0.001$ in both instances) and in contralateral untreated eyes $(\mathrm{p}<0.05$ and $\mathrm{p}<0.001$, respectively)

Conclusion Two weeks of laser-induced OHT induce an increase of microglia number and retraction of processes beyond the GCL both in OHT eyes as well as in contralateral normotensive untreated eyes. Differences between the two groups could help to elucidate glaucoma pathophysiology.(OFTARED Grant RD12/0034/0002 and RD12/0034/0014)

\section{- S008 / 3256}

Autophagy stimulus promotes HuR protein phosphorylation and SQSTM1/p62 protein up-regulation in ARPE-19 cells

AMADIOM (1), MARCHESIN (1), GOVONIS (1), PASCALEA (1),

KAARNIRANTA K (2)

(1) Dept of Drug Sciences, Pavia University, Pavia

(2) Dept. of Ophthalmology, School of Medicine, Eastern Finland University, Kuopio

Purpose Age-related macular degeneration (AMD) pathogenesis involves impaired protein degradation in retinal pigment epithelial (RPE) cells. Our recent observation reveals that the expression of autophagy receptor SQSTM1/p62 is positively regulated by HuR protein (mRNA-stabilizing human Embryonic Lethal Abnormal Vision protein) (Viiri et al. 8(7):e69563, 2013, PLoS One). In this study, we investigated the effects of AICAR (autophagy inducer, 5-aminoimidazole-4-carboxamide-1- $\beta$-D-ribofuranoside) with/without MG-132 (proteasome inhibitor) on HuR post-translational activation and p62 protein levels in ARPE-19 cells.

Methods ARPE-19 cells were treated with MG-132 $(1 \mu \mathrm{M})$ and/or AICAR $(2 \mathrm{mM})$ for increasing times (from $15 \mathrm{~min}$ to $2 \mathrm{~h}$ ) and subjected to cell fractionation. HuR and SQSTM1/p62 protein levels were measured by Western blotting. HuR phosphorylation in threonine residues was evaluated by Western blotting following immunoprecipitation. Results AICAR+MG-132 co-treatment induces early translocation of HuR protein from the nucleus to the cytoplasm (already after $15 \mathrm{~min}$ ). In this latter cellular fraction, after $2 \mathrm{~h}$ an increase of HuR protein phosphorylation (+94\% vs control) and an upregulation of p62 protein levels are observed ( $+85 \%$ vs control)

Conclusion In the cytoplasm of ARPE-19 cells AICAR+MG-132 co-treatment leads to HuR protein activation, which in turn may determine the increased p62 protein expression 
- S009 / 3257

Cytotoxicity of mesoporous silicon microparticles with different surface modifications on ARPE-19 cells

KORHONENE (1), RIIKONEN J (2), XU W (2), LEHTO VP (2), KAUPPINEN A (1,3) (1) University of Eastern Finland, Department of Ophthalmology, Kuopio

(2) University of Eastern Finland, Department of Applied Physics, Kuopio

(3) Kuopio University Hospital, Kuopio

Purpose Retinal pigment epithelial (RPE) cells play a crucial role in the pathogenesis of age-related macular degeneration (AMD). However, RPE is a challenging target in a therapeutical sense. In addition to its location, the possible hydrophobicity of drug molecules makes its medication even more challenging. Biodegradable porous silicon (PSi) microparticles could be a promising possibility for delivering hydrophobic drugs to RPE cells. In the present study, we aimed at testing the cytotoxicity of thermally oxidized (ToPSi) and thermally carbonized porous silicon (TCPSi) particles with and without additional surface modification with amino groups on human ARPE-19 cells Since the cytotoxicity of PSi particles cannot be tested by the commonly used MTT (3-[4,5-dimethylthiazol-2-yl]-2,5-diphenyltetrazolium bromide) assay due to nonspecific redox reactions, our aim was also to search for alternative assays for working with mesoporous silicon materials.

Methods ARPE-19 cells were treated with microparticles at several concentrations for $24 \mathrm{~h}$. Thereafter, cell viability was measured using a protease viability marker assay (CellTiter-FluorTM) and a lactate dehydrogenase (LDH) release assay. Cells were also evaluated ocularly under an inverted microscope.

Results All tested particles were well tolerated by ARPE-19 cells. CellTiter-Fluor assay seemed slightly more reliable than the LDH test.

Conclusion Our results show that the tested porous silicon particles did not cause major cytotoxicity on ARPE-19 cells, and CellTiter-Fluor assay, especially together with ocular examination, could be suitable for testing cytotoxicity in association with mesoporous silicon particles

\section{- S010}

A novel proteotoxic stress-associated mechanism for macular corneal dystrophy

KAARNIRANTA K (1), SZALAIE (2), SMEDOWSKI A (3), WYLEGALA E (3), EELSZEGHYS (4.

(1) Ophthalmology, Kuopio

(2) Ophthalmology, Debrecen

(3) Ophthalmology, Katowice

(4) Anatomy, Histology and Embryology, Debrecen

Purpose Macular corneal dystrophy is a rare autosomal recessive eye disease primarily affecting the corneal stroma. Abnormal deposits have been observed intra- and extracellularly in the stromal layer. In addition to the stromal keratocytes and corneal lamellae, deposits are also present in the basal epithelial cells, endothelial cells and Descemet's membrane. Misfolded proteins have a tendency to gather into aggregating deposits. We studied the interaction of molecular chaperones and proteasomal clearance in macular dystrophy human samples and in human corneal HCE-2 epithelial cells in this disease.

Methods Seven cases of macular dystrophy and 5 normal human corneal buttons were collected during corneal transplantation. Fluorescent immunohistochemistry on human donor corneal button was used to verify the spatial distribution pattern of selected Hsp70, SQSTM1/p62 and ubiquitin molecules, respectively. The expression level of these proteins were analysed in HCE-2 cells using western blotting and transmission electron microscopy, respectively.

Results Highly elevated Hsp70, SQSTM1/p62 and ubiquitin protein conjugates were observed in intracellular space of the epithelial cells in macular dystrophies. All the studied proteins were also highly elevated under proteasome inhibition in human corneal epithelial HCE-2 cells in cell cultures.

Conclusion We report a novel proteostatic regulatory mechanism that connects the molecular chaperone and proteasomal clearance system with the pathogenesis of macular corneal dystrophy.

- S012 / 3255

- S011 / 3254

Predegenerated Schwann cells promote neuroprotection and regeneration of retinal ganglion cells in ex vivo rat retinal explants

SMEDOWSKIA (1, 2, 3), PIETRUCHA-DUTCZAKM (1), KAARNIRANTA K $(3,4)$, LEWIN-KOWALIKJ $(1)$

(1) Department of Physiology, Medical University of Silesia, Katowice

(2) Clinical Department of Ophthalmology, Medical University of Silesia, Katowice

(3) Department of Ophthalmology, University of Eastern Finland, Kuopio

(4) Department of Ophthalmology, Kuopio University Hospital, Kuopio

Purpose To investigate neuroprotective and pro-regenerative potential of predegenerated Schwann cells towards RGCs in ex vivo retinal explants

Methods In this study we used two models of rat retinal explants culture - reversed explants from P2 Wistar rats pups and insert explants from 4 weeks old Wistar rats. Both types of explants were cultured in Neurobasal A medium with supplements (B-27, $\mathrm{N} 2$ and GlutaMax). In this way we prepared 32 insert explants (16 were cultured with 2 $\mu \mathrm{l}$ of Schwann cells suspension (104 cells) dropped on the each explants surface, another 16 served as control treated with $2 \mu \mathrm{l}$ of DPBS) and 30 reversed explants (10 of them were cultured in standard medium, 10 explants were with addition of $5 \mathrm{ng} / \mathrm{ml}$ BDNF into medium and 10 were co-cultured with 104 of Schwann cells). After proper culture time, explants were fixed and processed for immunostainings and stereological analyses.

Results In retinal explants cultured in inserts system, there was significantly higher density of RGCs in group treated with Schwann cells in comparison to PBS treated (independent samples t-test, $\mathrm{p}<0.05$ ). In reversed retinal explants, there were significant differences between groups expressed in mean and total length and surface of neurites, as well as in number and arboration rate of neurites (U-Mann Whitney test, $\mathrm{p}<0.05$ ). Immunofluorescent staining using GAP43 additionally revealed intense neurites outgrowth (also in explants from adult animals).
Pinosylvin protects retinal pigment epithelial cells from oxidative stress by activating Nrf2-mediated antioxidant defence system

KOSKELA A (1, 2), REINISALO M (1), HYTTINENJ (1), KAARNIRANTA K(1), KARJALAINENR (3)

(1) Department of Ophthalmology, University of Eastern Finland, Kuopio

(2) Department of Biology, Kuopio

(3) Department of Biology, University of Eastern Finland, Kuopio

Purpose The constant light exposure, high metabolic rate and high lipid concentration expose retinal pigment epithelial (RPE) cells to chronic oxidative stress, which may lead to RPE cell degeneration and evokes secondarily photoreceptor damage and visual loss. In this work, we assessed the protective role of wood derived polyphenol, pinosylvin (PS), in the prevention of oxidative stress and the molecular mechanisms behind these effects

Methods ARPE-19 cells were exposed either to PS or hydroquinone (HQ) or both simultaneoulsy. Toxicity and protective effects of PS against HQ-induced oxidative stress were determined by colorimetric cell viability test (MTT-test). Signalling mechanism of PS was studied by analyzing mRNA levels of Nrf2 (nuclear factor-erythroid 2-related factor-2) and its target genes heme oxygenase-1 (HO-1), glutathione S-transferase pi 1 (GSTP1) and sequestosome 1 (p62) by PCR. Moreover, Nrf2 and p62 were silenced by using siRNA technology.

Results PS treatment protected ARPE-19 cells from HQ, when analyzed by MTT assay. PS treatment significantly increased the expression of $\mathrm{HO}-1$ that was abolished by Nrf2 siRNA . PS did not protect p62 siRNA-treated cells from HQ induced oxidative stress.

Conclusion Our results suggest that PS treatment conferred protection against HQinduced oxidative stress through the induction of HO-1 in ARPE-19 cells. Consequently, stress-related diseases such as AMD. PS-stilbene compounds may possess health-promoting properties against oxidative 
- S013 / 1725

Influence of pressure on in vitro human corneal endothelial cells derived from human induced pluripotent stem cell (hIPSC)

JUMELLEC (1), SUFFEEN (1), FOREST F (1, 2), HE Z (1), BERNARD A (1), NANGOUM-FOSSO T (1), NAIGEONN (1), PERRACHE C (1), PEOC'HM (1,2) GAINP $(1,3)$, THURET G $(1,3,4)$

(1) Corneal Graft Biology, Engineering and Imaging Laboratory, EA2521, Federative Institute of Research in Sciences and Health Engineering, Faculty of Medicine, Jean Monnet University, Saint-Etienne

(2) Department of Pathology, University Hospital, Saint-Etienne

(3) Department of Ophthalmology, University Hospital, Saint-Etienne

(4) Institut Universitaire de France, Bd St Michel, Paris

Purpose Human induced pluripotent stem cells (hIPSC) have infinite self-renewal capacity and can differentiate into somatic cells. We obtained hIPSC-derived corneal endothelial-like cells (CE-likeC) using a sequential supply of growth factors. As, in vivo, the functionality of CEC may depend on various environmental factors, including intraocular pressure, we investigated whether pressure could influence the hIPSC differentiation into $\mathrm{EC}$

Methods We developed a specific device for pressurizing cell cultures placed in a $\mathrm{CO} 2$ incubator. The device consists of a pump injecting the gas mixture from the incubator into a sealed container equipped with a pressure sensor and an electronic control system. hIPSC-derived CEC were cultured in the differentiation medium for 1 week either in a standard incubator at atmospheric pressure or under a pressure of 20 $\mathrm{mmHg}$. The medium was changed every 2 days. Differentiation was determined by cell morphology analysis and ionic pumps immunostaining (CLCN3, VDAC3, SLC4A and $\mathrm{Na}+/ \mathrm{K}+/$ ATPase) and tight junctions (ZO-1).

Results At D8, the 2 cultures showed similar endothelial morphology, ZO-1 and SLC4A, but expression of ionic pumps CLCN3, VDAC3, and Na+/K+/ATPase were increased under pressure.

Conclusion The device developed is fully functional. Our results show that, in vivo, a pressure of $20 \mathrm{mmHg}$ did not modify the cell morphology. However, it tends to modulate the expression of some ionic pumps. Pressure level seems to be an important parameter in the differentiation into $\mathrm{EC}$. Considering this, it could improve the EC functionality, i.e. deswelling capacityGrants: ABM2013, IUF2012-2017 and postdoc UJM 2013

\section{- S015 / 1727}

Ocular chronic graft versus host disease after allogeneic hematopoietic stem cell transplantation - preliminary data

JEPPESEN H (1, 2), LINDEGAARD J (2), SENGELOEV H (3), JULIAN HO (2), PRAUSE J (4), HEEGAARDS $(2,4)$

(1) Dept of ophthalmology, Copenhagen

(2) Dept of ophthalmology, Glostrup

(3) Dept of hematology, Copenhagen

(4) Dept of neuroscience and pharmacology, Eye pathology section, University of Copenhagen, Copenhagen

Purpose Allogeneic hematopoietic stem cell transplantation(HSCT) is a curative therapy for a number of malignant and non-malignant hematological diseases. Allogeneic HSCT can be performed in several ways; donor cells can be obtained from bone marrow(BMT), peripheral blood(PBSCT) or cord blood(CBT). Ocular chronic graft versus host disease(cGVHD) is a major contributor to long-term morbidity after HSCT. The purpose of this study was to report the frequency of ocular cGVHD after allogeneic HSCT.

Methods Retrospective examination of 233 charts of patients (adults $>15 y e a r s)$ who underwent consecutive allogeneic HSCT from January 2000-june 2011 at Copenhagen University Hospital (Rigshospitalet). All allogeneic HSCT in Denmark are performed at this hospital. All patients were examined ophthalmologically before the transplant, yearly after the transplant and ad hoc if any ophthalmic problems occurred. The diagnosis of ocular CGVHD was made according to the NIH consensus criteria.

Results Fifty-eight(25.3\%) fulfilled the diagnostic criteria of ocular cGVHD, 16 out of $78(20.5 \%)$ in the BMT group, 41 out of $152(27.0 \%)$ in the PBSCT group and 1 out of 3(33.3\%) in the CBT group. Malefemale ratio was 40:18(2.22) (in the cohort 146:87(1.68)). Median time of onset was 24.3 months after HSCT(range 4.9-146.1). In the cohort median age was 45.2 years(rage 15.2-71) at transplantation time and 55.5 years(18.43-69.0) when ocular cGVHD was diagnosed. Median time of follow-up was 24.8 months(range 0.4-147.6)

Conclusion Out of 233 patients who received allogeneic HSCT, 59(25.3\%) developed ocular CGVHD. The median time of onset was 24.3 months after transplantation and median age at onset was 55.5 years.
- S014 / 1726

Development of an ECM hydrogel for corneal tissue engineering

$\operatorname{AHEARNEM}(1,2)$

(1) Trinity Centre for Bioengineering, Trinty College Dublin, Dublin

(2) Department of Mechanical and Manufacturing Engineering, Trinity College Dublin, Dublin

Purpose Tissue engineering has been proposed as a method of dealing with the shortage of suitable tissue available for transplants. Many biomaterials under investigation for engineering corneal tissue lack the native corneas biochemical composition. The aim of this study was to fabricate and test a biomimetic hydrogel derived from corneal extracellular matrix (ECM) to be used for corneal tissue engineering.

Methods Porcine corneas were decellularized using several different techniques. The corneas were then freeze dried and milled into a fine powder. This ECM powder was dissolved using an acidic pepsin digest solution for 72 hours. A crosslinked hydrogel was formed after neutralizing the $\mathrm{pH}$ of the solution. Human corneal stromal cells were mixed into the hydrogels prior to gelation and cultured over 14 days. Biochemical assays, mechanical and optical testing, RT-PCR and immunohistochemical staining were performed on the cell seeded hydrogels. Rat-tail collagen hydrogels were used as controls.

Results The hydrogels were highly transparent and able to maintain stromal cell viability. When compared to standard collagen hydrogels, the ECM hydrogels retained corneal GAGs and appeared to enhance the cells native keratocyte phenotype. The amount of GAG present was dependent on the decellularization technique used. The ECM hydrogels exhibited similar viscoelastic characteristics to collagen hydrogels.

Conclusion A new type of hydrogel has been demonstrated that could be used for engineering corneal tissue. We plan further develop these hydrogels by optimizing the stromal decellularization process and examining different crosslinking techniques to improve the hydrogels mechanical strength and stiffness.

- S016 / 1728

Positive cultures in corneas stored with cold storage technique to be used for corneal grafting

JULIAN HO (1), LINDEGAARD J (1), HØJGAARD-OLSEN K (1), HEEGAARD S (2) (1) Eye Department, Glostrup Hospital, Copenhagen

(2) Eye Pathology Institute, Department of Neuroscience and Pharmacology, University of Copenhagen, Copenhagen

Purpose Tissue for cornea grafting processed in the US is stored using cold storage method whereas corneas processed in the $\mathrm{EU}$ is stored in organ culture.The two preservation techniques differ in technical aspects, evaluation techniques, storage time and microbiological safety.Both techniques are considered to result in similar graft survival.Due to low availability of Danish donor tissue, we have used US donor tissue for more than seven years in Denmark.

Methods Routine microbiological culturing of the cornea-scleral ring was performed at the time of DSAEK surgery

Results From April 2013 to March 2014 a total of 11 donor rims tested after grafting were positive for bacteria or fungi with the cold storage technique. Microbiological testing of donor rims stored with organ culture were all negative. Total number of grafts, $\mathrm{n}=210$. Six candida, one MRSA, two E. Faecium and two E.coli were cultured. Two eyes had severe and vision threatening infection (fungi). Four eyes showed primary graft failure. Five eyes showed no signs of infection.

Conclusion Corneas prepared with the cold storage technique were more prone to microbiological contamination, some with vision-threatning fungal infection. Antimycotics are now added to the current hypothermic storage solution. 
- S017

In vivo corneal confocal microscopy allows direct observation of microbiological features in infectious keratitis

WYLEGALA E (1), SMEDOWSKI A (1), TARNAWSKA D (1,2), NOWINSKA A (1) DOBROWOLSKID (1)

(1) Clinical Department of Ophthalmology, Faculty of Medicine and Department of Dentistry in Zabrze, Medical University of Silesia, Katowice

(2) Department of Biophysics and Molecular Physics, Institute of Physics, University of Silesia, Katowice

Purpose To differentiate microbiological features of infectious keratitis in corneal epithelium.

Methods We performed in vivo confocal microscopy (HRT III, Rostock Cornea Module) in 190 patients with diagnosed infectious keratitis of various etiology - 72 viral, 66 bacterial, 34 fungal (fibrillar and candidal) and 18 amoebal keratitis. For corneal scans we used 400x lens and 63x magnification. Microscopic analysis of epithelial layer was performed to distinguish structures of epithelial cells morphology, inflammatory cells and potential microbes.

Results In viral keratitis, due to size of viral particles, highly specific secondary changes within epithelial cells were observed in form of "owl eyes" - an equivalent of active virus replication in cells. In bacterial keratitis we observed structures of bacteria forming streptococcal configuration (diagnosed bacteriologically as Streptococcus pneumoniae and Enterococcus spp.), granular bacteria could not be easily distinguished because of similarity with leukocytes infiltration (diagnosed bacteriologically as Staphylococci) In fungal keratitis we detected fungi fibrilles (diagnosed mycologically as Fusarium spp.) as well as granular fungi - Candida spp. Amoebal keratitis presented presence of Acantamoeba cysts and trophozoits.

Conclusion In vivo analysis of corneal epithelial histology shows high specificity and sensitivity in infectious keratitis diagnosis. Except describing corneal structure and inflammatory cells, it makes possible to distinguish microbiological features of keratitis.

- S019

Comparison of corneal power and astigmatism in normal eyes using 4 different devices

HAN KE, LEE HK, HWANG HS, SHIN MC

Ophthalmology, Gangwon-Do Chuncheon

Purpose To compare corneal power and astigmatism in normal subjects obtained from dual Scheimpflug analyzer (Galilei), single Scheimpflug analyzer (Pentacam), optical low coherence reflectometer (Lenstar) and autokeratometer (AR)

Methods Forty-eight eyes of 27 subjects were evaluated with 4 different devices. Steep flat and mean keratometry $(\mathrm{K})$ values and astigmatism magnitude were measured. Corneal power vectors, J0 and J45, were calculated using Jackson cross cylinder. Repeated-measured ANOVA with Bonferroni post hoc analysis was performed to compare the measurements, and correlation was accessed by Pearson correlation test. Agreement was assessed by Bland-Altman analysis and $95 \%$ limit of agreement (LoA) was calculated.

Results Mean K values measured by Galilei, Pentacam, Lenstar, and AR were $44.34 \pm$ 1.03 diopters (D), $44.52 \pm 1.03$ (D), $44.30 \pm 0.98$ (D), and $44.44 \pm 0.92$ (D), respectively. Steep K, J0, and 45 did not show significant differences (all $\mathrm{p}>0.05$ ), however, mean $\mathrm{K}$, flat $\mathrm{K}$, and astigmatism were significantly different $(\mathrm{p}=0.002,0.002,0.005$, respectively) All measured parameters were significantly correlated with each other (Pearson correlation test, all $\mathrm{p}<0.05$ ). For corneal power and astigmatism, 95\% LoA varied from 1 to 2 (D).

Conclusion Even though the measurements of corneal power and astigmatism were correlated with each other, some values showed significant differences and the agreements were not good. Therefore, the measurements of each device couldn't be used interchangeably in clinical practice
- S018

Role of SD-OCT in the diagnosis and management of a late-onset corneal LASIK flap tear secondary to screwdriver trauma

ESTEBAN O (1), RODRIGUEZ NA (2), ASCASO FJ (1), SOLANAS S (3)

(1) Ophthalmology, HCU "Lozano Blesa", Zaragoza

(2) Ophthalmology, Hospital de Navarra, Pamplona

(3) Radiology, Hospital Reina Sofia, Tudela

Purpose Traumatic flap displacement is a known possible complication after LASIK. It occurs most commonly in the first $24 \mathrm{~h}$ following surgery in approximately $1 \%-2 \%$ of cases. However, late flap dislocations have also been reported. We present a case of traumatic flap displacement occurring four years after the initial LASIK surgery.

Methods Interventional case report showing the role of spectral domain optical coherence tomography (SD-OCT) in the management of the LASIK flap dislocation.

Results A 29-year-old man presented with blurred vision, photophobia, and pain after trauma to his left eye caused by a screwdriver. He had a history of moderate myopia and had undergone uneventful bilateral LASIK 10 years previously. On examination, he had a 180-degree traumatic corneal flap dislocation with an inverted tear in the temporal cornea. We performed a precise exploration by SD-OCT after repositioning the corneal flap and a disposable contact lens, used as a therapeutic bandage, was placed on the cornea. Topical antibiotics and glucocorticoids were administered. One month postoperatively, his previous visual acuity had recovered and the dislodged flap showed an epithelial ingrowth, which did not require removal.

Conclusion Early recognition, precise control by SD-OCT and reposition the flap along with hydrating the flap together with a contact lens, topical antibiotics and steroids may be a good treatment option. Late traumatic flap dehiscence is a rare complication which demonstrates the vulnerability the flap-bed stromal interface how a potential zone of weakness for many years following the procedure. Patients should be advised to visit ophthalmologist for injury after LASIK.

\section{- S020}

presbyopia and hyperopia treatment by Presbylasik using a central multifocal excimer laser profile with a micromonovision

$\operatorname{SAIBN}(1), \operatorname{ABRIEUN}(1), B E R G U I G A M(1)$, RAMBAUD C (1), FENOLLAND JR (1), BONNEL S (2), FROUSSART-MAILLE F (3), RIGAL-SASTOURNEJC (3)

(1) Ophthalmology, HIA Percy, Paris

(2) Paris

(3) Ophthalmology, Paris

Purpose To analyze the efficacity and satisfaction of treatment by hyperopic presbyopia with a new in situ keratomileusis (LASIK) procedure, Supracor( Technolas Perfect Vision)

Methods This retrospective study included 58 eves of 29 patients treated with a new central multifocal presbyLASIK with a micromonovision using the Technolas 217P excimer laser between september 2011 to september 2013. Mean patient age was 55 years (range 47 to 62 years). The mean preoperative spherical equivalent refraction was 1.3D. The main outcome measure were uncorrected distance visual acuity (UDVA) and uncorrected reading ability, aberrometry, the bump and the satisfaction of the patients.

Results The mean binocular UDVA was $0.09 \operatorname{logMAR}-/+0.15$ for distance vision and $0.22 \log \mathrm{MAR}+/-0.11$ for near vision the day postoperatively , $0.04 \log \mathrm{MAR}+/-0.14$ for distance vision and $0.17 \log M A R+/-0.12$ for near vision at 1 months, $0.15 \log M A R+/-0.23$ and $0.19 \log M A R+/-0.18$ respectively at 3 months and $-0.02 \log M A R+/-0.06$ and $0.18 \log \mathrm{MAR}+/-0.11$ respectively at 6 months. The mean bump was $2.56+/-0.9 \mathrm{D}$. The median total RMS increased from $0.30 \mu \mathrm{m}+/-0.07$ preoperatively to $0.46 \mu \mathrm{m}+/-$ 0.14 postoperatively $(\mathrm{p}<0.01) .3$ patients had a retreatment : 2 in the dominant eye to improve the UDVA of distance vision and 1 in the nondominant eye to improve near vision. $86 \%$ of patients report an independance of glasses for daily activities using near intermediate and distance vision. 100\% of patients would recommend these procedure to their friends.

Conclusion Central Presbylasik with micromonovision provided a high level of independance for near, intermediate and far vision. 
- S021

Distribution of orbscan indices in a young population of Iran

YEKTA AA (1, 2), HASHEMI H (3), KHABAZKHOOB M $(4,3)$,

YAZDANIN (1), OSTADIMOGHADDAM H (1,2), DERAKHSHAN A (5), MOUSAVIM (5), HERAVIANI (1, 2), NOROUZIRADR R (6), YEKTA R (7)

(1) Department of Optometry, Mashhad University of Medical Sciences, Mashhad

(2) Refractive Errors Research Center, Mashhad University of Medical Sciences, Mashhad

(3) Noor Ophthalmology Research Center, Noor Eye Hospital, Tehran

(4) Department of Epidemiology, Faculty of Public Health, Shahid Beheshti University of Medical, Tehran

(5) Khatam-al-Anbia Eye Hospital, Mashhad University of Medical Sciences, Mashhad

(6) Dezful University of Medical Sciences, Dezful

(7) Fadak clinic, Mashhad

Purpose To determine the distribution of keratometric indices, corneal thickness, anteriorchamber depth, pupil diameter and corneal diameter by Orbscan in a young Iranian population

Methods In this cross-sectional study, samples were selected through multistage clustersampling from among the students of Mashhad University of Medical Sciences, Iran. Afterperforming refraction and retinoscopy, Orbscan was used for imaging.

Results Of 1330 selected students, 1121 participated in the study. After applying the exclusioncriteria, final analysis was performed on 1051 subjects. The mean age of thesubjects was $26.1 \pm 3.2$ years (19-34 years old). The mean and $95 \%$ confidence interval (CI) ofmaximum keratometry, minimum keratometry, pupil diameter corneal diameter, anteriorchamber depth, and central corneal thickness were 44.5(44.444.6), 43.1(43.0-43.2), 4.3(4.3-4.4),11.7(11.7-11.7), 3.7(3.6-3.7) and 550.5 (548.4-552.6), respectively. After adjusting with age and spherical equivalent (SE), the maximum and minimum keratometry, thinnest corneal thickness were higher in females while the corneal diameter and anterior chamber depth were more in males. Corneal diameter and anterior chamber depth showed significant changes with age. The SE was only correlated significantly with maximum keratometry and anterior chamber depth.

Conclusion The results of this study showed the normal ranges and averages of some Orbscan indices in a young Iranian population. The insignificant changes of the corneal diameter and anterior chamber depth with age and the differences between males and females in the evaluated indices should be considered in refractive surgery.

- S023

Expression of HGF/c-Met in control and keratoconus corneas

YOU J (1), ROUFAS A (1,2), WENL (1), HODGE C (1,3), SUTTONG (1,3), MADIGANM (4)

(1) Save Sight Institute, University of Sydney, Sydney

(2) Westmead Hospital Eye Clinic, Westmead Hospital, Westmead

(3) Vision Eye Institute Chatswood, Chatswood

(4) Optometry and Vision Science, University of NSW, Kensington

Purpose Keratoconus $(\mathrm{KC})$ is the most common primary degenerative corneal disease with a prevalence of around 1 in 2000 worldwide. A recent genome-wide association study on potential genes associated with $\mathrm{KC}$ identified a significant association between $\mathrm{KC}$ and two single nucleotide polymorphisms in the promoter region of the HGF gene. The HGF/c-Met pathway protects cells from apoptosis and is important to maintain epithelial homeostasis. In this study, we investigated if the $\mathrm{HGF} / \mathrm{c}$-Met pathway is involved in $\mathrm{KC}$ by comparing $\mathrm{HGF} / \mathrm{c}-\mathrm{Met}$ protein expression in control and $\mathrm{KC}$ samples. Methods HGF and c-Met immunoreactivities in control $(\mathrm{n}=5)$ and $\mathrm{KC}(\mathrm{n}=10)$ corneas were imaged by confocal microscopy.Basal tears were collected by capillary micropipettes from control and $\mathrm{KC}$ patients ( $\mathrm{n}=5$ each), and HGF protein expression determined by Western blot. Intracellular c-Met (the membrane bound receptor for HGF) expression was detected by extracting proteins from both control and $\mathrm{KC}$ corneal epithelium ( $\mathrm{n}=5$ each) followed by Western blot.

Results HGF and c-Met were expressed in control and KC corneas. However,in KC obvious cytoplasmic staining of basal epithelium was noted compared to the more diffuse cytoplasmic epithelial immunostaining in control corneas. Western blot results showed that lower levels of HGF in KC tears compared to control tears, and the intracellular c-Met expression was also weaker in KC cell lysates compared to controls.

Conclusion We find that $\mathrm{HGF} / \mathrm{c}$-met expression patterns were altered between $\mathrm{KC}$ and control corneas, and their expression levels were reduced in $\mathrm{KC}$ tears and corneal epithelium cell lysates suggesting a role for this pathway in the pathogenesis of KC.Funding: Medical School of Sydney University, Sydney Eye Hospital Foundation, Lions NSW Eye Bank

\section{- S022}

Structure of Collagen fibril and proteoglycans at different depth of corneal stroma of CXL treated rats

AKHTARS (1), SMEDOWSKI A (2), KHANA (1), KAARNIRANTA K (3)

ALMUBRAD T (1)

(1) Cornea Research Chair, Optometry, Riyadh

(2) Ophthalmology Clinic, Silesia

(3) Ophthalmology, University of Eastern Finland, Kuopio

Purpose In the present study we assess the effects of collagen cross-linking on ultrastructure organization of collagen fibrils (CF) and proteoglycans (PGs) in a rat model.

Methods The corneas were treated with a standard cross linking (CXL) method with isotonic riboflavin. Scelral-corneal rings were removed one week after the treatment. All samples were fixed in $2.5 \%$ glutaraldehyde containing cuprolinic blue in sodium acetate buffer and processed for electron microscopy.

Results The CXL rat corneal stroma thickness increased compared to the normal rat corneal stromal thickness. The collagen fibril diameter $(29.28 \pm 3.42 \mathrm{~nm})$ in the anterior stroma of CXL rats was larger compared to the CF diameter $(27.76 \pm 3.77 \mathrm{~nm})$ of the anterior stroma of control rats. Within the CXL rat corneal stroma, the CF diameter $(29.28 \pm 3.42 \mathrm{~nm})$ and the interfibrillar spacing $(43.01 \pm 3.32 \mathrm{~nm})$ in the anterior stroma were larger compared to the diameter $(26.07 \pm 2.8 \mathrm{~nm})$ and interfibrillar spacing $(41.27 \pm 3.52 \mathrm{~nm})$ in the posterior stroma. The PGs mean area was larger in the posterior stroma $(260.1 \pm 282.395 \mathrm{~nm} 2)$ than the PGs mean area in the anterior stroma $(209.98 \pm 177.0 \mathrm{~nm} 2)$ within the corneal stroma of the CXL rat.

Conclusion The present study demonstrates that CXL leads to an increase in the CF diameter and interfibrilar spacing and reduces the PGs area in the anterior stroma of the CXL rats. The changes in the collagen fibril diameter could be due to an increase in the spacing between the microfibrils within the collagen fibrils. The increase in interfibrillar spacing could be due to an increase in hydration in the corneal stroma.

- S024

Observational comparative study of different predictive scores of infraclinic keratoconus in refractive laser surgery in case of recused patients

CHENY T, ABRIEU M, RAMBAUD C, BERGUIGA M, SENDOND, THOUVENINR, FROUSSART-MAILLE F, RIGAL-SASTOURNEIC ophtalmology, Clamart

Purpose INTRODUCTION : The surgical laser treatment of refractive disorders is becoming more and more prevalent. Preoperative evaluation means are multiple and all seem promising in the detection of infraclinic keratoconus. Our purpose was to compare the different scores. SETTING : Review of cases, ophthalmology and refractive surgery unit, Percy Military Hospital, Clamart, France.

Methods MATHERIAL AND METHODS : Retrospective monocentric observational study, conducted between november 2013 and may 2014. Each patient benefited from an exhaustive corneal topography assessment : Pentacam" and a Belin/ Ambrosio ehancement (P/B), Orbscan" and SCORE analyzer by Gatinel \& Saad $(\mathrm{O} / \mathrm{S})$, and an Ocular Response Analyzer (ORA). A systematic analysis of the following was conducted : the thinnest point of pachymetry (TP), the value of maximum posterior elevation (MPE), the I-S ratio (IS), the maximum keratometry (K max), the presence of SRAX, and the enantiomorphism.

Results RESULTS : 36 myopic patients, recused from laser refractive surgery, were included, or 72 eyes. Suspect cases added up to 31 patients for $\mathrm{P} / \mathrm{B}, 32$ for $\mathrm{O} / \mathrm{S}$, and 27 for ORA (or respectively 52,55 and 40 eyes). There were 9 cases of discordance between the different scores. The averages were respectively : $502+/-47 \mu \mathrm{m}$ for P, $26.81+/-7.46 \mu \mathrm{m}$ for MPE, $0.78+/-0.55$ for IS, $44.6+/-1.8 \mathrm{D}$ for K-max.

Conclusion CONCLUSION : Each of the exams and indices studied led us to detect infraclinic keratoconus, but in discordant cases, a more detailed analysis of the topographic map remains essential. 
- S025

Fluorophotometry study of riboflavin in iontophoresis procedure and conventional cross linking

DANIEL E (1, 2), CASSAGNEM (3), BONNINN (1,2), BOREL A (4) VIENNET A (5) COUL ANGEONLM (1) MONNEYRONN (1) MALECAZEF(3), CHIAMBARETTA F $(1,2)$

(1) Ophthalmology, CHU G.Montpied, Clermont-Ferrand

(2) R2D2 EA7281, Medical Biochemistry Laboratory, School of Medicine,Auvergne University, Clermont-Ferrand

(3) Purpan Hospital, Toulouse

(4) Ophthalmology, CH Jacques Lacarin, Vichy

(5) Ophthalmology, Nice

Purpose A longitudinal observational multicentric study is conducted in Ophthalmology departments of Gabriel Montpied Hospital in Clermont-Ferrand and Purpan Hospital in Toulouse. The aim of our study is to evaluate and compare the variations of riboflavin concentration in vivo through fluorophotometry in the anterio segment, during iontophoresis procedure (I-CXL) and conventional cross-linking (C-CXL) for keratoconus patients.

Methods Inclusion criteria are patients from 18 to 40 years old, with progressive keratoconus and a corneal pachymetry higher than $400 \mu \mathrm{m}$ at the thinnest point. Exclusion criteria are patients with central corneal opacities or patients with previous history of corneal disease or corneal surgery. The use of Fluorotron Master FM- $2^{\circ}$ for anterior segment fluorophotometry allows us to study the concentration of fluorescent molecules in the cornea and in the anterior chamber, with an automated processing.

Results Corneal concentration of riboflavin increases during instillation until a plateau is reached for both procedures. Though, the measurements for I-CXL show a lower corneal concentration of riboflavin, about 50\% less than the concentration in C-CXL. More results are to come and will be presented during the EVER meeting.

Conclusion This is the first in vivo study of riboflavin corneal concentration kinetic in keratoconus patients. This fluorophotometry study needs to be complemented by the results of current clinical studies

- S027

Translation of the therapeutic approach of scleral collagen crosslinking (SXL) from animal to human

RATTUNDE U (1), KORBER N (1, 2), GOHLER C (1,2), KOCHC $(1,2,3)$ KARL A (1, 2), SCHULDT C (4), REICHENBACHA (2), FRANCKEM $(1,2)$ WIEDEMANN P (3), ISELI HP (5)

(1) Translational Centre for Regenerative Medicine, Leipzig

(2) Paul-Flechsig-Institute for Brain Research, Leipzig

(3) University Hospital, Dept. of Ophthalmology, Leipzig

(4) Institute of Soft Matter Physics, Leipzis

(5) Limmat Augenzentrum AG, Zuerich

Purpose In our research project "collagen crosslinking of scleral tissue (SXL)," the application of riboflavin and blue light has been proved as a possible therapeutic method to increase scleral stiffness. Nevertheless, it is necessary to develop a new application system applicable in human.

Methods Rheology was used to investigate changes of sclera stiffness after SXI treatment. An adapted and modified blue light irradiations system has been developed to apply various blue light intensities.

Results As a proof of principle, we demonstrate a dose dependent increase of scleral stiffness after application of various blue light energy dose rates. Increased biomechanical stiffness could be induced with light dose rates up to $200 \mathrm{~mW} / \mathrm{cm}^{2}$ However, the light application system used for animal surgery has serious limitations. The animal experiments revealed relevant technical parameters and helps to design a suitable substance and application system for usage in human.

Conclusion The results confirm the idea that SXL by riboflavin and blue light irradiation is an applicable method to increase biomechanical stiffness of scleral tissue and therefore, it might be a treatment for pathologic myopia. For the therapeutic application in patients a special substance application and irradiation system has to be refined and developed further.

\section{- S026}

Scleral collagen crosslinking (SXL) by riboflavin and blue light inhibits eye growth of young rabbits

KORBER N (1, 2), GOHLER C (1,2), KOCH C (1,3), KARL A (1, 2), PENK A (4) HUSTER D (4) REICHENBACHA (2) FRANCKEM $(1,2)$ WIEDEMANNP (3) $\operatorname{ISELI~HP~(5)~}$

(1) Translational Centre for Regenerative Medicine, Leipzig

(2) Paul-Flechsig-Institute for brain research, Leipzig

(3) University Hospital, Dept. of Ophthalmology, Leipzig

(4) Institute of Medical Physics and Biophysics, Leipzig

(5) Limmat Augenzentrum AG, Zuerich

Purpose The application of riboflavin and blue light (for Collagen Crosslinking of scleral tissue; SXL) was proved as a possible therapeutic method to increase scleral stiffness and therefore, to inhibit eye growth in young rabbits.

Methods For eye growth examination the sclera of young rabbit eyes were treated with riboflavin and $10 \mathrm{~mW} / \mathrm{cm}^{2}$ blue light. MR-Imaging and ultrasonic A scans were used to measure effects of SXL treatment on eye growth. Safety and efficacy parameters were determined using immunohistochemical and histological methods.

Results Immunohistochemical and histological examinations showed no neurodegenerative side effects or signs of glial reactivity in retinal tissue beyond a light intensity of $400 \mathrm{~mW} / \mathrm{cm}^{2}$. Young animals treated with a minimal effective light energy dose of $10 \mathrm{~mW} / \mathrm{cm}^{2}$ displayed a significant reduction of their eye growth. The eye growth inhibition after SXL treatment maintained for at least 24 weeks.

Conclusion The results confirm the idea that scleral collagen crosslinking by riboflavin/blue light is an applicable method to inhibit eye growth and therefore, it might be a treatment for pathologic myopia.

\section{- S028}

Characterization of extracellular matrix remodelling enzymes in the sclera after collagen cross linking (SXL)

GOHLER C (1, 2), KARL A (1), KORBER N (1, 2), KOCHC (2, 3), REICHENBACHA (1), WIEDEMANN P (3), FRANCKEM $(1,2)$

(1) Paul Flechsig Institute of Brain Research, University of Leipzig, Leipzig

(2) Translational Centre for Regenerative Medicine, University of Leipzig, Leipzig

(3) Department of Ophthalmology, Eye Hospital, University of Leipzig, Leipzig

Purpose High myopia is characterised by excessive eye growth and mechanically weakened, thinned and stretched scleral tissue. Enhanced chemical cross linking of collagen fibrils in the sclera (SXL) is a promising therapeutic approach to stiffen the sclera and to prevent eye globe elongation. Therefore, it is important to understand the role of collagen fibril producing fibroblasts and the extracellular matrix remodelling enzymes in normal and myopic eyes and after SXL treatment.

Methods We examined scleral tissue of normal rabbit eyes and after SXL treatment by means of histology and electron microscopy. We characterised expression profiles of cellular markers (e.g. Vimentin) and of relevant enzymes (e.g. MMPs) by means of RT-PCR analysis and immunohistochemistry in cell cultures from human and rabbit scleral tissue.

Results We observed altered scleral collagen fibril profiles and activated scleral fibroblasts - signs of remodelling processes after SXL treatment. Fibroblasts are capable to produce and secrete collagen and different matrix-degrading and regulatory enzymes (e.g. MMP1-3 and 9; TIMP1-4; LOX)

Conclusion Significant remodelling processes were induced after SXL treatment and the role of each specific contributor/enzyme has to be determined for an efficient SXL treatment.increases scleral stiffness. We want to investigate the influence of relevant enzymes on remodelling processes after SXL to optimize the new and promising therapeutic approach 
- S029 / 2434

Reproducibility of keratometric measurements obtained with OCULUS $^{\oplus}$ Pentacam $^{\circledR}$ HR in the evaluation of keratoconus progression

SILVA N (1,2), VIEIRA L (1,3), ANJOS R (1), ALVESN (1), MADURO V (1), FEIJAO J (1), CANDELARIA P (1)

(1) Centro Hospitalar Lisboa Central, Lisboa

(2) Centro Hospitalar do Funchal, Funchal

(3) Hospital Divino Espirito Santo, Ponta Delgada

Purpose To determine the reproducibility of keratometric parameters obtained by OCULUS" Pentacam "HR in the evaluation of keratoconus progression.

Methods Thirty nine eyes of 25 patients from the Cornea Department of the Centro Hospitalar de Lisboa Central were included in the study. Fourteen eyes presented with clinical and topographic diagnosis of keratoconus (stage 1-3) and were defined as the "keratoconus without ring" group; 9 eyes had already an intrastromal ring implanted and were defined as the "keratoconus with ring"; the remaining 16 eyes, without clinical or topographic evidence of disease, were used as controls. Every eye was submitted to identical methodology: 5 consecutive keratometric measurements obtained with OCULUS $^{\circ}$ Pentacam HR; central keratometry, SKmax, minimal pachymetry and anterior chamber depth were used to determine the existence of statistically significant reproducibility.

Results Overall significant measurement variability was obtained in the "keratoconus without ring" group (Km 0.129 D vs 0.0375 D, SKmax 0.507 D vs 0.0625 D, Minpach $5.429 \mu \mathrm{m}$ vs $2.5625 \mu \mathrm{m}, \mathrm{ACD} 0.024 \mathrm{~mm}$ vs $0.0194 \mathrm{~mm}$ ), with highest variability in the subgroup of patients with more advanced disease. In the "keratoconus with ring" group statistically significant variability was found for all parameters except for anterio chamber depth (Km 0.289 D vs 0.0375 D, SKmax 0.444 D vs 0.0625 D, Minpach 4.333 $\mu \mathrm{m}$ vs $2.5625 \mu \mathrm{m}$, ACD $0.024 \mathrm{~mm}$ vs $0.0194 \mathrm{~mm}$ ).

Conclusion Keratometric measurements obtained with OCULUS" Pentacam ${ }^{\circ} \mathrm{HR}$ should be assessed cautiously in the evaluation of keratoconus progression, with or without intrastromal ring; the use of single measurements is unadvised.

- S031 / 2436

Mathematical model of corneal reendothelialization after inadvertent descemetorhexis

TRONEM (1, 2), JULIENNE R (1, 2), RANNOU K (3), BERNARD A (1), HEZ (1), $\operatorname{GAVET} Y(3), \operatorname{THURET} G(1,4), \operatorname{GAINP}(1,2)$

(1) Corneal Graft Biology, Engineering and Imaging Laboratory, EA2521, Federative Institute of Research in Sciences and Health Engineering, Faculty of Medicine, Jean Monnet University, Saint-Etienne

(2) Ophthalmology dpt, University Hospital, Saint-Etienne

(3) Ecole Nationale Supérieure des Mines, Saint-Etienne

(4) Institut Universitaire de France, Bd St Michel, Paris

Purpose To report a case of inadvertent descemetorhexis during phacoemulsification and our conservative approach based on the endothelial cells (EC) self-healing capacity

Methods A 85-year-old man presented with an unusual complication of cataract surgery. During visco injection, the Descemet was accidentally dissected and removed during the capsulorhexis. On the next day, a massive corneal edema was observed. Based on the self-healing capacity of the residual peripheral ECs, we proposed to observe the natural course. The posterior corneal surface geometry was measured in OCT (CASIASS-1000) and the residual endothelial area was calculated from a mathematical hemiellipsoid model. Considering the EC density (ECD) from the fellow eye, we estimated the theoretical residual EC pool and thus the final post redistribution ECD in the patient. Results The theoretical ECD after endothelial cell redistribution was 1680 cells $/ \mathrm{mm} 2$, reinforcing the wait and see strategy. Visual acuity improved from counting fingers initially to $20 / 40$ at 8 months. The central cornea was clear and $540 \mu \mathrm{m}$ thick. Specular microscopy and confocal microscopy confirmed the repopulation of the central defect by the ECs over the denuded posterior stroma. The central ECD measured at 5 months and 8 months were 550 and 516 cells $/ \mathrm{mm} 2$ respectively.

Conclusion The ECD was less than expected suggesting a higher traumatic loss and a larger defect. The originality lies in the mathematical modeling of the spontaneous selfhealing capacity of the endothelium, deferring penetrating or endothelial keratoplasty. Interestingly, despite the 85 years old, ECs keep important migration and enlargement capacities. Grant: ANR/CORRIMO3D
- S030 / 2435

Peripheral neuropathy in chronic renal failure: an in vivo confocal microscopy study

VIEIRA L (1), ANJOS R (1), SOUSA H (2), SOUSA A (3), BORGES A (2), BRANCOP (2) ALVES N(1), MADUROV (1), CANDELARIA P (1)

(1) Centro Hospitalar Lisboa Central - Ophthalmology, Lisboa

(2) Centro Hospitalar Lisboa Ocidental - Nephrology, Lisboa

(3) Centro Hospitalar Lisboa Central - Neurology, Lisboa

Purpose To analyse corneal nerve morphology alterations in patients with chronic renal failure by in vivo corneal confocal microscopy (CCM).

Methods A prospective study was conducted in 30 patients ( 30 eyes) with pre-dialysis chronic renal failure (CRF) and 30 aged-matched healthy volunteers (30 eyes). A complete ophthalmologic examination, esthesiometry (Cochet-Bonnet esthesiometer), in vivo CCM (Heidelberg Retina Tomograph II/Rostock Cornea Module) and neurological evaluation were performed.

Results Corneal sensation was reduced in pre-dialysis CRF patients compared with control subjects. Nerve fiber density, length and branch density were reduced and tortuosity was increased in the CRF group $(\mathrm{p}<0,05)$. These parameters were related with severity of neuropathy

Conclusion In vivo CCM may be a valuable method for diagnosis of uremic neuropathy and may be used to grade its severity.

- S032 / 2437

Investigation of anterior chamber flare and corneal Langerhans cells in rheumatoid arthritis and in dry eye

MARSOVSZKY L, BAUSZ M, NÉMETH J, RESCHM

Department of Ophthalmology, Semmelweis University, Budapest

Purpose To examine the anterior chamber flare and corneal Langerhans cells (LC)in rheumatoid arthritis (RA) and compare the results to those found in patients with dry eye disease (DED) and in age matched control (C).

Methods 15 patients with RA (11 women, és 4 men, mean age: 67.3+8.6 years) 20 DED patients (11 women, 9 men, mean age: $57.2 \pm 14.4$ years) and 15 control subjects (8 women, 7 men, mean age: $49.6 \pm 19.1$ years) were enrolled in this study. Anterior chamber flare was evaluated using Kowa FC-600 laser flare meter and corneal LC densities were investigated at the corneal centre with confocal microscopy (Heidelberg Retina Tomograph with Rostock Cornea Modul (HRT II RCM)) in all groups.

Results There was a significant difference in the flare values between RA and DED patients $(6,65 \mathrm{f} / \mathrm{ms}$ in RA and 4,75 f/ms in DED $(\mathrm{p}<0.01)$. Central LC density was: $57.6 \pm 34.4 / \mathrm{mm} 2$ in RA and $52.3 \pm 31.3 / \mathrm{mm} 2$ in DED respectively. Both values were significantly lower than that of in control $(24.6 \pm 18.6 / \mathrm{mm} 2, \mathrm{p}<0.05)$

Conclusion The blood aqueous barrier might be altered in RA even without signs of clinically significant ocular inflammation. This alteration in the blood aqueous barrier might contribute to the accumulation of LCs at the corneal centre. The greater LC density and flare values found in RA might represent novel players in the pathogenesis of dry eve. 
- S033

Influence of pterygium excision surgery on corneal biomechanical properties

CARAMELLO C (1), MATEO OROBIA AJ (2), PÉREZ D (1), IBAÑEZJ (1), HERRERA L (1) ZABADANIK (1), MARTINEZ M (1), DEL BUEYMA (1) PINILLA I (1), ASCASO J (1)

(1) HCU Lozano Blesa, Zaragoza

(2) Hospital Universitario Miguel Servet, Zaragoza

Purpose To evaluate biomechanical corneal changes after pterygium excision surgery. Methods Prospective, interventional study in which 22 patients with unilateral pterygium were enrolled. Patients underwent pterigyum excision surgery with conjunctival autograft transplantation. The following corneal biomechanical properties were studied using Ocular Response Analyzer (ORA), preoperatively and postoperatively: Corneal Resistance Factor (CFR), Corneal Hysteresis (CH), Goldman correlated intraolcular pressure (IOPg) and Corneal Compensated IOP (IOPcc).

Results CRF increased after pterygium excision surgery. Nevertheless, CRF returned to normal values at day 30. There was a decrease in $\mathrm{CH}$ parameters at day 7 and at day 30 compared with preoperative values. There was an increase in IOP, IOPg and IOPcc postoperatively.

Conclusion $\mathrm{CH}$ decreases after pterygium surgery probably because of the elimination of a biomechanical reinforcement provided by the fibrovascular tissue that invades the anterior stroma. The postoperative decrease in $\mathrm{CH}$ could influence the IOP measure, which could be infraestimated using the Golman applanation technique. ORA postoperavtive exploration provides more precise IOP values.

\section{- S035}

Adenovirus keratoconjunctivitis in relation with refractive surgery. Effects and treatment of sequelae

PEREZ NAVARRO I, DEL BUEY MA, HERERRA L, CARAMELLOC, ALMENARA C, IDOATE A, MARTINEZ M, ESTEBAN O, PEREZ GARCIA D, PINILLA I

Ophthalmology. Hopital Clinico Lozanano Blesa, Zaragoza

Purpose Adenovirus keratoconjunctivitis (AK)can cause corneal opacities that sometimes remain in time, compromising the vision of the patient. The objetive is to show the different morphologic types of corneal affection as a consequence of AK in patients who have received refractive surgery,and to evaluate the application of refractive keratectomy with excimer laser as a possible treatment of the opacities and visual impairment caused.

Methods We analyze three examples of patients with visual impairment caused by corneal opacities secondary to adenovirus keratoconjunctivitis. The first case presents central nummular opacities without refractive history; opacities are treated,by photoablation myopic and associated hyperopic. The second case presents central corneal opacities after adenovirus keratoconjunctivitis, 10 years after PRK practice, causing myopic regression;the visual and refractive result emerges after a new laser photoablation. The third case shows the peculiar corneal fibrosis which appears after AK in a patient who received past LASIK treatment

Results In the cases of opacification due to superficial corneal fibrosis, good visual and refractive results are obtained through the use of superficial photoablation with excimer laser, with the associated application of mitomycin C

Conclusion The morphology of the lesions observed as a consequence of AK varies in patients with no history of refractive surgery, and in patients with a history of refractive surgery Lasik and surface techniques. Refractive PTK is an alternative to the treatment
- S034

Late diagnosis of donor-to-host herpes infection in a descemetic deep anterior lamellar keratoplasty (dDALK) procedure for keratoconus: case report.

MENICACCI F (1), SARNICOLA E (1), MENICACCI C (1), SARNICOLA V (2)

(1) Department of Ophthalmology, University of Siena, Siena

(2) Department of Ophthalmology, Misericordia Hospital, Grosseto

Purpose To report a case of donor herpetic infection after descemetic deep anterior lamellar keratoplasty (dDALK) using the big-bubble technique in a patient with no previous history of herpes simplex virus type 1 (HSV-1) infection.

Methods A 39 year old man with a 15-year history of keratoconus underwent an uncomplicated dDAL.K. Graft failure treated with excisional dDALK happened 3 times. Culture of failed donor corneas, bandage contact lens and stitches were tested for fungus and bacteria in all three explanted tissues. Polvmerase chain reaction (PCR) for HSV-1 DNA was performed only on the last failed corneal graft.

Results Four weeks after intervention patient developed a primary graft failure with corneal melting. Emergency DALK procedure was performed. Cultures were negative for fungus and bacteria. Graft failure occurred again during the early postoperative period. Another DALK procedure was performed. Cultures were again negative in removed tissues. In eight days the patient developed a third graft failure with corneal melting, so that a new DALK procedure had to be performed. PCR was positive for HSV-1 DNA. Systemic acyclovir prevented a recurrence of infection and at the 6-month and 1 year follow-up, no signs of corneal graft failure were detected.

Conclusion HSV infection should be considered in the differential diagnosis with bacterial and fungal infection in early graft failure after DALK procedure. DALK allowed the repetition of corneal transplantation four times and provided enough endothelial cell density at 6-month of follow-up.

\section{- S036}

Laser in situ keratomileusis: epidemiology and risk factors of flap folds

SENDOND (1), RAMBAUD C (1), ABRIEUM (1), BERGUIGA M (1),

FROUSSART-MAILLE F (2), RIGAL-SASTOURNE JC (2)

(1) Refractive Surgery Unit, Percy Military Hospital, Paris

(2) Refractive Surgery Unit, Percy Military Hospital, Clamart

Purpose Flap wrinkles can be seen in $0,2-4 \%$ of LASIK cases. Our purpose is to evaluate flap folds through 7 femto-second cases and make a review of the literature on the epidemiology.

Methods Retrospective monocentric review of 822 eyes who had flap created with he IntraLase FS60 femtosecond laser one month follow up were included between January 2012-January 2014 in the Ophthalmology and Refractive Surgery Unit, Percy Military Hospital. Patients had an exhaustive examination with uncorrected (UDVA) and corrected distance visual acuities, spherical equivalent and mean keratometry $(\mathrm{Km})$ were compared preoperatively and postoperatively. Flap thickness were measured with SD-OCT and optical aberrations on Zyoptix Wavefront System.

Results 7 folds of 6 patients were observed ( $0.85 \%$ of LASIK procedures), 4 were horizontal, 1 vertical and 2 oblique. No trauma was found. All the folds involved the visual axis. Their UDVA was $+0,2 \log$ MAR (from $+0,4$ to 0 ). Mean spherical equivalent decreased from -4,9 D to - $0,43 \mathrm{D}$, mean Km changed from $43 \mathrm{D}$ to $39.75 \mathrm{D}$. Postoperative optical aberrations measured at $4.57 \mu \mathrm{m}(+/-2)$. Mean thickness measured to $120 \mu \mathrm{m}(+/-8)$. The main causes are early trauma, flap desiccation and contraction, intraoperative misalignment, flap thinness, large photo-ablation of myopia and cuts with microkeratome. The folds concern throughout its thickness and are the result of hyper-hydration of proteoglycans, resulting in irregularity of Bowman's membrane and flap deformation

Conclusion Flap folds are a potentially sight-threatening complication. Rigorous surgical technique, education and post-operative follow-up would reduce their frequency, identify and treat them quickly to increase visual performance. 
- S037

Treatment of corneal ulcers in ocular surface inflammation

LAZREGS

cabinet lazreg, Dar el Beida

Purpose severe ocular surface inflammation can be lead to different diseases and can cause severe corneal involvement

Methods 5 cases of ocular surface inflammation with severe corneal ulcers resistant to conventional treatment , all treated with à new matrix therapy agent (cacicol 20\%) 1 drop a day every other day for 4 weeks, with biomicroscopic records at D0 , D3, D7, D 15 and D30.

Results patients were aged between 18 to 66 years old, ocular surface inflammation was due to GVHD, severe Sjögren's Syndrome, Atopic keratoconjuctivitis, ocular pymphygoide and Stevens Johnson syndrome, all ulcers were chronic and rebels to different treatment, at D7 we observed a decrease on VAs pain scale at D15 in all cases a total epitheliazitation of the ulcers,

Conclusion CACICOL $20^{\circ}$ is a new ophthalmic device, derived from RGTA based matrix therapy (large biopolymers engineered to replace heparan sulfates), wich is a revolution in treatment of chronic ulcers, in our cases it has a double effect antalgic and regenerating;

\section{- S038}

Treatment of alkali-injured corneas by nanofibers seeded with mesenchymal stem cells or loaded with cyclosporine a

HOLAN V, CEJKOVA J, CEJKA C, TROSAN P, ZAJICOVA A Department of Transplantation Immunology, Institute of Experimental Medicine, Prague

Purpose Purpose. To test a possibilty to treat the early corneal inflammation occuring after alkali injury by nanofiber scaffolds seeded with mesenchymal stem cells (MSCs) or loaded with cyclosporine A (CsA).

Methods Methods. The alkali was applied on the cornea of the rabbit eyes. The eyes were rinsed with the excess of physiological solution and covered with nanofiber scaffold seeded with rabbit bone marrow-derived MSCs or with nanofibers loaded with CsA. At different time intervals after the injury the eyes were analyzed macroscopically, immunohistochemically and biochemically.

Results Results. Treatment of alkali-injured eyes with MSCs or CsA significantly attenuated the local inflammatory reaction. The therapeutic effects were characterized clinically, according to a reduced infiltration with inflammatory cells, by a lower neovascularization and by decreased expression of genes for proinflammatory molecules IL-1beta, IL-2, IFN-gamma and iNOS and for vascular endothelial growth factor. These anti-inflammatory effects were accompanied by a more rapid regeneration of the corneal epithelium and by increased expression of gene for cytokeratin K12.

Conclusion Conclusion. The results demonstrate that MSC-seeded or CsA-loaded nanofiber scaffolds represent a promising therapeutic tool for the treatment of alkaliinduced ocular surface injuries

\section{- S039 / 4775 \\ Bioengineering of endothelial grafts using femtosecond Laser cut corneal lamellae or collagen lenticules, endothelialized with} immortalized or hIPSC-derived endothelial cells

FOREST F (1, 2), HE Z (1), MOULARD A (1), SUFFEE N (1), FOREST F (1, 2), DUMOLLARDIM (1,2), PEOC'HM (1,2), GAIN P (1,3), THURET G $(1,4,5$

(1) Corneal Graft Biology, Engineering and Imaging Laboratory, EA2521, Federative

Institute of Research in Sciences and Health Engineering, Faculty of Medicine, Jean

Monnet University, Saint-Etienne

(2) Department of Pathology, University Hospital, Saint-Etienne

(3) Ophthalmology dpt, University Hospital, Saint-Etienne

(4) Ophthalmology, University Hospital, Saint-Etienne

(5) Institut Universitaire de France, Bd St Michel, Paris

Purpose The bioengineering of endothelial grafts appears as a realistic solution to reduce the global scarcity for corneal donation worldwide. We focused our strategies of endothelial bioengineering on the endothelialization of human corneal stromal lamellae with hiPSC-derived endothelial cells.

Methods Human corneas unsuitable for graft but with a normal stroma, were cut into 4 to 7 lamellae of $<100 \mu \mathrm{m}$ thickness with a femtosecond laser (FsL), decellularized from their keratocytes using an optimized protocol (Ethanol/DNAse/SDS) and stored at $+4^{\circ} \mathrm{C}$. They were compared with collagen I compressed thin discs (RAFT) already described as carrier. Endothelialization was performed with immortalized endothelia cells (ECs) (BAG12) and hIPSC-derived ECs (also presented during this congress). Efficiency was assessed by determining endothelial cell density (ECD), morphology after alizarin red staining and SEM and immunolabeling for $\mathrm{Na} / \mathrm{K}$-ATPase expression. Transparency was measured using our customized transparometer

Results both carriers were evenly endothelialized by B4G12 with similar alizarin red staining and $\mathrm{Na} / \mathrm{K}$ ATPase expression patterns but higher ECD for stromal lamellae. Transparency was better for stromal lamellae. hIPSC-derived ECs gave similar characteristics

Conclusion Decellularized Fsl cut stromal lamellae seem better than collagen 1 compressed lenticules for the endothelial bioengineering using hISPC-derived ECs. Unsuitable human cornea could be therefore "recycled" to increase the pool of available grafts. Grants: ABM2013, AIRE2013

\section{- S040 / 4776}

3D reconstruction and segmentation methods for endothelial cell count of stored corneas

BERNARD A (1), HOR G (1), TIFFET T (1), RANNOU K (2), GAVET Y (2) PINOLIJC (2), ACQUART S (3), GAIN P (1, 4), THURET G $(1,4,5)$

(1) Corneal Graft Biology, Engineering and Imaging Laboratory, EA2521, Federative Institute of Research in Sciences and Health Engineering, Faculty of Medicine, Jean Monnet University, Saint-Etienne

(2) Ecole Nationale Supérieure des Mines, Saint-Etienne

(3) Eye Bank, French Blood Centre, Saint-Etienne

(4) Department of Ophthalmology, University Hospital, Saint-Etienne

(5) Institut Universitaire de France, Bd St Michel, Paris

Purpose Measurement of the endothelial cell density (ECD) is the main criterion to validate the quality of corneal grafts in eye banks. Because of corneal curvature and of deep storage-induced endothelial folds, parallax errors may result in ECD overestimation. We designed a software with $3 \mathrm{D}$ reconstruction and optimized segmentation methods to improve cell count reliability

Methods After acquisition of endothelial Z stacks by conventional motorized bright field microscopy, a depth map was obtained by researching maximized measurements of the focus in images (shape-from-focus method). Texture of the surface was built by summing image parts presenting the right focus leadind to an all-in-focus image. By calculating first derivative in all directions of depth map, precise estimation of cells in folds surfaces could be found. In order to improve and facilitate the cell count, a segmentation method based on watershed technique was applied on the texture image. Taking account of the new 3D area, a precise corrected ECD ( $\mathrm{CECD}$ ) could be calculated. We validated the cECD by comparison with ECD obtained manually by an expert. Two types of images were used: the Keratotest (cells boundaries engraved on a quartz wafer) and real endothelial images. Two parameters were compared: quality of cells borders recognition (correct shapes) and ECD.

Results Comparison results showed the relevance of these algorithms for both criteria. Automation of processing allowed gain of time compared to manual estimation

Conclusion 3D endothelial count can be useful to improve corneas selection in eye banks.Grant: ANR2012 CORIMMO3D 
- S041 / 4777

Comparison of ultrathin manual and automated stripping of the endothelium in Fuchs endothelial dystrophy

DUNCOMBE A (1), MATHIEU B (2), ROSSI P (1), TOUBEAU D (1), GUEUDRY J (1), MURAINEM (1)

(1) Ophtalmologie, Rouen

(2) Ophtalmologie, Dijon

Purpose This study compares two techniques of preparation of the endothelial graft: the DSAEK (Descemet Stripping Automated Endothelial Keratoplasty) and an ultrathin endothelial graft manually prepared.

Methods A randomised prospective study is being conducted at the Hospital of Rouen, France. Patients presenting a Fuchs dystrophy are included in two groups. Patients from Group A receive an ultrathin manually prepared endothelial graft and patients from Group B receive a DSAEK. In order to improve the power of our study, one patient from each group is grafted on the same day, with a corneal endothelium coming from the same donor. Improvement of visual acuity is the main outcome measure. Vitality of the graft, refractive modifications induced by surgery and complications are also studied.

Results Currently, 22 patients with a follow up of 6 months have been included. At inclusion, patients from Group A and B were not statistically different. Six months after surgery, mean best corrected visual acuity has significantly improved in both groups but is statistically better in Group A (0,18 $\log$ Mar in Group A versus 0,31 $\log$ Mar in Group B $(\mathrm{p}<0,05))$. Mean endothelial cell density is 1686 cells in Group A statistically more than in Group B ( 1260 cells) $p<0,05$. There were no refractive modifications in either group. There was no episode of graft rejection.

Conclusion Ultrathin endothelial graft seems to give better results than DSAEK in Fuchs dystrophy in terms of visual acuity and endothelial cell density. It could be a good alternative to DMEK (Descemet membrane endothelial keratoplasty) because it is easier to prepare and inject than DMEK and should then be favoured when DMEK is not possible
- S042 / 4778

Surgeon graft less viable endothelial cells than the eye bank cell count suggests

NEFZAOUIC (1, 2), TRONEMC (1, 2), HEZ (1), BERNARD A (1), PISELLIS (1), ACQUART S (3) THURETG $(1,4)$ GAIN P (1)

(1) Corneal Graft Biology, Engineering and Imaging Laboratory, EA2521, Federative Institute of Research in Sciences and Health Engineering, Faculty of Medicine, Jean Monnet University, Saint-Etienne

(2) Ophthalmology dpt, University Hospital, Saint-Etienne

(3) Eye Bank, French Blood Centre, Saint-Etienne

(4) Institut Universitaire de France, Bd St Michel, Paris

Purpose it is universally admitted that a very early important postoperative decrease in ECD occurs after all types of corneal graft, generally attributed to surgical traumatism. This presumed cell loss is calculated between eye bank ECD (ebECD) and post op cell count done in recipients by specular microscopy (SM). Aim: to redefine the very early postop cell loss.

Methods 1/Prospective series of consecutive penetrating keratoplasty for all-comer indications with post-op follow-up by SM (SP3000-P) performed as soon as possible and at least at D15, M1 and M3. This series provided reasonable quantification of the gap between ebECD and very early postop ECD in patients. 2/Ten pairs of corneas with comparable left/right ebECD were randomized: one cornea was grafted, the other was stored similarly and its viable ECD was measured after triple labelling with Hoechst/Ethidium/calcein-AM (IOVS2011 Pipparelli) and quantification with CorneaJ (Cornea2014 Bernard). Graft and vECD measurement were done simultaneously, Relationship between $\mathrm{vECD}$ and early postop ECD were studied.

Results For series 1, a gap of 623 (98-1479) cells/mm2 (median (range)) between ebECD and early postop was measured. For series 2, vECD and early postop ECD of paired corneas were well correlated $(\mathrm{r}=0.7, \mathrm{P}<0.05)$, with a difference of only 143 (5-359) cells between them.

Conclusion These 2 complementary series demonstrated that ebECD provided to surgeons overestimate the number of viable CEC grafted to patients. Taking account only the $\mathrm{vECD}$ at the end of storage (just before graft) and the postop ECD measured as early as possible, we show that the presumed initial very important cell loss is fictive and that the model of postop ECD decrease should be revisited.

- S044

Diquafosol sodium ophthalmic solution for the treatment of dry eye in soft contact lens wearers

YAMADA M (1), SHIGEYASU C (1, 2), FUKUIM (2)

(1) Kyorin University Eye Center, Tokyo

(2) National Tokyo Medical Center, Tokyo

Purpose Ocular dryness, experienced by up to $75 \%$ of soft contact lens (SCL) wearers, is the main reason for its discontinuation. The aim of the study is to evaluate the efficacy of topical application of diquafosol sodium for the treatment of dry eye in SCL wearers. In addition to clinical tests, we used a wheat germ agglutinin conjugate of fluorescein (F-WGA) and fluorophotometry to evaluate the glycocalyx in the ocular surface in vivo. Methods Twelve SCL wearers with dry eye symptoms ( $31.3 \pm 6.8$ year-old) were treated with 3\% diquafosol eyedrops for 4 weeks. The clinical tests included tear breakup time (BUT), Schirmer test, fluorescein staining score, and subjective symptoms evaluated by the Dry Eye Related Quality of Life Score (DEQS). Five minutes after a 5\% F-WGA solution was applied to the eye, fluorescent intensities in the central cornea were measured by fluorophotometry.

Results When comparing before and after diquafosol treatment for 4 weeks, BUT $(3.6 \pm 2.2 \mathrm{sec}$ to $5.0 \pm 2.1 \mathrm{sec}, \mathrm{p}=0.003)$, kerato-conjunctival staining score $(2.8 \pm 1.8$ to $2.1 \pm 1.4, \mathrm{p}=0.045)$ and DEQS score $(19.2 \pm 12.7$ to $10.6 \pm 12.5, \mathrm{p}=0.0032)$ showed statistically significant improvements (Wilcoxon signed-rank test). F-WGA fluorescent intensities in the central cornea $(571.8 \pm 227.8$ to $794.6 \pm 219.4, \mathrm{p}<0.0001)$ significantly increased, and had a significant correlation with BUT

Conclusion Fluorophotometric measurement of WGA staining appears to reflect the glycocalyx in the corneal epithelium, and therefore can be a good indicator of the ocular surface wettability. Topical application of diquafosol sodium improved subjective svmptoms of SCL wearers with dry eye, possibly by enhancing tear film stability and reducing damage of ocular surface epithelia. points, $100 \%$ improvement or stability of visual acuity with an average gain of -0.23 MAR acuity and $100 \%$ improvement or stability of the Oxford score with a mean gain of 1,917 points.

Conclusion Despite its limited size, this cohort of patients treated with sclero-corneal lenses is promising. Whenever possible, this approach should be considered in patients experiencing KCS refractory to standard treatments in cGVHD. 
- S045

Ocular protection index is related to ocular surface disease index in dry eye disease patients

BADIANR (1, 2), UTHEIM TP (2, 3), RÆDERS (2, 4), UTHEIM Ø (2, 3, 5), CHENX $(2,6)$, STOIANOVIC A $(2,6,7)$, EIDET IR $(2,8)$

(1) Department of Ophthalmology, Innlandet Hospital Trust, Oslo

(2) The Norwegian Dry Eye Clinic, Oslo

(3) Unit of Regenerative Medicine, Department of Medical Biochemistry, Oslo

(4) SynsLaser Kirurgi, Tromsoe and Oslo

(5) Department of Ophthalmology, Oslo University Hospital, Oslo

(6) SynsLaser Kirurgi, Tromsoe and Oslo

(7) Department of Ophthalmology, University Hospital of North Norway, Tromsoe

(8) Unit of Regenerative Medicine, Department of Medical Biochemistry, Oslo University Hospital, Oslo

Purpose To assess symptoms and signs in a Norwegian cohort of dry eye disease (DED) patients by investigating the relationship between Ocular Protection Index (OPI) and Ocular Surface Index (OSDI).

Methods DED patients of mixed etiologies were consecutively included in the study and defined as OPIhigh $(\mathrm{OPI} \geq 1)$ or OPIlow $(\mathrm{OPI}<1)$. All patients received an extensive ophthalmological work-up. Groups were compared by using analysis of covariance (ANCOVA).

Results Three hundred and twenty nine (329) DED patients were included. Seventyfour of the 329 patients (22\%) were OPIlow, whereas 255 were OPIhigh (68\%). The two groups did not differ from each other with respect to either age $(P=0.063)$, gender $(\mathrm{P}=0.099)$ or in the number of systemic prescription drugs used ( $\mathrm{P}=0.586)$. After controlling for the effects of gender, age and the number of systemic prescription drugs used OPIlow patients had significantly higher OSDI scores $(16.9 \pm 2.0)$ than OPIhigh patients $(13.5 \pm 1.1)(\mathrm{P}=0,002)$.

Conclusion A low OPI is related to a higher symptom load, as measured by the OSDI questionnaire.

\section{- S047}

Evaluation of self-report questionnaires as predictive tests for obtaining pathological schirmer i, tear film break-up time and ocular protection index scores in dry eye disease patients

BADIANR (1, 2), UTHEIM TP (2, 3), RÆDER S (2, 4), UTHEIM Ø (2, 3, 5), CHEN X (2, 4), EIDET IR $(2,3)$

(1) Department of Ophthalmology, Innlandet Hospital Trust, Oslo

(2) The Norwegian Dry Eye Clinic, Oslo

(3) Unit of Regenerative Medicine, Department of Medical Biochemistry, Oslo University Hospital, Oslo

(4) SynsLaser Kirurgi, Tromsoe and Oslo

(5) Department of Ophthalmology, Oslo University Hospital, Oslo

Purpose To assess if the ocular surface disease index (OSDI) and McMonnie's selfreport questionnaires can be used as predictive tests for obtaining pathological Schirmer I, tear film break-up time (TFBUT) and ocular protection index (OPI) scores in a Norwegian cohort of dry eye disease (DED) patients.

Methods Two hundred and fifty-two patients with DED of mixed etiology were consecutively included in the study. Pathological Schirmer I, TFBUT, and OPI scores were defined as $<10 \mathrm{~mm},<10 \mathrm{~s}$ and $<1$, respectively. All patients received an extensive ophthalmological work-up and completed the OSDI and the McMonnie's questionnaires. Receiver-operating characteristics (ROC) analysis was used to assess the ability of the OSDI and McMonnie's questionnaires to predict pathological scores.

Results Maximal sensitivity (0.58) and specificity $(0.67)$ for predicting a pathological OPI score was obtained by using 15.7 as OSDI cutoff value (area under the ROC curve $0.63 ; \mathrm{P}=0.003)$. For Schirmer I, the maximal sensitivity $(0.58)$ and specificity $(0.60)$ for predicting a pathological score was obtained when using 13.6 as the OSDI cutoff value (area under the ROC curve 0.59; $\mathrm{P}=0.015$ ). The McMonnies questionnaire could not predict either the pathological OPI or Schirmer I scores. Neither OSDI nor McMonnie's questionnaires were able to predict pathological TFBUT scores

Conclusion Cutoff values for the OSDI, but not the McMonnie's questionnaire can be defined to predict pathological Schirmer I and OPI scores in DED patients with moderate sensitivity and specificity.

\section{- 5046}

The effects of lower lid laxity on the response to dry eye treatment

LEEN (1), YIM H (2)

(1) Ophthalmology, Incheon St.Mary's hospital, Seoul

(2) Ophthalmology, Incheon St.Mary's hospital, Incheon

Purpose To compare the responses to dry eye treatment of patients sorted by the degree of lower lid laxity.

Methods Sixty patients were grouped into three groups according to the degree of lower lid laxity. Tear break-up times (TBUT), Schirmer test (ST) scores, ocular surface disease index (OSDI) scores, and changes in OSDI score in each group were compared, before and at 3 months after treatment.

Results TBUT, ST, and OSDI scores were not different among the three groups at baseline. TBUT improved in each group at 3 months after treatment, and no differences between groups were found. ST scores were not increased after treatment, while OSDI were improved to $22.57 \pm 5.243,31.16 \pm 11.353$, and $37.85 \pm 13.342$ in the no, moderate, and high laxity groups, respectively; these improvements were statistically significant $(p=0.003,<0.001,<0.001$, respectively). Patients with greater than moderate lower lid laxity saw the smallest improvement in response to dry eye treatment, as assessed by change in OSDI score ( $\mathrm{p}=0.005$ vs. moderate laxity group, $\mathrm{p}=0.005$ vs. no laxity group).

Conclusion Lower lid laxity is one of the factors contributing to the manifestation of dry eye symptoms, independently of TBUT and ST scores.

\section{- S048}

Clinical performance of preservative-free, hypotonic solution of $\mathbf{0 . 1 5 \%}$ sodium hyaluronate for mild to moderate dry eye

PURSLOW C $(1,2)$

(1) Cardiff University, Cardiff

(2) Plymouth University, Plymouth

Purpose The viscoelastic and hydration properties of Hyaluronic Acid (HA) make it the ingredient of choice in modern topical formulations for relief of dry eye symptoms, but there are few that present it as a hypotonic preparation without preservatives, both properties known to be beneficial for dry eye management. This study was designed to investigate the patient satisfaction and clinical performance of Hyabak (R) (Laboratoires Thea, France) in a group of volunteers with mild to moderate dry eye symptoms.

Methods Thirty volunteers (8M, 22F; mean age 66.6 (SD 9.9) years) with mild to moderate dry eye symptoms, as assessed by the Ocular Surface Disease Index (OSDI) questionnaire), were recruited from an older population around Plymouth, UK. Participants were assessed at baseline and after 10 days of treatment with Hyabak (4 drops daily to each eye). The following was recorded at each visit: bulbar and limbal redness, corneal and conjunctival staining, tear film stability, ocular comfort using visual analogue scales (VAS) and OSDI scores.

Results Ocular comfort and OSDI scores showed significant improvements by day 10 (by $35 \%$ and $34 \%$ respectively; $\mathrm{p}<0.001$ ). Average cornea and conjunctival staining decreased by $50 \%$ and $56 \%$ (both $\mathrm{p}<0.001$ ), respectively. Improvements in bulbar and limbal redness were significant $(\mathrm{p}<0.001)$, and the improvement in tear film stability after 10 days was statistically significant $(\mathrm{p}<0.001)$ but clinically small (average increase +1.1 seconds)

Conclusion Statistically and clinically significant improvements in the key clinical ndicators of success, including patient comfort, can be seen after only ten days using this preservative-free, hypotonic formulation in mild to moderate dry eye.

Commercial interest 
- S049

Immediate impact of hyaluronic and methylcellulose artificial tears on tear film stability

WYLEGALA E (1), SMEDOWSKIA (1), TARNAWSKA D $(1,2)$, CIESLIK T (3), WEGLARZ B (1), NOWINSKA A (1), DOBROWOLSKID (1)

(1) Clinical Department of Ophthalmology, Faculty of Medicine and Department of Dentistry in Zabrze, Medical University of Silesia, Katowice

(2) Department of Biophysics and Molecular Physics, Institute of Physics, University of Silesia, Katowice

(3) Department of Ophthalmology with Surgery Unit, Provincial Hospital, Kielce

Purpose To evaluate immediate impact of hyaluronic and methylcellulose artificial tears on tear film stability.

Methods We performed keratography with tear film analyze in 21 volunteers using Keratograph 4 (Oculus, Germany). After base measurement, volunteers received topical artificial tears drops containing $0.15 \%$ Sodium Hyaluronate into right eye and 0.5\% Carboxyl Methylcellulose into left eye. After 15 and 30 minutes keratography measurements were repeated.

Results In keratography analyzes we observed 4 types of tear film instability, short-time break-up on small area or big area, long-time break-up on small or big area. Mean size of tear meniscus in right eyes before drops was $0.3 \pm 0.1 \mathrm{~mm}$ and $0.32 \pm 0.09 / 0.33 \pm 0.09$ $\mathrm{mm} 15 / 30$ minutes after hyaluronic drops respectively ( $\mathrm{p}<0.05$, Kruskal-Wallis test). In left eyes tear meniscus size was $0.32 \pm 0.1 / 0.33 \pm 0.1 / 0.34 \pm 0.1 \mathrm{~mm}$ respectively before and after methylcellulose drops ( $\mathrm{p}<0.05$, Kruskal-Wallis test). First and mean non-invasive break-up-time in right eyes was 10.7/16.8 sec before drops and 12/18; 12/17 - 15 and 30 minutes after hyaluronic drops application ( $\mathrm{p}>0.05$, Kruskal-Wallis test). In left eyes these parameters were $12 / 16 ; 13 / 18$ and $13 / 17 \mathrm{sec}$ respectively before and after methylcellulose drops ( $\mathrm{p}>0.05$, Kruskal-Wallis test)

Conclusion Immediate impact of hyaluronic and methylcellulose artificial tears on tear film seems to be related more to increase of tear meniscus size, not to tear film break-up time or break-up surface. Keratograhy measurements allow for detail analyzes of tear film state, which includes not only break-up time but also break-up surface, which could be novel indicator for tear film stability impossible to measure in classic slit lamp.

\section{- S051}

Structure and microanalysis of tear film ferning of camel tears, human tears and refresh plus

AKHTAR S, MASMALI A, KHANA, ALMUBRAD T

Cornea Research Chair, Optometry, King Saud University, Riyadh

Purpose We investigated the tear ferning pattern and microelements of the tear film of camel and compare them with human tears and fresh plus eye lubricant.

Methods The tear film pattern was investigated by light and scanning electron microscope. A small drop (1 $\mu \mathrm{l})$ of tears was placed on a glass slide at 23 degrees temperature and humidity of less than $45 \%$. When the tears were dried, they were observed under the light microscope, Olympus BX1. The dried drop was also used to take images from an environmental scanning electron microscope. The microanalysis of the elements was done by using the STEM, JEOL 1400 transmission electron microscope. The Masmali grading scale for tear ferning was used to grade the ferning patterns

Results Light microscopy observations showed that the tear film pattern was surround by a thick peripheral homogenous layers which contained small oily droplets. The peripheral and central ferning contained very thin branches. The tear ferning at the periphery was between grades 1 to 2 whereas tear ferning pattern of the centre was between grades 0 to 1 . The tear ferning pattern of human tears and fresh plus were between grades 1 to 2 . The scanning electron microscope showed very tiny crystals in between the tear ferning. Edax analysis showed that mass percentage of chloride as highest in the camel tears and mass percentage of potassium was more than in the human tears but less than in the 'Reresh plus' lubricant.

Conclusion Our observations suggest that camel tears are better quality than human tears and 'Refresh Plus' lubricant. This suggests that camels may have some extra tear compositions in their eyes, which help the animal to avoid the dryness in their eyes in

\section{- S050}

Comparison of the lubricant eye drops Optive ${ }^{\circ}$, Vismed $^{\circ}$ and Cationorm $^{\circ}$ on the corneal healing process in an ex-vivo model of the anterior ocular segment, the Ex Vivo Eye Irritation Test (EVEIT) System.

DUTESCU R, PINHEIRO R, PANFIL C, SCHRAGEN

ACTO e.V. RWTH University, Aachen

Purpose Evaluation of the impact of lubricant eye drops on the corneal healing process and corneal toxicity.

Methods Optive and Cationorm were tested regarding corneal irritability and effect on corneal healing against Vismed and $0.01 \%$ benzalkonium chloride (BAK) as negative and positive control. Formulas were applied hourly over 3 days on rabbit corneas cultured on artificial anterior chambers (Ex-Vivo Eye Irritation Test (EVEIT) system). Five corneae were used for each substance except for BAK $(n=1)$. Initially, four corneal abrasions $\left(2-5.4 \mathrm{~mm}^{2}\right)$ were induced. All defects were monitored by fluorescein stains and photographes.

Results Optive and Vismed showed a complete corneal healing on day 2. In one cornea (Optive) erosion reoccurred on day 3. Erosion sizes of Cationorm treated corneae increased significantly from $12.20 \mathrm{~mm}^{2}$ to a subtotal erosion of $51.89 \mathrm{~mm}^{2}$ on day 3 . Histology revealed epithelial loss and severe alterations of the superficial stroma for Cationorm. Glucose and lactate concentrations were about unchanged after application of Optive and Vismed. In contrast, Cationorm and BAK treated cornea showed a significant increase in lactate concentrations.

Conclusion Vismed application led to a rapid corneal healing. Whether the toxicity seen for Optive in one cornea is a valid result should be examined in a long term application study. Cationorm shows considerable corneal toxicity that could be caused by its additive, cetalkonium chloride. Otherwise, Cationorms electrostatic properties lead to a drug film on the area of epithelial loss that could hinders epithelial cell migration and adhesion to heal the lesion.

Commercial interes

\section{- S052}

Effects of a tear supplement containing hyaluronic acid (HA) on tear film thickness in healthy subjects

WIRTHM (1), SCHMIDL D (1, 2), KAYA S (3), NAPORA K (1) WERKMEISTER R (4), GARHOFER G (1), SCHMETTERER L (1, 4)

(1) Department of Clinical Pharmacology, Medical University of Vienna, Vienna

(2) Department of Ophthalmology and Optometry, Medical University of Vienna

(3) University Clinic of Ophthalmology and Optometry, Paracelsus Medical University Salzburg, Salzburg

(4) Center for Medical Physics and Biomedical Engineering, Medical University of Vienna, Vienna

Purpose We have recently introduced a new technique for the measurement of tear film thickness (TFT) using ultra-high resolution optical coherence tomography (OCT) in humans. In the present study we hypothesized that the effect of a hyaluronic acid (HA) containing lubricant on TFT can be evaluated using this technique after single instillation.

Methods 16 healthy volunteers were included into the present study. The design was randomized, double-masked and placebo controlled. Subjects randomly received in one eve HA-containing eye drops ( $0.15 \%$, Olixia", Croma Pharma, Austria) and in the other eye $\mathrm{NaCl} 0.9 \%$. Measurement of TFT was performed at baseline and thereafter every 10 minutes until 60 minutes after start of instillation. A repeated measures ANOVA model was employed to study statistically significant differences

Results 8 female and 8 male subjects with a mean age of $26.8 \pm 4.8$ years were included in this study. Baseline TFT was $5.0 \pm 0.5 \mu \mathrm{m}$ in placebo and $4.8 \pm 0.5 \mu \mathrm{m}$ in HA treated eyes. A significant effect of HA containing eyedrops on TFT was seen over time ( $\mathrm{p}=0.014) .10$ minutes after instillation TFT increased to $5.5 \pm 0.8 \mu \mathrm{m}$ in the HA containing eyedrops and remained almost stable in the placebo group $(5.1 \pm 0.8 \mu \mathrm{m})$. Post hoc analysis revealed that TFT was increased up to 30 minutes after instillation and then returned to baseline. Conclusion The results of the present study indicate that HA containing eyedrops increase pre-corneal TFT for as long as 30 minutes in healthy subjects. This effect is not een with $\mathrm{NaCl}$ administration. Our data also indicate that measurement of TFT with ultra-high resolution OCT is a promising technique to study residence time of eye drops at the ocular surface. 
- S053

Substitutes of fetal bovine sera for the culture of human corneal limbal stem cells

INIGO PORTUGUÉS A (1, 2), MEANA A (1,2), VAZQUEZ N (1, 2)

CHACONM (1,2) RIESTRA ANAC (1,2) ORIVEG (3) ANITUA E (3)

MERAYO-LLOVESJ $(1,2)$

(1) Fundación de Investigación Oftalmológica / Instituto Oftalmológico Fernández-Vega, Oviedo

(2) Universidad de Oviedo, Oviedo

(3) Biotechnology Institute, Vitoria

Purpose We analyze the potential use of PRGF-Endoret and human sera (HS) as a substitute of xenogeneic sera in corneal cell cultures.

Methods Plasma rich in growth factors (PRGF) eye drop and HS was obtained at our local blood bank from human blood of healthy donors after informed consent. Briefly PRGF was purified using the Endoret kit in Ophthalmology. HS was obtained according to standardized protocols. Limbal stem cells were obtained from explants of 2-3mm in diameter of the limbal region of corneoscleral tissue and cultured in culture medium containing 10\% PRGF-Endoret", HS or fetal bovine serum (FBS) for 7 days. Afterwards, cells were fixed and growth area was quantified. Immunocytochemistry for p.63, ABCG-2 and cytokeratin was also performed in order to check their immunological markers

Results PRGF-Endoret", HS and FBS, cell cultures showed a positive expression in p.63, ABCG-2 and cytokeratin. However some differences were found between cultures, limbal stem cell cultures supplemented with PRGF-Endoret" and HS showed a better growth area in contrast with the ones supplemented with FBS. Moreover, there was cellular growth in more of the explants cultured in PRGF-Endoret" and HS compared to FBS supplemented cultures.

Conclusion PRGF-Endoret" and HS seems to improve the cellular growth and, while maintaining the p63 and ABCG-2 expression compared to FBS supplemented cultures. Therefore, PRGF-Endoret" or HS could be used as a substitute of xenogeneic sera, not only as an allogeneic product but also as an autologous product for the culture and expansion of corneal limbal stem cells.

\section{- S055}

Engineering the corneal stroma: The effect of a threedimensional environment and growth factor supplementation on keratocyte phenotype

SIDNEY LE (1), DUNPHY SE (2), ROSE FRAJ (2), HOPKINSON A (1)

(1) Academic Ophthalmology, Nottingham

(2) Tissue Engineering and Drug Delivery, Nottingham

Purpose A major challenge of producing a tissue-engineered cornea is the recapitulation of the complex environment of the stroma. The resident cells of the stroma are keratocytes; a quiescent, dendritic cell type, responsible for maintaining the extracellular matrix. Upon injury, or during in vitro culture in foetal bovine serum (FBS), keratocytes differentiate into a fibroblastic repair phenotype. Differentiating these fibroblasts back to keratocytes is crucial in stromal tissue-engineering. Methods previously suggested are three-dimensional environments and serum-free medium.

Methods Primary human corneal stromal cells (CSC) were cultured to achieve a fibroblastic cell type reminiscent of a mesenchymal stem cell. CSC were seeded in poly(lactic-co-glycolic acid) (PLGA) microfibre scaffolds. Constructs were maintained under 4 media conditions: 20\% FBS; $2 \%$ FBS; insulin-transferrin-selenium supplementation (ITS); and bFGF and TGF- $\beta 3$ supplementation (GF). Proliferation, cell phenotype, gene expression and matrix production were assessed.

Results Scaffolds supported cell adhesion and proliferation in all media types. Gene expression and immunocytochemistry showed that scaffolds in ITS and GF media promote the keratocyte phenotype, with increased expression of CD34 and ALDH3A1. Collagen-I deposition was seen to the greatest extent in GF cultures. Quantitative analysis showed expression of proteoglycans was greatest in GF

Conclusion PLGA microfibre scaffolds allow infiltration of CSCs and promote a keratocyte phenotype. This effect is augmented with the use of serum-free medium and growth factor supplementation, leading to improved ECM production and deposition.

\section{- S054}

Decreased basal tear in aged mice is linked to morphological and functional changes in corneal sensory nerve fibers

INIGO PORTUGUÉS A (1, 2), ALCALDE I (1,2), GONZÁLEZ-GONZÁLEZO (1, 2), GALLARJ (3), BELMONTE C (1,3) MERAYO-LLOVES J (1, 2)

(1) Fundación de Investigación Oftalmológica / Instituto Oftalmológico Fernández-Vega Oviedo

(2) Universidad de Oviedo, Oviedo

(3) Instituto de Neurociencias. Universidad Miguel Hernández-CSIC, San Juan de Alicante

Purpose To determine whether the effects of aging on density, morphology and functional activity of corneal cold sensory nerve fibers are associated with changes in basal tearing rate.

Methods TRPM8-EYFP and TRPM8-KO mice of different ages ranging from 90 to 720 postnatal davs were studied. Basal tearing was measured in anesthetized animals, using phenol red threads. Corneal nerves expressing EYFP protein were identified in whole mount corneas using immunohistochemical techniques. Also, trigeminal ganglia (TG) were processed for immunofluorescence techniques against TrkA and CGRP. Extracellular electrical activity of single sensory nerve endings of the corneal surface was recorded in excised and superfused eyes.

Results The number of TRPM8+ fibers and nerve terminals decreased with age. Also, fibers of p90 mice were morphologically different from those of p720 TRPM8EYFP mice resembling those found in TRPM8-KO mice. Basal tearing rate decreased in parallel with the reduction in number of TRPM8+ fibers. Two different populations of sensory nerve endings responding to cold were distinguished electrophysiologically: Low and High-threshold cold terminals. With aging, the incidence of low-threshold cold nerve endings decreased while more high-threshold nerve endings were found. Also, a population of non-peptidergic and another of peptidergic TRPM8+ neurons were distinguished in the TG.

Conclusion Corneal cold sensory fibers and terminals of TRPM8-EYFP mice developed parallel changes in number and morphology, functional activity and basal tearing rate during aging, thus supporting the hypothesis that cold thermoreceptor activity and basal tearing are functionally associated.

\section{- S056}

Plasmapheresis and autologous serum eye-drops in the treatment of acute ocular complications from toxic epidermal necrolysis (Lyell syndrome)

PINNA A (1), NUVOLI E (1), BLASETTI F (1), POSADINU MA (2)

(1) Department of Surgical, Microsurgical and Medical Sciences, Section of Ophthalmology, University of Sassari, Sassari

(2) Burn Unit, ASL1 - Sassari, Sassari

Purpose Toxic epidermal necrolysis (TEN) is a rare, life-threatening, drug-induced, mucocutaneous disease, which can affect the ocular surface. The purpose of this study was to evaluate the acute ocular complications from TEN and investigate on the efficacy of plasmapheresis and serum-eyedrops in the treatment of this condition.

Methods A retrospective chart review of all Burn Unit patients admitted from 20092013 identified 7 TEN patients ( 2 men, 5 women; mean age: $69.6 \pm 20.3$ years). TEN was associated with allopurinol use in 4 patients, with gefitinib in 1 , and with trimethoprim/ sulphamethoxazole in 1. In the remaining patient, the causative agent was not identified. Acute bilateral ocular complications were observed in 5 patients; 3 showed mild ocular surface inflammation, whereas 2 had severe pseudomembranous conjunctivitis with corneal ulcers. All TEN patients were immediately treated with plasmapheresis.

Results In the 2-3 weeks after plasmapheresis, there was a marked improvement of the patients' general condition. In those with mild ocular surface inflammation, the use of preservative-free artificial tears and steroids was beneficial. In the 2 with pseudomembranous conjunctivitis and corneal ulcers, treatment with autologous serum eye-drops and preservative-free artificial tears was effective, resulting in corneal epithelium healing and mild conjunctival scarring.

Conclusion Not all TEN patients have ocular complications at onset. Plasmapheresis may be life-saving and contribute to reduce ocular surface inflammation. Autologous serum eye-drops prepared after plasmaferesis may be helpful in the management of the acute ocular complications caused by TEN. 


\section{- S057}

In vivo confocal microscopy of mucous membrane pemphigoid, a new tool for the diagnosis. Reporting 6 cases

JULIENNER (1, 2), TRONEMC (1, 2), HAOUASM (2), PERROT IL (3), LABEILLEB B (3), GRIVET D (2), CINOTTIE (3), CAMBAZARD F (3), THURET G $(1,2,4)$

(1) Corneal Graft Biology, Engineering and Imaging Laboratory, EA2521, Federative Institute of Research in Sciences and Health Engineering, Faculty of Medicine, Jean Monnet University, Saint-Etienne

(2) Department of Ophthalmology, University Hospital, Saint-Etienne

(3) Department of Dermatology, University Hospital, Saint-Etienne

(4) Institut Universitaire de France, Bd St Michel, Paris

Purpose Mucous membrane pemphigoid (MMP), previously known as cicatricial pemphigoid, is an autoimmune disease characterized by linear deposition of IgG and IgA along the epithelial basement membrane zone in direct immunofluorescence (DIF). DIF requires mucosal biopsy, which can worsen the condition by triggering an inflammatory response of the mucosa, especially the conjunctiva. We assess in vivo confocal microscopy (IVCM) for the detection of the pathognomonic basement membrane cleavage zone (BMCZ) in MMP

Methods Six patients (4 M, $2 \mathrm{~W}$ ), suffering from ocular and/or oral MMP were tested with IVCM, using the handheld Vivascope 3000. Three patients had ocular involvement, 2 oral involvement, and 1 had both

Results Mean age was 76 years old. Out of the 4 patients with ocular involvement, 2 patients had active conjunctivitis and 2 patients had cicatricial lesions. A BMCZ was identified in both patients with active conjunctivitis, while none was observed in those with cicatricial lesions. All 3 patients with oral involvement had desquamative gingivitis with marked $\mathrm{BMCZ}$.

Conclusion IVCM is non-invasive and reliable for the diagnosis of MMP. Conjunctival fornix biopsy is at high risk of symblepharon. These biopsies are often performed blindly as the pathological zones are barely visible clinically. IVCM enables the observation of the whole inflammatory surface of the conjunctiva and gum. To our knowledge, these are the first 2 cases of patients with conjunctival BMCZ clearly identified with IVCM.

\section{- $\mathbf{S 0 5 9}$}

Looking at the eyelids of $\mathbf{4 8 8 4}$ patients in Germany, Poland and Spain: the MEIBUM* survey

KAERCHER T (1), ZAGORSKIZ (2), BENITEZ DEL CASTILLO JM (3)

(1) Augenarztpraxis, Heidelberg

(2) Eye Surgery Centre, Naleczow

(3) Department Ophthalmology, Hospital Clinico San Carlos, Madrid

Purpose To assess the incidence and characteristics of eyelid disorders during ophthalmology consultations.

Methods Multicenter, epidemiological survey implemented in 9 European countries (2012 - 2014). The participating ophthalmologists were asked to include the next 10 patients attending their consultations, whatever the motive of the visit.A 2 page questionnaire recorded: reason for visiting, ocular history, symptoms, examination of eyelids and ocular surface, diagnosis of MGD or Dry eye, impact on daily life, management of eyelid disorders.

Results 4884 patients were analyzed within the 3 first countries; Germany (375), Poland (2584), Spain (1925). Mean age was 57.5. 80\% of patients had some ocular history mainly dry eve (31\%). Diagnosis of MGD was established in $55 \%$ of patients and dry eve in $64 \%$. The impact of eyelid disorder on daily life was mainly on vision for $60 \%$ of the patients, on daily activities/work (57\%), on leisure (46\%), but also on emotions ( $22 \%$ ) and sleep (19\%).Eyelid disorders were treated by eyelid hygiene recommendations; warming $(42 \%)$, cleansing $(61 \%)$, massage $(47 \%)$ and eye drops for dry eye $(82 \%)$. Nutritional supplements were recommended in only $32 \%$ of patients. $92 \%$ of the patients declared at least one symptom related to ocular surface. Conjunctival hyperemia was found at examination in $62 \%$ of patients. Fluorescein staining was positive on conjunctiva in $10 \%$ and on cornea (SPK) in $23 \%$.

Conclusion MGD was diagnosed in more than half of the patients with a strong link to dry eye ( $\mathrm{p}$-value $<0.001$ ). The impact of eyelid disorders on daily life is notable. Dry eye seems to be taken in charge more often than MGD. This survey highlights the need to take a closer look at the patients' eyelids.

Commercial interest
- S058

Role of PACAP for impairment of cornea epithelial wound healing

SEKI T (1, 2, 3), NAKAMACHI T (4, 3), FARKAS J (3), WATANABE J (3),

IZUMI S (2), SHIODA S (3)

(1) Tamagawa Eye Clinic, Tokyo

(2) Ophthalmology Showa University School of Medicine, Tokyo

(3) Anatomy Showa University School of Medicine, Tokyo

(4) Graduate School of Science and Engineering, University of Toyama, Toyama

Purpose Corneal epithelium disorder causes by infection, chemical burn, use of contact lens and eye drops like benzalkonium chrolide. Pituitary adenylate cyclaseactivating peptide, PACAP is known to have trophic effects on various tissues. PACAP also exists in eye tissues, however, the effects on corneal epithelial wound healing is still unknown. The aim of this study is to investigate the therapeutical effects of PACAP on wound healing of mouse cornea.

Methods Cornea injury was performed in adult C57BL/6 male mice. One $\mathrm{mm}$ in diameter wounds were created on the cornea with a biopsy punch and the epithelium was removed. PACAP or saline were dropped on the eyes in every two hours until 12 hours post injury. Photographs were taken $0 \mathrm{~h}, 12 \mathrm{~h}$ and $24 \mathrm{~h}$ post injury with fluorescein staining. We also examined immunostaining or RT-PCR against PAC1 receptor in the corneal tissue.

Results We found that the area and perimeter of corneal injury were significantly decreased in PACAP-treated group compared with saline-treated group. In this time, the most effective does was 10-11M. The expression of PAC1 receptor was detected in the corneal epithelium with RT-PCR and immunostaining. PACAP-induced corneal healing was inhibited by co-administration of PAC1 receptor antagonist, PACAP 6-38. Conclusion These results suggest that PACAP stimulates the corneal wound healing via PAC1 receptor.

- S060

Epidemiology of corneal ulceration and ocular surface disease among patients with Graves'orbitopathy

KLYSIK A

Department of Ophthalmology of the Medical University of Lodz, Lodz

Purpose To determine the incidence of corneal ulceration and ocular surface disease among patients with active and inactive stage of Graves' orbitopathy.

Methods 678 patients with clinical evidence of moderate or severe Graves' orbitopathy remaining under the care of Department of Ophthalmology of the Medical University of Lodz from January 2004 to April 2014. Average duration of the disease was 6.8 years. Average duration of follow-up was 6.6 years. Episodes of corneal ulceration and ocular surface symptoms were analyzed.

Results Corneal ulceration occurred in 2 patients with active stage of orbitopathy, and 6 patient in an inactive stage. One patient in active stage of the disease developed central corneal ulceration, and 7 patients ( 1 in the active stage, and 6 in an inactive stage) developed peripheral corneal ulceration. 5 patient developed ulceration at 9 óclock in the right eye and 3 oclock in the left and 2 patients developed inferior ulcer at 6 oclock. The incidence rate of corneal ulceration in moderate to severe Graves' orbitopathy is $0.174 \%$. Ocular surface symptoms were noted in $26 \%$

Conclusion Corneal ulceration is a very rare complication of Graves' orbitopathy, more commonly occurring in the inactive stage of the disease. Ocular surface disease occurs in about one fourth of patients in an inactive stage of moderate and severe disease. 
- S061 / 4455

Anti-evaporative mechanism of wax esters: implications for the function of tear fluid

PAANANEN RO, RANTAMÄKIAH, HOLOPAINEN

Helsinki Eye Lab, Department of Ophthalmology, University of Helsinki, Helsinki

Purpose Our aim was to explain the molecular level basis of the ability of wax ester (WE) films to retard evaporation close to their bulk melting temperature. We studied the surface behavior of behenyl palmitoleate (BP), a WE representing the most abundant meibum WEs.

Methods Isotherm and isochor measurements coupled with imaging by Brewster angle microscopy (BAM) were used to study BP films at the air-water interface. In addition, evaporation rates through BP films were measured.

Results Close to room temperature, BP formed solid, non-spreading islands when applied to the air-water interface. Between 35 and $38{ }^{\circ} \mathrm{C}$, a fluid, effectively spreading monolayer phase coexisted with solid domains of BP. At higher temperatures, BP formed completely fluid films. Mean molecular areas of the fluid and solid films were determined to be approximately $50 \AA 2$ and $18 \AA 2$, respectively. The thickness of a solid BP monolayer was determined to be $42 \pm 6 \AA$ by BAM measurements. A fluid BP monolayer only had minimal evaporation resistance, while a solid monolayer retarded evaporation by $>50 \%$

Conclusion Below $35{ }^{\circ} \mathrm{C}$, BP assumes an extended conformation at the air-water interface, similar to the bulk crystal structure of WEs. The extended conformation allows tight packing of hydrocarbon chains, which hinders the permeation of water molecules and leads to the evaporation retarding effect. Although the solid islands effectively retard evaporation, they do not spread at low temperatures and water evaporation proceeds through the uncovered areas of the surface. Coexistence of fluid and solid monolaver phases between 35 and $38^{\circ} \mathrm{C}$ allows the film to spread, leading to a complete coverage of the interface by the solid phase and effective evaporation retardation.

\section{- S063 / 4457 \\ Changes of inflammatory cytokines and tear osmolarity in systemic sclerosis after treatment with liposomes sprayed}

GAGLIANOC (1, 2), AMATOR (1,2), FALLICOM (2), TOROM (2), AVITABILE T (2), FOTIR (3)

(1) NEST (Neurovisual Science Technology), Catania

(2) Ophthalmic Clinic, Catania

(3) Rheumatology Unit, Catania

Purpose To investigate the effect of treatment with a liposomal spray solution in patients with systemic sclerosis (SSc) and severe dry eye by measuring the levels of inflammatory cytokines and tear osmolarity.

Methods Twenty-two subjects (12M, 10F) aged 45.1+/-7.1 years participated in this prospective, randomised, double-masked investigation. DES was identified in patients with SSc according to the classification of Dry Eye Disease of International Dry Eye Workshop (2007) and tear osmolarity values (TearLab system) according to recommendations of the manufacturer with a cutoff value of $308 \mathrm{mOsm} / \mathrm{L}$ (3). Liposomal spray (nebulized, two sprays each, on the eyelids, 3 times per day for 3 weeks) with vitamin $\mathrm{A}$ and $\mathrm{E}$ was applied to one eye, and an equal volume of saline spray (control) applied to the contralateral eye. Non-invasive tear film stability (NIBUT), osmolarity and inflammatory cytokines (IL-1 $\beta$, IL6, TNF- $\alpha, I F N-\gamma$ ) were evaluated at baseline, and at 5, 10, 15 days after spray solution application.

Results A highly significant reduction $(\mathrm{p}<0.01-0.001)$ was detected in the inflammatory cytokine levels and osmolarity in tears of eye treated with the spray liposomes solution in comparison with fellow eye treated with sprayed saline solution. There were significant differences among the 2 eyes concerning tear osmolarity $(\mathrm{p}<0.001)$ and TBUT $(\mathrm{p}<$ $0.05)$ scores.

Conclusion This study showed the ability of liposomal spray application to modify the pro-inflammatory cytokine profile and to increase stability characteristics of the preocular tear film in severe dry eye of SSc patients.This effect could be due to the antiinflammatory mechanism of the vitamins and also to the barrier effect of liposomes.
- S062 / 4456

Systematic allergy investigation of patients with red eyes

MUNKSJ (1, 2), GARVEY LH (2), HEEGAARD S (3, 1), HANSEN KS (2, 4), SKOV L (5), ZACHARIAE C (5), MENNÉ T (5), JULIAN HO (1), HALKJÆR LB (2), JOHANSENJD (6)

(1) Department of Ophthalmology, Glostrup Hospital, University of Copenhagen, Copenhagen (2) Allergy Clinic, Gentofte Hospital, University of Copenhagen, Copenhagen

(3) Eye Pathology Institute, Department of Neuroscience and Pharmacology, University of Copenhagen, Copenhagen

(4) Department of Pediatrics, Herlev Hospital, University of Copenhagen, Copenhagen

(5) Department of Dermato-Allergology, Gentofte Hospital, University of Copenhagen, Copenhagen

(6) National Allergy Research Centre, Department of Dermato-Allergology, Gentofte Hospital, University of Copenhagen, Copenhagen

Purpose "Red eyes" is a common patient complaint. Determining the underlying cause is often difficult as differential diagnoses are numerous. Allergic causes may be easily overlooked as the diagnosis relies on specialized investigation. We wanted to determine the prevalence of relevant allergy in patients with "red eyes", where initial ophthalmological consultation did not result in a definite diagnosis.

Methods A multidisciplinary cooperation was established between ophthalmologists, allergologists, dermatologists and pediatricians. The investigation algorithm was based on clinical presentation. Patients with eye symptoms only, or eye and skin symptoms, underwent dermatological investigation comprising patch testing with European baseline series, eye drops and skin prick tests with inhalant allergens and the patients' own ophthalmological products. Patients with eye and airway symptoms were reviewed by allergologists and underwent skin prick tests and specific IgE analysis for relevant allergens and lung function tests. Children were reviewed by pediatric allergologists. Multidisciplinary conferences were held regularly and patients were reviewed and cross-referred if relevant.

Results In total 58 patients ( 21 males and 37 females) with "red eyes" were included over a one year period. In 21 patients (36\%) a relevant allergy was found, diagnosing contact allergy in 15 patients ( 9 perfume, 3 phenylephrine, 3 others) and IgE-mediated inhalant allergies in 6 patients. In 37 patients (64\%) no allergy was found, and of these 10 patients were diagnosed with rosacea.

Conclusion Multidisciplinary cooperation and systematic investigation resulted in identifying relevant allergy in $36 \%$ of patients presenting with "red eyes".

\section{- S064 / 4458}

Morphogenesis of eyelid meibomian gland

GUEDIRA TARIGHT G(1), TARIGHT N (1), SEVESTRE H (2), COPIN H (3),

BREMOND-GIGNACD $(1,4)$

(1) Ophthalmology, University Hospital of Amiens, Saint Victor Center, Amiens

(2) Pathology Department, University Hospital of Amiens, Amien

(3) Biology Reproduction and cytogenetic department, Amiens

(4) CNRS FR 3636, University Paris V, Paris

Purpose Dysfunction of Meibomian gland is the primary cause of dry eye. The purpose of our study is to understand embryogenesis for a better understanding of the pathophysiology of these diseases.

Methods Eyelids were obtained from 22 fetuses formalin fixated of 10-34 weeks gestational age (GA) with approval of Ethics Committee These 88 evelids were processed with sections $5 \mu \mathrm{m}$ thick and analyzed by light microscopy after staining with hematoxylin-phloxine-saffron and PAS reaction. For each sample, different morphological criteria were investigated: evaluation of glandular bud, sebaceous gland differentiation, arborization of the gland, opening of excretory duct.

Results Histological analysis revealed that up to 13 weeks of GA, the fetal lid consists of fine vascular structures in a loose mesenchyme, under a very thin epithelium. At 14 weeks of GA, a first process of the excretory duct is individualized as a cell invagination extending from the eyelid margin. Sebaceous cell differentiation is identified at 15 weeks of GA. The excretory canal keeps its maturation to appear as a small cellular cord, unique and full around 16 weeks of GA, glands begin its buds arborization at 19 weeks of GA. This channel gradually widens and expands, appearing always blocked by a plug of squamous cells and keratin cuticle at 22 weeks of GA. The plugging lasts until 34 weeks of GA.

Conclusion Glandular structures involved in tear secretion appear in fetus around fourteen weeks of gestation. The Meibomian glands extend its maturation after birth Further studies will allow a better understanding of the physiopathology of dry eye. 
- S065 / 4425

Cytological features of inflammatory cells infiltration indicates the etiology of infectious keratitis

SMEDOWSKIA (1), TARNAWSKA D (1,2), WYLEGALAE (1)

(1) Clinical Department of Ophthalmology, Faculty of Medicine and Department of Dentistry in Zabrze, Medical University of Silesia, Katowice

(2) Department of Biophysics and Molecular Physics, Institute of Physics, University of Silesia, Katowice

Purpose To analyze histological findings of infectious keratitis in corneal epithelium including inflammatory cells infiltrations.

Methods We performed in vivo confocal microscopy (HRT III, Rostock Corne Module) in 190 patients with diagnosed infectious keratitis of various etiology - 72 viral, 66 bacterial, 34 fungal, 18 amoebal keratitis. For corneal scans we used 400x lens and 63x magnification. Microscopic analysis included inflammatory cells infiltration - number size and shape of cells; superficial corneal nerve plexus (number and characteristics of nerve fibers) and cytology of epithelial cells. Described features were compared between groups.

Results Inflammatory cells densities showed no differences between groups, however there was clearly different ratio of round/dendritic inflammatory cells, respectively 0.07 8.0, 1.0, 1.2 for viral, bacterial, fungal and amoebal keratitis (U-Mann Whitney test, $\mathrm{P}<0.05)$. Additionally inflammatory cells presented various stage of polimorphism and polimegatism depended on keratitis type. Differences were observed also in nerve fiber numbers ( $\mathrm{U}-\mathrm{Mann}$ Whitney test, $\mathrm{P}<0.05$ ) and epithelial layer architecture.

Conclusion In vivo analysis of corneal epithelial histology shows high specificity and sensitivity in infectious keratitis diagnosis. Correct diagnosis of infectious keratitis is often difficult. In vivo microscopic analysis of both epithelial layer and cytology of inflammatory infiltration might provide fast and highly specific differentiation of keratitis etiology
- S066 / 4426

Anti-glaucoma drugs and their additives, testing the influence of excipients on the corneal healing process in the EX-Vivo Eye Irritation Test (EVEIT) system

DUTESCU M, PINHEIRO R, PANFIL C, SCHRAGEN ACTO e.V. RWTH University, Aachen

Purpose This study examined the impact of anti-glaucoma medications and their additives on the corneal healing process and corneal toxicity.

Methods BAK-free PG analogues (Lumigan UD, Monoprost, Taflotan Sine), soft preserved Travatan BAK free, as well as polyquaternium-1 (0.001\%) and Macrogolglycerolhydroxystearate 40 (5\%) were tested regarding corneal irritability and effect on corneal healing against HYLO COMOD $(1 \mathrm{mg} / \mathrm{mL}$ sodium hyaluronate) as positive and $0.02 \%$ benzalkonium chloride (BAK) as negative control. Formulas were applied over three davs, six times daily on rabbit corneas cultured on an artificial anterior chamber the Ex Vivo Eye Irritation Test (EVEIT) system. Initially, four corneal abrasions (2.5-4.5 mm2) were induced. All defects were monitored by fluorescein stains and photographs.

Results For BAK, the corneal erosion size increased from $14.16 \mathrm{~mm}^{2}$ to 88.89 $\mathrm{mm}^{2}$. A delay of corneal healing is evident for Monoprost $\left(14.84 \mathrm{~mm}^{2}\right.$ to $\left.9.45 \mathrm{~mm}^{2}\right)$ and Macrogolglycerolhydroxystearate $40\left(11.58 \mathrm{~mm}^{2}\right.$ to $\left.1.56 \mathrm{~mm}^{2}\right)$ in comparison to a complete healing for HYLO COMOD, Lumigan, Taflotan Sine, Travatan and polyquaternium- $1^{\circ}$. For both Monoprost and macrogolglycerolhydroxystearate 40, a bright opaque halo around the remaining erosion area could be noticed. Histology revealed severe alterations of the superficia stroma. Cornea tested without erosions show no corneal toxicity of macrogol 4000

Conclusion Macrogolglycerolhydroxystearate 40, an additive found in Monoprost shows corneal toxicity solely in case of corneal erosions. This excipient is a known skin irritant. Therefore, the use of Monoprost in patients with epithelial defects should be questioned

Commercial interest

\section{- S068 / 4428}

- S067 / 4427

3D reconstruction of corneal endothelial cell shape: new insight in structure-function relationships

HEZ (1), BERNARD A (1), DEFOE D (2), PISELLIS (1), ACQUART S (3) FOREST F (1, 4), DUMOLLARD JM $(1,4)$, PEOC'HM $(1,4), \operatorname{GAINP}(1)$, THURET G $(1,5)$

(1) Corneal Graft Biology, Engineering and Imaging Laboratory, EA2521, Federative Institute of Research in Sciences and Health Engineering, Faculty of Medicine, Jean Monnet University, Saint-Etienne

(2) Department of Biomedical Sciences, James H Quillen College of Medicine, East Tennessee State University, Johnson City

(3) Eye Bank, French Blood Centre, Saint-Etienne

(4) Department of Pathology, University Hospital, Saint-Etienne

(5) Institut Universitaire de France, Bd St Michel, Paris

Purpose Corneal endothelial cells (CECs) support the pump-leak theory explaining the maintenance of corneal transparency. CEC are highly polarized with a pole facing aqueous humor having an hexagonal shape bordered by apical tight-junctions that control permeability. The interdigitated shape of basolateral membranes, described in the late 70's, are much less known although they bear most of the pumping sites. Aim: to revisit the shape of CEC with modern microscopy and 3D reconstruction.

Methods Optimized methods of immunolocalization (fixation and antigen retrieval) were applied on flat mounted whole cornea (fresh or after organ culture). The subcellular structures were distinguished by visualizing apical pole with actin, myosin and ZO1; lateral membranes with NCAM (CD56), connexion 43, vimentin and tubulin, and the basal pole with integrin. Stacks of high magnification images were acquired with a confocal microscope with a $150 \mathrm{~nm}$ pitch. 3D reconstruction was performed with imageJ using convolve and Gaussian blur filters, and a model was defined.

Results A 3D map of all markers was obtained. In particular, CD56 perfectly outlined the basolateral membranes of $C E C$. The $3 \mathrm{D}$ model highlighted the dramatic increase in membrane surface due to basolateral expansions

Conclusion CD56 staining pattern and especially the presence of numerous interdigitations should be considered as a hallmark for functional CEC and their study should therefore be integrated in quality controls of CEC production for regenerative medicine processes. Modifications of this 3D structure should also be studied in endothelial diseases. Grant: IFRESIS, St-ETIENNE METROPOLE

\section{Terrien's marginal degeneration: Nordic collaborative study}

KURKINENM (1), DROLSUM L (2), FAGERHOLM P (3), HIORTDAL J (4) HOLOPAINENJ (5)

(1) Helsinki University Hospital, Helsinki

(2) Ullevål University Hospital, Oslo

(3) Linköping University, Department of Clinical and Experimental MedicineOphtalmology, Linköping

(4) Aarhus University Hospital, Aarhus Universitet, Aarhus

(5) Helsinki Eye Lab, University of Helsinki, Helsinki

Purpose The objective of our study is to describe the clinical andimaging features of Terrien's marginal degeneration (TMD) andsubsequently to define the clinical diagnostic criteria for TMD.Lastly we aim to detect the natural course of this disease.

Methods Our study design is cross-sectional and includes TMD patientsdiagnosed in Helsinki University Eye Hospital, Aarhus UniversityHospital, Sahlgrenska University Hospital and Oslo University Hospitalby corneal specialists. Analysis include gender distribution, age,symptoms, as well as clinical, topographical and imaging findings. Theanalysis is based on descriptive variables due the limited sample size

Results We present here clinical findings of Finnish TMD patients. Allpatients were asymptomatic and did not suffer from any systemicautoimmune diseases. Topographical analysis showed high astigmatismdue to severe corneal thinning as evidenced by optical coherencetomography imaging.

Conclusion TMD is a bilateral corneal disease. Patients are commonlyasymptomatic and visit an ophthalmologist because of deterioratedvision due to increasing astigmatism. TMD is thus diagnosed bycoincidence. TMD does not seem to be associated with any systemicautoimmune diseases and it is commonly non-progressing. 
- 5069

Spatial statistical analysis of the human corneal endothelial mosaic

RANNOUK (1), GUERREROP (1), GAVET Y (1), PINOLIJC (1), THURET G $(2,3,4)$ GAINP $(2,4)$

(1) Ecole Nationale Supérieure des Mines, Saint-Etienne

(2) Corneal Graft Biology, Engineering and Imaging Laboratory, EA2521, Federative

Institute of Research in Sciences and Health Engineering, Faculty of Medicine, Jean

Monnet University, Saint-Etienne

(3) Department of Ophthalmology, University Hospital, Saint-Etienne

(4) Institut Universitaire de France, Pari

Purpose The human corneal endothelium is a monolayer of flat hexagonal cells, which do not regenerate and are responsible for the maintenance of cornea transparency. The aim of our study is to provide a statistical characterization of their spatial distribution.

Methods The data consisted of manually segmented human corneal endothelial cells from specular microscopy images of patients and light microscopy of stored corneas. Each cell was summarized by its mass centre, and the spatial repartition was analysed with the Ripley's K and L functions and the Baddeley-vanLieshout's J function, that quantify the distance distribution between centres. This analysis was compared with two classical random point processes (Poisson, hard-core)

Results Ripley's and Baddeley-vanLieshout's functions allowed us to determine if the mass centres were distributed in a complete random or uniform way, or if spatial clusters did occur. Quantitative comparisons were made between young and old corneal endothelial mosaics.

Conclusion The perspectives of this mathematical modeling are to simulate cells mosaics behaviour over both space and time in different conditions such as normal aging, dystrophies, and after keratoplasty.Grant: ANR2012 CORIMMO3D

- S071

Red light has the capacity to blunt corneal endothelial cell damage in situ.

NÚNEEZ ÄLVAREZ C, OLMO-AGUADO S, MERAYO-LLOVES J, OSBORNE NN Fundación de Investigación Oftalmológica, Oviedo

Purpose To cause damage to rat corneal endothelial cells in situ and determine whether exposure to red light blunts this influence.

Methods Corneal endothelial cells from one eye in anesthetised rats were damaged by placing a needle in the anterior chamber and elevating the intraocular pressure (IOP, approximately $130 \mathrm{~mm} \mathrm{Hg}$ ) for 60 minutes in the dark. In some instances the cornea was exposed to red light (2500 lux). Rats were either killed immediately or after periods of up to 15 days. Flat mounts and transverse sections of cornea were processed for the localisation of tight junctional protein ZO-1 and JC-1 to monitor mitochondrial health with their degree of depolarization indicated by changes in the red to green intensity of fluorescence.

Results Raise IOP causes damage to the mosaic appearance of corneal endothelial cells clearly indicated by staining for ZO-1. Exposure to red light during elevation of IOP reduced the amount of patchy damage to endothelial cells. Corneal endothelial cells appear green when stained with JC-1 but after 60 minutes of raised IOP the cytoplasm of many cells appear red. Both the numbers and intensity of red endothelial cells increased in JC-1 stained corneas exposed to red light. Three days after an insult of raised IOP, some red JC-1 staining was present only in corneas exposed to red light.

Conclusion Raised IOP causes damage and disruption of corneal endothelial cells. This process is blunted by red light. JC-1 staining suggests that endothelial cell mitochondria are activated by raised IOP (presumably as a protective mechanism) and that this process is enhanced by red light.

\section{- S070}

Innovative, non-contact wide field imaging of corneal endothelium

ABERRA GUEBROU S (1), HEZ (2), GUILHEM M (1), PINOLIJC (1), GAIN P (2, 3 , THURET G $(2,3,4)$

(1) Ecole Nationale Supérieure des Mines, Saint-Etienne

(2) Corneal Graft Biology, Engineering and Imaging Laboratory, EA2521, Federative Institute of Research in Sciences and Health Engineering, Faculty of Medicine, Jean Monnet University, Saint-Etienne

(3) Department of Ophthalmology, University Hospital, Saint-Etienne

(4) Institut Universitaire de France, Bd St Michel, Paris

Purpose Wide field imaging of corneal endothelial cells remains challenging since the development of non-contact specular microscopes that all have a narrow field of observation (approx $0.15 \mathrm{sqmm}$ ). Nevertheless a "back to the future" with wide field imaging will definitely help clinicians to better assess the endothelial reserve of their patients and eve bankers to better the quality of the stored corneas. Aim: to present an innovative non-contact optical device able to provide wide field endothelial images. Methods We developed a new imaging prototype based on an hybrid numericaloptical proces

Results Images with a field of about $1 \mathrm{~mm} 2$ were obtained on stored human corneas. Conclusion Further developments are ongoing to assess the application domain of this new microscope, especially on patients.Grant: ANR2012 CORIMMO3D

\section{- S072}

In vivo and ex vivo confocal microscopy of eyelid melanoma: case report

HAOUASM (1, 2), GUILLEMOT C (2), GRIVET D (2), CINOTTIE (3) PERROT IL (3), LABEILLE B (3), CAMBAZARD F (3), FOREST F (3, 1) DUMOLLARDIM (4, 1), PEOC'HM (4, 1), GAIN P $(1,2)$, THURET G $(1,2,5)$

(1) Corneal Graft Biology, Engineering and Imaging Laboratory, EA2521, Federative Institute of Research in Sciences and Health Engineering, Faculty of Medicine, Jean Monnet University, Saint-Etienne

(2) Department of Ophthalmology, University Hospital, Saint-Etienne

(3) Department of Dermatology, University Hospital, Saint-Etienne

(4) Department of Pathology, University Hospital, Saint-Etienne

(5) Institut Universitaire de France, Bd St Michel, Paris

Purpose We report the first description of a malignant melanoma (MM) of the eyelid margin examined by in vivo and ex vivo reflectance confocal microscopy (IVCM and EVCM)

Methods A 65-year-old patient with a pigmented cutaneous tumor of the left upper eyelid margin was evaluated by clinical, dermoscopical and IVCM examination which suggested the diagnosis of MM. IVCM was performed with the handheld vivascope 3000 (Caliber/Mavig). Excision of the tumor with lateral margins of $2 \mathrm{~mm}$ was performed

Results The fresh excised tissue was examined by EVCM (vivascope $2500 \mathrm{ML}$ ) which found features of MM and showed that surgical margins were tumor-free. The diagnosis of the MM was confirmed by the histopathological examination.

Conclusion In addition to the clinical examination and dermoscopy, IVCM added evidence to the MM diagnosis and EVCM allowed to evaluate the excision margins before the histopathological examinations. Therefore IVCM and EVCM can provide to the clinician important elements to improve the diagnosis of $\mathrm{MM}$ and the management of the eyelid cutaneous melanocytic tumors. 
- S073

Secondary, vocational crystalline keratopathy - case report

WYLEGALA E (1), SMEDOWSKI A (1), JANISZEWSKA D (1)

TARNAWSKA D (1, 2), NOWINSKA A (1), DOBROWOLSKID (1)

(1) Clinical Department of Ophthalmology, Faculty of Medicine and Department of Dentistry in Zabrze, Medical University of Silesia, Katowice

(2) Department of Biophysics and Molecular Physics, Institute of Physics, University of Silesia, Katowice

Purpose To present case of secondary, vocational crystalline keratopathy in patient with history of long-time exposure to glass wool.

Methods The patient was consulted in Ophthalmology Outpatient Clinic because of suspicion of crystalline keratopathy of unknown origin. The following tests were performed: basic slit lamp examination, corneal scans using OCT Visante (Zeiss, Germany) and corneal confocal microscopy in vivo (Rostock Cornea Module Heidelberg Engineering Retina tomograph III).

Results On a slit lamp examination we described macroscopic crystal deposits within epithelial layer. In the images from the OCT Visante - hiperreflective deposits obscuring other structures of the cornea were present only in epithelial and subepithelial layers. Crystals were not crossing Bowman membrane border. The corneal confocal microscopy images described monomorphic needle-like crystals without arborations, covered by epithelial cells and infiltrating epithelial layer, epithelial basal membrane and Bowman membrane. Anterior and posterior stroma presented no abnormalities. On anamnesis patient reported history of 25 years of occupational exposure to glass wool. Additionally we excluded other possible causes of crystalline keratopathy, such as hypercholesterolemia, hiperuricemia, cystinosis and hematopoietic disorders.

Conclusion Corneal confocal microscopy is a useful tool for the detection and differentiation of corneal keratopathies of uncertain etiology.

- S075

Circumferential lymphangiectasia haemorrhagica conjunctivae: a rare case of spontaneous resolution

ASCASO FJ (1), HUERVA V (2), TRAVESET A (2), SANCHEZ MC (2)

(1) Department of Ophthalmology, Hospital Clinico Universitario, Zaragoza

(2) Department of Ophthalmology, Hospital Universitari Arnau de Vilanova, Lleida

Purpose Lymphangiectasia haemorrhagica conjunctivae (LHC) is a rare condition in which the lymphatic channels in the bulbar conjunctiva are dilated and filled with blood. LHC occurs as the result of a connection between conjunctival lymphatic and blood vessels. There have only been a few reported cases of spontaneous attacks followed by rapid resolution. Our purpose is to present the first reported case of LHC involving 360 degrees of bulbar conjunctiva.

Methods This case report shows the characteristics of LHC, such as segmental constrictions and balloon-shaped dilations of blood-filled lymphatic vessels in the conjunctiva. Anterior segment photographs confirmed that the blood resolved spontaneously in our patient without requiring any further intervention.

Results A 36-year-old woman complained of spontaneous ocular redness, showing a circumferential engorgement of the bulbar conjunctival vessels consistent with the diagnosis of LHC. One month later, varicose tortuous and transparent lymphatic vessels at the site of previously blood filled vessels were noted as linear bulbar swelling with the classic "string of pearls" appearance. No recurrence was observed after 18 months follow-up. Surgical excision or laser treatment was not necessary.

Conclusion This case is exceptional because of the anatomic location, the full hemorrhagic component of the dilated vessels and the absence of recurrences following its complete resolution. Ophthalmologists should be aware of the possibility of LCH when examining conjunctival hemorrhages.

\section{- S074}

The effects of paclitaxel on angiogenesis and lymphangiogenesis in the mouse cornea.

JASIELSKA M (1, 2), KOCIOKN (2), BRÄUTIGAM M (2), JOUSSENM (2), PLEYER U (2) MAIER AK(2)

(1) Department of Vitreoretinal Surgery of Medical University in Lublin, Lublin

(2) Charité - Universitätsmedizin Berlin, Berlin

Purpose Paclitaxel is a cytoskeletal agent that interacts with mitotic activity and inhibits microtuble disassembly. Consequently its antiproliferative effect has raised interest as antiangiogenic compound and is currently used in oncology and cardiology. Therefore we investigated the effects of paclitaxel on the angiogenesis and lymphangiogenesis process in a murine corneal neovascularization model

Methods The mouse model of suture-induced corneal neovascularization was used to assess the anti-hemangiogenic and anti-lymphangiogenic effect of paclitaxel by topical application, paclitaxel coated suture. As positive control we used dexamethason eye drops and as negative control - albumin eye drops. Application of eye drops was performed $(3 \mathrm{x} / \mathrm{day})$ for 2 weeks beginning at the day of the suture-treatment. Corneal neovascularization formation (angiogenesis and lymphangiogenesis) was quantified on corneal flatmounts stained with CD31 and LYVE-1.

Results Topical applications of paclitaxel significantly inhibited the outgrowth of lymphatic vessels. In contrast, we did not detect an anti-angiogenic effect of paclitaxel on corneal neovascularization, although the extend of corneal neovascularization was significantly reduced in animals treated with dexamethason (positive control),

Conclusion Topical application of paclitaxel inhibits lymphangiogenesis in the cornea. This finding suggests a potential role of paclitaxel in the treatment of corneal diseases.

\section{- S076}

Anterior stromal puncture with Nd: YAG laser for recurrent corneal erosion

KO BY, KIM SY

Department of Ophthalmology, Konyang University College of Medicine, Daejeon

Purpose To evaluate the clinical results of anterior stromal puncture (ASP) using neodymium:yttrium-aluminum-garnet (Nd:YAG) laser for refractory recurrent corneal erosion (RCE)

Methods A total of nine eyes from nine patients with unilateral RCE showing poor response to conservative therapy who were treated with Nd:YAG laser ASP from Mar to Dec 2013 were studied. The causes and frequency of erosions and the spot numbers and total energy of the Nd:YAG laser were recorded. The energy setting of the laser was 0.2 or $0.3 \mathrm{~mJ}$ per shot. Slit-lamp examination, refraction, times of laser, recurrence, complications were reviewed.

Results The mean preoperative attack frequency was six. The average total energy of the laser was $5.3 \mathrm{~mJ}$. Two of the 9 eyes received additional Nd:YAG laser treatment. The mean follow-up time was 8.5 months. All of the treated eyes had no evidence of recurrence after operation during follow-up. There was no significant change in refraction. No specific complication except mild corneal haze was observed.

Conclusion Nd:YAG laser ASP is a safe and effective procedure to treat refractory RCE in a short period of time. 
- S077

Distribution, molecular structure and functional analysis of carnitine transporter (SLC22A5) in canine lens epithelial cells

OCHIAI H

Research Institute of Biosciences, Sagamihara Kanagawa

Purpose While carnitine has been reported to have an anti-oxidative role on the ocular surface, there has been no report on the existence of a carnitine transporter (SLC22A5) in the lens. Therefore, we investigated the carnitine transport activity of canine lens epithelial cells (LEC) and determined the molecular structure of canine SLC22A5.

Methods The primary lens epithelial cells of dog were transfected with the expression plasmid DNA of large $\mathrm{T}$ antigen from replication origin-defective simian virus 40 (SV40), then cloned using glass a cylinder. The transport activities of carnitine in lens epithelial cells were measured. The cDNA sequences of SLC22A 5 were also determined.

Results The carnitine transport activity was $7.16+0.48 \mathrm{pmol} / \mathrm{mg}$ protein $/ 30 \mathrm{~min}$ Butyrobetaine, the analogue of carnitine, reduced $30 \%$ of the activity at 50 microM. A coding sequence of canine carnitine transporter was 1694 bp long and was predicted to encode 557 amino acid polypeptides. The deduced amino acid sequence of canine carnitine transporter showed $>80 \%$ similarity to that of mouse and human. Western blot analysis detected the band at $60 \mathrm{kDa}$ in the membrane of lens epithelial cells.

Conclusion Lens epithelial cells possess SLC22A5. The high content of carnitine in the lens is possibly transported from aqueous humor by SLC22A5.

\section{- 5079}

Caspase- 3 in ultraviolet radiation cataract

TALEBI ZADEHN, YUZ, KRONSCHLÄGER M, SÖDERBERG P Neuroscience, Uppsala

Purpose To determine the evolution of active caspase- 3 protein expression in normal lens and in the lens exposed to low dose UVR-300 nm

Methods Forty Sprague-Dawley rats were unilaterally exposed in vivo to $1 \mathrm{~kJ} / \mathrm{m} 2 \mathrm{UVR}$ $300 \mathrm{~nm}$ for 15 minutes. At 0.5, 8, 16, and 24 hours after the UVR exposure, the exposed and non-exposed lenses were removed and processed for immunohistochemistry. The number and distribution of the cells labeled for active caspase- 3 in the exposed and contralateral non-exposed lenses were compared among the four different time points after the exposure

Results In normal lenses, active caspase-3 expression was abundant in the anterior pole of the lens. The probability of a radial spatial distribution of the labelling was fitted to a logistic model. The increased rate of active caspase- 3 expression was at $23 \pm 3$ cell-1 and the inflection point was estimated at cell number $114 \pm 3(0.95 \% \mathrm{CI})$.Active caspase- 3 expression was higher in the exposed lenses $(95 \% \mathrm{CI}=0.18 \pm 0.02)$. The mean differences between the exposed and contralateral non-exposed lenses were $0.17 \pm 0.02$ $0.20 \pm 0.03,0.21 \pm 0.03$, and $0.11 \pm 0.04(95 \% \mathrm{CI})$ for the $0.5,8,16$, and 24 hour time groups, respectively. There were no differences in the expression of caspase-3 between the 0.5 and 24 -hour groups or between the 8 and 16 -hour groups $(95 \% \mathrm{CI}=-0.02 \pm 0.03)$. There was a difference when comparing the 0.5 and 24 hours groups to the 8 and 16 hours groups $(95 \% \mathrm{CI}=0.06 \pm 0.03)$

Conclusion Active caspase- 3 is present in normal lens epithelial cells, with more expression at the anterior pole of the lens and less expression towards the periphery. After UVR exposure, the expression of active caspase- 3 in the lens epithelium increased. The peak of expression was detected approximately 16 hours after the exposure.

\section{- S078}

Anterior lens epithelial cells attachment to the basal lamina

ANDJELIC S (1), DRASLAR K (2), HVALA A (3), LOPICN (4), STRANCAR J (4), HAWLINA M (1)

(1) Eye Hospital, University Medical Centre, Ljubljana

(2) Department of Biology, Biotechnical Faculty, University of Ljubljana, Ljubljana

(3) Department of Pathology, Medical Faculty, University of Ljubljana, Ljubljana

(4) Laboratory of Biophysics, Condensed Matter Physics Department, "Jožef Stefan" Institute, Ljubljana

Purpose The lens epithelium consisting of anterior lens epithelial cells (aLECs) is the first physical and biological barrier in the lens and the primary site of active transport and permeability. As the function of the lens epithelium is associated with the structural organization of the aLECs and the contacts the basal part of the aLECs have with the basal lamina (BL), we studied the aLEC structure, in particular aLECs attachment to the BL.

Methods The aLCs (BL and associated aLECs) were obtained from routine uneventful cataract surgery, prepared for and studied by scanning electron microscopy (SEM), transmission electron microscopy (TEM) and confocal microscopy.

Results SEM shows that the basal surface of the aLECs $(\sim 10-15 \mathrm{~mm})$ in contact with the $\mathrm{BL}$ is wrinkled $(\sim 1-3 \mathrm{~mm})$ and also the aLECs extensions $(\sim 0.5-3 \mathrm{~mm})$ attached to the BL. Confocal microscopy images of the basal sections of the aLECs after membrane staining also suggest that the basal part of aLECs is wrinkled $(\sim 1-3 \mathrm{~mm})$. TEM shows in the aLECs basal parts, toward BL, the structures that look like entanglement $(\sim 1-4 \mathrm{~mm})$. In cases where there is a swelling of the cytoplasm and offset of the aLECs from the BL, individual pins $(\sim 0.5-2 \mathrm{~mm})$ that extend to the BL are visible by TEM.

Conclusion We provide detail evidence about the structural organization of the aLECs, in particular about their basal side which is in contact with the BL. This is supported by the complementary use of 3 techniques, SEM, TEM and confocal microscopy, each of them showing the same morphological features, the extensions and the entanglements of the aLECs cytoplasmic membrane at the border with the BL. The basal surface of the aLECs is increased. It suggests the functional importance of the contact between aLECs and BL.

\section{- S080}

Ocular heat flow associated with threshold in vivo exposure to $1090 \mathrm{~nm}$ infrared radiation for cataract induction

YUZ (1), SCHULMEISTER K (2), TALEBIZADEHN (1), KRONSCHLÄGERM (1), SÖDERBERGP (1)

(1) Neuroscience/Ophthalmology, Uppsala

(2) Seibersdorf Labor $\mathrm{GmbH}$, Seibersdorf

Purpose To investigate the heat flow in the ocular media after in vivo exposure to 1090 $\mathrm{nm}$ IRR at the radiant exposure intended for cataract induction.

Methods Six-weeks-old albino SD rats were anesthetized and pupil-dilated prior to exposure. The animals were divided into two groups of 12 . All animals were unilaterally exposed to $197 \mathrm{~W} / \mathrm{cm} 2$ IRR at $1090 \mathrm{~nm}$ within the dilated pupil for $8 \mathrm{~s}$. In one group, temperature was recorded with the thermocouples placed at the limbus, in the vitreous behind lens and on the sclera next to the optic nerve, respectively in the exposed eye. In the other group, one thermocouple was placed at the limbus and another on the sclera next to the optic nerve in the exposed eye. Temperature was recorded from the end of the exposure till temperature back to the baseline

Results In the first group, the induced temperature rise was, $95 \% \mathrm{CI}, 11 \pm 3{ }^{\circ} \mathrm{C}$ at the limbus, $16 \pm 6^{\circ} \mathrm{C}$ in the vitreous behind lens and $16 \pm 7^{\circ} \mathrm{C}$ on the sclera next to the optic nerve in the exposed eye. The rate constant was $2.4 \pm 0.4 \times 10-2 \mathrm{~s}-1$ at the limbus, $2.6 \pm 0.3 \times 10-2 \mathrm{~s}-1$ in the vitreous behind lens, and $2.9 \pm 0.6 \times 10-2 \mathrm{~s}-1$ on the sclera next to the optic nerve in the exposed eye (d.f. $=11)$. In the second group, the temperature rise was $9 \pm 1{ }^{\circ} \mathrm{C}$ at the limbus and $26 \pm 10^{\circ} \mathrm{C}$ on the sclera next to the optic nerve in the exposed eye. The rate constant was $2.4 \pm 0.3 \times 10-2 \mathrm{~s}-1$ at the limbus and $4.3 \pm 1.1 \times 10-2 \mathrm{~s}-1$ on the sclera next to the optic nerve in the exposed eye (d.f.=11)

Conclusion An exposure to $197 \mathrm{~W} / \mathrm{cm} 21090 \mathrm{~nm}$ IRR for $8 \mathrm{~s}$ induces a temperature increase of $10^{\circ} \mathrm{C}$ at the limbus and $25^{\circ} \mathrm{C}$ close to the retina. The temperature fall in the anterior ocular segment is twice rapid in the posterior segment of the eye. 
- S081

Comparative study of the incidence of macula edema in healthy and diabetic patients after uncomplicated cataract surgery

MIRACHTSIS T (1), GEORGIADOUI (1), SIOULIS C (1), MARKOU C (2)

LAKES (3), MOSCHOU V (3), GEORGIADISN (3)

(1) General Army Hospital, Thessaloniki

(2) E.N.T. Department Aristotle University, AHEPA, Thessaloniki

(3) 1st Ophthalmogical Department Aristotle University, AHEPA, Thessaloniki

Purpose To report the incidence of macula edema and its correlation with $\mathrm{CDE}$ (Cumulative Dissipated Energy) data in healthy and diabetic patients after cataract surgery.

Methods 168 patients (105 healthy and 63 diabetic) underwent phacoemulsification and IOL implantation. Preoperatively medical history, biometric parameters were recorded and fundus photographs were taken. Phaco metrics were recorded after each surgery. Postoperatively fundus fluorescein angiography was performed between the 2nd and 6th month follow up. Patients with indications of macula thickness changes were evaluated with optical coherence tomography

Results a) Macular edema was recorded in 7 out of 63 diabetic patients (11,1\%) and in 5 out of 105 healthy patient $(4,8 \%)$. This difference is not significant statistically $(p=0,122>0,05)$. b) There was a significant correlation between CDE values and the occurrence of CME in both groups ( $\mathrm{p}=0,0001665<0,05$.) c) Values of $\mathrm{CDE}>36,32$ are predictors for $\mathrm{CME}(\mathrm{p}=0,006<0,050)$.

Conclusion a) Improved surgical techniques have decreased the incidence of postoperative macula edema.b) There was no statistically significant difference in the incidence of CME between the 2 study groups.c) CDE (Cumulative Dissipated Energy) value is an important factor that indicates the phacoemulsification energy and time needed.

\section{- S082}

Statin use and increased risk of cataract surgery

ERIE J (1), CHAMBERLAIN A (2), HODGE D (2)

(1) Department of Ophthalmology, Mayo Clinic, Rochester

(2) Department of Health Sciences Research, Rochester

Purpose To determine the association of statin use with the risk of cataract surgery.

Methods This is a retrospective population-based, case-control study. The Rochester Epidemiology Project was used to identify eligible residents of Olmsted county, Minnesota, USA. Cases included 6,024 county residents aged 50 years and older who had first-eye cataract surgery between January 1, 2004 and December 31, 2011. Controls included residents who never had cataract surgery and were matched to cases by age, sex, and index date within 1 month of surgery. Statin medication prescriptions for at least 1 year before the surgery date (cases) or index date (controls) were examined. Odds ratios (ORs) and corresponding confidence intervals (CIs) were estimated by using logistic regression models.

Results In the cataract surgery cohort, 2,557 (42\%) were statin users compared to $2,038(34 \%)$ in the matched control cohort $(\mathrm{P}<0.0001)$. Statin use of 1 or more years was associated with an increased risk of cataract surgery $(\mathrm{OR}=1.44 ; 95 \% \mathrm{CI}, 1.34$ $1.55 ; \mathrm{P}<0.0001)$. The associations were similar in women $(\mathrm{OR}=1.52 ; 95 \% \mathrm{CI}, 1.38-1.68$; $\mathrm{P}<0.0001)$ and men $(\mathrm{OR}=1.34 ; 95 \% \mathrm{CI}, 1.20-1.51 ; \mathrm{P}<0.0001)$, and remained significant after adjusting for diabetes, oral corticosteroid use, and selective serotonin reuptake inhibitor use $(\mathrm{P}<0.003)$.

Conclusion This study provides additional evidence that statin use is associated with an increased risk of cataract surgery in men and women, and underscores the likelihood of this unintended outcome in the large segment of the population that uses statins.

\section{- S084}

Changes of corneal endothelium in diabetes patients after cataract phacoemulsification surgery by confocal microscopy

Preliminary study of the association between corneal histocytological changes and surgically induced astigmatism after phacoemulsification

ZHAOG, LIN I, XU O

Ophthalmology, Qingdao

Purpose SIA was one of the factors that influences the desirable refractive outcome, and it was related to the length, type, location, structure of the incision and to the suture closure technique, etc. The aim was to evaluate the association of corneal histocytological changes with surgically induced astigmatism (SIA) after phacoemulsification.

Methods The study enrolled 68 cases of cataract patient (68 eyes). Corneal histocytological parameters at corneal incision, central cornea and contralateral incision obtained by confocal microscope through focusing(CMTF) were compared preoperatively and 1 week, 2weeks, 1 month, 3 months and 6 months postoperatively. These biometric factors included the endothelial cell density, keratocyte density of posterior stroma layer, and the morphological changes. SIA was calculated by Jaffe's vector analysis.

Results 1 From preoperatively to 1 week, 2 weeks, 1 month, 3 months and 6 month postoperatively, the endothelail cell density was decreased significantly $(\mathrm{p}<0.05) .2$ The histocytological observations indicated that the morphology changed significantly postoperatively at the corneal incision, including the cell absent area, wave-like area, dot-like and mass-like hyperreflection, stripe-like absent area, in the endothelial layer and the keratocyte activation, microfolds, irregular hyporeflective or hyperreflective belt, and a little dot-like hyperreflection in the posterior stroma layer. 3 The reduction of the endothelail cell density at the cormeal incision at 1 week, 2 weeks, 1 month postoperatively, were positively correlated with SIA

Conclusion The reduction of endothelail cell density and the histocytological changes at the corneal incision were associated with SIA
LIN J, ZHAOG

Ophthalmology, Qingdao

Purpose To observe the changes of corneal endothelium in diabetes patients before and after cataract phacoemulsification surgery with confocal microscopy for exploring the variation and influencing factors

Methods Phacoemulsification were randomly divided into 50 aged related cataract patients with diabetesmellitus and 50 patients with senile cataract.Cataract phacoemulsification and intraocular lens implantation were performed central corneal thickness the corneal endothelial cell density coefficient of variation and percentage of hexagonal cell were measured before surgery 1 week 1 month and 3 months after surgery by confocal microscopy

Results Before surgery there was no statistical difference between the two groups of central corneal thickness corneal endothelial cell density coefficient of variation and percentage of hexagonal cell Compared with pre operation the central corneal thickness and coefficient of variation were increased post operation but the corneal endothelial cell density and percentage of hexagonal cell decreased gradually. The central corneal thickness of diabetes group was more significantly than control group 1 week 3 months after surgery, and the corneal endothelial cell density was less significantly than control group. One week 3 months after surgery the percentage of hexagonal cell was less than control group. The coefficient of variation was significantly higher than control group.

Conclusion Aged \related cataract patients with diabetes mellitus were much more poor tolerability on cataract phacoemulsification surgery. Compared with senile cataract patients the corneal endothelium injury was more serious and the velocity and validity of recovery were lower comparatively 
- S085

Comparison of two different surgical treatments of presbyopia for hyperopic patients over 55 years old: Presbylasik (Supracor) and Prelex (presbyopic lens exchange)

MOYAL L, ABRIEU M, FROUSSART-MAILLE F, RIGAL-SASTOURNE IC Hôpital d'Instruction des Armées Percy, Paris

Purpose To compare the efficiency and safety of two surgical treatments of presbyopia, Presbylasik and presbyopic lens exchange (Prelex), and to assess patients' satisfaction with both procedures.

Methods It is a retrospective monocentric clinical trial between 06/2011 and 03/2014 comparing implantation of diffractive multifocal intraocular lenses (Finevision Micro F, PhysIOL) and Supracor (Technolas Perfect Vision, GmbH)

Results Forty-two eyes of 21 patients were treated by 3 physicians, with a mean followup of 3 months. Twenty-six eyes of 13 hyperopic/presbyopic patients were included for Supracor and 16 eyes of 8 patients for Prelex. Patients were aged over 55. Mean uncorrected binocular distance (DV) and near vision (NV) visual acuity (VA) in the Supracor group were respectively $0,03(-0,2-0,1) \operatorname{LogMar}$ and $0,23(0,06-0,36)$ one week post-operatively and $0,031(-0,2-0,1)$ and $0,166(0,06-0,36) 3$ months postoperatively. In the Prelex group, the mean uncorrected one week binocular VA was $0,025(0-0,1)$ for DV and $0,165(0,06-0,18)$ for NV and the three months VA was 0 $(-0,1-0,1)$ and $0,105(0,06-0,18)$ for DV and NV. All Prelex patients were spectaclefree at all distances, whereas 4 Supracor patients required spectacles for NV postoperatively. Patients' satisfaction was high in both groups. Patients reported more halos in the Prelex group (75\%). One eye required intraocular lens exchange and 4 eyes $(16,7 \%)$ required a new corneal procedure in the Supracor group.

Conclusion Both surgeries have effective and safe modalities in hyperopic and presbyopic patients. Prelex procedure seems to be more appropriate for patients over 55 yo.

\section{- S086}

Lens capsular bag dislocated into the anterior chamber following calcific phacolysis in an immunocompromised patient.

ALMENARA MICHELENA C (1), RODRIGUEZ A (2), ASCASO FJ (1), SOLANAS S (3). MAYAR (2) ANDONEGUIJ (2)

(1) Ophthalmology, Zaragoza

(2) Ophthalmology, Pamplona

(3) Radiology, Tudela

Purpose Calcific phacolvsis, a rare clinicopathologic entity that develops in longstanding unilateral traumatized or cataractous lenses, results in complete liquefaction of cortex, which escapes through an intact lens capsular bag. Cytomegalovirus (CMV) retinitis is associated with high risk of cataract but calcific phacolysis had never been described in patients with this opportunistic infection

Methods Interventional case report showing a calcific phacolytic process with dislocation of lens capsular bag into the anterior chamber.

Results A 48-year-old man with a history of HIV, chronic hepatitis C, and CMV chorioretinitis, presented with left eye pain and redness for three days. Slit-lamp biomicroscopy revealed the presence into the anterior chamber of a free-floating lens capsular bag associating calcified lens particles. The patient underwent irrigation and aspiration to remove this spontaneously dislocated material and symptoms resolved after one-month topical antibiotic-steroid therapy.

Conclusion Phacolysis has been described as the breakdown of a hipermature cataract, causing an antigenic reaction to the proteins released into the anterior chamber through an intact lens capsular bag, in this case displaced anteriourly. Although it is thought to be a slow process, sometimes a cataract can rapidly mature and become phacolytic. To the best of our knowledge, this appears to be the first case of spontaneous phacolvsis over the course of only three days in a patient with ocular history positive for a CMV chorioretinitis 15 years ago.

\section{- $\mathrm{S088}$}

A case series of late postoperative capsular block syndrome treated by Nd:YAG laser posterior capsulotomy

\section{evaluation of visual acuity and macular retinal thickness.}

LOMBARDOS (1), TINELLIC (2), ANTONIAZZIER (3)

(1) University Eye Clinic, Pavia

(2) Clinical Epidemiology and Biometric Unit, Scientific Direction, IRCCS Policlinico San Matteo Foundation, Pavia

(3) IRCCS Policlinico San Matteo Foundation, Pavia

Purpose To investigate the clinical outcomes of Bromfenac ophthalmic solution 0.09\% dosed twice daily (BID) for 2 weeks following Neodymium:YAG (Nd:YAG) laser posterior capsulotomy in terms of best-corrected visual acuity (BCVA) and macular retinal thickness.

Methods 35 eyes with posterior capsular opacification were recruited, treated with Nd:YAG laser capsulotomy and subsequently with Bromfenac 0.09\% BID for 2 weeks. Eyes with pre-existing macular pathology were excluded. BCVA, a complete ophthalmologic assessment, macular scans by spectral domain optical coherence tomography (SD-OCT Cirrus, Zeiss) were performed before YAG laser and after 2 weeks.

Results Post-operative BCVA at 2 weeks was significantly better than pre-operative one $(\mathrm{p}<0.001)$. Likewise post-operative signal strength (SS) of OCT images improved $(\mathrm{p}<0.001)$. There were no statistically significant differences between pre- and postoperative macular retinal thickness. After multivariate analysis controlling for potential confounders (age, sex, total energy, SS), a significant decrease in macular thickness was shown in superior and nasal sectors $(\mathrm{p}<0.5)$.

Conclusion Nd:YAG capsulotomy results in a measurable improvement in BCVA and in SS, allowing a more reliable examination of the posterior segment. It is also a safe procedure, resulting in no change in macular thickness. Bromfenac BID is effective for reducing ocular inflammation associated to laser surgery and for preventing the related complications (first of all macular cystoid edema). From a multivariate analysis Bromfenac even seems to determine a decreased macular thickness in some areas, due to a therapeutic efficacy and perhaps to an easier dosage with increased compliance.
MARTINEZ VELEZM (1), HUERVA V (2), SANCHEZ MC (2), ASCASO FJ (1), SOLDEVILA J (2)

(1) Ophthalmology, Hospital Clinico Universitario "Lozano Blesa", Zaragoza (2) Ophthalmology, Hospital Arnau Vilanova, Lleida

Purpose To evaluate the clinical characteristics of the unusual late postoperativecapsular block syndrome (CBS) and the effect of neodymium:yttriumaluminium-garnet(Nd:YAG) laser posterior capsulotomy on visual acuity, refractive error as well as itspossible complications

Methods Retrospective cohort study. Twelve eyes of 11patients with late CBS who had undergone Nd:YAG laser posterior capsulotomy werereviewed. A complete examination including Scheimpflug camera andanterior segment optical coherence tomography (AS-OCT) imaging had beenperformed before and after posterior capsulotomy

Results BCVA increased in 11 cases (91.7\%). Only one eye showed a 0.5 diopter hyperopic shift following posterior capsulotomy. There were no post-laser complications such as increased IOP or cystoid macular edema. In all patients, the posterior capsule was vaulted posteriorly and could not be seen clearly. AS-OCT confirmed CBS in the studied cases, demonstrating a distended capsular bag. Rotating Scheimpflug imaging examination showed the white substance located behind the IOL

Conclusion Late CBS is usually not accompanied by shallow anterior chamber forward IOL displacement or raised IOP. Nd:YAG laser posterior capsulotomy is a useful treatment and the prognosis of patients with this complication appears favorable. Although the AS-OCT measurements are easier to get than Scheimplug imaging, both are useful to visualize the distended capsular bag containing the white material and the IOL position 
- S089

Impact of lens thickness on hyper mature cataract surgical complications: preliminary results of a prospective study

ADDOU-REGNARD M (1), FAJNKUCHEN F (2), CHAINE G (2), GIOCANTI-AUREGANA (2)

(1) Ophtalmology, Avicenne Hospital, Bobigny

(2) Avicenne Hospital, Bobigny

Purpose There are no objective parameter allowing to predict the risk of surgical difficulties in white hyper mature cataracts. Lens density can be assessed by pentacam, but this tool is not commonly available. Our purpose was to assess the correlation between lens thickness (LT) and surgery complications.

Methods We conducted a prospective, observational, and monocentric study. We included all patients operated on for white hyper mature cataract from january 2013 to march 2014. We selected patients with a visual acuity (VA) lower than 20/200 on the Monoyer scale. We excluded patients suspect of zonula fibers weakness. Morphological features assessed were LT, axial length, depth of anterior chamber and vitreous length, measured by A or B ulrasound scan. The other parameters assessed were the surgical duration, complications during surgery, VA and corneal edema at the seventh day after surgery (analogic scale from 0 to 3 )

Results We included 30 eves of 29 patients. Mean LT was $4.11+0.64 \mathrm{~mm}$, with a median of $3,89 \mathrm{~mm}$. The Pearson correlation coefficient (PCC) between LT and operative duration was 0.27 . The PCC between lens thickness and length of anterior chamber was -0.32 . The PCC between LT and BCVA at the 7th day after surgery was -0.53 . Two groups were defined: group 1 with $\mathrm{LT}<3.89 \mathrm{~mm}$, and group 2 with LT $>$ $3.89 \mathrm{~mm}$. Mean length of the anterior chamber were respectively 3.48 and $3.02 \mathrm{~mm}$ $(\mathrm{p}=0.01)$. Three per operative complications were noted.

Conclusion LT in white hyper mature cataract did not seem to be correlated with duration of the surgery. However, it seemed to be inversely correlated with the depth of anterior chamber and with visual acuity at the 7th day.

- S091 / 1776

Change in intraocular pressure in the early period after cataract phacoemulsification

YUGAY MARIA, RYABTSEVA ALLA

Moscow Regional Research Clinical Institute, Moscow

Purpose Described decrease of IOP after phaco is $2-5 \mathrm{~mm} \mathrm{Hg}$. At the same time in the early period after cataract extraction frequent increase in IOP is described. The purpose of our study was to analyse the dynamics of IOP after uncomplicated phacoemulcification

Methods The study included 67 eyes of 66 patients who underwent uncomplicated phaco with foldable IOL implantation . All patients underwent tonography resulting tonographic IOP ( $\mathrm{Po}$ ) and ease outflow coeffitient $(\mathrm{C})$, a study of corneal - compensated IOP (IOPcc) and Goldmann IOP (IOPg) with Ocular Response Analyzer. Which were repeated before surgery, the first two days, 2 weeks, 1 and 3 months after operation.

Results Average Po before surgery was 15,67 $\pm 0,85 \mathrm{~mm} \mathrm{Hg}$, IOPcc 15,91 $\pm 0,69 \mathrm{~mm}$ $\mathrm{Hg}$ and IOPg $14,51 \pm 0,78 \mathrm{~mm} \mathrm{Hg}$. On the first day after surgery IOPcc increased by 2,84 $\mathrm{mm} \mathrm{Hg}$, and IOPg by $1.53 \mathrm{~mm} \mathrm{Hg}$. Two weeks after surgery Po decreased from baseline by $3.45 \mathrm{~mm} \mathrm{Hg}$, IOPcc still increasing by $0.62 \mathrm{~mm} \mathrm{Hg}$, IOPg also further increased by $1.44 \mathrm{~mm} \mathrm{Hg} .1$ month after surgery Po virtually unchanged compared with data from 2 weeks, IOPcc returned to preoperative values, IOPg reduced compared with the preoperative figures by $1.0 \mathrm{~mm} \mathrm{Hg}$. Finally, after 3 months Po became lower then preoperative by $3.23 \mathrm{~mm} \mathrm{Hg}$, IOPcc reduced compared with preoperative by $1.51 \mathrm{~mm}$ $\mathrm{Hg}$, and IOPg by $1.92 \mathrm{~mm} \mathrm{Hg}$

Conclusion IOP rise after phaco begins on the first postoperative day, reaching a maximum in 2 weeks. Then towards the end of the first month after surgery IOP returns to preoperative values. IOP reduction 1,5-2 $\mathrm{mm} \mathrm{Hg}$ starts after decrease of postoperative reactive syndrome 1 month after surgery

\section{- S090}

Simplified topical anaesthetic protocol for ambulatory cataract surgery: safety and satisfaction

FERTE A (1), BATTA B (1), TRECHOT F (1), FUCHS-BUDER T (2), ANGIOI K (1)

(1) Department of Ophthalmology, CHU de Nancy, Nancy

(2) Departement of Anaesthesiology, CHU de Nancy, Nancy

Purpose Background and goal of study: The aim of our study was to assess safety and efficiency of cataract surgery (CS) under topical anaesthesia alone, i.e. without preanaesthetic evaluation and without direct presence of an anaesthesiologist. To this end we assessed incidence of preoperative patients' anxiety, perioperative adverse events and patients' and surgeons' satisfaction.

Methods Materials and methods: Patients undergoing CS under topical anaesthesia were included during one month. Anaesthesiologist and anaesthesia nurse were present in the area and could intervene in case of an adverse event. Patients' anxiety was scored using Amsterdam Preoperative Anxiety \& Information Scale (APAIS), their satisfaction with Iowa Satisfaction with Anaesthesia Scale (ISAS). Surgeons' satisfaction was scored with a VAS from 0 to 10 (0: surgery not possible \& 10: excellent surgical conditions).

Results Results and discussion: 124 consecutive patients were included; mean age was $71( \pm 9.4)$. Mean APAIS I was 6.4/20 ( \pm 3.7$)$. Mean APAIS II was $3.1( \pm 1.8)$. Mean ISAS score was 5.5/6 $( \pm 0.6)$, indicating a high patients' satisfaction. Surgeons' satisfaction score was $8.9 / 10( \pm 1.7) .23$ adverse events occurred, 16 of them of required intervention: 5 supplemental regional anaesthesia, 6 iv-analgesia, 5 management of hypertension.

Conclusion Conclusion: These preliminary data suggest that simplified topical anaesthesia protocol for ambulatory CS seems to be feasible and safe, as long as an anaesthesia team is present in the area to intervene if needed.

\section{- S092}

Simulation of stray light with a phakic intraocular lens with a central hole

EPPIG T, LANGENBUCHER A

Institute of Experimental Ophthalmology, Saarland University, Homburg/Saar

Purpose Posterior chamber phakic intraocular lenses (pIOL) have been equipped with several holes to facilitate aqueous flow into the anterior chamber. The latest generation is equipped with a small hole in the center of the optic. The purpose of this study was to investigate whether a central cylindrical hole may increase intraocular stray light.

Methods We established a hyperopic (+4 D) model eye in the ray tracing software ASAP based on the Liou Brennan model eye (LBME). A pIOL was placed between the iris and the crystalline lens with a central thickness of $0.25 \mathrm{~mm}$. We created one pIOL model with and without central hole of 360 microns in diameter. Beams were projected to the eye at visual field angles up to $70^{\circ}$ temporally. Intensity distribution was compared at retinal plane between the lens with and the lens without the hole.

Results The PIOL model with central hole showed increased intraocular stray light at retinal plane originating from light sources in the peripheral visual field. When projecting light from a half visual field only, stray light is present in the entire visual field. However, intensity of stray light is rather small compared to direct rays. The pIOL without central hole showed stray light originating from interface reflections which are projected to retinal plane

Conclusion Stray light caused by the pIOL central hole showed a negligible effect on visual acuity in an ideal environment. However, glare in the peripheral visual field e g. traffic lights) might affect visual acuity due to an increase in intraocular stray light. Stray light caused by the central hole is more likely to appear in hyperopic eyes due to increased pIOL central thickness. 
- S093

Pseudophakic photopsia - Optical simulations with a model eye to evaluate IOL design and material

LANGENBUCHER A, EPPIG T

Institute of Experimental Ophthalmology, Saarland University, Homburg/Saar

Purpose Photopic phenomena (photopsia) gain in relevance and may become key problems after modern cataract surgery. The purpose of this study is to evaluate potential influencing factors for these optical phenomena using ray tracing techniques in a model eye.

Methods A schematic model eye was used for simulations with professional optic design software (ASAP). The crystalline lens was replaced by an intraocular lens implant. We varied the lens' edge design (edge radius from $0.00 \mathrm{~mm}(0.05 \mathrm{~mm}) 0.15$ $\mathrm{mm}$ ), refractive index (1.55 and 1.46), axial and lateral position (each $\pm 0.5 \mathrm{~mm}$ ), as well as the pupil diameter $(2.5,3.5$ and $4.5 \mathrm{~mm})$. Retinal illuminance was simulated for a Ganzfeld perimeter sphere ( $\mathrm{d}=300 \mathrm{~mm}, 250^{\circ}$ field of view) with 107 rays emitting in a Lambertian distribution.

Results Positive photopsia could be observed at the central and paracentral nasal visual field originating from total internal reflection at the lens' optics edge, which becomes more intense with higher refractive index and smaller edge radius or larger peripheral thickness of the lens. Negative photopsia was seen at the far temporal visual field as a gap in illuminance originating by a gap between overlaying light pathways of primary image and direct path. Photopsia is less pronounced with larger pupil size and axial or lateral displacement of the lens.

Conclusion Photopsia is not a new phenomenon in pseudophakic eyes, but with lenses with a high refractive index (smaller incision) and sharp optic edges (for prevention of secondary cataract) the intensity of artificial light phenomena and gaps photopsia might become the main reason for patient dissatisfaction after cataract surgery.

\section{- S095}

Long term results of toric intraocular lens implantation for correction of astigmatism in cataract patients with corneal ectasia

PARIKAKIS E (1), CHATZIRALLII (1), STRATOS E (1), PEPONIS V (1) KARAGIANNIS D (1) DAVID G(2) MITROPOULOS P (1)

(1) Ophthalmiatreio Eye Hospital, Athens

(2) Private practice doctor, Athens

Purpose Our purpose was to examine the long-term efficacy of toric intraocular lens (IOL) implantation in cataract patients with high astigmatism due to corneal ectasia, who underwent phacoemulsification cataract surgery.

Methods Seven cases, in which phacoemulsification with toric IOL implantation was used to correct high astigmatism in cataract patients with topographically stable keratoconus or PMD, were reported. Objective and subjective refraction, visual acuity measurement and corneal topography were performed in all cases before and after cataract surgery

Results In all cases, there was a significant improvement in visual acuity, as well as refraction, which remained stable over time. Specifically, in subjective refraction, all patients achieved visual acuity from $7 / 10$ to $9 / 10$ with up to -2.50 cyl. Corneal topography also remained stable. Post-operative follow-up was 22-32 months.

Conclusion Cataract surgery with toric IOL implantation seems to be safe and effective in correcting astigmatism and improving visual function, in cataract patients with topographically stable keratoconus or PMD.

\section{- S094}

Clinical outcomes after implantation of multifocal toric intra ocular lenses.

THOUVENINR (1), ABRIEU M (1), BERGUIGA M (1), RAMBAUD C (1)

CHENYT (1) SENDOND (2) FROUSSART-MAILLEF(2)

RIGAL-SASTOURNEJC (1)

(1) Ophtalmology, Clamart

(2) Ophtalmology, Paris

Purpose To assess clinical outcomes and optical performances of refractive cataract surgery with mutifocal toric intraocular lens (IOL).

Methods This was a monocentric, retrospective review of cases, conducted at the Ophtalmology and Refractive Unit Percy, military Hospital, Clamart, FRANCE. This study included eyes presenting preoperative regular corneal astigmatism of 1D or greater, who underwent cataract surgery with implantation of a +3,75D multifocal toric hydrophilic IOL, by 2 differents surgeons. Ten eyes of seven patients were operated between 2012 and 2014. At the one month postoperative visit, we analysed visual acuity at various distances, spherical équivalent, refractive cylinder, spectacle independance, halos perception and patients satisfaction.

Results The mean spherical équivalent was $-0,4 \mathrm{D}$ at one month. Mean pre-operative réfractive cylinder increased from -2,89D (ranged from -1 to -6,75D) to -0,67D (ranged from $-0,5$ to $-1,0 \mathrm{D})$. Mean monocular uncorrected visual acuities were 7,42/10 at $4 \mathrm{~m}$ (Monoyer) and -0,28 $\log$ MAR at preferred near $(40 \mathrm{~cm})$. Mean corrected visual acuities were $9,56 / 10$ at $4 \mathrm{~m}$ and $-0,18 \log$ MAR at near. 8 of 10 patients reported freedom from corrective wear, and 6 of 10 complained about halos perception. The mean score that patients rated their uncorrected visual acuity was 7,3 +- 0,6 on a scale of 0 to 10 .

Conclusion One month after implantation of a multifocal toric IOL, patients had significantly reduced spherical équivalent and refractive cylindre. They achieved good visual acuity across distances permiting spectacle independance for most of them.

\section{- S096}

Indexing of cataract surgery video by content based video retrieval

MARTIANO DH (1), CHARRIERE K (2), LAMARD M (2), COCHENER B (1)

(1) Ophthalmology CHRU Brest, Brest

(2) LATIM, Brest

Purpose Many surgical computer-aided projects have emerged in recent years, but none interested in cataract surgery. The aim of our study was to develop a method able to recognize in real time the video stream, surgical phases clearly identified and thus to classify them in a database

Methods Over a period of 2 years, we have adopted a first series of 60 videos of cataract surgery, performed by six different surgeons. The operating parameters were recorded in postoperative dedicated form. Our first method characterized videos in 9 tasks : incision, OVD injection, capsulorhexis, hydrodissection , phacoemulsification , epi core implementation, OVD aspiration and closure. We developed specifically for this study, a novel characterization method using a granular system at several levels. To recognize in real time during the surgical phase, we used search algorithms for video content. The parameters studied were primarily based on visual content. Finally, we used the calculation of the area under the ROC curve for the quantification measure.

Results The average video length was $15.25+/-7$ minutes [ 10.05-28 ] . 8 videos were atypical (implemented iris hooks, toric IOL). The mean area under the curve was 0.806 $+/-0.1$ [0563-0937] for the analysis of complete sequences.

Conclusion We choose to measure granularity in 4 different levels : procedure, phase, step, activity. These recognition system appear as a significant progress towards the construction of smart systems for surgery. Our method gives good quality results for sequencing our database video cataract surgery. And apart from some interest to have a database by automated grading, we can imagine the development of a device to support the initiation of surgical novice. 
- 5097 / 1777

Comparison of optical and psychophysical methods to measure intraocular straylight

PENNOS A, GINIS HS, ARTAL P

Laboratorio De Optica, University of Murcia, Murcia

Purpose To quantify intraocular forward scattering (straylight) by using three different methods in a group of normal subjects.

Methods Intraocular straylight was measured in the dominant eve of 10 emmetropic subjects without any known ocular pathology ( $30 \pm 5$ years).Two different, commercially available, scatter filters (Black Pro-Mist $1 / 4$ and Black Pro-Mist $1 / 2$ from Tiffen) where used in order to recreate the effects of higher scattering. Three measurement techniques were used:a commercially available straylight meter (C-quant, Oculus),a prototype of an optical double-pass based method (Sigma) and a psychophysical method that we developed.We used a Bayesian method (quick-CSF) in a range of spatial frequencies ( 2 to 20 cycles/degree) with 50 2-alternative forced choice trials.Each set of trials was repeated 2 times for each condition (with and without glare).As a glare source, a fluorescent lamp was positioned concentrically to the test area where the sinusoidal gratings were projected.All tests were presented on a screen placed at 1 meter distance from the subject and the test area was covering a total of 6.4 degrees of visual field.For Sigma and C-Quant tests,the logarithm of Scatter coefficient was determined, whereas for the q-CSF measurements the difference between the areas below the log contrast sensitivity curves was used as scatter metrics.

Results In the three methods, the effects of straylight were accordingly increasing with the addition of the scatter filters. A correlation between all three methods was verified.

Conclusion These results suggest the potential use of an even simplified method based on the q-CSF to evaluate straylight complementing other existing methods.

\section{- 5099}

Central nervous system abnormalities in retinoblastoma patients

DE FRANCESCOS (1), DE LUCA M (1), GALLUZZI P (2), HADIISTILIANOU T (1)

(1) Referral Center for Retinoblastoma, Siena

(2) Unit of Neuroradiology, Siena

Purpose Presence of CNS abnormalities on MR images in a large group of patients with retinoblastoma (rtb) is evaluated. Mental retardation and congenital brain anomalies are reported in patients with rtb, mostly in combination with $13 q$ deletion syndrome. Pinealoblastoma is the most important and "life threatening" condition associated with hereditary rtb, but recent studies suggest an association with pineal cysts. This association is important because some pinealoblastomas mimic pineal cysts.

Methods CNS MR images of 420 consecutive patients with rtb from 2000 to 2014 were evaluated by neuroradiologists for tumors, structural anomalies, myelinization, and coincidental findings. Clinical records were reviewed for laterality, heredity, and the presence of the $13 q$ del syndrome.

Results Patients with bilateral and unilateral proved RB1-germline mutation were $42(48.2 \%)$ out of 87 hereditary cases. 9 patients had 13q deletion syndrome. Normal findings on brain MR images were seen in 307 out of 420 patients. 1 pinealoblastoma was detected in a patient with hereditary $\mathrm{rtb}$, one arachnoid cyst in a sporadic unilateral RTB girl, 1 cerebral and corpus callosum atrophy and 3 pineal cysts were detected (2 non hereditary, 1 in $13 q$ deletion syndrome). Corpus callosum agenesis was found in 3 patients (2 13q deletion syndrome, 1 hereditary RTB), corpus callosum hypoplasia in 3 patients (2 twins, 1 sporadic RTB), both in combination with 13q deletion syndrome.

Conclusion Pinealoblastoma is associated with hereditary retinoblastoma, and structural brain abnormalities are associated not only with 13q deletion syndrome patients. Pineal cysts can be detected in patients with sporadic rtb and/or with 13q deletion syndrome.

\section{- S098}

Comparative study of anterior segment measurement with rotating dual Scheimpflug analyzer and optical low coherence reflectometry

ASCASO FI (1), HUERVA V (2), SOLDEVILA J (2), LAVILLA L (1)

(1) Department of Ophthalmology, Hospital Clinico Universitario, Zaragoza

(2) Department of Ophthalmology, Hospital Universitari Arnau de Vilanova, Lleida

Purpose The aim of the study was to compare the anterior segment measurements obtained with optical low coherence reflectometry (Lenstar LS 900, version 1.1, HaagStreit, Koeniz, Switzerland) and rotating dual Scheimpflug analyzer (Galilei Dual Scheimpflug Analyzer versión 3, Ziemer Ophthalmic Systems, Port, Switzerland).

Methods Prospective, comparative, observational study in which 59 eyes of 30 patients who needed a preoperative study for a refractive procedure or cataract surgery were sudied with both the Lenstar 900 and Galilei systems. Central corneal thickness (CCT), anterior chamber depth (ACD), horizontal limbal distance (white-to-white distance; WTW), pupil diameter (PD), as well as the keratometric readings at the steepest meridian (Ks), the flattest meridian (Kf), the corneal astigmatism power, and the plus astigmatic cylinder axis were measured. Degree of agreement between Ks, Kf, astigmatism power, cylinder axis, CCT, ACD, WTW and PD were the main outcome measures.

Results Ks, Kf, corneal astigmatism power, cylinder axis, and WTW measurements with the Lenstar and the Galilei system showed narrow 95\% limits of agreement (LoA), which implies good agreement ( $>>0.05$, Bland-Altman plot analysis). In contrast, range and 95\% LoA were significantly different for CCT, ACD and PD values ( $\mathrm{p}<0.05$, BlandAltman plot analysis)

Conclusion In clinical practice, the Lenstar and the Galilei system can be used interchangeably for WTW measurements and K readings but not for CCT, ACD and PD values.

\section{- S100 / 4641}

Vascularized vitreous seeding regression following systemic and intravitrealchemotherapy for advanced "late" retinoblastoma

HADIISTILIANOU T (1), DE FRANCESCOS (1), BORRIM (1), MICHELI L (1), GALIMBERTID (2), CAINIM (2), GALLUZZI P (3), MENICACCI F (4)

(1) Ocular Oncology, Ophthalmology unit, Siena

(2) Paediatrics, Siena

(3) Neuroradiology, Siena

(4) Ophthalmology unit, Siena

Purpose To report the regression of vascularized vitreous seeding in advanced "late" retinoblastoma

Methods Case report8 year old girl with bilateral disease. The affected eye was the left eve (group D - ABC classification- and group VB -Reese Classification-) while the right eye presented two peripheral retinomas

Results Complete remission of the massive vascularized vitreous seeding was achieved and multiple retinal tumors regressed following six cycles of systemic chemotherapy (three drugs-carboplatin etoposide and vincristine) and four intravitreal injections with Melphalan (20 micrograms).Retinal tumors presented type I regression (calcified tumors) while massive vitreous seeding was replaced by spidernet fibrotic formations.

Conclusion This case highlights the successful combination of three-drugs systemic chemotherapy with intravitreal melphalan in advanced "late" retinoblastoma presenting with vascularized vitreous seeding and multiple retinal tumors. 
- S101

Survival in uveal melanoma spanish patients: 1992-2012

SAORNIL ALVAREZM (1), ALONSO P (1), GARCIA-ALVAREZ C (1), MUNOZM (2), ALMARAZ A (3), LOPEZ-LARA F (4)

(1) Intraocular Tumors unit, Hospital Clinico Universitario, Valladolid

(2) Research unit. Hospital Clinico Universitario, Valladolid

(3) Epidemiology. Medicine School, Valladolid

(4) Intraocular Tumors Unit. Hospital Clinico Universitario, Valladolid

Purpose To analize the specific survival of spanish patients diagnosed and treated of uveal melanoma in a national referral unit and compare the results with the previously published in other population

Methods Patients diagnosed of choroidal and ciliary body melanoma between 1992 and 2012 were included. Epidemiologic and general data, clinical and tumor characteristics histopathologic features, survival status and cause of death were collected prospectively in a database Statistical analysis was descriptive for baseline clinical features; survival was analysed by Kaplan-Meier curves and multivariate Cox's regression model.

Results 480 patients were included. Mean follow up of 58.6 months; mean age of 61.7 in males (48.5\%) and 62.6 in females, arising in choroid 95.6\%. Iris color was grey in 19\%, and brown in $43.8 \%$. Most of the tumors were medium size (40.4\%) without neither extraocular $(94.6 \%)$ nor systemic $(98.7 \%)$ extension.Brachytherapy was performed in $43.4 \%$, observation in $36.4 \%$ and enucleation in $18.2 \%$. Predominant histologic types were mixed (39.1\%) and fusiform (34.3\%). Thirty nine patients died during the follow up time due to metastatic melanoma. Survival rate at 5 and 10 years was $87.7 \%$ and 85.5\% Patients with grey/blue iris presented worse survival at 5 years (75.6\%) than hazel (91.4\%). No deaths were registered in patients with small size; for medium and large survival was $91.2 \%$ and $64 \%$. Factors with prognostic significance were: ciliary body location, extraocular extension, epithelioid type and grey/blue iris color

Conclusion The present study show a better survival than previously published series. Lighter iris color as showed prognostic value.These results may be due to epidemiologic differences.

- $\mathbf{S 1 0 3}$

The coexistence of uveal melanoma and non-pigment ciliary body epithelium adenoma in one eye

MARKIEWICZ A (1), BOGDALIA (1), ROMANOWSKA-DIXONB (1), POCIEJ W (2), KOWAL J (2), DEBICKA-KUMELA M (2), JAKUBOWSKA B (2)

(1) Ophthalmology Jagiellonian University, Kraków

(2) Ophthalmology, Kraków

Purpose To report case series of coexistence of the non-pigmented ciliary body epithelium adenoma and uveal melanoma in one eye.

Methods Retrospective analysis of case series.

Results In Department of Ophthalomology and Ocular Oncology in Cracow we have found 3 patients with coexistence of uveal melanoma and non-pigmented ciliary body epithelium adenoma in some eye. All patients were treated because of diagnosis uveal melanoma . Non-pigmented ciliary body epithelium adenoma was detected accidently in histopatological examination. Before histopatological test there were no suspicion of ciliary body adenoma. In 1 case melanoma was located in iris and in 2 cases in choroid. We performed iris melanoma excision in patient with iris melanoma. First patient with choroid melanoma were treated with brachytherapy and next with enucleation because of neovascular, secondary glaucoma. Second patient with choroidal melanoma in spite extraocular extention disagreed with enucleation in the beginning. But after I-125 brachytherapy with excision of extraocular infiltration patient decided on enucleation. In histopathologycal examination choroidal melanoma and adenoma were detected.

Conclusion Adenoma of non-pigmented ciliary body epithelium is a very rare tumor It is more rarely with coexistence with uveal melanoma in one eye
- S102

Preliminary results of proton radiotherapy for choroidal melanoma in Departhment of Ophthalmology Jagiellonian University in Kraków

MARKIEWICZ A (1), ROMANOWSKA-DIXON B (1), BOGDALI A (1), KOWAL J (2), DEBICKA-KUMELA M (2), JAKUBOWSKA B (2)

(1) Department of Ophthalmology Jagiellonian University Collegium Medicum Krakow, Kraków

(2) Department of Ophthalmology and Ocular Oncology University Hospital in Krakow, Kraków

Purpose Analysis of all cases choroidal melanoma treated with proton beam radiotherapy (PBRT) in 2011-2013 in Krakow.

Methods The retrospective analysis of 39 patients with choroidal melanoma treated with proton beam in Krakow in 2011-2013 was performed. There were 20 (51\%) women and $19(49 \%)$ men in mean 53,7 years $(24-74)$. . The largest tumor diameter ranged from $4,6 \mathrm{~mm}$ to $16.2 \mathrm{~mm}$, and tumor thickness from $1.4 \mathrm{~mm}$ to $10.3 \mathrm{~mm}$.Treatment was delivered in 4 fractions on 4 consecutive davs. All patients received the dose of 60 CGE (Cobalt Grey Equivalent) given to PTV (planning target volume).

Results The tumour had regressed in 20 patients (51\%) and remained stable in 19 patients (49\%). The large tumours in 4 cases were removed during vitrectomy (endoresection). One tumor was excised with exoresection. In the case of 1 patient, despite intraocular tumour regression occurring the choroidal melanoma had spread multifocally into the orbit, which necessitated orbit exenteration. In this case tumor cells in scleral vessels were detected in histopathologycal examination. The results ensured that the eyeballs of 38 patients (99\%) could be saved. Visual acuity improved in 5 cases, was stable in 21 and decreased in 14 patientsThe follow-up period

Conclusion Proton beam radiotherapy is an effective method for treating patients with choroidal melanoma. This method ensures an eyeball preservation rate of $99 \%$, with the vision function of $67 \%$ patients being saved. Decreasing visual function was caused by choroidal melanomas location close to macula and/or optic nerve disc in our group it were in $19(49 \%)$ cases.

\section{- S104 / 4248}

Optico-reconstructive surgery after blockexcision of iridociliar tumors

SAAKYANS (1), ANDREEVA T(1), CHENTSOVA E (2)

(1) Ocular Oncology and Radiology, Moscow

(2) Oculoplastic and reconstructive surgery, Moscow

Purpose To estimate the possibilities and indication for optico-reconstructive surgery after blockexcision of iridociliar tumors.

Methods 68 patients with iridociliary tumors and complicated cataract were treated. 41 patients underwent combined surgery - block excision with cataract extraction (IOL implantation performed in 37 cases). In 14 of these patients iridectomy was performed using a tunnel corneal incision (in cases of small tumors of iris pupillary margin) followed by simultaneous phacoemulsification+IOL. The other patients underwent cataract extracapsular extraction. Simultaneous operations were carried out only in benign tumors.27 patients underwent delayed cataract extraction after blockexcision ( 9 patients with malignant tumors, 18 patients - benign). In 5 cases surgery were performed with implantation of artificial iris, in 17 cases - with IOL implantation.

Results In all cases we detected increase the visual acuity, depending on the type of surgery and the tumor's sizes.

Conclusion Indications for simultaneous optico-reconstructive surgery are benign tumors, for delayed - even malignant ones. In cases of small benign tumors of iris papillary margin simultaneous surgery could be done using FEC + IOL, for large benign tumors (no more than $1 / 3$ of iris) - with simultaneous cataract extracapsular extraction, if more than $1 / 3$ of iris - delayed surgery with implantation of artificial iris. 
- S105

Clinical features, instrumental diagnostics and surgical treatment of orbital rhabdomyosarcoma in children

SAAKYANS, AMIRYAN A, IVANOVA O, TATSKOVR

Ocular Oncology and Radiology, Moscow

Purpose To present the clinico-instrumental features and surgical approaches in orbital rhabdomiosarcoma in children

Methods 15 children in the age from 4 month to 14 years ( 4 girls and 11 boys) were treated in our clinic. All the patients underwent both clinical and instrumental examinations, including CT-scan, high frequency ultrasonography. The surgical treatment was performed in all cases - tumor excision or incision biopsy. The morphological verification of the tumors's type was dedicated in all children embryonal (13 children) and alveolar ( 2 children) rhabdomyosarcomas.

Results In all case the progressive proptoses with vision decreasing in short period of time were noticed (from 2 weeks to 4 month). CT scan demonstrated a solid mass with accurate margins, localized in different part of the orbit (mainly under the upper roof - in 6 cases) with infiltration of rectus or oblique extraocular muscles. High frequency ultrasonography with Doppler scanning showed a hyporeflective lesion with hypervascular internal vascularization. In 6 cases the tumor excision were performed (all these tumors were covered with thin pseudocapsula and has less infiltration of orbital tissues).

Conclusion The examination of rhabdomyosarcoma should be complex, based on anamnesis data, clinical features and instrumental investigations results. The type of surgical treatment should be chosen depending on the tumor infiltration of orbital tissues.
- S106 / 4247

Identification and characterization of melanin in choroidal melanoma by electron paramagnetic resonance spectroscopy (EPR)

ROMANOWSKA DIXON B (1), KLONOWSKA A (1), PLONKA P (2), SARNA T (2) (1) Ophthalmological Department of Jagiellonian University, Krakow (2) Faculty of Biochemistry, Biophysics and Biotechnology, Krakow

Purpose The purpose of this study was to determine the quantity and type of melanin in choroidal melanomas in correlation with patient's clinical characteristics.

Methods Melanoma-containing samples were obtained after enucleation surgery, performed because advanced choroidal melanoma was diagnosed, not qualified to preserving eye therapy.Frozen samples were examined at liquid nitrogen temperature by EPR spectroscopy.

Results Examination showed that choroidal melanoma cells had a low eumelanin to pheomelanin index. In most of investigated melanoma-containing samples the presence of pheomelanin was determined. Also strict correlation between patient's clinical characteristics such as skin phototype, iris and hair color and melanin type in tumor were observed. No correlation however were found between histopathological type and quality and quantity of melanin in choroidal melanoma. Macroscopic analysing cases of choroidal melanoma were characterized by wide pigment variations. The presence of tide correlation between tumor color and type of melanin was observed.

Conclusion Pheomelanin fenotype occurred in tumors with low content of pigment. Tumors intensively pigmented (black, dark brown) belong to eumelanin tumors.
- S107

Intravitreal Ranibizumab for the treatment of choroidal neovascularization associated with choroidal nevus

HERRERA L (1), ZABADANI K (1), ALMENARA C (1), CARAMELLO C (1), PEREZ NAVARRO I (1), ASCASO J (1), CAMINAL J (2), MARTINEZ M (1), ESTEBANO (1) CRISTOBAL J(1)

(1) Ophthalmology Department, Lozano Blesa University Hospital, Zaragoza

(2) Ophthalmology Department, Bellvitge University Hospital, Barcelona

Purpose To report our visual outcomes in a series of five cases of classic choroidal neovascularization $(\mathrm{CNV})$ associated with choroidal nevus which were treated with intravitreal ranibizumab injections.

Methods Interventional case series. Each patient underwent a complete ophthalmic evaluation, including fluorescein angiography and spectral domain optical coherence tomography (SD-OCT). Data were retrospectively analyzed to evaluate both clinical and anatomical results following intravitreal treatment.

Results The CNV was juxtafoveolar in four cases and extrafoveolar in one case Intravitreal ranibizumab therapy was used in all five cases by using 2-5 injections (mean 3.4 injections). Moreover, two cases required other therapies: photodynamic therapy (PDT) in one case of juxtafoveolar CNV and laser photocoagulation in a case of extrafoveolar CNV. After a mean follow-up of 18.6 months, final best-corrected visual acuity (BCVA) increased in two cases, decreased in two cases and remained stable in one case There were no adverse effects associated with ranibizumab injections. All choroidal nevi remained stable, showing no signs of malignancy.

Conclusion Intravitreal ranibizumab injections appear to be a safe and effective treatment modality for CNV associated with choroidal nevus

\section{- S108}

An aggressive small choroidal melanoma

LAUWWERS N, DE GROOT V, DE KEIZER RJW

UZA Ophthalmology, Edegem

Purpose To describe a small non diffuse choroidal melanoma with extraocular extension.

Methods Case-report

Results We describe the case of a 50year-old patient presenting with visual loss and metamorphopsia. Fundus examination revealed a suprapapillary trilobular choroidal mass with inhomogenous pigmentation and lipofuscin. The optic nerve was slightly oedematous. On fluorescein angiography, the lesion revealed pin-points and exsudation. Ultrasound sonography (20Mhz) showed a peripapillary lesion of $3 \mathrm{~mm}$ thickness. MRI was performed and confirmed the diagnosis of choroidal melanoma. No extraocular extension was seen on MRI or ultrasound imaging. Because of optic nerve oedema we decided to perform an enucleation. On pathology, the lesion measured 9mm diameter and $3 \mathrm{~mm}$ thickness. It was mostly composed of epithelioïd cells and it showed scleral, perineural, optic nerve and periocular soft tissue invasion. Complementary orbital radiotherapy was performed. Until now, after one year follow-up, the patient has shown no metastasis or local recurrence.

Conclusion Extraocular invasion in a small thickness non diffuse choroidal melanoma is rare In times of eye-sparing choroidal melanoma treatment, the ophthalmologist should not forget the possibility of extra-ocular involvement, even in small tumors. 
- S109

Melanomalytic glaucoma secondary to iris melanoma

AJAMIL RODANES S (1), GARCIA-ALVAREZ C (1), SAORNIL ALVAREZM (1), LOPEZ-LARA F (1), FRUTOS-BARAJA JM (1), QUINONESM (2)

(1) Intraocular Tumors Unit - University Hospital, Valladolid

(2) pathology - University Hospital, Valladolid

Purpose To report on an unusual form of adult onset iris melanoma as melanomalytic glaucoma.

Methods A 35-year-old white male with a history of unilateral ocular hypertension and visual loss for six months.

Results At first visit,Best Corrected Visual Acuity was 80/20 in the right eye (OD) and $20 / 20$ in the left one (OS).Intraocular pressure was $40 \mathrm{mmHg}$ in the $\mathrm{OD}$ and $10 \mathrm{mmHg}$ in OS.Anterior segment examination of the OD showed a melanotic elevated lesion on the base of the iris from 8:00 to 9:00.Gonioscopy revealed extension into the anterior chamber angle and that a dense, brown, pigmented band at the filtration portion of the trabecular meshwork.Ophthalmoscopy demonstrated glaucomatous changes to the right optic disc.The patient was unresponsive to medical treatment for glaucoma. The clinical differential diagnosis was melanocytoma, nevus and melanoma.Excisional biopsy (iridocycloresection) was performed. Light microscopy showed proliferation of fusiform cells with pleomorphic nuclei in the iris stroma, immunopositive for HMB45 and Melan-A.The final hystopathologic diagnosis was iris melanoma with dispersion of pigment in anterior-chamber and secondary melanomalytic glaucoma. Plaque radiotherapy was performed as a

Conclusion In patients presenting unilateral glaucoma and increased pigmentation in the trabecular meswork it is crucial to perform imaging studies, such as ultrasound, in order to rule out the presence of an intraocular tumor. Early diagnosis improves the patients visual prognosis and the morbidity of treatments. Resection by iridectomy with adjuvant epiescleral brachytheraphy is effective in controlling the disease.

\section{- S111}

White center retinal hemorrhages revealing acute lymphoblastic leukemia

BENLAHBIB M, BENCHERIFA F, HAJII Z, BOULANOUAR A, BERRAHO A Ophtalmologie B, Hôpital des spécialités, Rabat

Purpose Acute lymphoblastic leukemia is a severe affection related to invasion of the bone marrow by immature haematopoietic cells of the lymphoid lineage. Retinal hemorrhages with white center are secondary to a specific pathophysiological mechanism. They are exceptional when revealed acute leukemia.

Methods Through clinical observation of a case of acute lymphocytic leukemia revealed by retinal hemorrhages with white center, we recall the pathophysiological mechanisms and clinical aspects of this rare retinal disease.

Results A 16 years old child consult for myodesopsia in his left eye. The ophtalmological examination show a correct visual acuity 10/10 P2 in the right eye and 9/10 P2 in the left. We note a discolored conjunctiva with vascular occlusions. The examination of the fundus of eyes reveal a diffuse retinal hemorrhages with white center. The blood count analysis show ; $120.000 / \mathrm{mm} 3 \mathrm{WBC}, \mathrm{Hb} 3.5 \mathrm{~g} / \mathrm{dl}$ and platelet $37.000 / \mathrm{mm} 3$. The diagnosis of acute lymphoblastic leukemia is made. The child is cared for in a pediatric oncology service. He received a blood transfusion and chemotherapy with complete remission at the last control.

Conclusion Ophthalmologic examination has a major interest in the diagnostic and therapeutic management of this disease.
- S110 / 4648

Suspicious choroidal mass - an interdisciplinary challenge

GRAEFFE, GRIESHABER M, MEYERP

Eye Clinic, Basel

Purpose To highlight the importance of interdisciplinary work up based on a patient with presumed choroidal metastatic of unknown primary tumor:

Methods Clinical-pathological case report.

Results A 57 year old woman was referred to our clinic because of a progressive vision loss in her right eye for seven days. Loss of vision field and light flashes followed 3 days later. General medical history was inconspicuous and last general check-up including gynaecologic examination performed by general practitioner was apparently normal. At first presentation, a yellowish choroidal mass in the inferior part of fundus was found clinically. Further diagnostic exams like ultrasound, OCT, fluorescence angiography and MRI of the orbit were suspicious for a choroidal metastasis of unknown primary tumor. An immediate interdisciplinary work-up with gynaecologists and oncologist was performed; histology of a punch biopsy of the left mammae confirmed the suspected finding of a multiple low differentiated invasive growing ductal carcinoma. Furthermore, metastasis of the lung and axillary lymph nodes were found. Because of the multiple metastases, a combined chemotherapy and radiation therapy was initiated.

Conclusion Choroidal mass is indicative for metastasis which requires immediate interdisciplinary work-up even in the absence of a positive medical history. Since biopsy of the choroidal mass should not be performed, the diagnosis was made by the biopsy of the primary tumor, showing a metastasized aggressive breast tumor. Thanks to efficient interdisciplinary work up, local and systemic therapy saved vision in her right eye and best palliative care was offered.

\section{- $\mathrm{S} 112$}

Case of orbital mucosa-associated lymphoid tissue lymphoma accompanied with refractory serous retinal detachment

OSHITARI T (1), OTA S (1), KISHIMOTO T (2), YAMAMOTO S (1)

(1) Ophthalmology and Visual Science, Chiba University Graduate School of Medicine, Chiba

(2) Molecular Pathology, Chiba University Graduate School of Medicine, Chiba

Purpose We present a case of orbital mucosa-associated lymphoid tissue lymphoma accompanied with refractory serous retinal detachment.

Methods A 44-year-old man who developed uveitis in the right eye was referred to Chiba National Hospital. His visual acuity was 0.5 , and the IOP was $34 \mathrm{mmHg}$ OD. Keratic precipitates and inflammatory cells were observed in the anterior chamber. Fundus examinations showed a serous retinal detachment (SRD) and a hyperemic disc. B-mode ultrasonography showed a thickened choroidal membrane and a retrobulbar mass. The MRI findings showed that the mass had T1- and T2-low intensities. Although topical steroid treatments improved the inflammation, the SRD was not improved. Because the oral steroid administration did not reduce the size of the retrobulbar mass, he was referred to the Chiba University Hospital.

Results Two month later, the retrobulbar mass had increased, and a partial resection biopsy combined with flow cytometry was performed. The final diagnosis was mucosaassociated lymphoid tissue lymphoma. The intraocular IL-10 was not measurable. After R-CHOP chemotherapy, the tumor size decreased but the SRD was not improved. Not only lymphoma cell infiltration but also choroidal circulatory disturbance may be associated with the development of the SRD.

Conclusion Retrobulbar malignant lymphomas accompanied by refractory SRD are very rare and difficult to diagnose without biopsy. But, early diagnosis is more helpful in deciding the appropriate therapy. 
- S113

Suggestion of optimal response criteria in patients with ocular adnexal mucosa associated lymphoid tissue lymphoma

JUNG S, PAIK JISUN, YANG SW

Department of ophthalmology, Seoul

Purpose Ocular adnexal mucosa-associated lymphoid tissue (MALT) lymphoma (OAML) has been recognized as most common primary orbital malignancy. However little was known about the response criteria for OAML. Our aim was to sugges response criteria for nonconjunctival OAML, the response evaluation of which using the conventional response criteria is inappropriate.

Methods A retrospective chart review of 34 eves from 30 patients diagnosed with nonconjunctival OAML was conducted, focusing on the change in tumor size based on linear bi-dimensional, and three-dimensional methods in magnetic resonance imaging (MRI) of the orbit The maximum tumor response period of each case was investigated, and the expected optimal response period was calculated using regression analysis.

Results In the 30 evaluable patients, the median time taken for the maximum tumor response was 6 months (range, 3-18 months). More than 75\% of patients attained a maximal tumor response in 6 months after initial therapy (median follow-up period, 30 months; range, 15-77 months). Based on the regression analysis, it took 4.7 months for the maximum diameter $(2 \mathrm{r}$ ) of the tumor to decrease by $50 \%$ of the initial lesion size.

Conclusion We cautiously suggest that the optimal response could be defined as a $50 \%$ reduction of the maximum diameter within 6 months of treatment initiation and that only observation without additional therapy is adequate for nonconjunctival OAML if an optimal response is achieved.

- S115 / 4447

HSP90 inhibitors and conjunctival melanoma

MADIGANM (1,2), OUAH X (2), MCALPINE S (3), CONWAY RM (2)

(1) Optometry \& Vision Science, UNSW, Sydney

(2) Save Sight Institute Uni Sydney, Sydney

(3) Chemistry UNSW, Sydney

Purpose To investigate the expression of HSP90 in human conjunctival melanoma (CM) cell lines and primary human melanocytes, and to assess the effects of HSP90 inhibitors on cell growth and proliferation.

Methods CM cell lines (CRMM1 and 2; CM2005.1) and primary human melanocytes ( $\mathrm{n}=3, \mathrm{P} 2$ to P4) were immunolabelled for HSP90, and visualised using immunofluorescence and confocal microscopy. HSP90 expression was also examined in cell lysates from CM cell lines (CRMM1 and 2; CM2005.1) and primary melanocytes $(\mathrm{n}=3)$. The effect of several HSP90 inhibitors (17DMAG, SM252 and SM122) on cell growth and viability was assessed at $24 \mathrm{hr}$.

Results CRMM1 and 2, and CM2005.1 cells showed strong cytoplasmic expression of HSP90, with low levels of cytoplasmic expression in primary melanocytes. This was confirmed using western blot. The HSP90 inhibitors induced dose-dependent cell death in CM cells, 17DMAG (water soluble) being most effective (LD50 at 24 hrs 30uM compared with 50um for SM122 and SM122).Primary human melanocytes viability was not significantly reduced by the HSP90 inhibitors.

Conclusion HSP90 is a widely expressed chaperone protein that can regulate cell growth, survival and angiogenesis pathways. CM cell lines expressed high levels of HSP90 protein compared to melanocytes, consistent with a recent study of primary conjunctival melanoma specimens, that found over-expressed HSP90 in CM, particularly recurrent tumours. HSP90 inhibitors may be useful in inhibiting CM growth alone or in combination therapies. Funding: National Foundation for Medical Research \& Innovation (MCM \& RMC); Lions NSW Eyebank

\section{- S114}

Caruncular naevi investigated by in vivo confocal microscopy: reporting the first 5 cases

IULIENNER (1, 2), GRIVET D (2), CINOTTI E (3), PERROT IL (3)

LABEILLE B (3) CAMBAZARD F(3) FOREST F (4). DUMOLLARD IM (1,4)

PEOC'HM $(1,4)$, GAIN P $(1,2)$, THURET G $(1,2,5)$

(1) Corneal Graft Biology, Engineering and Imaging Laboratory, EA2521, Federative

Institute of Research in Sciences and Health Engineering, Faculty of Medicine, Jean Monnet University, Saint-Etienne

(2) Department of Ophthalmology, University Hospital, Saint-Etienne

(3) Department of Dermatology, University Hospital, Saint-Etienne

(4) Department of Pathology, University Hospital, Saint-Etienne

(5) Institut Universitaire de France, Bd St Michel, Paris

Purpose During body screening of melanocytic lesions, naevi of the eyelids, conjunctiva and caruncula, although accessible to the dermatologist, are often missed out. These tumors have similar characteristics and prognosis as oral or genital ones. We report the first cases of caruncular naevi observed by in vivo confocal microscopy (IVCM)

Methods Five patients, 4 men and 1 woman, who presented with a caruncular melanocytic lesion, were examined by IVCM, using the handheld dermatological IVCM Vivascope 3000 (Lucid Inc, NY, MAVIG GmbH, Germany) equipped with $830 \mathrm{~nm}$ class IB laser.

Results Mean age was 39 years old (25-71). The tumors which were $2.2 \mathrm{~mm}$ width on average $(0.5-3.0 \mathrm{~mm})$ were present since 4 years on average (1-10). Two patients had family history of skin melanoma. IVCM showed hyperreflective round cells that were either isolated or grouped within the lamina propria. No abnormal cells were observed. Despite the benign IVCM aspect, a surgical excision biopsy was performed on 4 patients, for definitive histopathology or esthetic reasons. All 4 were compound naevus on histopathology. The remaining patient had no progression at 6 months follow up

Conclusion The caruncular site is inaccessible to both dermatoscopic imaging and standard dedicated ophthalmological IVCM. The Vivascope 3000 overpass this site limitation thanks to the small head of the camera and its handy and portable dimensions. In all cases, the IVCM imaging correlated with histopathology. This is the first reported series of caruncular melanocytic lesions explored in IVCM.Grant: GIRCI Rhone Alpes Auvergne 2012

\section{- S116 / 4446}

Gene expression in conjunctival melanoma cell lines treated with the histone deacetylase (HDAC) inhibitor valproic acid (VPA)

MUNOZ-ERAZO L (1, 2), MADIGANM (3), CONWAYRM (2)

(1) Department of Pathology, Charles Perkins Centre, University of Sydney., Sydney

(2) Save Sight Institute, University of Sydney, Sydney

(3) Optometry \& Vision Science, UNSW, Sydney

Purpose To investigate the differential regulation of genes in conjunctival melanoma cell lines (CRMM1 and CM2005.1) treated with valproic acid (VPA), a class I and II histone deacetylase (HDAC) inhibitor:

Methods Based on previous viability assays, cell lines were treated with VPA CRMM1:3mM, CM2005.1:5mM). At 24 and $72 \mathrm{~h}$ post-treatment, RNA was extracted and processed for RT-PCR using defined gene-array microfluidic cards. Gene functions chosen for investigation related to HDACs, cell cycle (cyclins e.g. CCND2, CCNA1) cell death (caspases), melanocyte function (PAX6), increased susceptibility to malignant transformation (BAP1, GNAQ) and DNA damage (GADD45A)

Results Upregulation of HDACs 1,2,3,46, SIRT2 and GADD45A occurred in CRMM1 cells by 24h but only after 24h for CM2005.1. Both CRMM1 and CM2005.1 showed increased CCND2 after VPA treatment, albeit higher in CM2005.1 ( $>700$-fold by $72 \mathrm{~h}$, compared to 22-fold in CRMM1). CCNA1 was increased at 24 and $72 \mathrm{~h}$ in CRMM1 ( $>200$ fold 24h). For CM2005.1 cells, CCNA1 was initially downregulated and upregulated by $72 \mathrm{~h}$ ( $\sim 30$-fold)

Conclusion The delayed increase in GADD45A (DNA damage gene) in CM2005.1 cells compared with CRMM1 cells, is consistent with our toxicity assays showing CRMM1 more responsive to VPA than CM2005.1. The differences of CCND2 and CCNA1 fold-changes (which are partially regulated by HDACs) between the two cell lines, may explain their dissimilar responses to VPA.Funding - NFMRI 
- S117

Merkel cell carcinoma: therapeutic discussion about two cases

BAILLIEUL A (1), RICHET M (1), DESSERRE J (1), MAURAGE CA (2)

LABALETTE P (1)

(1) Ophtalmology, Lille

(2) Anatomopathology, Lille

Purpose Merkel cell carcinoma is a rare cutaneous neuroendocrine tumor. Its incidence is estimated 0.34/100000 in the U.S. Caucasian population. The Merkel cell carcinoma is a tumor of rapid change with a high malignant potential. Indeed, the survival rate at 3 years was estimated at $68 \%$. Eyelid localization is meanwhile $5-10 \%$ of Merkel cell carcinoma. The median age of diagnosis is 69 years. It is then in the form of an inflammatory nodule that may be confused with a chalazion.

Methods We present two clinical cases of Merkel cell carcinoma of the eyelid, initially confused with chalazion. The staging was performed by PET scan. The first case was classified T1N0M0 carcinoma, the second a classified TxN0M0 carcinoma. Both cases underwent surgical excision healthy margin. The first case is a simple excision, the second flap rotation of the lower eyelid. During follow up the second case received radiotherapy for loco regional reached.

Results There is no current consensus for the treatment of Merkel cell carcinoma in the eyelid location. Its management is complex and must be multidisciplinary. Mohs micrographic surgery is the technique of choice. To secure tumor margins, radiotherapy seems to be an interesting adjuvant treatment for tumors larger than $2 \mathrm{~cm}$. The sentinel nodetechnique can avoid unnecessary lymphadenectomy. Combination of radiotherapy and sentinel lymph node procedure may improved survival.

Conclusion Merkel cell carcinoma is probably a virally induced cancer: a recent prospective study shows a significant association between infection with polyomavirus $\mathrm{MCV}$ and Merkel cell carcinoma. New therapy should happen quickly in the future.

\author{
- S118 / 4449 \\ Aneurysmal bone cyst: an exceptional entity in the orbit \\ LEMAITRE S (1), ZMUDA M (2), JACOMET PV (2), BERGES O (2), \\ GALATOIRE O (2) \\ (1) Institut Curie, Paris \\ (2) Fondation ophtalmologique Rothschild, Paris
}

Purpose Aneurysmal bone cyst is a rare benign bone neoplasm. The most common affected anatomical sites are vertebral column and long bones. We report two uncommon cases of orbital aneurysmal bone cyst.

Methods Two patients presented with a sudden left proptosis and ocular motility restriction.Case 1 is a 74-year-old female whose visual acuity is perception of light in the left eye due to severe papilledema. Imaging studies of the orbit reveal a large cystic frontal bone tumor associated with a subperiosteal hematoma causing eyeball and optic nerve compression. A preoperative arteriography shows a moderate vascular blush. Drainage of the haematoma is performed. A local recurrence with hematoma formation occurred two years after the first surgery.Case 2 is a 45 -year-old female. Imaging studies reveal a smaller cystic frontal bone tumour associated with a subperiosteal hematoma. The patient achieved full visual recovery after the drainage of the hematoma with no recurrence after treatment.

Results In both cases the aneurysmal bone cyst was revealed by an acute orbital compartment syndrome due to a subperiosteal hemorrhage. A biopsy of the tumor within the frontal bone was performed during surgery and submitted to pathology for examination. Aneurysmal bone cyst secondary to another primary bone lesion was therefore excluded

Conclusion Aneurysmal bone cysts are tumors of unknown cause which occur more often in the first 2 decades of life. Complete surgical resection is not always possible and the local recurrence rate is $20 \%$.
- S119 / 4448

Periorbital necrotizing fasciitis in a 69 year old patient. Medical management and use of hyperbaric oxygen as further adjunctive therapy

PORTALIOU DM (1), KOUROUPAKI A (1), KABANAROU S (1), KARVELA I (2), FERETIS E (1) XIROU T(1)

(1) Ophthalmology Department, Red Cross Hospital, Athens

(2) Ophthalmology Department, 'Agios Savvas' Hospital, Athens

Purpose To report a case of periorbital necrotizing fasciitis in a 69 year old patient that was successfully managed with the use of intravenous antibiotics and surgical debridement. In addition to the usual treatment modalities, Hyperbaric Oxygen (HBO) was used as further adjunctive therapy

Methods A 69 year old male presented with profound swelling over the right eye and right side of forehead. On admission the physicians made the diagnosis of septic shock. Patient had no history of alcohol abuse, insect bite, infection, recent penetrating injury, blunt trauma, face surgery or known immunosuppression conditions such as diabetes mellitus. Orbital CT showed a predominantly preseptal soft-tissue swelling consistent with the diagnosis of periorbital necrotizing fasciitis

Results Patient started on empiric treatment with broad-spectrum intravenous antibiotics that modified according to blood culture results few days later. Surgical debridement and microbiologic evaluation of the necrotic tissue were performed. Streptococcus pyogenes and Staphylococcus aureus were isolated from tissue samples. Patient was further treated with twenty sessions of Hyperbaric Oxygen Therapy. Complete resolution of the infection occurred six weeks after initial diagnosis.

Conclusion Treatment of periorbital necrotizing fasciitis with intravenous antibiotics and surgical debridement can be further enhanced with Hyperbaric Oxygen sessions with promising results.

\section{- $\mathrm{S} 120$}

Case of relapsing polychondritis with different ocular inflammations

NANA F, TOSHIYUKI O, SHUICHI Y

Ophthalmology and Visual Science, Chiba University Graduate School of Medicine,

Chiba

Purpose We present a case of relapsing polychondritis with orbital cellulitis.

Methods A 71-year-old man had age-related macular degeneration and had been treated by an intravitreal injection of anti-VEGF in another hospital. Three months later, he noted that he was deaf in both ears. At the same time, he developed scleritis in the right which was improved by a topical steroids. At the first visit to our hospital, he had a reddish swelling in the left evelid with pain. There was also a reddish swelling of the right uricula. CT examinations showed maxillary sinusitis, and he was diagnosed with left orbital cellulitis. He was treated with intravenous antibiotics, and the left orbital cellulitis rapidly improved but he developed right scleritis and left gonitis. Blood tests showed an elevation of ESR and CRP, and positive antinuclear antibody. MRI showed bilateral posterior scleritis and right auricular perichondritis.

Results Because he had auricular perichondritis and cochleovestibular disorders, auricular biopsy was performed, and an infiltration of lymphocytes into the periauricular tissue was found histologically. Taken together, he was diagnosed with relapsing polychondritis. Oral predonisolone treatment was performed by a consulting rheumatologist, and both ocular and auricular inflammations improved. He continues with the oral predonisolone

Conclusion Although ocular complications of relapsing polychondritis are rare, polychondritis is a life-threatening disease. Thus, ophthalmologists should consider relapsing polychondritis in patients with both ocular and auricular inflammation. 
- S121 / 3648

Secondary Intraocular lymphoma of the iris following a

succussefully treated diffuse large B-cell lymphoma of the orbit

MEYER P, GRAEFFE

Eye Clinic, Basel

Purpose To report clinical findings of a rare case of lymphoma with iris involvement and therapeutic approach with local application of rituximab

Methods Case report

Results $A \neg n$ 80-year old patient reported blurred vision in the right eye for one week After dacryodacryocystorhinostomy he suffured from decreased vision, chemosis and double vision on downgaze. MRI of the head revealed a right orbital tumor mass, arising from a distinct tumor mass of the right maxillary and ethmoid sinuses. Biopsy revealed CD5-positive, diffuse large B-cell lymphoma. The patient was managed with tumor debulking and systemic chemotherapy. At completion of therapy he remained in remission until presentation of a cutaneous relapse on the lower leg with simultaneous eve discomfort.On ocular examination the visual acuity was hand motion in the right eve with normal Intraocular pressure.The right eye showed diffuse keratic precipitates and moderate cells and flare in the anterior chamber.From 8 to 1 oclock the iris showed diffuse thickening of the stroma and irregular pupil.Examination with ultrasound biomicroscopy showed involvement of the anterior part of the cilliary body. Posterior segment examination was unremarkable. Aspiration of the anterior chamber showed CD20 positive, atypical lymphocytes, compatible with the previously diagnosed non-Hodgkin's lymphoma. After local application of rituximab three weeks later the thickening of the iris tissue had resolved.

Conclusion Lymphomatous involvement oft he iris should be considered in the differential diagnosis of a severe anterior uveitis in elderly patients with known aggressive systemic lymphoma. An additional local application of rituximab may be suggested in isolated iris lymphoma. 


\section{All authors index}

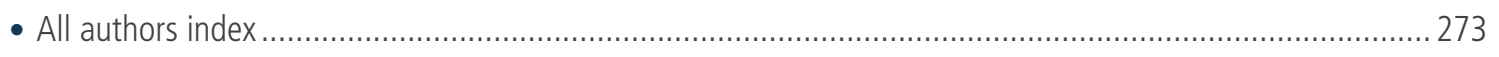


$274 \quad$ ALL AUTHOR INDEX

All authors of abstracts are listed alphabetically.

One letter and three digit numbers refer to posters.

Four digit numbers refer to oral presentations.

The digit numbers marked in bold indicate a first author abstract.

ABEGÃO PINTO L: 3473

ABERRA GUEBROU S: S070

ABOLBASHARI F: T007

ABOU SAMRA W: 3611

ABRAHAM S: 3214

ABRIEU M: S024, S036, S085, S094

ABRIEU N: S020

ABU EL ASRAR A: 3652, 4741

ACAR N: 2223, 3722, 1664

ACQUART S: S040, S042, S067, 1724, 4427, 4776, 4778

ADAM R: F096

ADAMS D: T077

ADDOU-REGNARD M: S089

AERTS J: 3421

AERTS L: F014

AGARD E: F015

AGUDO M: 2424

AGUDO-BARRIUSO M: S007, 2243

AGUIRRE G: 3264

AHEARNE M: S014, 1726

AHMADI HOSSEINI M: F029, T007

AHO S: 1731

AIFA A: 4421

AJAMIL RODANES S: F112, S109, 4477

AKESBI J: F096

AKHTAR S: S022, S051, 3252

ALBERT R: 1671, 3252

ALCALDE I: 054

ALESSANDRINI L: 4244

ALI Y: 3446

ALLENDE-MUNIIOZ MJ: 4253

ALMARAZ A: S101

ALMENARA C: F031, F036, F063, S035, S107, T098, T099

ALMENARA MICHELENA C: F038, S086, F088

ALMOALLEM B: T081

ALMUBRAD T: S022, S051, 3252

AL MUHTASEB H: $\mathbf{F 0 3 3}$

AL-OBEIDAN S: T081

ALONSO L: F050

ALONSO P: S101

ALVAREZ L: T075

ALVES N: S029, S030, T058, 2433, 2434, 2435, 2648

AMADIO M: S008, 3256, 3475

AMADO D: 3673,3674

AMAOUCHE N: 4772

AMARAL A: T051, 1735

AMATO R: S063, 4457

AMIRYAN A: S105, 4444

AMOAKU WM: F075, 4742

AMORIM A: 2445

ANDERSSON GRÖNLUND M: 3454

ANDJELIC S: S078, 3253

ANDONEGUI J: 5086

ANDRE H: T068, S002, T067

ANDRE M: F079
ANDREEVA T: S104, 4248

ANDROUDI S: 1642

ANG L: T025, T024

ANGIOI K: S001, S090, T085, 4674

ANITUA E: 5053

ANJOS R: T058, S029, S030, T107, 2433, 2648, 2434, 2435, 3673

ANSARI E: T014

ANTONIAZZI ER: S087

APTEL F: T043, T044, T038, T042, 3613, 3615

ARAPI I: 1643, 2251, 3654

ARAÚJO A: 2443

ARBOUR JD: F104

ARCKENS L: 3421

ARNASON S: F010

ARNDT C: F103, 4675

ARNOULD S: T064

ARONSSON M: 5002

ARTAL P: S097, 4255, 1777

ARVEILER B: 2661, 2663

ASATRYAN S: 3664

ASCASO F): S075, S098, F031, F038, F063, S018, S086, S088, T098, T105, T106, T114, 3411, 3771

ASCASO J: F036, S033, $\mathrm{S} 107$

ASLAM T: T010, 2533

ASMUS F: 3471,3472

ASOKLIS R: F024, F054, S003, S004

ASSELAIN B: 4445

ATAULLAH S: 3647

ATHERTON S: 3243

AUDENAERT K: 4224

AUDO I: 2764, 3764

AUGSTEN R: 3718

AUGUSTIN A: 4534

AUGYTE A: 5004

AUMAITRE O: F079

AUNG T: 3425

AVADHANAM VS: 4636, 4432, 4435

AVILES M: 2424

AVILES-TRIGUEROS M: S006

AVILÉS-TRIGUEROS M: 1673

AVITABILE T: S063, 4457

AYCHOUA N: 3423

AZEVEDO-PINTO S: 3476

AZIMI A: F029, T001

AZIZ A: T091

BADAL J: F033

BADIAN R: S045, S047

BAE HW: T021, T029

BAGGER M: 4245

BAGNOLI P: F005, F006

BAHNERS T: 4631

BAIER D: F108, 4672

BAILLIEUL A: S117

BAILLIF S: F094

BAINBRIDGE J: 3214
BALDWIN M: F056

BAO Y: F076, F078, 3441

BARALE PO: 3764

BARATZ K: 3632

BARBEIRO S: 2443

BARBIER J: 3647

BARCATALI MG: F099

BAREILLE M: 4635

BARILLA' D: T083

BARNARD A: 3761, 3763

BARRAQUER J: 4431

BARRAQUER R: 4253, 4655, 4751, 4251

BARREAU E: T077

BARRY P: 4753

BARZDZIUKAS V: F089

BASDEKIDOU C: 2212, 2211

BASSON W: T084

BATTA B: S090

BAUDONNET R: T062, 2647

BAUDOUIN C: T015

BAUSZ M: S032, 2437

BAUWENS M: T081, 4661

BAZIN L: F045

BEAUJEUX P: T085

BECHRAKIS N: $\mathbf{3 7 4 3}$

BEHL C: 2421

BEK T: F043, F044, 1611, 3715, 4744

BELAHDA A: F021

BELAOUDMOU R: 2475

BELLA A: T061

BELMONTE C: S054

BELTRAN W: 3264

BELVILLE C: 4423

BEMELMANS A: F023

BENALI ABDELLAH M: 4451

BENCHERIFA F: F082, S111

BENITEZ DEL CASTILLO JM: S059

BENLAHBIB M: F082, $\mathbf{S 1 1}$

BEN YAHIA S: 1743

BENZACKEN L: 3426

BERENDSCHOT TTJM: 4733

BERGES O: S118, 4449, 4772

BERGUIGA M: S020, S024, S036, S094

BERNABEI M: 3615

BERNARD A: S040, S013, S031, S042, S067, 4776, 1722,

$1725,2436,4427,4778$

BERNARDES R: $2443,2445,3663$

BERNOTAS G: T102

BERRAHO A: F082, S111

BERROD JP: 4674

BERTAUX AC: 4473

BERTONE C: T083

BESOMBES G: T030

BEUERMAN R: 2772, 4454

BEUTELSPACHER S: 3474

BEYTOUT J: F079 
BEZUNARTEA J: 1764

BHUMIKA S: F002

BIANCHI PE: T004, T083

BIARNÉS M: F033

BIDAUT GARNIER M: $\mathbf{0 5 4}$

BIKBOVA G: 2651

BINQUET C: 1664, 4671

BIRKELDH U: F060

BISCHOFF M: 4735

BITOUN P: 3426

BLANCHON L: 4423

BLANDAMURA S: 4244

BLANN A: F046

BLASETTI F: F051, S056

BLASIAK J: T072, 1765

BLATTEAU JE: T009

BLUEML C: 2652

BLUMEN-OHANA E: F096

BOCHNIA M: F013

BODAGHI B: F083, T053, T054, T095, 1745, 3446, 3443 BOEY PY: T047

BOGATYREVA E: T012

BOGDALI A: S102, S103

BOGNER B: 3431

BOLLAERTS I: F002, 4665

BONNABEL A: F071, 1731, 3661

BONNEL S: S020

BONNIN N: F079, S025, 4423

BONSHEK R: 3647

BORDERIE V: 4734

BOREL A: S025

BORGES A: S030, 2435

BORGES B: T058, T107, 2648

BORRELLI M: 2674

BORRI M: S100, 4641, 4642, 4643

BORRUAT F: T112, 4461, 4463, 3678, 4464

BORZENOK SA: 4233

BOSCHI A: 1653

BOSCIA F: F051

BOSTAN C: 3433

BOUAFIA K: 3426

BOUCHE-PILLON J: 4671

BOUDRY C: 4241

BOUHACINA BENMANSOUR A: T033

BOULAGNON C: 4675

BOULANOUAR A: F082, S111

BOULZE PANKERT M: 4635

BOURNIQUEL M: T087, T009

BOYA P: $\mathbf{2 4 2 2}$

BRACCO S: 4643

BRANCH MJ: 1721, 3251

BRANCO P: S030, 2435

BRASNU E: F083

BRAUN M: S001

BRAUTASET R: F030, F060, 1666

BRÄUTIGAM M: S074

BREIER M: 1761

BREMOND-GIGNAC D: S064, 2232, 2734, 3453, 4621, 4458, 4623

BRETILLON L: 3723, 1664, 2223, 3722

BRON AM: F071, T044, 1731, 3242, 4723, 1664, 2223, $3661,3722,4473,4671$

BROWN D: 4261
BRUGIER A: T009

BRUNEL N: 2623

BRUNETTE I: 3433, 4635

BRUYNS C: T056

BUCHER M: 3646

BUCOLO C: 3475

BURD H: 4651

BUSHUYEVA NN: T090

BUSIN M: 4732

BUSUTTIL A: 4432

BUZNYK O: 4634

BYHR E: 3454

CABEZON L: F031, F063

CAHUZAC A: 2215

CAINI M: S100, 4641

CALDIROLA P: 1733

CALIPPE B: 1754

CALLIADA F: T035

CÁMARA R: 1772

CAMBAZARD F: S057, S072, S114, 2432

CAMINAL J: S107

CAMMALLERI M: F005, F006

CANASTRO M: F105, 3665

CANDELARIA P: S029, S030, T058, 2433, 2434, 2435, 2648

CANOVAS-MARTINEZ I: S007

CARAMELLO C: F036, S033, F031, F038, F063, F088, S035, S107, T098, T099

CARAMELO F: 2443

CARAM-LELHAM N: 1733

CARBONARI G: 2251

CARDEN D: T010

CARLU C: 3672

CASAS P: F031, F036

CASINI G: F005

CASPERS L: T056

CASSAGNE M: 5025

CASSOUX N: 3245, 3742, 3443, 3645, 4445

CASTAGNA O: T009

CASTANHEIRA-DINIS A: 3674

CASTELO-BRANCO M: T103, 2445, 3671, 3676

CASU G: F051

CAUQUIL C: T077

CEGIELSKI M: F013

CEJKA C: $\mathrm{S} 038$

CEJKOVA J: S038

CELLINI M: T028

CENDRE R: S001

CHABANE H: 3274

CHACONN M: $\mathrm{S} 053$

CHAINE G: F053, S089, 4474

CHAMBARD JP: 3426

CHAMBERLAIN A: S082

CHAMPION E: F083, 3443

CHANG HR: $\mathbf{T 0 8 2}$

CHANG ML: F061

CHAO J: F076, F078, 3441

CHAPELLE P: 3426

CHAPRON T: T034, T039

CHAQUES-ALEPUZ V: F050

CHARFI H: F018

CHARRIERE K: S096

CHATZIRALLI I: F087, F095, F101, S095
CHAVEROT D: T043

CHEBIL A: F018

CHEMTOB S: F012

CHEN A: 4452

CHEN CX: F028

CHEN X: S045, S047

CHENTSOVA E: S104, 4248

CHENY T: S024, S094

CHERNINKOVA S: F022

CHERNOCK M: 3446

CHIAMBARETTA F: F066, F079, S025, 3272, 4423

CHIQUET C: 2214, 3613, 3615

CHIU CJ: F061

CHLEBDA E: F009

CHO B: T027, T040

CHODOSH J: 4436, 2644

CHOI J: F017

CHOI MINY: T065

CHUKRAYOV A: 4231

CHUN Y: F012

CHUNG SY: F017

CHYCZEWSKI L: T055, 2645

CIDECIYAN A: 3264

CIESLIK T: S049

CIMBALAS A: F054

CIMBOLINI N: T017

CINOTTI E: S057, S072, S114, 2432

CLARK WL: 4261

CLOCHE V: T085

COCA-PRADOS M: T075

COCHENER B: S096

COCHEREAU I: 4772

COHEN SY: F065, F066

COLCLOUGH T: T070

COLEST: 4435

COMAN C: T045

COMPTOUR A: 4423

CONART JB: 4674

CONCEIÇÃO G: 3476

CONSTABLE P: $\mathbf{3 2 6 1}$

CONWAY RM: S115, S116, 4446, 4447

COOK A: 3647

COPIN H: S064, 4458

COPPARD N: T110, T111, 2658

COPPIN J: T043

CORDEIRO M: 2622, 3721, 2445

CORREIA A: $\mathbf{2 4 4 3}$

COSCAS F: 2414, 2415

COSCAS G: 2411, 2413, 2414, 2415, 2416

COSTA L: T107, 3673

COULANGEON LM: S025

COUPLAND SE: 4243

CRAFT J: F016, T071

CRAIG JP: $\mathbf{4 4 5 2}$

CREUZOT-GARCHER C: F066, F071, 2615, 1664, 1731, 2223, 3661, 3722, 4473, 4671

CRISTOBAL J: F031, F038, F063, S107, T098, T106

CROUZET E: 4422

CRUZ N: F031, F063

C. SILVA AS: 3663

CTORI I: F109, 1665, 1663

CUNHA JP: T051, T107, 3674, 1735, 3673

CUNHA-VAZ J: 4532 
276 ALL AUTHOR INDEX

CURSIEFEN C: 3733

CUTHBERTSON F: 3414

CUTOLO C: T035

DABASIA P: 3422

DA CRUZ L: 2254

DAI Y: F032

DAIEN V: T115, 2654

DAIMON M: F114

DAL MONTE M: F005, F006

DAMATO B: 1622, 4243

DAMES C: 2642

D'AMICO RICCI G: F051

DAMJI KF: $\mathbf{2 7 2 4}$

DANIEL E: S025, 4423

DANIEL F: 4623

DANIEL S: 1664

DARUGAR A: 3443

DASCALU AM: T045

D'ATHIS P: 4473

DAVID G: S095

DAVIES JR: T069, 1767

DAVIES VJ: T069, 1767

DE BAERE E: T081, 4661, 4766

DE BATS F: 2211

DEBELLEMANIERE G: 1771, 1667

DEBICKA-KUMELA M: S102, S103

DECAUTIN D: 3645

DE DEYN PP: 4224

DE FARIA A: T009, T087

DEFOE D: S067, 4427

DE FRANCESCO S: S099, S100, 4642, 4641, 4643

DEGOUMOIS A: F096, T093, 2656

DE GROEF L: 3421

DE GROOT V: S108, 4224, 4223

DE HOZ R: S006, T108, 1673, 2245, 2424, 3675

DEKARIS I: $\mathbf{3 7 3 2}$

DE KEIZER RJW: $\mathbf{S 1 0 8}$

DEKEYSTER E: 3421, 4662

DE KLEIN JEMM: 4246

DE LAZZER A: 1731

DELBARRE M: T038, T034, T036, T039

DELBEKE P: T08

DELBOSC B: F011, 1667, 1771

DEL BUEY MA: S033, S035

DELCOURT C: F066

DE LEENEER K: T081

DELEMOTTE A: T073

DELGADO TIRADO S: T066

DELLAFIORE C: T035

DEL OLMO AGUADO S: Fo08

DELPORTE C: F110, 4748

DE LUCA M: S099

DELUCA MC: 4643

DENDALE R: 4445

DENION E: F099, T093, 2656

DENIS D: F021, T091

DENIS P: T044

DE OLIVEIRA MAIA JR O: F019, F084, F085

DE PLATER L: 3645

DERAKHSHAN A: S021

DE ROO A: 4663

DE SEZE J: 2262, 2264
DESJARDINS L: 2743, 4445

DESJARDINS M: F104

DESMANGLES PM: F070

DE SMIT E: T097

DESPREAUX R: F083

DESSERRE J: F069, S117

DEWISPELAERE R: T056

DE ZAEYTIJD J: T081

DICK A: F078, F076, 1641, 1646, 2253, 3244, 3441

DICKMAN MM: 4733

DIEBOLD MC: 4675

DIEP M: 3644

DIMIER-POISSON I: T059, 2646

DING J: 2675

DIRANI A: 2414

DIWO E: T053

DOAN S: 2676, 3271

DOBNER B: 2642

DOBROWOLSKI D: S017, S049, S073, 1634

DOLZ-MARCO R: F050

DOMNGANG NOCHE C: T061

DONALD J: 2441

DONATI G: 2412, 3614

DOOLEY I: $\mathbf{4 4 7 5}$

DORÓ Z: 1671

DOT C: F015, F039, F042

DOUGLAS RH: T069, 1767

DOUGLAS CAMERON I: 4635

DOUMA I: F015

DOURMAD P: F065

DRAGO F: 3475

DRAKOS E: F087

DRASLAR K: S078

DROLSUM L: S068, 4428

DUA A: 1721

DUA H: 2732, 3624, 3735, 3251, 3621, 4773

DUCASSE A: F103, 4675

DUHAMEL F: F012

DUMOLLARD JM: S039, S067, S072, S114, 1722, 1724 . 2432, 4427, 4775

DUNCOMBE A: S041, 4777

DUNPHY SE: S055

DUPUY C: T043

DUQUESROIX B: T080

DURIEUX P: T073

DUTA S: T045

DUTESCU M: S066, 4426

DUTESCU R: S050

DUTROW E: 3264

DZIEWISZEK W: F009, F013

EBELING M: 2272

ECKSTEIN A: T055, 2645

EDGAR D: 3422

EICHLER WOLFR: 3215

EIDET JR: S045, S047

EKSTROM M: T068

ELALFY M: 4773

EL CHEHAB H: F023, F015, T034, T036, T038, T039, 3232

ELDRED JA: 1773

ELENA P: T017

EL-HUSSEINY M: 3631

ELIAOU-MALRIEU C: 2654
ELLIOTT D: 4252

EL MATRI L: F018

EMPERADOR S: T114

EPPIG T: S092, S093

ERB C: T016

ERDIAKOV A: F077

ERIE J: $\mathbf{S 0 8 2}$

ERNOULD F: S043

ERRERA MH: T060

ESCRIBANO J: T075

ESTEBAN O: S018, T114, F031, F036, F038, F063, F088, S035, S107, T099

EYSTEINSSON T: F010

FACSKO A: F001, 3253

FADLALLAH A: 4772

FAGERHOLM P: S068, 4428

FAGJENBAUM M: T014

FAJNKUCHEN F: F047, F053, S089, 4474

FAKHOURY O: T091

FALLICO M: S063, 4457

FALLON T: F097

FARAJ LANA: 4773

FARDEAU C: F083, F091, 3443

FARIA M: F105, 3665

FARKAS J: 5058

FAUCHER C: 3672

FAUQUERT JL: 3273

FAVENNEC L: 4421

FAZILATI F: F029

FEIJAO J: S029, 2434

FEL A: T095

FELSZEGHY S: S010

FELTGEN N: 4263

FENOLLAND JR: S020, T034, T036, T039

FERAILLE L: T017

FERETIS E: S119, 4448

FERNÁNDEZ-ROBREDO P: 1764

FERNANDEZ-VEGA L: T075

FERREIRA C: 2445

FERREIRA J: T107, 3673, 3674

FERREIRA S: T103,3676, 3671

FERRERO A: 3661

FERRINGTON D: 2272

FERRIS J: 3223

FERTE A: S090

FÉSÜS L: 1671

FIGUEIRA J: 3663

FINIS D: 2674

FINK W: F062, F067

FINZI A: T028

FITZGERALD O: 3445

FLANAGAN JG: 3712

FLECK O: 1664

FLORES M: 1667, 1771

FOETS B: 4663

FONTAINE M: T086

FOREST F: S039, S013, S039, S067, S072, S114, 4775

$1722,1724,1725,2432,4427,4775$

FORGET K: 3433

FORNACIARI I: F005, F006

FORSTER V: 2623

FOTI R: S063, 4457

FOUCART V: T056 


\section{ALL AUTHOR INDEX}

FRANCKE M: S026, S027, S028

FRANCOIS A: 4421

FRIGERI IA: F006

FRIOT M: F011

FRIZZIERO L: 4244, 4442

FROGER N: T095, 2623

FROUNTZOU E: 3614

FROUSSART-MAILLE F: S020, S024, S036, S085, S094

FRUTOS-BARAJA JM: $\$ 109$

FRUTTIGER M: 2244, 2755

FSADNI M: 1733

FUCHS-BUDER T: S090

FUCHSIAGER-MAYRL G: F004

FUCHSLUGER T: 3432, 4731, 4631

FUKUI M: S044

GABISON E: T064, 4772

GACEK M: T076

GAGLIANO C: $\mathbf{S 0 6 3 , 4 4 5 7}$

GAGYI-PALFFY Z: 3474

GAIN P: S013, S031, S039, S040, S042, S067, S069, S070, S072, S114, 1722, 1724, 1725, 2432, 2436, 4422, $4427,4771,4775,4776,4778$

GAJECKA M: T078, 1762, 1766

GALATOIRE O: S118, 4449

GALETTO R: T064

GALGAUSKAS S: S003, S004

GALIMBERTI D: S100, 4641

GALINDO-ROMERO C: 2243, 2424

GALLAR J: S054, 4632

GALLEGO B: S006, 1673, 2245, 2424

GALLEGO-PINAZO R: F050

GALLENMÜLLER C: T111, 2658

GALLICE M: 2214, 3613

GALLUZZI P: S099, S100, 4641, 4643

GAMATIKOV B: T092, 2655

GAN N: T046

GARCIA M: T075

GARCIA-ALVAREZ C: S101, S109, 4645

GARCIA GARCIA L: $\mathbf{1 7 6 4}$

GARCIA-LAYANA A: 1764

GARCIA MARTIN ES: T108, 3675

GARHOFER G: F004, S052, T023, 2222

GARNIER F: T062, 2647

GARVEY LH: S062, 4456

GARWAY-HEATH D: 4721, 3422

GASCARD L: 3661

GASTAUD P: F094

GAUCHER D: T057, T086

GAUTHIER AS: F011

GAUTIER E: 1751

GAVET Y: S031, S040, S069, 2436, 4776

GAVRILOVA S: F077

GEAMANU A: T045

GEBOES K: 4741

GEERAERTS E: 4662

GEERLING G: 2674

GEISER M: 3615, 3613, 3614

GELFI C: 4644

GENSLER G: F061

GENTIEN D: 3645

GEORGIADIS N: S081

GEORGIADIS O: T037

GEORGIADOU I: S081
GEORGIEV R: F022

GEORGOPOULOS V: 173

GEROMETTA M: F056

GERSTNER B: 2652

GHOUBAY D: 4734

GICQUEL J: 2231, 2631, 2731

GIL P: T108, 3675

GILLEBERT C: T094, 2657

GINIS HS: S097, 1777

GIOCANTI-AUREGAN A: F065, F047, F053, S089, 4474

GIRAUD JM: T009, T038, T087, 3232

GIZZI C: T028

GLACET-BERNARD A: 1712

GLEBAUSKIENE B: T102

GODARD G: 3774

GOHLER C: S028, S026, S027

GOLOVIN A: 4232

GOMES N: 3663

GONI F: 4533

GONZALEZC: F064, F072, F073

GONZALEZ BUENDIA L: T074, T066, 1768

GONZÁLEZ-GONZÁLEZ O: S054

GONZALEZ-IGLESIAS H: T075

GONZALEZ SARMIENTO R: T066, T074, 1768

GOTO Y: T020

GOVONI S: S008, 3256, 3475

GOWEIDA M: 4774

GOYER B: 4635

GRABNER G: 3431

GRADINARU S: T045

GRAEFF E: S110, S121, 4648, 3648

GRALLERT H: 1761

GRAVANIS A: 1662

GRAW J: 1761, 3253

GREENWOOD J: 3214

GREINER J: 2673

GRESTI G: 3654

GRIESHABER M: S110, 4648

GRIFFITH M: 4634

GRILL E: 1761

GRILLO-ANTONELLI S: T017

GRISHINA E: 4646

GRIVET D: S057, S072, S114, 2432

GROSMAN-DZIEWISZEK P: F013

GRUENERT A: 3731

GRUNEWALD F: T030

GRUS F: 2423

GRZYBOWSKI A: T055, 3413, 3775, 4752, 4754, 2645

GUAGLIANO R: T083

GUALINO V: $\mathbf{4 4 1 2}$

GUDMUNDSSON J: T067

GUEDIRA TARIGHT G: S064, 4458

GUERRERO P: S069

GUEUDRY J: S041, T053, 4421, 4771, 4777

GUIGOU S: F106, 4472

GUILHEM M: S070

GUILLEMOT C: S072

GUILLOIS B: T093, 2656

GUILLONNEAU X: 1754

GÜMBEL H: 3233

GUMBELEVICIUS A: F089

GUTHOFF R: 3434
GUTHOFF RF: 4653

GUTMANN J: 4631

GUYADER V: F096

GUYTON D: T092, 2655

GUZIEWICZ K: 3264

HADIISTILIANOU T: S100, S099, 4641, 4643, 4642, 4644

HADOUX X: T003

HAGIWARA M: F007

HAJJAR C: F106, 4472

HAJJI Z: F082, S111

HALFELD FURTADO DE MENDONCA R: F019, F084, F085

HALKJÆR LB: S062, 4456

HAMAM R: 3442

HAMEL C: 2761, 3263

HAMMER GP: 1772

HAMMER M: 3718

HAN KE: $\mathbf{S 0 1 9}$

HANSEN KS: S062, 4456

HAOUAS M: S072, S057, 2432, 4422

HAOUCHINE B: 3426

HARDARSON S: $\mathbf{3 7 1 7}$

HARDCASTLE AJ: T070

HARDER B: F108, 4672

HARDY P: F012

HARJU M: T019

HAROON M: 3445

HART-HOLDEN N: T070

HASEGAWA N: F058

HASHEMI H: S021, T001

HA THI B: $\mathbf{1 7 2 4}$

HAUSWIRTH W: 3264

HAWLINA M: S078, 3253

HAYAJNEH J: 2674

HAYNES R: 3222

HE M: 3425

HE Z: S067, S013, S031, S039, S042, S070, 4427, 1722,

1724, 1725, 2436, 4422, 4771, 4775, 4778

HEEGAARD S: S015, S016, S062, 4647, 1727, 1728 , $4245,4441,4456$

HEGYI P: F001

HEIER M: 1761

HEIMANN H: 4243

HEISSIGEROVA J: F093

HEITMAR R: F046, 3716

HEITZ A: T088

HEITZ P: T057

HEITZ R: 3773

HELISALMI S: T072, 1765

HERAVIAN J: S021, T001, T007

HERBORT C: 1645, 1744, 3651

HERERRA L: S035

HERNÁNDEZ M: 1764

HEROLD J: 4432

HERON E: T054

HERRERA L: S107, T099, F031, F036, F038, F063, F088, S033, T098

HERRERO-MARTIN MD: T114

HEUSSEN FM: 4243

HEVE D: 2654

HILDEBRANDT L: 3444

HILTUNEN M: T072, 1765 
278 ALL AUTHOR INDEX

HIROTA Y: T020

HJORTDAL J: S068, 4428

HO H: 3425

HÖCK A: 1772

HODGE C: S023

HODGE D: S082

HOFMANN F: F108, 4672

HØJGAARD-OLSEN K: S016, 1728

HOLAN V: S038

HOLDER GE: 2664, 3464

HOLLANDER DA: 4454

HOLLANDERS K: 1661, 4664

HOLLE R: 1761

HOLMES JM: 2454

HOLOPAINEN J: S061, S068, 4428, 4455

HOMMER A: T023

HONG S: T021

HOOGHE S: T081

HOPKINSON A: S055, 1721, 2733, 3251

HOR G: S040, 4776

HOSOYA T: F007

HOSSEINI A: T022

HOUTMAN AC: $\mathbf{2 4 5 3}$

HOW A: 3425

HOWARD S: S005

HRARAT L: F091

HSAIRI A: F018

HTOON HM: T047

HU S: 1754

HUBAULT B: F103

HUBER M: 4735

HUBERT I: 4674

HUDSON C: 3712

HUERVA V: S075, S088, S098, T098

HUMBERT C: 4421

HUNTJENS B: F109, 1663, 1665

HUSTER D: S026

HVALA A: S078

HWANG HS: S019

HYSI P: 2462

HYTTI M: 1675

HYTTINEN J: S012, 3255

HYUNKYUNG KIM: T096

IAKYMENKO S: 4634

IANCU R: T045

IBANEZ J: S033, T106

IDOATE A: S035

IKUNO Y: 3472

INGLAVE G: 4636

INIGO PORTUGUÉS A: S053, $\mathbf{5 0 5 4}$

IONICA V: T011

IRION L: 3647

IRSCH K: T092, 2655

ISAICO R: F071, 4473

ISELI HP: S026, S027, 2715

ISLAM M: 4634

IVANOVA O: 105

IVKOVIC I: T095, 2623

IWABE S: 3264

IYER G: 4433

IZSVÁK Z: 1764

IZUMI S: S058
J-A SAHEL: 3764

JACOBS D: 2473

JACOBSON S: 3264

JACOMET PV: S118, 4449

JAGER M: 3245

JAKOBSSON G: 3454

JAKUBOWSKA B: S102, S103, 3641

JANG HJ: T082

JANISZEWSKA D: S073, 1631

JANSONIUS NM: F034, 3423, 3424

JANSSENS MSA: 3424

JANUNTS E: 3631

JANY B: T084

JASIELSKA M: S074

JEFFS N: 3214

JENSEN PS: F044

JENTSCH S: 3718

JEPPESEN H: S015, 1727

JIMENEZ DEL RIO B: T105, T106

JIMENEZ-LOPEZ M: S007, 2243

JIN H: F100

JOS: F035

JOHANSEN JD: S062, 4456

JOHNEN S: 1764

JOHNSON K: T055, 2645

JONAS J: F028, F032, F108, 4724, 4747, 3474, 4672

JONQUA F: T084, 4623

JONVAL L: 4473

JOO CHOUN: T065

JØRGENSEN A: 4441

JOUROT P: 3426

JOUSSEN A: 3435

JOUSSEN M: S074

JULIAN HO: S016, S015, S062, 1728, 1727, 4456

JULIENNE R: S057, S114, S031, 4422, 2432, 2436

JUMELLE C: S013, 1725

JUNG KI: T048

JUNG S: $\mathbf{S 1 3}$

JUNG Y: T026

JUNGHANS B: 3644

JUNOY MONTOLIO FG: 3424

KAARNIRANTA K: S010, F052, S008, S011, S012, S022, T072, 2531, 1675, 1676, 1765, 3254, 3255, 3256

KABANAROU S: F020, F080, F092, S119, 4212, 4448

KADZIAUSKIENE A: S004

KAERCHER T: S059

KAESER PF: 4462, 4464

KAGMENI G: T06

KAHLERT CHR: F108, 4672

KAHLOUN R: 1743, 3655

KALIRAI H: 3754, 4243

KAM WR: 2675

KAMENAROVA K: F022

KAMPMANN S: 2652

KANELLAS D: F080

KANEVA R: F022

KANG HB: T008

KANG JW: T040

KANG S: F057

KAPOULA Z: 4623

KAPSALA Z: F111, F113, 4745, 4746

KARAGIANNIS D: F101, S095

KARDON R: 2263
KARJALAINEN R: S012, 3255

KARL A: S026, S027, S028

KAROLAK JA: T078, 1766

KARTTUNEN T: F052

KARVELA I: S119, 4448

KASER-EICHBERGER A: 3431

KATAMISH T: 4773

KATO A: F058

KATO T: F114

KATONA M: F001

KAUPPINEN A: S009, 2271, 1675, 3257

KAWASAKI A: 1654, 3463

KAWASAKI R: F114

KAWASAKI Y: F114

KAYA M: F043

KAYA S: S052

KAYAMA T: F114

KELLY JMF: T010

KEMAYOU D: T061

KESTELYN P: T081, 3633, 3772

KHABAZKHOOB M: S021, T001

KHAIRALLAH M: 1743, 3655

KHAN A: S022, S051, 3252

KIEKENS S: 4223

KIEL JW: 3431

KIILGAARD J: 4245, 4441

KILIC E: 4246

KIM CY: T021, T029

KIM HJ: F017

KIM J: T100

KIM IS: F048, T005

KIM MS: F048, T113

KIM SY: S076

KIM YT: T005

KINASZ R: T018

KING NJC: 2641

KINNUNEN K: F052

KIRAT O: 3252

KISHIMOTO T: S112

KIVELÄ T: T019, 1621, 1623, 2742, 3623, 3621, 4242, 4443

KLARSKOV ANDERSEN M: 4245

KLIMOVA A: F093

KLONOWSKA A: S106, 4247

KLOPSTOCK T: T110, T111, 2658

KLYSIK A: S060, 1774

KNAAPI L: 2444

KNOP E: 2471, 2671

KNOP N: 2671, 2471

KO BY: $\mathbf{S 0 7 6}$

KOCH C: S026, S027, S028

KOCIOK N: 5074

KODJIKIAN L: 2614, 4473

KOEHL A: 1667

KOEHRER P: F071, 1664, 1731, 4473, 4671

KOEV K: F022, F081

KOLKO M: 1674

KOMÁROMY A: 3264

KÖNIG C: 2674

KOOPMANS AE: 4246

KORBER N: S026, S027, S028

KORHONEN E: S009, 3257, 1675

KOROBELNIK J: F098, F066, 2532, 3472, 3533, 4673 4471 
KORT F: F018

KORZYCKA D: 1774

KOSKAS P: 4772

KOSKELA A: S012,3255

KOTAPATI RAGHUPATHY R: F016

KOTECHA A: 2721

KOURENTIS CHR: 4211

KOUROUPAKI A: F020, S119, 4448

KOUSAL B: T070

KOVALEVSKAYA M: F062, F067, T012

KOVARSKI C: $\mathbf{3 6 7 2}$

KOVSHUN E: 4232

KOWAL J: S102, S103

KRASTEL H: F108, 3474, 4672

KREFFT K: 3431

KREUGER M: F056

KRIAUCIUNIENE L: F089, T102

KRISTÓF E: 1671

KROMUS M: T023

KRONSCHLÄGER M: S079, S080, 1775

KU HC: F025

KUBICKA M: T078, 1762, 1766

KUCERA T: F093

KUCHARSKA AZ: F009

KUDRYAVTSEVA O: $\mathbf{4 7 4 4}$

KUHN D: 4736

KULINSKA K: T078, 1766

KUMAR A: 3647

KUOLIENE K: F024, F054

KURKINEN M: S068, 4428

KURUMTHOTTICAL M: F074

KURYSHEVA N: 2621

KUZNIAR A: F108, 4672

KVANTA A: S002, T067, T068

KWON HJ: F017

LABALETTE P: F069, S043, S117, T073

LABEILLE B: S057, S072, S114, 2432

LABETOULLE M: T063, T064, T077, 2233

LABIOD D: 3645

LABREUCHE J: T073

LAKE S: S081

LALLOUM F: 2413, 2415

LAM W: $\mathbf{4 7 5 5}$

LAMARD M: S096

LANDI G: 4644

LANDRÉ C: F094

LANGE C: 3214

LANGENBUCHER A: S093, S092, 3631, 4735

LANTERI S: T004

LAPLACE O: F096

LARSEN L: 4264

LASER-JUNGA H: 1772

LASSEAUX E: 2663

LATTANZIO F: T022

LAURENS C: F096

LAURICHESSE H: F079

LAURINAVICIUTE G: 5003

LAUWERS N: F014, $\mathbf{S 1 0 8}$

LAVA M: T035

LAVALETTE S: 1754

LAVIERS H: 4475

LAVILLA L: F038, S098
LAWRENSON J: 3415, 3422

LAZREG S: S037, 4451

LEABACK R: 4264

LEATHERBARROW B: 3647

LE DÛ B: F096

LEE EK: F025

LEE HK: S019

LEE HS: T065

LEE J: T100, T101, F102

LEE JH: F017

LEE JS: 2644, 2644

LEE JY: T027

LEE KH: F100

LEE M: T097

LEE MK: F025

LEE N: S046, T041

LEE S: F037, F049, T089, F035

LEE SY: T029, T021

LEE T: T024, T025

LEE Y: T032, T021, T029

LEFEVRE S: 4771

LE GOFF L: 4421

LEHESJOKI AE: 4242

LE HOANG P: F083, F091, T053, T054, T095, 3443

LEHTO VP: S009, 3257

LEINONEN M: 2444

LEITE-MOREIRA AF: 3476

LEITGEB R: 1613

LEMAITRE S: S118, 3645, 4449

LEMEE G: T059, 2646

LE MER Y: 4413

LEMIJ H: T015

LEMMENS K: F002, 4665

LEONCINI R: 4644

LEPAPE A: 2654

LEROY B: 3462, 4674

LEROY BP: T081, 2662, 2762, 3262, 3762, 4661, 4766

LESAGE F: F104

LESENS O: F079

LEVY C: 4445

LEVY O: F053

LEWIN-KOWALIK J: S011, 3254

LI J: 2441

LI T: F061

LIBERT S: F110, 4748

LIM BA: T046

LIM HS: F100

LIM SJ: T079

LIN J: S084, S083

LINDEGAARD J: S015, S016, 1727, 1728

LINDH S: 4242

LING Z: F032

LINKOHR B: 1761

LIPSKI D: T056

LISBOA M: T051, 1735

LISCH W: 3634

LISKOVA P: T070, 1633, 2463, 2763

LIU CSC: 4432, 4435, 4636

LIU L: $\mathbf{3 4 1 4}$

LIU X: 4635

LIU Y: 2675

LIUTKEVICIENE R: F089, T102
LLOBET L: T114

LOB F: T111, 2658

LOBO C: 3663

LOEWENSTEIN A: 1715, 2612

LÖFGREN S: 4622

LOMBARDO S: $\mathbf{S 0 8 7}$

LÓPEZ DEL VAL J: T106

LOPEZ-GALLARDO E: T114

LÓPEZ GÁLVEZ MI: F112, 4477

LOPEZ-LARA F: S101, S109, 4645

LOPIC N: S078

LORENZ B: 2652

LORENZ K: 4262

LOUKOVAARA S: 4476

LOURO C: 3674

LUBINSKI W: T018

LUHMANN U: 3214

LUK S: F074

LUMBROSO LE ROUIC L: 4445

LUMI X: 3253

LUX AL: T093, 2656

LUZ LEITÃO GUERRA R: F019

LYONS T: 2752

LYTVYNCHUK L: 1672

MA Y: T068

MACAULAY D: 3441

MACHALINSKA A: 3213

MACHIN C: 2446

MACKENSEN F: $\mathbf{3 4 4 4}$

MADIGAN M: S115, S023, S116, 3644, 4447, 2641, 4446

MADURO V: S029, S030, T051, T058, 1735, 2433, 2434, 2435,2648

MAEDA A: 1753

MAFFIA A: T083

MAGAZZINI S: F065

MAGOURITSAS G: T037, 1734

MAGRO L: S043

MAIER AK: S074

MAIER M: 2714

MAIER RF: 2652

MAJSTEREK I: T076

MAKOWIECKA K: 2446

MALECAZE F: S025

MALYSKO K: $\mathbf{F 0 5 4}$

MALYUGIN B: 4231, 4235

MAMMADZADA P: $\mathbf{T 0 6 7}$

MAMOULAKIS D: F113, 4746

MAMOURI R: F018

MAMUNUR R: 4443

MANASSEH N: 2213

MANCZAK H: 2474

MANGIORIS G: F059, F003

MANSOUR A: 3442

MANZANAS LEAL L: F112, 4477

MARCHESI N: S008, 3256

MARECHAL M: T039, T034, T036

MARIANI P: 3645

MARIE C: 1764

MARILL AF: T034, T036, T039

MARIOTTI C: $\mathbf{2 2 5 2}$

MARKIEWICZ A: S102, S103, 3641

MARKIEWICZ L: T076 
MARKKINEN S: 4242

MARKOU C: S081

MARQUES J: 2445

MARQUES-NEVES C: F105, 3665

MARQUINA I: F030, 1666

MARRAY IJ: T010

MARSOVSZKY L: S032, 2437

MARTIANO DH: $\mathbf{S 0 9 6}$

MARTIN AL: F003

MARTINEZ M: F031, F038, F063, F088, S033, S035, S107, T099

MARTINEZ-ROMERO I: T114

MARTINEZ VELEZ M: S088, T098

MARTIN-NAVARRO A: T114

MASCHI C: $\mathbf{3 7 4 4}$

MASMALI A: S051

MASSIN P: 2611, 4471, 4473

MATEO OROBIA AJ: F036, S033

MATEUS C: T103, 3671, 3676

MATHIEU B: S041, 4777

MATONTI F: F106, F021, T091, 4472, 2623

MATTER MA: F070

MATYSIAK A: 1762

MAUGET-FAYSSE M: 2211, 2213, 2215

MAURAGE CA: S117

MAYA R: 5086

MCALPINE S: S115, 4447

MCCULLOCH D: F016, T071

MCINTOSH OD: 3251

MCKECHNIE C: 4475

MCNAUGHT A: 3224, 3714

MEANA A: 053

MEENS W: 3424

MEHTA J: 4434

MEIER T: T110, T111, 2658

MEIER-GIBBONS F: T052, 1737

MEILLAT M: 1667

MEILLON C: F071

MEIRELES A: 3663

MEKKI MB: 2475

MELY R: 2472

MENDRINOS E: F003, F059

MENEY J: T077

MENICACCI C: S034

MENICACCI F: S034, S100, 4641, 4642, 4644

MENNÉ T: S062, 4456

MENSAH E: F055

MENTEK M: 3615

MERAYO-LLOVES J: S053, S054, S071

MERITE PY: F106, 4472

MERKOUDIS N: 4264

MESSENGER W: 3444

MESSMER EM: T080

METZ G: T110, T111, 2658

METZIG C: 4471

METZNER S: 2642

MEUNIER I: 3263

MEYER F: F106, 4472

MEYER P: S121, S110, 3648, 4648

MICARD E: 5001

MICHAEL R: 4251, 4253, 4655

MICHAU S: T115

MICHELI L: S100, 4644, 4641
MIDENA E: 4442, 4244

MIDENA G: 4442

MILANO G: T035

MILAZZO S: T084

MILLEC: T084

MILYUTKINA S: F062, F067

MINAKAKIS P: F080, F092

MINCHEVA Z: T077

MIOTTI H: 3672

MIRACHTSIS T: S081

MIRONOVA I: 4444

MISIUK HOJLO M: T018

MITROPOULOS P: F087, F095, F101, S095

MITRY D: F074

MIZOGUCHI T: 3425

MOCKO L: T018

MOGAVERO V: T004

MOHAMMAD G: 4741

MOHAMMADIAN M: F029

MOHAND-SAID S: 2446, 3764

MOHNEY B: 2452

MOKHTARI MA: 2475

MOLEIRO A: 3476

MOLNOS S: 1761

MOMBAERTS I: 3643

MONÉS J: F033

MONNEYRON N: S025

MONTANARI S: T084

MONTARD M: 1771

MONTEIRO-GRILLO M: F105, 3665

MONTENEGRO G: 4253

MONTEZUMA S: 2272

MONTOYA J: T114

MOONS L: F002, 3421, 4662, 4665

MORGADO M: 2443

MORGAN I: 2712

MORGAN JE: 2722

MORICE-PICARD F: 2663

MORIGGI M: 4644

MORISSE S: T059, 2646

MORIYUKI K: T020

MORIZE A: 4623

MOROOKA S: F007

MOROZZ: 4232

MORREALE BUBELLLA D: F027, T109

MORREALE BUBELLA R: F027, T109

MOSCHANDREAS J: F111, 4745

MOSCHONAS K: F020, T037, 1734

MOSCHOU V: 5081

MOSS S: 3214

MOSSER G: 4734

MOUALLEM A: 3443

MOUINGA A: T034, T036, T039

MOULARD A: S039, 4775

MOULIN A: 3646

MOUNIER A: F015

MOURIAUX F: T093, 4241, 2656

MOUSAVI M: S021

MOYAL L: S085

MUKHERJEE S: 2644

MULAK M: T018

MUMCUOGLU T: T031, T049
MUNIER F: 2746

MUNK SJ: S062, 4456

MUNOZ M: S101, 4645

MUNOZ-ERAZO L: S116, 2641, 4446

MUONA M: 4242

MURAINE M: S041, 4771, 4421, 4777

MURPHY C: 3445, 4453

MYSLIWIEC J: T055, 2645

NADAL-NICOLAS FM: S007

NADIM N: $\mathbf{3 4 1 2}$

NAIGEON N: S013, 1725

NAKAMACHI T: S058

NAKHAEE MA: T001

NAKHIANPOUR N: F029

NANA F: $\mathbf{S 1 2 0}$

NANGOUM-FOSSO T: S013, 1725

NAPORA K: S052

NARBERHAUS B: T114

NAWAZ M: 4741

NEFZAOUI C: S042, 4778

NEITZ J: 4311

NEMATI F: 3645

NÉMETH J: S032, 2437

NERI P: 1643, 2251, 3654

NETO E: F105, 3665

NEVALAINEN J: F034

NEVEU MM: 2664

NG J: T025

NIAVI S: 1662

NICCHIA GP: F006

NICOLAI M: 2252

NICOLAS M: 3646

NIEPON ML: F023

NIETO-LOPEZ L: S007

NÍ GABHANN J: 4453

NILSSON M: F026, F030, F060, 1666

NONGPIUR M: 3425

NORDMANN JP: F096

NOROUZIRAD R: S021

NORVYDAITE D: $\mathbf{S 0 0 3}$

NOUVEL-JAILLARD C: F023

NOWAK DM: T078, 1766

NOWINSKA A: S017, S049, S073, 1632

NOZAKI M: F058

NUIJTS R: 4733

NÚÑEZ ÄLVAREZ C: S071, F008

NUVOLI E: S056

NYSTRÖM A: 3454

O'BRIEN C: $\mathbf{2 7 7 4}$

OCHIAI H: S077

O'GRADY A: T070

OGURA Y: F058

OHBAYASHI M: F058

OHLENDORF A: 2713

OIZUMI T: F114

OKALIBE X: F076

OKUNO Y: F007

OLMO-AGUADO S: S071

OMRI S: F012

ONISHENKO NA: 4233

ORBAN GA: T094, 2657

ORGÜL S: T006, T013 


\section{ALL AUTHOR INDEX}

ORIVE G: S053

O'ROURKE M: 3445

ORSSAUD C: 4623

ORSSAUD O: 3672

ORTYL-MARKIEWICZ R: T018

OSBORNE NN: F008, S071, 2241, 3724

OSHITARI T: S1 12, 2651

OSMAN I: $\mathbf{2 4 3 1}$

OSTADIMOGHADDAM H: F029, T007, S021, T001

OTA S: $\$ 112$

OYE B: F076

OZAKI M: 3425

OZGE G: T031, T049

OZGONUL C: T031

OZONE D: F058

PÄÄKKÖNEN A: 1676

PAANANEN RO: S061, 4455

PADGETT E: F063

PAETZOLD J: F034

PAIK JISUN: S113

PALACIN M: T105

PALLIKARIS A: F111, 4745

PALLOT C: $\mathbf{4 4 7 3}$

PÅLSSON S: 3454

PANFIL C: S050, S066, 4426

PAPADAKI H: F113, 4746

PAPANDREOU I: F055, F097

PAPAPANOS P: F020

PAPAVASILEIOU E: 4475

PAQUES M: 3241, 2215

PARIDAENS D: 4246

PARIKAKIS E: F101, S095, F087, F095

PARK BJ: T040

PARK C: T026, T041

PARK H: T026

PARK J: F035, F049, T089

PARK SH: T008, T113

PARK YH: F102

PARRAT E: F106, 4472

PARROZZANI R: 4244, 4442

PARRY NRA: T010

PARSA C: $\mathbf{2 6 5 3}$

PASCALE A: S008, 3256, 3475

PASCHALIDIS T: F092

PASDELOUP D: T064

PASHTAEV A: 4235

PASHTAYEV NP: 4234

PASTOR IC: T066, T074, 1768

PASTOR IDOATE S: T066, T074, 1768

PASU S: F074

PASYECHNIKOVA N: 4634

PATEL SR: 3712

PATERNO JJ: T072, 1765

PATNAIK S: T071

PAVESIO C: 3446

PAWLOWSKA M: F090

PAWLOWSKI P: F090, T055, 2645

PEAN V: 3426

PEARCE I: 3532, 4262

PEDERSEN L: F043

PENAUD B: F053

PENG XY: F028
PENK A: S026

PENNOS A: S097, 1777

PEOC'H M: S013, S039, S067, S072, S114, 1722, 1724, $1725,2432,4427,4775$

PEPONIS V: F087, F095, F101, S095

PERARD L: T054

PEREIRA AC: T103, 3671, 3676

PEREIRA-SILVA P: 3476

PERERA S: 3425

PÉREZ D: S033, T099, T105, T106

PEREZ GARCIA D: F088, S035

PEREZ NAVARRO I: F088, S035, F031, F036, F063, S107, T098, T099

PERLAT A: T054

PERNEY P: T115

PERPETUA C: F105, 3665

PERRACHE C: S013, 1722, 1724, 1725

PERRINI P: 4244

PERRON MC: 4635

PERROT JL: S057, S072, S114, 2432

PETERS A: 1761

PETERS S: 3718

PETRAKI D: T110

PETROPOULOS I: F003, F070, F059, 1713

PETROVIC A: 3642

PETROVSKI G: 3253, 3751, 1671, 3252

PICAUD S: T095, 2623

PICCININI P: T004

PIELEN A: 4263

PIERI E: T091

PIETRUCHA-DUTCZAK M: S011, 3254

PIIPPO N: 1675

PILOTTO E: 4442

PILSON Q: 4453

PINAZO-DURAN MD: F050

PINHEIRO R: S050, S066, 4426

PINILLA I: F036, S033, S035, T099

PINILLA-CORTÉ L: 4253

PINNA A: S056, F051

PINOLI JC: S040, S069, S070, 4776

PINTO LA: 4676

PINTO M: T058, 2648

PINTO-BONILLA I: 2832

PIORECKI N: F009

PIPERNO S: 3645

PIRANI V: 1643, 2251, 3654

PIRVULESCU R: T045

PISANI F: F006

PISELLA P: T059, 2632, 2646

PISELLI S: S042, S067, 1722, 1724, 4427, 4778

PLANCHER C: 4445

PLEYER U: S074, 1644, 1742, 3621, 2642

PLONKA P: S106, 4247

POCIEJ W: S103

PODBORACZYNSKA-JODKO K: T018

POJDA-WILCZEK D: T002, 2442

POLAKOWSKI P: T078, 1766

POLGAR T: 1652

POMMIER S: F040, F106, 4472

POMORSKA M: T018

PONTIKOGLOU C: F113, 4746

PONZIN D: 3734

POPA CHERECHEANU A: T045
POPOVIC Z: F026

PORTALIER S: 3672

PORTALIOU DM: S119, T037, 4448, 1734

POSADINU MA: S056

POURJAVAN S: 4222

POURNARAS C: F003, F059, 1711, 2613, 3614, 3711 4411, 2412, 3611

POURNARAS JA: 4214, 4414, 4415

POZDEYEVA N: 4234

PRAUSE J: S015, 1727, 4441, 4647

PRÉVOST G: T057

PRIMAVERA L: 1643

PROENCA H: F105, 3665

PRONGUÉ A: F011

PROST M: T018

PROST-MAGNIN O: F106, 4472

PROULX S: 3433, 4635

PRUSAKIEWICZ J: F056

PRZYBYLOWSKA-SYGUT K: T076

PUCHE N: 1714

PURSLOW C: S048, 2672, 2831

QIU K: F034

QU L: 4474

QUAH X: S115, 4447

QUENDERA B: T103, 2445, 3671, 3676

QUINONES M: S109

QURESHI F: 3647

RÆDER S: S045, S047

RAIMONDI M: T004

RAIVIO V: 4242

RAJAIYA J: 2644

RAKONCZAY Z: F001

RAMASAMY P: 3445

RAMBAUD C: S020, S024, S036, S094

RAMIREZ AI: S006, T108, 1673, 2245, 2424, 3675

RAMIREZ JM: S006, T108, 1673, 2245, 2424, 3675

RAMKE M: 2644

RAMM L: 3718

RAMOA R: 4645

RAMONT L: F103

RANDON T: S043

RANNOU K: S069, S031, S040, 2436, 4776

RANTAMÄKI AH: S061, 4455

RATTUNDE U: S027

RAZAKANDRAINIBE R: 4421

RECALDE S: 1764

REICHENBACH A: S026, S027, S028, 2242

REINA M: T051, 1735

REINISALO M: S012, 3255

REIS A: 2445

REITSAMER HA: 3431

REKAS M: T018

RELPH J: S005

REMEIJER L: 4733

REMOND A: T095

RENARD JP: F023, T034, T036, T038, T039, T044, 3232 RENDU I: F021

RESCH M: S032, 2437

RESZEC J: F090, T055, 2645

REVOL C: 3441

RHIM WI: F025

RHO YJ: F057

RIBEIRO L: 2445 
RICHET M: S043, S117

RIESTRA ANAC: S053

RIGAL-SASTOURNE JC: S020, S024, S036, S085, S094, 3234

RIGOLE H: T115

RIIKONEN J: S009, 3257

RIMENSBERGER PC: F003

RITZ M: T088

RIVA C: 3611, 3614

ROBERT PY: T062, 2647

ROCHA DE SOUSA A: $\mathbf{3 4 7 6}$

RODALLEC T: F096

RODRIGO E: T010

RODRIGUES M: 3645

RODRIGUEZ A: S086

RODRIGUEZ JR: 1764

RODRIGUEZ NA: S018

RODRIGUEZ-CALVO PP: T075

RODRIGUEZ HERNANDEZ I: T066, T074, 1768

ROEHRICH H: 2272

ROJAS B: S006, T108, 2245, 1673, 2424, 3675

ROJAS J: T066, T074, 1768

ROKICKI D: T018

ROMANENKO DV: T090

ROMANIUK W: T018

ROMANOWSKA DIXON B: S106, S102, S103, 3641, 4247

ROMAN ROMAN S: 3645

ROMBACH M: 4654

ROORDA A: 2311

ROSE FRAJ: S055

ROSE K: 1763

ROSÉN R: F026

ROSENBAUM JT: 3444

ROSENBERG R: T036, T034, T039

ROSSI P: S041, 4777

ROUFAS A: S023

ROULAND I: T042, T030, T033, T043, T044, T073

ROUSSEAU A: T064, T077

ROYER B: F011

RUBERTO G: T004

RUIZ HIDALGO I: 4666

RUIZ-PESINI E: T114

RUNGE C: 343

RUSSO A: F042, F015

RYABTSEVA ALLA: S091, 1776

S A: 1672

SAAB S: 2223

SAADOUN D: T053, T054

SAAKYAN S: S104, S105, 4248, 4444

SAHANNE S: 4476

SAHEL J: T095, T110, 2211, 2213, 2446, 2623

SAIB N: $\mathbf{S 0 2 0}$

SAID DG: 4773

SAITTA A: 2252

SAKER S: 4742

SALAZAR JJ: S006, T108, 1673, 2245, 2424, 3675

SALEH M: 1667, 177

SALEHI S: 4631

SALIK D: F110, 4667, 4748

SALINAS-NAVARRO M: S007, 2424, 3421

SALMINEN A: 1675

SAMUDRE S: T022
SANCHEZ A: T099

SANCHEZ MC: S075, S088

SANDEMAN S: 4636

SANTANDER-TRENTINI F: F050

SANTOS A: T051, 1735, 3673, 3674

SAORNIL ALVAREZM: S101, S109, 4645

SAPIN V: 4423

SARNA T: S106, 4247

SARNICOLA E: S034

SARNICOLA V: S034

SAROSSY M: T003, T097, 2441

SASTRE X: 3645,4445

SAUER A: T086, T088

SAUER L: 3718

SAUVAN L: T091

SAWAMURA H: T094, 2657

SCEMAMA TIMSIT C: 2213

SCHAEFFEL F: 2711

SCHALENBOURG A: 2745, 3642, 3646

SCHEIDEMANN-WESP U: 1772

SCHERMAN D: 1764

SCHIEFER J: F034

SCHIEFER U: F034

SCHLEICHER M: F061

SCHLICHTENBREDE F: 3474

SCHLICKEISER S: 2642

SCHLINGEMANN R: 3531

SCHMETTERER L: F004, S052, T023, 2224, 2624 2833, 3612, 4722

SCHMIDL D: S052, T023, 3713

SCHMIDT-ERFURTH U: F004

SCHRADER S: 2674

SCHRAGE N: S050, S066, 4426

SCHROEDL F: 3431

SCHULDT C: S027

SCHULMEISTER K: S080

SCHWARTZC: 1667

SCOTT ROBER: 3231

SECHE E: T059, 2646

SEDAGHAT M: F029

SEIDLER STANGOVA P: F093

SEITZ B: 3631, 4735

SEKFALI R: T009

SEKI T: $\mathbf{S 0 5 8}$

SELTON J: 4674

SEMOUN O: 2415, 2413, 2414

SENDON D: S036, S024, S094

SENGELOEV H: S015, 1727

SENNLAUB F: 1755, 2753, 3211, 1754

SEO SJ: T021, T029

SEONG GJ: T021

SERRANHO P: 2443

SEVE P: T054

SEVESTRE H: S064, 4458

SHAH P: $\mathbf{3 2 2 1}$

SHAHIDI AM: 3712

SHARIFI F: T007

SHARKAWI E: $\mathbf{3 7 4 5}$

SHIGEYASU C: S044.

SHIN J: F086, F102

SHIN MC: S019

SHIN SY: T008, T113

SHIODA S: S058
SHOAIE-NIA K: F050

SHU X: T071, F016

SHUICHI Y: S120

SIDDIQUEI M: 4741

SIDNEY LE: S055, 3251

SIJNAVE D: 4424, 4761

SIKORAV A: 2413, 2415

SILVA E: T103, 3671, 3676

SILVA N: S029, T107, 2434

SILVA R: 3471

SILVA-GOMES R: 3476

SIMANTIRAKI D: F113, 4746

SIMÃO S: 3663

SIMONUTTI M: T095

SIMONYI S: 4454

SINGER S: 1772

SINHA D: 2273

SIOULIS C: S081

SJÖDELL L: 3454

SJÖSTRAND J: F026

SKALICKA P: T070

SKARPHEDINSDOTTIR S: F010

SKOV L: S062, 4456

SKUP M: F076, F078, 3441

SKVARCIANY I: $S 003$

SLATKEVICIENE G: T102

SLIESORAITYTE I: 2446

SMEDOWSKI A: S011, S065, T050, S010, S017, S022 S049, S073, 1736, 3254, 4425

SMETS RME: F014

SOBRADO-CALVO P: S007, 2243

SÖDERBERG P: S079, S080, 1775

SOLANAS S: S018, S086

SOLDEVILA J: S088, S098

SONG W: F017

SORG R: F078

SOUBRANE G: 1614, 4216, 4531

SOUIED E: F066, 2413, 2414, 2415

SOUSA A: S030, T107, 2433, 2435

SOUSA H: S030, 2435

SOZANSKI T: F009

SPALLONE L: T083

SPALTON DI: 1773

SPEEG-SCHATZC: T086, T088

SPILEERS W: 3461, 4664, 4761

STACHON T: 4735

STACHS O: 4652

STAHL A: 3216

STALMANS I: 1661, 1732, 1733, 3473, 4424, 4676, 4763, 4764

STALMANS P: 4676

STAM L: 3424

STANA D: T045

STECH S: S003, S004

STECK J: 2652

STEFANSSON E: 3436, 3622, 3621

STEMPER B: 4261, 4263

STENMAN G: 4647

STEPHAN S: F047

STERN ME: 4454

STEVENS A: 4221

STEWART EA: F075, 4742

STIEGER K: 2652 


\section{ALL AUTHOR INDEX}

STITT A: $\mathbf{2 7 5 4}$

STOJANOVIC A: S045

STORSBERG J: 4633

STRANCAR J: S078

STRATOS E: F101, S095

STRELKAUSKAITE E: F024, S004

STROHMAIER C: 3431

STRUBLE C: F056, $\mathbf{5 0 0 5}$

STRUPAITE R: F024

SUFFEE N: S013, S039, 1722, 1725, 4775

SUGANO A: F114

SUGITANI M: T020

SUHLER E: 3444

SULLIVAN DA: 2675, 3311

SUN X: F032

SUNARIC MEGEVAND G: $\mathbf{3 2 2 5}$

SURGUCH V: $\mathbf{3 6 6 4}$

SUTTON G: S023

SVELTO M: F006

SVOZILKOVA P: F093

SWIRE M: 3214

SYLVESTRE JP: F104

SZAFLIK I: T076, T076, T078, 1762, 1766

SZALAI E: S010

SZATMARY G: 1652

SZELAG A: F009, F013

SZENTMARY N: 4735, 363

SZUMNY A: F009

SZUMNY D: F009, F013

SZYMANEK K: T076

TADAYONI R: F065

TAEYOON LA: T096

TAHIR HJ: T010

TAHIRI H: F012

TAKEI A: T068

TALEBI ZADEH N: S079, S080, 1775

TÄLL M: 4242

TAMASAUSKAS A: T102

TAN D: 4434

TAN X: 4434

TANG I: T003

TARFAOUI N: T059, 2646

TARIGHT N: S064, 4458

TARKKA I: 1676

TARNAWSKA D: S017, S049, S065, S073, T050, 1736, 4425

TASSI F: T028

TASSIGNON MI: 3451

TATSKOV R: S105

TAWK M: T057

TAYLOR A: F061

TAYYARI F: 3712

TEN TUSSCHER M: 2451

TERLUK M: 2272

TESTER A: F056

THEOCHARIS IP: 3662

THERIAULT M: 3433

THIEME C: $\mathbf{2 6 4 2}$

THORNE J: 3441

THORP TAS: 4432

THOUVENIN R: S094, S024

THULEAU A: 3645

THUMANN G: 176
THURAU S: 3446

THURET G: S013, S031, S039, S040, S042, S057, S067 S069, S070, S072, S114, 1722, 1724, 1725, 2432 2436, 4422, 4427, 4771, 4775, 4776, 4778

THURET JY: 1724

TIAN J: T024

TIEULIE N: T054

TIFFET T: S040, 4776

TILLEUL J: 2413

TINELLI C: S087, T035

TING M: F055

TISSIER P: T015

TODD JT: T094, 2657

TODOROVA MG: T006, T013

TOKARZ P: T072, 1765

TOLD R: F004

TOLSTRUP ANDERSEN M: 4245

TONAEVA H: 4233

TONG LMG: 4454

TONNELET R: S001

TOPOUZIS F: 222

TORNQVIST K: $\mathbf{3 4 5 5}$

TORO M: S063, 4457

TORRICELLI A: 2771

TORVINEN P: T019

TOSHIYUKI O: S120

TÓTH M: 1671

TÓTH-MOLNÁR E: F001

TOUBEAU D: S041, 4771, 4777

TOUITOU V: T095, 3443

TRAVESET A: S075

TRECHOT F: S001, S090, T085

TRIVINO A: S006, T108, 1673, 2245, 2424, 3675

TRONE M: S031, S042, S057, 2436, 4422, 4778

TROSAN P: S038

TROST A: 343

TRUFFER F: 3613, 3615

TRUSSART R: F104

TSAI A: T047

TSAKIRIS K: F020

TSIKA C: F113, 1662, 4746

TSILIMBARIS M: F113, F111, 4746, 1662, 4745

TSIOTRA V: F095, F101

TUGAL-TUTKUN I: 3653

TUIL E: T080

TUMAHAI P: 1667

TUNDIA N: F076, F078, 344

TUNIK S: S002

TÜRKSEVER C: T006, T013

TURNBULL PR: 4452

TURUNEN J: 4242

TUULONEN A: $\mathbf{2 7 2 3}$

TUUMINEN R: 4476

TZOURIO C: 1664, 4671

UTHEIM Ø: S045, S047

UTHEIM TP: S045, S047

UUSITALO H: 2773

UUSITUPA M: T072, 1765

UZZAN J: 2416

VAARWATER J: 4246

VADALA M: T109

VAIKOUSIS E: F080, F092

VALERO B: T009, T087, 3232
VALESOVA L: T070

VALGAEREN H: 4762

VALIENTE-SORIANO FJ: S006, 1673, 2243, 2424, 3421

VALLET H: T054

VALTER K: 3644

VAN BERGEN T: 1733, 4763, 1661, 1732, 4424

VAN CALSTER J: F041, 2643

VAN CAUWENBERGH C: T081

VAN DAM D: 4224

VAN DEN BERG T: 2633, 4254

VANDENBERGHE R: T094, 2657

VAN DEN BIGGELAAR FJHM: 4733

VAN DEN OORD J: 4663

VAN DER LELII G: 4733

VANDERMEULEN J: T104, 3677

VANDERVEEN D: $\mathbf{3 4 5 2}$

VAN DE VELDE S: 1732, 4764

VANDEWALLE E: 1661, 1732, 1733, 3473, 4424, 4676

VAN GINDERDEUREN R: 3643, 2643

VAN HOUCKE J: F002

VAN HOVE I: F002

VAN IJCKEN WFJ: 4246

VAN KEER K: 3473

VAN LOOVEREN J: $\mathbf{4 7 6 5}$

VAN MARION FW: 4733

VANNONI D: 4644

VAN ROOIJ J: 4733

VAN SCHIL K: 4766

VASSEUR V: 2211, 2213, 2215

VASSO M: 4644

VATANDOUST S: T007

VÁZQUEZ N: S053

VENGLOVECZ V: F001

VERDIN H: T081

VERÉB Z: 1671

VERTICCHIO VERCELLIN A: T035

VICENTE A: T058, T107, 3673, 2648, 3674

VIDAL-SANZ M: S006, S007, 2424, 1673, 2243, 342

VIE A: F039, F015

VIEIRA L: S030, T051, T107, S029, T058, 1735, 2435 $2433,2434,2648,3673$

VIENNET A: S025

VIGNAL R: T009, 3232

VILLAIN M: T115, 2654

VILLEGAS PEREZ MP: S006, 2243, 1673, 2424

VINCENT AL: 2461

VIREVIALLE C: F096

VIVAR-LLOPIS B: F050

VIZVARI E: F001

VOßMERBÄUMER U: 1772

VOIDE N: T112, 3678

VOLK HD: 2642

VON HOLSTEIN S: 3755, 4647

VON LIVONIUS B: T111, 2658

VOTRUBA M: T069, 1767

VOTTONEN P: 1676

VUJOSEVIC S: 4442

WADT K: 4441

WAHLBERG RAMSAY M: F060

WALCZAK A: T076

WALLACE G: $\mathbf{3 7 5 3}$

WALRAEDT S: T081

WANG N: 4747 
284 ALL AUTHOR INDEX

WANG X: 3214

WANG Y: F028, 4747

WASYLUK J: T018

WATANABE J: S058

WEBER M: F066

WEGENER A: 1772

WEGLARZ B: S049

WEI B: 3666

WEI WB: F028

WEN L: S023

WERKMEISTER R: S052, 1612

WESCHLER B: T053

WEYMOUTH A: 2441

WIEDEMANN P: S026, S027, S028

WIERZBOWSKA J: T018

WIJDH RJH: 4733

WILLEKENS K: 4676, 3473

WILLERMAIN F: F110, T056, 1741, 4748

WILLOUGHBY C: $\mathbf{3 7 5 2}$

WILSKA R: 4242

WILSON S: 2771

WILSON SL: 1723

WIRTH M: S052

WIRYASAPUTRA S: $\mathbf{F 0 6 8}$

WITTRUP-JENSEN K: 4262

WOLFF B: 2211, 2213, 2215

WOLZT M: F004

WONG HT: T046

WONG T: T047, 3425, 3471

WONG W: 1752

WONG TEN YUEN A: T097

WORMSTONE IM: 1773

WOSTIYN P: 4224

WU YK: T092, 2655

WYCISÅ『O-GAWRON P: T002

WYLEGALA E: S017, S049, S073, S010, S065, T050, 1635, 3635, 1736, 4425

XIROU T: F020, F080, F092, S119, 4213, 4212, 4448

XU H: 2274, 2751

XU JIE: 4747

XU L: F028, 4747

XU Q: 5083

XU W: S009, 3257

XU YP: T046

YAHIAOUI S: 2475

YAKOUB-AGHA I: S043

YAMADA M: S044

YAMAMOTO H: T020

YAMAMOTO S: S112, 2651

YAMANE S: T020

YAMASHITA H: F114

YAN X: 3253

YANG SW: $\$ 113$

YANG Y: 3446

YASUKAWA T: F058

YAVUZYIGITOGLU S: 4246

YAZDANI N: S021

YEKTA AA: S021, T001, F029, T007

YEKTA R: S021, T001

YEOM M: F049

YEUNG A: 2234

YIM H: S046

YIN Y: 4445
YIP L: T024, T025

YIP WLL: T046

YONG KYV: T046

YONG SO: F068

YOO RM: T027

YOO YS: F102

YOSHIMURA N: F007

YOU J: $\mathbf{S 0 2 3}$

YOU Q: F028, 4747

YOUNIS S: F074

YOUSUF MA: 2644

YU S: T101

YUZ: S080, S079, 1775

YUBERO R: T108, 3675

YUGAY MARIA: S091, 1776

YUKIHIKO TAKAHASHI W: F019

YUM HR: T008, T113

YUNG Y: F086

YU-WAI-MAN P: 1651, 2261

ZABADANI K: F031, F063, F036, F038, S033, S107, T098, T099

ZACHARIAE C: S062, 4456

ZAGORSKI Z: S059

ZAGUIA F: 4635

ZAHN G: 1733

ZAJICOVA A: 5038

ZAKARIA N: $\mathbf{4 7 6 7}$

ZALESKA-ZMIJEWSKA A: T018

ZALIUNIENE D: T102

ZAMBARAKJI H: 4215, 4475

ZANGERL B: 3264

ZANON-MORENO V: F050

ZAOUALI S: 3655

ZAWADA M: 4735

ZEITZC: 3764

ZERBIB J: 2413, 2415

ZETTERBERG M: 3454

ZHANG FF: F061

ZHANG S: 3212

ZHAOC: F078

ZHAO G: S083, S084

ZHAO L: 3666

ZHOU T: 3613

ZHOU X: 2644

ZINCZUK M: F090

ZMUDA M: S118, 4449

ZOGRAFOS L: 2741, 2744, 3642, 3741

ZOZOLOU M: F080, F092 


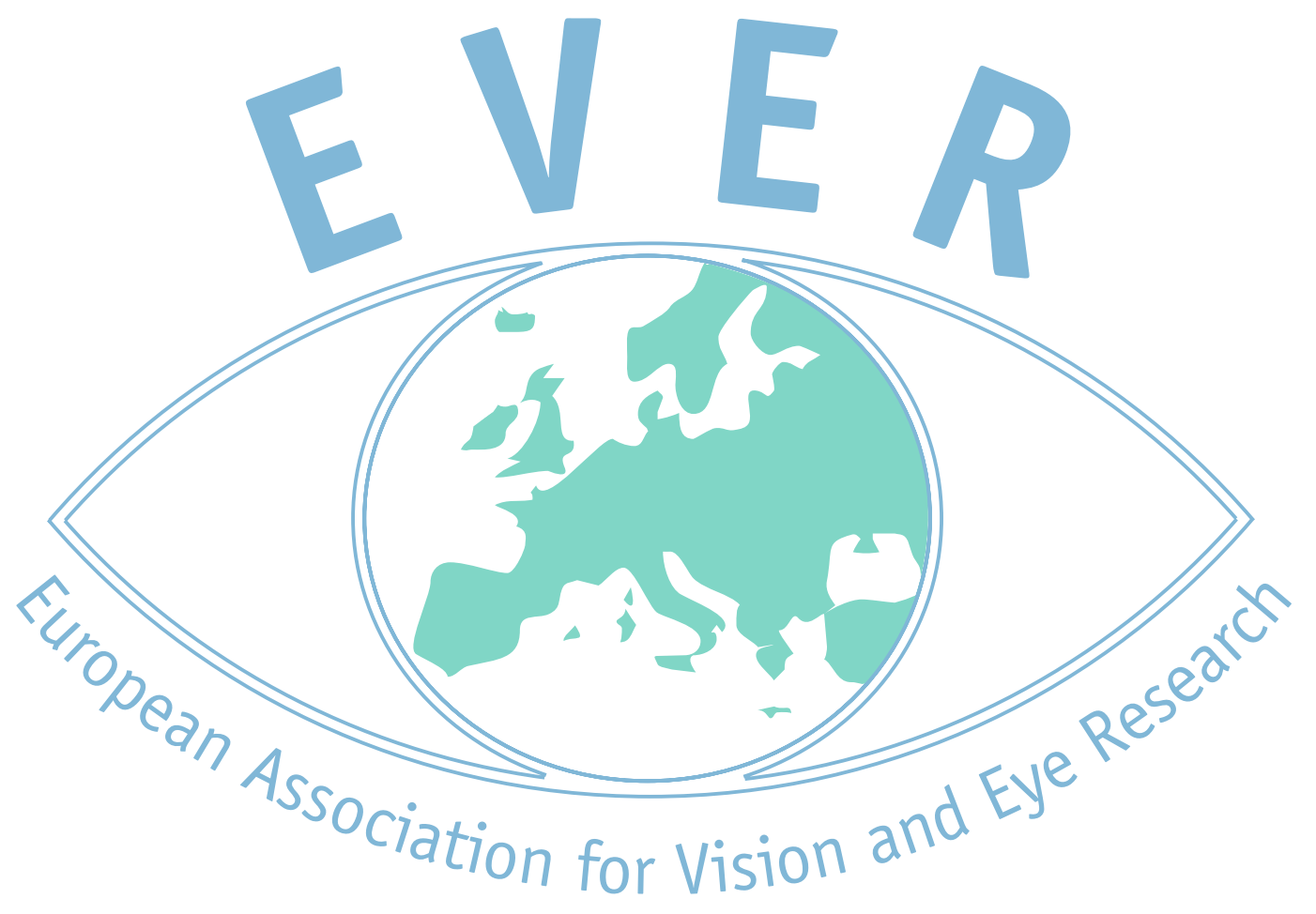

